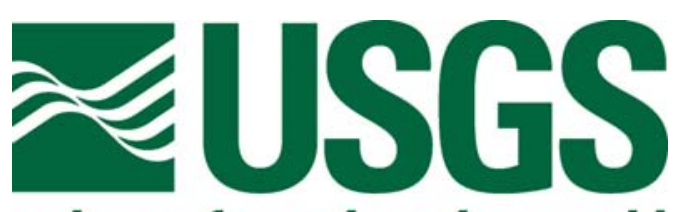

science for a changing world

\title{
Methodology and results for the assessment of oil and gas resources, National Petroleum Reserve, Alaska
}

by John H. Schuenemeyer ${ }^{1}$

Open-File Report 03-0118

2003

This report is preliminary and has not been reviewed for conformity with U.S. Geological Survey editorial standards or with the North American Stratigraphic Code. Any use of trade, firm, or product names is for descriptive purposes only and does not imply endorsement by the U.S. Government.

U.S. DEPARTMENT OF THE INTERIOR

U.S. GEOLOGICAL SURVEY

${ }^{1}$ Southwest Statistical Consulting, LLC, 960 Sligo St., Cortez, CO 81321 


\section{TABLE OF CONTENTS}

Introduction

Specification of the Input

Oil parameters

Gas parameters

Correlated attributes

Risking

Minimum size accumulations

The Play Simulation

Aggregation Methodology

Overview

Specifying the dependency

Generating a correlated sample

Results

References

Appendix A. Description of computer code

Appendix B. Glossary of assessment terms

\section{FIGURES}

1. Relationship of $F V F_{o}$, API gravity, and trap depth.

2. Relationship between $F V F_{g}$ and trap depth.

3. The play methodology.

\section{TABLES}

1, a - e. Assessment form details and instructions.

2. Equations used to compute co-products.

3. List of plays and number of Monte Carlo runs.

4. Assessor specified dependencies between plays.

5. Arithmetic averages of pairwise dependencies.

6. Adjusted correlation matrix.

7, a - f. Brookian Topset Play forms and resource estimates.

8, a - f. Brookian Clinoform North Play forms and resource estimates.

9, a - f. Brookian Clinoform Central Play forms and resource estimates.

10, a - f. Brookian Clinoform South-Shallow Play forms and resource estimates.

11, a - e. Brookian Clinoform South-Deep Play forms and resource estimates. 
12, a - f. Beaufortian Cretaceous Topset North Play forms and resource estimates.

13, a - e. Beaufortian Cretaceous Topset South Play forms and resource estimates.

14, a - e Beaufortian Upper Jurassic Topset Northeast Play forms and resource estimates.

15, a - f. Beaufortian Upper Jurassic Topset Southeast Play forms and resource estimates.

16, a - e. Beaufortian Upper Jurassic Topset Northwest Play forms and resource estimates.

17, a - f. Beaufortian Upper Jurassic Topset Southwest Play forms and resource estimates.

18, a - f. Beaufortian Lower Jurassic Topset Play forms and resource estimates.

19, a - f. Beaufortian Clinoform Play forms and resource estimates.

20, a - f. Brookian Topset Structural Play forms and resource estimates.

21, a - f. Torok Structural Play forms and resource estimates.

22, a - f. Ellesmerian Structural Play forms and resource estimates.

23, a - f. Thrust Belt Play forms and resource estimates.

24, a - f. Ellesmerian Ivishak Play forms and resource estimates.

25, a - f. Ellesmerian Echooka North Play forms and resource estimates.

26, a - f. Ellesmerian Echooka South Play forms and resource estimates.

27, a - f. Ellesmerian Lisburne North Play forms and resource estimates.

28, a - f. Ellesmerian Lisburne South Play forms and resource estimates.

29, a - f. Ellesmerian Endicott North Play forms and resource estimates.

30, a - f. Ellesmerian Endicott South Play forms and resource estimates.

31. Means of NPRA in-place oil, nonassociated gas, and derivatives by play.

32. Means of NPRA technically recoverable oil, nonassociated gas, and derivatives by play.

33. Aggregate summary of resource estimates for the NPRA. 


\begin{abstract}
Oil and gas resources in each of the 24 plays within the National Petroleum Reserve in Alaska (NPRA) were estimated using a play analysis. Assessors specified geologic attributes, risks, and number of prospects for each play. Some specifications established distributions, while others were given as single values. From this information, sizes of oil and gas accumulations were simulated using a Monte Carlo algorithm. The number of such accumulations considered in a given simulation run was obtained from the distribution of the number of prospects. Each prospect in each successful simulation run was risked. This process yielded size-frequency distributions and summary statistics for the various petroleum categories. Estimates of remaining resources from individual plays were then aggregated, and measures of uncertainty computed. Technically recoverable, undiscovered oil beneath the Federal part of NPRA likely ranges between 5.9 and 13.2 billion barrels, with a mean (expected) value of 9.3 billion barrels. Technically recoverable, undiscovered nonassociated natural gas for the same area likely ranges between 39.1 and 83.2 trillion cubic feet, with a mean (expected) value of 59.7 trillion cubic feet. Mean values of the corresponding associated dissolved gas and natural gas liquid are 10.3 trillion cubic feet and 1.4 billion barrels respectively.
\end{abstract}

\title{
INTRODUCTION
}

There are two commonly used methodologies for assessing undiscovered oil and gas in geologic plays. One is discovery process modeling, a statisticalgeological modeling procedure, which is used in more mature areas (Drew, Schuenemeyer, and Mast, 1995). A mature area is one that has a sufficient number of exploratory and discovery wells to ensure a degree of statistical regularity in an analysis. The other is subjective probability and risking, which is commonly used in frontier areas. Because there were relatively few discoveries in NPRA, the latter procedure was used in this assessment. In subjective probability assessments, experts specify input values for various parameters. In this assessment a Monte Carlo simulation algorithm was used to generate estimates of undiscovered resources.

The NPRA assessment was performed at the play level. For each play, assessors specified distributions needed to generate accumulations of oil and gas, and a distribution of the number of prospects expected to occur. They also specified risk factors. The methodology used in this assessment was a 
modified version of the methodology used in the U.S. Geological Survey's 1998 assessment of the 1002 Area of the Artic National Wildlife Refuge (ANWR) (Schuenemeyer, 1999). Improvements included modifications to the input form, fitting beta distributions to specified fractiles, and new petroleum engineering models. Minimum reservoir sizes for oil and for gas were established to facilitate estimation of the number of prospects. Approximate deposit size distributions were generated at the mean, median, and $5^{\text {th }}$ and $95^{\text {th }}$ levels of uncertainty. Play resources were allocated to Federal land based on estimated proportions of oil and gas resources.

Estimates of remaining resources from individual plays were aggregated into distributions of remaining resources for the total NPRA land area and for a subset, the Federal land area. The aggregation procedures also were modified from those used in the 1002-ANWR assessment.

This chapter begins with a discussion of the geologic and engineering input, which was specified by the assessors for each play and entered on the assessment form. Following this, the Monte Carlo simulation is presented. We conclude with a discussion of the aggregation procedure.

After presentation of the methodology, results will be summarized for each play and for the aggregate total of oil and nonassociated gas and various derivatives. Detailed discussion of the results is presented elsewhere on this Web site. Definition of terms is given in the Glossary.

\section{SPECIFICATION OF THE INPUT}

Information used by the assessment algorithm consisted of statistical models with parameters and assessor-specified distributions and constants. An assessment form, which was a modified version of that used in the 1998 1002-ANWR assessment, was used to capture the distributions and constants for each play. The input for oil, gas, and number of prospects and risk was specified on three Excel ${ }^{1}$ worksheets. The first worksheet (Table 1a) provided for the entry of hydrocarbon volume parameters for oil, the trap depth distribution, and oil accumulation characteristics. The second worksheet (Table $1 \mathrm{~b}$ ) provided for similar information for nonassociated gas. The third worksheet (Table 1c) was for the specification of the number

\footnotetext{
${ }^{1}$ Mention of a brand name is provided for clarification and does not constitute an endorsement by the U.S. Geological Survey or the author.
} 
of prospects, risking information, the proportional allocation of deposits between oil and gas, and the proportional land allocation. Many of the entries on the form are self-explanatory. Those that are not or those of special importance will be discussed here. Information provided by assessors in tables 1a, 1b, and 1c served as input to a series of Splus, v.6.0 (Insightful Corp., Seattle, WA) programs used to estimate resultant volumes of undiscovered resources. (Splus is a commercially available statistical computing package and programming language, which is functional in form and can be translated into other high level languages.) General instructions for completing these forms were provide to each assessor (Table 1d). In addition to specifying fractiles of a distribution, assessors were asked to specify its shape, which could range from highly right skewed to highly left skewed (Table 1e). This procedure served as a check on the specification of fractiles.

In order to avoid the considerable uncertainties associated with assessing a potentially large number of small prospects, which would be neither technically recoverable nor commercially viable in the foreseeable future, a minimum reservoir size or cutoff value was established for oil and gas. For oil, only deposits of at least $50 \mathrm{MMBO}$ in place were considered (Table 1c). For gas, only deposits of at least 250 BCF technically recoverable were considered. Because of differences in volumetric and expansion properties of oil and gas, separate minimum reservoir sizes were used. This issue is discussed in more detail in a subsequent subsection on minimum size accumulations.

\section{Oil Parameters}

Assessors specified distributions for the following oil hydrocarbon volume parameters (Table 1a):

net reservoir thickness, $N R T$, in feet, area of closure, $A C$, in thousands of acres, porosity, $P O R$, in percent, trap fill, $T F$ in percent, and trap depth, TD in thousands of feet.

Estimates of the lower truncation point (LTP), the $50^{\text {th }}$ (F50), and $5^{\text {th }}$ (F05) fractiles, and the maximum value were entered for $N R T, A C, P O R$, and $T F$.

The hydrocarbon pore volume (HPV) in percent was computed as 


$$
H P V=P O R-B V W
$$

where $B V W$, the bulk volume water, is defined as the product of $P O R$ and water saturation $\mathrm{S}_{\mathrm{w}}\left(B V W=P O R^{*} S_{w}\right)$. That is, $B V W$ is the fraction or percentage of rock volume, which is filled with water. (The analysis and discussion of $H P V$ is based upon written communication from Philip Nelson, U.S. Geological Survey.) The question we faced was, what fraction of the rock volume is filled with water (the $B V W$ ) and how much with hydrocarbon (called hydrocarbon pore volume or HPV)? These two values, given either as a percentage or as a fraction, must sum to the porosity, such that $B V W+H P V=P O R$. The porosity distribution is determined by the assessor from available core data, well logs, and analog fields. Because $B V W$ is the product of porosity and water saturation, the problem of determining $B V W$ reduces to the estimation of water saturation. In this assessment we adopted the same assumption used in the assessment of the Arctic National Wildlife Refuge (Nelson, 1999): that $B V W$ is constant in a given field. That assumption rests on the concept that $B V W$ is constant within a specified lithology above the water-hydrocarbon transition zone and implies that water saturation varies inversely with porosity. The oil and gas parameter assessment sheets (Table $1 \mathrm{a}$ and Table 1b, respectively) show the assignment of $B V W$ (called $P O R^{*} S w$ on the form) as the rightmost entry on the row labeled HYDROCARBON PORE VOLUME on the Oil and Gas worksheets. The value of $B V W$ is treated as a constant, which is then subtracted from the porosity distribution to yield the distribution of hydrocarbon pore volume. For example, in the northeast Beaufortian Upper Jurassic Topset Play, $B V W$ is 4 percent, the median porosity is 17 percent, and consequently the median hydrocarbon pore volume is 13 percent. $B V W$ was between 1 and 6 for the assessed NPRA plays.

Values of LTP were chosen so that the oil in place $(O I P)$ generated at these points would be approximately $50 \mathrm{MMBO}$. It was assumed that for $N R T$, $A C, P O R$, and $T F$ the population minimum was zero. (Note that LTP does not represent a specific fractile common to all attributes in a given play.) This information established guidelines concerning the values of NRT, TF, and the other parameters that "on average" constituted a $50 \mathrm{MMBO}$ in-place oil accumulation. Then assessors were given plots showing the shape of each histogram of the hydrocarbon volume parameters. Assessors modified their initial distributions, as necessary, so that the minimum accumulation size generated from these distributions would be within an order of magnitude of the cutoff. 
For TD and number of prospects (Table 1c) the LTP is the minimum value of the population distribution. The TD was adjusted before use in the model by adding average surface elevation (Table 1a).

The oil accumulation volume parameter distributions were intended to show the variation in characteristics of prospects across a play and not variation within a given prospect. The values chosen for the fractiles and other estimates were based upon field studies, geophysical and geochemical data, well logs, and analogy. Specific justifications are given in individual play description chapters.

Samples from these oil volume distributions and an estimate of the formation volume factor $\left(F V F_{o}\right)$ in reservoir barrels/stock tank barrels, rb/stb were combined to estimate OIP (in millions of barrels, MMBO), as:

$$
\mathrm{OP}=7.758 \times N R T \times A C \times H P V \times \mathrm{TF} \times 10^{-4} / F \mathrm{~F}_{o}
$$

An estimate of $F V F_{o}$, developed by Mahendra Verma, U.S. Geological Survey, (written communication and Reservoir Engineering Aspects chapter) is computed as follows. Let $p=1000 T D / 2$ and $t=19 T D+30$ where $p$ is pressure in pounds per square inch (psi) and $t$ is temperature in degrees $\mathrm{F}$. Then the solution specific gravity, sgg is

$$
s g g=((0.1402 \ln (p+14.7)-0.4227)+(0.1369 \ln (t)+0.0156)+(0.1704 \ln (a g)+0.1469)) / 3
$$

where $a g$ is API gravity as specified on the oil worksheet play form (Table 1a) and "In" in the above equation is log base e function. The associated gas to oil ratio GOR (cu. ft. per barrel at stp) is

$$
G_{u}=\operatorname{sgg}\left[\frac{(p+14.7) 10^{0.0125 a g}}{18 \times 10^{0.00091 t}}\right]^{1 / 0.83} .
$$

Then

$$
\begin{aligned}
o g & =141.5 /(\operatorname{ag}+131.5) \\
F & =G O R(\operatorname{sgg} / \mathrm{og})^{0.5}+1.25 t^{\prime}
\end{aligned}
$$


where og is oil gravity.

Finally

$$
F V F_{o}=0.972+0.000147 F^{1.175} \text {. }
$$

Oil FVF's are shown for the minimum, medium, and maximum API gravities in NPRA (Fig. ME1).

\section{Gas parameters}

The type of gas hydrocarbon volume attributes and characteristics, Table ME1b, which were used to compute the accumulation sizes of gas, are the same as for oil, namely NRT, AC, POR, TF, and TD. The fractile values specified for these attributes, however, sometimes differed from those for oil. A different algorithm was used to estimate the gas formation volume factor $F V F_{g}$.

The equation for the accumulation size of gas in place, GIP, (in billions of cubic feet, BCF) is:

$$
G I P=4.356 \times N R T \times A C \times H P V \times T F \times 10^{-6} \times F V F_{g}
$$

The $F V F_{g}$ was derived from a curve fit model based upon results derived by Mahendra Verma, U.S. Geological Survey, (written communication and Reservoir Engineering Aspects chapter) using the theory of Corresponding States:

$$
F V F_{g}=\frac{35.37415(p+14.7)}{(t+460) z}
$$

where $z$ is the gas compressibility factor. Because $z$ is nonlinear, we chose to fit the computed $F V F_{g}$ versus depth. The piecewise curve fitted model is:

$$
F V F_{g} \square\left\{\begin{array}{l}
752.2\left(1-\mathrm{e}^{-0.05728 T D}\right) \quad 0<T D \leq 5.67 \\
113.3+21.1 T D-0.812 T D^{2}+0.0116 T D^{3} \quad 5.67<T D \leq 30
\end{array}\right.
$$

where $T D$ is trap depth in thousands of feet. The form of the model is illustrated in Figure 2. 


\section{Correlated attributes}

The formulas for oil and gas accumulations OIP and GIP given in the previous section imply pairwise independence between all of the attributes. In some instances assessors chose to specify negative correlations between $N R T$ and $A C$ and between $N R T$ and $H P V$. To incorporate these correlation structures into the accumulation formulas, we used the following procedure:

1. Let $\mathrm{R}$ be a matrix whose elements are the assessor specified pairwise correlations. We assume $\mathrm{R}$ to be a $3 \times 3$ matrix. Also, let ns $=10,000$, where ns is the number of simulation runs.

2. We then see if $\mathrm{R}$ is permissible. A permissible matrix is one whose determinant is greater than or equal to zero. All assessor defined correlations resulted in permissible matrices. (See additional discussion in the section on Aggregation Methodology.)

3. If $\mathrm{R}$ is permissible, let $\mathrm{Ch}=\mathrm{Cholesky}(\mathrm{R})$, be the Cholesky factorization of $\mathrm{R}$.

4. Let Ua be a ns $x 3$ matrix, where each row of $U a$ is an independent set of uniform random numbers between -1 and 1 .

5. Let $\mathrm{Uac}=\mathrm{Ua} \times \mathrm{R}$.

6. Let $\operatorname{Ur}[, \mathrm{i}]=\operatorname{Rank}(\mathrm{Uac}[, \mathrm{i}]), \mathrm{i}=1,2,3$

7. The ranked values in the columns of Ur then represent the sample numbers of the accumulations (with accumulations sorted in ascending order) necessary to achieve the desired correlation.

\section{Risking}

Risk in the context of this study is the probability that a play or prospect would be unsuccessful because of the failure of one or more geologic attributes necessary to achieve success. Because it is natural to think of the likelihood of an attribute being present, we used the complement of risk, namely favorability. Thus, a favorability of one implies zero risk.

There are two favorability structures. One is play; the other is prospect. Prospect favorability was sometimes further subdivided into oil and gas. Each of these is the product of three attributes, however, play favorability refers to the product of attributes needed for a successful play, whereas, prospect probability refers to the product of those attributes associated with a randomly chosen prospect. The attributes that constitute these structures are 
charge, trap, and timing formation (Table 1c). Although the names of the attributes are the same at the play and prospect levels, there are six distinct attributes (or nine if separate oil and gas prospect favorability probabilities are specified). They are assumed to be pairwise independent of each other. Prospect attributes were assessed conditional upon the play being successful.

A successful play is one in which all three of the play level attributes necessary for a prospect of at least $50 \mathrm{MMBO}$ in-place oil or $250 \mathrm{BCF}$ of technically recoverable nonassociated gas are present. However, there is no guarantee that such a prospect will be found in a "successful play". A failure to find at least one deposit in a "successful play" can occur when few prospects are specified and/or the prospect favorability is low. Given a successful play, the number of prospects was drawn at random from the distribution of prospects specified in Table ME1c. The prospect favorability was then applied to each of these prospects. The mechanism to do this was to generate a $[0,1]$ continuous uniform pseudo-random number for each prospect selected. When the value of the random number did not exceed the prospect favorability, we accepted the prospect and relabeled it a deposit. Thus, the deposits generated in such a manner reflect an unconditional distribution, the risks associated with play and prospect having been applied. Assessment definitions were established and made available to the assessors to provide specific guidelines to allow them to differentiate between these two risks.

\section{Minimum size accumulations}

For oil, only prospects of at least $50 \mathrm{MMBO}$ in place (at the surface) were considered. For gas, only prospects of at least 250 BCF technically recoverable (at the surface) were considered. There are several reasons for the choice of a cutoff value. Resources in most small fields will not be technically recoverable and/or economic in the foreseeable future. Although the amount of the resource contribution of very small fields is dependent upon the population distribution of oil and/or gas fields, it is unlikely that they will contribute substantially to the NPRA resource base in the foreseeable future. In addition, there are technical reasons for specifying a cutoff. It is difficult to estimate the potentially large number of small fields with any degree of accuracy due to the coarse seismic grid (approximately 3 mi. x 6 mi.) in NPRA and lack of significant exploratory drilling. We did allow, however, for fields, which are now marginally economic to be considered if the price/cost ratio becomes more favorable. 
The reason for the choice of $50 \mathrm{MMBO}$ for oil was two fold. First, members of the assessment team believe that the overwhelming majority of fields containing less that $50 \mathrm{MMBO}$ in place, were not likely to technically recoverable and/or of economic interest in the foreseeable future. Second, a choice of a 50 MMBO cutoff was consistent with that used in the ANWR assessment Schuenemeyer (1999). The LTP's for the oil hydrocarbon attributes where chosen in part to yield an in-place accumulation of approximately $50 \mathrm{MMBO}$. For nonassociated gas, we diverted slightly from the procedure used in the ANWR assessment. In this assessment, a choice was made of a 250 BCF technically recoverable cutoff. Note that 300 BCF in-place nonassociated gas at the surface is the approximate energy equivalent of $50 \mathrm{MMBO}$, however, because of the expansive properties of gas, the equivalent oil to gas container size might not be appropriate. We saw previously that $F V F_{g}$ varies widely, say from 42 for a depth of 1,000 feet to 329 for a depth of 30,000 ft. (See M. Verma, Reservoir Engineering Aspects chapter for specific details on gas attributes.) Thus, the 250 BCF cutoff value was chosen as a guide to allow specification of geologically reasonable containers in such a manner most consistent with the knowledge base of the assessors. However, nonassociated gas accumulations resulting from the specification of the LTP's varied more widely from the cutoff value than those for oil.

This distribution (Table 1c) was specified in the same manner as the distributions for trap depth and hydrocarbon volume. As with trap depth, the LTP is an estimate of the minimum value of the population. From this distribution, the number of oil prospects equal to or greater than $50 \mathrm{MMBO}$ in place (and/or the number of nonassociated gas prospects equal to or greater than 250 BCF recoverable) was determined given a favorable play. If the probability of a favorable prospect is less than one, then the expected number of deposits will be less than the expected number of prospects.

\section{THE PLAY SIMULATION}

The methodology was based upon a Monte Carlo simulation. A series of functions, written in Splus6.0 and listed in appendix MEA, were used to implement the simulation. Results were stored in Excel spreadsheets. For each play, 10,000 simulations were run, conditioned on the play being favorable. For example, if the play probability was 0.80 , as in the Ellesmerian Structural Play (Table 22), then the expected total number of 
runs would be 10,000 divided by 0.80 or 12,500 . However, since the expected number of unsuccessful runs was 2,500 , we chose to run only the 10,000 potentially successful runs. The reason for choosing to run 10,000 simulations conditioned on a successful play was to obtain similar levels of precision on the summary statistics for all plays, even those that were highly risked. The uniform random number generator used for the simulations is an Splus.0 function called runif.

Input from tables 1a, 1b, and 1c was transferred to an Excel play form worksheet (not shown) and taken in to Splus6.0 using functions OGIn.fn and OGdata.fn.

All sampling occurred from the standard two-parameter beta distribution shown below

$$
f(x ; u, v)=B(u, v) x^{u}(1-x)^{v} \quad 0 \leq x \leq 1
$$

or a modified beta distributions, which were fit to the specified fractiles (function OGpare.fn). The method of fit was the Splus nonlinear estimation function nlmin, which uses a general quasi-Newton optimizer. The function being minimized (fmin) was

$$
(q \operatorname{beta}(0.50, p[1], p[2])-c o[1])^{2}+(q \operatorname{beta}(0.95, p[1], p[2])-c o[2])^{2}
$$

where qbeta is the Splus beta quantile function, $p[1]$ and $p$ [2] are initial estimates of the beta distribution parameters established from the user specified distribution shape (Table 1e), and $c$ [1] and $c$ [2] are the standardized (to 0 to 1 ) values of the 0.50 (F50 fractile) and 0.95 (F05 fractile) percentiles specified by the assessors. The result is the estimated parameters of the beta distribution. The use of a modified beta distribution only occurred in a few instances for the distribution of trap fill where an assessor specified a) an inverted j-shaped distribution with 0.50 probability occurring at the maximum value (Table 1e, shape 7), or b) an inverted jshaped distribution with 0.05 probability occurring at the maximum value Table 1e, shape 8). In case a) the fitted beta density function was

$$
f(x ; 5,1)=\left\{\begin{array}{l}
0.5 \operatorname{beta}(x ; 5,1), \quad 0 \leq x<1 \\
0.5 \quad x=1
\end{array}\right.
$$

In case $b$ the fitted beta distribution was 


$$
f(x ; 1, v)=\left\{\begin{array}{l}
0.95 \operatorname{beta}(x ; 1, v), \quad 0 \leq x<1 \\
0.05 \quad x=1
\end{array}\right.
$$

where $v=\ln (0.5) / \ln \left(1-x_{0.95}\right)$. Assessors were given the option of choosing other distributions if they did not feel these provided an adequate fit, however, none did.

Figure 3 is a flow chart for the simulation algorithm (function OGRa.fn). It begins with the big simulation loop, which was executed 10,000 times for each play; recall that 10,000 is the expected number of potentially favorable plays. Next, a sample was taken from the number of prospects distribution. This is a distribution conditioned on the volume of oil being at least 50 MMBO in-place or nonassociated gas being 250 BCF technically recoverable. Each prospect was randomly classified as oil or gas according to fraction oil specified by the assessor (Table 1c). The oil or gas prospect probability was then applied (Table 1c). For a successful prospect, now relabeled a deposit, the appropriate hydrocarbon volume parameters and depth were sampled and an in-place oil or technically recoverable nonassociated gas accumulation greater than the minimum reservoir size was computed. Associated-dissolved gas and natural gas liquids (NGL) from associated-dissolved gas and from nonassociated gas were also computed (Table 2). Technically recoverable quantities of oil were computed by multiplying the in-place volumes by the oil recovery factor (Tables 1a). Inplace quantities of gas were computed by dividing the technically recoverable volumes by the gas recovery factor (Table 1b). Detailed information about the deposits for each play run was summarized (function OGDepSum.fn). After 10,000 simulations, summary statistics for the play were computed (function OGPS.fn) and are presented in the Results Excel spreadsheet. In addition, size-frequency distributions for in-place and recoverable oil and nonassociated gas were computed (functions OGshist.fn and OGsfreq.fn) and are presented in a Size-Freq Excel spreadsheet.

\section{AGGREGATION METHODOLOGY}

\section{Overview}

Resource estimates from individual plays were aggregated to total Federal land and total land in NPRA. An aggregate distribution was constructed by 
sampling from the individual plays in a manner so as to estimate assessor specified dependencies between plays. Such dependencies may result from shared sources of charge, trap or timing. The basic concern in aggregating results is the effect that dependency has upon the spread of the aggregate distribution and thus on estimates of uncertainty. Failure to account for positive dependency would have resulted in estimates of uncertainty that were too narrow and thus would have created a higher level of confidence in results than would be warranted if the correct measure of dependency were used. Dependency does not affect the mean of the aggregate distribution, only the spread. The mean of the aggregate is simply the sum of the means of the plays to be aggregated.

The basic procedure used was to create a correlation matrix from assessorspecified dependencies, generate observations that have the specified correlation structure, rank the correlations, and then choose the samples to form an aggregate distribution. The 24 plays assessed in this NPRA assessment area are listed in Table ME3 and the corresponding expected number of total runs (including plays expected to be unsuccessful).

\section{Specifying the Dependency}

Assessors considered all possible pairs of the 24 plays being assessed. For each pair they assigned one of three values (low, medium or high) to the attributes of charge, trap, and timing (Tables 4). A high (positive) value assigned to charge between, say plays 1 and 2 might indicate a common mechanism charged both plays. Thus if the value of charge in play 1 was found to be high, the values of charge in play 2 would most likely be high. The three dependencies (charge, trap, and timing) were converted to correlations by assigning values of $0.1,0.5$, and 0.9 respectively to low, medium, and high entries. A single correlation matrix (Table 5) was then formed by taking the arithmetic average of the three correlation matrices. Negative dependencies were allowed, however, none were specified by the assessors.

There is a potential inconsistency associated with specifying correlations by pairs of plays, namely, some correlations impose restrictions on others. For example, suppose the correlation between plays A and B is 0.367 and that between plays $\mathrm{A}$ and $\mathrm{C}$ is 0.500 . Then range of correlation between plays $\mathrm{B}$ and $C$ is restricted in that not all values between -1 and +1 are permissible. In order to see if the $24 \times 24$ computed correlation matrix (Table 5) was 
permissible, a statistical procedure called eigenvalue analysis was performed. The minimum eigenvalue of a permissible correlation matrix would be equal to or greater than zero. The minimum eigenvalue of this matrix (Table 5) is -0.211 . Thus, a slight biasing factor, 0.212 , was applied to each of the 24 eigenvalues (function OGcorr.fn). Then the correlation matrix was reconstructed. The resultant correlation matrix, which was used for the remaining part of the analysis, is given in Table 6 . This procedure is similar to that used in the 1998 ANWR assessment (Schuenemeyer, J.H., 1999b). It is also virtually identical to the dependency scheme used in the USGS 1996 National Assessment (Gautier and other, 1995).

\section{Generating a Correlated Sample}

The adjusted correlation matrix (Table 6) was then used to induce the appropriate correlation structure in the data. A justification for this procedure was given previously. The algorithm is essential the same as that used to generate correlated samples of hydrocarbon volume attributes. The procedure is outlined below.

1. Let $\mathrm{R}$ be the $24 \times 24$ adjusted (permissible) correlation matrix of play dependencies. Also, let ns $=10,000$, where ns is the number of simulation runs.

2. Perform a Cholesky factorization on $\mathrm{R}$ to obtain a lower triangular matrix plus the diagonal matrix, call this $\mathrm{A}$, such that $\mathrm{AA}^{\prime}=\mathrm{R}$ (where $\mathrm{A}^{\prime}$ is the transpose of $\mathrm{A}$ ).

3. Let Ua be an ns $x 24$ matrix, where each row of $U a$ is an independent set of continuous uniform random numbers between -1 and 1 .

4. Let $U a c=U a x$.

5. Let $U r[, i]=\operatorname{Rank}(\operatorname{Uac}[, \mathrm{i}]), \mathrm{i}=1, \ldots, 24$.

6. Adjust the ranked matrix Ur by the total number of plays run (10,000/play probability).

7. The ranked values in the columns of Ur then represent the sample numbers of the play (with play recoverable barrels of oil equivalent sorted in ascending order) necessary to achieve the desired correlation.

Each element in the matrix Ur became a sample number. As previously discussed, only the expected number of successful plays was generated; however, sample numbers from unsuccessful plays were needed to generate samples for the aggregate distributions. For example, as previously noted, 
Ellesmerian Structural Play has 12,500 total expect number of runs, however, only 10,000 simulation runs were make. In sampling for aggregation, we take a simple random sample of 10,000 from a population of 12,500 runs, 2,500 of which were a priori unsuccessful. For those plays that consisted of 10,000 runs (i.e., the play probability was 1.0), such as the Brookian Topset Play, this procedure generated a permutation of the original data that imparted the appropriate correlation structure. A rank correlation structure was used because the oil and gas distributions differ widely among the 24 plays. The standard (Pearson) correlation coefficient is only meaningful when distributions are similar and in particular when they are symmetric. The sample numbers were generated by function OGcorr.fn.

The actual process of aggregation, performed by algorithm OGagg.fn, was straightforward. Samples were selected by row from matrix Ur and the corresponding values of oil or gas were obtained from the appropriate play and/or prospect file. There were 10,000 simulation runs in each of the play/prospect files. The unsuccessful runs resulting from a favorable play probability less than one were assumed to follow the actual 10,000 runs generated from the simulation for purposes of sampling. The sums at the desired levels of aggregation were written to a file. The aggregation procedure was repeated 10 times with different random samples (algorithm OGcorr.fn) from the play dependency correlation matrix to minimize the sensitivity of results to a particular sample. Ten aggregation runs were chosen because, for example, the mean of F05 for in-place oil was 54752 and the corresponding standard error was 84 , which is well within the precision limits for the assessment. The 10 aggregation runs were merged (Splus6.0 function rbind) resulting a total of 100,000 plays.

The estimates of uncertainty at the aggregate level were performed by function OGsummary.fn from the merged results. Summary results included the mean, standard deviation, and F95, F50, and F05 fractiles for in-place and recoverable oil and nonassociated gas for Federal and total lands.

For purposes of economic analysis it was desired to obtain and estimate of the field size distributions at each of the fractiles. To accomplish this and reduce sensitivity to the choice of a single fractile, the actual observation at the F95, F50, and F05 fractiles plus 10 observations on either side of this value were extracted and provide to Emil Attanasi, (Economics of Undiscovered Oil in Federal Lands in the NPRA chapter) for economic analysis. 


\section{RESULTS}

An overview of result is given by Bird (Overview, chapter) and Bird and Houseknech (2002). Detailed discussions of play results are incorporated in chapters by individual assessors. Table R1 references detailed play results and aggregate summaries.

Individual play results are presented in tables 7 through 30 . As an illustration we discuss the Brookian Topset Play results (table 7). This form contains the following worksheets:

- Oil (Table 7a)

- Gas (Table 7b)

- Play-prospect (Table 7c)

- Distn_O (Table 7d)

- Distn_G (Table 7d)

- Results (Table 7e)

- Size_Freq (Table 7f)

The first three of these worksheets (oil, gas, and play-prospect) are for the specification of input values and have been discussed in the methodology section. The next two (distn_O and distn_G) show the distribution of the fitted oil and gas hydrocarbon volume attributes, plus, trap depth and number of prospects. Sometimes these results are presented in a single sheet called distn. The results sheet shows density curves for unrisked oil and/or gas accumulation distributions and risked recoverable oil and/or gas for the play. The numerical results (table 7e, Results worksheet) show means, standard deviations, F95, F50, and F05 fractiles for in-place oil and nonassociated gas, and recoverable oil and gas and derivatives. The size frequency (table 7f, Size_freq worksheet) shows the distribution of number of deposits by size class and the distribution of recoverable oil and/or gas by size class.

Tables 31 and 32 show means by play for in-place and technically recoverable resources respectively. Means are presented for total and Federal land resources, comprising oil, nonassociated gas, associated dissolved gas (ADG) and natural gas liquids from ADG (NGL(adg)) and from nonassociated gas (NGL(nag)). 
Table 33 presents means, F95, F50, and F05 fractiles for the aggregate summary of all plays for in-place and technically recoverable oil and nonassociated gas in the total assessed area and in Federal lands. Means of the aggregated totals are given for NGL and ADG.

\section{REFERENCES}

Bird, K.J., and Houseknecht, D.W., 2002, U.S. Geological Survey 2002 petroleum resource assessment of the National Petroleum Reserve in Alaska (NPRA): U.S. Geological Survey Fact Sheet 045-02.

Drew, L.J., Schuenemeyer, J.H., and Mast, R.F., 1995, Application of the modified Arps-Roberts discovery process model to the 1995 U.S. National Oil and Gas Assessment: Nonrenewable Resources, v. 4, p. 242-252.

Gautier, D.L, Dolton, G.L., Takahashi, L.I., and Varnes, K.L., eds., 1995, 1995 National Assessment of the United States Oil and Gas Resources-Results, Methodology, and Supporting Data, U.S. Geological Survey Digital Data Series DDS-30.

Nelson, P.H., 1999, Petrophysical Properties, Chapter PP in The oil and gas resource potential of the 1002 Area, Arctic National Wildlife Refuge, Alaska, by ANWR Assessment Team, U. S. Geological Survey Open File Report 98-34.

Schuenemeyer, J.H., 1999a, Assessment results, in the oil and gas resource potential of the 1002 Area, Arctic National Wildlife Refuge, Alaska, U.S. Geological Survey Open File Report 98-34, CDROM.

Schuenemeyer, J.H., 1999b, Methodology, in the oil and gas resource potential of the 1002 Area, Arctic National Wildlife Refuge, Alaska, U.S. Geological Survey Open File Report 98-34, CDROM. 


\section{Appendix A. Brief description of Splus functions used in the NPRA resource assessment}

The programs are listed in the order they are to be used. Code for each program is available as text files elsewhere on this web site.

OGIn.fn

Calls OGdata.fn

Input:

play names file (AllocPR)

Output:

Basic Splus data files, xxdata, where $\mathrm{xx}$ is play name prefix

OGdata.fn

Gets data from Excel playwork worksheets

Input:

Excel play file name (from AllocPR)

Sheet sequence number of play worksheet (from AllocPR)

OGpare.fn

Fits beta distributions to oil/gas play attributes and generates

distribution plots. This function calls fmin the minimization function. Input:

Play name \& xxdata

Output: xxpar, file containing parameter estimates and other basic data and plots (see Distn worksheet in Excel play form)

OGRa.fn

Generates fully risked oil/gas deposits; each play is 10,000 runs.

Input is xxpar \& a random number seed

Output: xxres (this is the basic detailed output file)

soila.fn (function called by OGRa.fn)

Contains the formula to generate oil accumulations

Input is number of oil accumulations to be generated, number

of prospects, parameter file xxpar, sample numbers, and

correlation between attributes

Output is accumulation sizes 
sgasa.fn (function called by OGRa.fn-similar to soila.fn except generates gas accumulations.

\section{OGDepSum.fn}

Generates play file and deposit summary stats and plots Input: play name, xxres

Output: xxplay; one record for each successful simulation run

OGPS.fn

Summary stats (mean, std dev, quantiles) for each oil/gas commodity Input: play name, xxplay, xxdata Output: xxpss (summary stats; goes to Result sheet in Excel play form)

OGsfhist.fn

Creates summary information for oil/gas size-frequency bar plots Input: xxres, xxdata, og (og=1 for oil \& $=2$ for gas)

Output: $x x y c n t$, where $y=0$ for oil and $g$ for gas

OGsfreq.fn

Generates size-frequency bar plots for oil and gas

Input: play name, xxycnt, og

Output: plots (see Size_Freq worksheet in Excel play form)

OGcorr.fn

Checks user specified $24 \times 24$ correlation matrix to see if it is permissible and adjusts correlations if necessary Input: user specified correlation matrix, AllocPR, random number seed

Output: Adjusted correlation matrix (CodepAdjB) or sample number for aggregating plays

OGagg.fn

Aggregates play results

Input: Sample number file, AllocPR, tf ( $=1$ for total land, $=2$ for Federal land)

Output: file containing aggregate results for oil/gas commodities and corresponding sample numbers

OGsummary.fn 
Summarizes aggregate file; means, quantiles

Input: aggregate file

Output: summary file (to aggregation results Excel worksheet)

\section{Appendix B. Definitions for NPRA Assessment Form}

The following definitions were updated by J.H. Schuenemeyer, as appropriate, from those by R. Charpentier, Definitions in The Oil and Gas Resource Potential of the 1002 Area, Arctic National Wildlife Refuge, Alaska, by ANWR Assessment Team, U.S. Geological Survey Open-File Report 98-34.

Hydrocarbon Volume Attributes: Distributions were specified for five attributes (listed below) used to calculate the volumes of oil and gas accumulations in the simulation program and in economic scenarios. Because the simulation program calculates an accumulation size using one randomly sampled number from each fitted distribution, spread in the distributions reflects variability between accumulations. Even though some attributes (net reservoir thickness, porosity, and trap fill) could show variation within an individual accumulation that source of variability is beyond the level of detail used in this analysis. The distribution of porosity, for example, shows how the average porosity in an accumulation may vary from one accumulation to another. A sampled value of porosity should be viewed as the mean value in a given accumulation. All of the hydrocarbon volume attributes are conditional distributions -- conditional on both the play being favorable and the prospect being favorable. In other words, the uncertainty expressed in the specification of the hydrocarbon volume attributes is not intended to reflect the chance that such an attribute will be present. This is addressed by the risking. Rather, the spread in the distributions, reflects geologic uncertainty and lack of knowledge.

Net Reservoir Thickness: A distribution for net reservoir thickness (in feet) in accumulations. The distribution shows how the average net reservoir thickness may change from accumulation to accumulation. It is not the same as the prospect height because it only includes net thickness of reservoirquality rocks. It is also not the same as net pay thickness, because only some proportion of the reservoir rock contains hydrocarbons. (See trap fill.) 
Area of Closure: A distribution for area of trap closure (in thousands of acres) of accumulations.

Porosity: A distribution for average porosity (in percent) in accumulations. The distribution shows how the average porosity may change from accumulation to accumulation.

Trap Fill: A distribution for trap fill (in percent) in accumulations. It is the volumetric percent of the gross reservoir volume (area of closure times net thickness) containing hydrocarbons.

Trap Depth: A distribution for trap depth (in thousands of feet sub sea level) in accumulations. The distribution shows how the average trap depth changes from accumulation to accumulation. A correction factor for the average ground elevation in the play area is added to Trap Depth to facilitate calculations of depth-related engineering parameters such as reservoir temperature and pressure.

\section{Risking:}

Minimum Reservoir Size Oil: The smallest accumulation size being assessed, in this case 50 million barrels (MMBO) in-place at surface conditions. Smaller accumulations may exist in the play but are not being assessed.

Minimum Reservoir Size Nonassociated Gas: The smallest accumulation size being assessed, in this case 250 billion cubic feet (BCF) technically recoverable at surface conditions. Smaller accumulations may exist in the play but are not being assessed.

Number of Prospects: A distribution showing uncertainty in the number of drillable prospects for accumulations of the minimum size or larger. This distribution is conditional on the play being favorable.

Play Attributes: Three probabilities -- Charge (C), Trap (T), and Timing (F) -- that are used in calculating the Play Probability. 
Charge (C): The probability that there has been sufficient source rock, thermal history, and migration to allow for at least one accumulation of minimum size or larger somewhere within the play.

Trap (T): The probability of the occurrence of rocks containing suitable reservoir characteristics, sealing characteristics, and trap geometry capable of containing at least one accumulation of minimum size or larger somewhere within the play.

Timing (F): The probability that the timing of trap formation relative to hydrocarbon generation/migration was favorable for an accumulation of minimum size or larger somewhere within the play.

Play Probability: The probability that the play is favorable, i.e., that the play attributes are adequate to allow at least one accumulation of minimum size or larger. It is calculated as the product of the three play attributes -- Charge (C), Trap (T), and Timing (F) -- which are assumed to be pairwise independent. Favorability of the product of the three play attributes is necessary, but not sufficient, for the existence of an accumulation of minimum size or larger. With a small number of prospects, there is some probability that the play attributes are favorable, but just not present in any one prospect.

Play Risk: The probability that the play is unfavorable, i.e., that the play attributes are not sufficiently favorable to allow any accumulations of minimum size or larger. It is calculated as 1 minus the Play Probability.

Prospect Attributes: Three probabilities -- Charge (c), Trap (t), and Timing (f) -- that are used in calculating the Prospect Probability. All of them are conditional probabilities -- conditional on the play being favorable. Probabilities are expressed relative to a randomly chosen prospect. This can also be thought of as giving the proportion of prospects for which a particular condition is favorable. These differ from the play attributes. For example, one may be certain that there has been sufficient source rock, thermal history, and migration to allow at least one accumulation of minimum size or larger somewhere within the play $(\mathrm{C}=1.0)$ but estimate that only $50 \%$ of the prospects have had adequate migration paths open (c = $0.5)$. 
Charge (c): The probability (given that the play is favorable) that a randomly chosen prospect has been charged by fluids sufficient for an accumulation of minimum size or larger.

Trap (t): The probability (given that the play is favorable) that a randomly chosen prospect has suitable reservoir characteristics, sealing characteristics, and trap geometry capable of containing an accumulation of minimum size or larger.

Timing (f): The probability (given that the play is favorable) that a randomly chosen prospect has timing of trap formation relative to hydrocarbon generation/migration favorable for an accumulation of minimum size or larger.

Prospect Probability: The probability that a randomly chosen prospect is favorable (given that the play is favorable), i.e., that the prospect contains an accumulation of minimum size or larger. It is calculated as the product of the three prospect attributes -- Charge (c), Trap (t), and Timing (f) -- which are assumed to be pairwise independent. Favorability of the product of the three prospect attributes is both necessary and sufficient for the existence of an accumulation of minimum size or larger in a prospect. The prospect probability can also be thought of as giving the proportion of prospects that contain an accumulation of minimum size or greater.

Prospect Risk: The probability that a randomly chosen prospect is unfavorable, i.e., that the prospect does not contain an accumulation of minimum size or larger. It is calculated as 1 minus the Prospect Probability.

Fraction of Accumulations Being Oil: That proportion of the accumulations that will be simulated as oil accumulations as opposed to nonassociated gas accumulations.

\section{Miscellaneous:}

Accumulation: Trapped hydrocarbons in contiguous pools of a particular play. In this assessment only accumulations of minimum size or larger are being assessed. 
Field: One or more accumulations whose projections on the earth's surface are the same or overlap. All the pools in an accumulation are of the same play, but a field may include pools of different plays. The simulation methodology for plays estimates accumulation sizes. Fields are important principally in the economic part of the analysis.

Play: A geologically homogeneous collection of accumulations (discovered and undiscovered) and prospects. Homogeneous is, of course, a relative term and the amount of variability acceptable within a play can vary with data available and methodology used. The accumulations within a play generally share similar source rocks, migration pathways, timing of relevant events, trapping mechanisms, and hydrocarbon types.

Play Area: A geographic area that includes all the discovered accumulations (if any) and all the prospects (if any) in a particular play.

Prospect: A drillable feature that may contain trapped hydrocarbons. 
Figure 1. Oil formation volume factor versus trap depth for selected gravities.

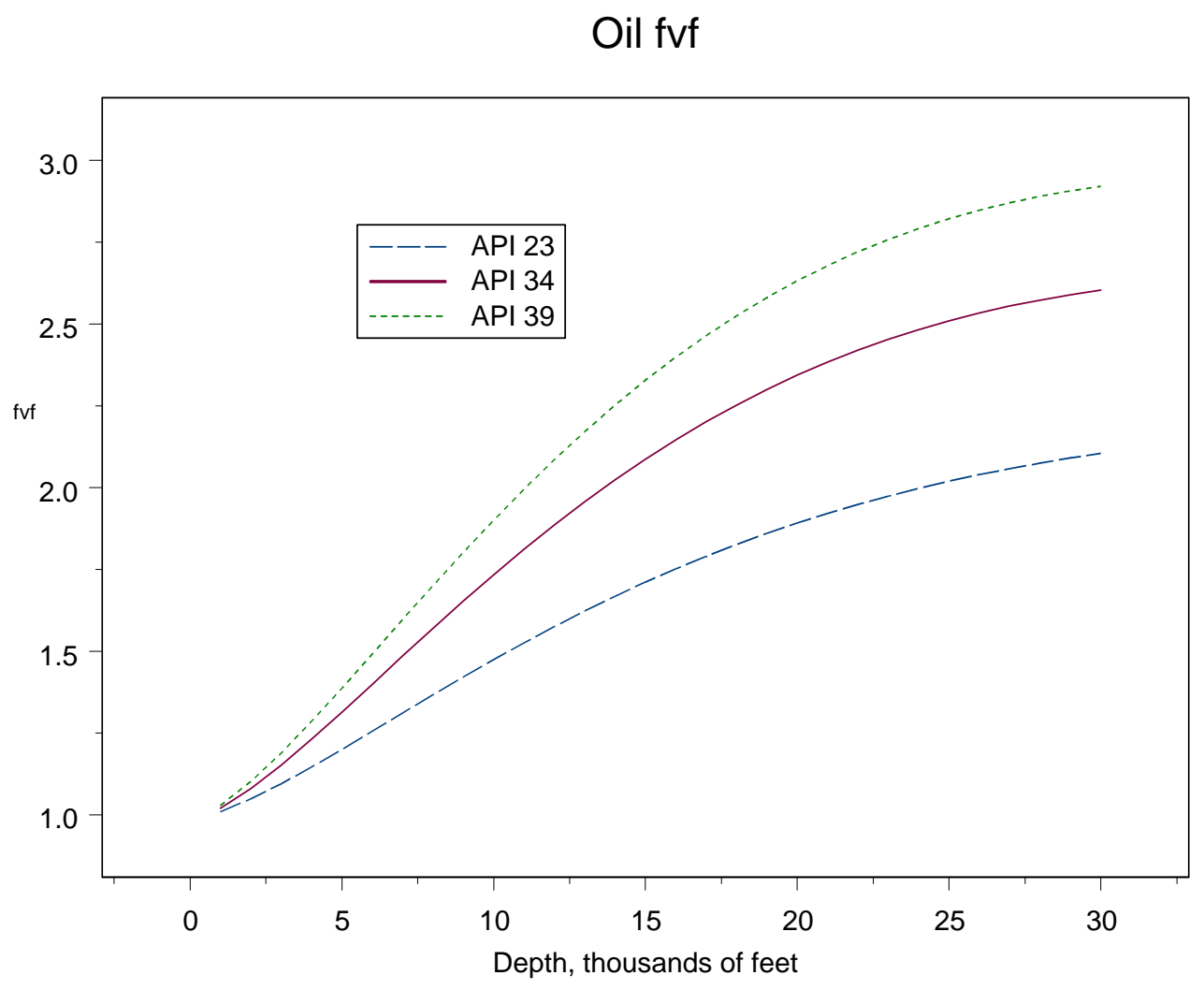


Figure 2. Gas formation volume factor versus trap depth.

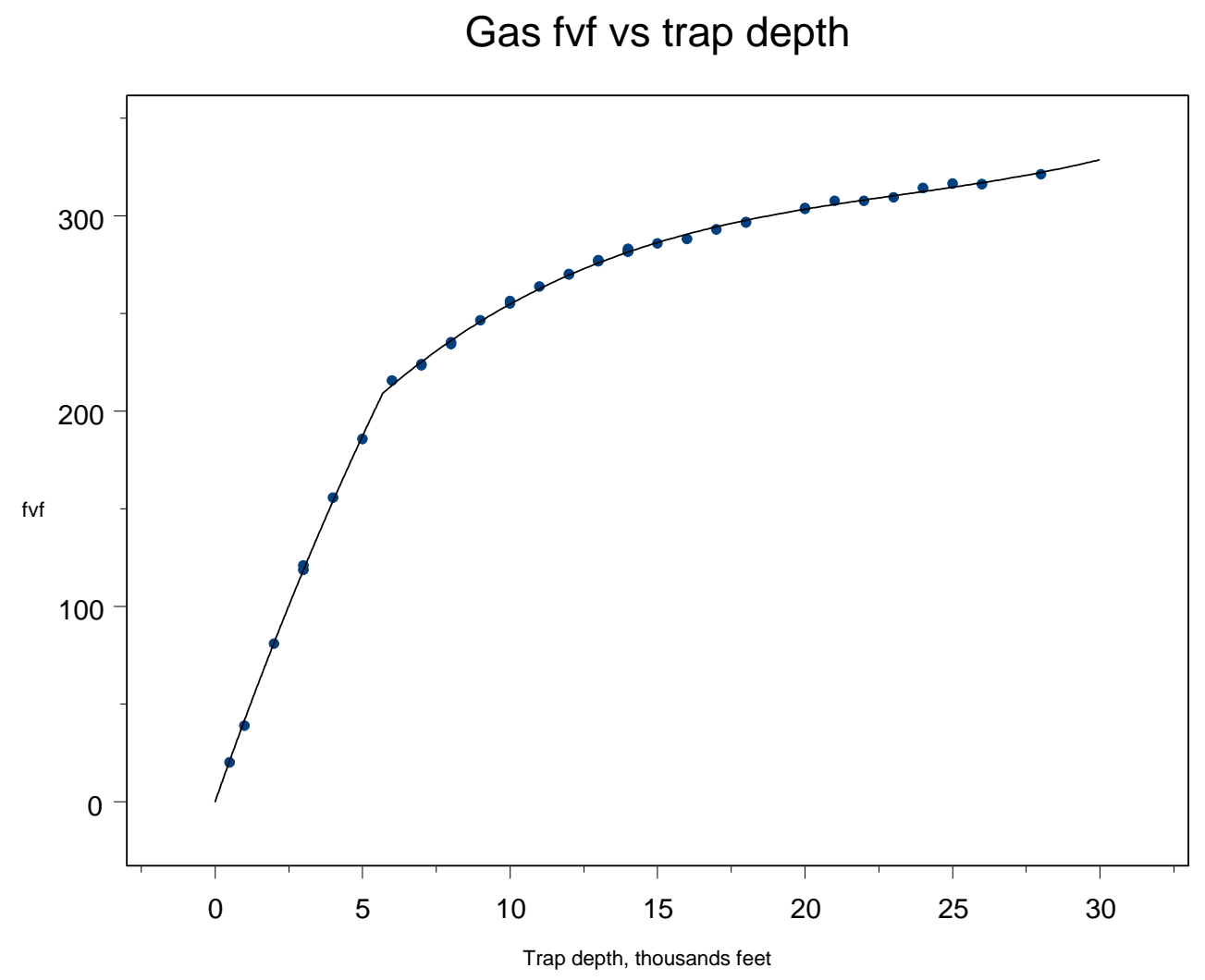


Figure 3. General flow chart for simulation algorithm.

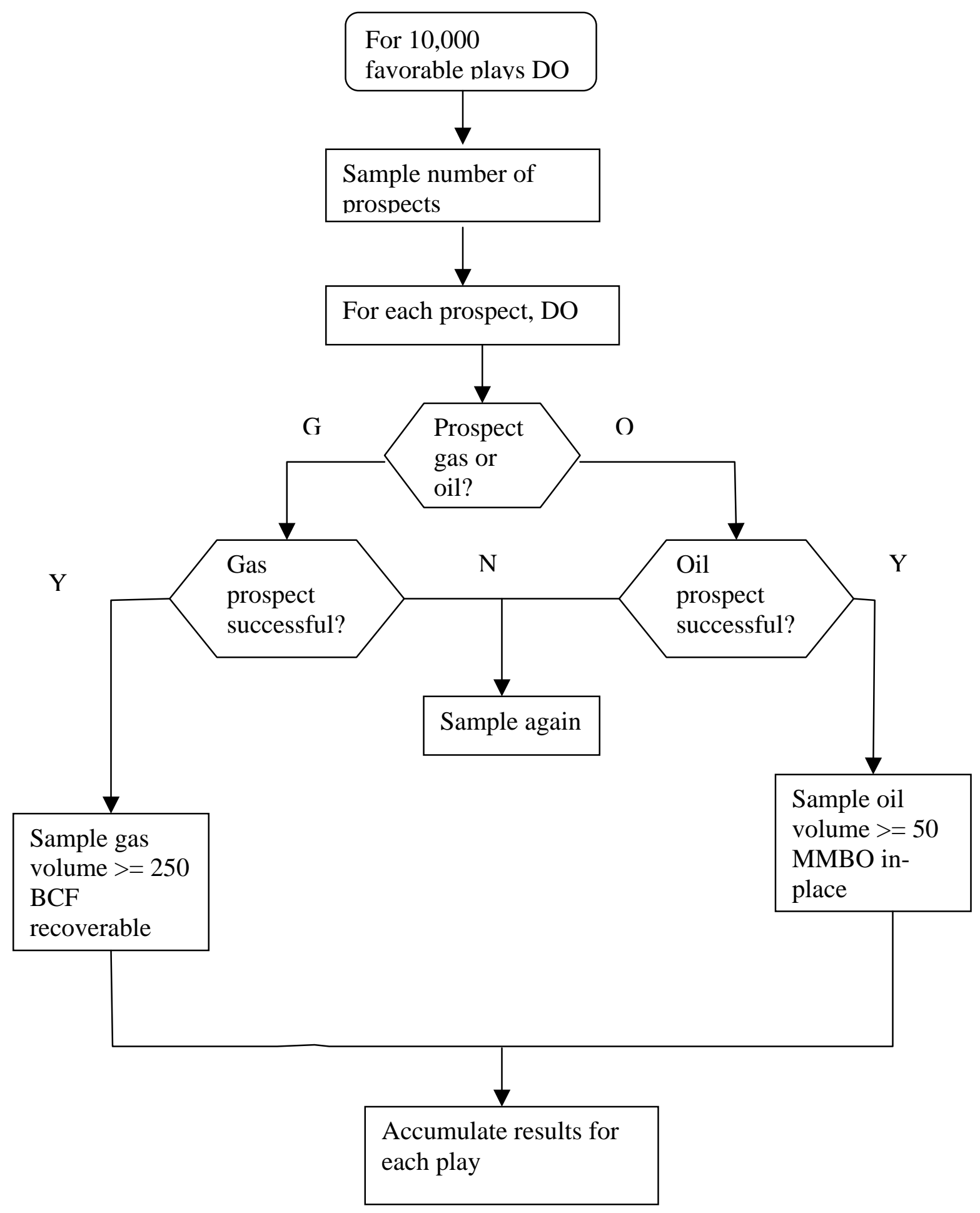


Table 1a. Assessment form for oil.

NPRA Assessment Form-2001
\begin{tabular}{|c|} 
PLAY:|EXAMPLE PLAY \\
\hline Play area: $1830410^{3}$ Acres \\
\hline
\end{tabular}

\section{OIL ACCUMULATION VOLUME PARAMETERS}

\begin{tabular}{|c|c|c|c|c|c|c|c|}
\hline \multirow[b]{2}{*}{ ATTRIBUTES } & \multirow{2}{*}{\begin{tabular}{|l|} 
Est Shape \\
$(1$ to 5$)$
\end{tabular}} & \multicolumn{4}{|c|}{ PROB OF AND GREATER THAN } & \multirow{2}{*}{$\begin{array}{l}\text { Knowledge } \\
\text { Level } 1-3^{5}\end{array}$} & \\
\hline & & LTP & 0.50 & 0.05 & Max & & \\
\hline \multirow{4}{*}{$\begin{array}{l}\text { NET RESERVOIR THICKNESS } \\
\text { AREA OF CLOSURE }^{2} \\
\text { POROSITY }^{3,4} \\
\text { TRAP FILL }^{3}\end{array}$} & 2 & 25 & 50 & 75 & 200 & \begin{tabular}{r|}
2 \\
\end{tabular} & \\
\hline & 2 & 3 & 5 & 10 & 20 & 2 & \\
\hline & 1 & 15 & 18 & 19 & 21 & 2 & \\
\hline & 3 & 40 & 50 & 75 & 100 & 2 & Enter POR*Sw \\
\hline \multirow{3}{*}{\multicolumn{2}{|c|}{$\begin{array}{l}\text { HYDROCARBON PORE VOL } \\
\text { Approx mm bbl (fvf }=1 \text { ) } \\
\text { Recov mm bbl at surface }\end{array}$}} & 9 & 12 & 13 & 15 & & 6 \\
\hline & & 17.5 & 116.4 & 567.3 & 4654.8 & & \\
\hline & & 4.7 & 31.2 & 152.1 & 1248.1 & & \\
\hline \multicolumn{7}{|c|}{$\begin{array}{l}\text { 1-thickness in feet, 2-thousands of acres, 3-percent, 4-correlation between Porosity and Water Saturation }=-1.0 \\
\text { 5-Knowledge Level: } 1=\text { High, 2=Medium, 3=Low; LTP=Left Truncation Point }\end{array}$} & \\
\hline \multirow{2}{*}{$\begin{array}{l}\text { TRAP DEPTH (in } 1000 \mathrm{ft} \text { ) } \\
\text { (from sea level) }\end{array}$} & \begin{tabular}{r|}
4 \\
\end{tabular} & $1 \mid$ & 5 & 9 & 10 & 1 & \\
\hline & & iface to sea & el correctio & $(1000 \mathrm{ft}):$ & 0.492 & & \\
\hline
\end{tabular}

\section{OIL ACCUMULATION CHARACTERISTICS}

Oil recovery factor $\%$

Type of reservoir-drive (check any that apply):

FVF (Formation volume factor, rb/stb):

\begin{tabular}{|lr|}
\hline Pressure(psi) & 2500 \\
temp(deg F) & 125 \\
SolGasGr & 0.705 \\
F & 717 \\
\hline
\end{tabular}

GOR (Associated gas to oil ratio, cu.ft./bbl, at stp):

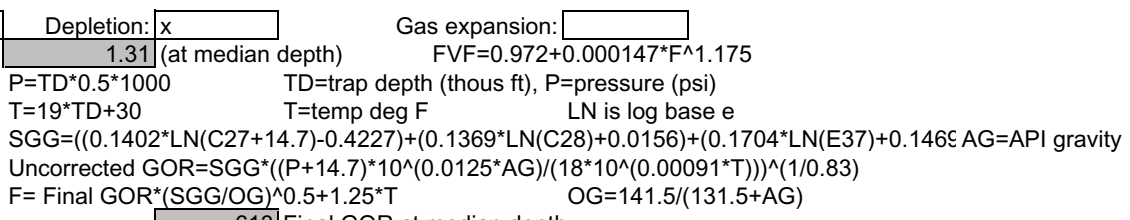

Oil quality parameters:
API gravity Sulfur content of oil

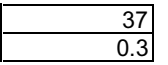
Oil Grav (ratio) 0.840

Associated gas quality parameters:

Hydrogen sulfide \%

CO2 contamination \% Other inert gases:

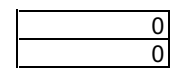

Name:

Name:

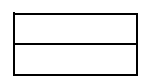

Percent:

Percent:

TIME OF TRAP DEVELOPMENT

STRATIGRAPHIC COMPONENT (Ma)

STRUCTURAL COMPONENT (Ma)

\begin{tabular}{|r|r|r|}
\multicolumn{1}{l|}{ BEGIN } & PEAK & END \\
\hline 120 & 115 & 95 \\
\hline & & \\
\hline
\end{tabular}

Assessor's Name: Dave Houseknecht

Date of Data Entry MM/DD/YYYY:

Date of Simulation Run MM/DD/YYYY:

10/19/01

$1 / 16 / 02$

Note: only enter play name and assessor's name on Oil worksheet 
Table 1b. Assessment form for gas.

NPRA Assessment Form-2001

PLAY: Example play

NONASSOCIATED GAS ACCUMULATION VOLUME PARAMETERS

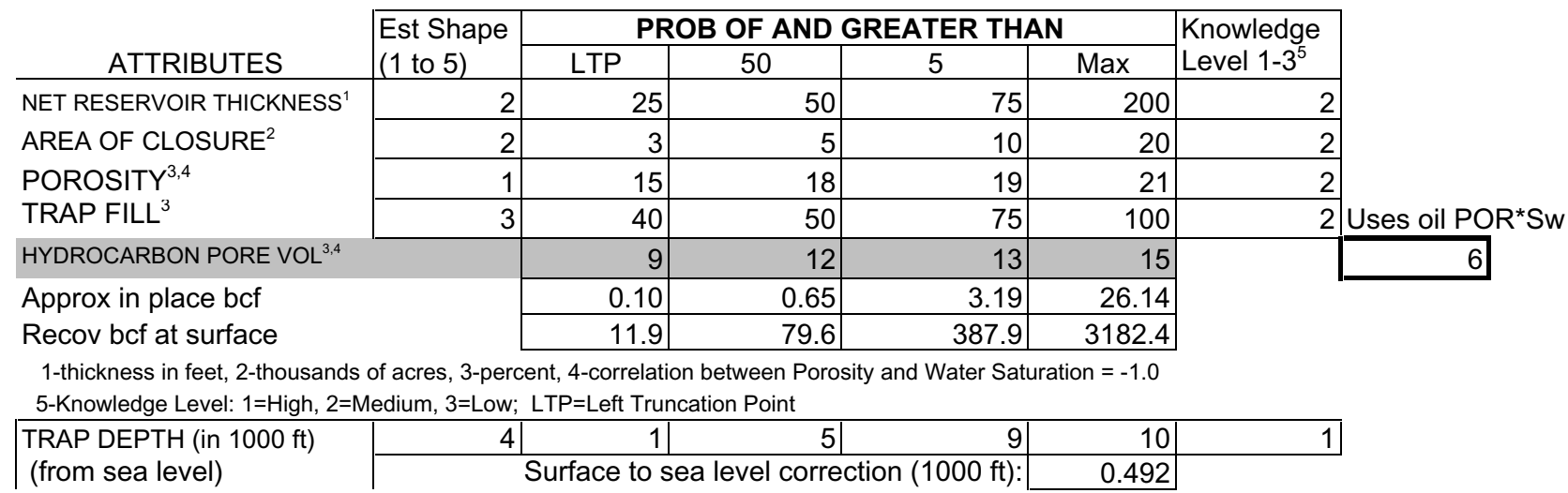

\section{NONASSOCIATED GAS ACCUMULATION CHARACTERISTICS}

NA Gas recovery factor \%

Type of reservoir-drive (check any that apply):

$$
\text { Water: }
$$

Gas expansion:

Natural gas liquids plus condensate to non-associated gas (bbls/million cf) (in place):

$$
\text { NGL-NAG }=1.785^{\star} \mathrm{TD}
$$

$$
8.9 \text { (at median) }
$$

Non-associated gas quality parameters:

Hydrogen sulfide \% CO2 contamination \% Other inert gases:

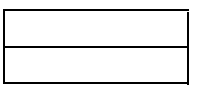

$$
\begin{array}{ll}
\text { Name: } & \\
\text { Name: } & \text { Percent: } \\
& \text { Percent: }
\end{array}
$$

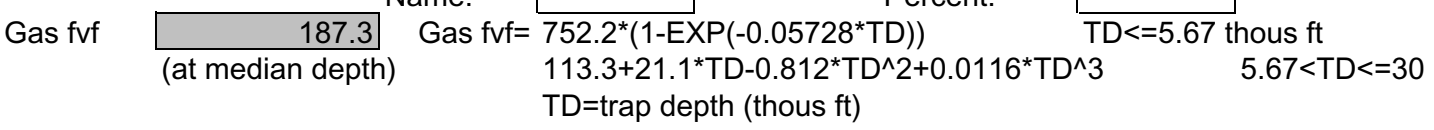

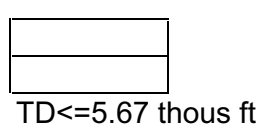

\begin{tabular}{l} 
TIME OF TRAP DEVELOPMEN BEGIN $\quad$ PEAK \\
STRATIGRAPHIC COMPONENT (Ma) \\
STRUCTURAL COMPONENT (Ma) \\
\hline
\end{tabular}

Assessor's Name: Dave Houseknecht Date of Data Entry MM/DD/YYYY:

Date of Simulation Run MM/DD/YYYY: 
Table 1c. Assessment form for risking.

NPRA Assessment Form-2001

Play: Example play

\section{RISKING}

MINIMUM ACCUMULATION SIZE, MAS (Millions of BBL in place)

50

PRERISKED FREQUENCY DISTRIBUTION (Oil plus Gas)

\begin{tabular}{l|r|r|r|r|r|l|}
\cline { 3 - 7 } & \multicolumn{4}{c|}{} & \multicolumn{3}{c|}{ PROB OF AND GREATER THAN } & Knowledge \\
NUM OF PROSPECTS & Est Shape & Min & 50 & 5 & Max & Level 1-3 \\
\hline > MINIMUM SIZE & 2 & 25 & 50 & 75 & 100 & 3 \\
\hline
\end{tabular}

5-Knowledge Level: 1=High, 2=Medium, 3=Low; LTP=Left Truncation Point

\section{ATTRIBUTES}

$\begin{array}{ll}\text { PLAY } & \text { CHARGE }(C) \\ \text { ATTRIBUTES } & \text { TRAP (T) } \\ & \text { TIMING (F) }\end{array}$

Probability that play contains at least 1 reservoir $>=$ minimum size $(C x T \times F)$

$\begin{array}{ll}\text { PROSPECT } & \text { CHARGE (c) } \\ \text { ATTRIBUTES } & \text { TRAP (t) } \\ & \text { TIMING (f) }\end{array}$

Probability that a randomly chosen prospect is favorable (cxtxf)

Play Attributes x Prospect Attributes (CxTxFxcxtxf)

FRACTION OF ACCUMULATIONS BEING OIL

Fraction NA Gas=1-Fraction(Oil)

\section{PROBABILITY OF FAVORABLE}
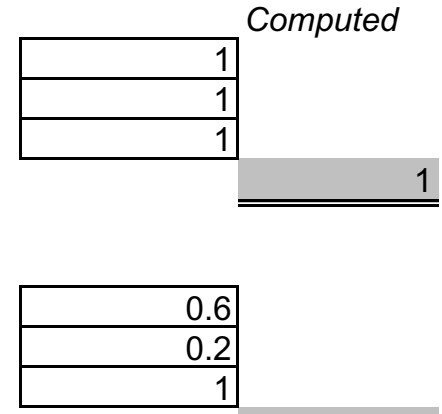

1

0.9

Allocation (percent):

\begin{tabular}{|l|r|r|r|}
\cline { 2 - 4 } \multicolumn{1}{c|}{} & \multicolumn{1}{c|}{ Land } & \multicolumn{1}{c|}{ Oil } & \multicolumn{2}{c|}{ Gas } \\
\hline Federal & 90 & 86 & 92 \\
\hline State & 6 & 10 & 5 \\
\hline Native & 4 & 4 & 3 \\
\hline
\end{tabular}

Assessor's Name: Date of Data Entry MM/DD/YYYY:

Date of Simulation Run MM/DD/YYYY:

Dave Houseknecht

$10 / 19 / 01$

$1 / 16 / 02$ 


\section{Table 1d. Instructions for NPRA Assessment Form-2001}

\section{Contents (worksheets)}

Instr - contents and general instructions

Shapes - examples of distribution shapes

Oil - Oil accumulation volume parameters (for assessor input)

Gas - Gas accumulation volume parameters (for assessor input)

Play-Prospect (PP)- Prerisked frequency distribution, play risks, and prospect risks (for assessor input)

Playwork - worksheet for plays (for data transfer to Splus algorithms)

Distn - fitted distribution of oil and gas hydrocarbon attributes

Results - summary statistics and plots for play results

Size-Freq - size frequency distribution and historgrams

Stats - summary stats for sampled distributions of oil/gas hydrocarbon volume parameters

\section{Suggested order to specify volume parameters, trap depth, and frequency distributions}

1 Specify your perception of the general form of (untruncated) distribution using attached Shapes worksheet

2 Specify Left Truncation Point (LTP)

* For oil and gas hydrocarbon parameters, this value should be consistent with minimum accumulation size (MAS), i.e., these should generate an accumulation approximately equal to the MAS. You will be provided feedback to ensure consistency with MAS.

* In general the LTP will be greater than zero.

* Note, we are assuming that the population distribution begins at or very near zero. If this is not true, a shift parameter will need to be specified

LTP is the minimum value for Trap Depth and Number of Prospects

3 Specify Max. This must be a finite value. It is to be the maximum as opposed to say the 1 fractile.

4 Specify the median (50th fractile). Note that this value is with respect to the untruncated distribution. The kth fractile, defined as Fk, where $(0<=k<=100)$, is a value such that the probability that a randomly chosen value, say $X$ is $>F k=0.01^{*} k$, i.e., $P(X>F k)=0.01^{*} k$.

In the Sheet worksheet, for the Right (positive) skewed density distribution F05 $=0.52$

5 Specify the 5th fractile with respect to the untruncated distribution.

In the Shapes worksheet examples, the value of the attribute $\mathrm{X}$ is between 0 and 1 .

Probability density, cumulative density, and F95, F50, and F05 fractiles are given.

The LTP is assumed to be F95 in the Shapes worksheet but need not be so. The MAS should guide the choice of the LTP

MAS - Minimum Accumulation Size

LTP - Left Truncation Point

\section{Dependencies}

The model assumes pairwise independence between all oil and gas accumulations attributes except that the correlation between proosity and water saturation is assumed to be -1.0

We assume that number of prospects (Play-Prospect worksheet) is independent of Oil and Gas hydrocarbon parameters

Protocol for Entering Distributions \& Feedback

1 Use actual data when possible (assessor decides that a given data set is representative of a play)

2 Specify fractiles as indicated above At this stage feedback (graphs and statistics) on individual attributes is given to assessors

3 Assessors modify distributions as appropriate After receiving modified attribute distributions (oil or gas hydrocarbon parameters), an oil/gas accumulation size distribution will be generated. If the minimum accumulation is $<25 \mathrm{MMBOE}$ or $>60 \mathrm{MMBOE}$ (for a $50 \mathrm{MMBOE} M A S$ ) the assessor will be informed and may chose to modify hydrocarbon attributes. If the minimum is below $25 \mathrm{MMBOE}$ one or more LTP for the hydrocarbon volume parameters may be too low. If the maximum is above $60 \mathrm{MMBOE}$, one or more LTP's for the hydrocarbon volume parameters may be set too high. 


\section{Table 1e. Distribution shapes specified in the NPRA assessment form.}

Shape 1

Probability Density

J-shaped $($ mode $=0)$

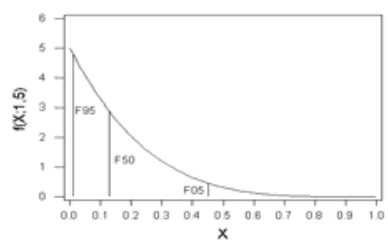

Shape 2

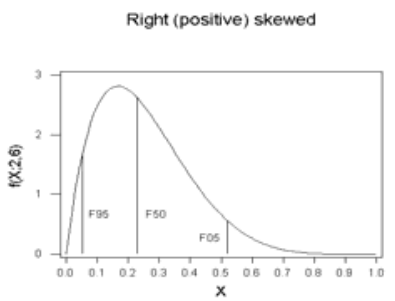

Shape 3

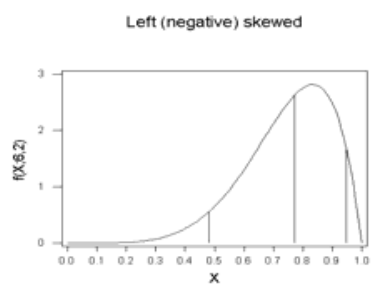

Shape 4

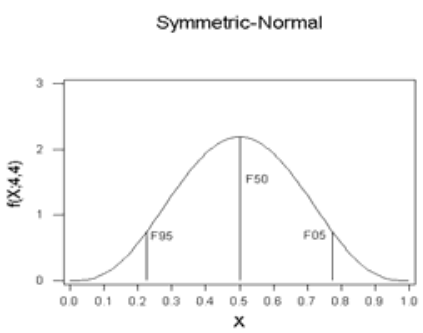

Shape 5

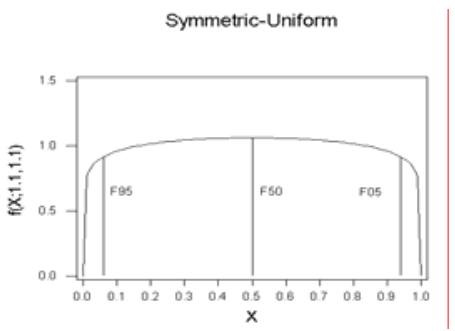

Shape 6 Inverse J

Shape 7 Inverse $\mathrm{J}$ for TrapFill with F50,F05 and MAX $=100$ (50\% of probability at 100$)$

Shape 8 Inverse $\mathrm{J}$ for TrapFill with $\mathrm{F} 05, \mathrm{MAX}=100$ (5\% of probability at 100$)$

Shape 9 No variability; estimate is a single number
Cumulative Density

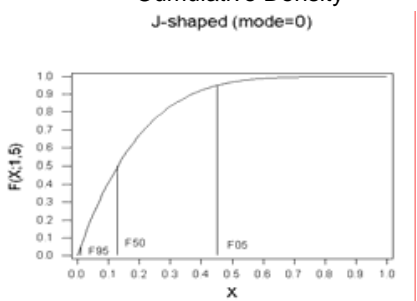

\begin{tabular}{|r|r|}
\hline F95 & 0.01 \\
\hline F50 & 0.13 \\
\hline F05 & 0.45 \\
\hline
\end{tabular}

Mean $\quad 0.33$

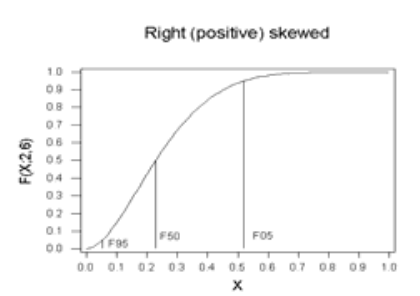

\begin{tabular}{|r|r|}
\hline F95 & 0.05 \\
\hline F50 & 0.23 \\
\hline F05 & 0.52 \\
\hline \multicolumn{2}{|c|}{} \\
\hline Mean & 0.25 \\
\hline
\end{tabular}

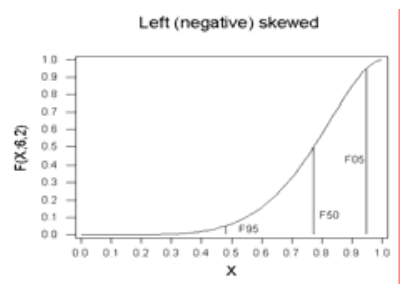

\begin{tabular}{|l|l|}
\hline F95 & 0.48 \\
\hline F50 & 0.77 \\
\hline F05 & 0.95 \\
\hline \multicolumn{2}{|c|}{ Mean } \\
\hline
\end{tabular}

Mean 0.75
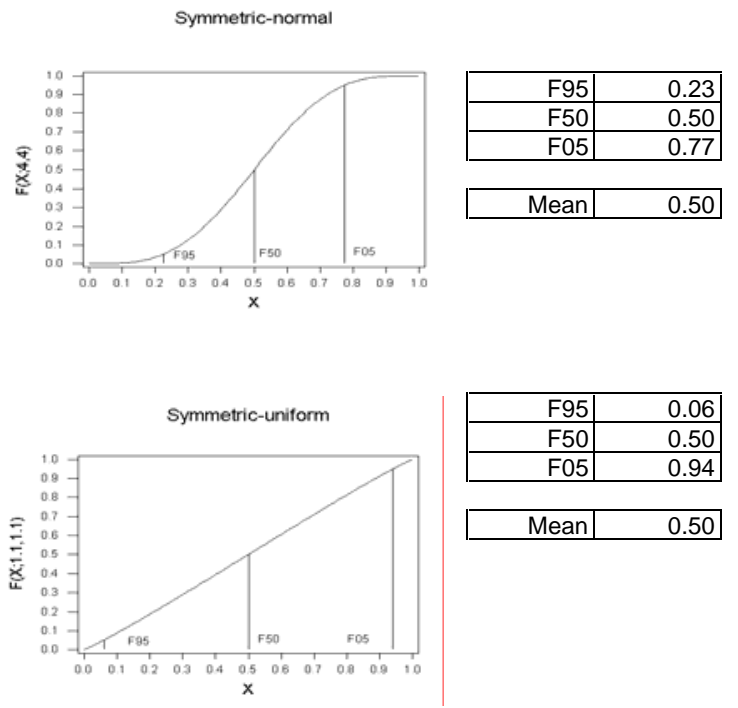

$\begin{array}{ll}\text { F95 } & 0.99 \\ \text { F50 } & 0.87 \\ \text { F05 } & 0.55 \\ & \\ \text { Mean } & 0.67\end{array}$


Table 2. Equations used to compute co-products in the NPRA assessment.

Oil deposit:

ADG (associated-dissolved gas, in BCFG)

$\mathrm{ADG}=\mathrm{GOR} * \mathrm{Oil} * 10^{-3}$ where GOR is the associated gas to oil ratio (see Table ME1a) and the oil is either in-place or recoverable in MMBO.

NGL-ADG (natural gas liquid from ADG, in MMBO)

$\mathrm{NGL}-\mathrm{ADG}=\mathrm{NGLR} * \mathrm{ADG} * 10^{-3}$ where NGLR is the natural gas liquid to associated gas ratio (see Table ME1a).

Non-associated natural gas deposit:

NGL-GAS (natural gas liquid from non-associated gas, in MMBO) NGL-GAS $=$ NGL-NAG $*$ NAG $* 10^{-3}$ where NGL-NAG is the natural gas liquid to nonassociated gas ratio (see Table ME1b) and NAG is nonassociated gas (in BCFG). 
Table 3. Play name and total Monte Carlo runs for each play in the NPRA assessment

\begin{tabular}{|l|r|}
\multicolumn{1}{c}{ Play Name } & Total Runs \\
\hline \hline Beaufortian Clinoform & 11,111 \\
\hline Beaufortian Cretaceous Topset North & 11,111 \\
\hline Beaufortian Cretaceous Topset South & 10,000 \\
\hline Beaufortian Lower Jurassic Topset & 11,111 \\
\hline Beaufortian Upper Jurassic Topset NE & 10,000 \\
\hline Beaufortian Upper Jurassic Topset NW & 10,000 \\
\hline Beaufortian Upper Jurassic Topset SE & 10,000 \\
\hline Beaufortian Upper Jurassic Topset SW & 10,000 \\
\hline Brookian Clinoform Central & 10,000 \\
\hline Brookian Clinoform North & 10,000 \\
\hline Brookian Clinoform South-Deep & 12,500 \\
\hline Brookian Clinoform South-Shallow & 10,000 \\
\hline Brookian Topset & 10,000 \\
\hline Brookian Topset Structural & 10,000 \\
\hline Ellesmerian Echooka North & 50,000 \\
\hline Ellesmerian Echooka South & 20,833 \\
\hline Ellesmerian Endicott North & 18,519 \\
\hline Ellesmerian Endicott South & 16,667 \\
\hline Ellesmerian Lisburne North & 18,519 \\
\hline Ellesmerian Lisburne South & 18,519 \\
\hline Ellesmerian Structural & 12,500 \\
\hline Ellesmerian Thrust Belt & 13,889 \\
\hline Ellesmerian-Ivishak & 11,111 \\
\hline Torok Structural & 11,111 \\
\hline
\end{tabular}


Table 4. Assessor specified dependencies between plays in the NPRA assessment.

\begin{tabular}{|c|c|c|c|c|c|c|c|c|c|c|c|c|c|c|c|c|c|c|c|c|c|c|c|c|}
\hline & Charge-Trap-Timing & 1 & 2 & 3 & 4 & 5 & 6 & 7 & 8 & 9 & 10 & 11 & 12 & 13 & 14 & 15 & 16 & 17 & 18 & 19 & 20 & 21 & 22 & 23 \\
\hline \multicolumn{25}{|c|}{1 Brookian Topset } \\
\hline & Brookian Topset Structural & M-M-L & & & & & & & & & & & & & & & & & & & & & & \\
\hline 3 & Brookian Clinoform North & H-L-H & M-L-L & & & & & & & & & & & & & & & & & & & & & \\
\hline 4 & Brookian Clinoform Central & H-L-H & H-L-L & $\mathrm{H}-\mathrm{H}-\mathrm{H}$ & & & & & & & & & & & & & & & & & & & & \\
\hline 5 & Brookian Clinoform South-Shallow & M-L-H & H-L-L & M-H-H & M-H-H & & & & & & & & & & & & & & & & & & & \\
\hline 6 & Brookian Clinoform South-Deep & M-L-H & M-L-L & M-H-H & M-H-H & M-H-H & & & & & & & & & & & & & & & & & & \\
\hline 7 & Torok Structural & M-L-L & H-M-H & L-L-L & H-M-L & H-M-L & H-M-L & & & & & & & & & & & & & & & & & \\
\hline 8 & Beaufortian Cretaceous Topset North & M-L-L & L-L-L & M-L-M & L-L-L & L-L-L & L-L-L & L-L-L & & & & & & & & & & & & & & & & \\
\hline 9 & Beaufortian Cretaceous Topset South & L-L-L & L-L-L & L-L-L & L-L-L & L-L-L & H-L-L & M-L-L & M-M-H & & & & & & & & & & & & & & & \\
\hline 10 & Beaufortian Upper Jurassic Topset NW & L-L-L & L-L-L & L-L-L & L-L-L & L-L-L & L-L-L & L-L-L & M-L-M & L-L-M & & & & & & & & & & & & & & \\
\hline 11 & Beaufortian Upper Jurassic Topset NE & L-L-L & L-L-L & L-L-L & L-L-L & L-L-L & L-L-L & L-L-L & M-L-M & L-L-M & $\mathrm{H}-\mathrm{H}-\mathrm{H}$ & & & & & & & & & & & & & \\
\hline 12 & Beaufortian Upper Jurassic Topset SW & L-L-L & L-L-L & L-L-L & L-L-L & L-L-L & L-L-L & L-L-L & M-L-M & M-L-M & H-H-H & $\mathrm{H}-\mathrm{H}-\mathrm{H}$ & & & & & & & & & & & & \\
\hline 13 & Beaufortian Upper Jurassic Topset SE & L-L-L & L-L-L & L-L-L & L-L-L & L-L-L & L-L-L & L-L-L & M-L-M & M-L-M & H-H-H & H-H-H & $\mathrm{H}-\mathrm{H}-\mathrm{H}$ & & & & & & & & & & & \\
\hline 14 & Beaufortian Lower Jurassic Topset & M-L-L & L-L-L & L-L-L & L-L-L & L-L-L & L-L-L & L-L-L & M-L-M & L-L-M & M-L-M & M-L-M & L-L-M & L-L-M & & & & & & & & & & \\
\hline 15 & Beaufortian Clinoform & M-L-L & M-L-L & L-L-L & L-L-L & L-L-L & L-L-L & L-L-L & H-L-M & L-L-M & H-M-H & H-L-H & $\mathrm{H}-\mathrm{H}-\mathrm{H}$ & $\mathrm{H}-\mathrm{H}-\mathrm{H}$ & M-L-H & & & & & & & & & \\
\hline 16 & Ellesmerian-Ivishak & M-L-M & L-L-L & L-L-L & L-L-L & L-L-L & L-L-L & L-L-L & M-L-H & L-L-L & L-L-L & L-L-L & L-L-L & L-L-L & H-L-H & L-L-M & & & & & & & & \\
\hline 17 & Ellesmerian Endicott North & L-L-L & L-L-L & L-L-L & L-L-L & L-L-L & L-L-L & L-L-L & L-L-L & L-L-L & L-L-L & L-L-L & L-L-L & L-L-L & L-L-L & L-L-L & L-L-L & & & & & & & \\
\hline 18 & Ellesmerian Endicott South & L-L-L & L-L-L & L-L-L & L-L-L & L-L-L & L-L-L & L-L-L & L-L-L & L-L-L & L-L-L & L-L-L & L-L-L & L-L-L & L-L-L & L-L-L & L-L-L & $\mathrm{H}-\mathrm{H}-\mathrm{H}$ & & & & & & \\
\hline 19 & Ellesmerian Echooka North & L-L-L & L-L-L & L-L-L & L-L-L & L-L-L & L-L-L & L-L-L & L-L-L & L-L-L & L-L-L & L-L-L & L-L-L & L-L-L & L-L-L & L-L-L & M-L-M & M-L-M & M-L-M & & & & & \\
\hline 20 & Ellesmerian Echooka South & L-L-L & L-L-L & L-L-L & L-L-L & L-L-L & L-L-L & L-L-L & L-L-L & L-L-L & L-L-L & L-L-L & L-L-L & L-L-L & L-L-L & L-L-L & M-L-M & M-L-M & M-L-M & H-H-H & & & & \\
\hline 21 & Ellesmerian Lisburne North & L-L-L & L-L-L & L-L-L & L-L-L & L-L-L & L-L-L & L-L-L & L-L-L & L-L-L & L-L-L & L-L-L & L-L-L & L-L-L & L-L-L & L-L-L & M-L-M & M-M-M & M-M-M & H-M-H & H-M-H & & & \\
\hline 22 & Ellesmerian Lisburne South & L-L-L & L-L-L & L-L-L & L-L-L & L-L-L & L-L-L & L-L-L & L-L-L & L-L-L & L-L-L & L-L-L & L-L-L & L-L-L & L-L-L & L-L-L & M-L-M & M-M-M & M-M-M & H-M-H & H-M-H & $\mathrm{H}-\mathrm{H}-\mathrm{H}$ & & \\
\hline 23 & Ellesmerian Structural & L-L-L & L-L-M & L-L-L & L-L-L & L-L-L & L-L-L & L-L-M & L-L-L & L-L-L & L-L-L & L-L-L & L-L-L & L-L-L & L-L-L & L-L-L & L-L-L & L-L-L & M-L-L & L-L-L & L-L-L & L-L-L & L-M-L & \\
\hline 24 & Ellesmerian Thrust Belt & L-L-L & M-L-M & L-L-L & L-L-L & L-L-L & L-L-L & M-L-M & L-L-L & L-L-L & L-L-L & L-L-L & L-L-L & L-L-L & L-L-L & M-L-L & L-L-L & L-L-L & L-L-L & L-L-L & L-L-L & L-L-L & L-L-L & \\
\hline
\end{tabular}

Enter L, M, H for Low, Medium \& High 
Table 5. Arithmetic averages of pairwise dependencies in the NPRA assessment.

\begin{tabular}{|c|c|c|c|c|c|c|c|c|c|c|c|c|c|c|c|c|c|c|c|c|c|c|c|c|}
\hline & Average of Charge-Trap-Timing & 1 & 2 & 3 & 4 & 5 & 6 & 7 & 8 & 9 & 10 & 11 & 12 & 13 & 14 & 15 & 16 & 17 & 18 & 19 & 20 & 21 & 22 & 23 \\
\hline \multicolumn{25}{|c|}{1 Brookian Topset } \\
\hline 2 & Brookian Topset Structural & 0.37 & & & & & & & & & & & & & & & & & & & & & & \\
\hline 3 & Brookian Clinoform North & 0.63 & 0.23 & & & & & & & & & & & & & & & & & & & & & \\
\hline 4 & Brookian Clinoform Central & 0.63 & 0.37 & 0.90 & & & & & & & & & & & & & & & & & & & & \\
\hline 5 & Brookian Clinoform South-Shallow & 0.50 & 0.37 & 0.77 & 0.77 & & & & & & & & & & & & & & & & & & & \\
\hline 6 & Brookian Clinoform South-Deep & 0.50 & 0.23 & 0.77 & 0.77 & 0.77 & & & & & & & & & & & & & & & & & & \\
\hline 7 & Torok Structural & 0.23 & 0.77 & 0.10 & 0.50 & 0.50 & 0.50 & & & & & & & & & & & & & & & & & \\
\hline 8 & Beaufortian Cretaceous Topset North & 0.23 & 0.10 & 0.37 & 0.10 & 0.10 & 0.10 & 0.10 & & & & & & & & & & & & & & & & \\
\hline 9 & Beaufortian Cretaceous Topset South & 0.10 & 0.10 & 0.10 & 0.10 & 0.10 & 0.37 & 0.23 & 0.63 & & & & & & & & & & & & & & & \\
\hline 10 & Beaufortian Upper Jurassic Topset NW & 0.10 & 0.10 & 0.10 & 0.10 & 0.10 & 0.10 & 0.10 & 0.37 & 0.23 & & & & & & & & & & & & & & \\
\hline 11. & Beaufortian Upper Jurassic Topset NE & 0.10 & 0.10 & 0.10 & 0.10 & 0.10 & 0.10 & 0.10 & 0.37 & 0.23 & 0.90 & & & & & & & & & & & & & \\
\hline 12 & Beaufortian Upper Jurassic Topset SW & 0.10 & 0.10 & 0.10 & 0.10 & 0.10 & 0.10 & 0.10 & 0.37 & 0.37 & 0.90 & 0.90 & & & & & & & & & & & & \\
\hline 13 & Beaufortian Upper Jurassic Topset SE & 0.10 & 0.10 & 0.10 & 0.10 & 0.10 & 0.10 & 0.10 & 0.37 & 0.37 & 0.90 & 0.90 & 0.90 & & & & & & & & & & & \\
\hline 14 & Beaufortian Lower Jurassic Topset & 0.23 & 0.10 & 0.10 & 0.10 & 0.10 & 0.10 & 0.10 & 0.37 & 0.23 & 0.37 & 0.37 & 0.23 & 0.23 & & & & & & & & & & \\
\hline 15 & Beaufortian Clinoform & 0.23 & 0.23 & 0.10 & 0.10 & 0.10 & 0.10 & 0.10 & 0.50 & 0.23 & 0.77 & 0.63 & 0.90 & 0.90 & 0.50 & & & & & & & & & \\
\hline 16 & Ellesmerian-Ivishak & 0.36 & 0.10 & 0.10 & 0.10 & 0.10 & 0.10 & 0.10 & 0.50 & 0.10 & 0.10 & 0.10 & 0.10 & 0.10 & 0.63 & 0.23 & & & & & & & & \\
\hline 17 & Ellesmerian Endicott North & 0.10 & 0.10 & 0.10 & 0.10 & 0.10 & 0.10 & 0.10 & 0.10 & 0.10 & 0.10 & 0.10 & 0.10 & 0.10 & 0.10 & 0.10 & 0.10 & & & & & & & \\
\hline 18 & Ellesmerian Endicott South & 0.10 & 0.10 & 0.10 & 0.10 & 0.10 & 0.10 & 0.10 & 0.10 & 0.10 & 0.10 & 0.10 & 0.10 & 0.10 & 0.10 & 0.10 & 0.10 & 0.90 & & & & & & \\
\hline 19 & Ellesmerian Echooka North & 0.10 & 0.10 & 0.10 & 0.10 & 0.10 & 0.10 & 0.10 & 0.10 & 0.10 & 0.10 & 0.10 & 0.10 & 0.10 & 0.10 & 0.10 & 0.37 & 0.37 & 0.37 & & & & & \\
\hline 20 & Ellesmerian Echooka South & 0.10 & 0.10 & 0.10 & 0.10 & 0.10 & 0.10 & 0.10 & 0.10 & 0.10 & 0.10 & 0.10 & 0.10 & 0.10 & 0.10 & 0.10 & 0.37 & 0.37 & 0.37 & 0.90 & & & & \\
\hline 21 & Ellesmerian Lisburne North & 0.10 & 0.10 & 0.10 & 0.10 & 0.10 & 0.10 & 0.10 & 0.10 & 0.10 & 0.10 & 0.10 & 0.10 & 0.10 & 0.10 & 0.10 & 0.37 & 0.50 & 0.50 & 0.77 & 0.77 & & & \\
\hline 22 & Ellesmerian Lisburne South & 0.10 & 0.10 & 0.10 & 0.10 & 0.10 & 0.10 & 0.10 & 0.10 & 0.10 & 0.10 & 0.10 & 0.10 & 0.10 & 0.10 & 0.10 & 0.37 & 0.50 & 0.50 & 0.77 & 0.77 & 0.90 & & \\
\hline 23 & Ellesmerian Structural & 0.10 & 0.23 & 0.10 & 0.10 & 0.10 & 0.10 & 0.23 & 0.10 & 0.10 & 0.10 & 0.10 & 0.10 & 0.10 & 0.10 & 0.10 & 0.10 & 0.10 & 0.23 & 0.10 & 0.10 & 0.10 & 0.23 & \\
\hline 24 & Ellesmerian Thrust Belt & 0.10 & 0.37 & 0.10 & 0.10 & 0.10 & 0.10 & 0.37 & 0.10 & 0.10 & 0.10 & 0.10 & 0.10 & 0.10 & 0.10 & 0.23 & 0.10 & 0.10 & 0.10 & 0.10 & 0.10 & 0.10 & 0.10 & 0.37 \\
\hline
\end{tabular}


Table 6. Adjusted correlation matrix for plays in the NPRA assessment.

\begin{tabular}{rl|}
\multicolumn{1}{c|}{ Adjusted Average Charge-Trap-Timing } \\
& Brookian Topset \\
2 & Brookian Topset Structural \\
3 & Brookian Clinoform North \\
4 & Brookian Clinoform Central \\
5 & Brookian Clinoform South-Shallow \\
6 & Brookian Clinoform South-Deep \\
7 & Torok Structural \\
8 & Beaufortian Cretaceous Topset North \\
9 & Beaufortian Cretaceous Topset South \\
10 & Beaufortian Upper Jurassic Topset NW \\
11 & Beaufortian Upper Jurassic Topset NE \\
12 & Beaufortian Upper Jurassic Topset SW \\
13 & Beaufortian Upper Jurassic Topset SE \\
14 & Beaufortian Lower Jurassic Topset \\
15 & Beaufortian Clinoform \\
16 & Ellesmerian-lvishak \\
17 & Ellesmerian Endicott North \\
18 & Ellesmerian Endicott South \\
19 & Ellesmerian Echooka North \\
20 & Ellesmerian Echooka South \\
21 & Ellesmerian Lisburne North \\
22 & Ellesmerian Lisburne South \\
23 & Ellesmerian Structural \\
24 & Ellesmerian Thrust Belt \\
\hline
\end{tabular}
1 0.31 $\begin{array}{ll}0.31 & \\ 0.52 & 0.19\end{array}$ $\begin{array}{lll}0.52 & 0.19 & \\ 0.52 & 0.31 & 0.74\end{array}$ $\begin{array}{llll}0.52 & 0.31 & 0.74 & \\ 0.41 & 0.31 & 0.64 & 0.64\end{array}$ $\begin{array}{lllll}0.41 & 0.19 & 0.64 & 0.64 & 0.64\end{array}$ $\begin{array}{llllll}0.19 & 0.64 & 0.08 & 0.41 & 0.41 & 0.41\end{array}$ $\begin{array}{lllllll}0.19 & 0.08 & 0.31 & 0.08 & 0.08 & 0.08 & 0.08\end{array}$ $\begin{array}{llllllll}0.08 & 0.08 & 0.08 & 0.08 & 0.08 & 0.31 & 0.19 & 0.52\end{array}$ $\begin{array}{lllllllll}0.08 & 0.08 & 0.08 & 0.08 & 0.08 & 0.08 & 0.08 & 0.31 & 0.19\end{array}$ $\begin{array}{lllllllllll}0.08 & 0.08 & 0.08 & 0.08 & 0.08 & 0.08 & 0.08 & 0.31 & 0.19 & \\ 0.08 & 0.08 & 0.08 & 0.08 & 0.08 & 0.08 & 0.08 & 0.31 & 0.19 & 0.74 \\ 0.08 & 0.08 & 0.08 & 0.08 & 0.08 & 0.08 & 0.08 & 0.31 & 0.31 & 0.74 & 0\end{array}$ $\begin{array}{lllllllllll}0.08 & 0.08 & 0.08 & 0.08 & 0.08 & 0.08 & 0.08 & 0.31 & 0.19 & 0.74 & \\ 0.08 & 0.08 & 0.08 & 0.08 & 0.08 & 0.08 & 0.08 & 0.31 & 0.31 & 0.74 & 0.74\end{array}$ $\begin{array}{lllllllllllll}0.08 & 0.08 & 0.08 & 0.08 & 0.08 & 0.08 & 0.08 & 0.31 & 0.31 & 0.74 & 0.74 & \\ 0.08 & 0.08 & 0.08 & 0.08 & 0.08 & 0.08 & 0.08 & 0.31 & 0.31 & 0.74 & 0.74 & 0.74\end{array}$ $\begin{array}{lllllllllllll}0.19 & 0.08 & 0.08 & 0.08 & 0.08 & 0.08 & 0.08 & 0.31 & 0.19 & 0.31 & 0.31 & 0.19 & 0.19\end{array}$ $\begin{array}{llllllllllllll}0.19 & 0.19 & 0.08 & 0.08 & 0.08 & 0.08 & 0.08 & 0.41 & 0.19 & 0.64 & 0.52 & 0.74 & 0.74 & 0.41\end{array}$ $\begin{array}{lllllllllllllll}0.30 & 0.08 & 0.08 & 0.08 & 0.08 & 0.08 & 0.08 & 0.41 & 0.08 & 0.08 & 0.08 & 0.08 & 0.08 & 0.52 & 0.19\end{array}$ $\begin{array}{lllllllllllllllll}0.08 & 0.08 & 0.08 & 0.08 & 0.08 & 0.08 & 0.08 & 0.08 & 0.08 & 0.08 & 0.08 & 0.08 & 0.08 & 0.08 & 0.08 & 0.08\end{array}$ $\begin{array}{lllllllllllllllllll}0.08 & 0.08 & 0.08 & 0.08 & 0.08 & 0.08 & 0.08 & 0.08 & 0.08 & 0.08 & 0.08 & 0.08 & 0.08 & 0.08 & 0.08 & 0.08 & 0.74\end{array}$ $\begin{array}{llllllllllllllllllll}0.08 & 0.08 & 0.08 & 0.08 & 0.08 & 0.08 & 0.08 & 0.08 & 0.08 & 0.08 & 0.08 & 0.08 & 0.08 & 0.08 & 0.08 & 0.08 & 0.74 & \\ 0.08 & 0.08 & 0.08 & 0.08 & 0.08 & 0.08 & 0.08 & 0.08 & 0.08 & 0.08 & 0.08 & 0.08 & 0.08 & 0.08 & 0.08 & 0.31 & 0.31 & 0.31\end{array}$ $\begin{array}{lllllllllllllllllll}0.08 & 0.08 & 0.08 & 0.08 & 0.08 & 0.08 & 0.08 & 0.08 & 0.08 & 0.08 & 0.08 & 0.08 & 0.08 & 0.08 & 0.08 & 0.31 & 0.31 & 0.31 & \\ 0.08 & 0.08 & 0.08 & 0.08 & 0.08 & 0.08 & 0.08 & 0.08 & 0.08 & 0.08 & 0.08 & 0.08 & 0.08 & 0.08 & 0.08 & 0.31 & 0.31 & 0.31 & 0.74\end{array}$ $\begin{array}{llllllllllllllllllllllll}0.08 & 0.08 & 0.08 & 0.08 & 0.08 & 0.08 & 0.08 & 0.08 & 0.08 & 0.08 & 0.08 & 0.08 & 0.08 & 0.08 & 0.08 & 0.31 & 0.41 & 0.41 & 0.64 & 0.64\end{array}$ $\begin{array}{lllllllllllllllllllll}0.08 & 0.08 & 0.08 & 0.08 & 0.08 & 0.08 & 0.08 & 0.08 & 0.08 & 0.08 & 0.08 & 0.08 & 0.08 & 0.08 & 0.08 & 0.31 & 0.41 & 0.41 & 0.64 & 0.64 & 0.74\end{array}$ $\begin{array}{llllllllllllllllllllll}0.08 & 0.19 & 0.08 & 0.08 & 0.08 & 0.08 & 0.19 & 0.08 & 0.08 & 0.08 & 0.08 & 0.08 & 0.08 & 0.08 & 0.08 & 0.08 & 0.08 & 0.19 & 0.08 & 0.08 & 0.08 & 0.19\end{array}$ 24 Ellesmerian Thrust Belt

Minimum eigenvalue $=\mathbf{- 0 . 2 1 1}$ Bias Adjustment $=0.212$ 
Table 7a: Input values for oil accumulations in the Brookian Topset Play

NPRA Assessment Form-2001

PLAY: Brookian Topset

Play area: $\quad 1830410^{3}$ Acres

\section{OIL ACCUMULATION VOLUME PARAMETERS}

\begin{tabular}{|c|c|c|c|c|c|c|c|}
\hline \multirow[b]{2}{*}{ ATTRIBUTES } & \multirow{2}{*}{$\begin{array}{l}\text { Est Shape } \\
(1 \text { to } 5)\end{array}$} & \multicolumn{4}{|c|}{ PROB OF AND GREATER THAN } & \multirow{2}{*}{$\begin{array}{l}\text { Knowledge } \\
\text { Level } 1-3^{5}\end{array}$} & \\
\hline & & LTP & 0.50 & 0.05 & $\operatorname{Max}$ & & \\
\hline \multirow{4}{*}{$\begin{array}{l}\text { NET RESERVOIR THICKNESS } \\
\text { AREA OF CLOSURE }^{2} \\
\text { POROSITY } \\
\text { TRAP FILL } \\
\text { TRA.4 }\end{array}$} & 2 & 25 & 50 & 75 & 200 & 2 & \\
\hline & 2 & 3 & 5 & 10 & 20 & 2 & \\
\hline & 1 & 15 & 18 & 19 & 21 & 2 & \\
\hline & 3 & 40 & 50 & 75 & 100 & 2 & Enter $\mathrm{POR}^{*} \mathrm{Sw}$ \\
\hline \multicolumn{2}{|l|}{ HYDROCARBON PORE VOL } & 9 & 12 & 13 & 15 & & 6 \\
\hline \multirow{2}{*}{\multicolumn{2}{|c|}{$\begin{array}{l}\text { Approx mm bbl }(f v f=1) \\
\text { Recov mm bbl at surface }\end{array}$}} & 17.5 & 116.4 & 567.3 & 4654.8 & & \\
\hline & & 4.7 & 31.2 & 152.1 & 1248.1 & & \\
\hline \multicolumn{8}{|c|}{ 1-thickness in feet, 2-thousands of acres, 3-percent, 4-correlation between Porosity and Water Saturation $=-1.0$} \\
\hline \multirow{2}{*}{$\begin{array}{l}\text { TRAP DEPTH (in } 1000 \mathrm{ft} \text { ) } \\
\text { (from sea level) }\end{array}$} & 4 & 1 & 5 & 9 & 10 & 1 & \\
\hline & & Irface to sea & vel correctic & (1000 ft): & 0.492 & & \\
\hline
\end{tabular}

\section{OIL ACCUMULATION CHARACTERISTICS}

Oil recovery factor \% 35

Type of reservoir-drive (check any that apply):

FVF (Formation volume factor, rb/stb):

\begin{tabular}{|lr|}
\hline Pressure(psi) & 2500 \\
temp(deg F) & 125 \\
SolGasGr & 0.705 \\
F & 717 \\
\hline
\end{tabular}

Depletion: $x$

Gas expansion:

GOR (Associated gas to oil ratio, cu.ft./bbl, at stp):

(at median depth)

$F V F=0.972+0.000147^{\star} F^{\wedge} 1.175$

$\mathrm{P}=\mathrm{TD}^{\star} 0.5^{\star} 1000 \quad \mathrm{TD}=$ trap depth (thous $\mathrm{ft}$ ), $\mathrm{P}=$ pressure $(\mathrm{psi})$

$$
T=19 * T D+30 \quad T=\text { temp deg } F \quad L N \text { is log base } e
$$

$S G G=\left(\left(0.1402^{*} \mathrm{LN}(\mathrm{C} 27+14.7)-0.4227\right)+\left(0.1369^{*} \mathrm{LN}(\mathrm{C} 28)+0.0156\right)+\left(0.1704^{*} \mathrm{LN}(\mathrm{E} 37)+0 . \mathrm{AG}=\mathrm{API}\right.\right.$ gravity Uncorrected $\mathrm{GOR}=\mathrm{SGG}^{\star}\left((\mathrm{P}+14.7)^{\star} 10^{\wedge}\left(0.0125^{\star} \mathrm{AG}\right) /\left(18^{\star} 10^{\wedge}\left(0.00091^{\star} \mathrm{T}\right)\right)\right)^{\wedge}(1 / 0.83)$

$\mathrm{F}=$ Final $\mathrm{GOR}^{\star}(\mathrm{SGG} / \mathrm{OG})^{\wedge} 0.5+1.25^{\star} \mathrm{T}$

$O G=141.5 /(131.5+A G)$

\begin{tabular}{r|r} 
& 613 \\
\hline
\end{tabular}

NGLR (Natural gas liquids to associated gas ratio, bbls/million cu.ft., at $\operatorname{stp})=4.5985^{\star} \exp \left(.1711^{\star} T D\right.$ )

10.8 (median depth)

Oil quality parameters:

$$
\text { API gravity }
$$

Sulfur content of oil

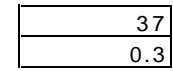

Oil Grav (ratio)

0.840

Associated gas quality parameters:

Hydrogen sulfide $\%$

CO2 contamination \%

Other inert gases:
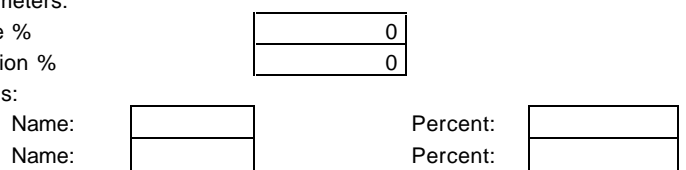

TIME OF TRAP DEVELOPMENT

STRATIGRAPHIC COMPONENT (Ma)

STRUCTURAL COMPONENT (Ma)

\begin{tabular}{|r|r|r|}
\multicolumn{1}{l|}{ BEGIN } & \multicolumn{1}{c}{ PEAK } & \multicolumn{2}{c|}{ END } \\
\hline 120 & 115 & 95 \\
\hline & & \\
\hline
\end{tabular}

Assessor's Name: Dave Houseknecht

Date of Data Entry MM/DD/YYYY:

Date of Simulation Run MM/DD/YYYY:

Note: only enter play name and assessor's name on Oil worksheet 
Table 7b: Input values for nonassociated gas accumulations in the Brookian Topset Play NPRA Assessment Form-2001

PLAY: Brookian Topset

\section{NONASSOCIATED GAS ACCUMULATION VOLUME PARAMETERS}

\begin{tabular}{|c|c|c|c|c|c|c|c|}
\hline \multirow[b]{2}{*}{ ATTRIBUTES } & \multirow{2}{*}{$\begin{array}{l}\text { Est Shape } \\
(1 \text { to } 5)\end{array}$} & \multicolumn{4}{|c|}{ PROB OF AND GREATER THAN } & \multirow{2}{*}{$\begin{array}{l}\text { Knowledge } \\
\text { Level } 1-3^{5} \\
\end{array}$} & \\
\hline & & LTP & 50 & 5 & Max & & \\
\hline \multirow{4}{*}{$\begin{array}{l}\text { NET RESERVOIR THICKNESS } \\
\text { AREA OF CLOSURE }^{1} \\
\text { POROSITY } \\
\text { TRAP FILL }^{3}\end{array}$} & 2 & 25 & 50 & 75 & 200 & 2 & \\
\hline & 2 & 3 & 5 & 10 & 20 & 2 & \\
\hline & 1 & 15 & 18 & 19 & 21 & 2 & \\
\hline & 3 & 40 & 50 & 75 & 100 & 2 & Uses oil POR ${ }^{\star}$ Sw \\
\hline \multicolumn{2}{|l|}{ HYDROCARBON PORE VOL ${ }^{3,4}$} & 9 & 12 & 13 & 15 & & 6 \\
\hline \multirow{2}{*}{\multicolumn{2}{|c|}{$\begin{array}{l}\text { Approx in place bcf } \\
\text { Recov bcf at surface }\end{array}$}} & 0.10 & 0.65 & 3.19 & 26.14 & & \\
\hline & & 11.9 & 79.6 & 387.9 & 3182.4 & & \\
\hline
\end{tabular}

1-thickness in feet, 2-thousands of acres, 3-percent, 4-correlation between Porosity and Water Saturation = -1.0

5-Knowledge Level: 1=High, 2=Medium, 3=Low; LTP=Left Truncation Point

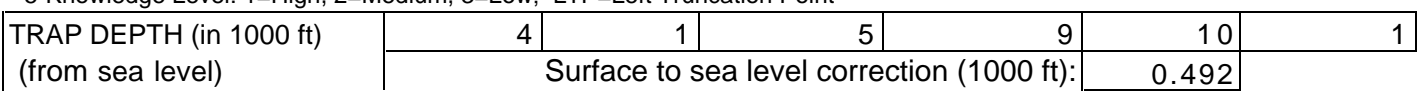

\section{NONASSOCIATED GAS ACCUMULATION CHARACTERISTICS}

NA Gas recovery factor \% 65

Type of reservoir-drive (check any that apply):

$$
\text { Water: }
$$

Gas expansion:

Natural gas liquids plus condensate to non-associated gas (bbls/million cf) (in place): NGL-NAG $=1.785^{\star} \mathrm{TD}$

$$
8.9 \text { (at median) }
$$

Non-associated gas quality parameters: Hydrogen sulfide \% CO2 contamination \% Other inert gases: Name: Name:

Gas fvf 187.3 (at median depth)

Name:

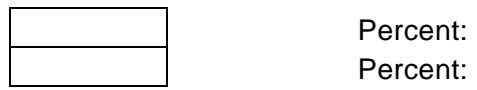

Percent:

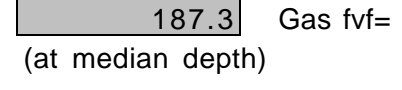

$113.3+21.1^{*} T D-0.812^{*} T^{\wedge} 2+0.0116^{*} T^{\wedge}{ }^{\wedge} 3$

$\mathrm{TD}=$ trap depth (thous $\mathrm{ft}$ )

\begin{tabular}{l} 
TIME OF TRAP DEVELOPMEN BEGIN PEAK \\
STRATIGRAPHIC COMPONENT (Ma) \\
STRUCTURAL COMPONENT (Ma) \\
\hline
\end{tabular}

Assessor's Name: Dave Houseknecht Date of Data Entry MM/DD/YYYY: Date of Simulation Run MM/DD/YYYY: 
Table 7c: Input risking values for the Brookian Topset Play

NPRA Assessment Form-2001

Play: Brookian Topset

\section{RISKING}

MINIMUM ACCUMULATION SIZE, MAS (Millions of BBL in place)

PRERISKED FREQUENCY DISTRIBUTION (Oil plus Gas)

NUM OF PROSPECTS

$>$ MINIMUM SIZE

PROB OF AND GREATER THAN

Knowledge

\begin{tabular}{|r|r|r|r|r|r|}
\hline Est Shape & Min & 50 & 5 & Max & Level 1-3 \\
\hline 2 & 25 & 50 & 75 & 100 & 3 \\
\hline
\end{tabular}

5-Knowledge Level: 1=High, 2=Medium, 3=Low; LTP=Left Truncation Point

\section{ATTRIBUTES}

PLAY

ATTRIBUTES

CHARGE (C)

TRAP (T)
TIMING (F)

Probability that play contains at least 1 reservoir $>=$ minimum size (CXTXF)

PROSPECT

ATTRIBUTES

CHARGE (c)

TRAP ( $\mathrm{t})$

TIMING ( $f$ )

Probability that a randomly chosen prospect is favorable (cxtxf)

Play Attributes $x$ Prospect Attributes (CxTxFxcxtxf)

FRACTION OF ACCUMULATIONS BEING OIL

Fraction NA Gas=1-Fraction(Oil)
PROBABILITY OF FAVORABLE

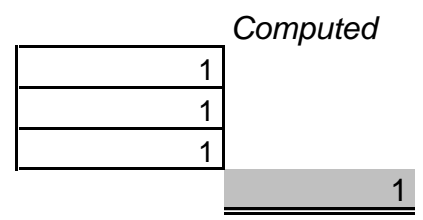

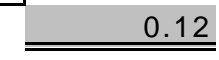

0.12

0.12

0.9

\begin{tabular}{|l|r|r|r|}
\cline { 2 - 4 } \multicolumn{1}{c|}{} & \multicolumn{1}{c|}{ Land } & \multicolumn{1}{c|}{ Oil } & \multicolumn{2}{c|}{ Gas } \\
\hline Federal & 90 & 86 & 92 \\
\hline State & 6 & 10 & 5 \\
\hline Native & 4 & 4 & 3 \\
\hline
\end{tabular}

Assessor's Name:

Dave Houseknecht

Date of Data Entry MM/DD/YYYY:

Date of Simulation Run MM/DD/YYYY:

$10 / 19 / 01$

$1 / 16 / 02$ 
Table 7d: Distribution of fitted hydrocarbon volume attributes, trap depth, and number of prospects for the Brookian Topset Play
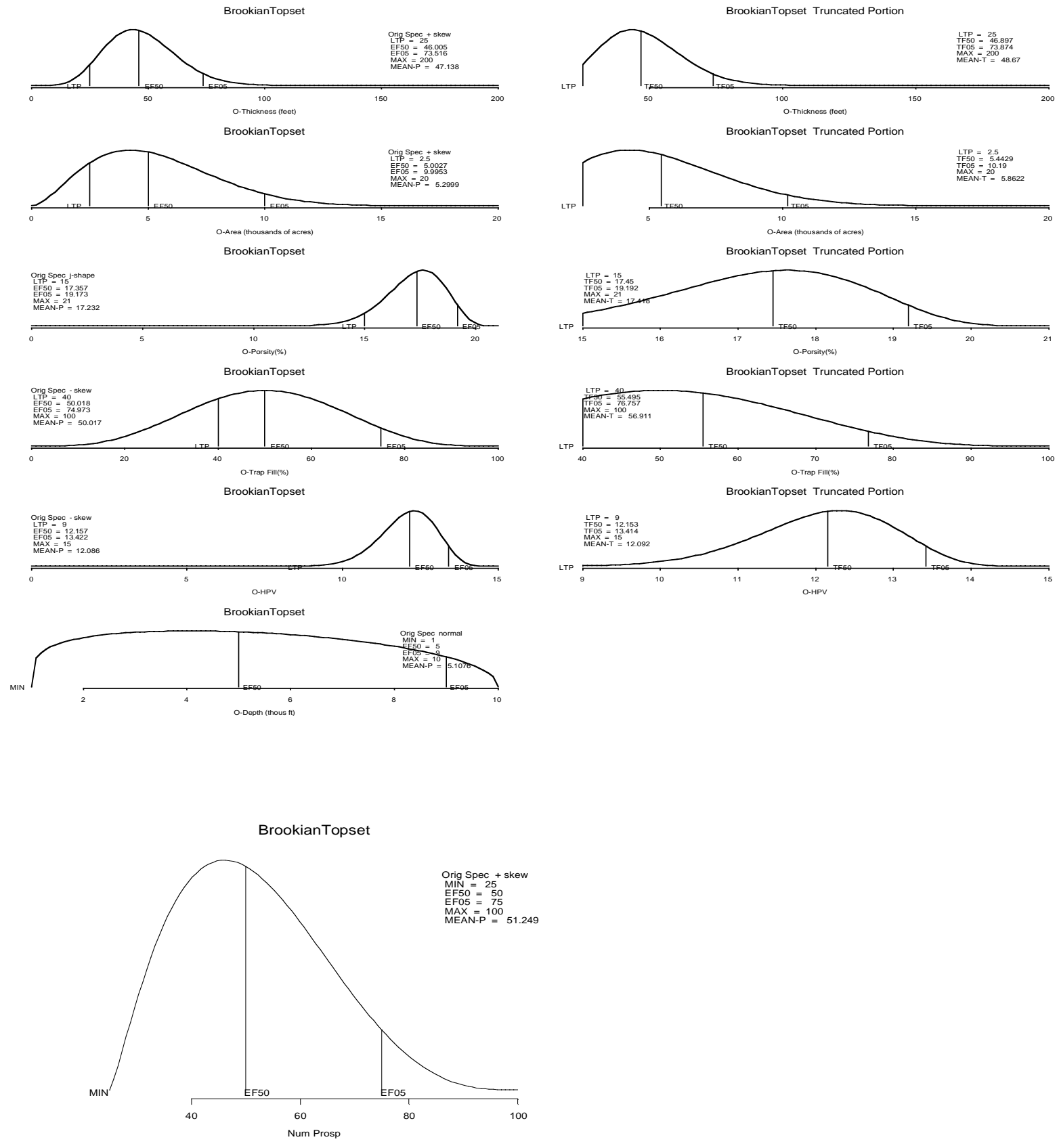
Table 7e: Estimated undiscovered oil and gas resources for the Brookian Topset Play Deposit stats

Brookian Topset
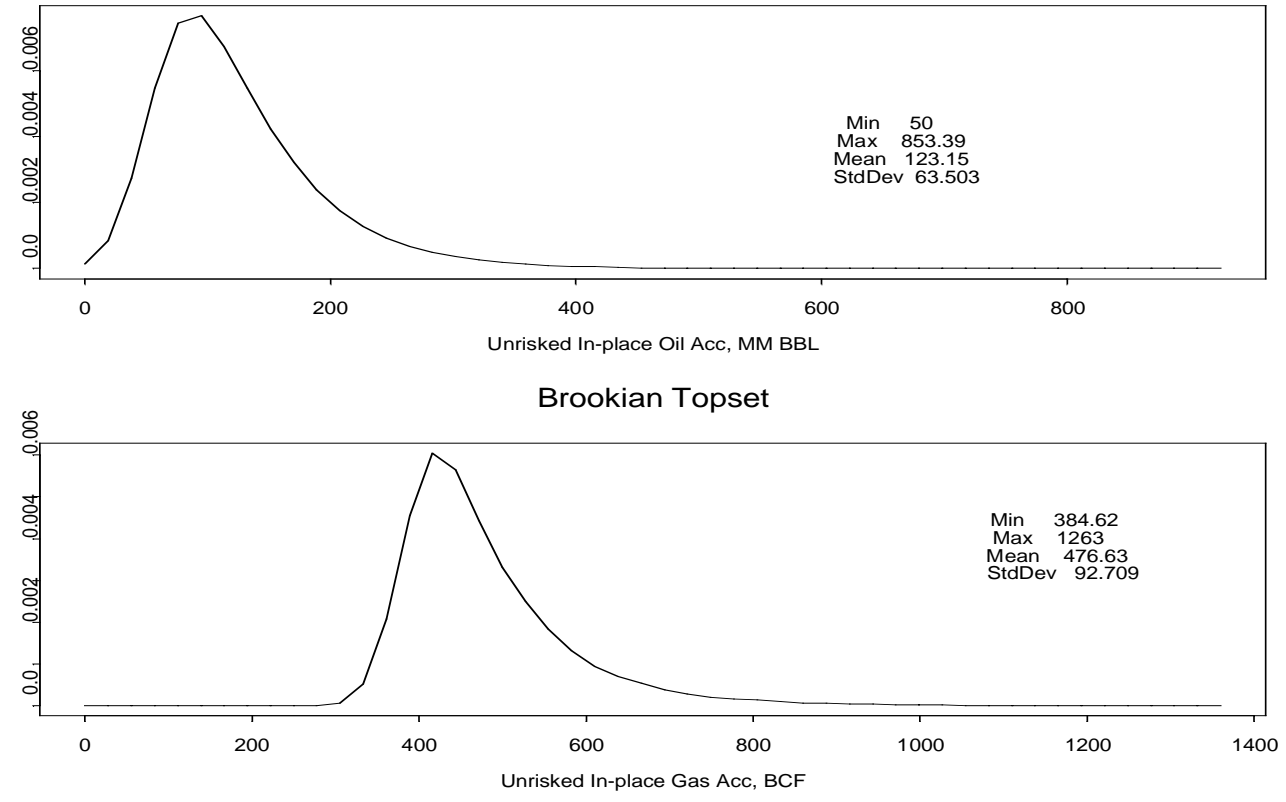

\begin{tabular}{|c|c|c|c|c|c|c|c|}
\hline $\begin{array}{l}\text { Play Stats } \\
\text { Resource }\end{array}$ & Unit & Mean & StdDev & F95 & F50 & F05 & $\operatorname{Max}$ \\
\hline In-place oil & MMBO & 683.05 & 358.23 & 174.00 & 637.65 & 1329.23 & 2480.13 \\
\hline In-place NA gas & BCFG & 295.72 & 388.72 & 0.00 & 0.00 & 1044.66 & 3630.99 \\
\hline Recov oil & MMBO & 239.07 & 125.38 & 60.90 & 223.18 & 465.23 & 868.05 \\
\hline Recov assoc diss gas & BCFG & 150.94 & 81.83 & 36.22 & 140.58 & 299.35 & 590.58 \\
\hline Recov NA gas & BCFG & 192.22 & 252.67 & 0.00 & 0.00 & 679.03 & 2360.14 \\
\hline Recov NGL (ADG) & MMBO & 2.90 & 1.55 & 0.71 & 2.71 & 5.72 & 11.07 \\
\hline Recov NGL (NAG) & MMBO & 2.59 & 3.50 & 0.00 & 0.00 & 9.58 & 30.68 \\
\hline Num oil deposits & & 5.55 & 2.64 & 2.00 & 5.00 & 10.00 & 19.00 \\
\hline Num NA gas deposits & & 0.62 & 0.80 & 0.00 & 0.00 & 2.00 & 6.00 \\
\hline
\end{tabular}

Play Totals Brookian Topset
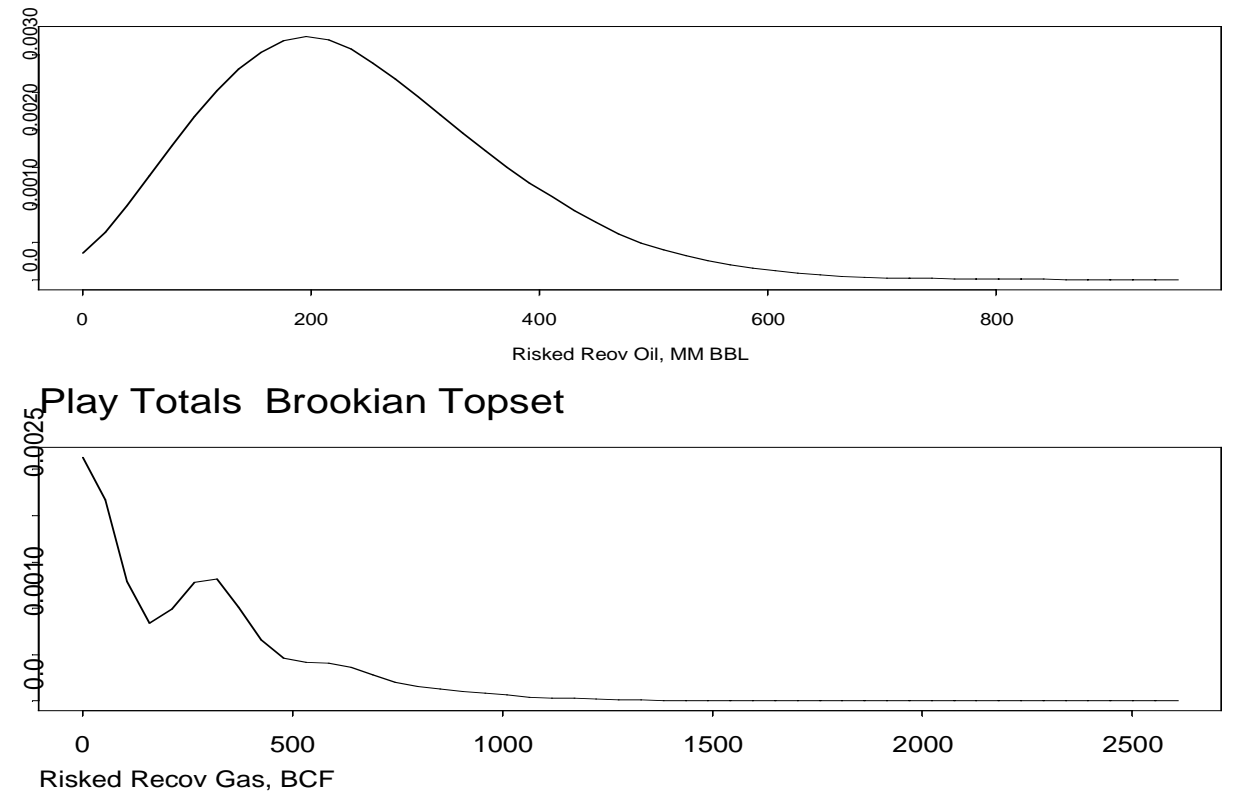
Table 7f: Estimated undiscovered oil and gas resources for the Brookian Topset Play showing number of deposits and volumes by accumulation size class.

\begin{tabular}{|c|c|c|c|c|c|}
\hline Start size class & Oil deposits & Oil (MMBO) & Start size class & Gas deposits & NA Gas (BCFG) \\
\hline 0 & 0.00 & 0.00 & 0 & 0.00 & 0.00 \\
\hline 8 & 0.00 & 0.00 & 48 & 0.00 & 0.00 \\
\hline 16 & 2.10 & 52.12 & 96 & 0.00 & 0.00 \\
\hline 32 & 2.62 & 117.57 & 192 & 0.56 & 163.31 \\
\hline 64 & 0.78 & 63.57 & 384 & 0.06 & 28.84 \\
\hline 128 & 0.04 & 5.77 & 768 & 0.00 & 0.08 \\
\hline 256 & 0.00 & 0.06 & 1536 & & \\
\hline 512 & & & 3072 & & \\
\hline Totals & 5.55 & 239.09 & & 0.62 & 192.24 \\
\hline
\end{tabular}
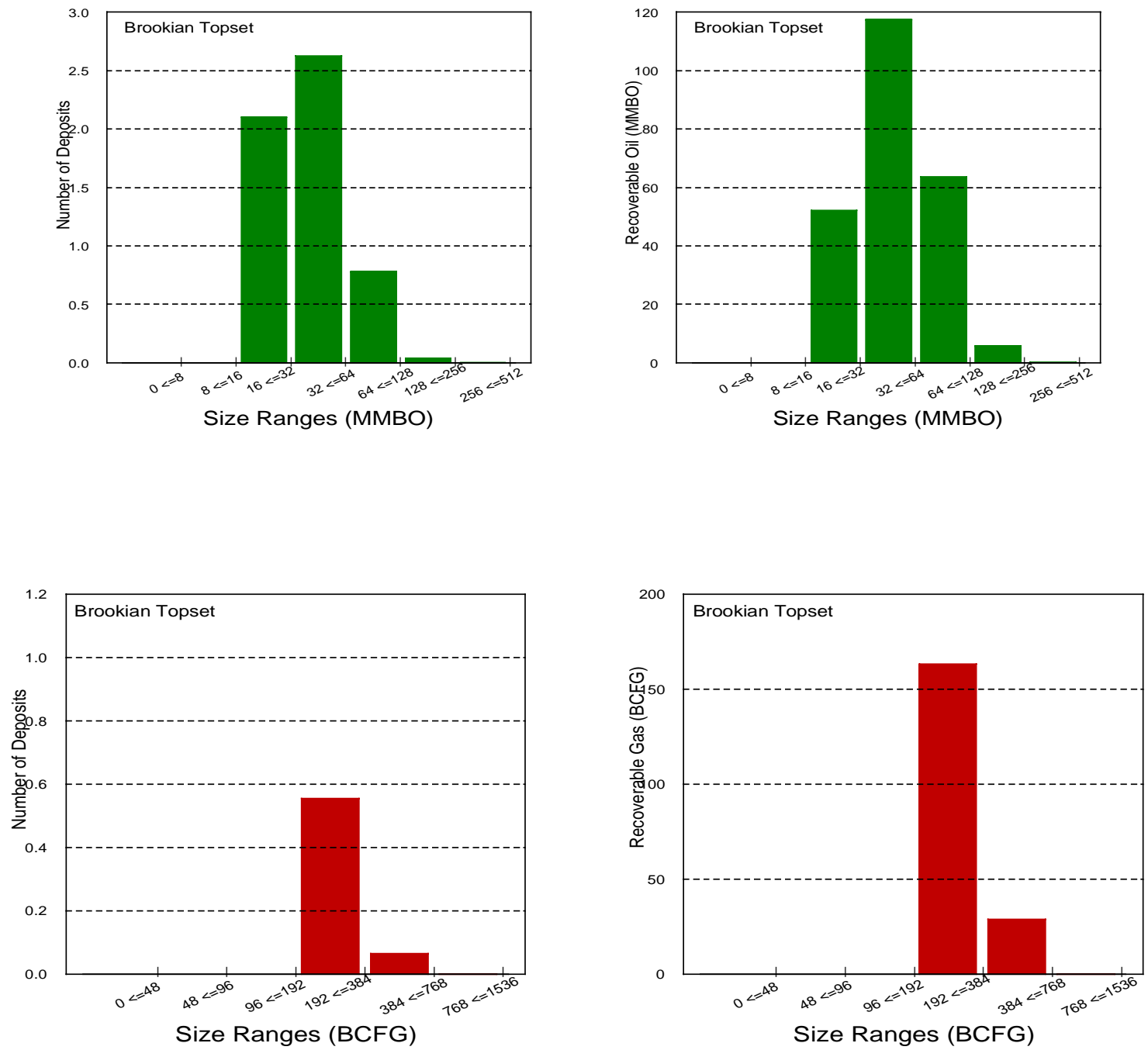
Table 8a: Input values for oil accumulations in the Brookian Clinoform North Play

NPRA Assessment Form-2001

PLAY: Brookian Clinoform North

Play area: $\quad 6791 \mid 10^{3}$ Acres

\section{OIL ACCUMULATION VOLUME PARAMETERS}

\begin{tabular}{|c|c|c|c|c|c|c|c|}
\hline \multirow[b]{2}{*}{ ATTRIBUTES } & \multirow{2}{*}{$\begin{array}{l}\text { Est Shape } \\
(1 \text { to } 5)\end{array}$} & \multicolumn{4}{|c|}{ PROB OF AND GREATER THAN } & \multirow{2}{*}{$\begin{array}{l}\text { Knowledge } \\
\text { Level } 1-3^{5}\end{array}$} & \\
\hline & & LTP & 0.50 & 0.05 & Max & & \\
\hline \multirow{4}{*}{$\begin{array}{l}\text { NET RESERVOIR THICKNESS } \\
\text { AREA OF CLOSURE }^{1} \\
\text { POROSITY }^{3,4} \\
\text { TRAP FILL }^{3}\end{array}$} & \begin{tabular}{|r|}
2 \\
\end{tabular} & 25 & 60 & 100 & 200 & 2 & \\
\hline & 2 & 3 & 5 & 15 & 30 & 2 & \\
\hline & 1 & 12 & 18 & 19 & 21 & 2 & \\
\hline & 7 & 80 & 100 & 100 & 100 & 2 & Enter POR*Sw \\
\hline \multirow{3}{*}{\multicolumn{2}{|c|}{$\begin{array}{l}\text { HYDROCARBON PORE } \mathrm{VOL}^{3,4} \\
\text { Approx mm bbl }(\mathrm{fvf}=1) \\
\text { Recov } \mathrm{mm} \text { bbl at surface }\end{array}$}} & 6 & 12 & 13 & 15 & & 6 \\
\hline & & 23.3 & 279.3 & 1512.8 & 6982.2 & & \\
\hline & & 5.5 & 66.4 & 359.8 & 1660.6 & & \\
\hline \multicolumn{7}{|c|}{$\begin{array}{l}\text { 1-thickness in feet, 2-thousands of acres, 3-percent, 4-correlation between Porosity and Water Saturation = }-1.0 \\
\text { 5-Knowledge Level: 1=High, 2=Medium, 3=Low; LTP=Left Truncation Point }\end{array}$} & \\
\hline \multirow{2}{*}{$\begin{array}{l}\text { TRAP DEPTH (in } 1000 \mathrm{ft} \text { ) } \\
\text { (from sea level) }\end{array}$} & 4 & 3 & 7 & 9 & 10 & 1 & \\
\hline & \multicolumn{4}{|c|}{ Surface to sea level correction (1000 ft): } & 0.074 & & \\
\hline
\end{tabular}

\section{OIL ACCUMULATION CHARACTERISTICS}

Oil recovery factor \% 35

Type of reservoir-drive (check any that apply):

FVF (Formation volume factor,

\begin{tabular}{|lr|} 
Pressure(psi) & 3500 \\
temp(deg F) & 163 \\
SolGasGr & 0.732 \\
F & 1013 \\
\hline
\end{tabular}
(stb):

(2)

GOR (Associated gas to oil ratio, cu.ft./bbl, at stp):

Depletion: $\mathrm{x}$ Gas expansion:

1.47 (at median depth) $\quad F V F=0.972+0.000147^{\star} F^{\wedge} 1.175$

$\mathrm{P}=\mathrm{TD}^{*} 0.5^{*} 1000 \quad \mathrm{TD}=$ trap depth (thous $\mathrm{ft}$ ), $\mathrm{P}=$ pressure $(\mathrm{psi})$

$T=19 * T D+30 \quad T=$ temp deg $F \quad L N$ is log base $e$

$S G G=\left((0.1402 * L N(C 27+14.7)-0.4227)+\left(0.1369^{*} \mathrm{LN}(\mathrm{C} 28)+0.0156\right)+(0.1704 * \mathrm{LN}(\mathrm{E} 37 \mathrm{AG}=\mathrm{API}\right.$ gravity

Uncorrected $\mathrm{GOR}=\mathrm{SGG}^{*}\left((\mathrm{P}+14.7)^{*} 10^{\wedge}\left(0.0125^{\star} \mathrm{AG}\right) /\left(18^{*} 10^{\wedge}\left(0.00091^{*} \mathrm{~T}\right)\right)\right)^{\wedge}(1 / 0.83)$

$\mathrm{F}=$ Final $\mathrm{GOR}^{\star}(\mathrm{SGG} / \mathrm{OG})^{\wedge} 0.5+1.25^{\star} \mathrm{T}$

$O G=141.5 /(131.5+A G)$

$$
\text { 866 Final GOR at median depth }
$$

NGLR (Natural gas liquids to associated gas ratio, bbls/million cu.ft., at stp) $=4.5985^{\star} \exp \left(.1711^{\star}\right.$ TD)

15.2 (median depth)

Oil quality parameters:

API gravity

Sulfur content of oil

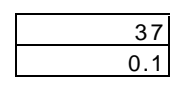

Oil Grav (ratio)

0.840

Associated gas quality parameters:

Hydrogen sulfide \%

CO2 contamination \%

Other inert gases:

Name:

Name:
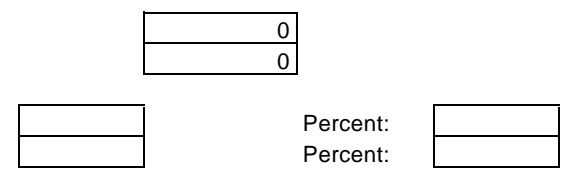

TIME OF TRAP DEVELOPMENT

STRATIGRAPHIC COMPONENT (Ma)

STRUCTURAL COMPONENT (Ma)

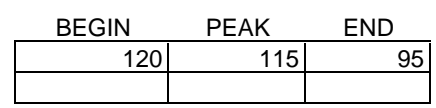

Assessor's Name: Dave Houseknecht

Date of Data Entry MM/DD/YYYY:

Date of Simulation Run MM/DD/YYYY:

$10 / 19 / 01$

Note: only enter play name and assessor's name on Oil worksheet 
Table 8b: Input values for nonassociated gas accumulations in the Brookian Clinoform North Play

NPRA Assessment Form-2001

PLAY: Brookian Clinoform North

NONASSOCIATED GAS ACCUMULATION VOLUME PARAMETERS

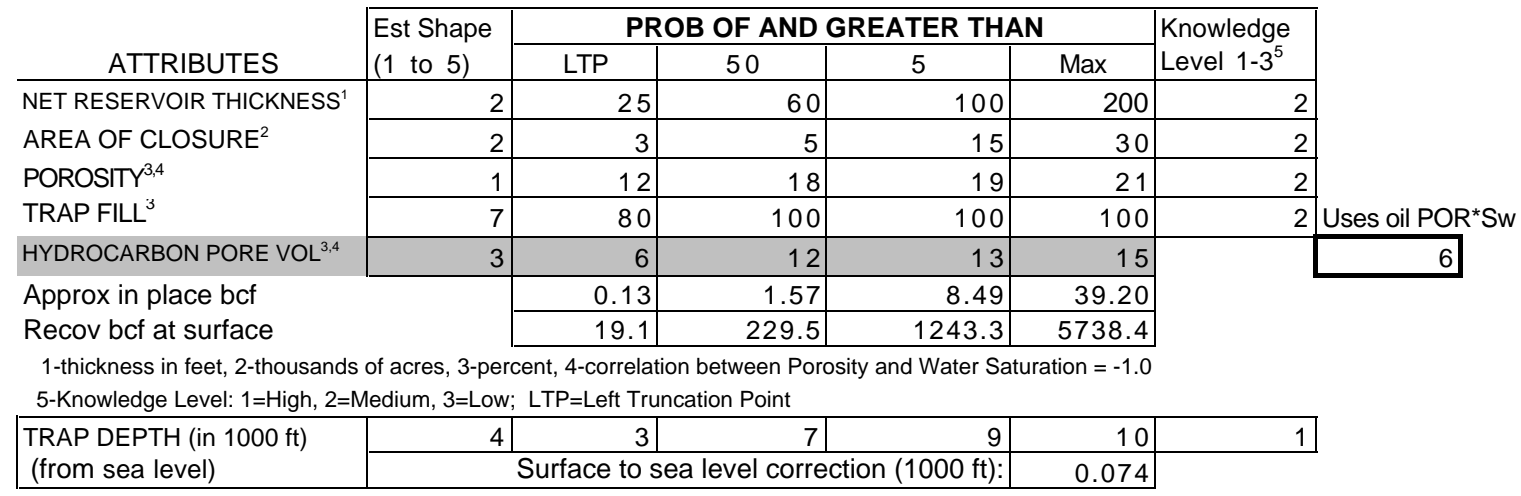

\section{NONASSOCIATED GAS ACCUMULATION CHARACTERISTICS}

NA Gas recovery factor \% 65

Type of reservoir-drive (check any that apply):

Water:

Gas expansion:

Natural gas liquids plus condensate to non-associated gas (bbls/million cf) (in place): NGL-NAG $=1.785^{\star} T D$

12.5 (at median)

Non-associated gas quality parameters:

Hydrogen sulfide \%

CO2 contamination \%

Other inert gases:

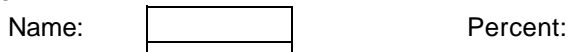

Name:

Percent:

Gas fvf

$$
225.2
$$

Gas

(at median depth)

Gas $\mathrm{fvf}=752.2^{*}\left(1-\mathrm{EXP}\left(-0.05728^{*} \mathrm{TD}\right)\right)$

$113.3+21.1^{\star} T D-0.812^{*} T^{\wedge} 2+0.0116^{*} T^{\wedge} 3$

$\mathrm{TD}=$ trap depth (thous $\mathrm{ft}$ )

\begin{tabular}{|c|c|c|c|}
\hline TIME OF TRAP DEVELOPMEN1 & BEGIN & PEAK & END \\
\hline STRATIGRAPHIC COMPONENT (Ma) & & & \\
\hline STRUCTURAL COMPONENT (Ma) & & & \\
\hline
\end{tabular}

Assessor's Name: Dave Houseknecht

Date of Data Entry MM/DD/YYYY:

Date of Simulation Run MM/DD/YYYY:

$10 / 19 / 01$

$1 / 18 / 02$ 
Table 8c: Input risking values for the Brookian Clinoform North Play NPRA Assessment Form-2001

Play: Brookian Clinoform North

\section{RISKING}

MINIMUM ACCUMULATION SIZE, MAS (Millions of BBL in place)

50

PRERISKED FREQUENCY DISTRIBUTION (Oil plus Gas)

NUM OF PROSPECTS

$>$ MINIMUM SIZE

\begin{tabular}{|c|c|c|c|c|c|}
\hline & \multicolumn{4}{|c|}{ PROB OF AND GREATER THAN } & \multirow{2}{*}{$\begin{array}{l}\text { Knowledge } \\
\text { Level } 1-3^{5}\end{array}$} \\
\hline Est Shape & Min & 50 & 5 & Max & \\
\hline 2 & 25 & 50 & 75 & 100 & 3 \\
\hline
\end{tabular}

5-Knowledge Level: 1=High, 2=Medium, 3=Low; LTP=Left Truncation Point

\section{ATTRIBUTES}

PLAY

ATTRIBUTES

\section{TIMING (F)}

Probability that play contains at least 1 reservoir $>=$ minimum size $(C x T x F)$

PROSPECT

ATTRIBUTES

CHARGE (c)

TRAP (t)

TIMING ( $f$ )

Probability that a randomly chosen prospect is favorable (cxtxf)

Play Attributes x Prospect Attributes (CxTxFxcxtxf)

FRACTION OF ACCUMULATIONS BEING OIL

Fraction NA Gas=1-Fraction(Oil)
PROBABILITY OF FAVORABLE

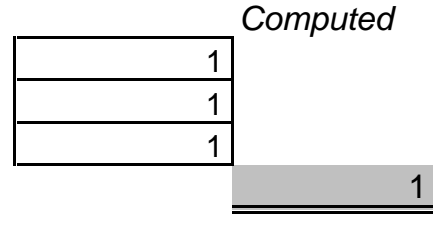

0.9

Allocation (percent):

\begin{tabular}{|l|r|r|r|}
\cline { 2 - 4 } \multicolumn{1}{c|}{} & \multicolumn{1}{c|}{ Land } & \multicolumn{1}{c|}{ Oil } & \multicolumn{2}{c|}{ Gas } \\
\hline Federal & 79 & 80 & 82 \\
\hline State & 13 & 15 & 13 \\
\hline Native & 8 & 5 & 5 \\
\hline
\end{tabular}

Assessor's Name:

Dave Houseknecht

Date of Data Entry MM/DD/YYYY:

Date of Simulation Run MM/DD/YYYY:

$10 / 19 / 01$

$1 / 18 / 02$ 
Table 8d: Distribution of fitted hydrocarbon volume attributes, trap depth, and number of prospects for the Brookian Clinoform North Play.
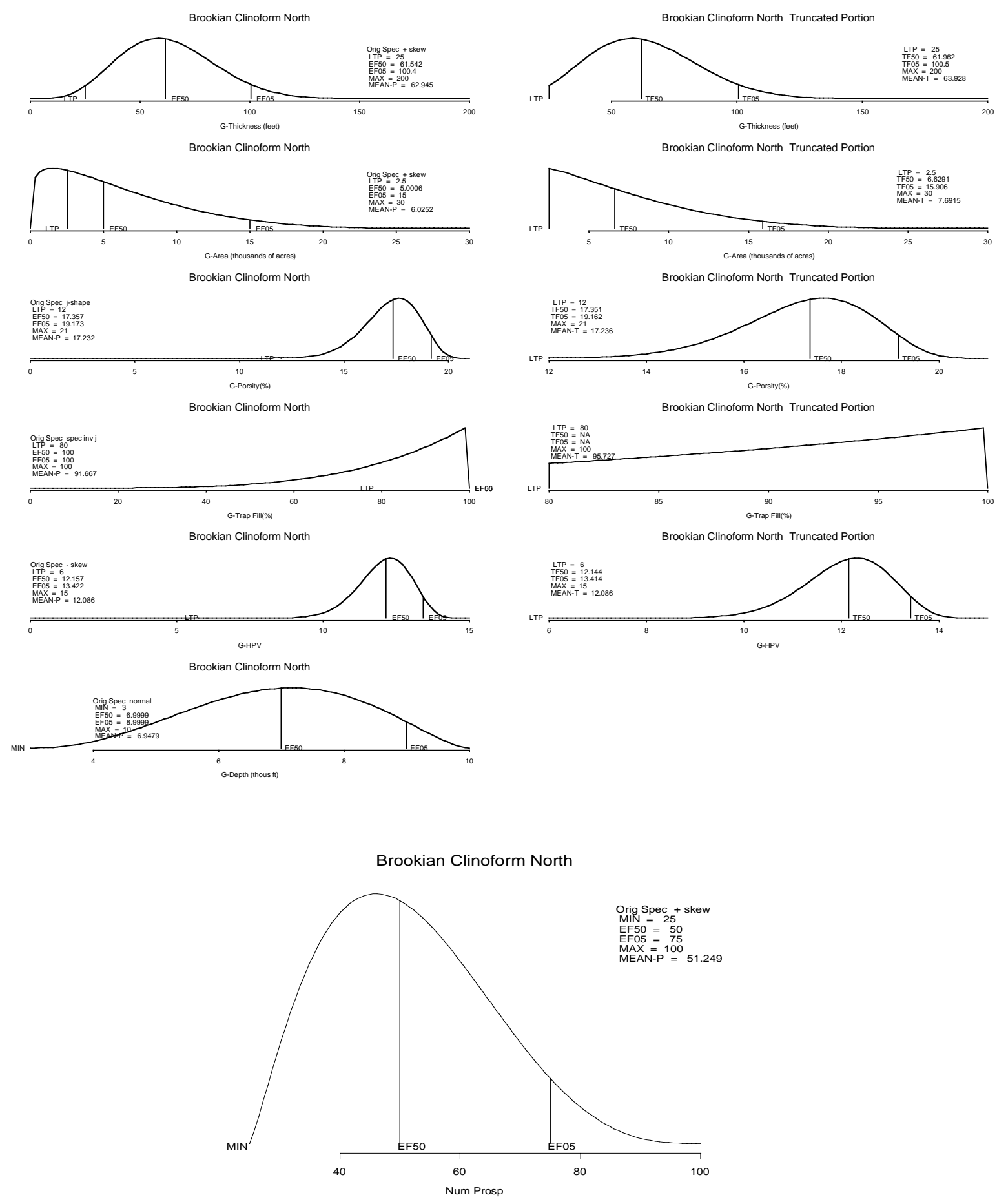
Table 8e: Estimated undiscovered oil and gas resources for the Brookian Clinoform North Play Deposit

Brookian Clinoform North

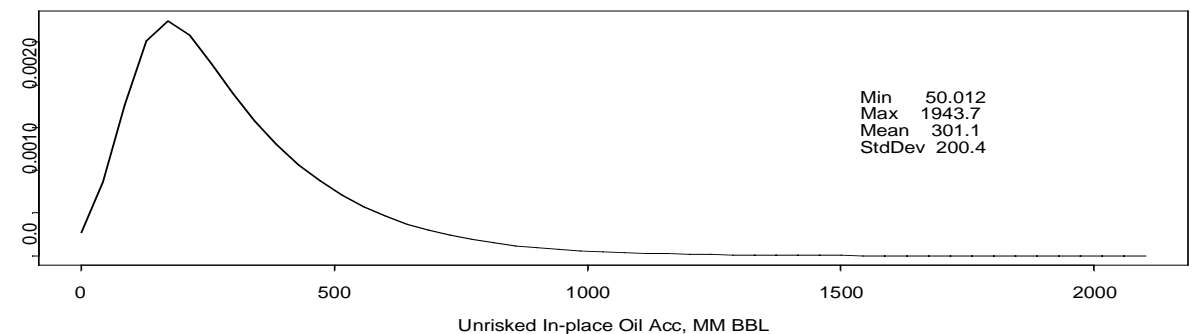

Unrisked In-place Oil Acc, MM BBL

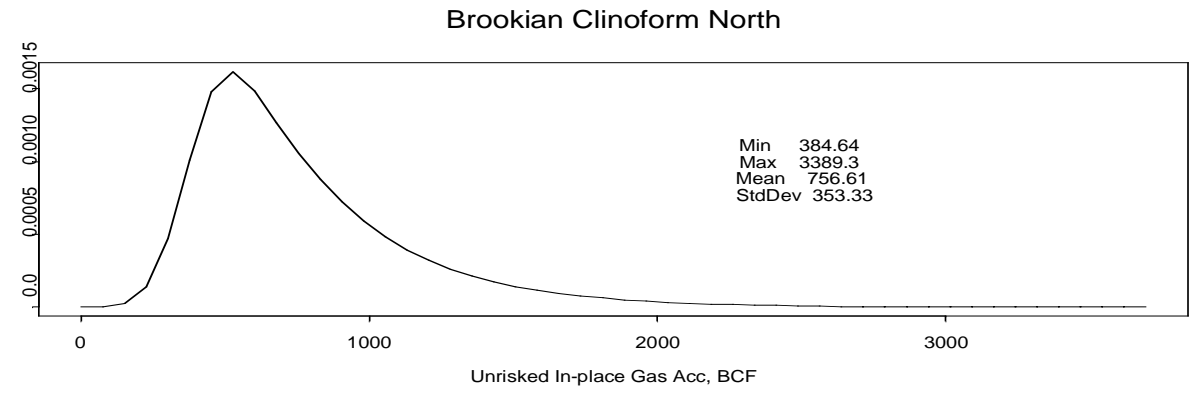

\begin{tabular}{|c|c|c|c|c|c|}
\hline \multicolumn{6}{|l|}{$\begin{array}{l}\text { Play } \\
\text { Play Stats }\end{array}$} \\
\hline Resource & Mean & StdDev & F95 & $\mathrm{F} 50$ & F05 \\
\hline In-place oil & 3732.23 & 1516.20 & 1538.27 & 3564.19 & 6449.03 \\
\hline In-place NA gas & 1036.98 & 1015.72 & 0.00 & 809.42 & 3028.58 \\
\hline Recov oil & 1306.28 & 530.67 & 538.40 & 1247.47 & 2257.16 \\
\hline Recov assoc diss gas & 1112.34 & 452.99 & 459.77 & 1062.75 & 1929.85 \\
\hline Recov NA gas & 674.04 & 660.22 & 0.00 & 526.12 & 1968.58 \\
\hline Recov NGL (ADG) & 20.12 & 8.22 & 8.27 & 19.20 & 35.05 \\
\hline Recov NGL (NAG) & 8.64 & 8.62 & 0.00 & 6.64 & 25.67 \\
\hline Num oil deposits & 12.40 & 4.45 & 6.00 & 12.00 & 20.00 \\
\hline Num NA gas deposits & 1.37 & 1.22 & 0.00 & 1.00 & 4.00 \\
\hline
\end{tabular}

Play Totals Brookian Clinoform North

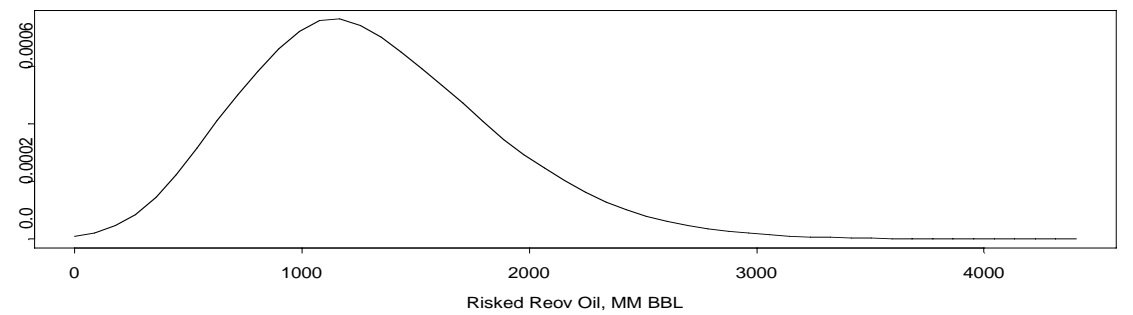

Play Totals Brookian Clinoform North

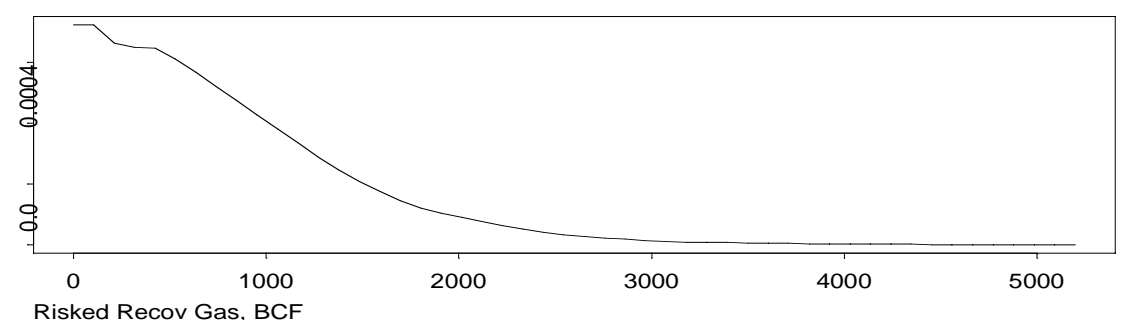


Table 8f: Estimated undiscovered oil and gas resources for the Brookian Clinoform North Play showing number of deposits and volumes by accumulation size class.

\begin{tabular}{|c|c|c|c|c|c|}
\hline Start size class & Oil deposits & Oil (MMBO) & Start size class & Gas deposits & NA Gas (BCFG) \\
\hline 0 & 0.00 & 0.00 & 0 & 0.00 & 0.00 \\
\hline 8 & 0.00 & 0.00 & 48 & 0.00 & 0.00 \\
\hline 16 & 0.71 & 18.78 & 96 & 0.00 & 0.00 \\
\hline 32 & 3.42 & 165.74 & 192 & 0.57 & 175.59 \\
\hline 64 & 4.78 & 440.51 & 384 & 0.65 & 341.77 \\
\hline 128 & 2.97 & 516.87 & 768 & 0.16 & 150.75 \\
\hline 256 & 0.51 & 160.63 & 1536 & 0.00 & 5.99 \\
\hline 512 & 0.01 & 3.75 & 3072 & & \\
\hline Totals & 12.40 & 1306.28 & & 1.37 & 674.10 \\
\hline
\end{tabular}
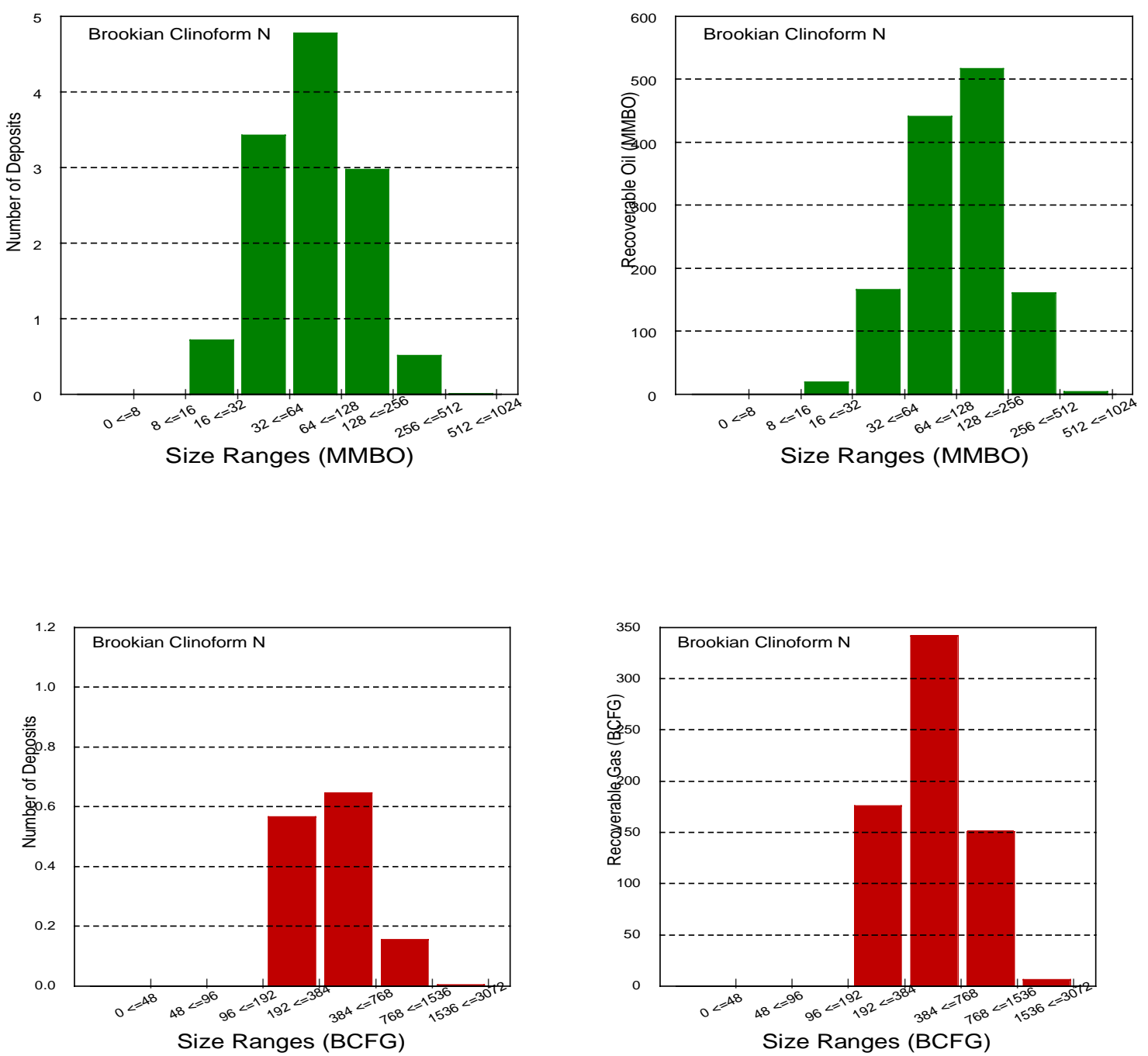
Table 9a: Input values for oil accumulations in the Brookian Clinoform Central Play

NPRA Assessment Form-2001

PLAY: Brookian Clinoform Central

\begin{tabular}{lr|r|r|} 
Play area: $\quad 6528$ & $10^{3}$ Acres \\
\hline
\end{tabular}

\section{OIL ACCUMULATION VOLUME PARAMETERS}

\begin{tabular}{|c|c|c|c|c|c|c|c|}
\hline \multirow[b]{2}{*}{ ATTRIBUTES } & \multirow{2}{*}{$\begin{array}{l}\text { Est Shape } \\
(1 \text { to } 5)\end{array}$} & \multicolumn{4}{|c|}{ PROB OF AND GREATER THAN } & \multirow{2}{*}{$\begin{array}{l}\text { Knowledge } \\
\text { Level } 1-3^{5}\end{array}$} & \multirow[b]{6}{*}{ Enter POR*Sw } \\
\hline & & LTP & 0.50 & 0.05 & Max & & \\
\hline \multirow{4}{*}{$\begin{array}{l}\text { NET RESERVOIR THICKNESS } \\
\text { AREA OF CLOSURE }^{1} \\
\text { POROSITY } \\
\text { TRAP FILL }^{3}\end{array}$} & \begin{tabular}{r|}
2 \\
\end{tabular} & 40 & 70 & 150 & 300 & 2 & \\
\hline & 2 & 4 & 6 & 18 & 35 & 2 & \\
\hline & 3 & 10 & 16 & 19 & 20 & 2 & \\
\hline & 7 & 80 & 100 & 100 & 100 & 2 & \\
\hline HYDROCARBON PORE VOL & 2 & 4 & 10 & 13 & 14 & & 6 \\
\hline \multirow{2}{*}{$\begin{array}{l}\text { Approx mm bbl (fvf }=1 \text { ) } \\
\text { Recov mm bbl at surface }\end{array}$} & & 39.7 & 325.8 & 2723.1 & 11404.3 & & \\
\hline & & 8.1 & 66.5 & 556.2 & 2329.2 & & \\
\hline \multicolumn{8}{|c|}{ 1-thickness in feet, 2-thousands of acres, 3-percent, 4-correlation between Porosity and Water Saturation $=-1.0$} \\
\hline \multirow{2}{*}{$\begin{array}{l}\text { TRAP DEPTH (in } 1000 \mathrm{ft} \text { ) } \\
\text { (from sea level) }\end{array}$} & \begin{tabular}{|r|}
4 \\
\end{tabular} & 9 & 10 & 11 & 12 & 1 & \\
\hline & Su & ice to sea & el correctio & $(1000 \mathrm{ft}):$ & 0.246 & & \\
\hline
\end{tabular}

\section{OIL ACCUMULATION CHARACTERISTICS}

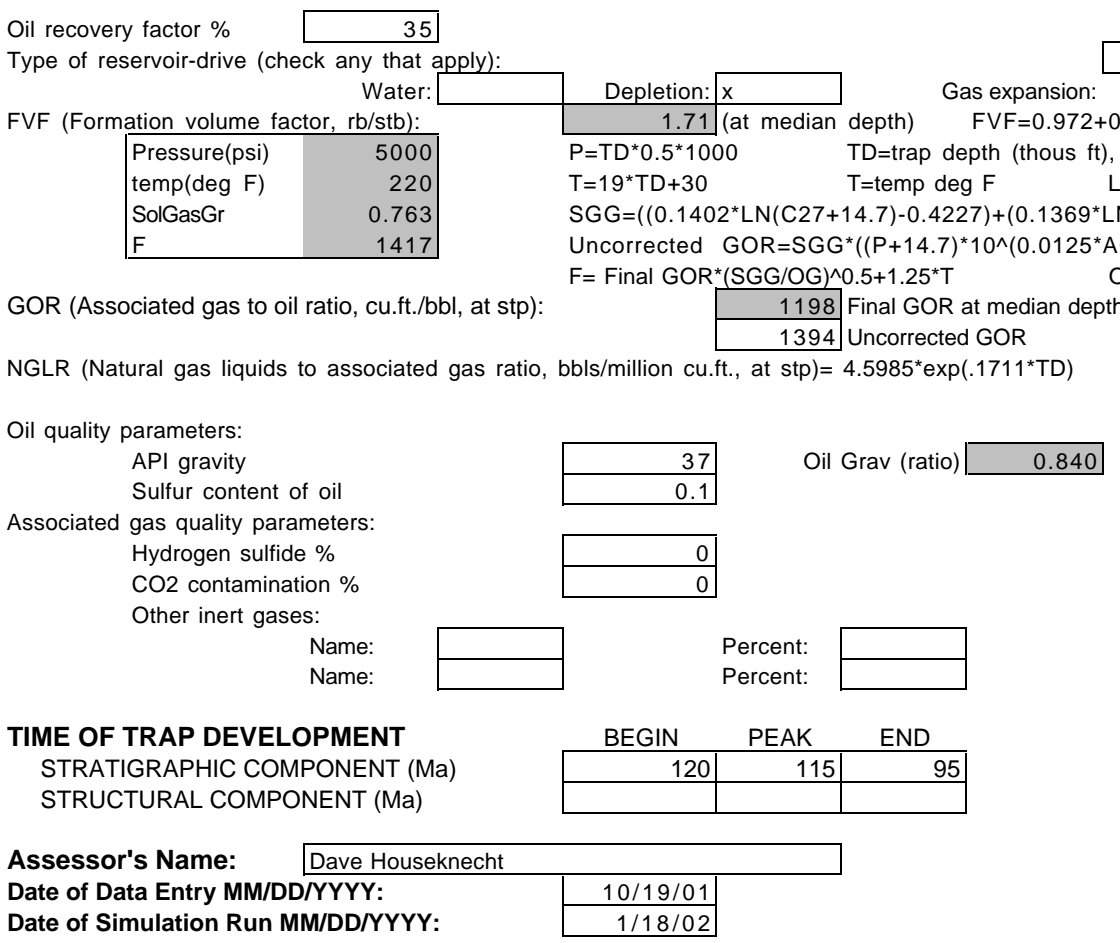

Note: only enter play name and assessor's name on Oil worksheet 
Table 9b: Input values for nonassociated gas accumulations in the Brookian Clinoform Central Play

NPRA Assessment Form-2001

PLAY: Brookian Clinoform Central

\section{NONASSOCIATED GAS ACCUMULATION VOLUME PARAMETERS}

\begin{tabular}{|c|c|c|c|c|c|c|c|}
\hline \multirow[b]{2}{*}{ ATTRIBUTES } & \multirow{2}{*}{$\begin{array}{l}\text { Est Shape } \\
(1 \text { to } 5)\end{array}$} & \multicolumn{4}{|c|}{ PROB OF AND GREATER THAN } & \multirow{2}{*}{$\begin{array}{l}\text { Knowledge } \\
\text { Level } 1-3^{5}\end{array}$} & \\
\hline & & LTP & 50 & 5 & Max & & \\
\hline \multirow{2}{*}{$\begin{array}{l}\text { NET RESERVOIR THICKNESS } \\
\text { AREA OF CLOSURE }\end{array}$} & 2 & 40 & 70 & 150 & 300 & 2 & \\
\hline & 2 & 4 & 6 & 18 & 35 & 2 & \\
\hline \multirow{2}{*}{$\begin{array}{l}\text { AREA OF CLOSURE }{ }^{2} \\
\text { POROSITY',4 } \\
\text { TRAP FILL }^{3}\end{array}$} & 3 & 10 & 16 & 19 & 20 & 2 & \\
\hline & 7 & 80 & 100 & 100 & 100 & 2 & Uses oil POR ${ }^{\star} \mathrm{S} w$ \\
\hline \multicolumn{2}{|l|}{ HYDROCARBON PORE VOL ${ }^{3,4}$} & 4 & 10 & 13 & 14 & & 6 \\
\hline \multirow{2}{*}{\multicolumn{2}{|c|}{$\begin{array}{l}\text { Approx in place bcf } \\
\text { Recov bcf at surface }\end{array}$}} & 0.22 & 1.83 & 15.29 & 64.03 & & \\
\hline & & 39.1 & 320.6 & 2679.5 & 11221.9 & & \\
\hline \multicolumn{7}{|c|}{$\begin{array}{l}\text { 1-thickness in feet, 2-thousands of acres, 3-percent, 4-correlation between Porosity and Water Saturation }=-1.0 \\
\text { 5-Knowledge Level: 1=High, 2=Medium, 3=Low; LTP=Left Truncation Point }\end{array}$} & \\
\hline \multirow{2}{*}{$\begin{array}{l}\text { TRAP DEPTH (in } 1000 \mathrm{ft} \text { ) } \\
\text { (from sea level) }\end{array}$} & 4 & 10 & 12 & 13 & 14 & 1 & \\
\hline & \multicolumn{4}{|c|}{ Surface to sea level correction $(1000 \mathrm{ft})$ : } & 0.246 & & \\
\hline
\end{tabular}

\section{NONASSOCIATED GAS ACCUMULATION CHARACTERISTICS}

NA Gas recovery factor \% 65

Type of reservoir-drive (check any that apply):

Water:

Gas expansion:

Natural gas liquids plus condensate to non-associated gas (bbls/million cf) (in place): NGL-NAG $=1.785^{*}$ TD $\quad 21.4$ (at median)

Non-associated gas quality parameters: Hydrogen sulfide \% CO2 contamination \% Other inert gases:

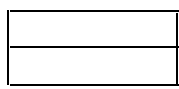

Name: Percent: Name:

Gas fvf 269.6 (at median depth)

Percent:

$=752.2^{*}\left(1-\operatorname{EXP}\left(-0.05728^{*} \mathrm{TD}\right)\right) \quad T \mathrm{~T}<=5.67$ thous $\mathrm{ft}$

$113.3+21.1^{*} T D-0.812^{*} T^{\wedge} 2+0.0116^{*} T^{\wedge} 3 \quad 5.67<T D<=30$

$\mathrm{TD}=$ trap depth (thous $\mathrm{ft}$ )

TIME OF TRAP DEVELOPMENT

STRATIGRAPHIC COMPONENT (Ma) STRUCTURAL COMPONENT (Ma)

\begin{tabular}{|c|c|c|}
\hline BEGIN & PEAK & END \\
\hline 120 & 115 & 95 \\
\hline
\end{tabular}

Assessor's Name: Dave Houseknecht Date of Data Entry MM/DD/YYYY:

Date of Simulation Run MM/DD/YYYY:

$10 / 19 / 01$


Table 9c: Input risking values for the Brookian Clinoform Central Play

NPRA Assessment Form-2001

Play: Brookian Clinoform Central

\section{RISKING}

MINIMUM ACCUMULATION SIZE, MAS (Millions of BBL in place)

50

PRERISKED FREQUENCY DISTRIBUTION (Oil plus Gas)

NUM OF PROSPECTS

$>$ MINIMUM SIZE

\begin{tabular}{|c|c|c|c|c|c|}
\hline & \multicolumn{4}{|c|}{ PROB OF AND GREATER THAN } & \multirow{2}{*}{$\begin{array}{l}\text { Knowledge } \\
\text { Level } 1-3^{5}\end{array}$} \\
\hline Est Shape & Min & 50 & \begin{tabular}{|l|l}
5 & \\
\end{tabular} & Max & \\
\hline 2 & 30 & 55 & 80 & 110 & 3 \\
\hline
\end{tabular}

5-Knowledge Level: 1=High, 2=Medium, 3=Low; LTP=Left Truncation Point

\section{ATTRIBUTES}

PLAY

ATTRIBUTES

CHARGE (C)

TRAP (T)
TIMING (F)

Probability that play contains at least 1 reservoir $>=$ minimum size $(C x T \times F)$

PROSPECT

ATTRIBUTES

CHARGE (c)

$\operatorname{TRAP}(\mathrm{t})$

TIMING (f)

Probability that a randomly chosen prospect is favorable (cxtxf)

Play Attributes $x$ Prospect Attributes (CxTxFxcxtxf)

FRACTION OF ACCUMULATIONS BEING OIL

Fraction NA Gas=1-Fraction(Oil)
PROBABILITY OF FAVORABLE

Computed

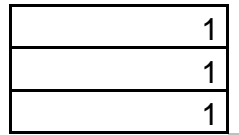

1 1

0.5

Allocation (percent):

\begin{tabular}{|l|r|r|r|}
\cline { 2 - 4 } \multicolumn{1}{c|}{} & \multicolumn{1}{c|}{ Land } & \multicolumn{1}{c|}{ Oil } & \multicolumn{2}{c|}{ Gas } \\
\hline Federal & 97 & 98 & 94 \\
\hline State & 1 & 1 & 3 \\
\hline Native & 2 & 1 & 3 \\
\hline
\end{tabular}

Assessor's Name:

$$
\text { Dave Houseknecht }
$$

Date of Data Entry MM/DD/YYYY:

Date of Simulation Run MM/DD/YYYY:

$10 / 19 / 01$

$1 / 18 / 02$ 
Table 9d: Distribution of fitted hydrocarbon volume attributes, trap depth, and number of prospects for the Brookian Clinoform Central Play

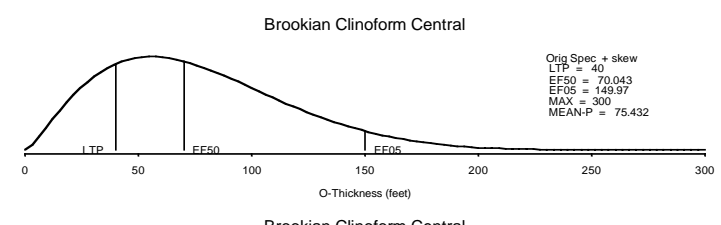

Brookian Clinoform Central

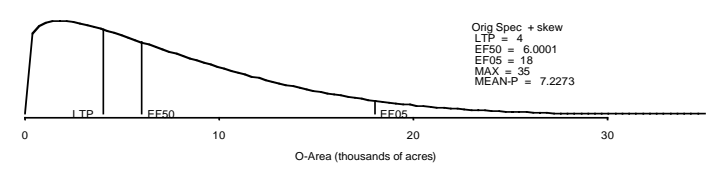

Brookian Clinoform Central

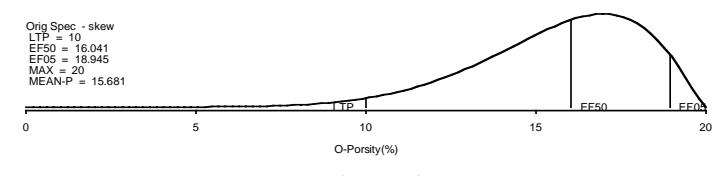

Brookian Clinoform Central
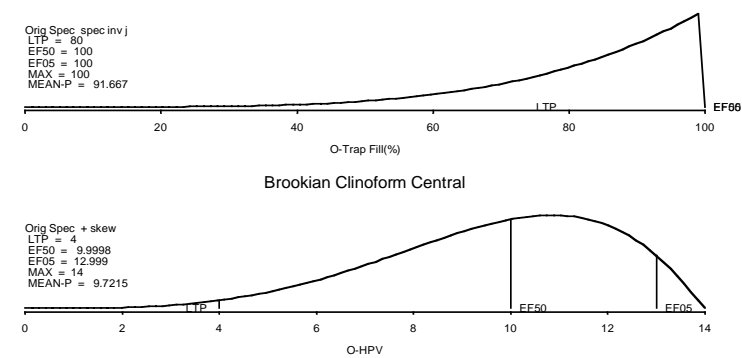

Brookian Clinoform Central

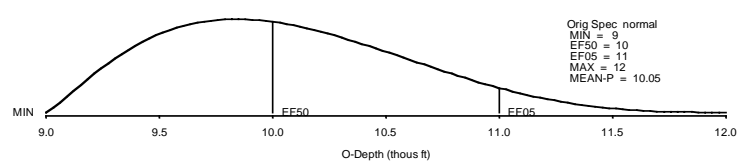

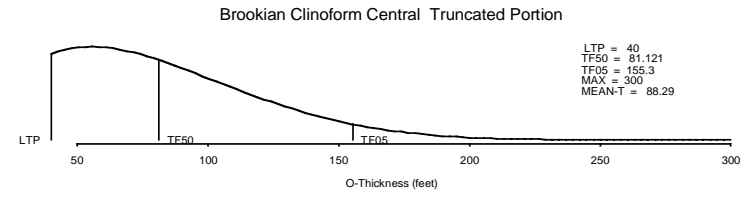

Brookian Clinoform Central Truncated Portion

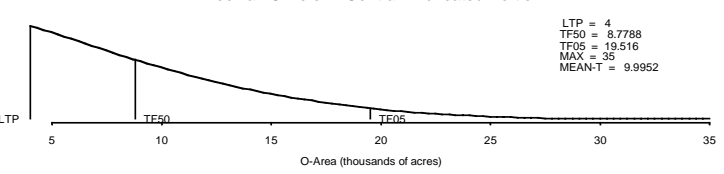

Brookian Clinoform Central Truncated Portion

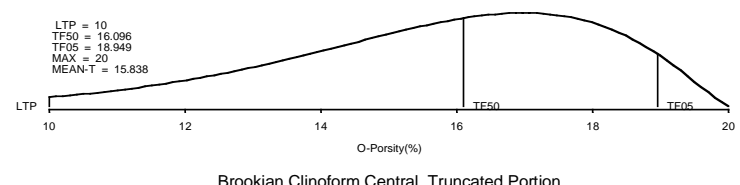

Brookian Clinoform Central Truncated Portion

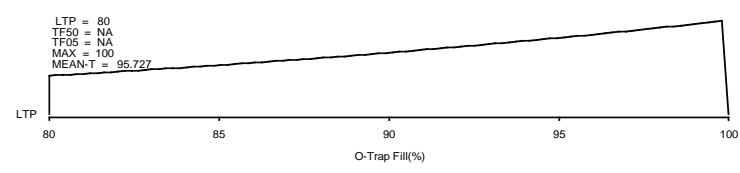

Brookian Clinoform Central Truncated Portion

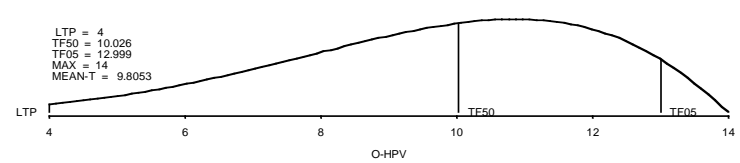

\section{Brookian Clinoform Central}

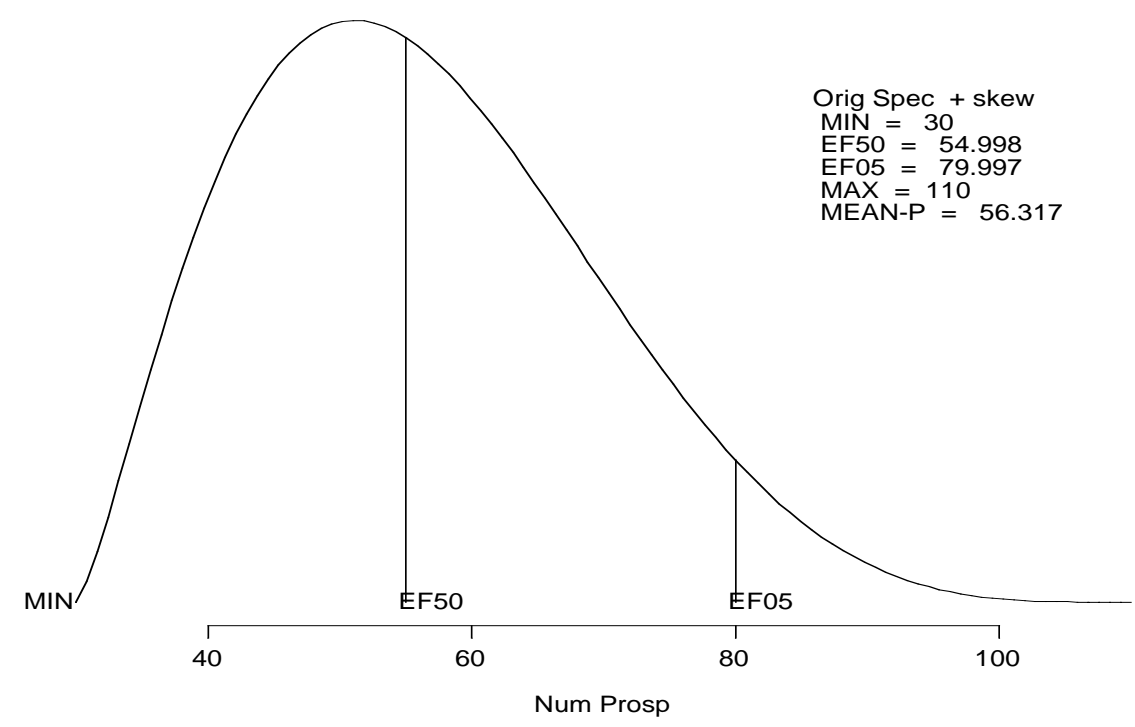


Table 9e: Estimated undiscovered oil and gas resources for the Brookian Clinoform Central Play Deposit

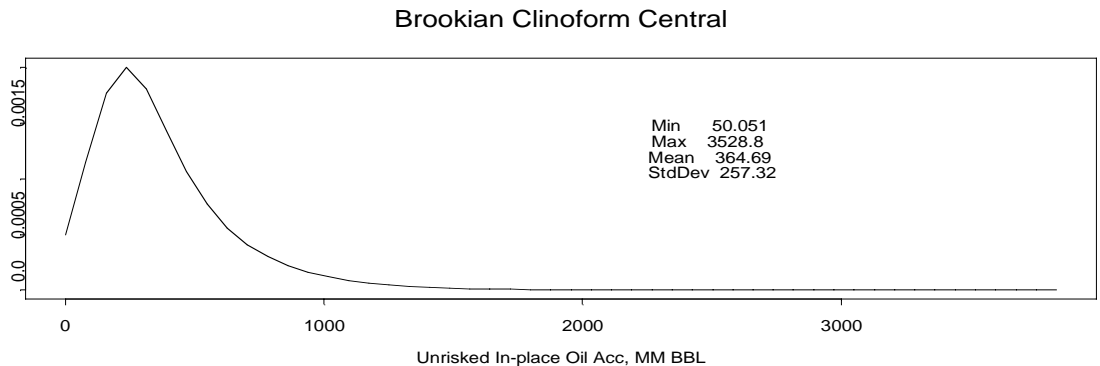

Brookian Clinoform Central

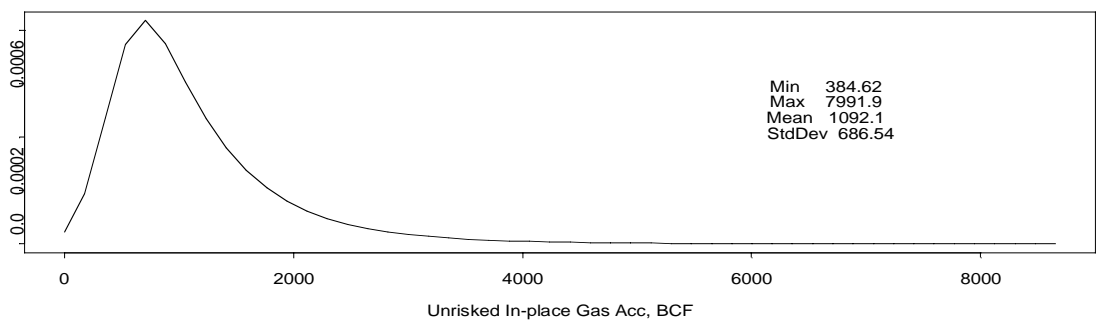

Play
$\begin{aligned} & \text { Play Stats } \\
& \text { Resource }\end{aligned}$
\begin{tabular}{|l|rrrrr||}
\hline In-place oil & Mean & StdDev & F95 & F50 & F05 \\
\hline In-place NA gas & 2779.12 & 1361.81 & 853.59 & 2618.31 & 5282.73 \\
Recov oil & 8315.29 & 3900.45 & 2778.00 & 7860.89 & 15501.38 \\
Recov assoc diss gas & 972.69 & 476.63 & 298.75 & 916.41 & 1848.96 \\
Recov NA gas & 1230.43 & 602.86 & 378.59 & 1154.45 & 2335.82 \\
Recov NGL (ADG) & 5404.94 & 2535.29 & 1805.70 & 5109.58 & 10075.89 \\
Recov NGL (NAG) & 26.07 & 12.79 & 8.02 & 24.44 & 49.46 \\
Num oil deposits & 118.26 & 55.54 & 39.36 & 111.86 & 220.54 \\
Num NA gas deposits & 7.62 & 3.16 & 3.00 & 7.00 & 13.00 \\
\hline RNS & 7.61 & 3.13 & 3.00 & 7.00 & 13.00 \\
\hline
\end{tabular}

A gas deposits

RNS $=77$

Play Totals Brookian Clinoform Central

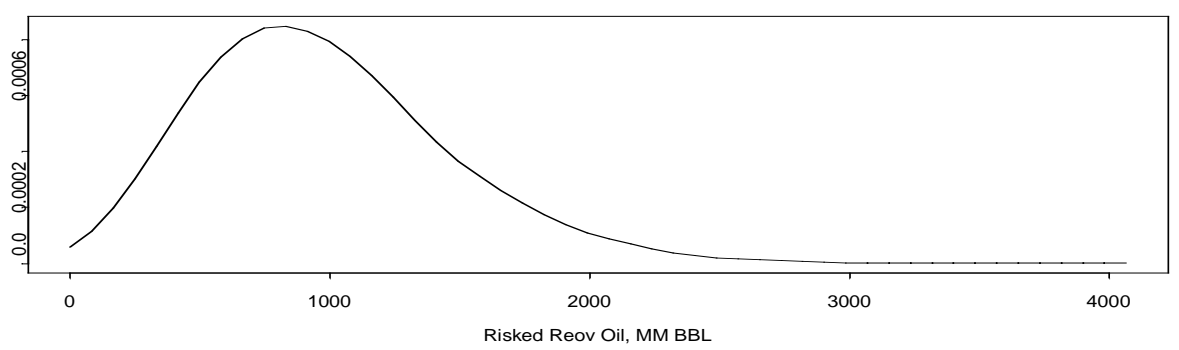

Play Totals Brookian Clinoform Central

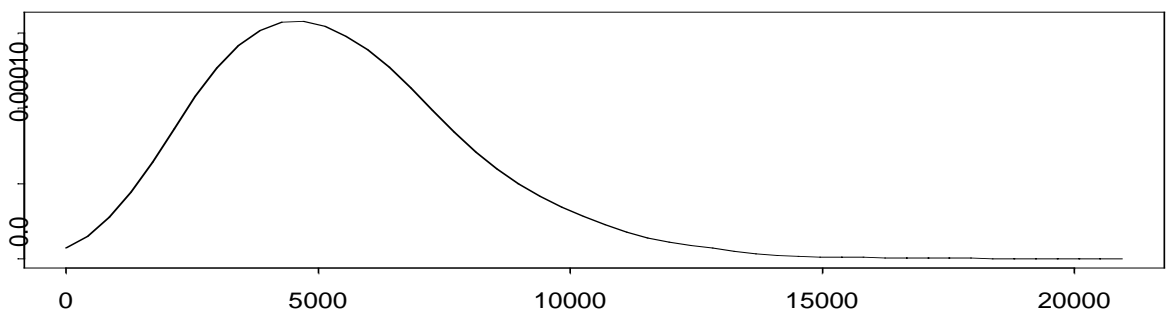

Risked Recov Gas, BCF 
Table 9f: Estimated undiscovered oil and gas resources for the Brookian Clinoform Central Play showing number of deposits and volumes by accumulation size class

\begin{tabular}{|rrrrrr} 
Start size clOil deposits Oil (MMBO_ & Start size clias deposits & Gas $(B C F G)$ \\
0 & 0.00 & 0.00 & 0 & 0.00 & 0.00 \\
8 & 0.00 & 0.00 & 48 & 0.00 & 0.00 \\
16 & 0.24 & 6.51 & 96 & 0.00 & 0.00 \\
32 & 1.59 & 78.22 & 192 & 1.75 & 553.41 \\
64 & 2.96 & 275.68 & 384 & 3.42 & 1883.98 \\
128 & 2.16 & 381.29 & 768 & 2.01 & 2093.69 \\
256 & 0.63 & 208.94 & 1536 & 0.42 & 822.99 \\
512 & 0.04 & 21.71 & 3072 & 0.01 & 51.42 \\
1024 & 0.00 & 0.45 & & & \\
\hline Totals & 7.62 & 972.79 & & 7.61 & 5405.48 \\
\hline
\end{tabular}
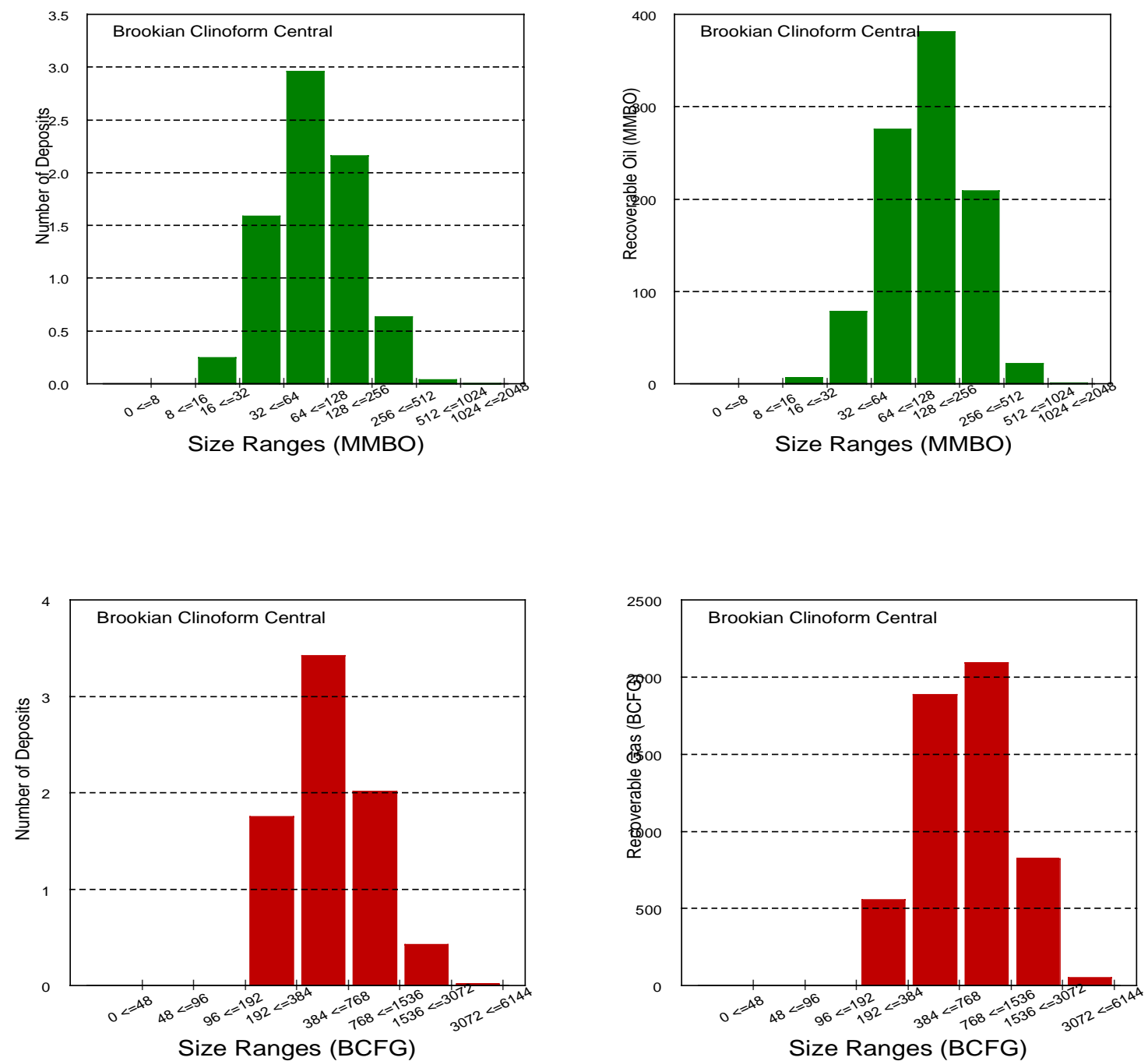
Table 10a: Input values for oil accumulations in the Brookian Clinoform South Shallow Play NPRA Assessment Form-2001

PLAY: Brookian Clinoform South Shallow

Play area: $\quad 7491 \mid 10^{3}$ Acres

\section{OIL ACCUMULATION VOLUME PARAMETERS}

\begin{tabular}{|c|c|c|c|c|c|c|c|}
\hline \multirow[b]{2}{*}{ ATTRIBUTES } & \multirow{2}{*}{$\begin{array}{l}\text { Est Shape } \\
(1 \text { to } 5)\end{array}$} & \multicolumn{4}{|c|}{ PROB OF AND GREATER THAN } & \multirow{2}{*}{$\begin{array}{l}\text { Knowledge } \\
\text { Level } 1-3^{5}\end{array}$} & \multirow{6}{*}{ Enter $\mathrm{POR}^{*} \mathrm{Sw}$} \\
\hline & & LTP & 0.50 & \multirow{2}{*}{0.05} & Max & & \\
\hline \multirow{4}{*}{$\begin{array}{l}\text { NET RESERVOIR THICKNESS } \\
\text { AREA OF CLOSURE }^{2} \\
\text { POROSITY }^{3,4} \\
\text { TRAP FILL }^{3}\end{array}$} & \begin{tabular}{r|}
2 \\
\end{tabular} & 50 & 75 & & 400 & 2 & \\
\hline & 2 & 5 & 7 & 20 & 40 & 2 & \\
\hline & 4 & 10 & 14 & 18 & 20 & 2 & \\
\hline & 7 & 80 & 100 & 100 & 100 & 2 & \\
\hline HYDROCARBON PORE VOL & 4 & 4 & 8 & 12 & 14 & & 6 \\
\hline \multirow{2}{*}{\multicolumn{2}{|c|}{$\begin{array}{l}\text { Approx } \mathrm{mm} \text { bbl ( } \mathrm{fvf}=1 \text { ) } \\
\text { Recov } \mathrm{mm} \text { bbl at surface }\end{array}$}} & 62.1 & 325.8 & 3723.8 & 17377.9 & & \\
\hline & & 14.1 & 74.2 & 848.1 & 3958.0 & & \\
\hline \multicolumn{7}{|c|}{$\begin{array}{l}\text { 1-thickness in feet, 2-thousands of acres, 3-percent, 4-correlation between Porosit } \\
\text { 5-Knowledge Level: 1=High, 2=Medium, 3=Low; LTP=Left Truncation Point }\end{array}$} & \\
\hline \multirow{2}{*}{$\begin{array}{l}\text { TRAP DEPTH (in } 1000 \mathrm{ft} \text { ) } \\
\text { (from sea level) }\end{array}$} & \begin{tabular}{r|}
2 \\
\end{tabular} & 3 & 6 & 9 & 10 & 1 & \\
\hline & \multicolumn{4}{|c|}{ Surface to sea level correction $(1000 \mathrm{ft})$ : } & 1.044 & & \\
\hline
\end{tabular}

\section{OIL ACCUMULATION CHARACTERISTICS}

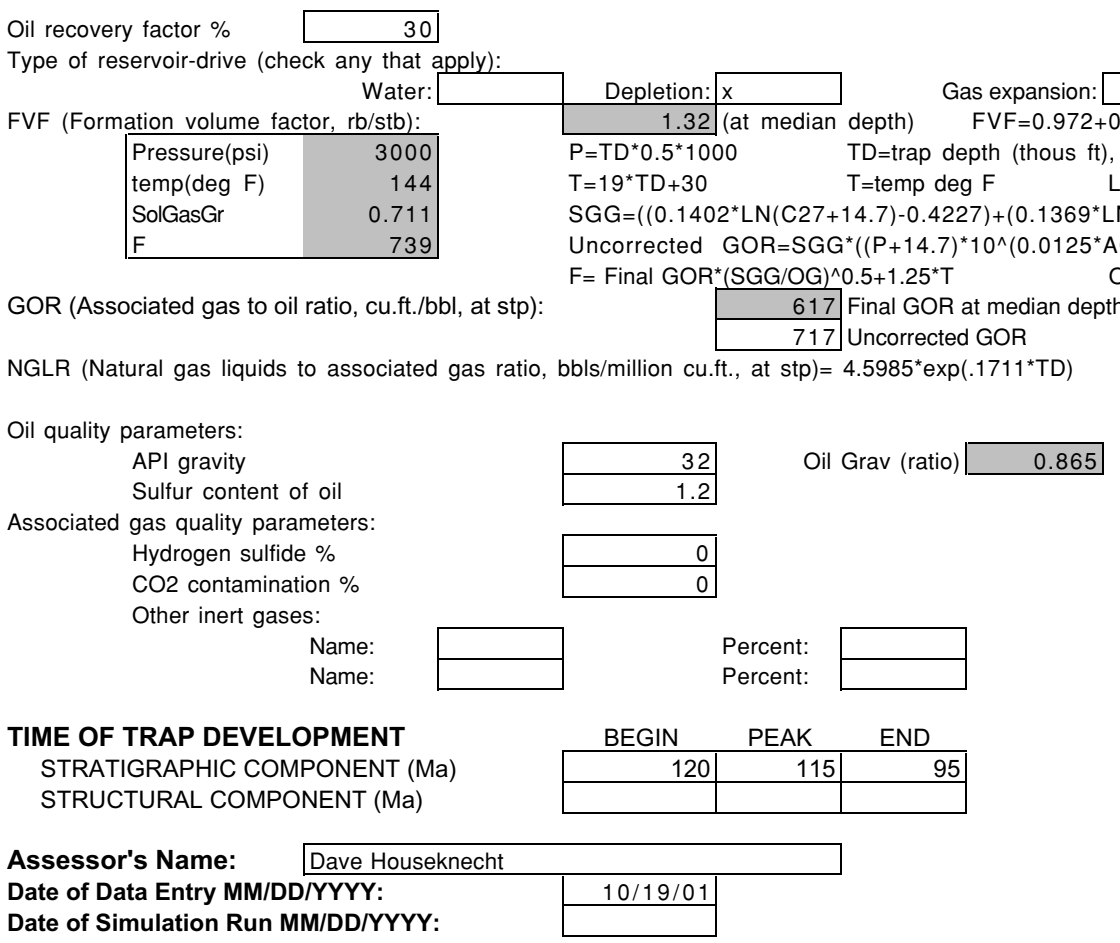

Note: only enter play name and assessor's name on Oil worksheet 
Table 10b: Input values for nonassociated gas accumulations in the Brookian Clinoform South Shallow Play NPRA Assessment Form-2001

PLAY: Brookian Clinoform South Shallow

\section{NONASSOCIATED GAS ACCUMULATION VOLUME PARAMETERS}

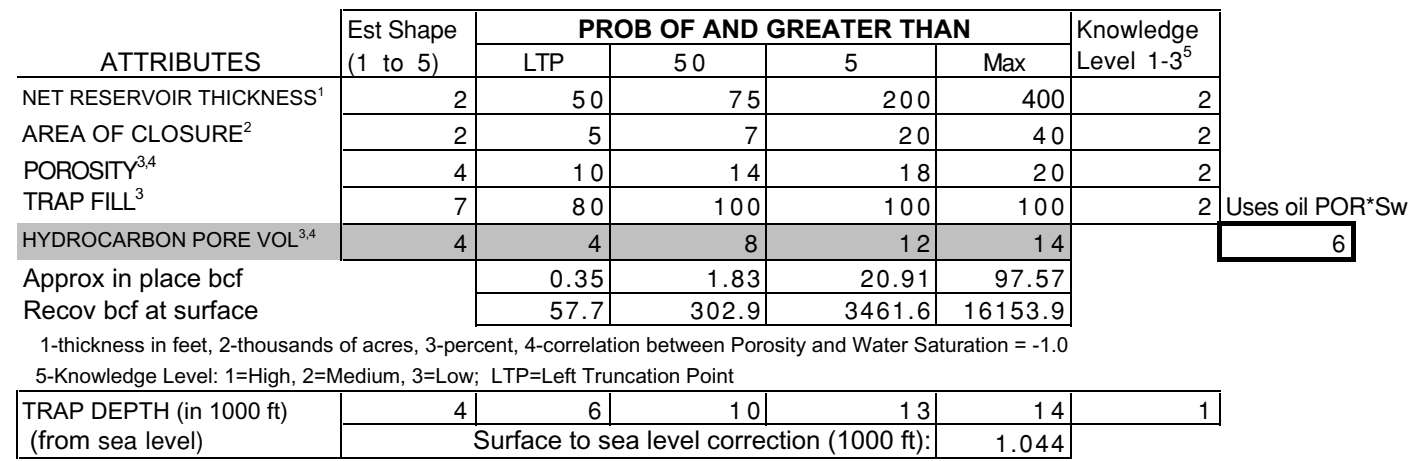

\section{NONASSOCIATED GAS ACCUMULATION CHARACTERISTICS}

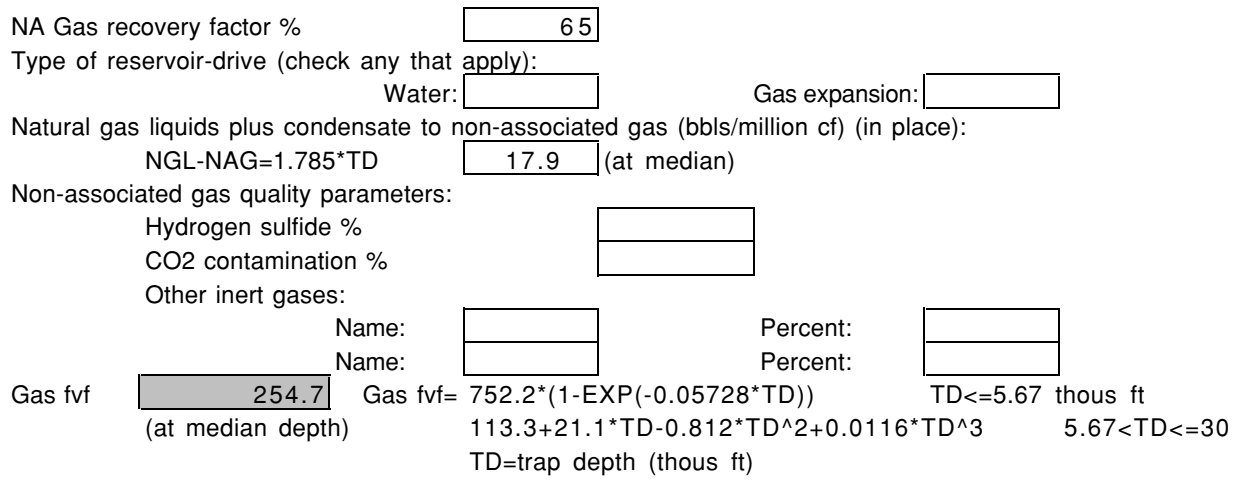

\begin{tabular}{|c|c|c|}
\hline TIME OF TRAP DEVELOPMEN 1 & PEAK & END \\
\hline STRATIGRAPHIC COMPONENT (Ma) & & \\
\hline STRUCTURAL COMPONENT (Ma) & & \\
\hline
\end{tabular}

\begin{tabular}{l|l|} 
Assessor's Name: & \multicolumn{2}{|l|}{ Fred Geoknow } \\
Date of Data Entry MM/DD/YYYY: & $1 / 1 / 00$ \\
Date of Simulation Run MM/DD/YYYY: & $1 / 1 / 00$ \\
\hline
\end{tabular}


Table 10c: Input risking values for the Brookian Clinoform South Shallow Play NPRA Assessment Form-2001

Play: Brookian Clinoform South Shallow

\section{RISKING}

MINIMUM ACCUMULATION SIZE, MAS (Millions of BBL in place)

PRERISKED FREQUENCY DISTRIBUTION (Oil plus Gas)

\begin{tabular}{|c|c|c|c|c|c|c|}
\hline \multirow{3}{*}{$\begin{array}{l}\text { NUM OF PROSPECTS } \\
>\text { MINIMUM SIZE }\end{array}$} & & \multicolumn{4}{|c|}{ PROB OF AND GREATER THAN } & \multirow{2}{*}{$\begin{array}{l}\text { Knowledge } \\
\text { Level } 1-3^{5}\end{array}$} \\
\hline & Est Shape & Min & 50 & 5 & Max & \\
\hline & 2 & 20 & 40 & 60 & 100 & 3 \\
\hline
\end{tabular}

5-Knowledge Level: 1=High, 2=Medium, 3=Low; LTP=Left Truncation Point

\section{ATTRIBUTES}

PLAY

ATTRIBUTES

CHARGE (C)

TRAP (T)
TIMING (F)

Probability that play contains at least 1 reservoir $>=$ minimum size $(C x T x F)$

PROSPECT

ATTRIBUTES

CHARGE (c)

$\operatorname{TRAP}(\mathrm{t})$

TIMING (f)

Probability that a randomly chosen prospect is favorable (cxtxf)

Play Attributes x Prospect Attributes (CxTxFxcxtxf)

FRACTION OF ACCUMULATIONS BEING OIL

Fraction NA Gas=1-Fraction(Oil)
PROBABILITY

\section{OF FAVORABLE}

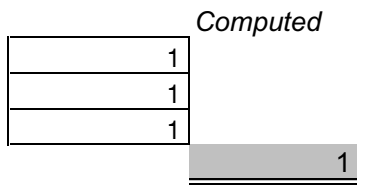

\begin{tabular}{r|}
\hline 0.7 \\
\hline 0.2 \\
\hline 1 \\
\hline
\end{tabular}

0.14

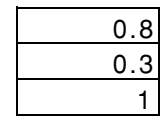

Allocation (percent):

\begin{tabular}{|l|r|r|r|}
\cline { 2 - 4 } \multicolumn{1}{c|}{} & \multicolumn{1}{c|}{ Land } & \multicolumn{1}{c|}{ Oil } & \multicolumn{1}{c|}{ Gas } \\
\hline Federal & 99 & 100 & 100 \\
\hline State & 0 & 0 & 0 \\
\hline Native & 1 & 0 & 0 \\
\hline
\end{tabular}

Assessor's Name:

Dave Houseknecht

Date of Data Entry MM/DD/YYYY:

Date of Simulation Run MM/DD/YYYY: 
Table 10d: Distribution of fitted hydrocarbon volume attributes, trap depth, and number of prospects for the Brookian Clinoform South Shallow Play

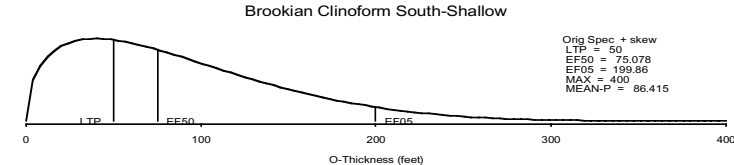

Brookian Clinoform South-Shallow
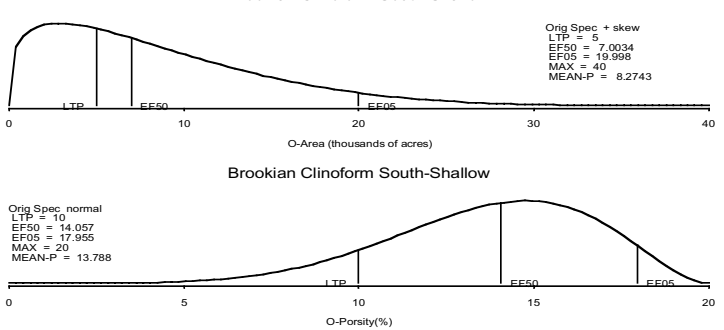

Brookian Clinoform South-Shallow

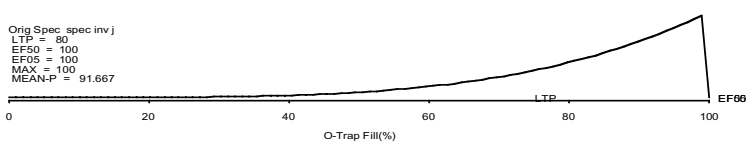

Brookian Clinoform South-Shallow

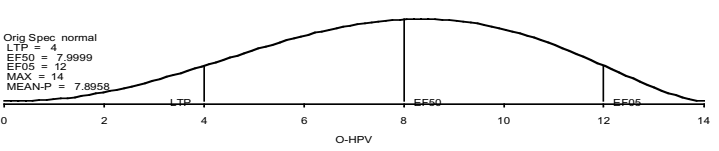

Brookian Clinoform South-Shallow

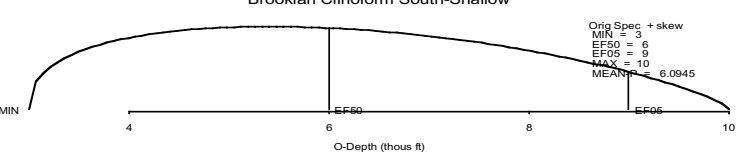

rookian Clinoform South-Shallow

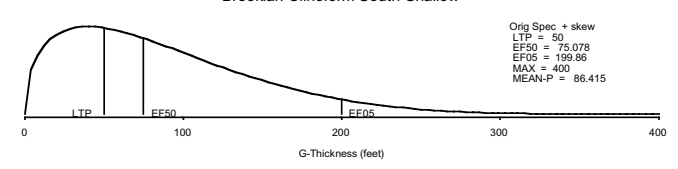

Brookian Clinoform South-Shallow

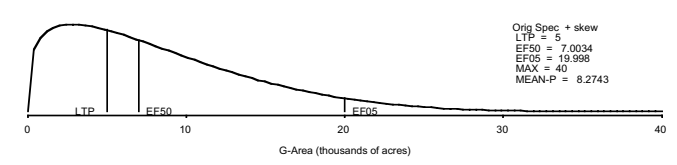

Rrnokian C.linnform Snut th-Shallow
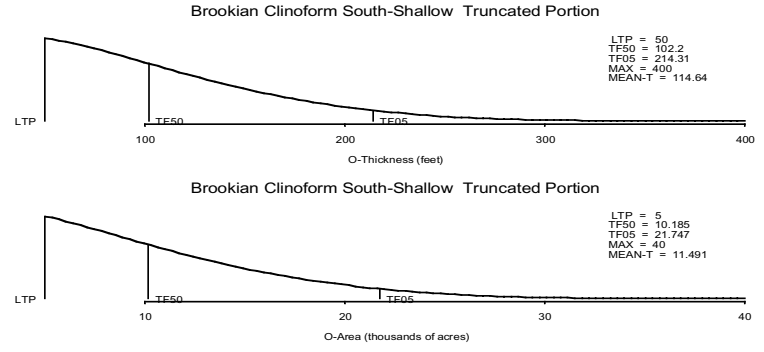

Brookian Clinoform South-Shallow Truncated Portion

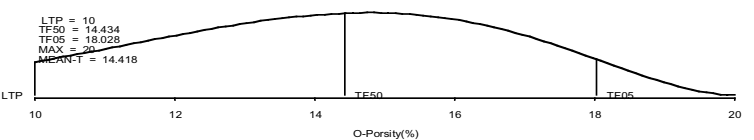

Brookian Clinoform South-Shallow Truncated Portion

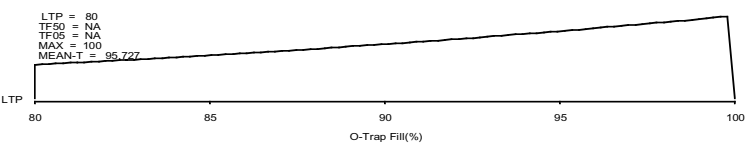

Brookian Clinoform South-Shallow Truncated Portion

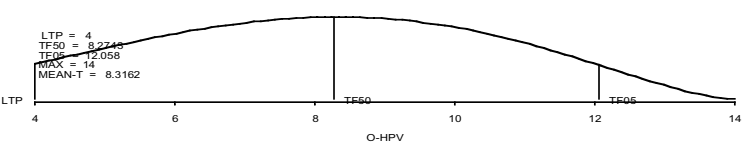

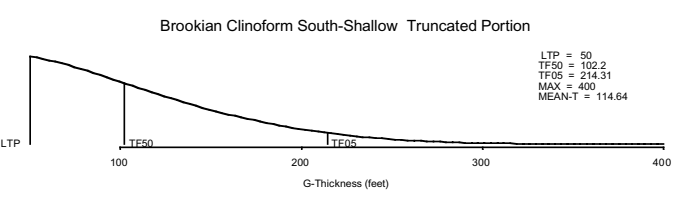

Brookian Clinoform South-Shallow Truncated Portion

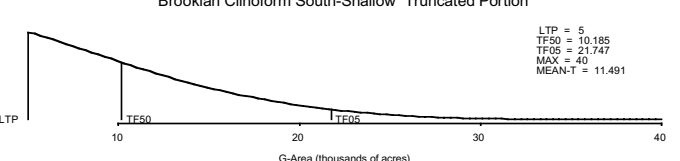

Rrnnkian C.linnform Snuth_Shallnw Truncated Pnrtion 

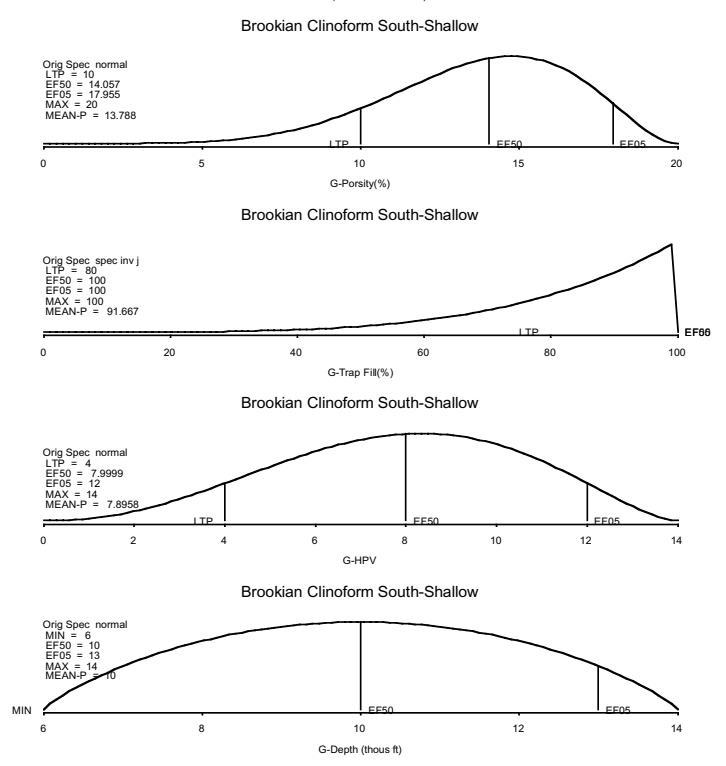

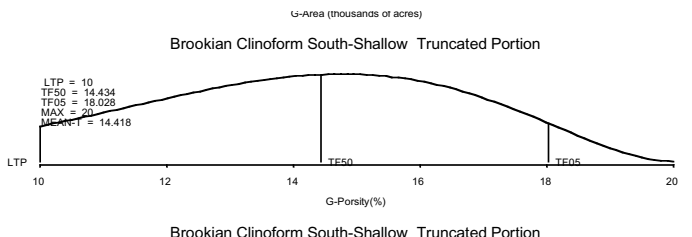

Brookian Clinoform South-Shallow Truncated Portion

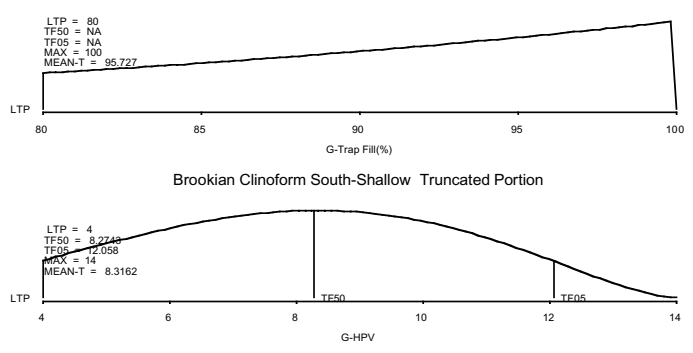

Brookian Clinoform South-Shallow

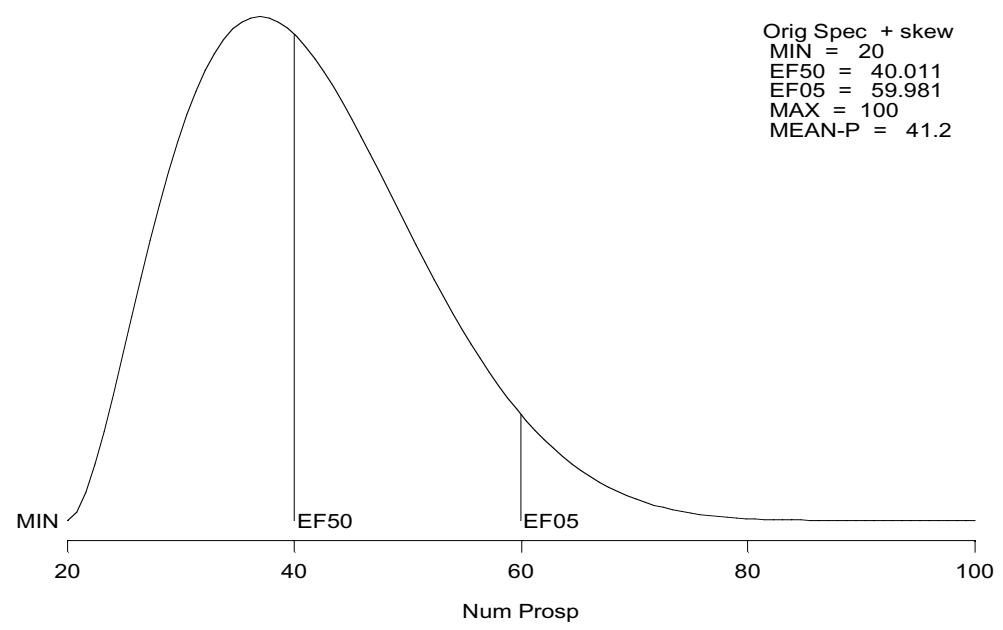


Table 10e: Estimated undiscovered oil and gas resources for the Brookian Clinoform S-Shallow Play Deposit

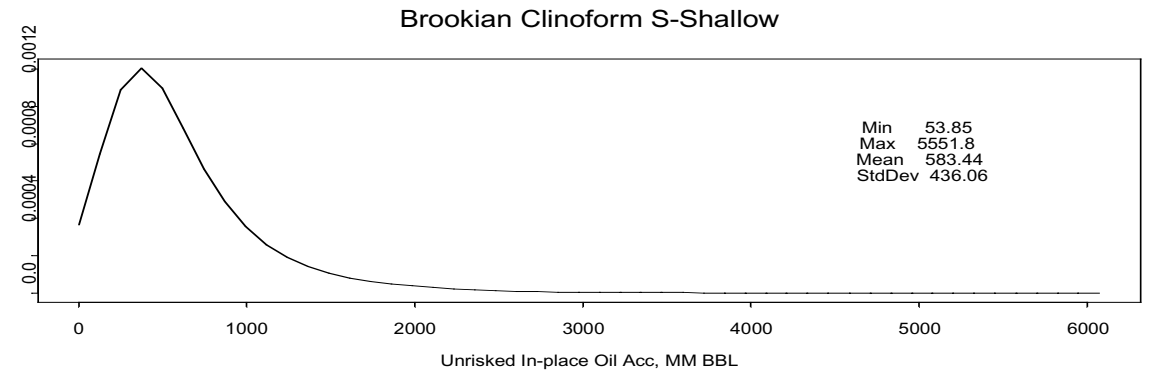

Brookian Clinoform S-Shallow

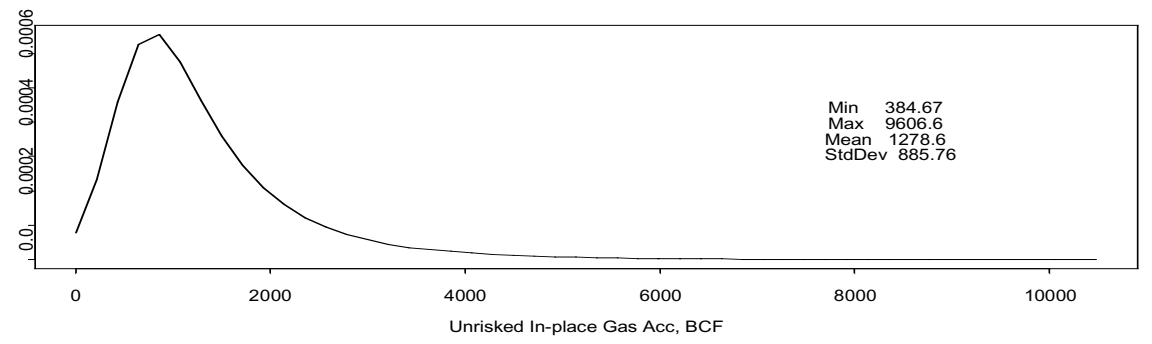

\begin{tabular}{|c|c|c|c|c|c|}
\hline \\
\hline \multicolumn{6}{|l|}{$\begin{array}{l}\text { Play } \\
\text { Play Stats }\end{array}$} \\
\hline \multirow{2}{*}{$\frac{\text { Resource }}{\text { In-place oil }}$} & Mean & StdDev & F95 & F50 & F05 \\
\hline & 1692.03 & 1295.53 & 0.00 & 1433.27 & 4178.98 \\
\hline \multirow{4}{*}{$\begin{array}{l}\text { In-place NA gas } \\
\text { Recov oil } \\
\text { Recov assoc diss gas } \\
\text { Recov NA gas }\end{array}$} & 3699.35 & 2757.00 & 0.00 & 3175.92 & 8936.19 \\
\hline & 507.61 & 388.66 & 0.00 & 429.98 & 1253.70 \\
\hline & 362.10 & 279.09 & 0.00 & 308.34 & 907.46 \\
\hline & 2404.58 & 1792.05 & 0.00 & 2064.34 & 5808.52 \\
\hline \multirow{2}{*}{$\begin{array}{l}\text { Recov NGL (ADG) } \\
\text { Recov NGL (NAG) }\end{array}$} & 8.08 & 6.30 & 0.00 & 6.80 & 20.31 \\
\hline & 47.87 & 36.36 & 0.00 & 40.81 & 117.36 \\
\hline \multirow{2}{*}{$\begin{array}{l}\text { Num oil deposits } \\
\text { Num NA gas deposits }\end{array}$} & 2.90 & 1.81 & 0.00 & 3.00 & 6.00 \\
\hline & 2.89 & 1.79 & 0.00 & 3.00 & 6.00 \\
\hline
\end{tabular}

RNS=341

Play Totals Brookian Clinoform S-Shallow
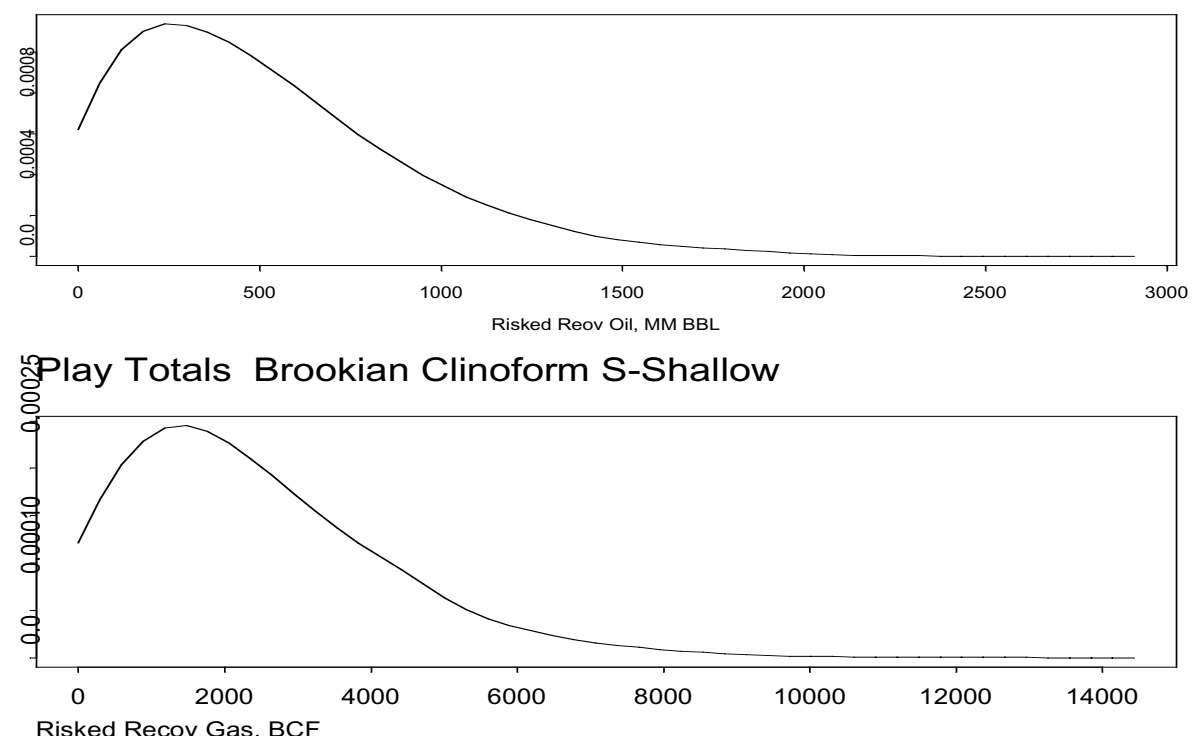
Table 10f: Estimated undiscovered oil and gas resources for the Brookian Clinoform S-shallow Play showing number of deposits and volumes by accumulation size class

\begin{tabular}{rrrrrr} 
Start size class & & Oil deposits & Oil (MMBO) Start size class & Gas deposits & NA Gas (BCFG) \\
0 & 0.00 & 0.00 & 0 & 0.00 & 0.00 \\
8 & 0.00 & 0.00 & 48 & 0.00 & 0.00 \\
16 & 0.03 & 0.87 & 96 & 0.00 & 0.00 \\
32 & 0.35 & 17.73 & 192 & 0.51 & 163.06 \\
64 & 0.94 & 88.62 & 384 & 1.20 & 670.31 \\
128 & 1.03 & 186.67 & 768 & 0.89 & 939.50 \\
256 & 0.47 & 160.12 & 1536 & 0.27 & 537.10 \\
512 & 0.08 & 50.58 & 3072 & 0.03 & 93.61 \\
& 0.00 & 3.08 & & 0.00 & 1.24 \\
\hline Totals & 2.90 & 507.66 & & 2.89 & 2404.82 \\
\hline
\end{tabular}
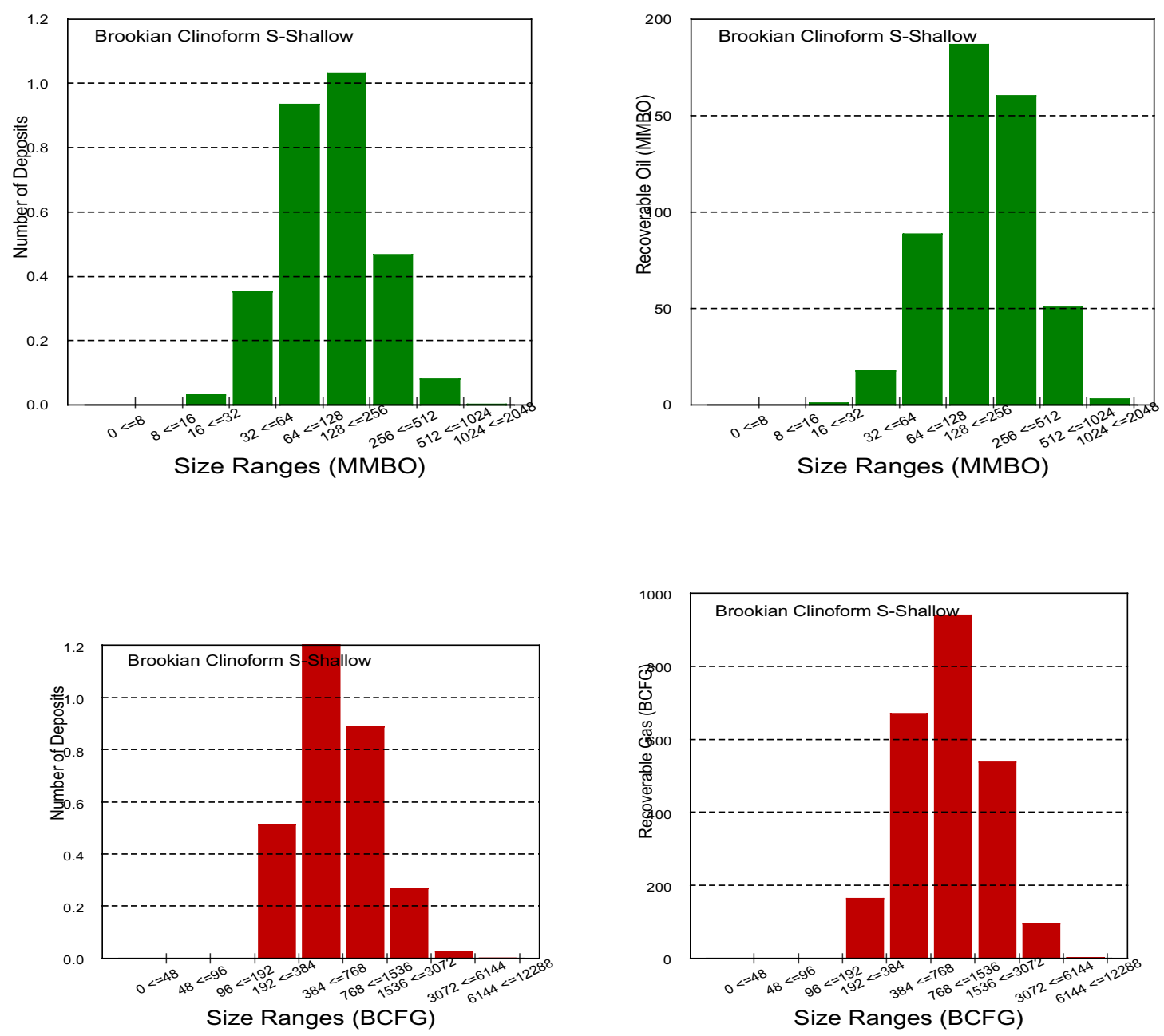
Table 11a: Input values for nonassociated gas accumulations in the Brookian Clinoform South Deep Play NPRA Assessment Form-2001

PLAY: Brookian Clinoform South Deep

Play area: $\quad 7491 \mid 10^{3}$ Acres

\section{NONASSOCIATED GAS ACCUMULATION VOLUME PARAMETERS}

\begin{tabular}{|c|c|c|c|c|c|c|c|}
\hline & & \multirow{2}{*}{\multicolumn{4}{|c|}{ PROB OF AND GREATER THAN }} & \multirow{3}{*}{$\begin{array}{l}\text { Knowledge } \\
\text { Level } 1-3^{5} \\
\end{array}$} & \\
\hline \multirow{2}{*}{ ATTRIBUTES } & \multirow{2}{*}{$\begin{array}{l}\text { Est Shape } \\
(1 \text { to } 5)\end{array}$} & & & & & & \\
\hline & & \begin{tabular}{l|l} 
LTP &
\end{tabular} & 50 & 5 & Max & & \\
\hline \multirow{4}{*}{$\begin{array}{l}\text { NET RESERVOIR THICKNESS } \\
\text { AREA OF CLOSURE }^{1} \\
\text { POROSITY }^{3,4} \\
\text { TRAP FILL }^{3}\end{array}$} & 2 & 50 & 75 & 200 & 400 & 2 & \\
\hline & 2 & 5 & 7 & 20 & 40 & 2 & \\
\hline & 4 & 8 & 11 & 14 & 17 & 2 & \\
\hline & 7 & 80 & 100 & 100 & 100 & 2 & $\mathrm{POR}^{\star} \mathrm{Sw}$ \\
\hline \multicolumn{2}{|l|}{ HYDROCARBON PORE VOL $\mathrm{L}^{3,4}$} & 2 & 5 & 8 & 11 & & 6 \\
\hline \multirow{2}{*}{\multicolumn{2}{|c|}{$\begin{array}{l}\text { Approx in place bcf } \\
\text { Recov bcf at surface }\end{array}$}} & 0.17 & 1.14 & 13.94 & 76.67 & & \\
\hline & & 32.4 & 212.8 & 2593.6 & 14264.6 & & \\
\hline \multicolumn{8}{|c|}{ 1-thickness in feet, 2-thousands of acres, 3-percent, 4-correlation between Porosity and Water Saturation $=-1.0$} \\
\hline \multirow{2}{*}{$\begin{array}{l}\text { TRAP DEPTH (in } 1000 \mathrm{ft} \text { ) } \\
\text { (from sea level) }\end{array}$} & 2 & 11 & 15 & 20 & 24 & & \\
\hline & \multicolumn{4}{|c|}{ Surface to sea level correction $(1000 \mathrm{ft})$ : } & 1.044 & & \\
\hline
\end{tabular}

\section{NONASSOCIATED GAS ACCUMULATION CHARACTERISTICS}

NA Gas recovery factor \% 65

Type of reservoir-drive (check any that apply):

$$
\text { Water: }
$$

Gas expansion: $\mathrm{x}$

Natural gas liquids plus condensate to non-associated gas (bbls/million cf) (in place): NGL-NAG $=1.785^{\star} \mathrm{TD}$

$$
26.8 \text { (at median) }
$$

Non-associated gas quality parameters:

Hydrogen sulfide \%

CO2 contamination \%

Other inert gases:

Name:

Name:

Gas fvf$$
286.3
$$

Gas

(at median depth)

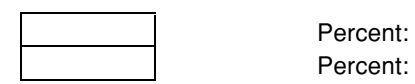

$752.2^{\star}\left(1-\mathrm{EXP}\left(-0.05728^{*} \mathrm{TD}\right)\right)$

$113.3+21.1^{*} T D-0.812^{*} T^{\wedge} 2+0.0116^{*} T^{\wedge} 3 \quad 5.67<T D<=30$

\begin{tabular}{|c|c|c|c|}
\hline TIME OF TRAP DEVELOPMENT & BEGIN & PEAK & END \\
\hline STRATIGRAPHIC COMPONENT (Ma) & 120 & 115 & 95 \\
\hline STRUCTURAL COMPONENT (Ma) & & & \\
\hline
\end{tabular}

$\mathrm{TD}=$ trap depth (thous $\mathrm{ft}$ )

Assessor's Name: Dave Houseknecht

Date of Data Entry MM/DD/YYYY:

Date of Simulation Run MM/DD/YYYY: 
Table 11b: Input risking values for the Brookian Clinoform South Deep Play NPRA Assessment Form-2001

Play: Brookian Clinoform South Deep

\section{RISKING}

MINIMUM ACCUMULATION SIZE, MAS (recov bcf)

PRERISKED FREQUENCY DISTRIBUTION (Oil plus Gas)

NUM OF PROSPECTS

$>$ MINIMUM SIZE

PROB OF AND GREATER THAN

\begin{tabular}{|r|r|r|r|r|r|r|}
\hline Est Shape & \multicolumn{4}{|c|}{ PROB OF AND GREATER THAN } & \multicolumn{2}{l|}{$\begin{array}{l}\text { Knowledge } \\
\text { Level } 1-3^{5}\end{array}$} \\
\hline 2 & 30 & 50 & 5 & 80 & 120 & 3 \\
\hline
\end{tabular}

5-Knowledge Level: 1=High, 2=Medium, 3=Low; LTP=Left Truncation Point

\section{ATTRIBUTES}

PLAY

ATTRIBUTES

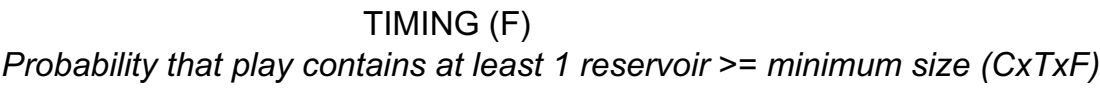

CHARGE (C)

TRAP (T)

PROSPECT

ATTRIBUTES

CHARGE (c)

$\operatorname{TRAP}(\mathrm{t})$

TIMING (f)

Probability that a randomly chosen prospect is favorable (cxtxf)

Play Attributes x Prospect Attributes (CxTxFxcxtxf)

FRACTION OF ACCUMULATIONS BEING OIL

Fraction NA Gas=1-Fraction(Oil)
PROBABILITY OF FAVORABLE

1

Computed

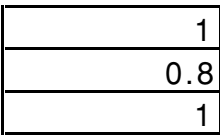

1

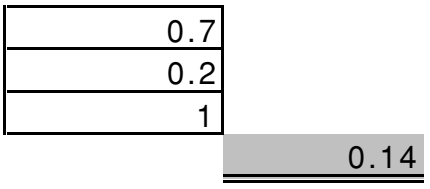

0.112

Allocation (percent):

\begin{tabular}{|l|r|r|r|}
\cline { 2 - 4 } \multicolumn{1}{c|}{} & \multicolumn{1}{c|}{ Land } & Oil & \multicolumn{1}{c|}{ Gas } \\
\hline Federal & 99 & & 100 \\
\hline State & 0 & & 0 \\
\hline Native & 1 & & 0 \\
\hline
\end{tabular}

Assessor's Name:

Dave Houseknecht

Date of Data Entry MM/DD/YYYY:

Date of Simulation Run MM/DD/YYYY:

$10 / 19 / 01$

$1 / 18 / 02$

0

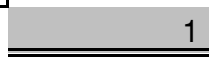


Table 11c: Distribution of fitted hydrocarbon volume attributes, trap depth, and number of prospects for the Brookian Clinoform South-Deep Play
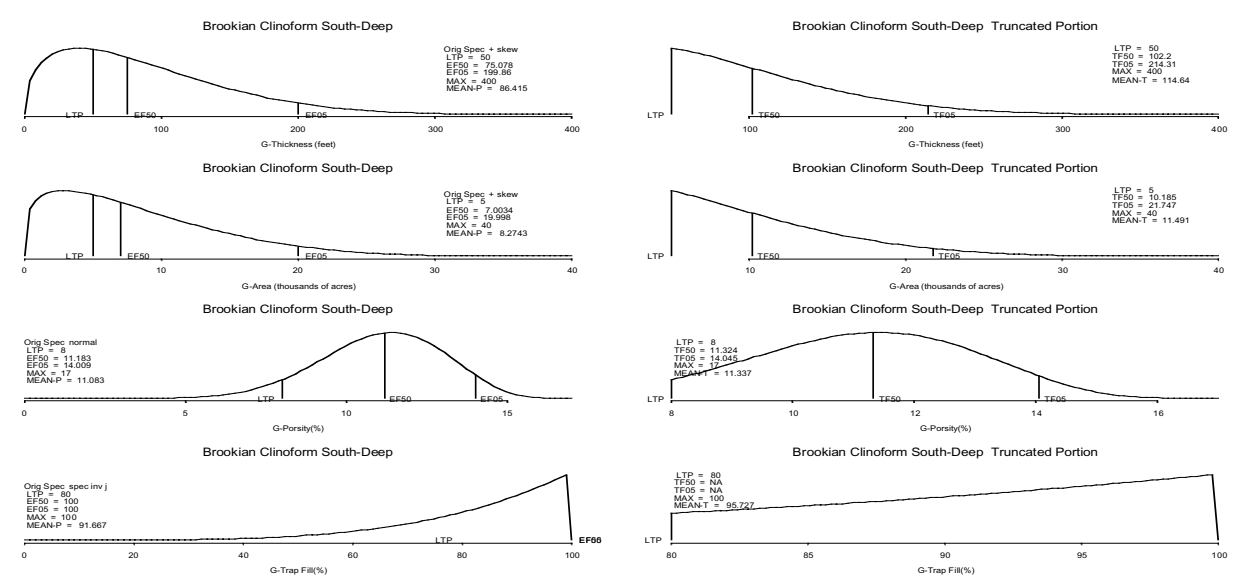

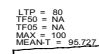
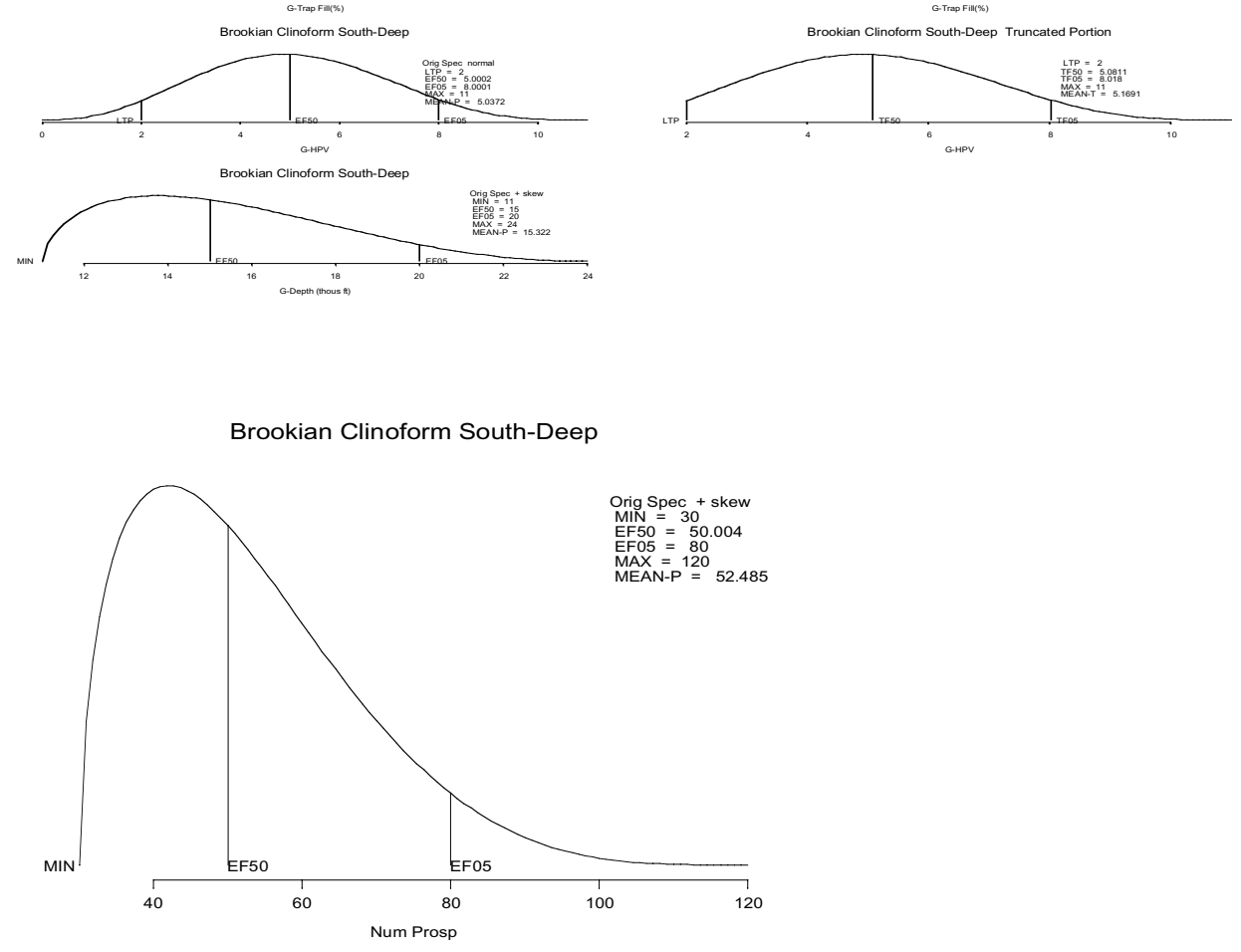
Table 11d: Estimated undiscovered oil and gas resources for the Brookian Clinoform S-Deep Play Deposit

Brookian Clinoform S-Deep

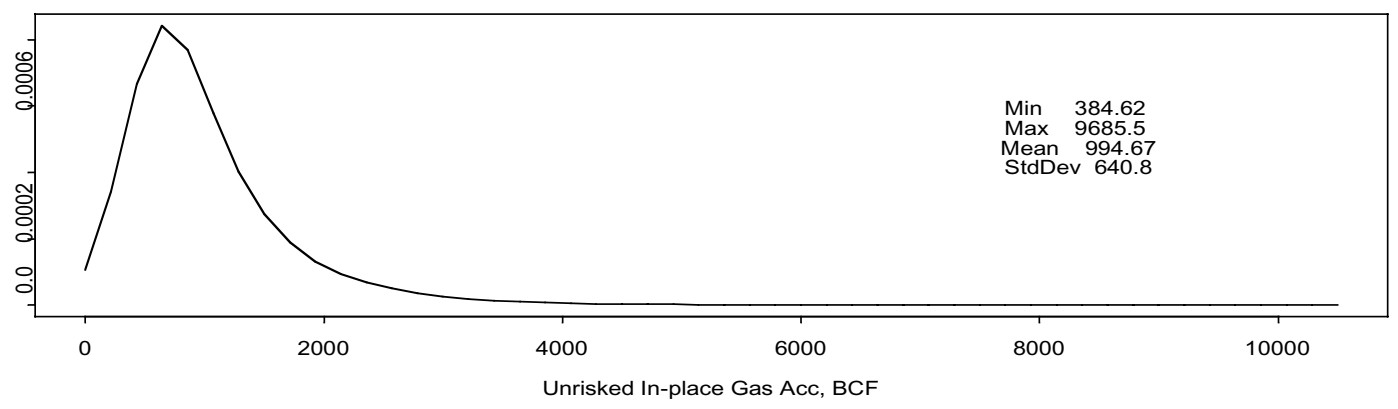

Play

Play Stats

Resource

In-place oil

In-place NA gas

Recov oil

Recov assoc diss gas

Recov NA gas

Recov NGL (ADG)

Recov NGL (NAG)

Num oil deposits

Num NA gas deposits

$\mathrm{RNS}=87$

\begin{tabular}{|rrrrr|}
\hline Mean & StdDev & F95 & F50 & F05 \\
5827.81 & 4374.21 & 0.00 & 5703.46 & 13531.67 \\
& & & & \\
3788.08 & 2843.24 & 0.00 & 3707.25 & 8795.58 \\
111.40 & 84.27 & 0.00 & 108.53 & 259.42 \\
5.86 & 4.11 & 0.00 & 6.00 & 13.00 \\
\hline
\end{tabular}

Play Totals Brookian Clinoform S-Deep

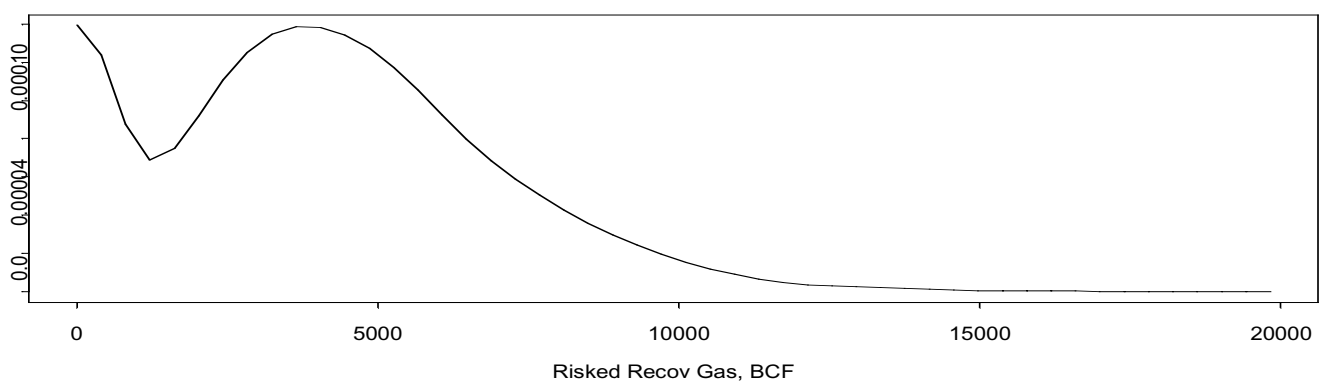


Table 11e: Estimated undiscovered oil and gas resources for the Brookian Clinoform S-Deep Play showing number of deposits and volumes by accumulation size class

\begin{tabular}{rrrrrr} 
Start size class & Oil deposits & Oil (MMBO) Start size class & Gas deposits & NA Gas (BCFG) \\
0 & & 0 & 0.00 & 0.00 \\
8 & & 48 & 0.00 & 0.00 \\
16 & 96 & 0.00 & 0.00 \\
32 & & 192 & 1.69 & 530.09 \\
64 & & 384 & 2.66 & 1442.62 \\
128 & & 768 & 1.26 & 1305.48 \\
256 & & 1536 & 0.25 & 475.74 \\
512 & & 3072 & 0.01 & 33.95 \\
& & & 0.00 & 0.50 \\
\hline Totals & 0.00 & 0.00 & & 5.86 & 3788.38 \\
\hline
\end{tabular}
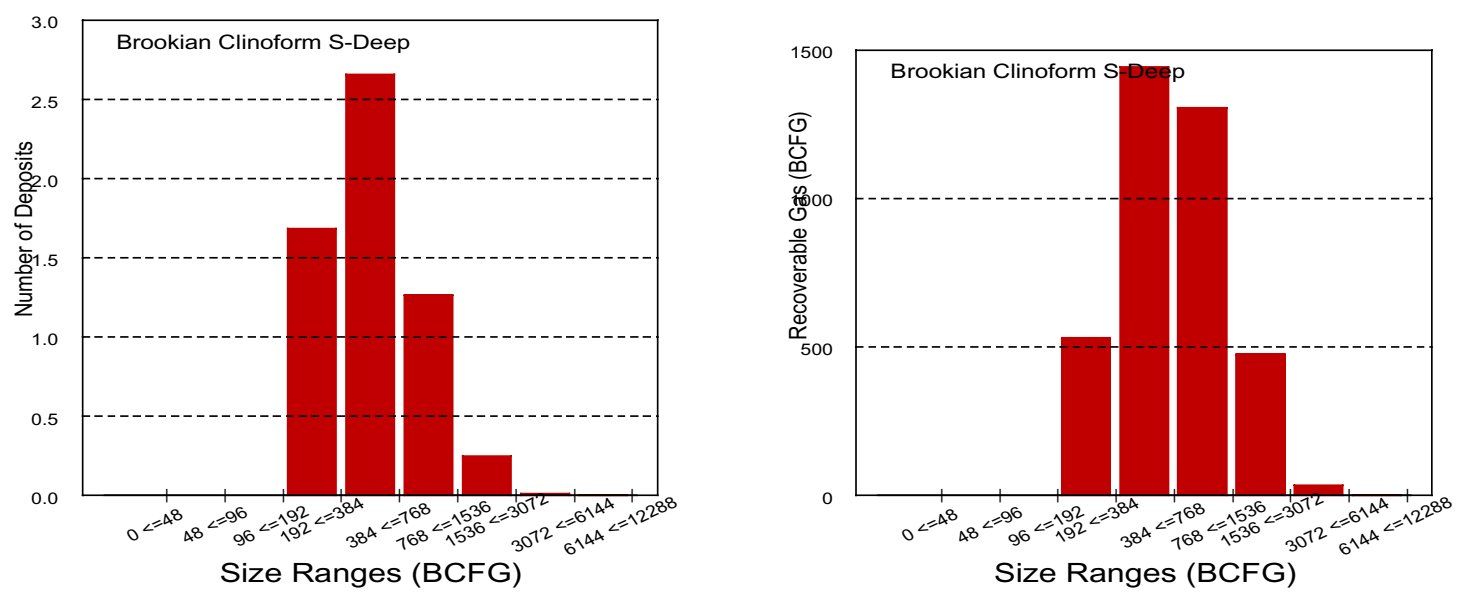
Table 12a: Input values for oil accumulations in the Beaufortian Cretaceous Topset North Play NPRA Assessment Form-2001

PLAY: Beaufortian Cretaceous Topset North

Play area: $\quad 7,879 \mid 10^{3}$ Acres

\section{OIL ACCUMULATION VOLUME PARAMETERS}

\begin{tabular}{|c|c|c|c|c|c|c|c|}
\hline \multirow[b]{2}{*}{ ATTRIBUTES } & \multirow{2}{*}{$\begin{array}{l}\text { Est Shape } \\
(1 \text { to } 5)\end{array}$} & \multicolumn{4}{|c|}{ PROB OF AND GREATER THAN } & \multirow{2}{*}{$\begin{array}{l}\text { Knowledge } \\
\text { Level } 1-3^{5}\end{array}$} & \multirow[b]{6}{*}{ Enter $\mathrm{POR}^{\star} \mathrm{Sw}$} \\
\hline & & LTP & 0.50 & 0.05 & Max & & \\
\hline \multirow{4}{*}{$\begin{array}{l}\text { NET RESERVOIR THICKNESS } \\
\text { AREA OF CLOSURE }^{2} \\
\text { POROSITY } \\
\text { TRAP FILL }\end{array}$} & 2 & 15 & 20 & 30 & 100 & 2 & \\
\hline & 2 & 4 & 5 & 10 & 50 & 3 & \\
\hline & 4 & 15 & 18 & 20 & 22 & 2 & \\
\hline & 6 & 50 & 75 & 99 & 100 & 2 & \\
\hline HYDROCARBON PORE VOL ${ }^{3,4}$ & 3 & 10 & 13 & 15 & 17 & & 5 \\
\hline \multirow{2}{*}{$\begin{array}{l}\text { Approx mm bbl (fvf=1) } \\
\text { Recov mm bbl at surface }\end{array}$} & & 23.3 & 75.6 & 345.6 & 6594.3 & & \\
\hline & & 5.7 & 18.7 & 85.2 & 1626.2 & & \\
\hline \multicolumn{8}{|c|}{ 1-thickness in feet, 2-thousands of acres, 3-percent, 4-correlation between Porosity and Water Saturation $=-1.0$} \\
\hline \multirow{2}{*}{$\begin{array}{l}\text { TRAP DEPTH (in } 1000 \mathrm{ft} \text { ) } \\
\text { (from sea level) }\end{array}$} & \multirow{2}{*}{$\frac{3}{s}$} & 3 & 8 & 9 & 10 & 1 & \\
\hline & & \multicolumn{3}{|c|}{ Surface to sea level correction $(1000 \mathrm{ft})$ : } & 0.107 & & \\
\hline
\end{tabular}

\section{OIL ACCUMULATION CHARACTERISTICS}

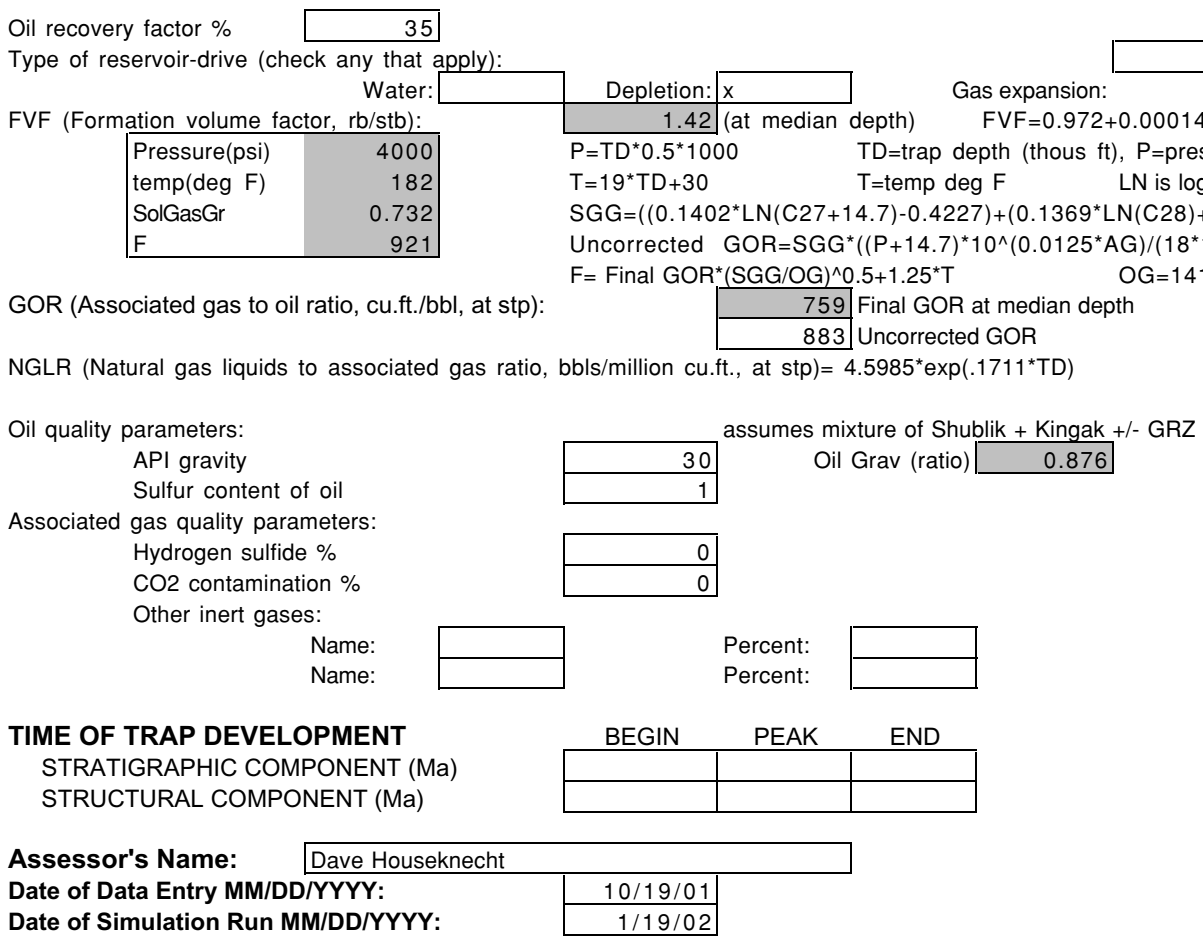

Note: only enter play name and assessor's name on Oil worksheet 
Table 12b: Input values for nonassociated gas accumulations in the Beaufortian Cretaceous Topset North Play NPRA Assessment Form-2001

PLAY: Beaufortian Cretaceous Topset North

NONASSOCIATED GAS ACCUMULATION VOLUME PARAMETERS

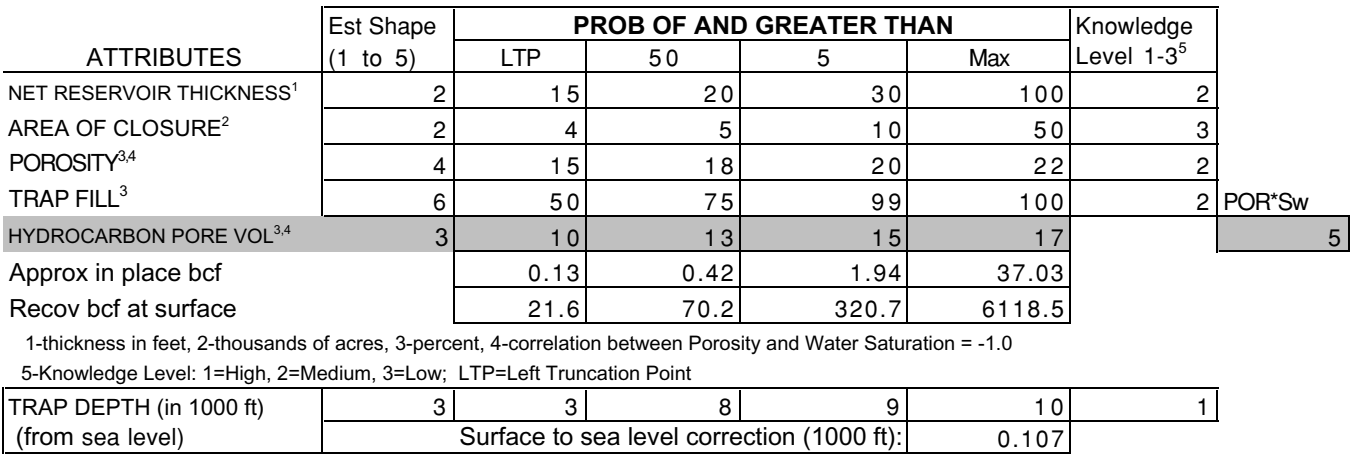

\section{NONASSOCIATED GAS ACCUMULATION CHARACTERISTICS}

NA Gas recovery factor \% 70

Type of reservoir-drive (check any that apply): Water:

Gas expansion:

Natural gas liquids plus condensate to non-associated gas (bbls/million cf) (in place): NGL-NAG $=1.785^{\star} \mathrm{TD}$

14.3 (at median)

Non-associated gas quality parameters: Hydrogen sulfide \% CO2 contamination \% Other inert gases:

$$
\begin{aligned}
& \text { Name: } \\
& \text { Name: }
\end{aligned}
$$$$
\begin{array}{r|}
\hline 236.1 \\
\text { (at median depth) }
\end{array}
$$

Gas

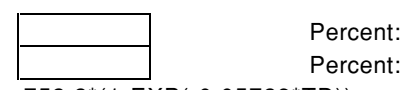

$752.2^{*}\left(1-\operatorname{EXP}\left(-0.05728^{*} \mathrm{TD}\right)\right)$

$113.3+21.1^{*} T D-0.812^{*} T^{\wedge} 2+0.0116^{*} T^{\wedge} 3$

$\mathrm{TD}=$ trap depth (thous $\mathrm{ft}$ )

TIME OF TRAP DEVELOPMENT

STRATIGRAPHIC COMPONENT (Ma)

STRUCTURAL COMPONENT (Ma)

\begin{tabular}{|l|l|l|}
\multicolumn{1}{l}{ BEGIN } & \multicolumn{1}{l}{ PEAK } & END \\
\hline & & \\
\hline & & \\
\hline
\end{tabular}

Assessor's Name: Dave Houseknecht

Date of Data Entry MM/DD/YYYY:

Date of Simulation Run MM/DD/YYYY:

$10 / 19 / 01$

$1 / 19 / 02$ 
Table 12c: Input risking values for the Beaufortian Cretaceous Topset North Play NPRA Assessment Form-2001

Play: Beaufortian Cretaceous Topset North

\section{RISKING}

MINIMUM ACCUMULATION SIZE, MAS (Millions of BBL in place)

PRERISKED FREQUENCY DISTRIBUTION (Oil plus Gas)

\begin{tabular}{l|r|r|r|r|r|r|}
\cline { 3 - 7 } & \multicolumn{4}{c|}{ PROB OF AND GREATER THAN } & Knowledge \\
NUM OF PROSPECTS & Est Shape & Min & 50 & 5 & Max & Level $1-3^{5}$ \\
\hline MINIMUM SIZE & 2 & 15 & 30 & 45 & 90 & 3 \\
\hline
\end{tabular}

5-Knowledge Level: 1=High, 2=Medium, 3=Low; LTP=Left Truncation Point

\section{ATTRIBUTES}

$\begin{array}{ll}\text { PLAY } & \text { CHARGE (C) } \\ \text { ATTRIBUTES } & \text { TRAP (T) } \\ & \text { TIMING (F) }\end{array}$

Probability that play contains at least 1 reservoir >= minimum size (CxTxF)

$\begin{array}{ll}\text { PROSPECT } & \text { CHARGE }(\mathrm{c}) \\ \text { ATTRIBUTES } & \text { TRAP }(\mathrm{t}) \\ & \text { TIMING }(\mathrm{f})\end{array}$

Probability that a randomly chosen prospect is favorable (cxtxf)

Play Attributes x Prospect Attributes (CxTxFxcxtxf)

FRACTION OF ACCUMULATIONS BEING OIL

Fraction NA Gas=1-Fraction(Oil)

Allocation (percent):

\begin{tabular}{|l|r|r|r|}
\cline { 2 - 4 } \multicolumn{1}{c|}{} & \multicolumn{1}{c|}{ Land } & \multicolumn{1}{c|}{ Oil } & \multicolumn{2}{c|}{ Gas } \\
\hline Federal & 82 & 75 & 78 \\
\hline State & 11 & 16 & 14 \\
\hline Native & 7 & 9 & 8 \\
\hline
\end{tabular}

Assessor's Name:

Dave Houseknecht

Date of Data Entry MM/DD/YYYY:

Date of Simulation Run MM/DD/YYYY:

\section{PROBABILITY}

OF FAVORABLE
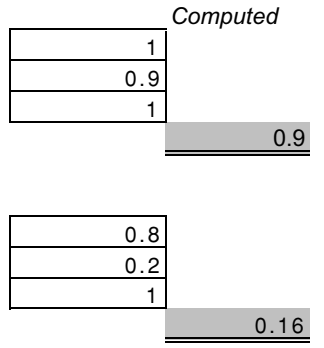

0.144
0.7

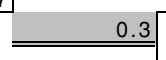


Table 12d: Distribution of fitted hydrocarbon volume attributes, trap depth, and number of prospects for the Beaufortian Cretaceous Topset North Play Beau Cret Top North
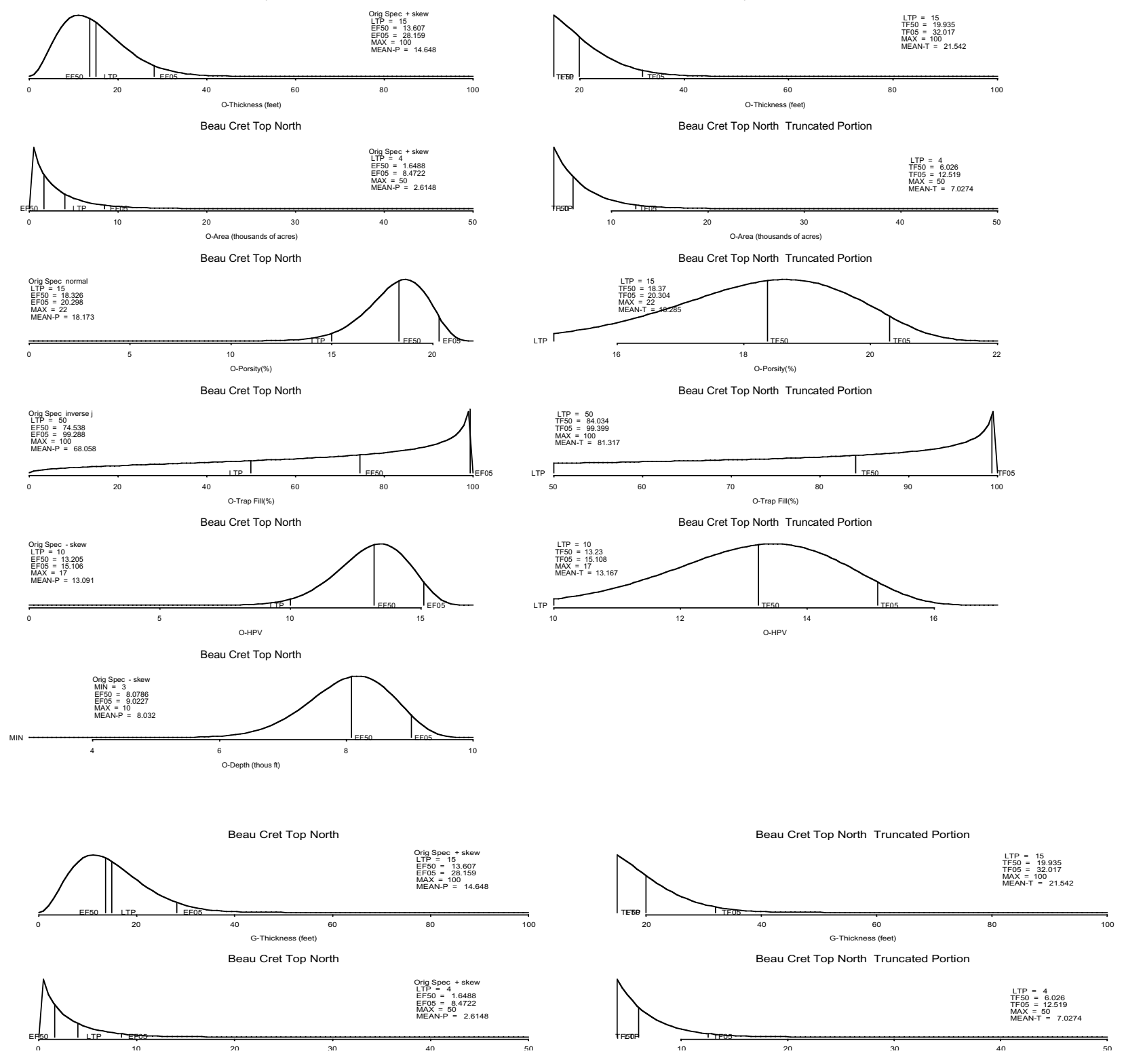

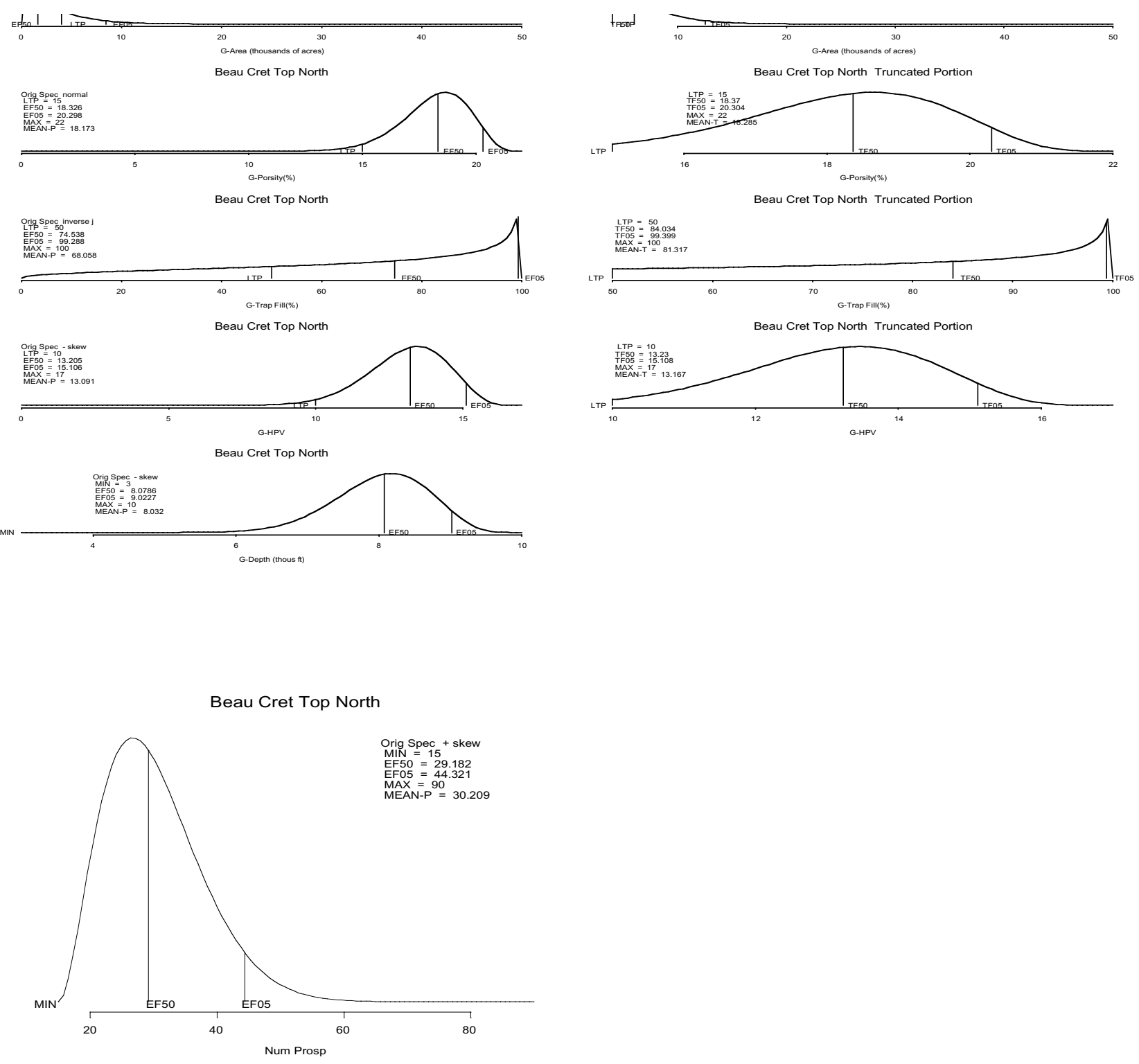
Table 12e: Estimated undiscovered oil and gas resources for the Beaufortian Cretaceous Topset North Play Deposit

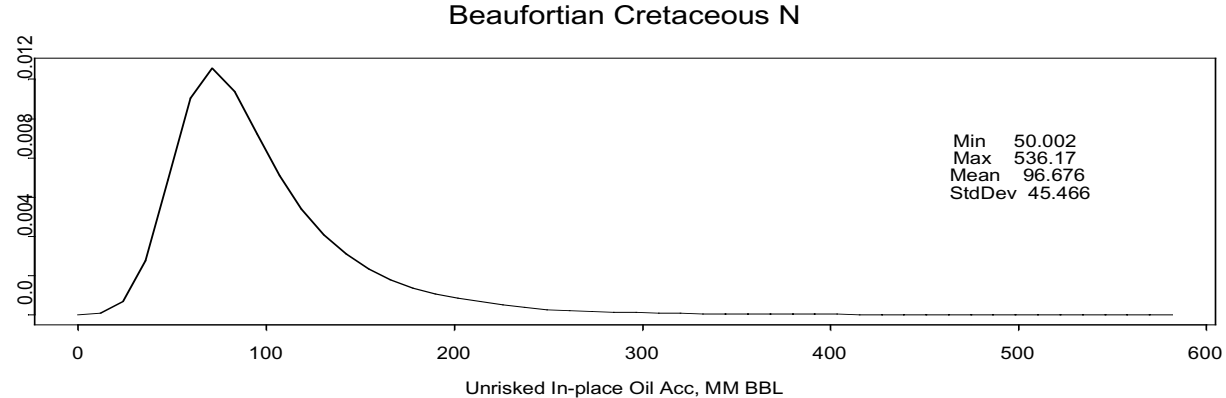

Beaufortian Cretaceous N

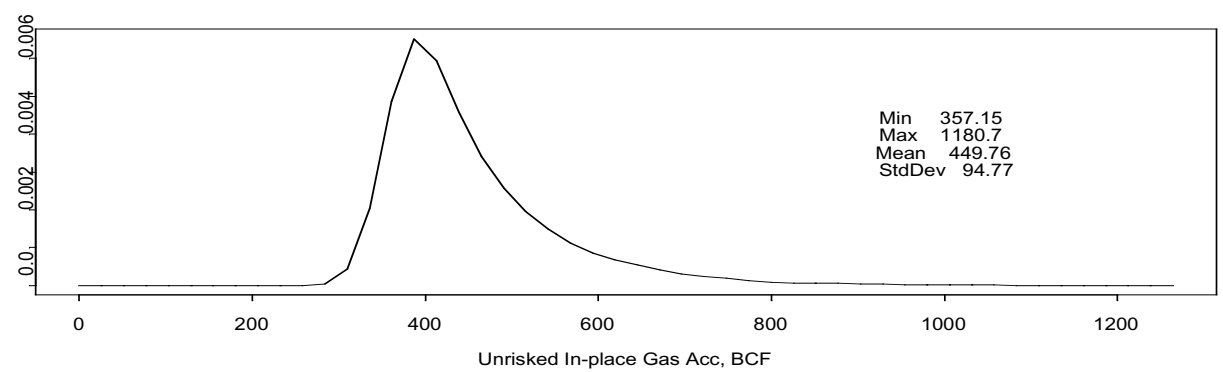

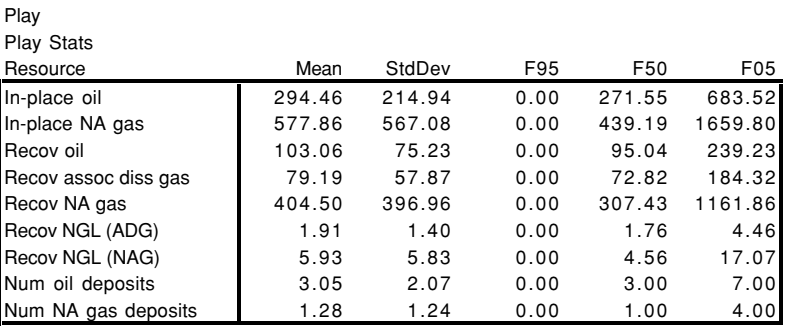

RNS $=442$

Play Totals Beaufortian Cretaceous Topset N

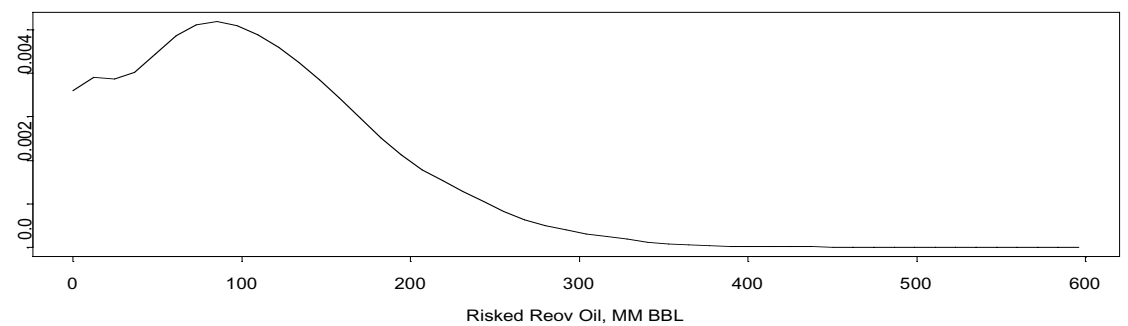

Play Totals Beaufortian Cretaceous Topset N

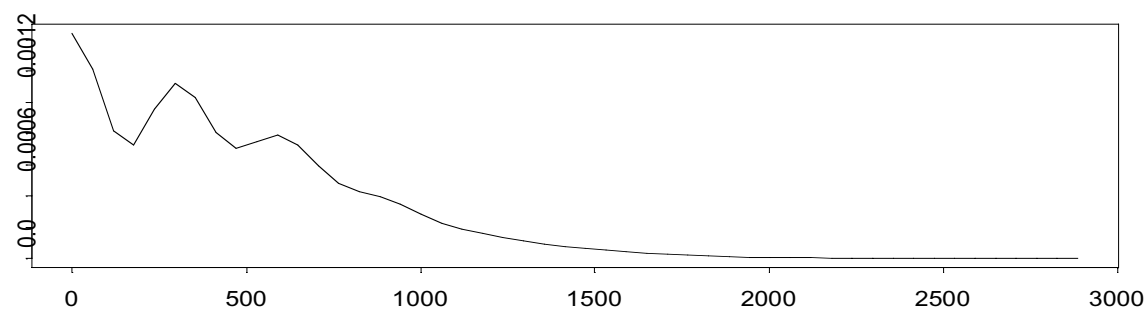

Risked Recov Gas, BCF 
Table 12f: Estimated undiscovered oil and gas resources for the Beaufortian Cretaceous Topset North Play showing number of deposits and volumes by accumulation size class

\begin{tabular}{rrrrrr} 
Start size class & Oil deposits & Oil (MMBO_Start size class & Gas deposits & NA Gas (BCFG) \\
0 & 0.00 & 0.00 & 0 & 0.00 & 0.00 \\
8 & 0.00 & 0.00 & 48 & 0.00 & 0.00 \\
16 & 1.79 & 43.07 & 96 & 0.00 & 0.00 \\
32 & 1.09 & 46.72 & 192 & 1.12 & 329.79 \\
64 & 0.16 & 12.61 & 384 & 0.16 & 74.46 \\
128 & 0.00 & 0.66 & 768 & 0.00 & 0.29 \\
256 & \multicolumn{5}{r}{} \\
\hline Totals & 3.05 & 103.07 & 1536 & 1.28 & 404.53 \\
\hline
\end{tabular}
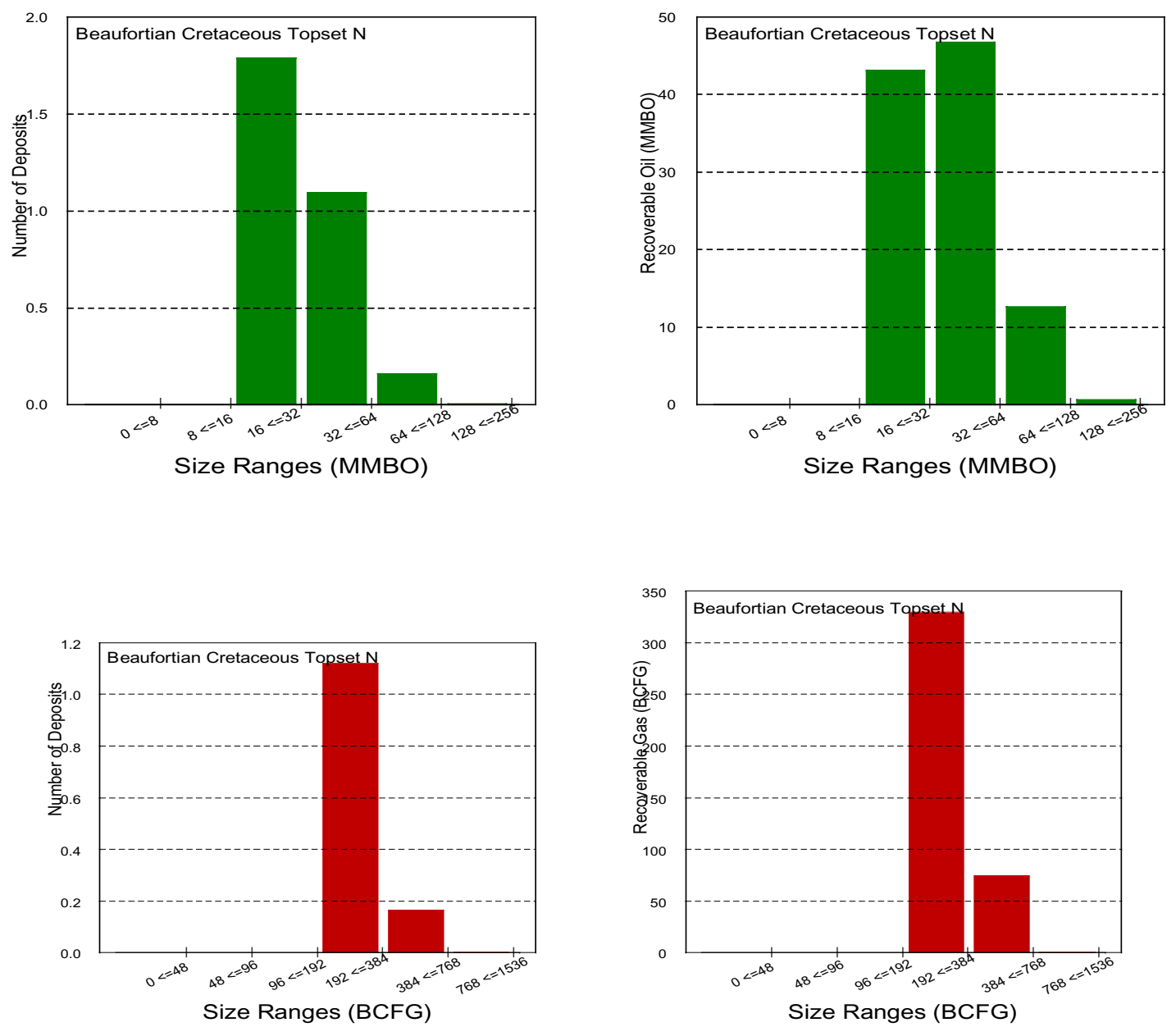
Table 13a: Input values for nonassociated gas accumulations in the Beaufortian Cretaceous Topset South Play NPRA Assessment Form-2001

PLAY: Beaufortian Cretaceous Topset South

\begin{tabular}{ll|l|l|} 
Play area: & 6,831 & $10^{3}$ Acres \\
\hline
\end{tabular}

NONASSOCIATED GAS ACCUMULATION VOLUME PARAMETERS

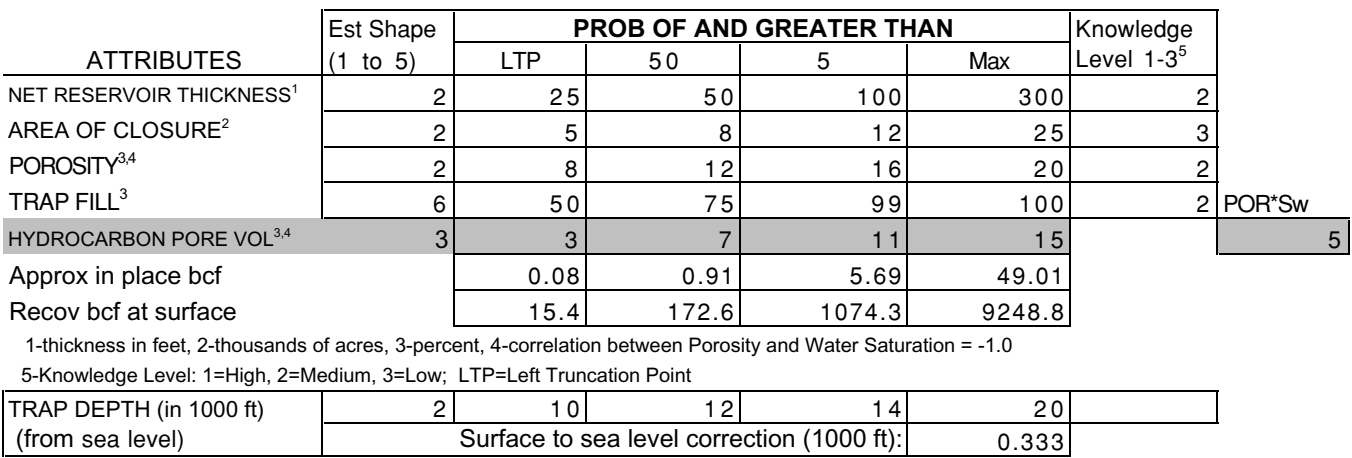

\section{NONASSOCIATED GAS ACCUMULATION CHARACTERISTICS}

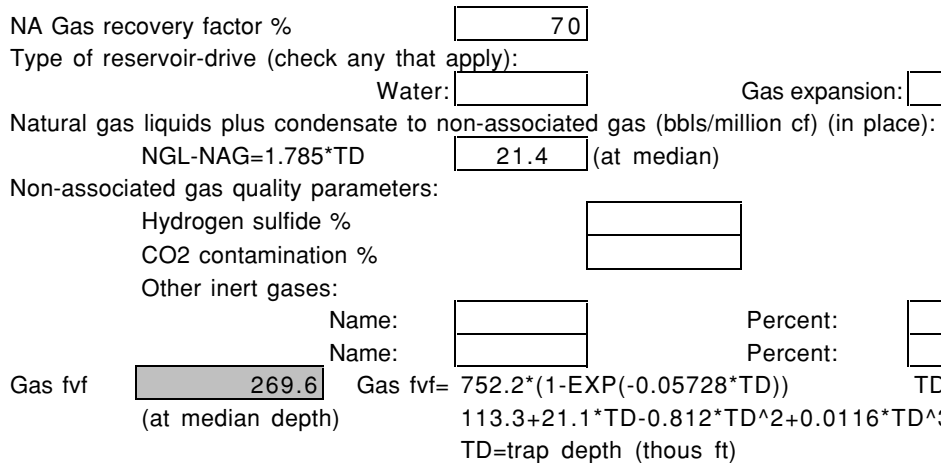

Gas fvf (at median depth)

$$
\mathrm{TD}=\text { trap depth (thous } \mathrm{ft} \text { ) }
$$

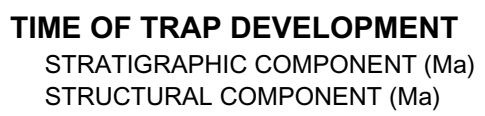

\begin{tabular}{|l|l|l|}
\multicolumn{1}{l}{ BEGIN } & PEAK & END \\
\hline & & \\
\hline & & \\
\hline
\end{tabular}

Assessor's Name: Dave Houseknecht Date of Data Entry MM/DD/YYYY:

Date of Simulation Run MM/DD/YYYY: 
Table 13b: Input risking values for the Beaufortian Cretaceous Topset South Play NPRA Assessment Form-2001

Play: Beaufortian Cretaceous Topset South

\section{RISKING}

MINIMUM ACCUMULATION SIZE, MAS (Millions of BBL in place)

PRERISKED FREQUENCY DISTRIBUTION (Oil plus Gas)

\begin{tabular}{|c|c|c|c|c|c|c|}
\hline \multirow{3}{*}{$\begin{array}{l}\text { NUM OF PROSPECTS } \\
>\text { MINIMUM SIZE }\end{array}$} & & \multicolumn{4}{|c|}{ PROB OF AND GREATER THAN } & \multirow{2}{*}{$\begin{array}{l}\text { Knowledge } \\
\text { Level } 1-3^{5}\end{array}$} \\
\hline & Est Shape & Min & 50 & 5 & Max & \\
\hline & 2 & 15 & 30 & 45 & 90 & 3 \\
\hline
\end{tabular}

5-Knowledge Level: 1=High, 2=Medium, 3=Low; LTP=Left Truncation Point

\section{ATTRIBUTES}

PLAY

ATTRIBUTES

CHARGE (C)

TRAP (T)
TIMING (F)

Probability that play contains at least 1 reservoir $>=$ minimum size (CxTXF)

PROSPECT

ATTRIBUTES

\section{CHARGE (c)}

$\operatorname{TRAP}(\mathrm{t})$

TIMING (f)

Probability that a randomly chosen prospect is favorable (cxtxf)

Play Attributes x Prospect Attributes (CxTxFxcxtxf)

FRACTION OF ACCUMULATIONS BEING OIL

Fraction NA Gas=1-Fraction(Oil)

\section{PROBABILITY}

\section{OF FAVORABLE}

Computed
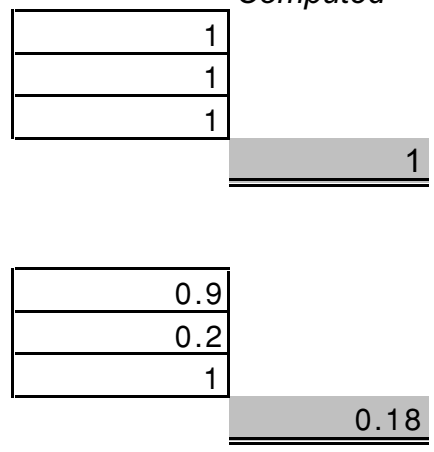

0.18

Allocation (percent):

\begin{tabular}{|l|r|r|r|}
\cline { 2 - 4 } \multicolumn{1}{c|}{} & \multicolumn{1}{c|}{ Land } & \multicolumn{1}{c|}{ Oil } & \multicolumn{2}{c|}{ Gas } \\
\hline Federal & 97 & 0 & 94 \\
\hline State & 2 & 0 & 3 \\
\hline Native & 1 & 0 & 3 \\
\hline
\end{tabular}

Assessor's Name:

Dave Houseknecht

Date of Data Entry MM/DD/YYYY:

Date of Simulation Run MM/DD/YYYY:

$10 / 19 / 01$

$1 / 19 / 02$

0

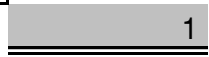


Table 13c: Distribution of fitted hydrocarbon volume attributes, trap depth, and number of prospects for the Beaufortian Cretaceous Topset South Play
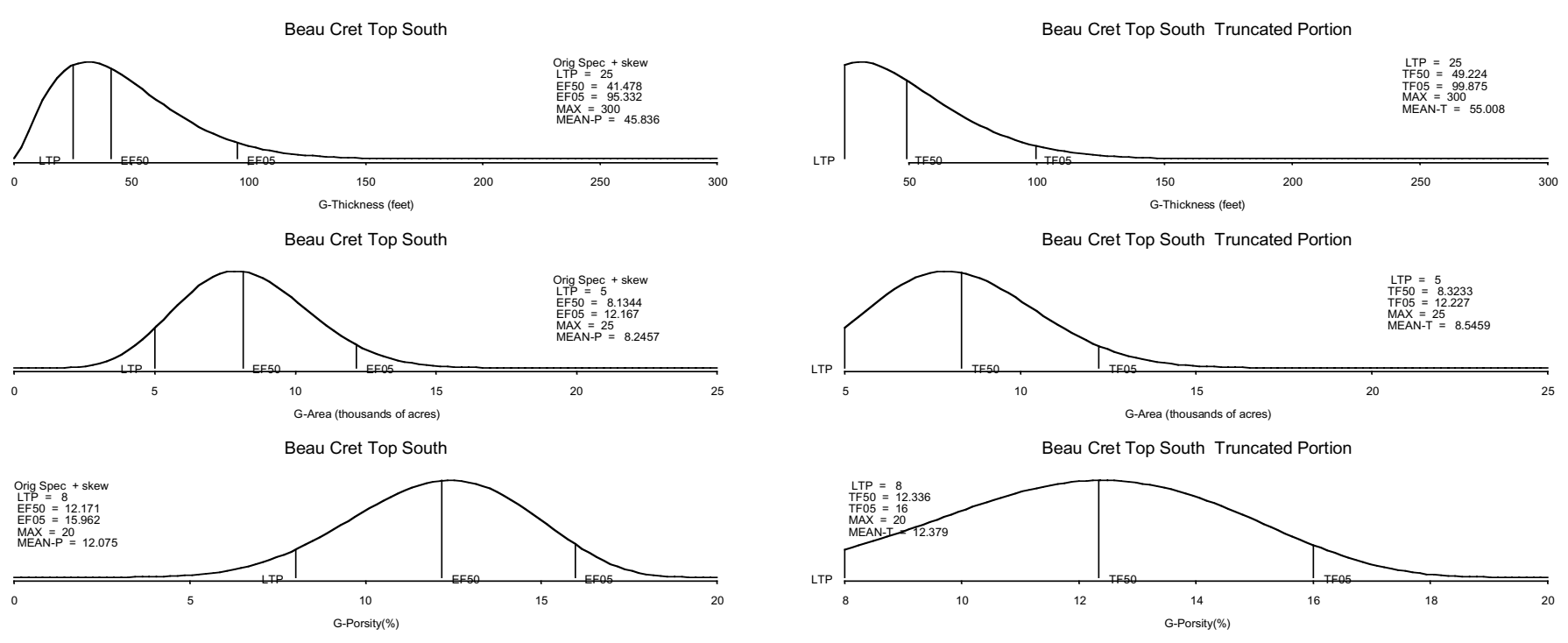

Beau Cret Top South
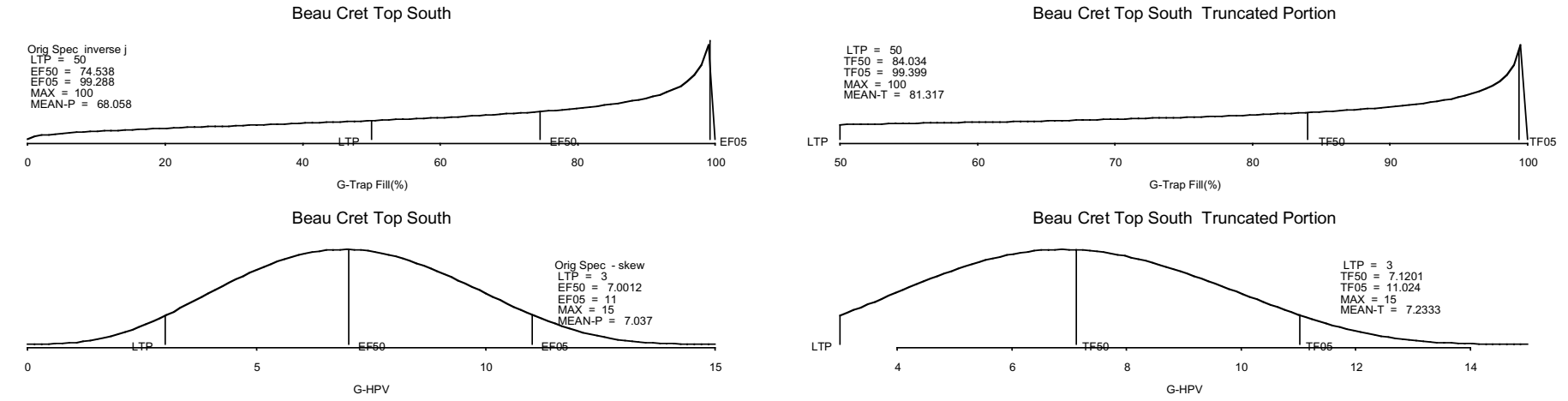

Beau Cret Top South

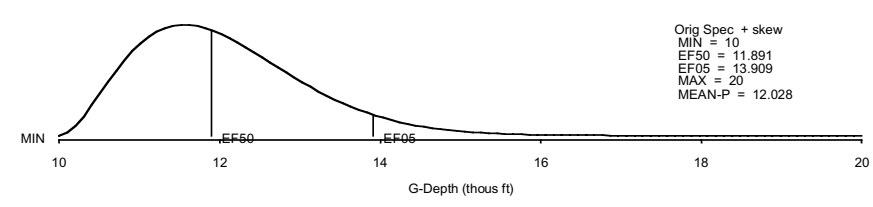




\section{Beau Cret Top South}

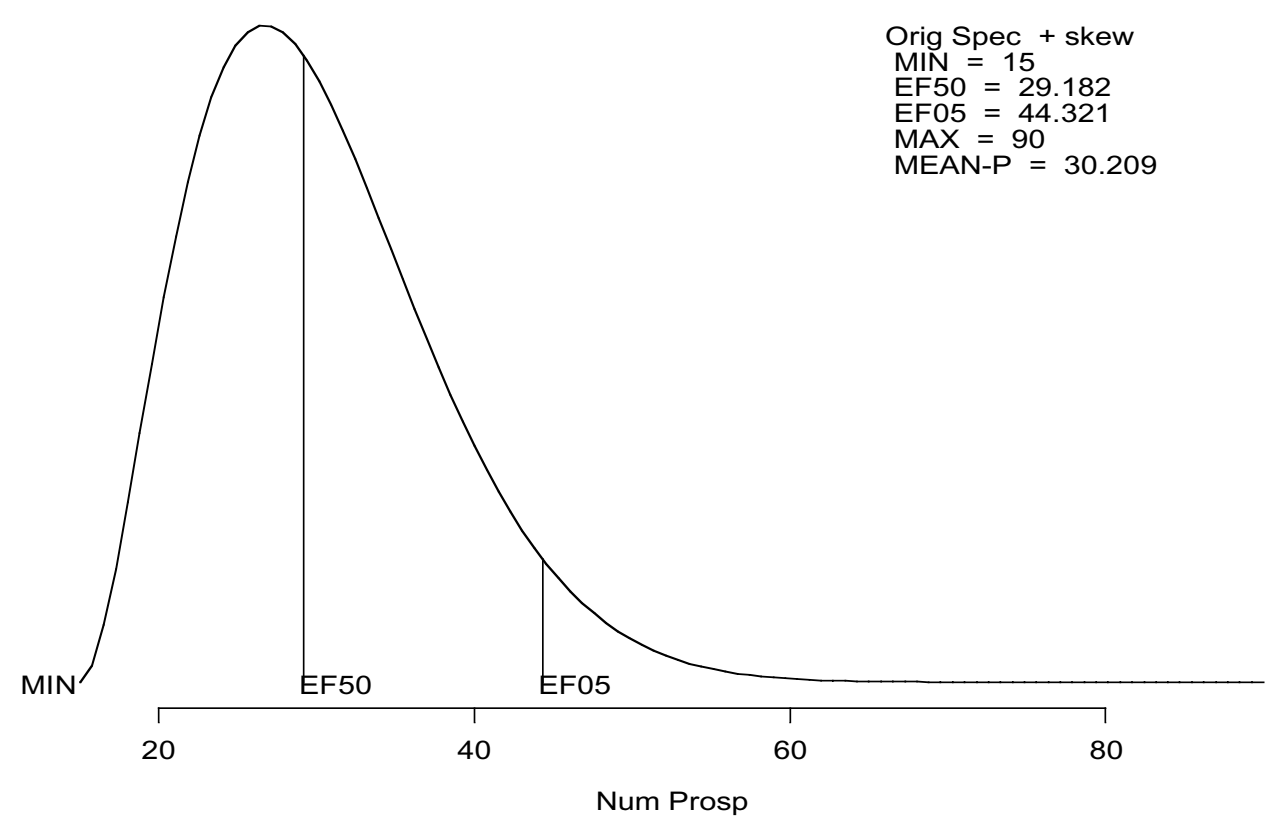


Table 13d: Estimated undiscovered oil and gas resources for the Beaufortian Cretaceous Topset South Play Deposit

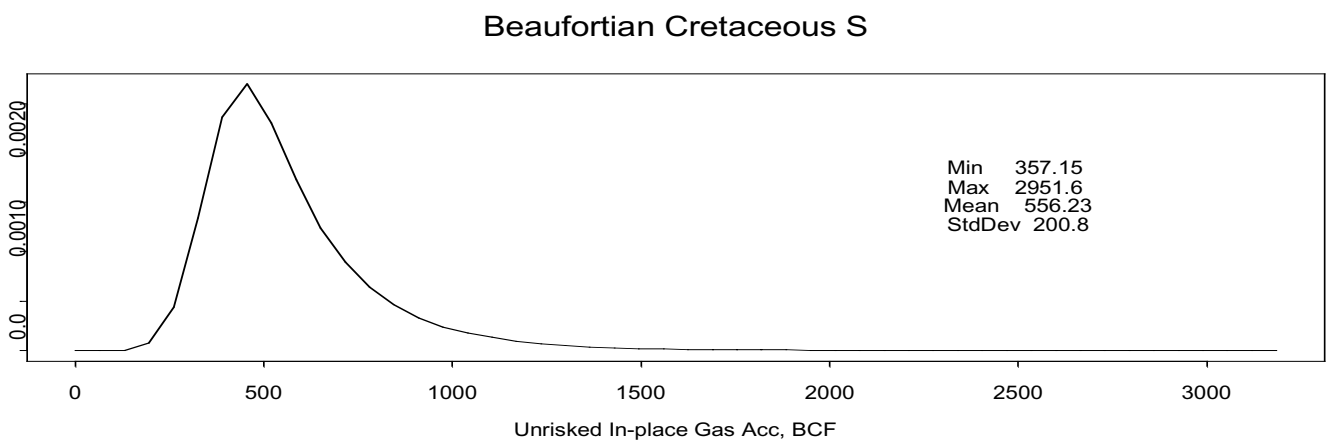

\begin{tabular}{|c|c|c|c|c|c|}
\hline Play & & & & & \\
\hline \begin{tabular}{|l|} 
Resource \\
In-place oil
\end{tabular} & Mean & StdDev & F95 & F50 & F05 \\
\hline $\begin{array}{l}\text { In-place NA gas } \\
\text { Recov oil }\end{array}$ & 3042.44 & 1469.32 & 906.77 & 2889.00 & 5720.36 \\
\hline $\begin{array}{l}\text { Recov assoc diss gas } \\
\text { Recov NA gas } \\
\text { Recov NGL (ADG) }\end{array}$ & 2129.71 & 1028.53 & 634.74 & 2022.30 & 4004.25 \\
\hline $\begin{array}{l}\text { Recov NGL (NAG) } \\
\text { Num oil deposits }\end{array}$ & 47.18 & 22.87 & 14.01 & 44.87 & 88.99 \\
\hline Num NA gas deposits & 5.47 & 2.52 & 2.00 & 5.00 & 10.00 \\
\hline
\end{tabular}

RNS $=981$

Play Totals Beaufortian Cretaceous Topset S

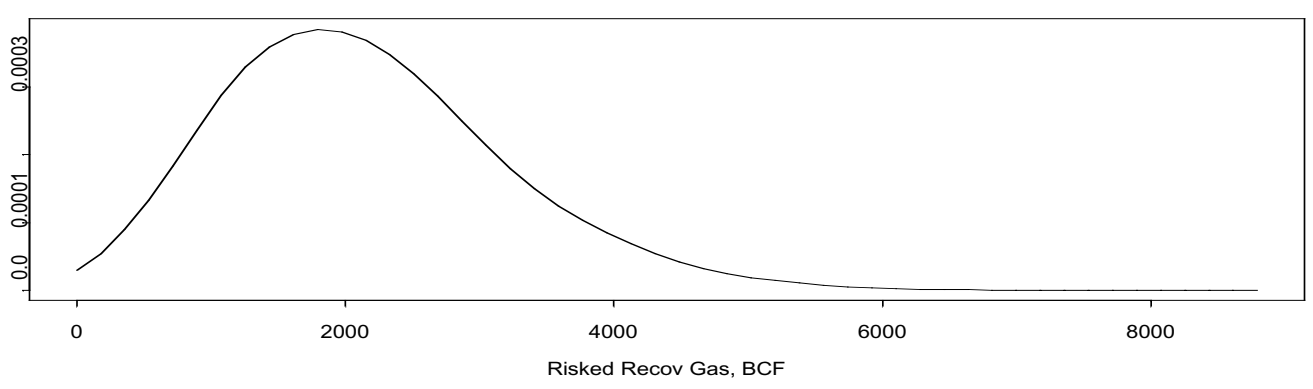


Table 13e: Estimated undiscovered oil and gas resources for the Beaufortian Cretaceous Topset South Play showing number of deposits and volumes by accumulation size class

\begin{tabular}{|c|c|c|c|c|c|}
\hline 0 & & & 0 & חم ח & חم ח \\
\hline 8 & & & 48 & 0.00 & 0.00 \\
\hline 16 & & & 96 & 0.00 & 0.00 \\
\hline 32 & & & 192 & 3.39 & 1038.84 \\
\hline 64 & & & 384 & 1.94 & 964.18 \\
\hline 128 & & & 768 & 0.14 & 124.92 \\
\hline 256 & & & 1536 & 0.00 & 1.98 \\
\hline 512 & & & 3072 & & \\
\hline Totals & 0.00 & 0.00 & & 5.47 & 2129.92 \\
\hline
\end{tabular}
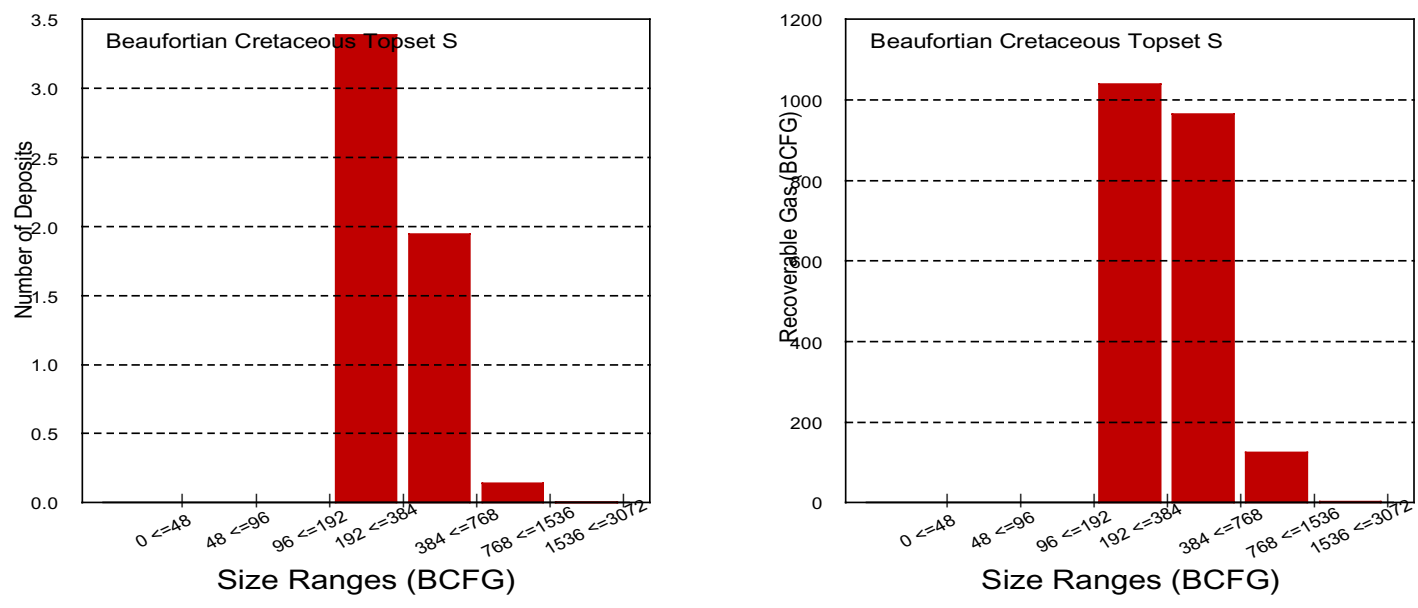
Table 14a: Input values for oil accumulations in the Beaufortian Upper Jurassic Topset NE Play NPRA Assessment Form-2001

PLAY: Beaufortian Upper Jurassic Topset NE

SubArea A (northeast) NE

Play area: $\quad 428110^{3}$ Acres

OIL ACCUMULATION VOLUME PARAMETERS

\begin{tabular}{|c|c|c|c|c|c|c|c|}
\hline \multirow[b]{2}{*}{ ATTRIBUTES } & \multirow{2}{*}{$\begin{array}{l}\text { Est Shape } \\
(1 \text { to } 5)\end{array}$} & \multicolumn{4}{|c|}{ PROB OF AND GREATER THAN } & \multirow{2}{*}{$\begin{array}{l}\text { Knowledge } \\
\text { Level } 1-3^{5} \\
\end{array}$} & \\
\hline & & LTP & 0.50 & 0.05 & Max & & \\
\hline \multirow{4}{*}{$\begin{array}{l}\text { NET RESERVOIR THICKNESS } \\
\text { AREA OF CLOSURE }^{1} \\
\text { POROSITY }^{3,4} \\
\text { TRAP FILL }\end{array}$} & \begin{tabular}{r|}
2 \\
\end{tabular} & 25 & 50 & 75 & 150 & 2 & \\
\hline & 2 & 2 & 8 & 25 & 40 & 3 & Alpine $\sim 28,000$ acres \\
\hline & 4 & 12 & 17 & 21 & 22 & 2 & Left skewed \\
\hline & 7 & 80 & 100 & 100 & 100 & 2 & Enter $\mathrm{POR}^{*} \mathrm{Sw}$ \\
\hline \multicolumn{2}{|l|}{ HYDROCARBON PORE VOL } & 8 & 13 & 17 & 18 & & \begin{tabular}{|r|}
4 \\
\end{tabular} \\
\hline \multirow{2}{*}{\multicolumn{2}{|c|}{$\begin{array}{l}\text { Approx mm bbl (fvf }=1) \\
\text { Recov mm bbl at surface }\end{array}$}} & 24.8 & 403.4 & 2472.9 & 8378.6 & & \\
\hline & & 7.4 & 119.5 & 732.3 & 2481.1 & & \\
\hline \multicolumn{8}{|c|}{ 1-thickness in feet, 2-thousands of acres, 3-percent, 4-correlation between Porosity and Water Saturation $=-1.0$} \\
\hline \multirow{2}{*}{$\begin{array}{l}\text { TRAP DEPTH (in } 1000 \mathrm{ft} \text { ) } \\
\text { (from sea level) }\end{array}$} & \begin{tabular}{|r|}
3 \\
\end{tabular} & 3 & 9 & 10 & 11 & 1 & \multirow[t]{2}{*}{ JSee SubAreas Tab } \\
\hline & & Surface to $\mathrm{s}$ & level correct & (1000 ft): & 0.102 & & \\
\hline
\end{tabular}

\section{OIL ACCUMULATION CHARACTERISTICS}

Oil recovery factor \% 50

Type of reservoir-drive (check any that apply):

WVF (Formation volume factor, rb/stb):
\begin{tabular}{|lr} 
Pressure(psi) & 4500 \\
temp(deg F) & 201 \\
SolGasGr & 0.757 \\
F & 1376 \\
\hline
\end{tabular}

GOR (Associated gas to oil ratio, cu.ft./bbl, at stp):

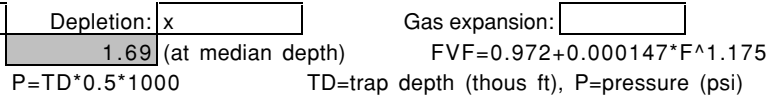

$T=19 * T D+30 \quad T=$ temp deg $F \quad L N$ is log base e

$S G G=\left(\left(0.1402^{*} \mathrm{LN}(\mathrm{C} 27+14.7)-0.4227\right)+\left(0.1369^{*} \mathrm{LN}(\mathrm{C} 28)+0.0156\right)+\left(0.1704^{*} \mathrm{LN}(\mathrm{E} 37)-\mathrm{AG}=\mathrm{API}\right.\right.$ gravity

Uncorrected GOR $=\mathrm{SGG}^{*}\left((\mathrm{P}+14.7)^{\star} 10^{\wedge}\left(0.0125^{\star} \mathrm{AG}\right) /\left(18^{\star} 10^{\wedge}\left(0.00091^{\star} \mathrm{T}\right)\right)\right)^{\wedge}(1 / 0.83)$

$\mathrm{F}=$ Final $\mathrm{GOR}^{\star}(\mathrm{SGG} / \mathrm{OG})^{\wedge} 0.5+1.25^{\star} \mathrm{T} \quad \mathrm{OG}=141.5 /(131.5+\mathrm{AG})$

\begin{tabular}{r|r|}
\cline { 2 - 2 } & 1178 \\
Uncorrected GOR
\end{tabular}

NGLR (Natural gas liquids to associated gas ratio, bbls/million cu.ft., at $\operatorname{stp})=4.5985^{\star} \exp \left(.1711^{\star}\right.$ TD)

21.4 (median depth)

Oil quality parameters:

API gravity

Sulfur content of oil

\begin{tabular}{r|}
\hline 39 \\
\hline 0.3 \\
\hline
\end{tabular}

Oil Grav (ratio)

0.830

Associated gas quality parameters

Hydrogen sulfide \%

CO2 contamination \%

Other inert gases:

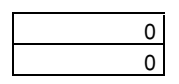

Name: $\quad$ Percent:

Name:

Percent:

TIME OF TRAP DEVELOPMENT

STRATIGRAPHIC COMPONENT (Ma)

STRUCTURAL COMPONENT (Ma)

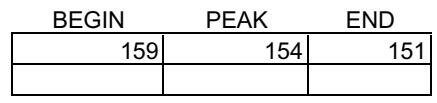

Assessor's Name: Dave Houseknecht

Date of Data Entry MM/DD/YYYY:

Date of Simulation Run MM/DD/YYYY:

$10 / 19 / 01$

$2 / 20 / 02$

Note: only enter play name and assessor's name on Oil worksheet 
Table 14b: Input risking values for the Beaufortian Upper Jurassic Topset NE Play NPRA Assessment Form-2001

Play: Beaufortian Upper Jurassic Topset NE

\section{RISKING}

MINIMUM ACCUMULATION SIZE, MAS (Millions of BBL in place)

50

PRERISKED FREQUENCY DISTRIBUTION (Oil plus Gas)

NUM OF PROSPECTS

$>$ MINIMUM SIZE

PROB OF AND GREATER THAN

Knowledge

\begin{tabular}{|r|r|r|r|r|r|}
\hline Est Shape & Min & 50 & 5 & Max & Level $1-3^{5}$ \\
\hline 2 & 30 & 60 & 90 & 120 & 3 \\
\hline
\end{tabular}

5-Knowledge Level: 1=High, 2=Medium, 3=Low; LTP=Left Truncation Point

\section{ATTRIBUTES}

PLAY

ATTRIBUTES

TIMING $(\mathrm{F})$
Probability that play contains at least 1 reservoir $>=$ minimum size $(C x T x F)$

CHARGE (C)

TRAP (T)

PROSPECT

ATTRIBUTES

CHARGE (c)

$\operatorname{TRAP}(\mathrm{t})$

TIMING (f)

Probability that a randomly chosen prospect is favorable (cxtxf)

Play Attributes x Prospect Attributes (CxTxFxcxtxf)

FRACTION OF ACCUMULATIONS BEING OIL

Fraction NA Gas=1-Fraction(Oil)

Allocation (percent):

\begin{tabular}{|l|r|r|l|}
\cline { 2 - 4 } \multicolumn{1}{c|}{} & \multicolumn{1}{c|}{ Land } & \multicolumn{1}{c|}{ Oil } & Gas \\
\hline Federal & 93 & 92 & \\
\hline State & 3 & 1 & \\
\hline Native & 4 & 7 & \\
\hline
\end{tabular}

Assessor's Name:

Date of Data Entry MM/DD/YYYY:

Date of Simulation Run MM/DD/YYYY:
PROBABILITY OF FAVORABLE

Computed

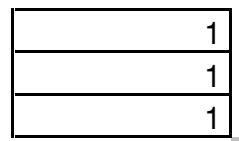

1
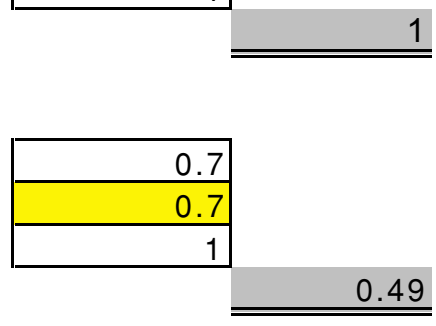

0.49

1.

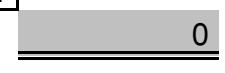


Table 14c: Distribution of fitted hydrocarbon volume attributes, trap depth, and number of prospects for the Beaufortian Upper Jurassic Topset NE Play
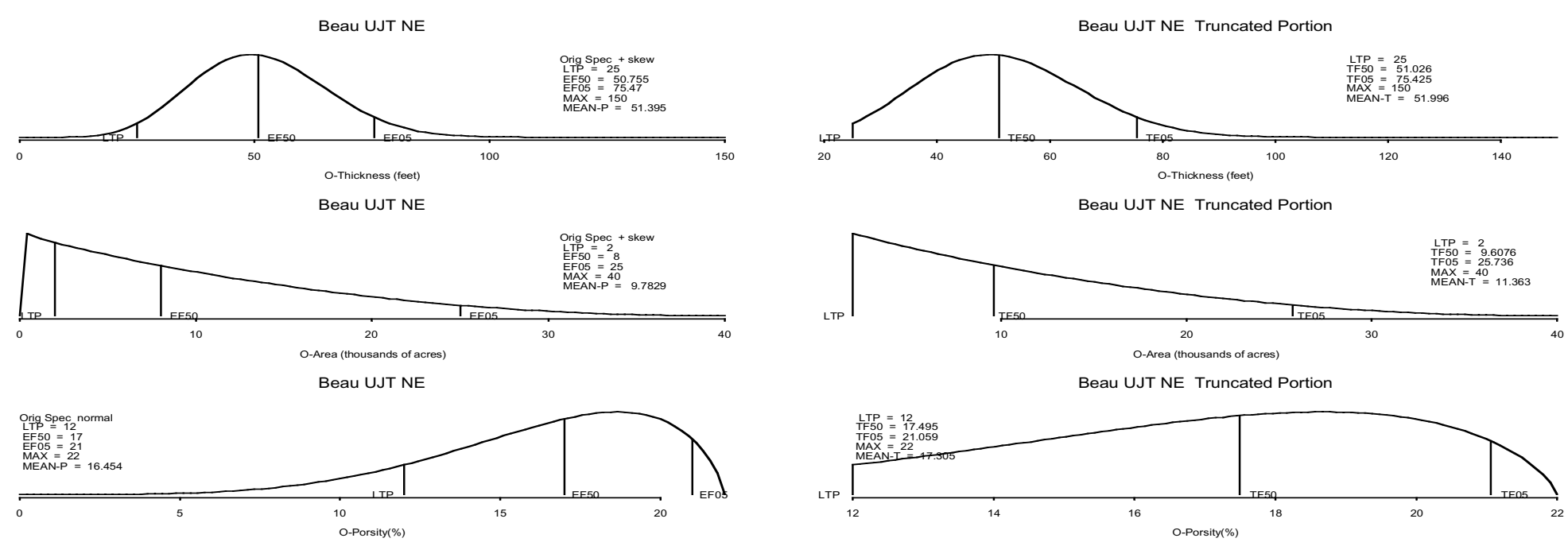

Beau UJT NE
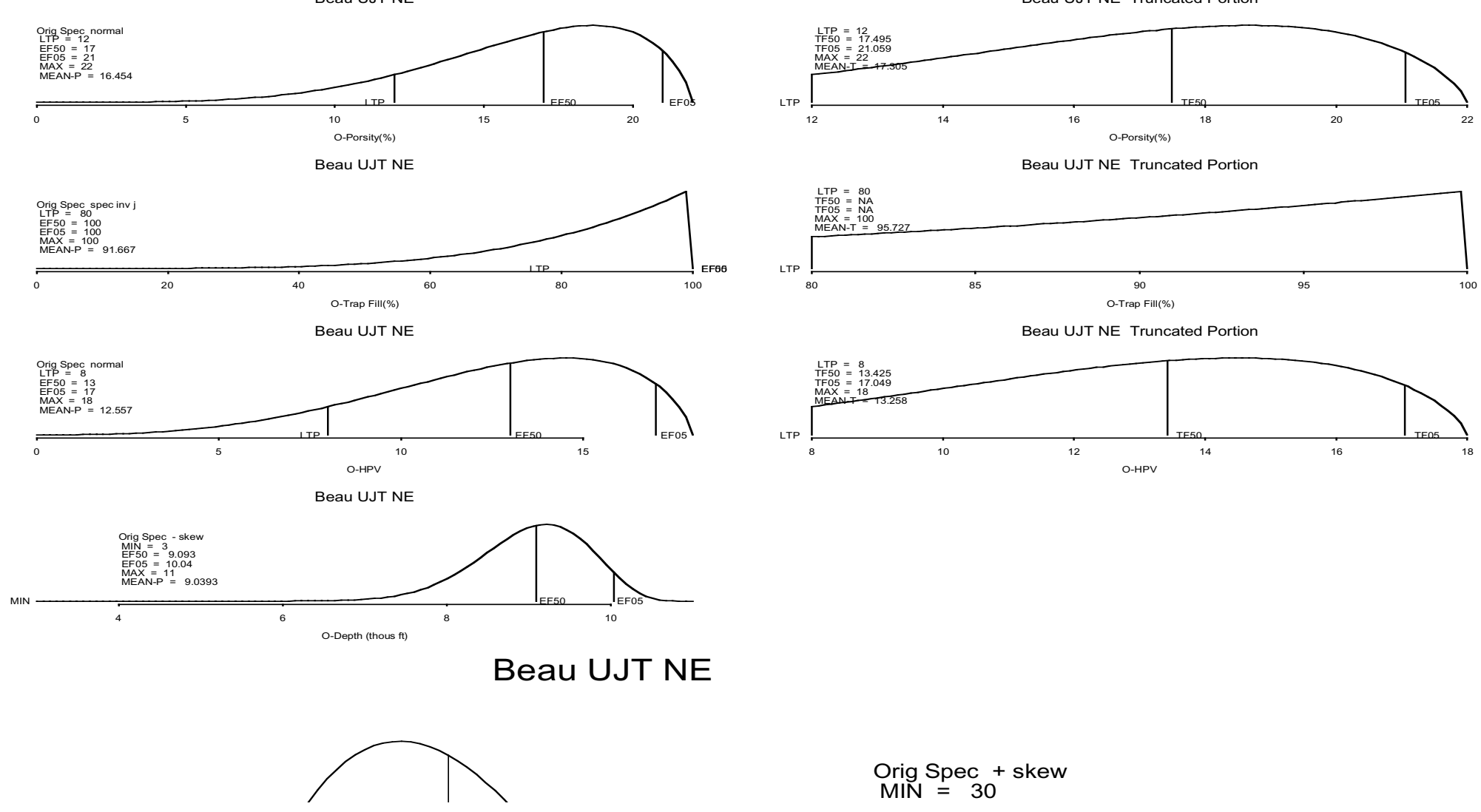


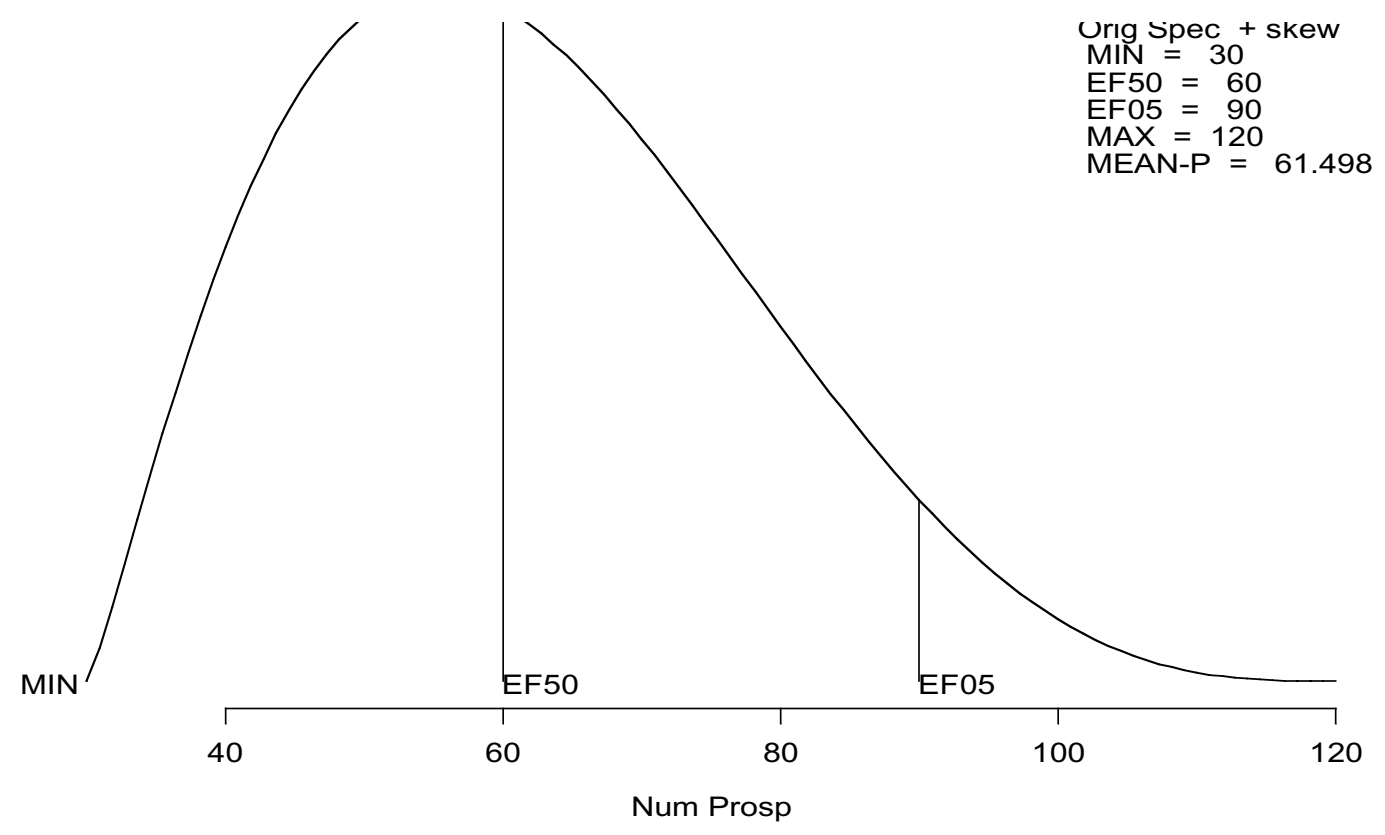


Table 14d: Estimated undiscovered oil and gas resources for the Beaufortian Upper Jurassic Topset NE Play

\begin{tabular}{rrrrrr} 
Start size class & & Oil deposits & Oil (MMBO_ Start size cl & Gas deposits & NA Gas (BCFG) \\
0 & 0.00 & 0.00 & 0 & \\
8 & 0.00 & 0.00 & 48 & \\
16 & 0.79 & 22.55 & 96 & \\
32 & 5.03 & 242.39 & 192 & \\
64 & 8.35 & 785.80 & 384 & \\
128 & 9.78 & 1793.59 & 768 & \\
256 & 5.57 & 1916.99 & 1536 & \\
512 & 0.67 & 411.67 & 3072 & \\
1024 & 0.00 & 3.05 & 6144 & \\
\hline Totals & 30.19 & 5176.05 & & \\
\hline
\end{tabular}
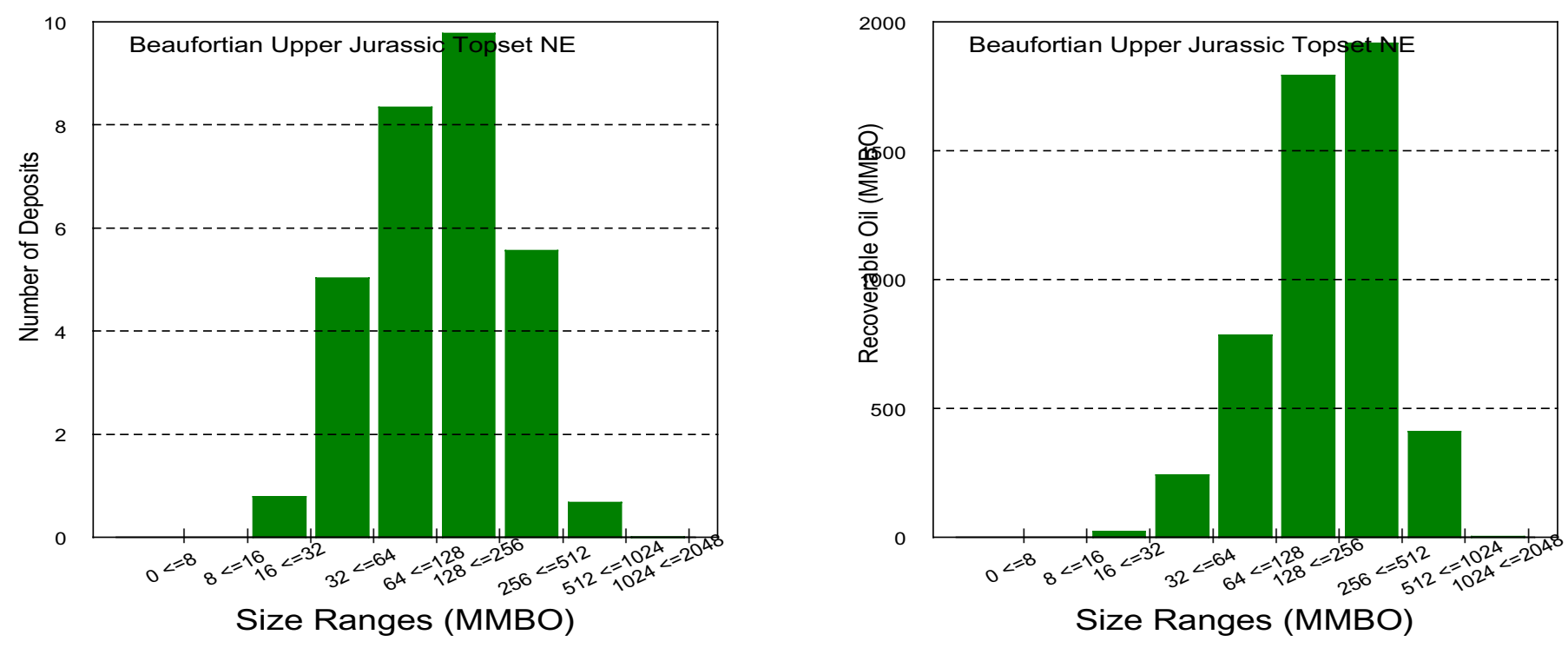
Table 15a: Input values for oil accumulations in the Beaufortian Upper Jurassic Topset SE Play NPRA Assessment Form-2001

PLAY: Beaufortian Upper Jurassic Topset SE $\quad$ SubArea B (eastcentral) SE

Play area: $\quad 1795 \mid 10^{3}$ Acres

OIL ACCUMULATION VOLUME PARAMETERS

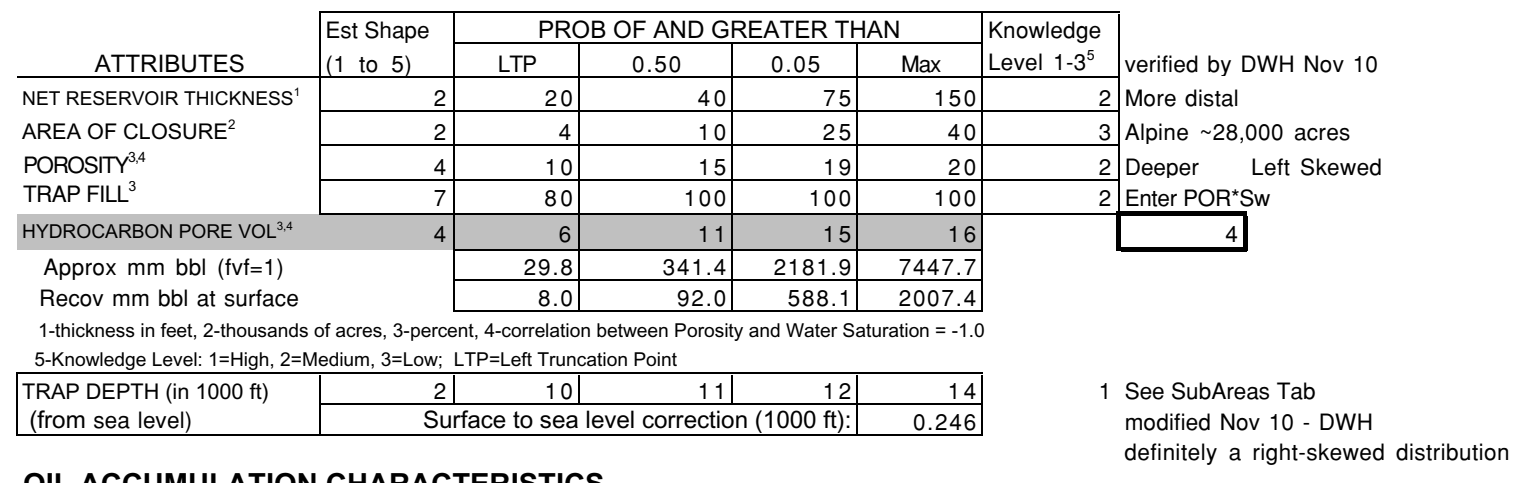

\section{OIL ACCUMULATION CHARACTERISTICS}

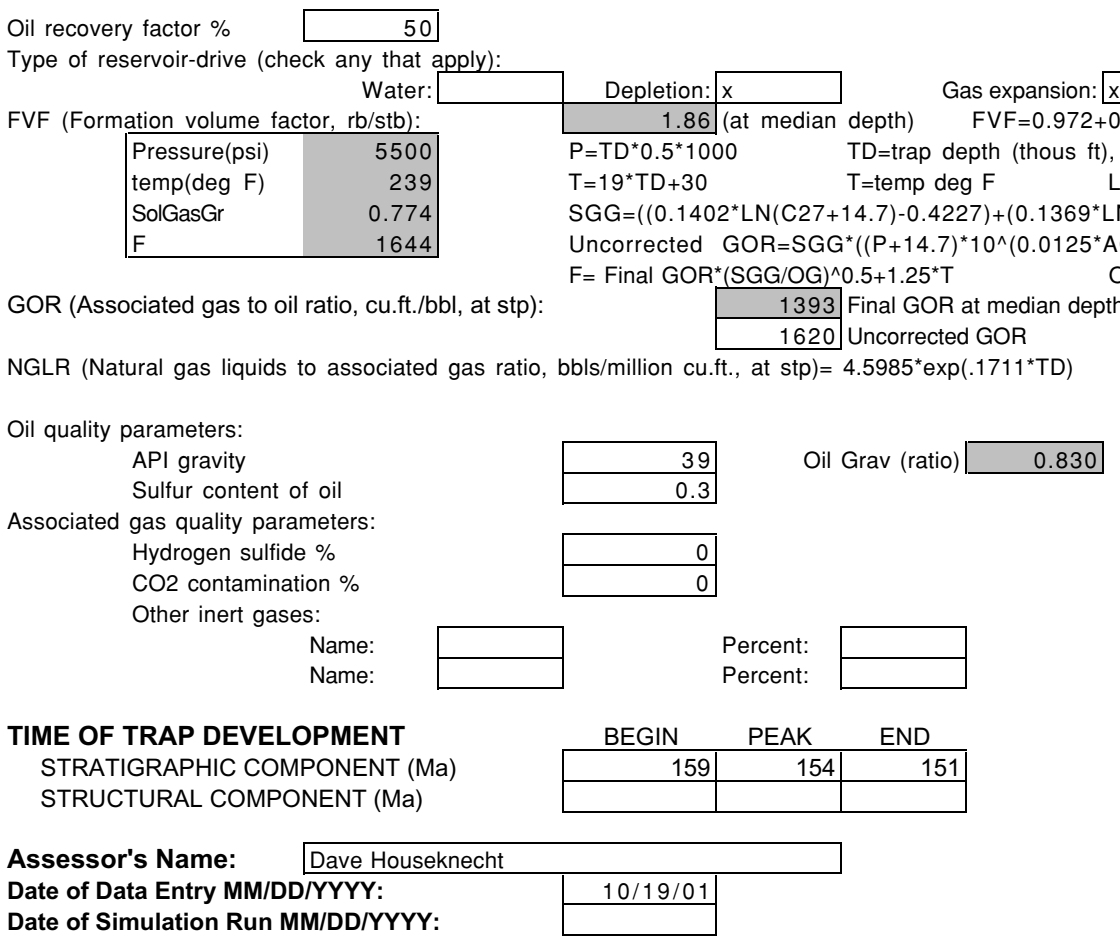

Note: only enter play name and assessor's name on Oil worksheet 
Table 15b: Input values for nonassociated gas accumulations in the Beaufortian Upper Jurassic Topset SE Play NPRA Assessment Form-2001
PLAY: Beaufortian Upper Jurassic Topset SE
SubArea B (eastcentral) SE

NONASSOCIATED GAS ACCUMULATION VOLUME PARAMETERS

\begin{tabular}{|c|c|c|c|c|c|c|c|}
\hline \multirow[b]{2}{*}{ ATTRIBUTES } & \multirow{2}{*}{\begin{tabular}{|l|} 
Est Shape \\
$(1$ to 5$)$
\end{tabular}} & \multicolumn{4}{|c|}{ PROB OF AND GREATER THAN } & \multirow{2}{*}{$\begin{array}{l}\text { Knowledge } \\
\text { Level } 1-3^{5} \\
\end{array}$} & \\
\hline & & LTP & 50 & 5 & Max & & \\
\hline \multirow{4}{*}{$\begin{array}{l}\text { NET RESERVOIR THICKNESS } \\
\text { AREA OF CLOSURE }^{1} \\
\text { POROSITY }^{3,4} \\
\text { TRAP FILL }^{3}\end{array}$} & 2 & 20 & 40 & 75 & 150 & 2 & \\
\hline & 2 & 4 & 10 & 25 & 40 & 3 & \\
\hline & 4 & 10 & 15 & 19 & 20 & 2 & \\
\hline & 7 & 80 & 100 & 100 & 100 & 2 & Uses oil POR ${ }^{\star} S w$ \\
\hline HYDROCARBON PORE VOL & 4 & 6 & 11 & 15 & 16 & & 4 \\
\hline \multirow{2}{*}{\multicolumn{2}{|c|}{$\begin{array}{l}\text { Approx in place bcf } \\
\text { Recov bcf at surface }\end{array}$}} & 0.17 & 1.92 & 12.25 & 41.82 & & \\
\hline & & 30.0 & 343.7 & 2196.7 & 7498.2 & & \\
\hline \multicolumn{7}{|c|}{$\begin{array}{l}\text { 1-thickness in feet, 2-thousands of acres, 3-percent, 4-correlation between Porosity and Water Saturation }=-1.0 \\
\text { 5-Knowledge Level: } 1=\text { High, 2=Medium, 3=Low; LTP=Left Truncation Point }\end{array}$} & \\
\hline \multirow{2}{*}{\begin{tabular}{l|} 
TRAP DEPTH (in $1000 \mathrm{ft}$ ) \\
(from sea level)
\end{tabular}} & 2 & 10 & 13 & 16 & 20 & 1 & \\
\hline & \multicolumn{4}{|c|}{ Surface to sea level correction $(1000 \mathrm{ft})$ : } & 0.246 & & \\
\hline
\end{tabular}

\section{NONASSOCIATED GAS ACCUMULATION CHARACTERISTICS}

NA Gas recovery factor \% 65

Type of reservoir-drive (check any that apply):

$$
\text { Water: }
$$

Gas expansion:

Natural gas liquids plus condensate to non-associated gas (bbls/million cf) (in place): NGL-NAG $=1.785^{\star} \mathrm{TD}$ 23.2 (at median)

Non-associated gas quality parameters: Hydrogen sulfide \% CO2 contamination \% Other inert gases:

$$
\text { Name: }
$$
Name:

Gas fvf

$$
275.9
$$
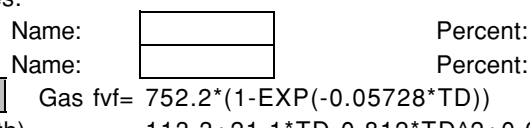

$$
\text { Percent: }
$$$$
113.3+21.1^{*} T D-0.812^{*} T^{\wedge} 2+0.0116^{*} T^{\wedge} 3 \quad 5.67<T D<=30
$$

$\mathrm{TD}=$ trap depth (thous $\mathrm{ft}$ )

TIME OF TRAP DEVELOPMEN 7 BEGIN
STRATIGRAPHIC COMPONENT (Ma)
STRUCTURAL COMPONENT (Ma)

Assessor's Name: David Houseknecht Date of Data Entry MM/DD/YYYY:

Date of Simulation Run MM/DD/YYYY: 
Table 15c: Input risking values for the Beaufortian Upper Jurassic Topset SE Play NPRA Assessment Form-2001

Play: Beaufortian Upper Jurassic Topset SE

\section{RISKING}

MINIMUM ACCUMULATION SIZE, MAS (Millions of BBL in place)

PRERISKED FREQUENCY DISTRIBUTION (Oil plus Gas)

NUM OF PROSPECTS

$>$ MINIMUM SIZE

\begin{tabular}{|r|r|r|r|r|r|}
\cline { 2 - 5 } \multicolumn{1}{c|}{} & \multicolumn{4}{|c|}{ PROB OF AND GREATER THAN } & \multicolumn{2}{l|}{$\begin{array}{l}\text { Knowledge } \\
\text { Est Shape }\end{array}$} & Min & 50 & 5 & Max & \\
\hline 2 & 10 & 18 & 28 & 40 & 3 \\
\hline
\end{tabular}

5-Knowledge Level: 1=High, 2=Medium, 3=Low; LTP=Left Truncation Point

\section{ATTRIBUTES}

PLAY

ATTRIBUTES

TIMING $(F)$

Probability that play contains at least 1 reservoir $>=$ minimum size $(C \times T \times F)$

PROSPECT

ATTRIBUTES

TIMING (f)

Probability that a randomly chosen prospect is favorable (cxtxf)

Play Attributes x Prospect Attributes (CxTxFxcxtxf)

FRACTION OF ACCUMULATIONS BEING OIL

Fraction NA Gas=1-Fraction(Oil)

\section{PROBABILITY} OF FAVORABLE

Computed

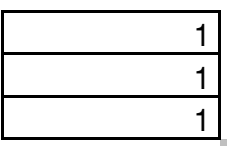

1

0

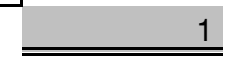

Allocation (percent):

\begin{tabular}{|l|r|r|r|}
\cline { 2 - 4 } \multicolumn{1}{c|}{} & \multicolumn{1}{c|}{ Land } & Oil & \multicolumn{1}{c|}{ Gas } \\
\hline Federal & 100 & & 100 \\
\hline State & 0 & & 0 \\
\hline Native & 0 & & 0 \\
\hline
\end{tabular}

Assessor's Name:

Dave Houseknecht

Date of Data Entry MM/DD/YYYY:

Date of Simulation Run MM/DD/YYYY:

$10 / 19 / 01$

$2 / 20 / 02$ 
Table 15d: Distribution of fitted hydrocarbon volume attributes, trap depth, and number of prospects for the Beaufortian Upper Jurassic Topset SE Play

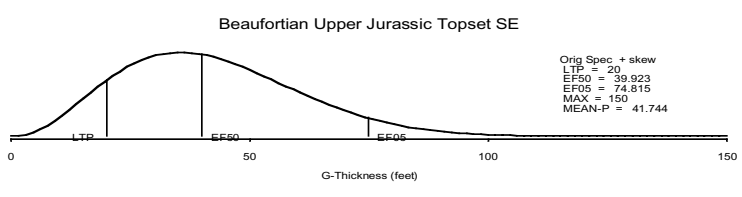

Beaufortian Upper Jurassic Topset SE

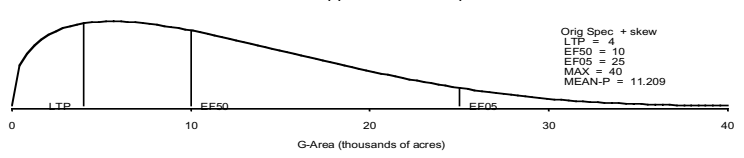

Beaufortian Upper Jurassic Topset SE

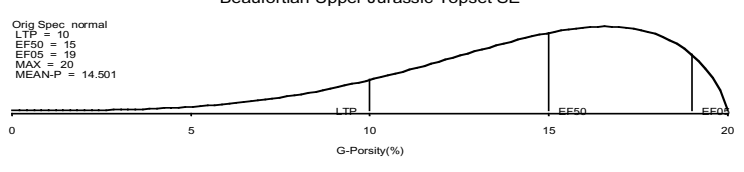

Beaufortian Upper Jurassic Topset SE

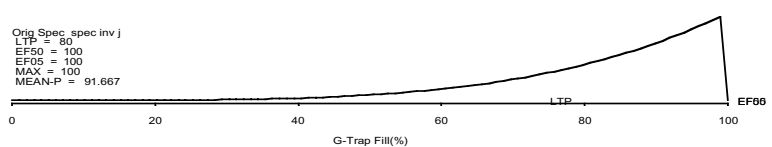

Beaufortian Upper Jurassic Topset SE

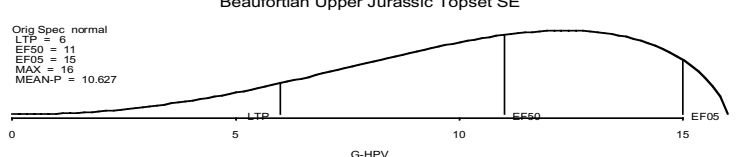

Beaufortian Upper Jurassic Topset SE

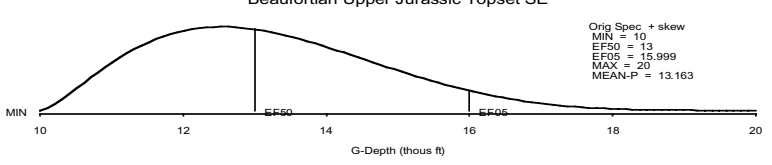

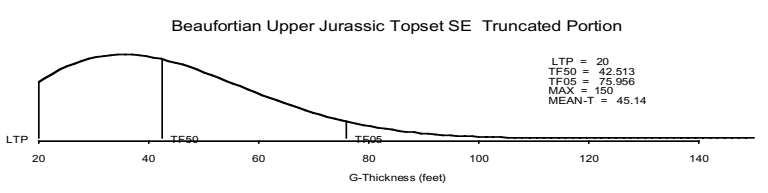

Beaufortian Upper Jurassic Topset SE Truncated Portion

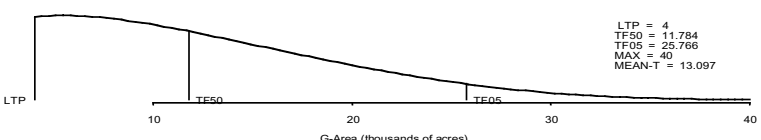

Beaufortian Upper Jurassic Topset SE Truncated Portion

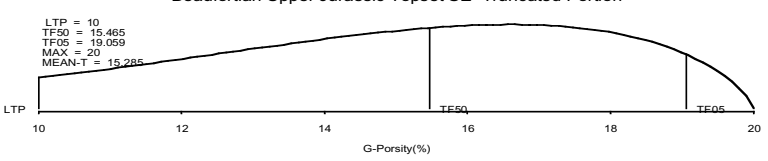

Beaufortian Upper Jurassic Topset SE Truncated Portion

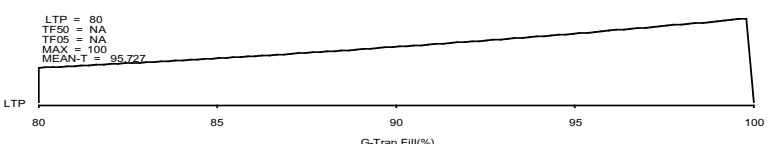

Beaufortian Upper Jurassic Topset SE Truncated Portion

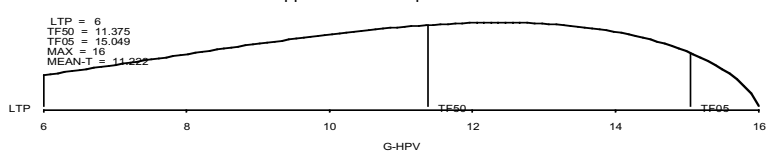


Beaufortian Upper Jurassic Topset SE

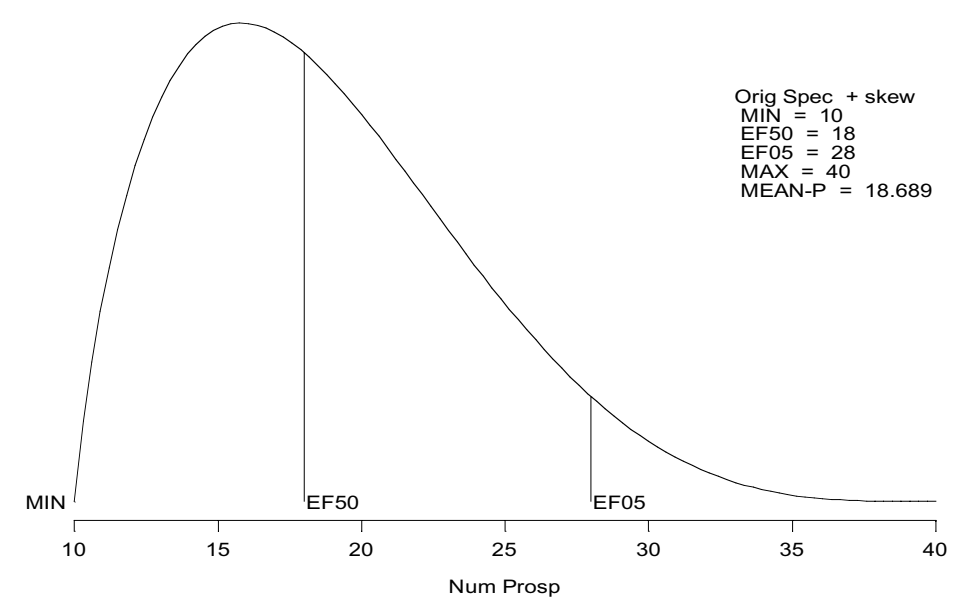


Table 15e: Estimated undiscovered oil and gas resources for the Beaufortian Upper Jurassic Topset SE Play Deposit

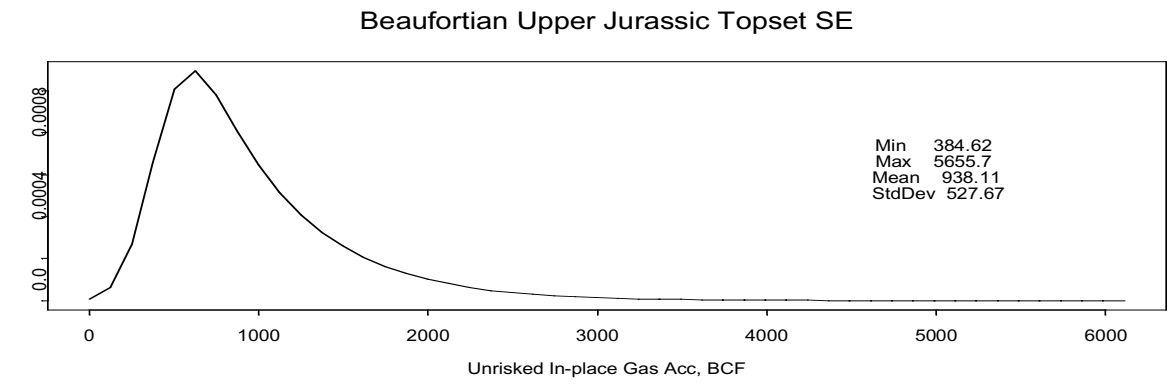

Play Stats
\begin{tabular}{|ll|rrrrr|} 
Resource & Unit & Mean & StdDev & F95 & F50 & F05 \\
\hline In-place oil & MMBO & 0.00 & 0.00 & 0.00 & 0.00 & 0.00 \\
In-place NA gas & BCFG & 7903.28 & 3305.13 & 3159.04 & 7520.49 & 13892.57 \\
Recov oil & MMBO & 0.00 & 0.00 & 0.00 & 0.00 & 0.00 \\
Recov assoc diss gas & BCFG & 0.00 & 0.00 & 0.00 & 0.00 & 0.00 \\
Recov NA gas & BCFG & 5137.13 & 2148.33 & 2053.38 & 4888.32 & 9030.17 \\
Recov NGL (ADG) & MMBO & 0.00 & 0.00 & 0.00 & 0.00 & 0.00 \\
Recov NGL (NAG) & MMBO & 123.53 & 52.21 & 48.69 & 117.36 & 218.62 \\
Num oil deposits & & 0.00 & 0.00 & 0.00 & 0.00 & 0.00 \\
Num NA gas deposits & & 8.42 & 3.14 & 4.00 & 8.00 & 14.00 \\
\hline
\end{tabular}

Play Totals Beaufortian Upper Jurassic Topset SE

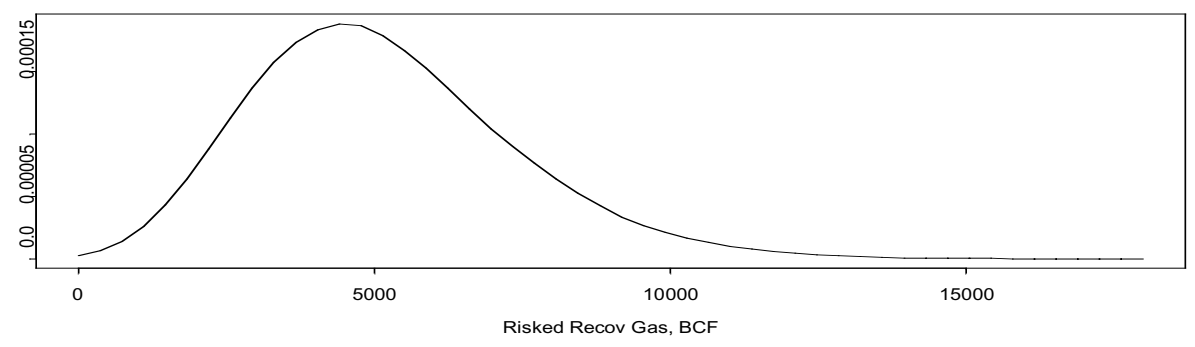


Table 15f: Estimated undiscovered oil and gas resources for the Beaufortian Upper Jurassic Topset SE Play showing number of deposits and volumes by accumulation size class

\begin{tabular}{rrrrrr} 
Start size class & Oil deposits & Oil (MMBO_Start size class & Gas deposits & NA Gas (BCFG) \\
& & 0 & 0.00 & 0.00 \\
8 & & 48 & 0.00 & 0.00 \\
16 & & 96 & 0.00 & 0.00 \\
32 & & 192 & 2.48 & 780.68 \\
64 & & 384 & 3.95 & 2147.71 \\
128 & & 768 & 1.80 & 1832.83 \\
256 & & 1536 & 0.20 & 371.78 \\
512 & 0.00 & 3072 & 0.00 & 4.65 \\
\hline Totals & 0.00 & & 8.43 & 5137.65 \\
\hline
\end{tabular}
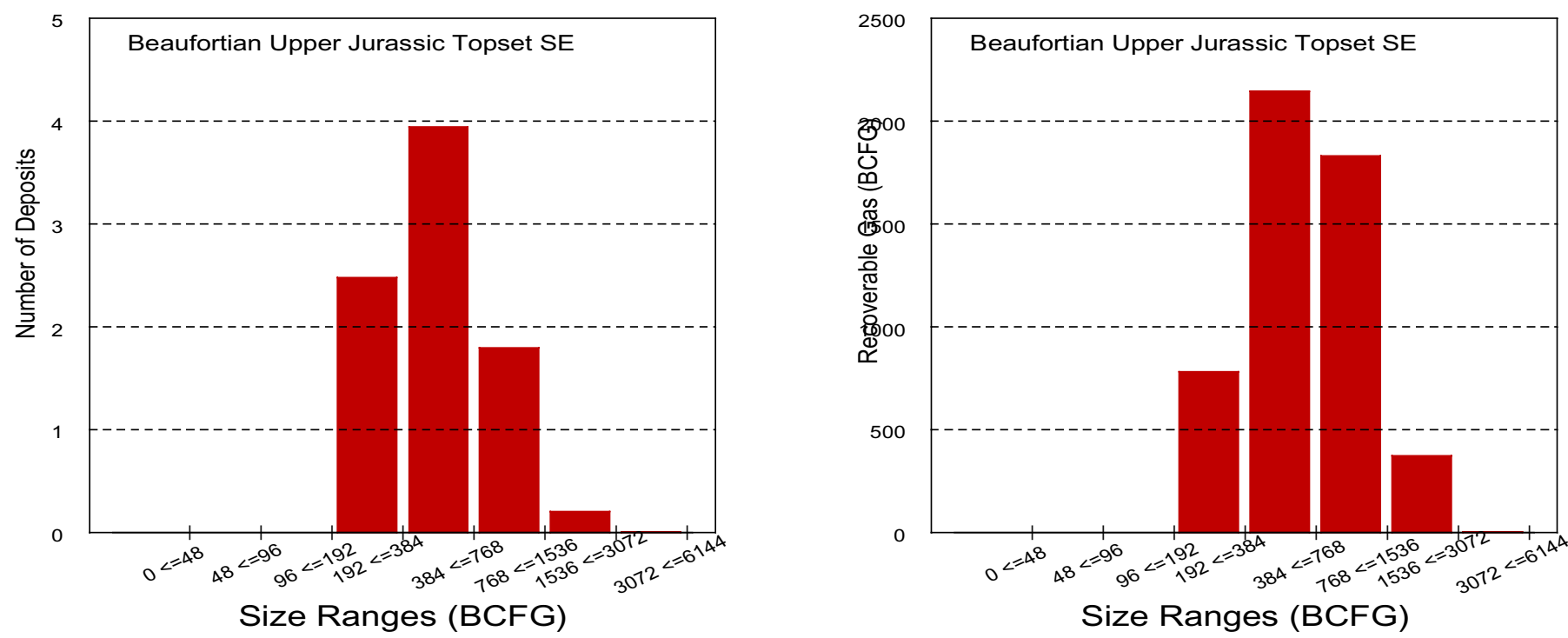
Table 16a: Input values for oil accumulations in the Beaufortian Upper Jurassic Topset NW Play NPRA Assessment Form-2001

PLAY: Beaufortian Upper Jurassic Topset NW

SubArea D (northwest) NW Play area: $2290.77 \mid 10^{3}$ Acres

\section{OIL ACCUMULATION VOLUME PARAMETERS}

\begin{tabular}{|c|c|c|c|c|c|c|c|}
\hline \multirow[b]{2}{*}{ ATTRIBUTES } & \multirow{2}{*}{$\begin{array}{l}\text { Est Shape } \\
(1 \text { to } 5)\end{array}$} & \multicolumn{4}{|c|}{ PROB OF AND GREATER THAN } & \multirow{2}{*}{$\begin{array}{l}\text { Knowledge } \\
\text { Level } 1-3^{5}\end{array}$} & \\
\hline & & LTP & 0.50 & 0.05 & Max & & \\
\hline \multirow{4}{*}{$\begin{array}{l}\text { NET RESERVOIR THICKNESS }^{1} \\
\text { AREA OF CLOSURE }^{2} \\
\text { POROSITY }^{3,4} \\
\text { TRAP FILL }^{3}\end{array}$} & 2 & 25 & 50 & 75 & 150 & 2 & \multirow{2}{*}{$\begin{array}{l}\text { Alpine Ave }=50 \\
\text { Alpine } \sim 28,000 \text { acres }\end{array}$} \\
\hline & 2 & 2 & 8 & 25 & 40 & 3 & \\
\hline & 4 & 12 & 17 & 21 & 22 & 2 & \multirow{2}{*}{$\begin{array}{l}\text { Left skewed } \\
\text { Enter POR*Sw }\end{array}$} \\
\hline & 7 & 80 & 100 & 100 & 100 & 2 & \\
\hline \multicolumn{2}{|l|}{ HYDROCARBON PORE VOL ${ }^{3,4}$} & 8 & 13 & 17 & 18 & & \multirow{6}{*}{ See SubAreas Tab } \\
\hline \multirow{2}{*}{\multicolumn{2}{|c|}{$\begin{array}{l}\text { Approx mm bbl (fvf }=1) \\
\text { Recov } \mathrm{mm} \text { bbl at surface }\end{array}$}} & 24.8 & 403.4 & 2472.9 & 8378.6 & & \\
\hline & & 7.4 & 119.5 & 732.3 & 2481.1 & & \\
\hline \multicolumn{7}{|c|}{$\begin{array}{l}\text { 1-thickness in feet, 2-thousands of acres, 3-percent, 4-correlation between Porosity and Water Saturation }=-1.0 \\
\text { 5-Knowledge Level: } 1=\text { High, 2=Medium, 3=Low; LTP=Left Truncation Point }\end{array}$} & \\
\hline \multirow{2}{*}{$\begin{array}{l}\text { TRAP DEPTH (in } 1000 \mathrm{ft} \text { ) } \\
\text { (from sea level) }\end{array}$} & \multirow{2}{*}{3} & 3 & 9 & 10 & 11 & 1 & \\
\hline & & \multicolumn{3}{|c|}{\begin{tabular}{rr|r|}
3 & 9 & 10 \\
urface to sea level correction $(1000 \mathrm{ft}):$
\end{tabular}} & 0.049 & & \\
\hline
\end{tabular}

\begin{tabular}{|r|r|r|r|}
\hline 20 & 40 & 75 & 150 \\
\hline 5 & 15 & 30 & 40 \\
\hline 12 & 17 & 21 & 22 \\
\hline 80 & 100 & 100 & 100 \\
\hline
\end{tabular}

\section{OIL ACCUMULATION CHARACTERISTICS}

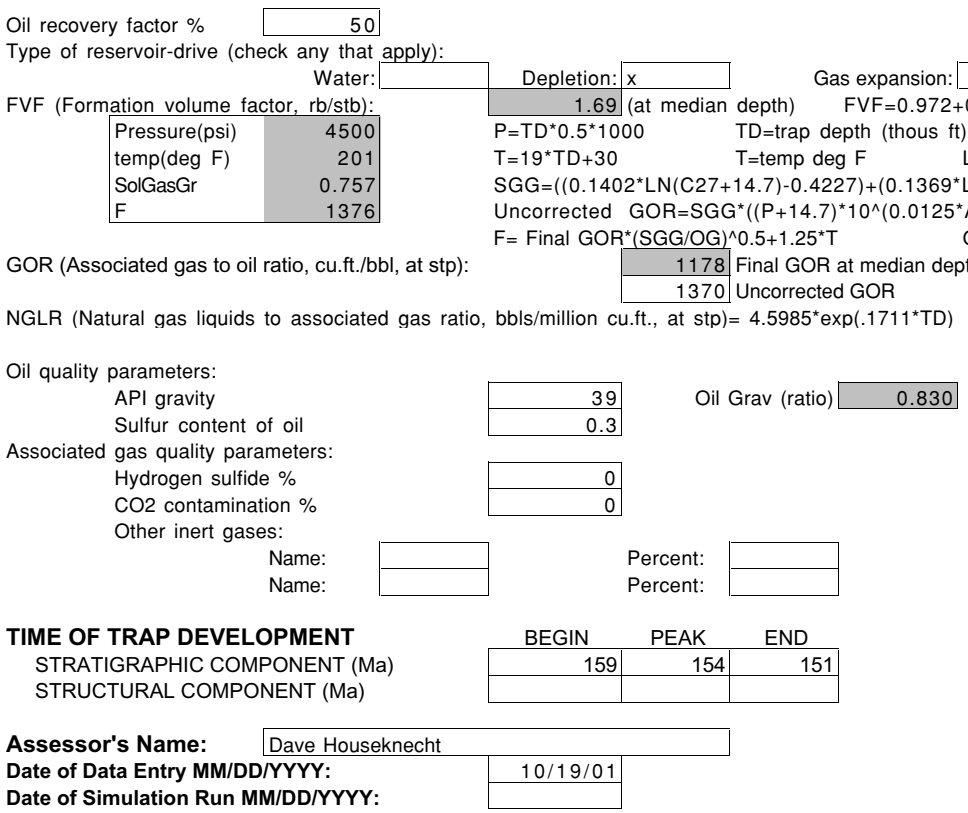

Note: only enter play name and assessor's name on Oil worksheet 
Table 16b: Input risking values for the Beaufortian Upper Jurassic Topset NW Play NPRA Assessment Form-2001

Play: Beaufortian Upper Jurassic Topset NW

\section{RISKING}

MINIMUM ACCUMULATION SIZE, MAS (Millions of BBL in place)

PRERISKED FREQUENCY DISTRIBUTION (Oil plus Gas)

\begin{tabular}{l|r|r|r|r|r|r|}
\cline { 3 - 7 } & \multicolumn{4}{c|}{ PROB OF AND GREATER THAN } & \multicolumn{1}{l|}{$\begin{array}{l}\text { Knowledge } \\
\text { Level } 1-3^{5}\end{array}$} \\
\hline
\end{tabular}

5-Knowledge Level: 1=High, 2=Medium, 3=Low; LTP=Left Truncation Point

\section{ATTRIBUTES}

PLAY

ATTRIBUTES

CHARGE (C)

TRAP (T)

TIMING (F)

Probability that play contains at least 1 reservoir $>=$ minimum size (CxTxF)

PROSPECT

ATTRIBUTES

CHARGE (c)

$\operatorname{TRAP}(\mathrm{t})$

TIMING (f)

Probability that a randomly chosen prospect is favorable (cxtxf)

Play Attributes x Prospect Attributes (CxTxFxcxtxf)

FRACTION OF ACCUMULATIONS BEING OIL
PROBABILITY

\section{OF FAVORABLE}
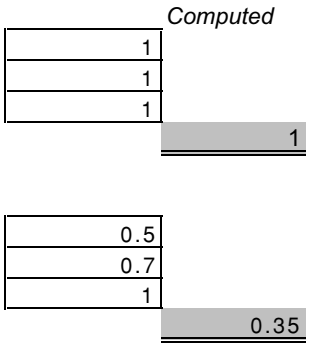

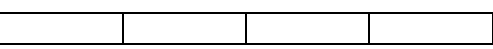

Fraction NA Gas=1-Fraction(Oil)

Allocation (percent):

\begin{tabular}{|l|r|r|l|}
\cline { 2 - 4 } \multicolumn{1}{c|}{} & \multicolumn{1}{c|}{ Land } & \multicolumn{1}{c|}{ Oil } & Gas \\
\hline Federal & 71 & 75 & \\
\hline State & 13 & 12 & \\
\hline Native & 16 & 13 & \\
\hline
\end{tabular}

Assessor's Name:

Dave Houseknecht

Date of Data Entry MM/DD/YYYY:

Date of Simulation Run MM/DD/YYYY: 
Table 16c: Distribution of fitted hydrocarbon volume attributes, trap depth, and number of prospects for the Beaufortian Upper Jurassic Topset NW Play
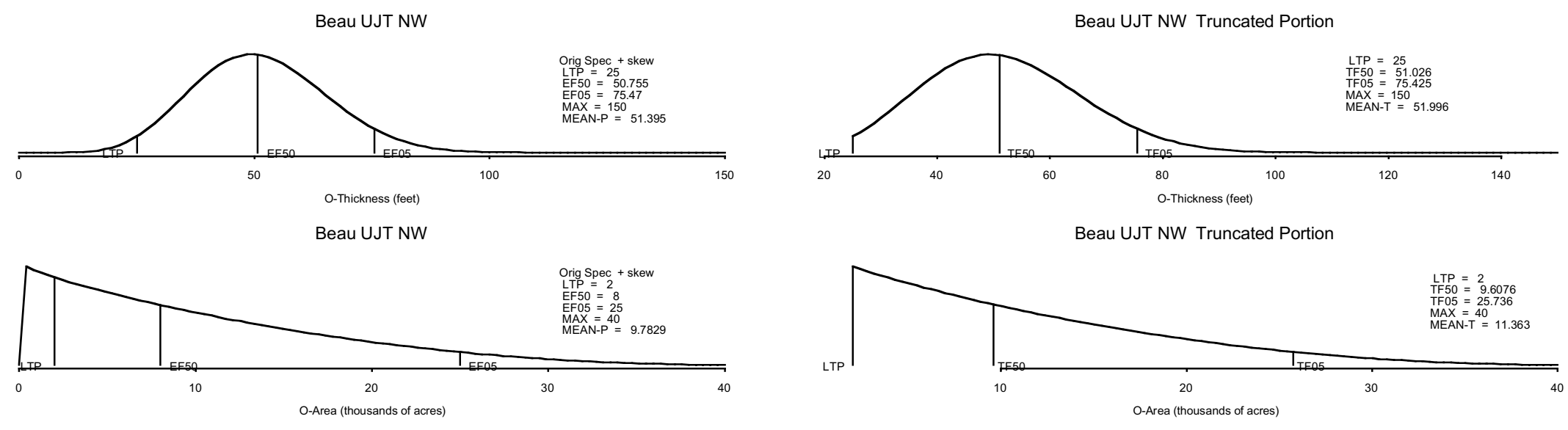

Beau UJT NW

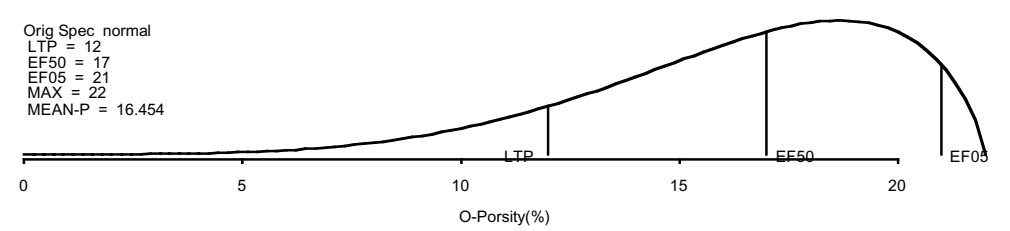

Beau UJT NW

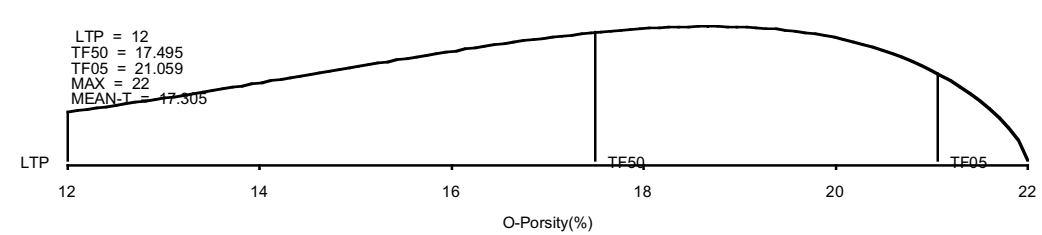

Beau UJT NW Truncated Portion

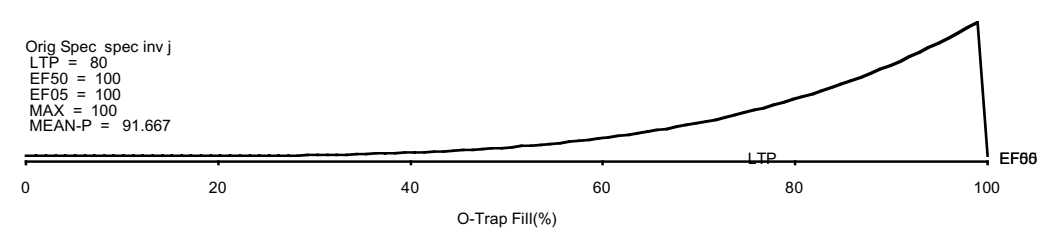

Beau UJT NW

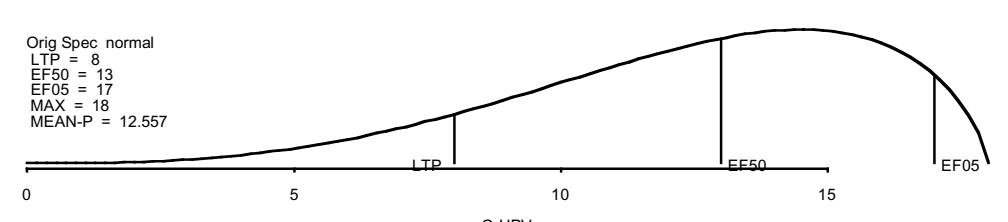

O-HPV

Beau UJT NW

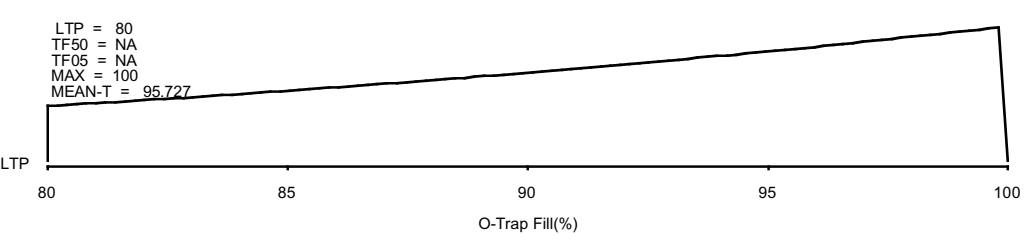

Beau UJT NW Truncated Portion

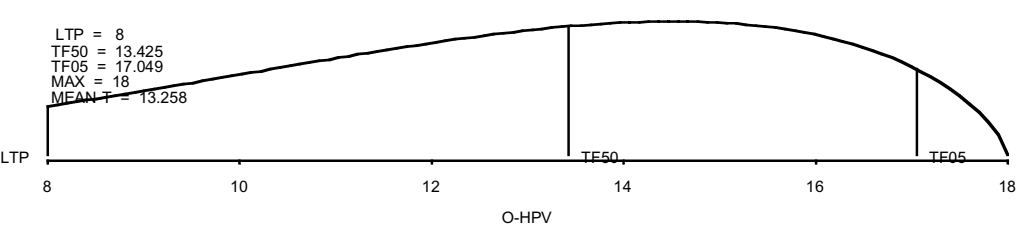

Orig Spec - skew
MiN

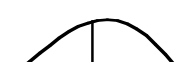




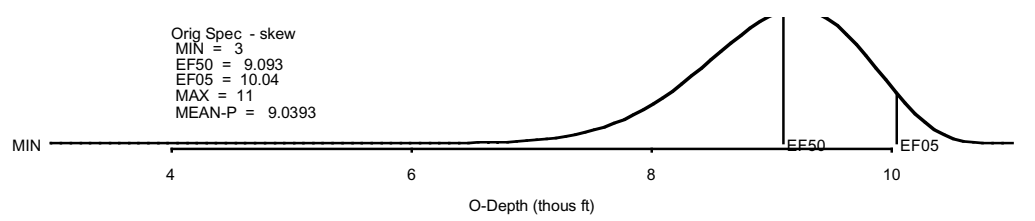

\section{Beau UJT NW}

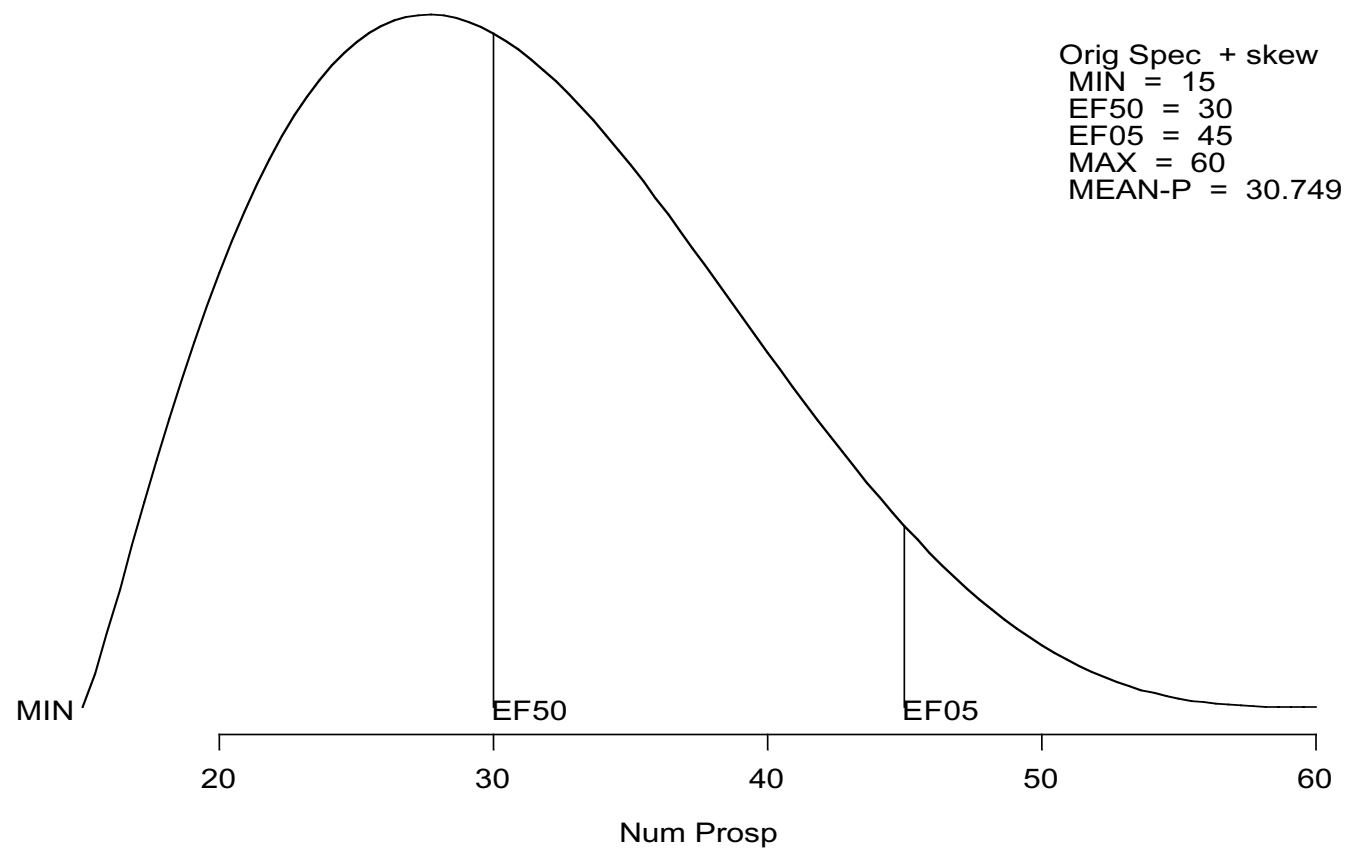


Table 16d: Estimated undiscovered oil and gas resources for the Beaufortian Upper Jurassic Topset NW Play

Deposit

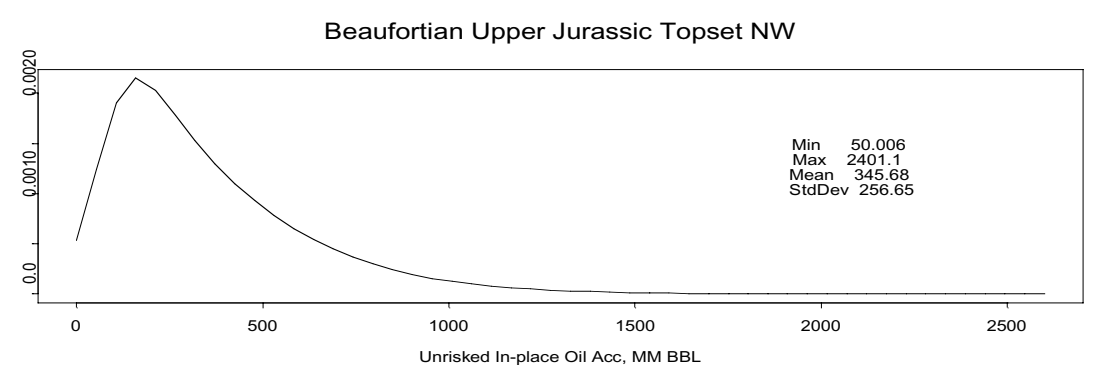

\begin{tabular}{|c|c|c|c|c|c|c|}
\hline \multicolumn{7}{|l|}{ Stats } \\
\hline source & Unit & Mean & StdDev & F95 & F50 & \\
\hline $\begin{array}{l}\text { In-place oil } \\
\text { In-place NA gas }\end{array}$ & $\begin{array}{l}\text { MMBO } \\
\text { BCFG }\end{array}$ & 3718.82 & 1573.33 & $\begin{array}{l}1466.58 \\
0.00\end{array}$ & 3540.26 & 6623 \\
\hline Recov oil & MMBO & 1859.41 & 786.67 & 733.29 & 1770.13 & \\
\hline $\begin{array}{l}\text { Recov assoc diss } 9 \\
\text { Recov NA gas }\end{array}$ & $\begin{array}{l}\text { BCFG } \\
\text { BCFG }\end{array}$ & 2253.03 & 953.60 & $\begin{array}{r}879.72 \\
0.00\end{array}$ & 2143.10 & 4003.3 \\
\hline Recov NGL (ADC & MMBO & 40.54 & 17.19 & 15.76 & 38.58 & 71.5. \\
\hline $\begin{array}{l}\text { Recov NGL (NAG) } \\
\text { Num oil deposits } \\
\text { Num NA gas deposits }\end{array}$ & MMBO & 10.76 & 3.85 & $\begin{array}{l}0.00 \\
5.00 \\
0.00\end{array}$ & 10.00 & $18 . \mathrm{C}$ \\
\hline
\end{tabular}

Play Totals Beaufortian Upper Jurassic Topset NW

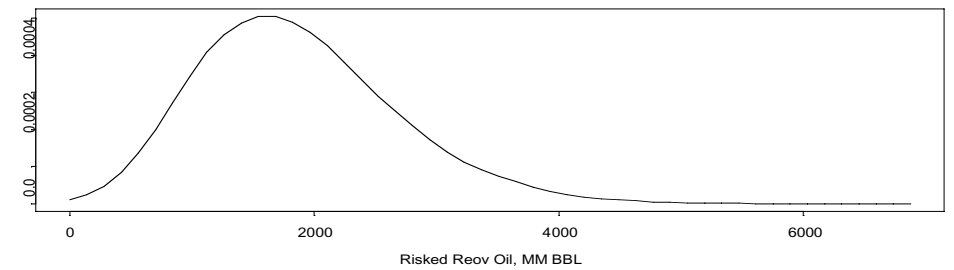


Table 16e: Estimated undiscovered oil and gas resources for the Beaufortian Upper Jurassic Topset NW Play showing number of deposits and volumes by accumulation size class

Start size clOil deposits Oil (MMBO_ Start size clias deposits Gas (BCFG)

\begin{tabular}{rrrrrr|}
0 & 0.00 & 0.00 & 0 & & \\
8 & 0.00 & 0.00 & 48 & & \\
16 & 0.28 & 8.16 & 96 & & \\
32 & 1.76 & 84.58 & 192 & & \\
64 & 2.99 & 281.39 & 384 & & \\
128 & 3.46 & 635.80 & 768 & & \\
256 & 2.02 & 696.21 & 1536 & & \\
512 & 0.25 & 152.58 & 3072 & & \\
1024 & 0.00 & 0.86 & & 0.00 & 0.00 \\
\hline Totals & 10.76 & 1859.60 & & 0.00 & \\
\hline
\end{tabular}
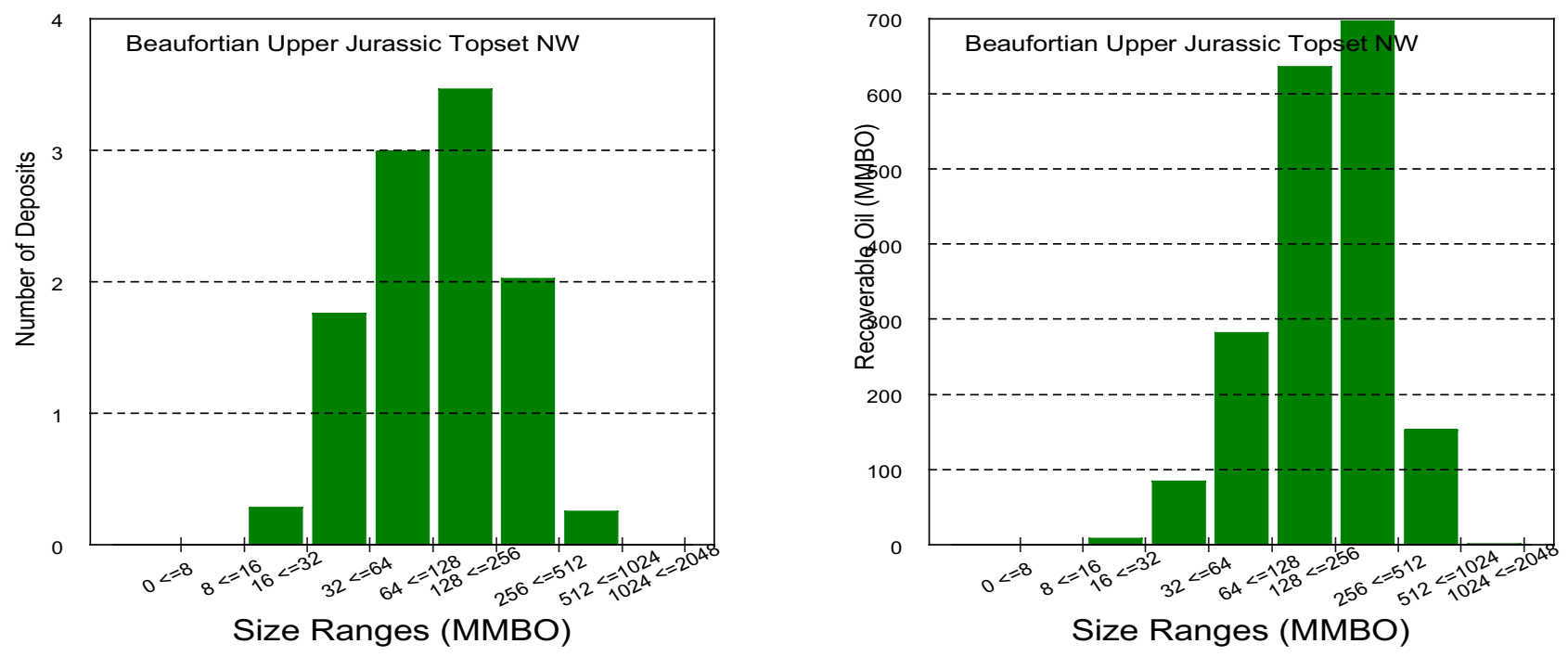
Table 17a: Input values for oil accumulations in the Beaufortian Upper Jurassic Topset SW Play NPRA Assessment Form-2001

$$
\begin{aligned}
& \text { PLAY: Beaufortian Upper Jurassic Topset SW } \\
& \text { Play area: } \quad 333510^{3} \text { Acres }
\end{aligned}
$$

\section{OIL ACCUMULATION VOLUME PARAMETERS}

\begin{tabular}{|c|c|c|c|c|c|c|c|}
\hline \multirow[b]{2}{*}{ ATTRIBUTES } & \multirow{2}{*}{\begin{tabular}{|l|} 
Est Shape \\
$(1$ to 5$)$
\end{tabular}} & \multicolumn{4}{|c|}{ PROB OF AND GREATER THAN } & \multirow{2}{*}{$\begin{array}{l}\text { Knowledge } \\
\text { Level } 1-3^{5}\end{array}$} & \multirow[b]{2}{*}{ verified by DWH Nov 10} \\
\hline & & LTP & \begin{tabular}{l|l}
0.50 \\
\end{tabular} & 0.05 & $\operatorname{Max}$ & & \\
\hline \multirow{4}{*}{$\begin{array}{l}\text { NET RESERVOIR THICKNESS }{ }^{1} \\
\text { AREA OF CLOSURE }{ }^{2} \\
\text { POROSITY,4. } \\
\text { TRAP FILL }^{3}\end{array}$} & 2 & 20 & 40 & 75 & 150 & 2 & Alpine Ave $=50$ \\
\hline & 2 & 4 & 10 & 25 & 40 & 3 & Alpine $\sim 28,000$ acres \\
\hline & 4 & 10 & 15 & 19 & 20 & & Left skewed \\
\hline & 7 & 80 & 100 & 100 & 100 & & Enter $\mathrm{POR}^{*} \mathrm{Sw}$ \\
\hline HYDROCARBON PORE VOL ${ }^{3.4}$ & 3 & 6 & 11 & 15 & 16 & & 4 \\
\hline \multirow{2}{*}{$\begin{array}{l}\text { Approx } \mathrm{mm} \text { bbl (fvf=1) } \\
\text { Recov mm bbl at surface }\end{array}$} & & 29.8 & 341.4 & 2181.9 & 7447.7 & & \\
\hline & & 8.0 & 92.0 & 588.1 & 2007.4 & & \\
\hline \multicolumn{8}{|c|}{$\begin{array}{l}\text { 1-thickness in feet, 2-thousands of acres, 3-percent, 4-correlation between Porosity } \\
\text { 5-Knowledge Level: } 1=\text { High, } 2=\text { =edium, } 3=\text { Low; LTP=Left Truncation Point }\end{array}$} \\
\hline \multirow{2}{*}{$\begin{array}{l}\text { TRAP DEPTH (in } 1000 \mathrm{ft} \text { ) } \\
\text { (from sea level) }\end{array}$} & \begin{tabular}{|r|}
2 \\
\end{tabular} & $10 \mid$ & $|11|$ & 12 & 14 & & See SubAreas Tab \\
\hline & & face to sea & vel correctio & $(1000 \mathrm{ft}):$ & 0.25 & & \\
\hline
\end{tabular}

$\begin{array}{ll}\text { OIL ACCUMULATION CHARACTERISTICS } & \text { modified Nov } 10 \text { - DWH } \\ \text { definitely a right-skewed distribution }\end{array}$

Oil recovery factor $\%$ 50

Type of reservoir-drive (check any that apply):

FVF (Formation volume factor, rb/stb):

\begin{tabular}{|lr|}
\hline Pressure(psi) & 5500 \\
temp(deg F) & 239 \\
SolGasGr & 0.774 \\
F & 1644 \\
\hline
\end{tabular}

Depletion: $x$

1.86 (at median depth) $\quad F V F=0.972+0.000147^{*} F^{\wedge} 1.175$

$=T D^{*} 0.5 * 1000 \quad T D=$ trap depth (thous $\mathrm{ft}$ ), $P=$ pressure $(\mathrm{ps})$

$S G G=\left(\left(0.1402^{*} \mathrm{LN}(\mathrm{C} 27+14.7)-0.4227\right)+\left(0.1369^{*} \mathrm{LN}(\mathrm{C} 28)+0.0156\right)+\left(0.1704^{*} \mathrm{LN}(\mathrm{E} A G=A P I\right.\right.$ gravity Uncorrected $\mathrm{GOR}=\mathrm{SGG}^{*}\left((\mathrm{P}+14.7)^{*} 10^{\wedge}\left(0.0125^{\star} \mathrm{AG}\right) /\left(18^{*} 10^{\wedge}\left(0.00091^{*} \mathrm{~T}\right)\right)\right)^{\wedge}(1 / 0.83)$

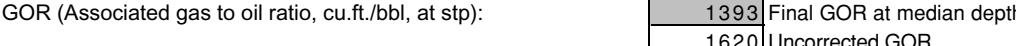
$F=$ Final $G O R^{*}(S G G / O G)^{\wedge} 0.5+1.25^{*} T$ $O G=141.5 /(131.5+A G)$

NGLR (Natural gas liquids to associated gas ratio, bbls/million cu.ft., at $s t p)=4.5985^{*} \exp \left(.1711^{*} \mathrm{TD}\right.$ )

30.2 (median depth)

Oil quality parameters:

API gravity $\quad$\begin{tabular}{r|r}
39 & Oil Grav (ratio) \\
\hline 0.830
\end{tabular}

Associated gas quality parameters:

Hydrogen sulfide \%

$\mathrm{CO} 2$ contamination \%

Other inert gases:

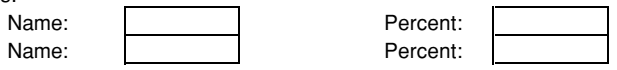

TIME OF TRAP DEVELOPMENT

STRATIGRAPHIC COMPONENT (Ma)

STRUCTURAL COMPONENT (Ma)

\begin{tabular}{|r|r|r|}
\multicolumn{1}{ll}{ BEGIN } & \multicolumn{1}{l}{ PEAK } & END \\
\hline 159 & 154 & 151 \\
\hline & & \\
\hline
\end{tabular}

Assessor's Name: Dave Houseknecht

Date of Data Entry MM/DD/YYYY:

Date of Simulation Run MM/DD/YYYY

10/19/01

Note: only enter play name and assessor's name on Oil worksheet 
Table 17b: Input values for nonassociated gas accumulations in the Beaufortian Upper Jurassic Topset SW Play NPRA Assessment Form-2001

\section{NONASSOCIATED GAS ACCUMULATION VOLUME PARAMETERS}

\begin{tabular}{|c|c|c|c|c|c|c|c|}
\hline \multirow{3}{*}{ ATTRIBUTES } & \multirow{3}{*}{$\begin{array}{l}\text { Est Shape } \\
(1 \text { to } 5)\end{array}$} & \multirow{2}{*}{\multicolumn{4}{|c|}{ PROB OF AND GREATER THAN }} & \multirow{3}{*}{$\begin{array}{l}\text { Knowledge } \\
\text { Level } 1-3^{5}\end{array}$} & \\
\hline & & & & & & & \\
\hline & & LTP & 50 & 5 & $\operatorname{Max}$ & & \\
\hline NET RESERVOIR THICKNESS ${ }^{1}$ & 2 & 20 & 40 & 75 & 150 & 2 & \\
\hline \multirow{3}{*}{$\begin{array}{l}\text { AREA OF CLOSURE } \\
\text { POROSITY } \\
\text { PO,4 } \\
\text { TRAP FILL }\end{array}$} & 2 & 4 & 10 & 25 & 40 & 3 & \\
\hline & 4 & 10 & 15 & 19 & 20 & 2 & \\
\hline & 7 & 80 & 100 & 100 & 100 & 2 & Uses oil POR ${ }^{*} \mathrm{Sw}$ \\
\hline HYDROCARBON PORE VOL ${ }^{3,4}$ & 3 & 6 & 11 & 15 & 16 & & 4 \\
\hline \multirow{2}{*}{$\begin{array}{l}\text { Approx in place bcf } \\
\text { Recov bcf at surface }\end{array}$} & & 0.17 & 1.92 & 12.25 & 41.82 & & \\
\hline & & 30.0 & 343.7 & 2196.7 & 7498.2 & & \\
\hline
\end{tabular}

1-thickness in feet, 2-thousands of acres, 3-percent, 4-correlation between Porosity and Water Saturation $=-1.0$

5-Knowledge Level: 1=High, 2=Medium, 3=Low; LTP=Left Truncation Point

TRAP DEPTH (in $1000 \mathrm{ft}$ )
(from sea level)

\begin{tabular}{r|r|r|}
2 & 10 & 13 \\
Surface to sea level correction $(1000 \mathrm{ft})$
\end{tabular}

20

\section{NONASSOCIATED GAS ACCUMULATION CHARACTERISTICS}

NA Gas recovery factor \%

Type of reservoir-drive (check any that apply):

Water: Gas expansion:

Natural gas liquids plus condensate to non-associated gas (bbls/million cf) (in place): NGL-NAG $=1.785^{\star} \mathrm{TD}$ 23.2 (at median)

Non-associated gas quality parameters:

Hydrogen sulfide \%

CO2 contamination \%

Other inert gases:

Gas fvf

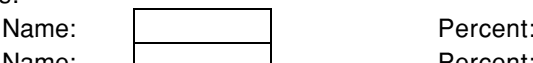

$\begin{array}{ll}\text { Name: } & \text { Percent: } \\ \text { Name: } & \text { Percent: }\end{array}$

Percent:

(at median depth)

$113.3+21.1^{\star} T D-0.812^{*} T D^{\wedge} 2+0.0116^{*} T^{\wedge} 3$

thous $\mathrm{ft}$

$\mathrm{TD}=$ trap depth (thous $\mathrm{ft}$ )

TIME OF TRAP DEVELOPMENT

STRATIGRAPHIC COMPONENT (Ma)

STRUCTURAL COMPONENT (Ma)

\begin{tabular}{|l|l|l|}
\multicolumn{1}{l|}{ BEGIN } & \multicolumn{1}{l}{ PEAK } & \multicolumn{1}{l|}{ END } \\
\hline & & \\
\hline & & \\
\hline
\end{tabular}

Assessor's Name: David Houseknecht

Date of Data Entry MM/DD/YYYY:

Date of Simulation Run MM/DD/YYYY:

$10 / 23 / 01$

$2 / 25 / 02$ 
Table 17c: Input risking values for the Beaufortian Upper Jurassic Topset SW Play NPRA Assessment Form-2001

Play: Beaufortian Upper Jurassic Topset SW

\section{RISKING}

MINIMUM ACCUMULATION SIZE, MAS (Millions of BBL in place)

50

PRERISKED FREQUENCY DISTRIBUTION (Oil plus Gas)

NUM OF PROSPECTS

$>$ MINIMUM SIZE

PROB OF AND GREATER THAN

\begin{tabular}{|r|r|r|r|r|r|}
\hline & \multicolumn{4}{|c|}{ PROB OF AND GREATER THAN } & \multicolumn{2}{l|}{$\begin{array}{l}\text { Knowledge } \\
\text { Est Shape }\end{array}$} & Min & 50 & 5 & Max $1-3^{5}$ \\
\hline 2 & 18 & 33 & 50 & 75 & 3 \\
\hline
\end{tabular}

5-Knowledge Level: 1=High, 2=Medium, 3=Low; LTP=Left Truncation Point

\section{ATTRIBUTES}

PLAY

ATTRIBUTES

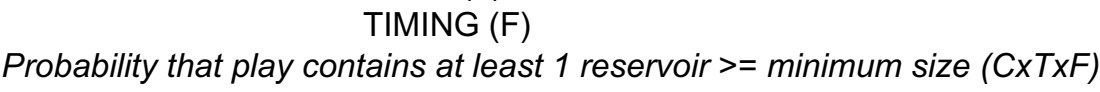

CHARGE (C)

TRAP (T)

PROSPECT

ATTRIBUTES

CHARGE (c)

TRAP (t)

TIMING (f)

Probability that a randomly chosen prospect is favorable (cxtxf)

Play Attributes x Prospect Attributes (CxTxFxcxtxf)

FRACTION OF ACCUMULATIONS BEING OIL

Fraction NA Gas=1-Fraction(Oil)
PROBABILITY OF FAVORABLE

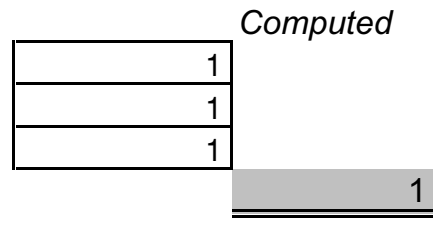

\begin{tabular}{r|}
\hline 0.5 \\
\hline 0.5 \\
\hline 1 \\
\hline
\end{tabular}

0

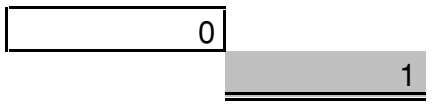

Allocation (percent):

\begin{tabular}{|l|r|r|r|}
\cline { 2 - 4 } \multicolumn{1}{c|}{} & \multicolumn{1}{c|}{ Land } & Oil & \multicolumn{2}{c|}{ Gas } \\
\hline Federal & 97 & & 93 \\
\hline State & 2 & & 5 \\
\hline Native & 1 & & 2 \\
\hline
\end{tabular}

Assessor's Name:

Dave Houseknecht

Date of Data Entry MM/DD/YYYY:

Date of Simulation Run MM/DD/YYYY:

$10 / 19 / 01$

$2 / 20 / 02$ 
Table 17d: Distribution of fitted hydrocarbon volume attributes, trap depth, and number of prospects for the Beaufortian Upper Jurassic Topset SW Play

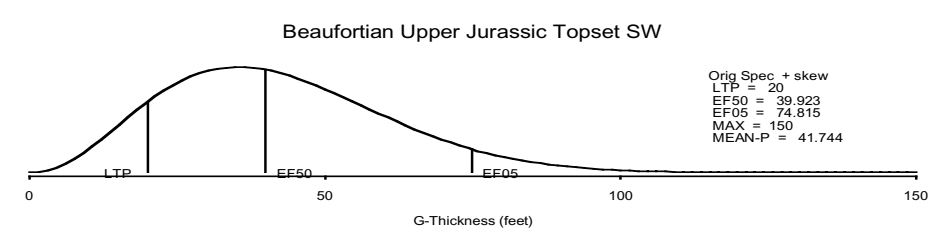

Beaufortian Upper Jurassic Topset SW
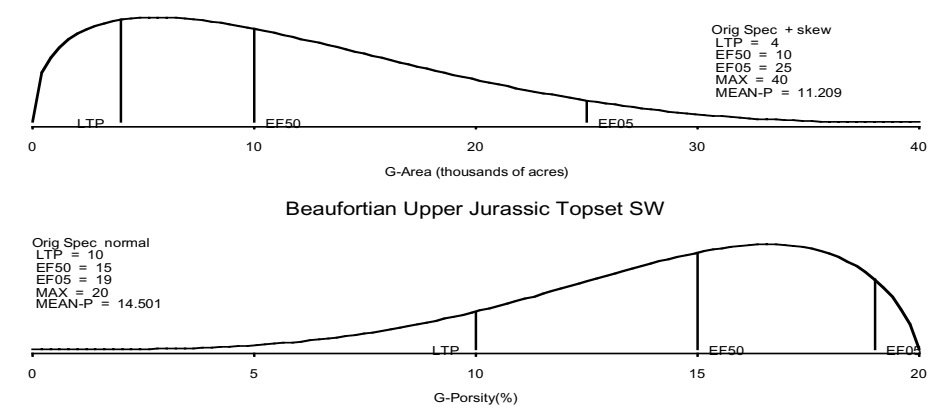

Beaufortian Upper Jurassic Topset SW

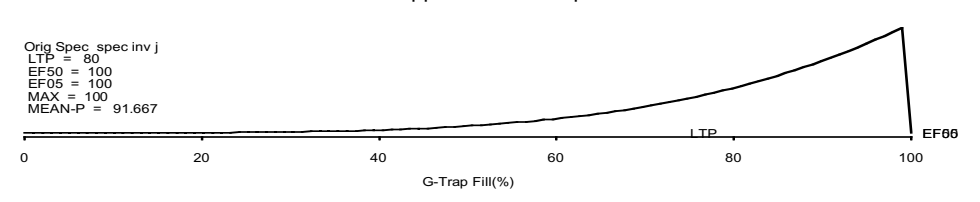

Beaufortian Upper Jurassic Topset SW

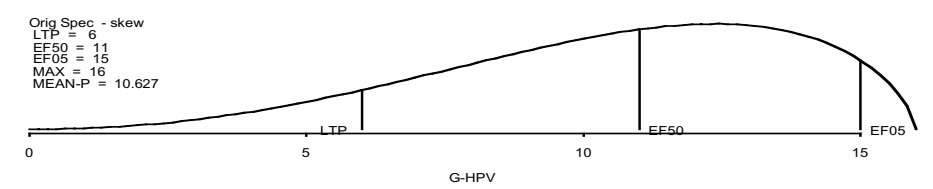

Beaufortian Upper Jurassic Topset SW

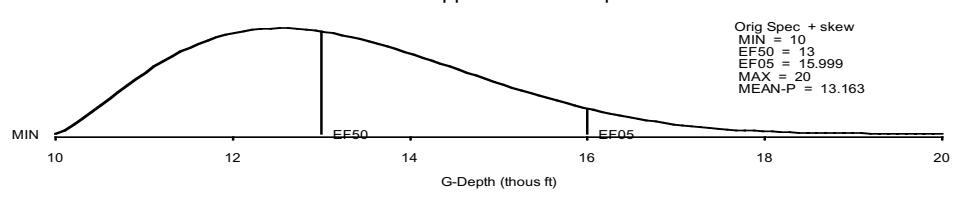

Beaufortian Upper Jurassic Topset SW Truncated Portion

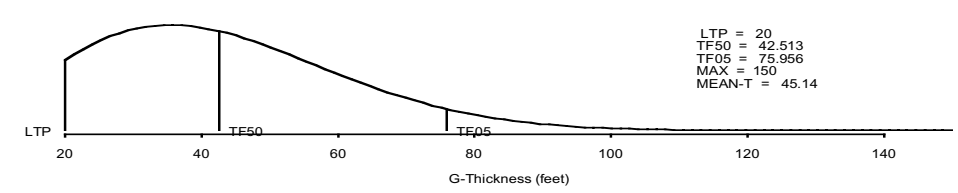

Beaufortian Upper Jurassic Topset SW Truncated Portion

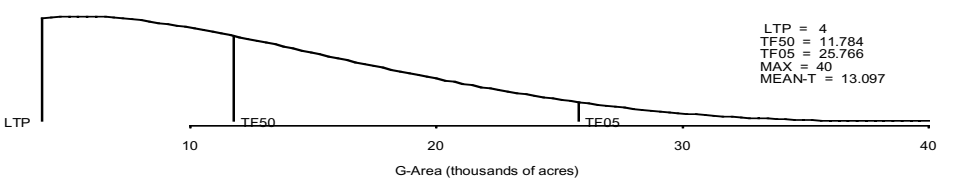

Beaufortian Upper Jurassic Topset SW Truncated Portion

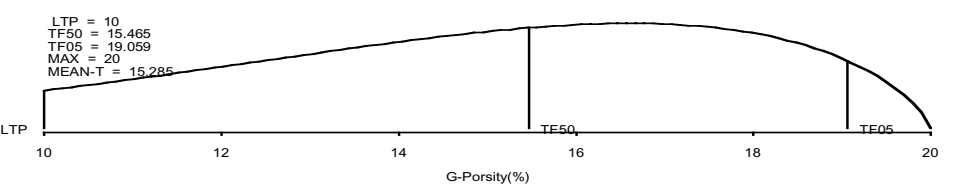

Beaufortian Upper Jurassic Topset SW Truncated Portion

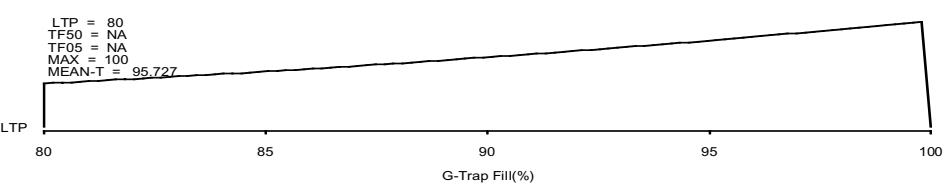

Beaufortian Upper Jurassic Topset SW Truncated Portion

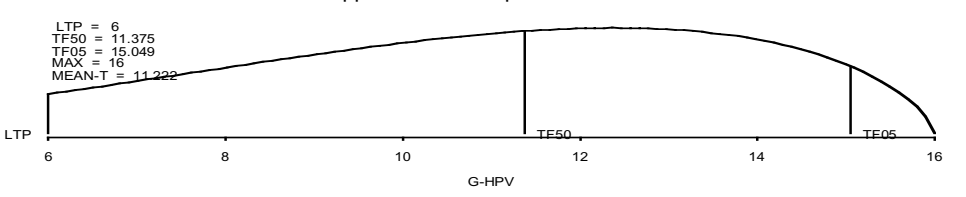


Beaufortian Upper Jurassic Topset SW

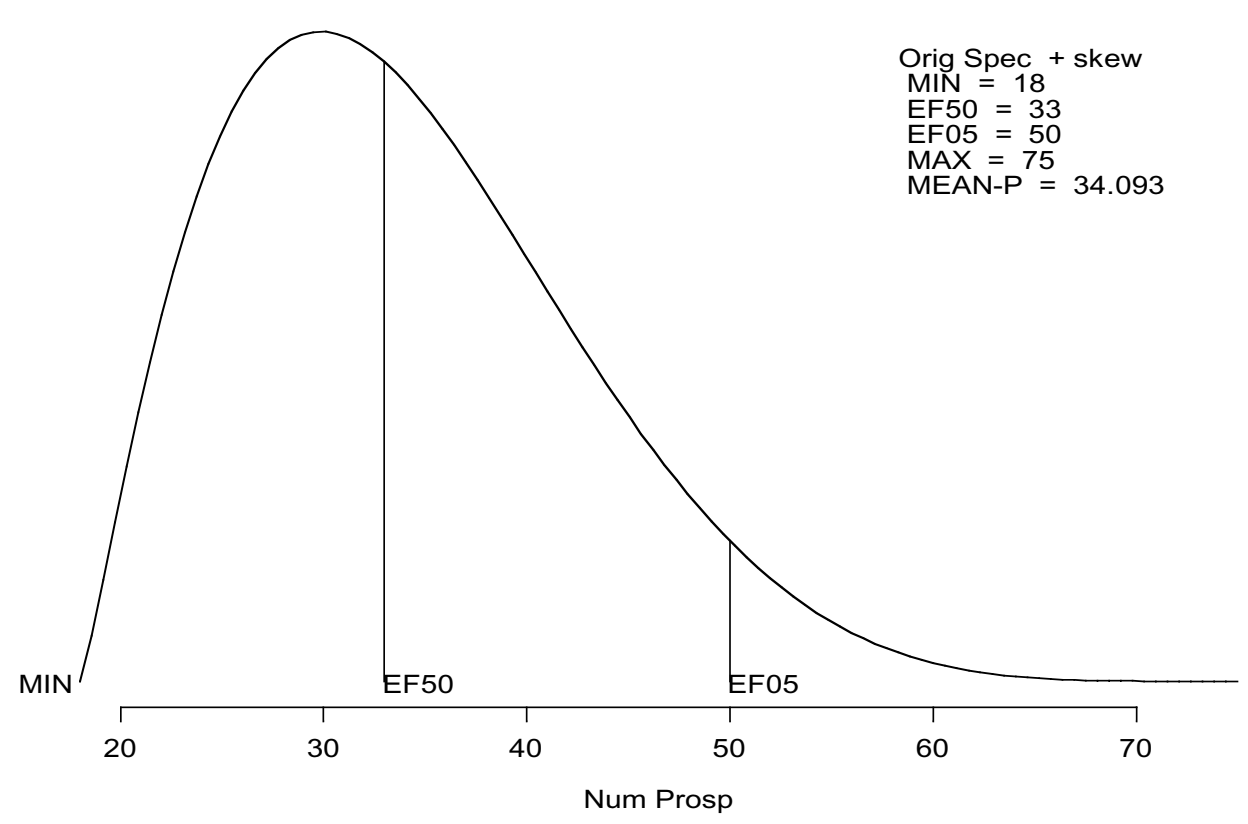


Table 17e: Estimated undiscovered oil and gas resources for the Beaufortian Upper Jurassic Topset SW Play Deposit

Beaufortian Upper Jurassic Topset SW

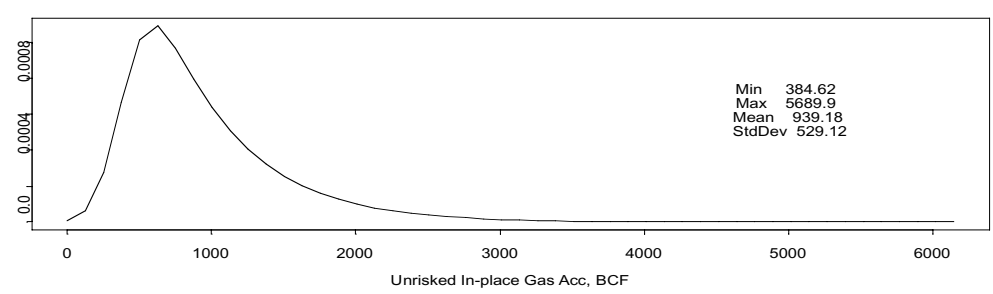

\begin{tabular}{|c|c|c|c|c|c|c|}
\hline \\
\hline Resource & Unit & Mean & StdDev & F95 & F50 & F05 \\
\hline In-place oil & MMBO & 0.00 & 0.00 & 0.00 & 0.00 & 0.00 \\
\hline In-place NA g & BCFG & 8030.01 & 3479.21 & 3089.60 & 646.46 & 14253.49 \\
\hline Recov oil & MMBO & 0.00 & 0.00 & 0.00 & 0.00 & 0.00 \\
\hline Recov assoc diss gas & $B C F G$ & 0.00 & 0.00 & 0.00 & 0.00 & 0.00 \\
\hline Recov NA gas & $B C F G$ & 5219.51 & 2261.49 & 2008.24 & 4970.20 & 9264.77 \\
\hline Recov NGL (ADG) & MMBO & 0.00 & 0.00 & 0.00 & 0.00 & 0.00 \\
\hline Recov NGL (NA & MMBO & 125.50 & 54.74 & 47.77 & 119.36 & 224.07 \\
\hline Num oil deposits & & 0.00 & 0.00 & 0.00 & 0.00 & 0.00 \\
\hline Num NA gas deposits & & 8.55 & 3.33 & 4.00 & 8.00 & 15.00 \\
\hline
\end{tabular}

Num NA gas deposits

Play Totals Beaufortian Upper Jurassic Topset SW

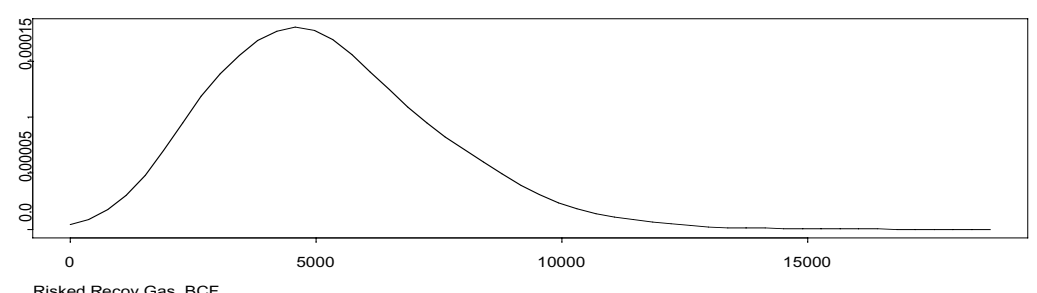


Table 17f: Estimated undiscovered oil and gas resources for the Beaufortian Upper Jurassic Topset SW showing number of deposits and volumes by accumulation size class

\begin{tabular}{rrrrrr|} 
Start size class & Oil deposits & Oil (MMBO_Start size class & Gas deposits & NA Gas (BCFG) \\
0 & & 0 & 0.00 & 0.00 \\
8 & & 48 & 0.00 & 0.00 \\
16 & & 96 & 0.00 & 0.00 \\
32 & & 192 & 2.51 & 788.24 \\
64 & & 384 & 4.01 & 2178.80 \\
128 & & 768 & 1.82 & 1861.04 \\
256 & & 1536 & 0.21 & 387.00 \\
512 & 0.00 & 0.00 & 3072 & 0.00 & 4.96 \\
\hline Totals & & & 8.55 & 5220.03 \\
\hline
\end{tabular}
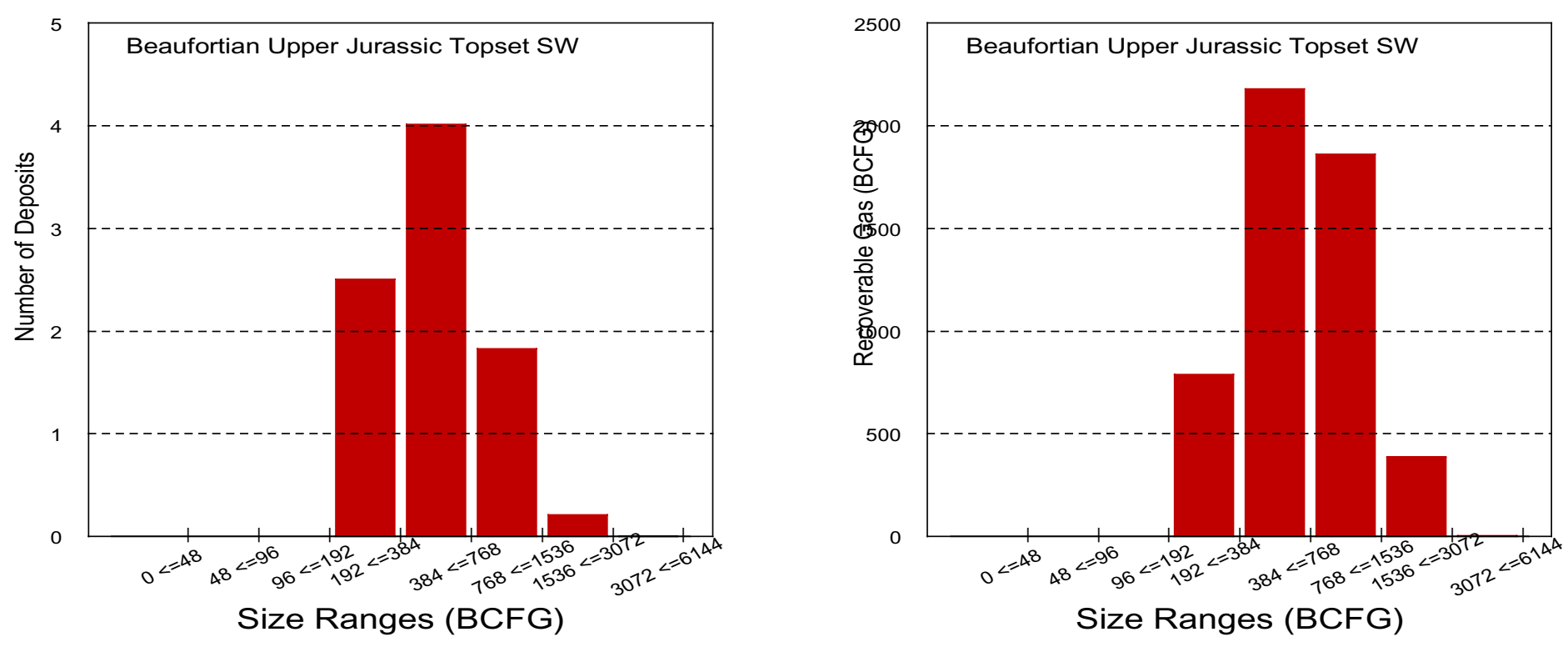
Table 18a: Input values for oil accumulations in the Beaufortian Lower Jurassic Topset Play NPRA Assessment Form-2001

PLAY: Beaufortian Lower Jurassic Topset

Play area: $\quad 9278 \mid 10^{3}$ Acres

\section{OIL ACCUMULATION VOLUME PARAMETERS}

\begin{tabular}{|c|c|c|c|c|c|c|c|}
\hline \multirow[b]{2}{*}{ ATTRIBUTES } & \multirow{2}{*}{$\begin{array}{l}\text { Est Shape } \\
(1 \text { to } 5)\end{array}$} & \multicolumn{4}{|c|}{ PROB OF AND GREATER THAN } & \multirow{2}{*}{$\begin{array}{l}\text { Knowledge } \\
\text { Level } 1-3^{5}\end{array}$} & \multirow[b]{6}{*}{ Enter POR*Sw } \\
\hline & & LTP & 0.50 & 0.05 & Max & & \\
\hline \multirow{4}{*}{$\begin{array}{l}\text { NET RESERVOIR THICKNESS } \\
\text { AREA OF CLOSURE }^{2} \\
\text { POROSITY }^{3,4} \\
\text { TRAP FILL }^{3}\end{array}$} & \begin{tabular}{r|}
2 \\
\end{tabular} & 15 & 30 & 60 & 100 & 2 & \\
\hline & 2 & 3 & 4 & 8 & 20 & 3 & \\
\hline & 4 & 15 & 18 & 20 & 22 & 2 & \\
\hline & 6 & 50 & 75 & 99 & 100 & 2 & \\
\hline \multicolumn{2}{|l|}{ HYDROCARBON PORE VOL } & 7 & 10 & 12 & 14 & & 8 \\
\hline \multirow{2}{*}{\multicolumn{2}{|c|}{$\begin{array}{l}\text { Approx } \mathrm{mm} \text { bbl }(\mathrm{fvf}=1) \\
\text { Recov } \mathrm{mm} \text { bbl at surface }\end{array}$}} & 12.2 & 69.8 & 442.4 & 2172.2 & & \\
\hline & & 3.0 & 17.0 & 107.9 & 529.9 & & \\
\hline \multicolumn{7}{|c|}{$\begin{array}{l}\text { 1-thickness in feet, 2-thousands of acres, 3-percent, 4-correlation between Porosity and Water Saturation }=-1.0 \\
\text { 5-Knowledge Level: } 1=\text { High, 2=Medium, 3=Low; LTP=Left Truncation Point }\end{array}$} & \\
\hline \multirow{2}{*}{$\begin{array}{l}\text { TRAP DEPTH (in } 1000 \mathrm{ft} \text { ) } \\
\text { (from sea level) }\end{array}$} & \begin{tabular}{r|}
2 \\
\end{tabular} & $3 \mid$ & 5 & 8 & 14 & 1 & \\
\hline & \multicolumn{4}{|c|}{ Surface to sea level correction (1000 ft): } & 0.113 & & \\
\hline
\end{tabular}

\section{OIL ACCUMULATION CHARACTERISTICS}

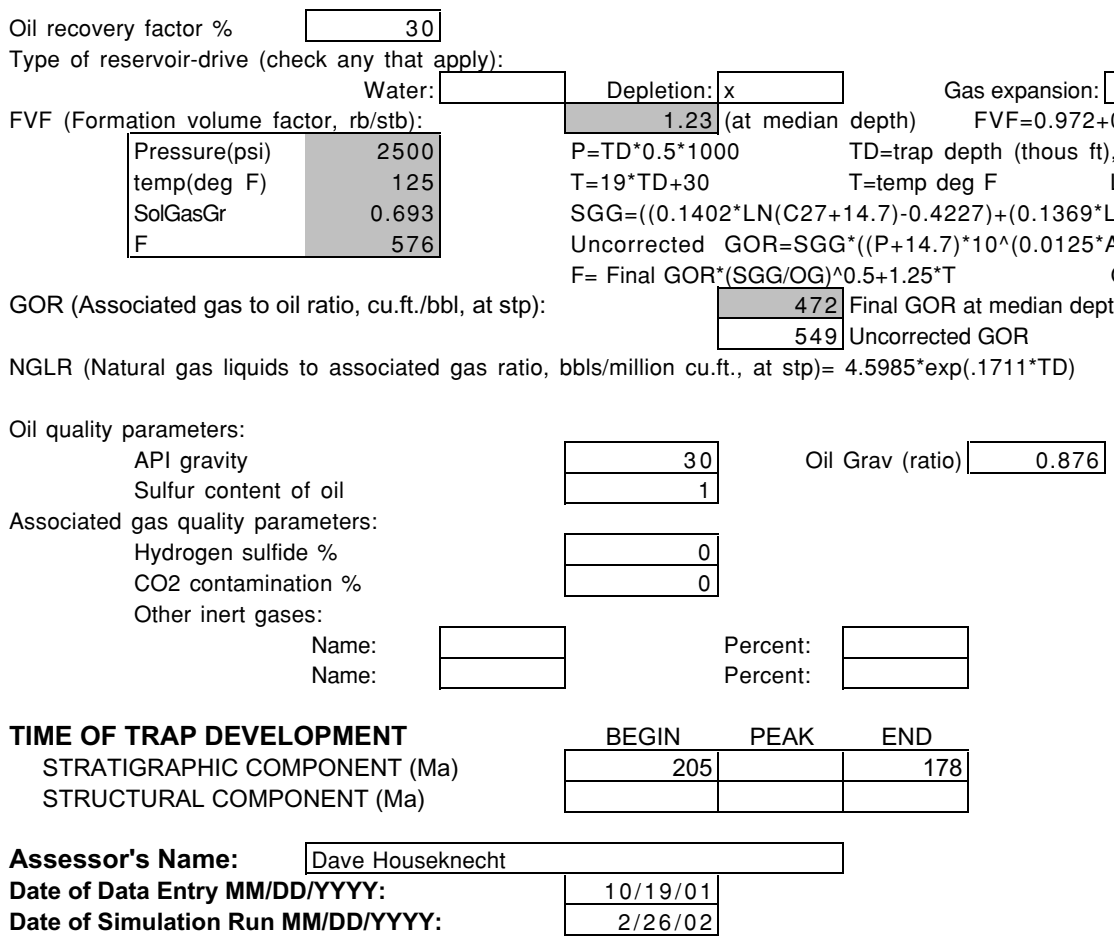

Note: only enter play name and assessor's name on Oil worksheet 
Table 18b: Input values for nonassociated gas accumulations in the Beaufortian Lower Jurassic Topset Play NPRA Assessment Form-2001

PLAY: Beaufortian Lower Jurassic Topset

\section{NONASSOCIATED GAS ACCUMULATION VOLUME PARAMETERS}

\begin{tabular}{|c|c|c|c|c|c|c|c|}
\hline & & \multirow{2}{*}{\multicolumn{4}{|c|}{ PROB OF AND GREATER THAN }} & \multirow{3}{*}{\begin{tabular}{|l|l|} 
Knowledge \\
Level $1-3^{5}$ \\
\end{tabular}} & \\
\hline \multirow{2}{*}{ ATTRIBUTES } & \multirow{2}{*}{$\begin{array}{l}\text { Est Shape } \\
(1 \text { to } 5)\end{array}$} & & & & & & \\
\hline & & \begin{tabular}{l|l} 
LTP & \\
\end{tabular} & 50 & $\begin{array}{ll}5 \\
\end{array}$ & $\operatorname{Max}$ & & \\
\hline \multirow{4}{*}{$\begin{array}{l}\text { NET RESERVOIR THICKNESS } \\
\text { AREA OF CLOSURE }^{2} \\
\text { POROSITY }^{3,4} \\
\text { TRAP FILL' }^{3}\end{array}$} & 2 & 15 & 30 & 60 & 100 & 2 & \\
\hline & 2 & 3 & 4 & 8 & 20 & 3 & \\
\hline & 4 & 15 & 18 & 20 & 22 & 2 & \\
\hline & 6 & 50 & 75 & 99 & 100 & 2 & From Oil \\
\hline \multicolumn{2}{|l|}{ HYDROCARBON PORE VOL } & 7 & 10 & 12 & 14 & & \begin{tabular}{|r|}
8 \\
\end{tabular} \\
\hline \multirow{2}{*}{\multicolumn{2}{|c|}{$\begin{array}{l}\text { Approx in place bcf } \\
\text { Recov bcf at surface }\end{array}$}} & 0.07 & 0.39 & 2.48 & 12.20 & & \\
\hline & & 10.5 & 60.2 & 381.2 & 1871.6 & & \\
\hline \multicolumn{7}{|c|}{$\begin{array}{l}\text { 1-thickness in feet, 2-thousands of acres, 3-percent, 4-correlation between Porosity and Water Saturation }=-1.0 \\
\text { 5-Knowledge Level: 1=High, 2=Medium, 3=Low; LTP=Left Truncation Point }\end{array}$} & \\
\hline \multirow{2}{*}{$\begin{array}{l}\text { TRAP DEPTH (in } 1000 \mathrm{ft} \text { ) } \\
\text { (from sea level) }\end{array}$} & \begin{tabular}{r|}
2 \\
\end{tabular} & 3 & 8 & 13 & 17 & 1 & \\
\hline & \multicolumn{4}{|c|}{ Surface to sea level correction (1000 ft): } & 0.113 & & \\
\hline
\end{tabular}

\section{NONASSOCIATED GAS ACCUMULATION CHARACTERISTICS}

NA Gas recovery factor \% 65

Type of reservoir-drive (check any that apply):

$$
\text { Water: }
$$

Gas expansion:

Natural gas liquids plus condensate to non-associated gas (bbls/million cf) (in place): NGL-NAG $=1.785^{\star} \mathrm{TD}$ 14.3 (at median)

Non-associated gas quality parameters: Hydrogen sulfide \% CO2 contamination \% Other inert gases:

$$
\text { Name: }
$$
Name:

Gas fvf

$$
236.1
$$
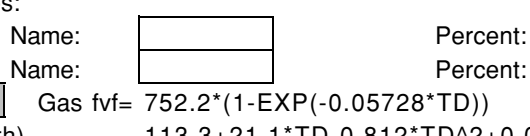

Percent:

$$
\begin{aligned}
& 113.3+21.1^{*} T D-0.812^{*} T D^{\wedge} 2+0.0116^{*} T^{\wedge} 3 \quad 5.67<T D<=30 \\
& T D=\text { trap depth (thous } \mathrm{ft} \text { ) }
\end{aligned}
$$

\section{TIME OF TRAP DEVELOPMENT \\ STRATIGRAPHIC COMPONENT (Ma) \\ STRUCTURAL COMPONENT (Ma)}

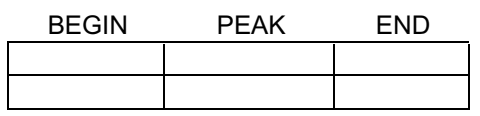

Assessor's Name: Dave Houseknecht Date of Data Entry MM/DD/YYYY:

Date of Simulation Run MM/DD/YYYY: 
Table 18c: Input risking values for the Beaufortian Lower Jurassic Topset Play NPRA Assessment Form-2001

Play: Beaufortian Lower Jurassic Topset

\section{RISKING}

MINIMUM ACCUMULATION SIZE, MAS (Millions of BBL in place)

50

PRERISKED FREQUENCY DISTRIBUTION (Oil plus Gas)

NUM OF PROSPECTS

$>$ MINIMUM SIZE

PROB OF AND GREATER THAN

Knowledge

\begin{tabular}{|r|r|r|r|r|r|}
\hline Est Shape & Min & 50 & 5 & \multicolumn{1}{|c|}{ Max } & Level $1-3^{5}$ \\
\hline 2 & 15 & 40 & 65 & 90 & 3 \\
\hline
\end{tabular}

5-Knowledge Level: 1=High, 2=Medium, 3=Low; LTP=Left Truncation Point

\section{ATTRIBUTES}

PLAY

ATTRIBUTES

TIMING $(\mathrm{F})$
Probability that play contains at least 1 reservoir $>=$ minimum size $(C x T x F)$

CHARGE (C)

TRAP (T)

PROSPECT

ATTRIBUTES

CHARGE (c)

TRAP (t)

TIMING (f)

Probability that a randomly chosen prospect is favorable (cxtxf)

Play Attributes x Prospect Attributes (CxTxFxcxtxf)

FRACTION OF ACCUMULATIONS BEING OIL

Fraction NA Gas=1-Fraction(Oil)
PROBABILITY OF FAVORABLE

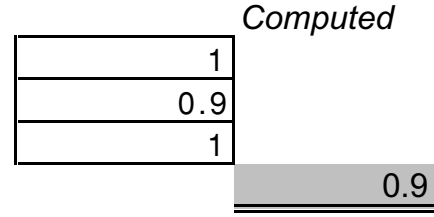

0.5

Allocation (percent):

\begin{tabular}{|l|r|r|r|}
\cline { 2 - 4 } \multicolumn{1}{c|}{} & \multicolumn{1}{c|}{ Land } & \multicolumn{1}{c|}{ Oil } & \multicolumn{2}{c|}{ Gas } \\
\hline Federal & 87 & 81 & 80 \\
\hline State & 7 & 12 & 9 \\
\hline Native & 6 & 7 & 11 \\
\hline
\end{tabular}

Assessor's Name:

Date of Data Entry MM/DD/YYYY:

Date of Simulation Run MM/DD/YYYY:

Dave Houseknecht

$10 / 19 / 01$

$2 / 26 / 02$ 
Table 18d: distribution of fitted hydrocarbon volume attributes, trap depth, and number of prospects for the Beaufortian Lower Jurassic Topset Play
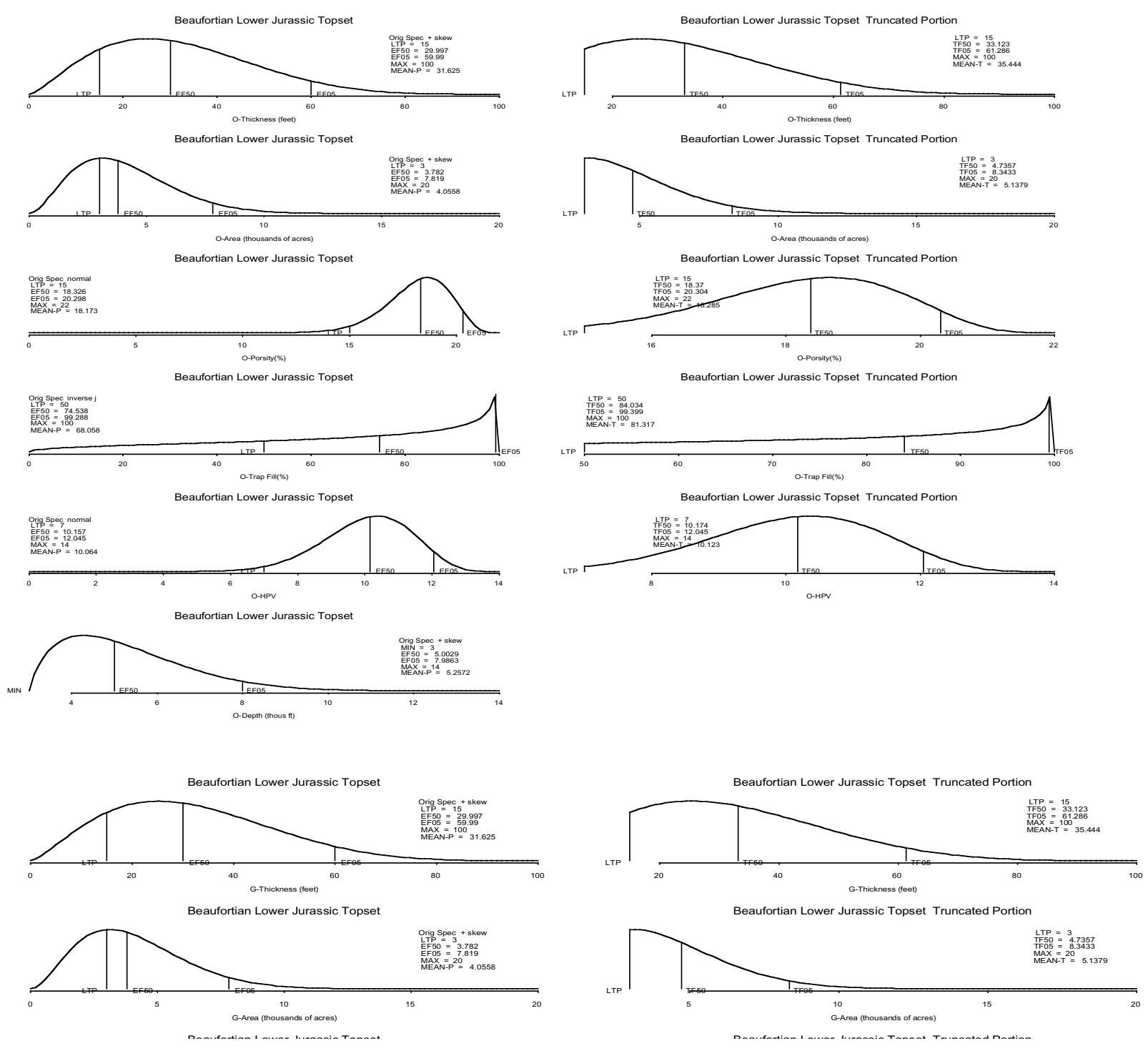
G.Area (thousands of acres)

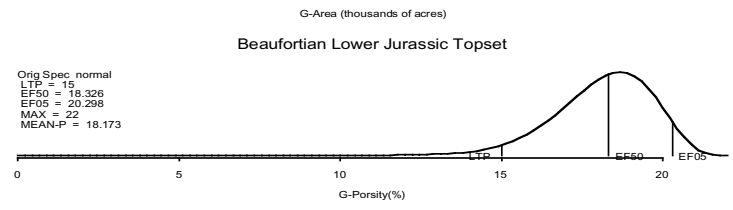

Beaufortian Lower Jurassic Topset

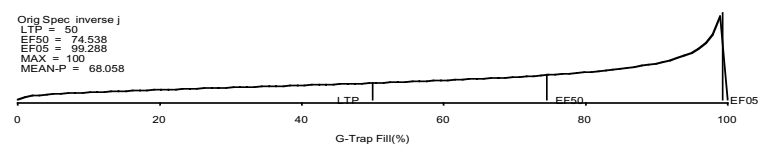

Beaufortian Lower Jurassic Topset

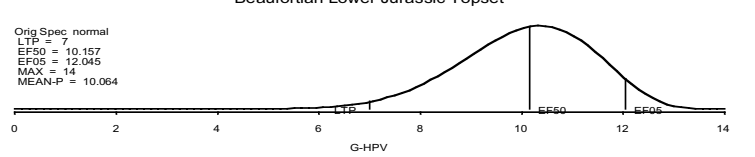

Beautorth Lowor Jurassic Topset

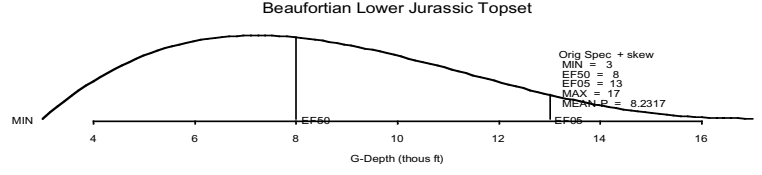

Beaufortian Lower Jurassic Topset

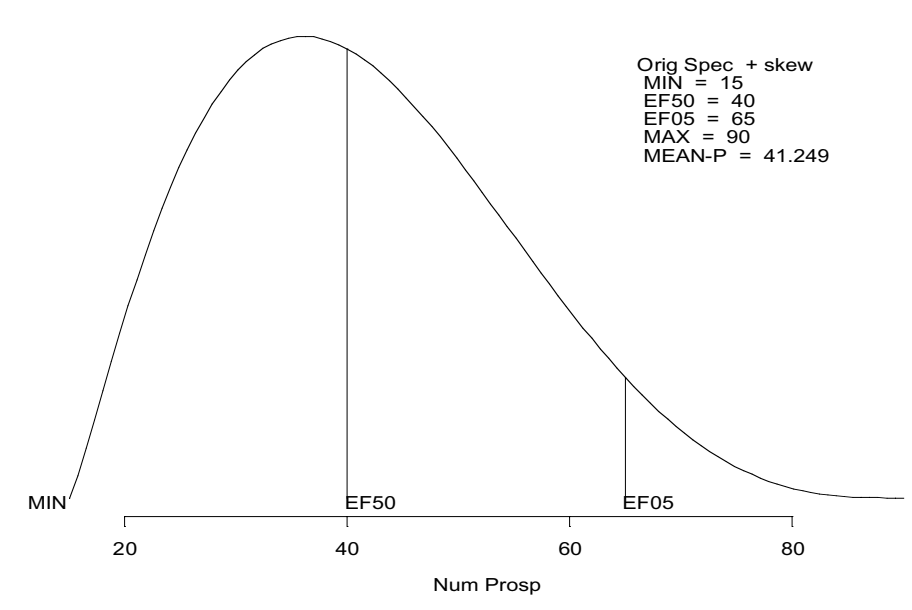

Beaufortian Lower Jurassic Topset Truncated Portion

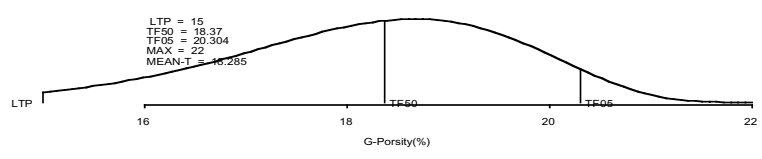

Beaufortian Lower Jurassic Topset Truncated Portion
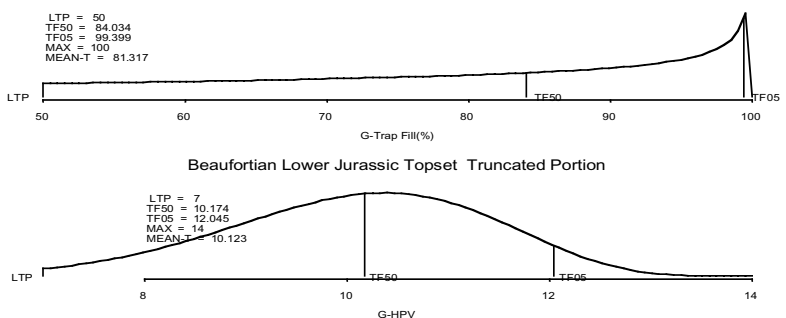
Table 18e: Estimated undiscovered oil and gas resources for the Beaufortian Lower Jurassic Topset Play Deposit

Beaufortian Lower Jurassic Topset

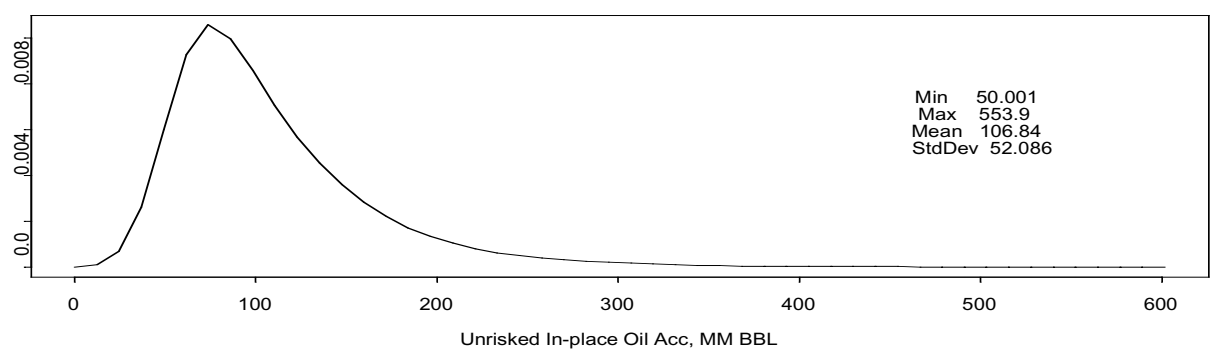

Beaufortian Lower Jurassic Topset

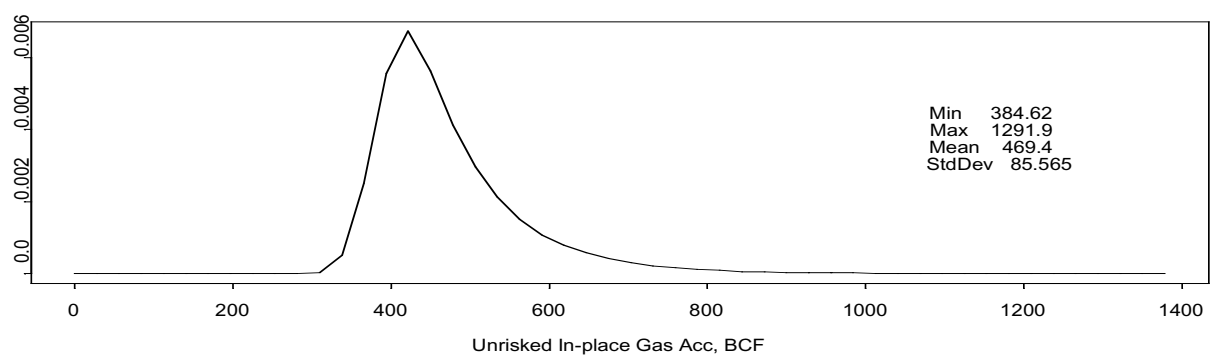

Play

Play Stats

Resource

In-place oil

In-place NA gas

Recov oil

Recov assoc diss gas

Recov NA gas

Recov NGL (ADG)

Recov NGL (NAG)

Num oil deposits

Num NA gas deposits

\begin{tabular}{l|rrrrr|} 
MMBO & 275.63 & 225.37 & 0.00 & 242.39 & 699.53 \\
BCFG & 1219.89 & 941.86 & 0.00 & 1082.50 & 2945.89 \\
MMBO & 82.69 & 67.61 & 0.00 & 72.72 & 209.86 \\
BCFG & 2.53 & 12.11 & 0.00 & 0.00 & 15.39 \\
BCFG & 792.93 & 612.21 & 0.00 & 703.62 & 1914.83 \\
MMBO & 0.96 & 0.79 & 0.00 & 0.83 & 2.45 \\
MMBO & 13.86 & 10.95 & 0.00 & 12.55 & 34.08 \\
& 2.58 & 1.97 & 0.00 & 2.00 & 6.00 \\
& 2.60 & 1.99 & 0.00 & 2.00 & 6.00
\end{tabular}

RNS $=774$

Play Totals Beaufortian Lower Jurassic Topset

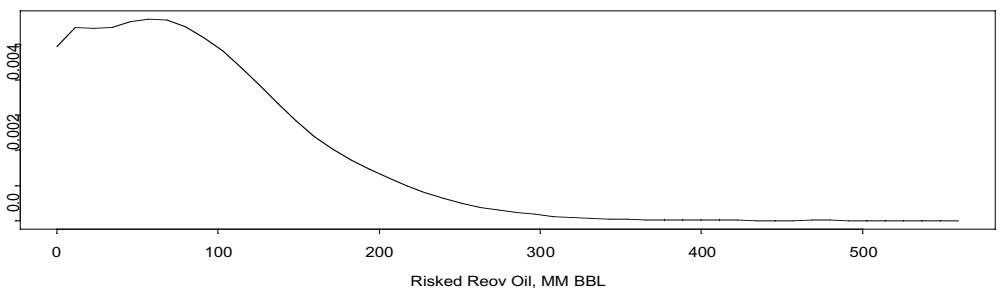

Play Totals Beaufortian Lower Jurassic Topset

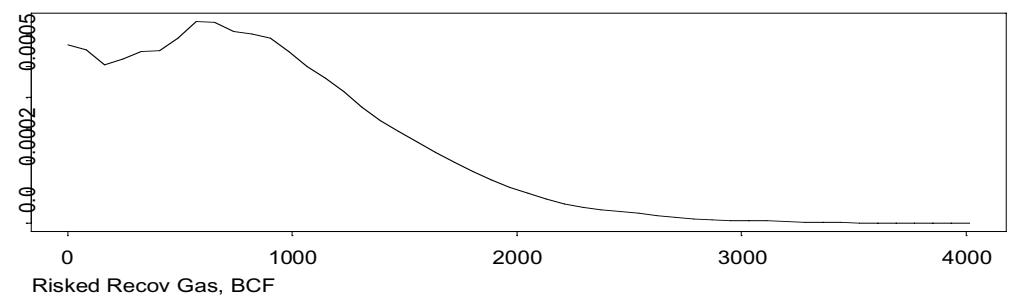


Table 18f: Estimated undiscovered oil and gas resources for the Beaufortian Lower Jurassic Topset Play showing number of deposits and volumes by accumulation size class

$\begin{array}{rrrrrr}\text { Start size class } & \text { Oil deposits } & \text { Oil (MMBO_Start size class } & \text { Gas deposits } & \text { NA Gas (BCFG) } \\ 0 & 0.00 & 0.00 & 0 & 0.00 & 0.00 \\ 8 & 0.11 & 1.70 & 48 & 0.00 & 0.00 \\ 16 & 1.49 & 34.51 & 96 & 0.00 & 0.00 \\ 32 & 0.87 & 37.00 & 192 & 2.37 & 690.91 \\ 64 & 0.12 & 9.24 & 384 & 0.23 & 101.86 \\ 128 & 0.00 & 0.25 & 768 & 0.00 & 0.22 \\ 256 & & & 1536 & & \end{array}$

\begin{tabular}{|r|r|r|r|r|}
\hline Totals & 2.58 & 82.70 & 2.60 & 792.99 \\
\hline
\end{tabular}
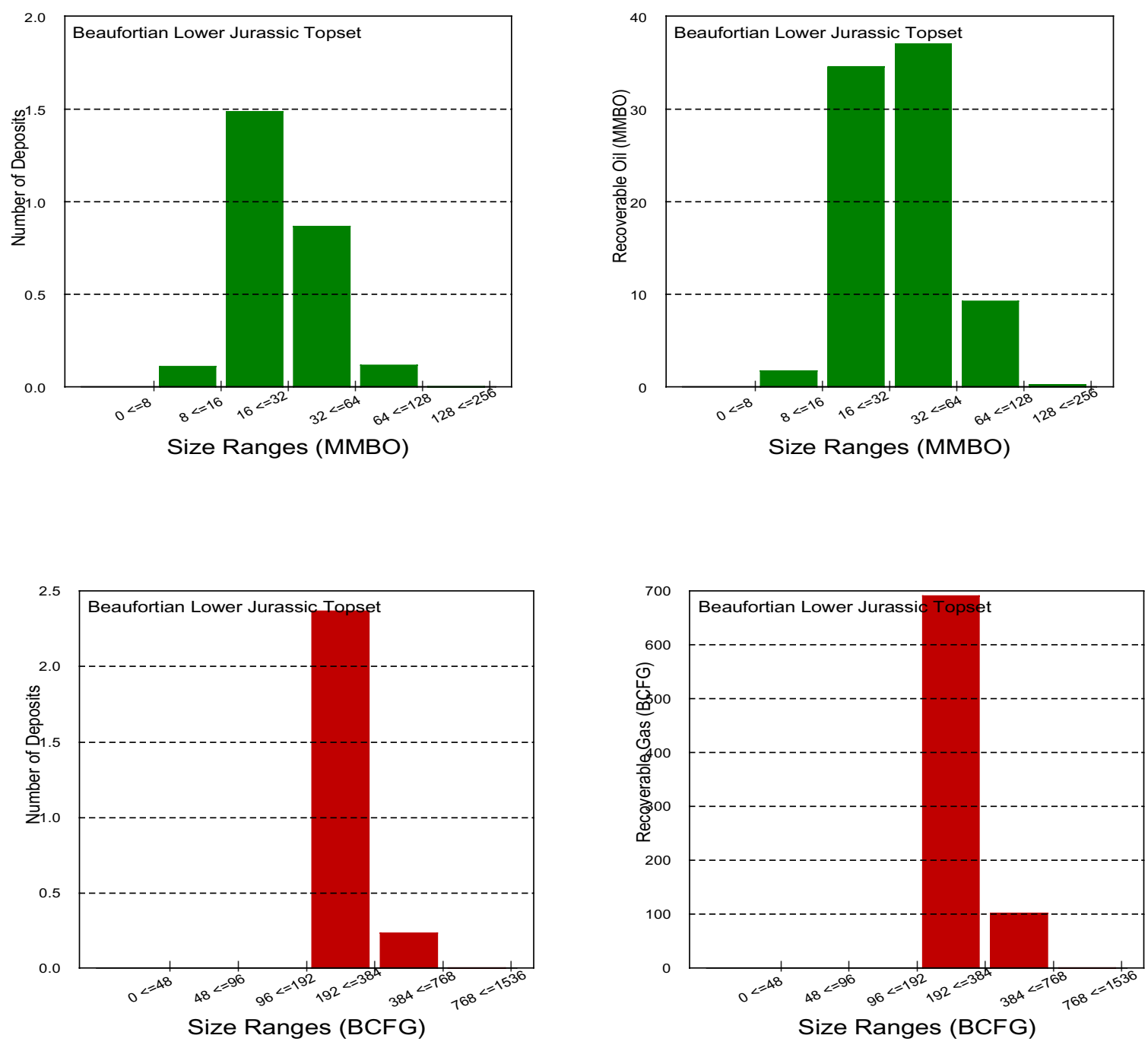
Table 19a: Input values for oil accumulations in the Beaufortian Clinoform Play NPRA Assessment Form-2001

PLAY: Beaufortian Clinoform

Play area: $\quad 7880 \mid 10^{3}$ Acres

\section{OIL ACCUMULATION VOLUME PARAMETERS}

\begin{tabular}{|c|c|c|c|c|c|c|c|}
\hline \multirow[b]{2}{*}{ ATTRIBUTES } & \multirow{2}{*}{$\begin{array}{l}\text { Est Shape } \\
(1 \text { to } 5)\end{array}$} & \multicolumn{4}{|c|}{ PROB OF AND GREATER THAN } & \multirow{2}{*}{$\begin{array}{l}\text { Knowledge } \\
\text { Level } 1-3^{5} \\
\end{array}$} & \\
\hline & & LTP & 0.50 & 0.05 & Max & & \\
\hline \multirow{4}{*}{$\begin{array}{l}\text { NET RESERVOIR THICKNESS }^{1} \\
\text { AREA OF CLOSURE }^{2} \\
\text { POROSITY }^{3,4} \\
\text { TRAP FILL }\end{array}$} & 2 & 15 & 40 & 70 & 150 & 3 & \\
\hline & 2 & 5 & 10 & 15 & 25 & 3 & \\
\hline & 4 & 8 & 13 & 17 & 19 & 3 & \\
\hline & 6 & 50 & 75 & 99 & 100 & 3 & Enter $\mathrm{POR}^{\star} \mathrm{Sw}$ \\
\hline \multicolumn{2}{|l|}{ HYDROCARBON PORE VOL ${ }^{3,4}$} & 3 & 8 & 12 & 14 & & 5 \\
\hline \multirow{2}{*}{\multicolumn{2}{|c|}{$\begin{array}{l}\text { Approx } \mathrm{mm} \text { bbl }(\mathrm{fvf}=1) \\
\text { Recov } \mathrm{mm} \text { bbl at surface }\end{array}$}} & 8.7 & 186.2 & 967.7 & 4073.0 & & \\
\hline & & 1.8 & 38.6 & 200.6 & 844.3 & & \\
\hline \multicolumn{8}{|c|}{ 1-thickness in feet, 2-thousands of acres, 3-percent, 4-correlation between Porosity and Water Saturation $=-1.0$} \\
\hline \multirow{2}{*}{$\begin{array}{l}\text { TRAP DEPTH (in } 1000 \mathrm{ft} \text { ) } \\
\text { (from sea level) }\end{array}$} & 2 & 8 & 9 & 10 & 12 & 2 & \\
\hline & & Surface to $\mathrm{s}$ & level correc & (1000 ft): & 0.431 & & \\
\hline
\end{tabular}

\section{OIL ACCUMULATION CHARACTERISTICS}

Oil recovery factor \% 35

Type of reservoir-drive (check any that apply):

FVF (Formation volume factor, rb/stb):
\begin{tabular}{|lr|}
\hline Pressure(psi) & 4500 \\
temp(deg F) & 201 \\
SolGasGr & 0.757 \\
F & 1376 \\
\hline
\end{tabular}

GOR (Associated gas to oil ratio, cu.ft./bbl, at stp):

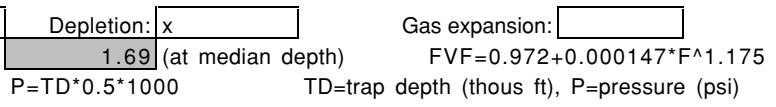

$\begin{array}{ll}\mathrm{P}=\mathrm{TD}^{*} 0.5^{*} 1000 & \mathrm{TD}=\text { trap depth (thous } \mathrm{ft} \text { ), } \mathrm{P}=\mathrm{pressure}(\mathrm{psi}) \\ \mathrm{T}=19^{*} \mathrm{TD}+30 & \mathrm{~T}=\text { temp deg } \mathrm{F} \text { is log base e }\end{array}$

$S G G=\left(\left(0.1402^{*} \mathrm{LN}(\mathrm{C} 27+14.7)-0.4227\right)+\left(0.1369^{*} \mathrm{LN}(\mathrm{C} 28)+0.0156\right)+\left(0.1704^{*} \mathrm{LN}(\mathrm{E} 37)-\mathrm{AG}=\mathrm{API}\right.\right.$ gravity

Uncorrected GOR $=\mathrm{SGG}^{*}\left((\mathrm{P}+14.7)^{\star} 10^{\wedge}\left(0.0125^{\star} \mathrm{AG}\right) /\left(18^{\star} 10^{\wedge}\left(0.00091^{\star} \mathrm{T}\right)\right)\right)^{\wedge}(1 / 0.83)$

$\mathrm{F}=$ Final $\mathrm{GOR}^{\star}(\mathrm{SGG} / \mathrm{OG})^{\wedge} 0.5+1.25^{\star} \mathrm{T} \quad \mathrm{OG}=141.5 /(131.5+\mathrm{AG})$

\begin{tabular}{r|r|}
\cline { 2 - 2 } & 1178 \\
Uncorrected GOR
\end{tabular}

NGLR (Natural gas liquids to associated gas ratio, bbls/million cu.ft., at $\operatorname{stp})=4.5985^{\star} \exp \left(.1711^{\star} T D\right.$ )

21.4 (median depth)

Oil quality parameters:

API gravity

Sulfur content of oil

\begin{tabular}{|r|}
\hline 39 \\
\hline 0.3 \\
\hline
\end{tabular}

Oil Grav (ratio)

0.830

Associated gas quality parameters:

Hydrogen sulfide $\%$

CO2 contamination \%

Other inert gases:

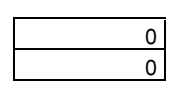

Name: $\quad$ Percent:

Name:

Percent:

TIME OF TRAP DEVELOPMENT

STRATIGRAPHIC COMPONENT (Ma)

STRUCTURAL COMPONENT (Ma)

\begin{tabular}{|r|r|r|}
\multicolumn{1}{l}{ BEGIN } & PEAK & \multicolumn{1}{c}{ END } \\
\hline 205 & & 178 \\
\hline & & \\
\hline
\end{tabular}

Assessor's Name: Dave Houseknecht

Date of Data Entry MM/DD/YYYY:

Date of Simulation Run MM/DD/YYYY:

$10 / 19 / 01$

$1 / 19 / 02$

Note: only enter play name and assessor's name on Oil worksheet 
Table 19b: Input values for nonassociated gas accumulations in the Beaufortian Clinoform Play NPRA Assessment Form-2001

PLAY: Beaufortian Clinoform

\section{NONASSOCIATED GAS ACCUMULATION VOLUME PARAMETERS}

\begin{tabular}{|c|c|c|c|c|c|c|c|}
\hline \multirow[b]{2}{*}{ ATTRIBUTES } & \multirow{2}{*}{$\begin{array}{l}\text { Est Shape } \\
(1 \text { to } 5)\end{array}$} & \multicolumn{4}{|c|}{ PROB OF AND GREATER THAN } & \multirow{2}{*}{$\begin{array}{l}\text { Knowledge } \\
\text { Level } 1-3^{5}\end{array}$} & \\
\hline & & \begin{tabular}{|l|l} 
LTP \\
\end{tabular} & 50 & 5 & Max & & \\
\hline \multirow{4}{*}{$\begin{array}{l}\text { NET RESERVOIR THICKNESS } \\
\text { AREA OF CLOSURE }^{2} \\
\text { POROSITY, } \\
\text { TRAP FILL }{ }^{3}\end{array}$} & 2 & 15 & 40 & 70 & 150 & 3 & \\
\hline & 2 & 5 & 10 & 15 & 25 & 3 & \\
\hline & 4 & 8 & 13 & 17 & 19 & 3 & \\
\hline & 6 & 50 & 75 & 99 & 100 & 3 & $\mathrm{POR}^{\star} \mathrm{Sw}$ \\
\hline \multicolumn{2}{|l|}{ HYDROCARBON PORE VOL ${ }^{3,4}$} & 3 & 8 & 12 & 14 & & 5 \\
\hline \multirow{2}{*}{\multicolumn{2}{|c|}{$\begin{array}{l}\text { Approx in place bcf } \\
\text { Recov bcf at surface }\end{array}$}} & 0.05 & 1.05 & 5.43 & 22.87 & & \\
\hline & & 8.6 & 183.2 & 952.3 & 4007.8 & & \\
\hline \multicolumn{7}{|c|}{$\begin{array}{l}\text { 1-thickness in feet, 2-thousands of acres, 3-percent, 4-correlation between Porosity and Water Saturation }=-1.0 \\
\text { 5-Knowledge Level: 1=High, 2=Medium, 3=Low; LTP=Left Truncation Point }\end{array}$} & \\
\hline \multirow{2}{*}{$\begin{array}{l}\text { TRAP DEPTH (in } 1000 \mathrm{ft} \text { ) } \\
\text { (from sea level) }\end{array}$} & \begin{tabular}{|r|}
2 \\
\end{tabular} & 8 & 12 & 16 & 18 & 2 & \\
\hline & \multicolumn{4}{|c|}{ Surface to sea level correction $(1000 \mathrm{ft}):$} & 0.431 & & \\
\hline
\end{tabular}

\section{NONASSOCIATED GAS ACCUMULATION CHARACTERISTICS}

NA Gas recovery factor \% 65

Type of reservoir-drive (check any that apply):

$$
\text { Water: Gas expansion: }
$$

Natural gas liquids plus condensate to non-associated gas (bbls/million cf) (in place):

NGL-NAG=1.785*TD 21.4 (at median)

Non-associated gas quality parameters:

Hydrogen sulfide \%

CO2 contamination \%

Other inert gases:
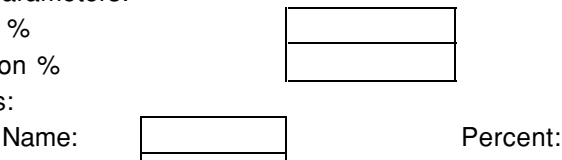
Name:
Percent:

Gas fvf

$$
\frac{269.6}{\text { (at median depth) }}
$$

Gas $f v f=752.2^{*}\left(1-\operatorname{EXP}\left(-0.05728^{*} T D\right)\right)$

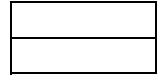

TD $<=5.67$ thous $\mathrm{ft}$

$$
113.3+21.1^{*} T D-0.812^{*} T^{\wedge} 2+0.0116^{*} T^{\wedge} 3 \quad 5.67<T D<=30
$$

$\mathrm{TD}=$ trap depth (thous $\mathrm{ft}$ )

TIME OF TRAP DEVELOPMENT

STRATIGRAPHIC COMPONENT (Ma)

STRUCTURAL COMPONENT (Ma)

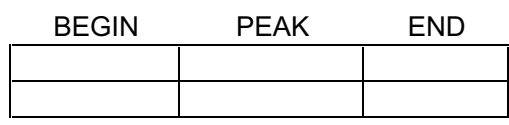

Assessor's Name: Dave Houseknecht

Date of Data Entry MM/DD/YYYY:

Date of Simulation Run MM/DD/YYYY:

$10 / 19 / 01$

$1 / 19 / 02$ 
Table 19c: Input risking values for the Beaufortian Clinoform Play

NPRA Assessment Form-2001

Play: Beaufortian Clinoform

\section{RISKING}

MINIMUM ACCUMULATION SIZE, MAS (Millions of BBL in place)

50

PRERISKED FREQUENCY DISTRIBUTION (Oil plus Gas)

\begin{tabular}{|c|c|c|c|c|c|c|}
\hline \multirow{3}{*}{$\begin{array}{l}\text { NUM OF PROSPECTS } \\
>\text { MINIMUM SIZE }\end{array}$} & & \multicolumn{4}{|c|}{ PROB OF AND GREATER THAN } & \multirow{2}{*}{$\begin{array}{l}\text { Knowledge } \\
\text { Level } 1-3^{5} \\
\end{array}$} \\
\hline & Est Shape & Min & 50 & 5 & $\operatorname{Max}$ & \\
\hline & 2 & 10 & 25 & 50 & 75 & +2 \\
\hline
\end{tabular}

5-Knowledge Level: 1=High, 2=Medium, 3=Low; LTP=Left Truncation Point

\section{ATTRIBUTES}

PLAY

ATTRIBUTES

CHARGE (C)
$\operatorname{TRAP}(\mathrm{T})$

TIMING (F)

Probability that play contains at least 1 reservoir $>=$ minimum size (CxTXF)

PROSPECT

ATTRIBUTES

TIMING (f)

Probability that a randomly chosen prospect is favorable (cxtxf)

Play Attributes x Prospect Attributes (CxTxFxcxtxf)

FRACTION OF ACCUMULATIONS BEING OIL

Fraction NA Gas=1-Fraction(Oil)
PROBABILITY

OF FAVORABLE

Computed

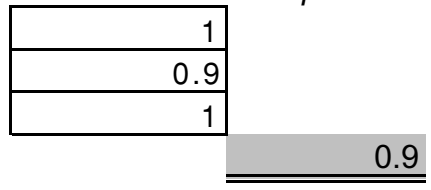

\begin{tabular}{r|}
\hline 1 \\
\hline 0.1 \\
\hline 1 \\
\hline
\end{tabular}

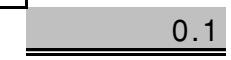

0.09

0.1

Allocation (percent):

\begin{tabular}{|l|r|r|r|}
\cline { 2 - 4 } \multicolumn{1}{c|}{} & \multicolumn{1}{c|}{ Land } & \multicolumn{1}{c|}{ Oil } & \multicolumn{2}{c|}{ Gas } \\
\hline Federal & 97 & 65 & 94 \\
\hline State & 2 & 20 & 4 \\
\hline Native & 1 & 15 & 2 \\
\hline
\end{tabular}

Assessor's Name:

Dave Houseknecht

Date of Data Entry MM/DD/YYYY:

Date of Simulation Run MM/DD/YYYY:

$10 / 19 / 01$

$1 / 19 / 02$ 
Table 19d: Distribution of fitted hydrocarbon volume attributes, trap depth, and number of prospects for the Beaufortian Clinoform Play
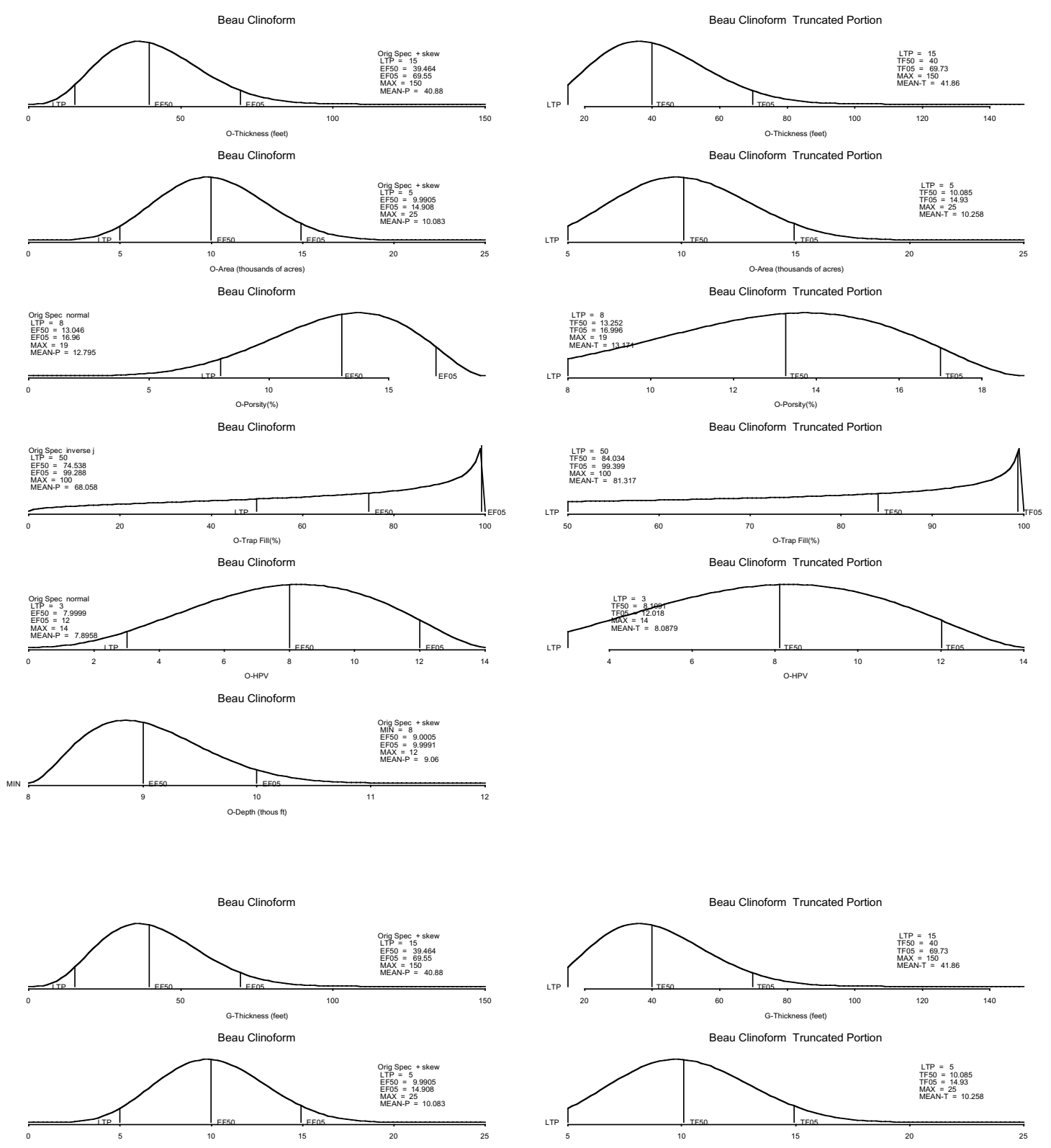

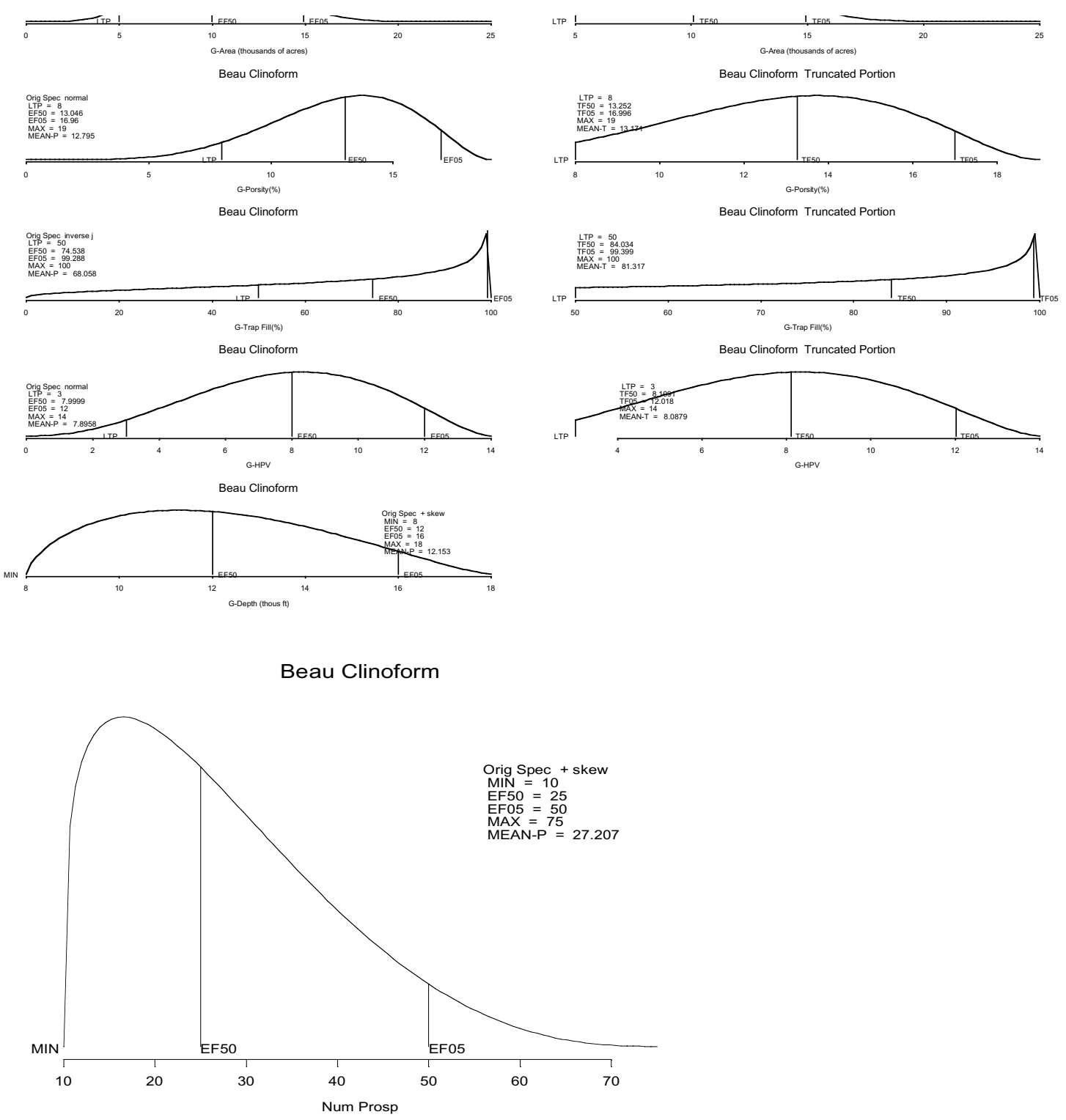
Table 19e: Estimated undiscovered oil and gas resources for the Beaufortian Clinoform Play Deposit

Beaufortian Clinoform
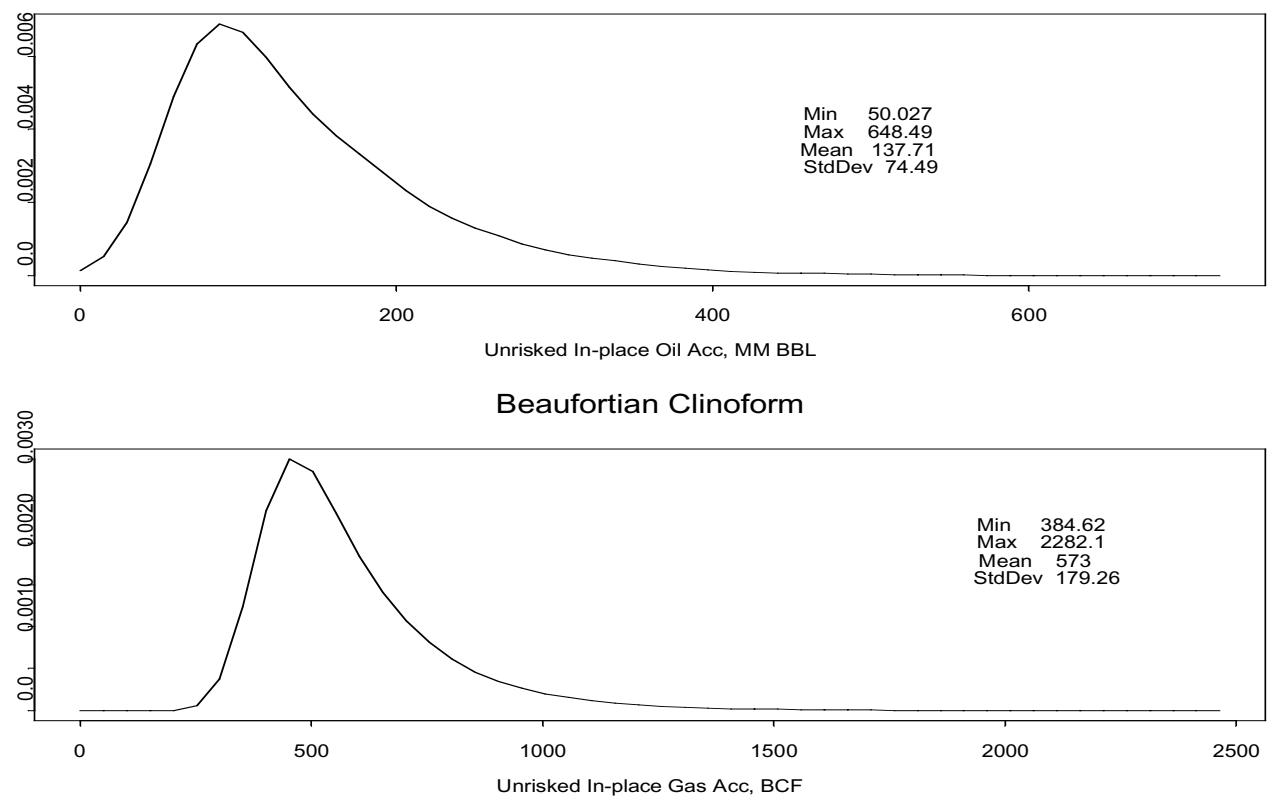

Play Stats
\begin{tabular}{|ll|rrrrr|} 
Resource & Unit & Mean & StdDev & F95 & F50 & F05 \\
\hline In-place oil & MMBO & 33.53 & 79.76 & 0.00 & 0.00 & 203.33 \\
In-place NA gas & BCFG & 1264.60 & 1106.59 & 0.00 & 1054.77 & 3354.29 \\
Recov oil & MMBO & 11.74 & 27.92 & 0.00 & 0.00 & 71.16 \\
Recov assoc diss gas & BCFG & 14.93 & 35.50 & 0.00 & 0.00 & 90.21 \\
Recov NA gas & BCFG & 821.99 & 719.28 & 0.00 & 685.60 & 2180.29 \\
Recov NGL (ADG) & MMBO & 0.27 & 0.65 & 0.00 & 0.00 & 1.66 \\
Recov NGL (NAG) & MMBO & 18.83 & 16.66 & 0.00 & 15.59 & 50.24 \\
Num oil deposits & & 0.24 & 0.51 & 0.00 & 0.00 & 1.00 \\
Num NA gas deposits & & 2.21 & 1.88 & 0.00 & 2.00 & 6.00 \\
\hline RNS & & & & & & \\
\hline
\end{tabular}

RNS $=774$

Play Totals Beaufortian Clinoform

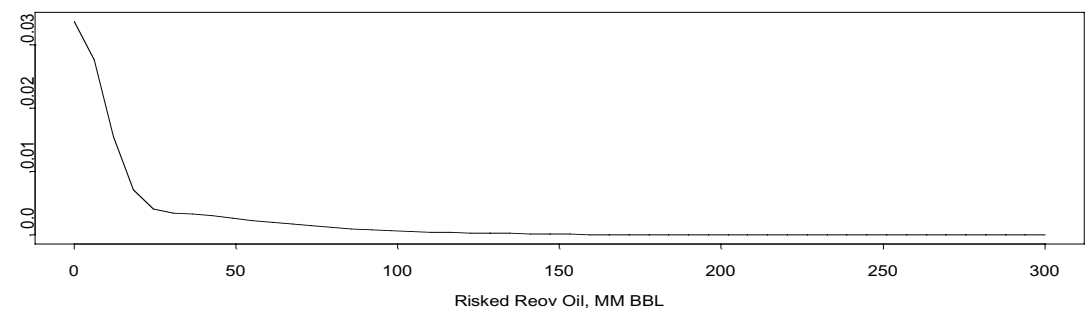

Play Totals Beaufortian Clinoform

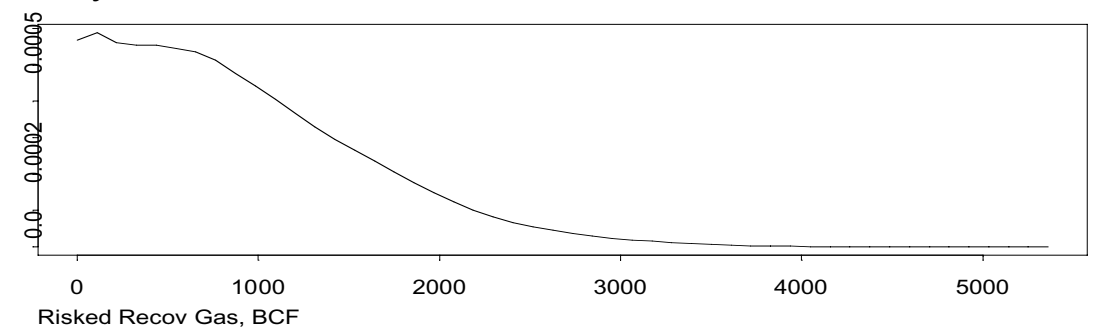


Table 19f: Estimated undiscovered oil and gas resources for the Beaufortian Clinoform Play showing number of deposits and volumes by accumulation size class

\begin{tabular}{rrrrrr} 
Start size class & Oil deposits & Oil (MMBO_Start size class & Gas deposits & NA Gas (BCFG) \\
0 & 0.00 & 0.00 & 0 & 0.00 & 0.00 \\
8 & 0.00 & 0.00 & 48 & 0.00 & 0.00 \\
16 & 0.08 & 1.98 & 96 & 0.00 & 0.00 \\
32 & 0.11 & 5.05 & 192 & 1.45 & 445.28 \\
64 & 0.05 & 4.18 & 384 & 0.73 & 354.49 \\
128 & 0.00 & 0.52 & 768 & 0.03 & 22.28 \\
256 & & & 1536 & & \\
\hline Totals & 0.24 & 11.74 & & 2.21 & 822.06 \\
\hline
\end{tabular}
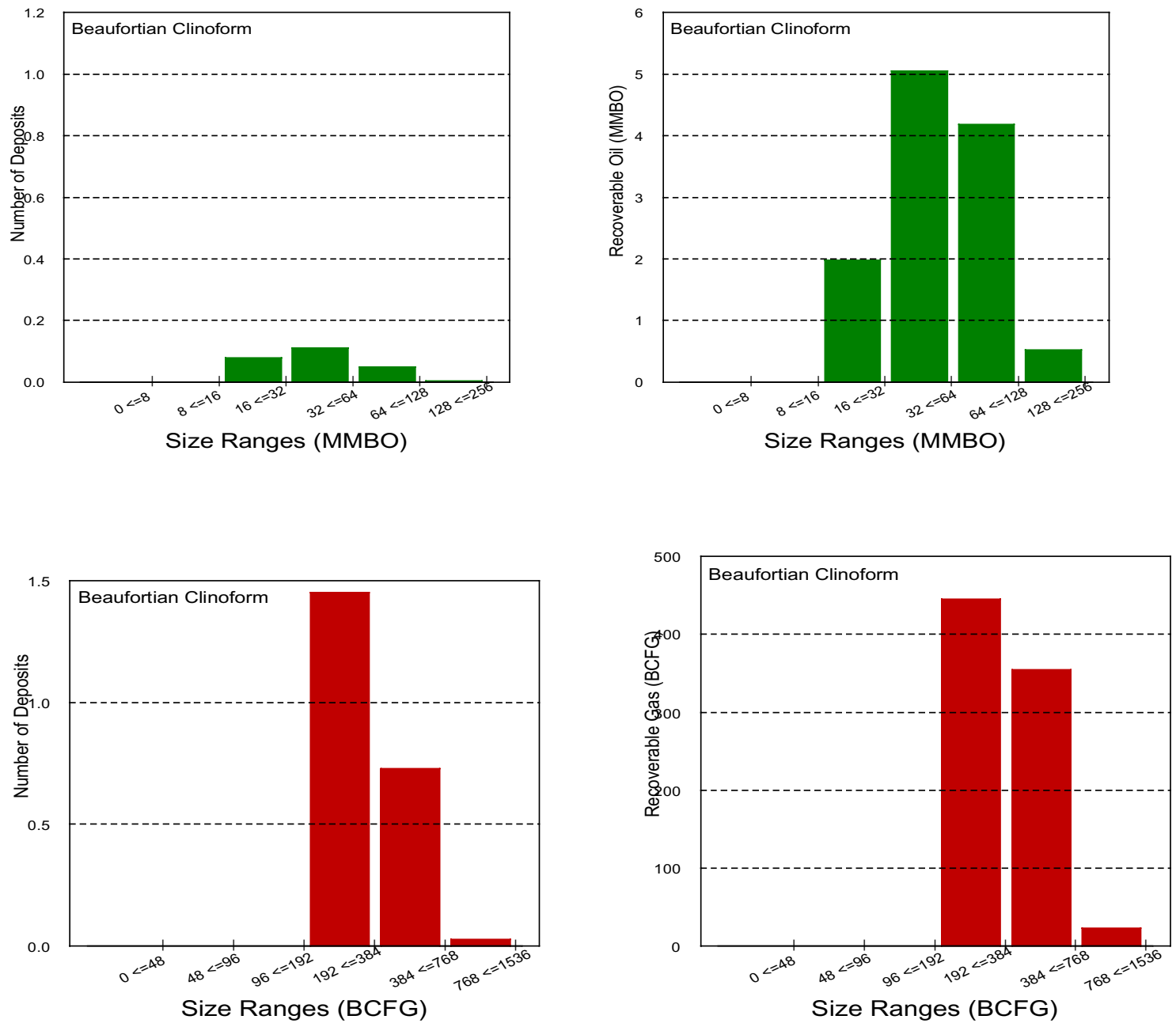
Table 20a: Input values for oil accumulations in the Brookian topset Structural Play NPRA Assessment Form-2001

PLAY: Brookian Topset Structural

Play area: $\quad 13388 \mid 10^{3}$ Acres

\section{OIL ACCUMULATION VOLUME PARAMETERS}

\begin{tabular}{|c|c|c|c|c|c|c|c|}
\hline \multirow[b]{2}{*}{ ATTRIBUTES } & \multirow{2}{*}{$\begin{array}{l}\text { Est Shape } \\
(1 \text { to } 6)\end{array}$} & \multicolumn{4}{|c|}{ PROB OF AND GREATER THAN } & \multirow{2}{*}{$\begin{array}{l}\text { Knowledge } \\
\text { Level } 1-3^{5}\end{array}$} & \\
\hline & & \begin{tabular}{|l|l} 
LTP \\
\end{tabular} & 50 & 5 & Max & & \\
\hline \multirow{4}{*}{$\begin{array}{l}\text { NET RESERVOIR THICKNESS } \\
\text { AREA OF CLOSURE }^{2} \\
\text { POROSITY } \\
\text { TRAP FILL }\end{array}$} & 2 & 25 & 100 & 400 & 500 & 2 & \begin{tabular}{l|l|l|}
-0.5 & Corr(NRT,HPV \\
\end{tabular} \\
\hline & 2 & 2 & 7 & 30 & 75 & 1 & \\
\hline & 4 & 12 & 14 & 17 & 20 & 2 & \\
\hline & 2 & 20 & 40 & 80 & 100 & 3 & Enter $\mathrm{POR}^{\star} \mathrm{Sw}_{\mathrm{w}}$ \\
\hline \multicolumn{2}{|l|}{ HYDROCARBON PORE VOL ${ }^{3,4}$} & 6 & 8 & 11 & 14 & & 6 \\
\hline \multirow{2}{*}{\multicolumn{2}{|c|}{$\begin{array}{l}\text { Approx mm bbl (fvf }=1 \text { ) } \\
\text { Recov mm bbl at surface }\end{array}$}} & 4.7 & 173.8 & 8192.4 & 40729.5 & & \\
\hline & & 1.5 & 56.4 & 2660.0 & 13224.4 & & \\
\hline \multicolumn{8}{|c|}{ 1-thickness in feet, 2-thousands of acres, 3-percent, 4-correlation between Porosity and Water Saturation $=-1.0$} \\
\hline \multirow{2}{*}{$\begin{array}{l}\text { TRAP DEPTH (in } 1000 \mathrm{ft} \text { ) } \\
\text { (from sea level) }\end{array}$} & 2 & 0.5 & 2 & 10 & 12 & 1 & \\
\hline & \multicolumn{4}{|c|}{ Surface to sea level correction $(1000 \mathrm{ft}):$} & 0.9 & & \\
\hline
\end{tabular}

\section{OIL ACCUMULATION CHARACTERISTICS}

Oil recovery factor \%

Type of reservoir-drive (check any that apply):

FVF (Formation volume factor, rb/stb):

\begin{tabular}{|lr|}
\hline Pressure(psi) & 1000 \\
temp(deg F) & 68 \\
SolGasGr & 0.634 \\
F & 271 \\
\hline
\end{tabular}

1000

(y).

\section{Depletion: $\mathrm{x}$ Gas expansion: \\ 1.08 (at median depth) $F V F=0.972+0.000147^{\star} F^{\wedge} 1.175$}

$\mathrm{P}=\mathrm{TD}^{*} 0.5^{*} 1000 \quad \mathrm{TD}=$ trap depth (thous $\mathrm{ft}$ ), $\mathrm{P}=$ pressure (psi)

$\mathrm{T}=19^{*} \mathrm{TD}+30 \quad \mathrm{~T}=$ temp deg $\mathrm{F} \quad \mathrm{LN}$ is log base e

$S G G=\left(\left(0.1402^{*} L N(C 27+14.7)-0.4227\right)+\left(0.1369^{*} L N(C 28)+0.0156\right)+\left(0.1704^{*} L N(E 3 A G=A P I\right.\right.$ gravity Uncorrected GOR $=S G^{*}\left((P+14.7)^{*} 10^{\wedge}\left(0.0125^{*} A G\right) /\left(18^{*} 10^{\wedge}\left(0.00091^{*} T\right)\right)\right)^{\wedge}(1 / 0.83)$

GOR (Associated gas to oil ratio, cu.ft./bbl, at stp):

$O G=141.5 /(131.5+A G)$

213 Final GOR at median depth

248 Uncorrected GOR

NGLR (Natural gas liquids to associated gas ratio, bbls/million cu.ft., at $\operatorname{stp})=4.5985^{\star} \exp \left(.1711^{*}\right.$ TD

6.5 (median depth)

Oil quality parameters:

API gravity

Sulfur content of oil

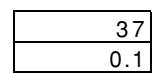

Oil Grav (ratio) 0.840

gas quality parameters:

Hydrogen sulfide \%

CO2 contamination \%

Other inert gases:

Name:

Name:

Percent:

Percent:

Umiat value

TIME OF TRAP DEVELOPMENT

STRATIGRAPHIC COMPONENT (Ma)

STRUCTURAL COMPONENT (Ma)

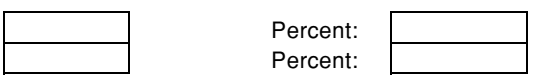

\begin{tabular}{r|r|r|}
\multicolumn{1}{c}{ BEGIN } & \multicolumn{1}{c}{ PEAK } & \multicolumn{2}{c}{ END } \\
\hline 108 & 100 & 97 \\
\hline 100 & 60 & 45 \\
\hline
\end{tabular}

Assessor's Name:

Chris Potter

Date of Data Entry MM/DD/YYYY:

Date of Simulation Run MM/DD/YYYY: 
Table 20b: Input values for nonassociated gas accumulations in the Brookian Topset Structural Play

PLAY: Brookian Topset Structural

\section{NONASSOCIATED GAS ACCUMULATION VOLUME PARAMETERS}

\begin{tabular}{|c|c|c|c|c|c|c|c|}
\hline \multirow[b]{2}{*}{ ATTRIBUTES } & \multirow{2}{*}{$\begin{array}{l}\text { Est Shape } \\
(1 \text { to } 6)\end{array}$} & \multicolumn{4}{|c|}{ PROB OF AND GREATER THAN } & \multirow{2}{*}{$\begin{array}{l}\text { Knowledge } \\
\text { Level } 1-3^{5}\end{array}$} & \\
\hline & & \begin{tabular}{|l|l|} 
LTP \\
\end{tabular} & 50 & 5 & Max & & \\
\hline \multirow{4}{*}{$\begin{array}{l}\text { NET RESERVOIR THICKNESS } \\
\text { AREA OF CLOSURE }^{1} \\
\text { POROSITY }^{3,4} \\
\text { TRAP FILL }^{3}\end{array}$} & 2 & 25 & 125 & 450 & 600 & 2 & $\begin{array}{ll}-0.5 & \text { Corr(NRT,HPV) } \\
\end{array}$ \\
\hline & 2 & 2 & 7 & 30 & 75 & 2 & \multirow{4}{*}{$\mathrm{POR}^{\star} \mathrm{Sw}$} \\
\hline & 4 & 10 & 14 & 17 & 20 & 2 & \\
\hline & 2 & 30 & 50 & 80 & 100 & 3 & \\
\hline \multicolumn{2}{|l|}{ HYDROCARBON PORE VOL ${ }^{3,4}$} & 4 & 8 & 11 & 14 & & \\
\hline \multirow{2}{*}{\multicolumn{2}{|c|}{$\begin{array}{l}\text { Approx in place bcf } \\
\text { Recov bcf at surface }\end{array}$}} & 0.03 & 1.52 & 51.75 & 274.43 & & \\
\hline & & 1.3 & 74.5 & 2528.0 & 13406.2 & & \\
\hline \multicolumn{7}{|c|}{$\begin{array}{l}\text { 1-thickness in feet, 2-thousands of acres, 3-percent, 4-correlation between Porosity and Water Saturation }=-1.0 \\
\text { 5-Knowledge Level: } 1=\text { High, 2=Medium, 3=Low; LTP=Left Truncation Point }\end{array}$} & \\
\hline \multirow{2}{*}{$\begin{array}{l}\text { TRAP DEPTH (in } 1000 \mathrm{ft} \text { ) } \\
\text { (from sea level) }\end{array}$} & 2 & 0.5 & 2 & 10 & 12 & 1 & \\
\hline & & Surface to $s$ & vel corre & (1000 ft): & 0.9 & & \\
\hline
\end{tabular}

\section{NONASSOCIATED GAS ACCUMULATION CHARACTERISTICS}

NA Gas recovery factor \% 60

Type of reservoir-drive (check any that apply):

$$
\text { Water: }
$$

Gas expansion:

Natural gas liquids plus condensate to non-associated gas (bbls/million cf) (in place):

\begin{tabular}{l|l}
$\mathrm{NGL}-\mathrm{NAG}=1.785^{\star} \mathrm{TD}$ & 3.6 \\
\end{tabular}

Non-associated gas quality parameters:

Hydrogen sulfide \%

CO2 contamination \%

Other inert gases:

$$
\text { Name: }
$$

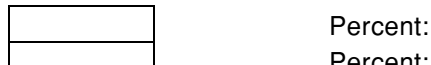

\section{Percent:}

Gas fvf 81.4

$$
\text { (at median depth) }
$$

$752.2^{*}(1-\operatorname{EXP}(-0.0$

$$
113.3+21.1^{*} T D-0.812^{*} T^{\wedge} 2+0.0116^{*} T D^{\wedge} 3
$$

TD=trap depth (thous $\mathrm{ft}$ )

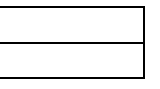

$\mathrm{TD}<=5.67$ thous $\mathrm{ft}$

$5.67<\mathrm{TD}<=30$

\begin{tabular}{|c|c|c|}
\hline BEGIN & PEAK & END \\
\hline \begin{tabular}{|c|}
108 \\
\end{tabular} & 100 & 97 \\
\hline 100 & 60 & 45 \\
\hline
\end{tabular}

For Nonassociated Gas Accumulation:

TIME OF TRAP DEVELOPMEN 7

STRATIGRAPHIC COMPONENT (M STRUCTURAL COMPONENT (Ma)

Assessor's Name: $\quad$ Chris Potter Date of Data Entry MM/DD/YYYY:

Date of Simulation Run MM/DD/YYYY: 
Table 20c: Input risking values for the Brookian Topset Structural Play

NPRA Assessment Form-2001

Play: Brookian Topset Structural

\section{RISKING}

MINIMUM ACCUMULATION SIZE, MAS (Millions of BBL in place)

PRERISKED FREQUENCY DISTRIBUTION (Oil plus Gas)

\begin{tabular}{l|r|r|r|r|r|r|}
\cline { 3 - 7 } & \multicolumn{4}{c|}{ PROB OF AND GREATER THAN } & Knowledge \\
NUM OF PROSPECTS & Est Shape & Min & F50 & F05 & Max & Level $1-3^{5}$ \\
\hline M MINIMUM SIZE & 1 & 70 & 105 & 130 & 180 & \\
\hline
\end{tabular}

5-Knowledge Level: 1=High, 2=Medium, 3=Low

\section{ATTRIBUTES}

PLAY

ATTRIBUTES

CHARGE (C)

TRAP/ROCK (T)
TIMING (F)

Probability that play contains at least 1 reservoir $>=$ minimum size $(C x T x F)$

PROSPECT

ATTRIBUTES

TIMING $(\mathrm{f})$
Probability that a randomly chosen

Probability that a randomly chosen prospect is favorable (cxtxf)

Play Attributes x Prospect Attributes (CxTxFxcxtxf)

FRACTION OF ACCUMULATIONS BEING OIL

Fraction NA Gas=1-Fraction(Oil)

\section{PROBABILITY}

OF FAVORABLE

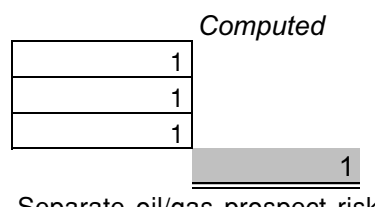

Separate oil/gas prospect risk

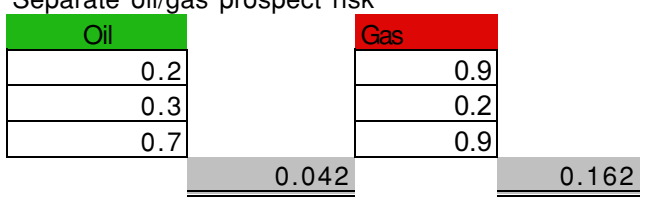

0.042

0.162

Allocation (percent):

\begin{tabular}{|l|r|r|r|}
\cline { 2 - 4 } \multicolumn{1}{c|}{} & \multicolumn{1}{c|}{ Land } & \multicolumn{1}{c|}{ Oil } & \multicolumn{2}{c|}{ Gas } \\
\hline Federal & 99 & 99 & 99 \\
\hline State & 0 & 0 & 0 \\
\hline Native & 1 & 1 & 1 \\
\hline
\end{tabular}

Assessor's Name:

$$
\text { Chris Potter }
$$

0.15 
Table 20d: Distribution of fitted hydrocarbon volume attributes, trap depth, and number of prospects for the Brookian Topset Structural Play
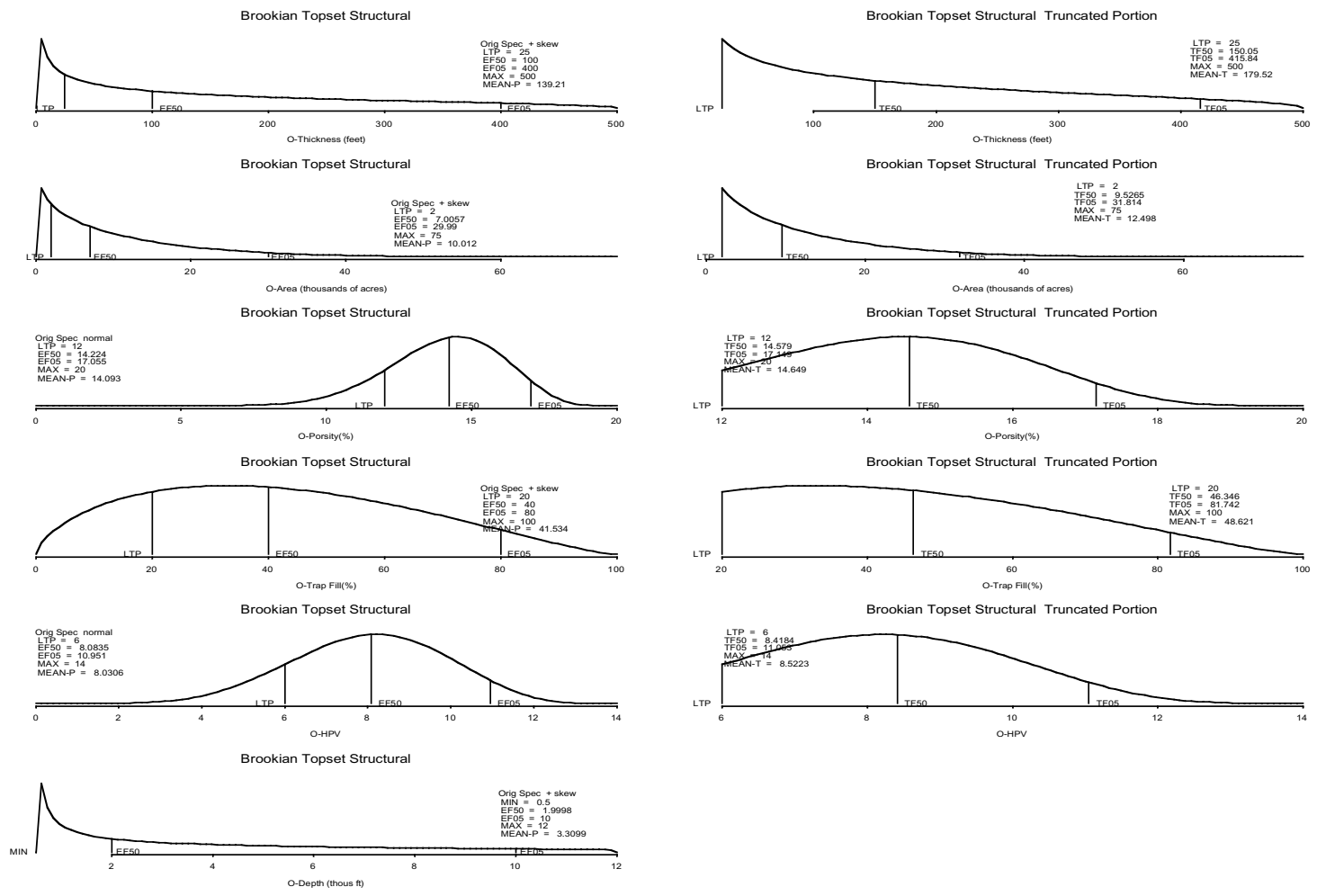

Brookian Topset Stru
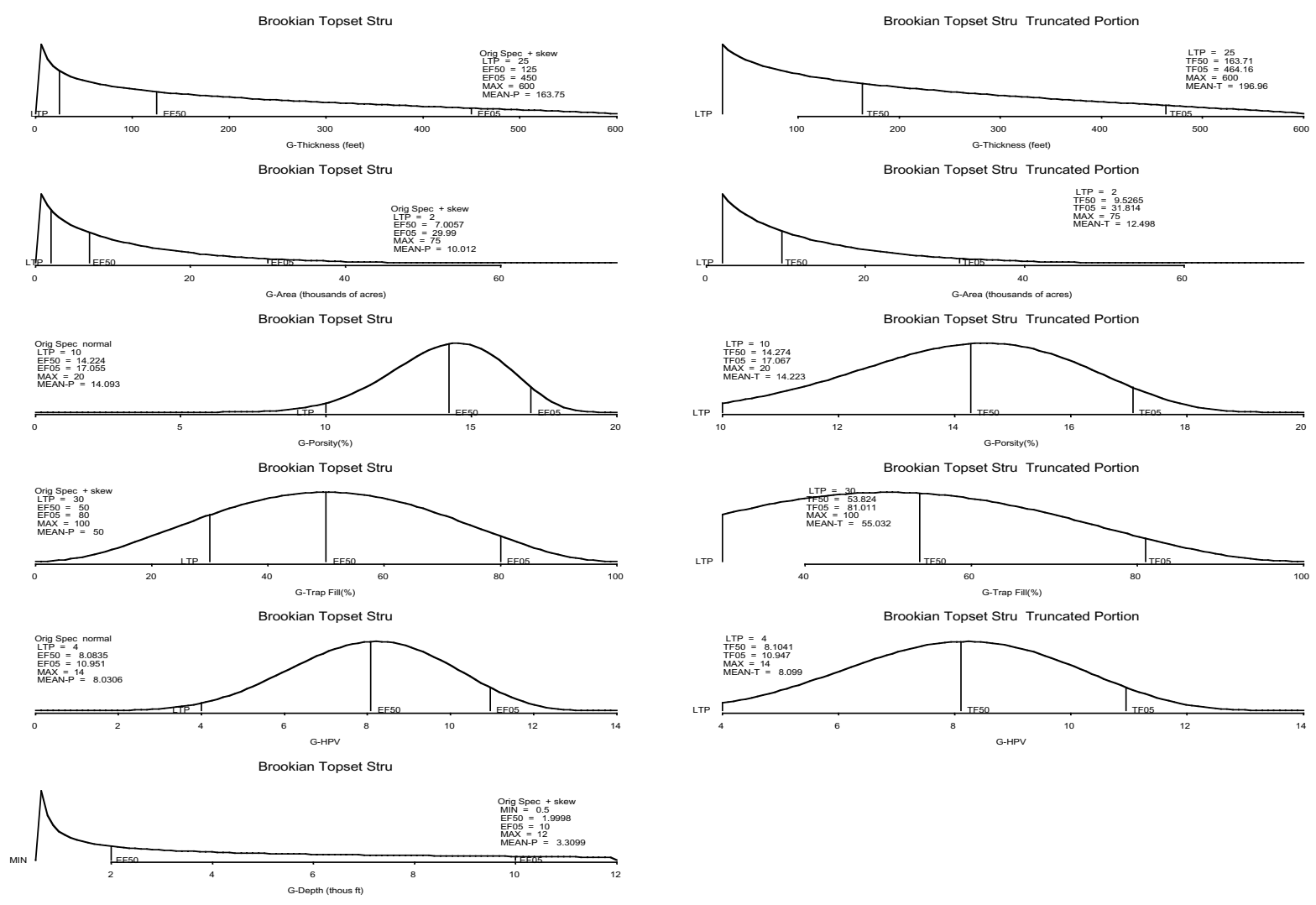
MiA
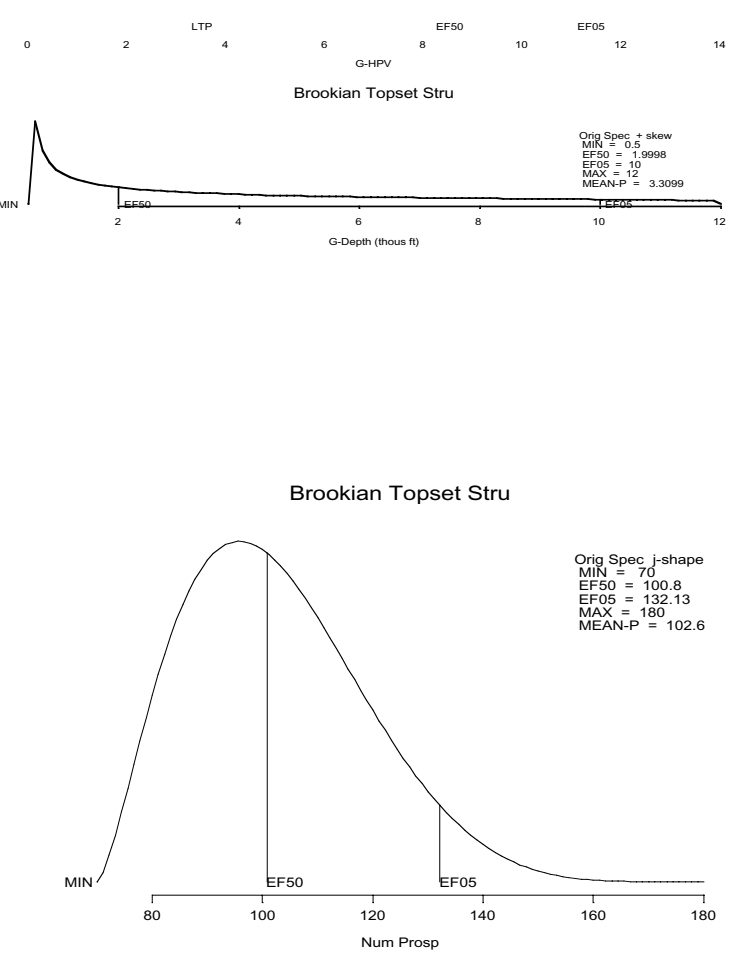
Table 20e: Estimated undiscovered oil and gas resources for the Brookian Topset Structural Play Deposit

Brookian Topset Structural

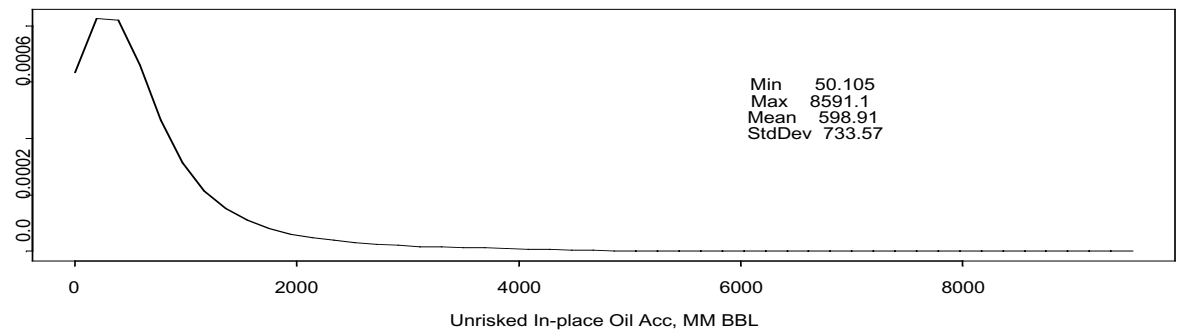

Brookian Topset Structural

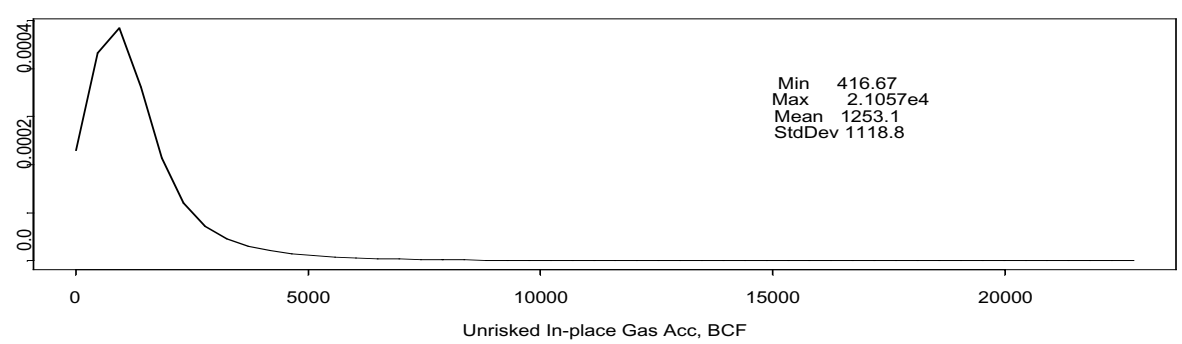

Play Stats
\begin{tabular}{|ll|rrrrr|} 
Resource & Unit & Mean & StdDev & F95 & F50 & F05 \\
\hline In-place oil & MMBO & 391.57 & 768.39 & 0.00 & 0.00 & 1867.81 \\
In-place NA gas & BCFG & 17676.13 & 6787.35 & 8001.89 & 16865.47 & 30019.64 \\
Recov oil & MMBO & 137.05 & 268.93 & 0.00 & 0.00 & 653.73 \\
Recov assoc diss gas & BCFG & 59.88 & 135.77 & 0.00 & 0.00 & 297.17 \\
Recov NA gas & BCFG & 10605.68 & 4072.41 & 4801.14 & 10119.28 & 18011.78 \\
Recov NGL (ADG) & MMBO & 1.37 & 2.91 & 0.00 & 0.00 & 6.55 \\
Recov NGL (NAG) & MMBO & 117.93 & 55.66 & 43.15 & 109.05 & 221.04 \\
Num oil deposits & & 0.65 & 0.81 & 0.00 & 0.00 & 2.00 \\
Num NA gas deposits & & 14.11 & 4.19 & 8.00 & 14.00 & 21.00 \\
\hline
\end{tabular}

Random number seed 887

Play Totals Brookian Topset Structural
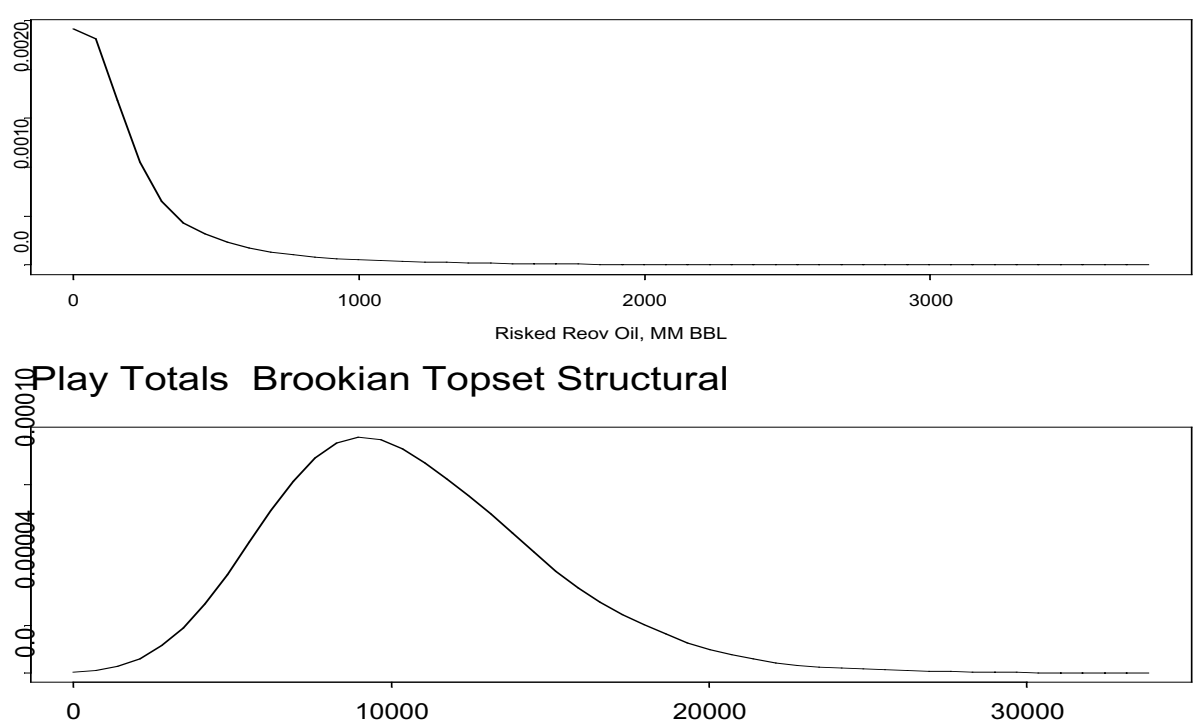

Risked Recov Gas, BCF 
Table 20f: Estimated undiscovered oil and gas resources for the Brookian Topset Structural Play showing number of deposits and volumes by accumulation size class

\begin{tabular}{rrrrrr} 
Start size class & & Oil deposits & Oil (MMBO) Start size class & Gas deposits & NA Gas (BCFG) \\
0 & 0.00 & 0.00 & 0 & 0.00 & 0.00 \\
8 & 0.00 & 0.00 & 48 & 0.00 & 0.00 \\
16 & 0.06 & 1.57 & 96 & 0.00 & 0.00 \\
32 & 0.12 & 5.81 & 192 & 4.37 & 1358.97 \\
64 & 0.16 & 14.49 & 384 & 5.44 & 2940.08 \\
128 & 0.15 & 26.83 & 768 & 2.98 & 3155.95 \\
256 & 0.10 & 37.22 & 1536 & 1.08 & 2227.84 \\
512 & 0.05 & 32.06 & 3072 & 0.21 & 834.11 \\
1024 & 0.01 & 17.23 & 6144 & 0.01 & 86.20 \\
2048 & 0.00 & 1.84 & 12288 & 0.00 & 2.52 \\
\hline Totals & 0.65 & 137.05 & \multicolumn{3}{c}{} \\
\hline
\end{tabular}
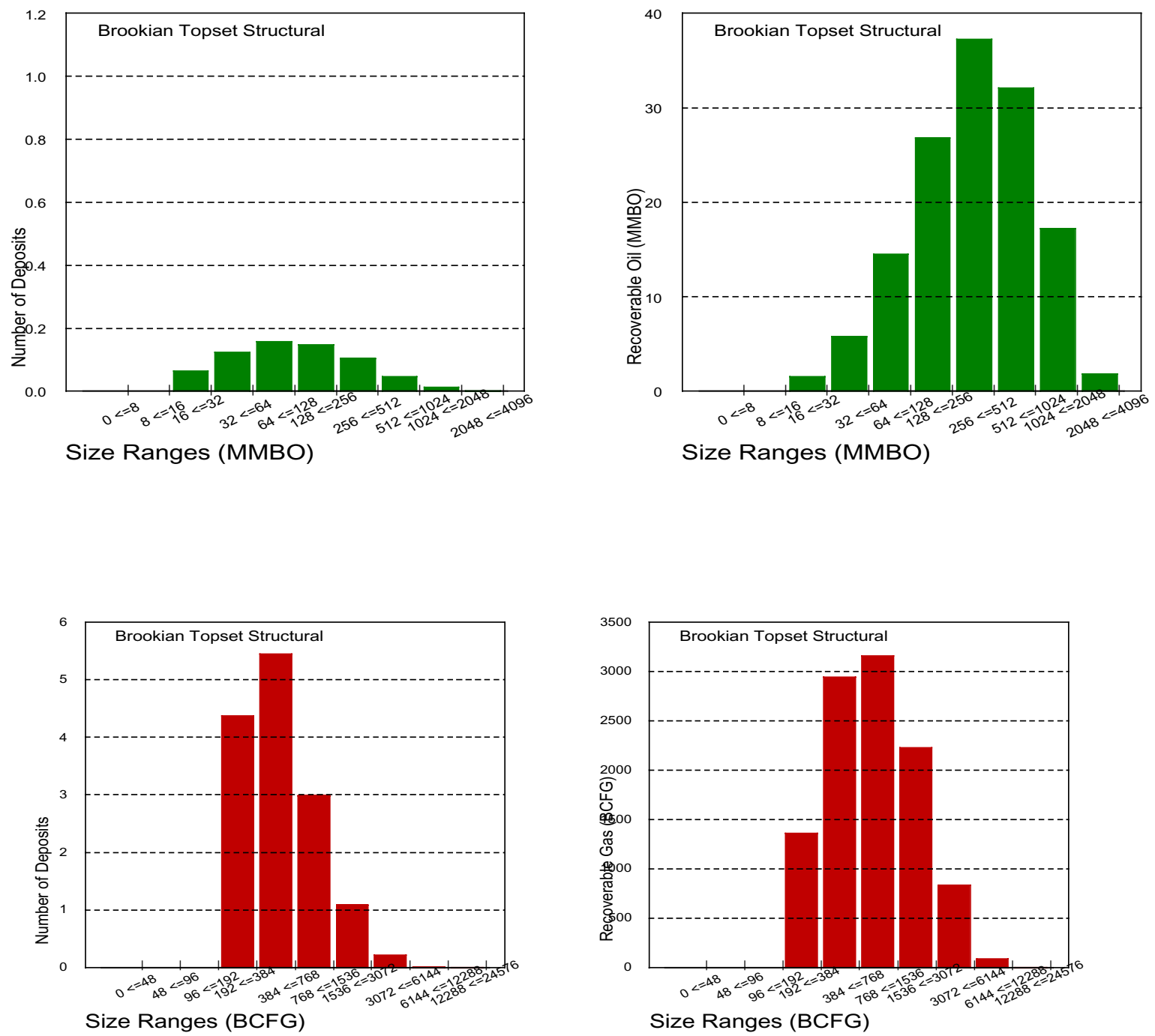
Table 21a: Input values for oil accumulations in the Torok Structural Play

NPRA Assessment Form-2001

PLAY: Torok Structural

Play area: $\quad 14,981 \mid 10^{3}$ Acres

\section{OIL ACCUMULATION VOLUME PARAMETERS}

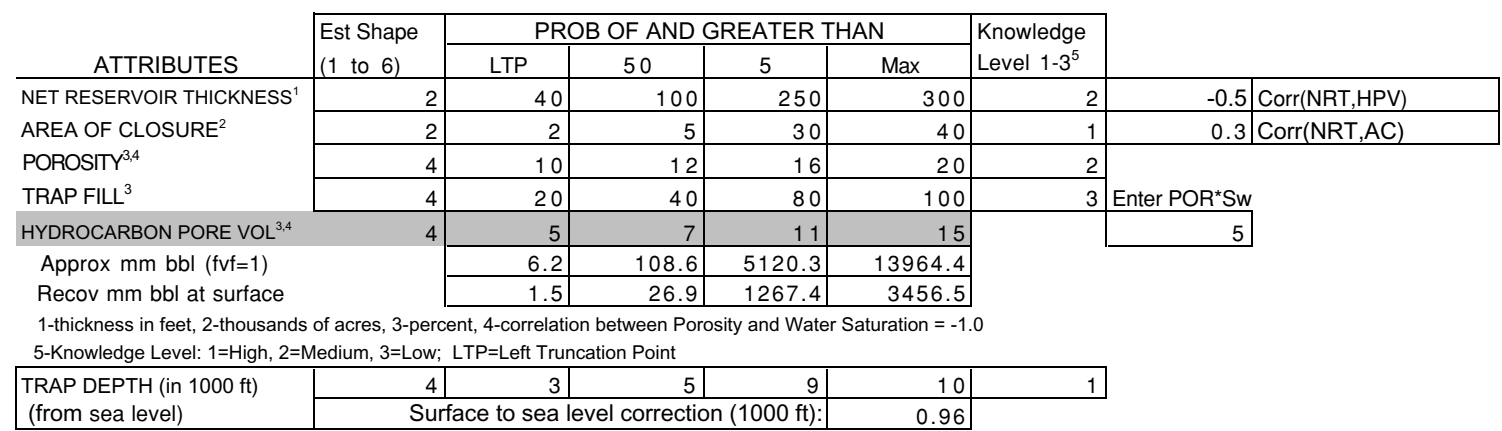

\section{OIL ACCUMULATION CHARACTERISTICS}

Oil recovery factor \% 30

Type of reservoir-drive (check any that apply):

FVF (Formation volume factor, rb/stb):

\begin{tabular}{|lr|}
\hline Pressure(psi) & 2500 \\
temp(deg F) & 125 \\
SolGasGr & 0.689 \\
F & 542 \\
\hline
\end{tabular}

GOR (Associated gas to oil ratio, cu.ft./bbl, at stp): Depletion: $x$

1.21 (at median depth) $\mathrm{FVF}=0.972+0.000147^{\star} \mathrm{F}^{\wedge} 1.175$

$\mathrm{P}=\mathrm{TD}^{*} 0.5^{*} 1000 \quad \mathrm{TD}=$ trap depth (thous $\mathrm{ft}$ ), $\mathrm{P}=$ pressure (psi)

$\mathrm{T}=19 * \mathrm{TD}+30 \quad \mathrm{~T}=$ temp deg $\mathrm{F} \quad \mathrm{LN}$ is log base e

$S G G=\left(\left(0.1402^{*} \mathrm{LN}(\mathrm{C} 27+14.7)-0.4227\right)+\left(0.1369^{*} \mathrm{LN}(\mathrm{C} 28)+0.0156\right)+\left(0.1704^{*} \mathrm{LN}(\mathrm{E} 37)+\mathrm{AG}=\mathrm{API}\right.\right.$ gravity Uncorrected GOR $=\mathrm{SGG}^{*}\left((\mathrm{P}+14.7)^{\star} 10^{\wedge}\left(0.0125^{\star} \mathrm{AG}\right) /\left(18^{\star} 10^{\wedge}\left(0.00091^{\star} \mathrm{T}\right)\right)\right)^{\wedge}(1 / 0.83)$

$F=$ Final $G^{\star}(S G G / O G)^{\wedge} 0.5+1.25^{\star} T \quad O G=141.5 /(131.5+A G)$

\begin{tabular}{|l|l|l|}
\cline { 2 - 2 } & 438 \\
\hline
\end{tabular}

NGLR (Natural gas liquids to associated gas ratio, bbls/million cu.ft., at $\operatorname{stp})=4.5985^{\star} \exp \left(.1711^{\star} T D\right.$ )

10.8 (median depth)

Oil quality parameters:

API gravity

Sulfur content of oil

\begin{tabular}{|r|r|}
\hline 28 \\
\hline 1.2 \\
$\quad$ Oil Grav (ratio) \\
\hline
\end{tabular}

Associated gas quality parameters:

Hydrogen sulfide \%

CO2 contamination \%

Other inert gases: $G=141.5 /(131.5+A G)$ 
Table 21b: Input values for nonassociated gas accumulations in the Torok Structural Play NPRA Assessment Form-2001

PLAY: Torok Structural

\section{NONASSOCIATED GAS ACCUMULATION VOLUME PARAMETERS}

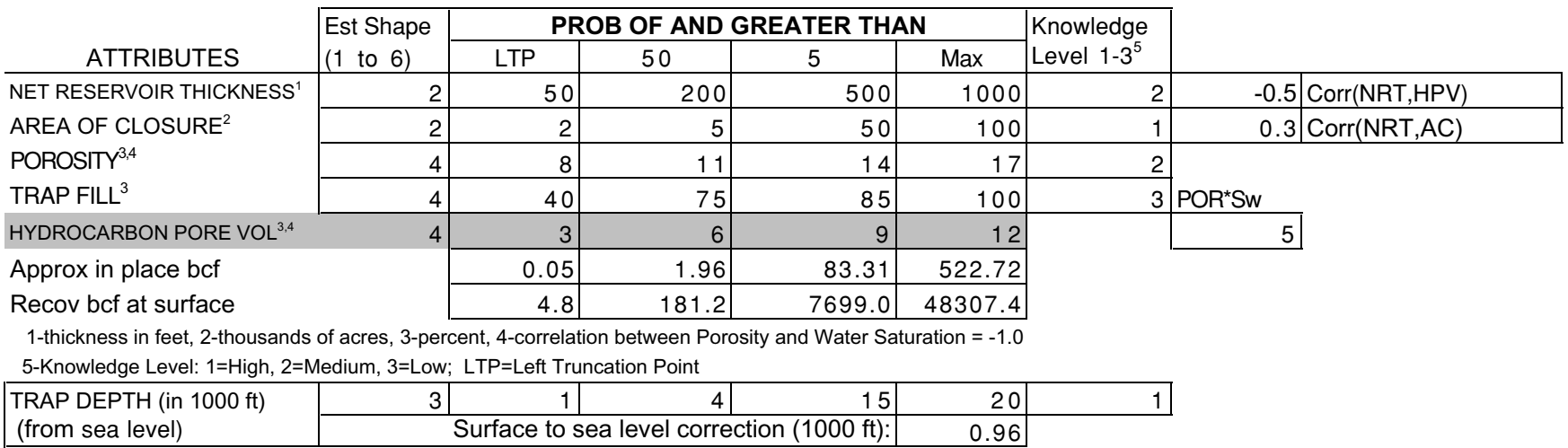

\section{NONASSOCIATED GAS ACCUMULATION CHARACTERISTICS}

NA Gas recovery factor \% 60

Type of reservoir-drive (check any that apply):

$$
\text { Water: }
$$

Gas expansion:

Natural gas liquids plus condensate to non-associated gas (bbls/million cf) (in place): NGL-NAG $=1.785^{\star} \mathrm{TD} \quad 7.1$ (at median)

Non-associated gas quality parameters:

Hydrogen sulfide \%

CO2 contamination \%

Other inert gases:

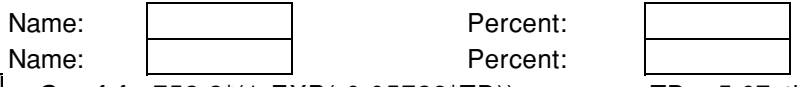

Gas fvf

Name:

Percent:

$$
\begin{aligned}
& \text { (at median depth) } \\
& \text { 752.2* }\left(1-\operatorname{EXP}\left(-0.05728^{*} T D\right)\right) \\
& T D<=5.67 \text { thous } \mathrm{ft} \\
& 113.3+21.1^{*} T D-0.812^{*} T^{\wedge} 2+0.0116^{*} T^{\wedge} 3 \quad 5.67<T D<=30 \\
& \mathrm{TD}=\text { trap depth (thous } \mathrm{ft} \text { ) }
\end{aligned}
$$

\begin{tabular}{|c|c|c|}
\hline BEGIN & PEAK & END \\
\hline 113 & 105 & 102 \\
\hline 100 & 60 & 45 \\
\hline
\end{tabular}

TIME OF TRAP DEVELOPMENT

STRATIGRAPHIC COMPONENT (Ma) STRUCTURAL COMPONENT (Ma)

Assessor's Name: Chris Potter Date of Data Entry MM/DD/YYYY:

Date of Simulation Run MM/DD/YYYY: 
Table 21c: Input risking values for the Torok Structural Play

NPRA Assessment Form-2001

Play: Torok Structural

\section{RISKING}

MINIMUM ACCUMULATION SIZE, MAS (Millions of BBL in place)

PRERISKED FREQUENCY DISTRIBUTION

NUM OF PROSPECTS

$>$ MINIMUM SIZE

\begin{tabular}{|r|r|r|r|r|r|}
\cline { 2 - 5 } \multicolumn{1}{c|}{} & \multicolumn{4}{c|}{ PROB OF AND GREATER THAN } & \multicolumn{2}{l|}{$\begin{array}{l}\text { Knowledge } \\
\text { Est Shape }\end{array}$} & Min & F50 & F05 & Max & Level $1-3^{5}$ \\
\hline 2 & 60 & 90 & 120 & 150 & 2 \\
\hline
\end{tabular}

5-Knowledge Level: 1=High, 2=Medium, 3=Low

\section{ATTRIBUTES}

PLAY

ATTRIBUTES

CHARGE (C)

TRAP/ROCK (T)
TIMING (F)

Probability that play contains at least 1 reservoir $>=$ minimum size $(C x T x F)$

PROSPECT

ATTRIBUTES

TIMING (f)
Probability that a randomly chosen

CHARGE (c)

TRAP ( $\mathrm{t}$ )

Play Attributes x Prospect Attributes (CxTxFxcxtxf)

FRACTION OF ACCUMULATIONS BEING OIL

Fraction NA Gas=1-Fraction(Oil)

\section{PROBABILITY}

OF FAVORABLE
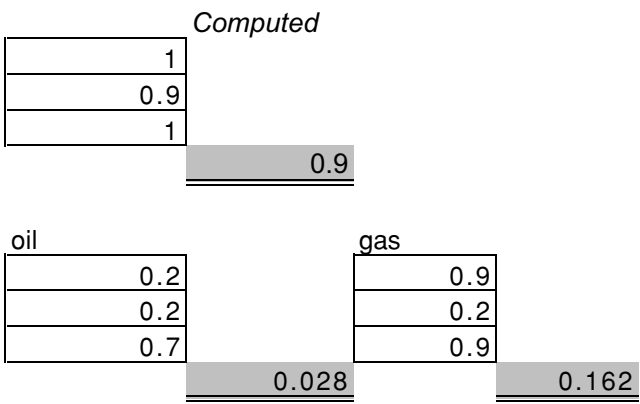

0.0252

0.1458

Allocation (percent):

\begin{tabular}{|l|r|r|r|}
\cline { 2 - 4 } \multicolumn{1}{c|}{} & \multicolumn{1}{c|}{ Land } & \multicolumn{1}{c|}{ Oil } & \multicolumn{1}{c|}{ Gas } \\
\hline Federal & 99 & 99 & 99 \\
\hline State & 0 & 0 & 0 \\
\hline Native & 1 & 1 & 1 \\
\hline
\end{tabular}

Assessor's Name:

Chris Potter

Date of Data Entry MM/DD/YYYY:

Date of Simulation Run MM/DD/YYYY:

$3 / 20 / 02$

$3 / 20 / 02$

0.1

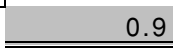


Table 21d: Distribution of fitted hydrocarbon volume attributes, trap depth and number of prospects for the Torok Structural Play
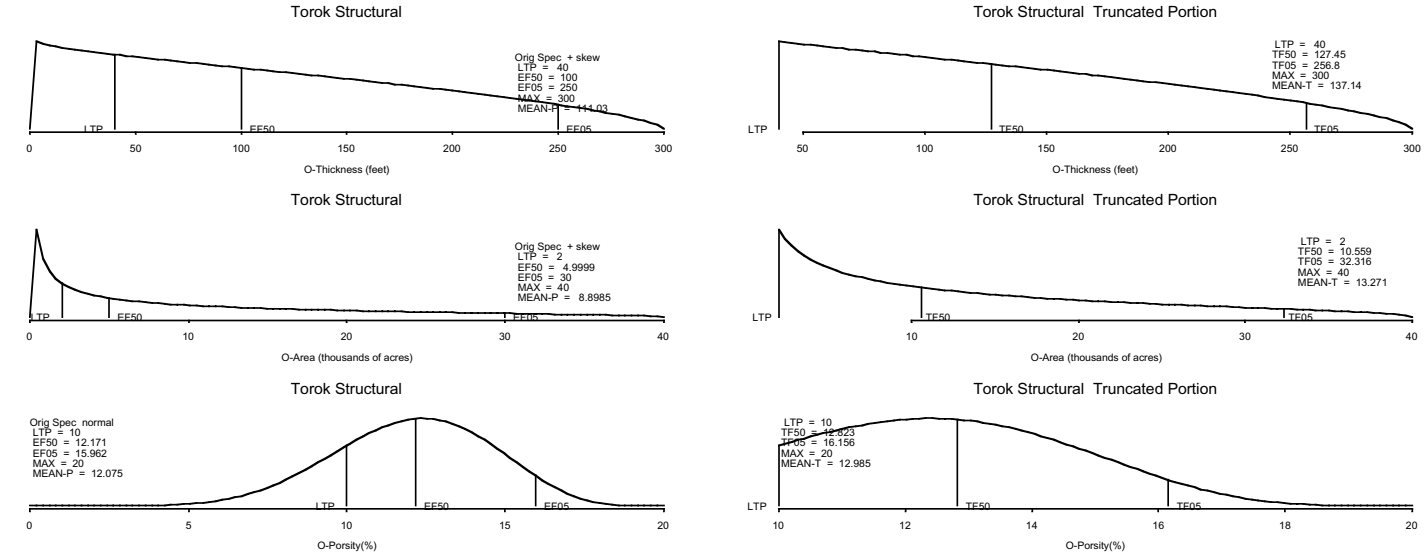

Torok Structural
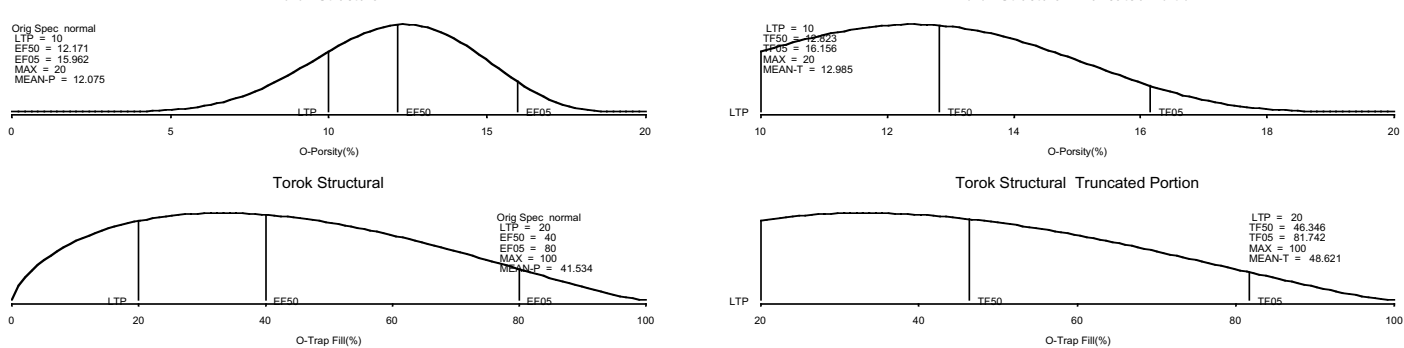

Torok Structural Truncated Portion
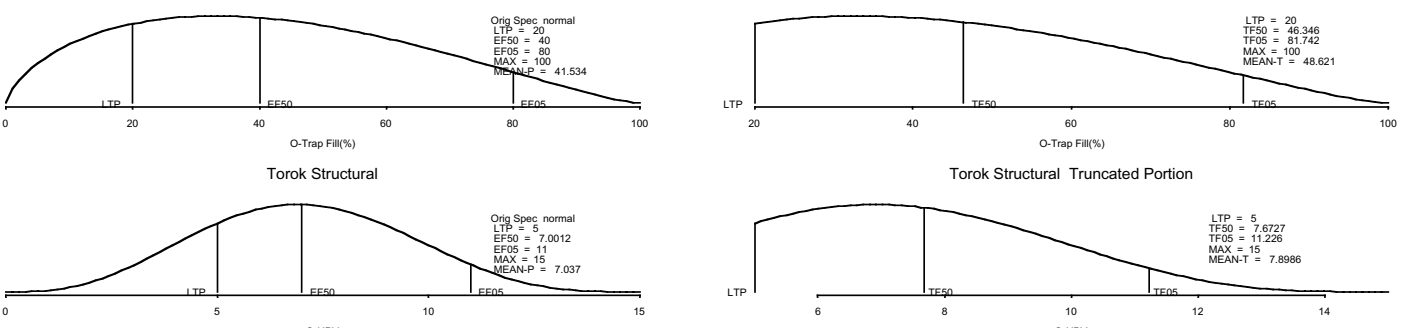

Torok Structural
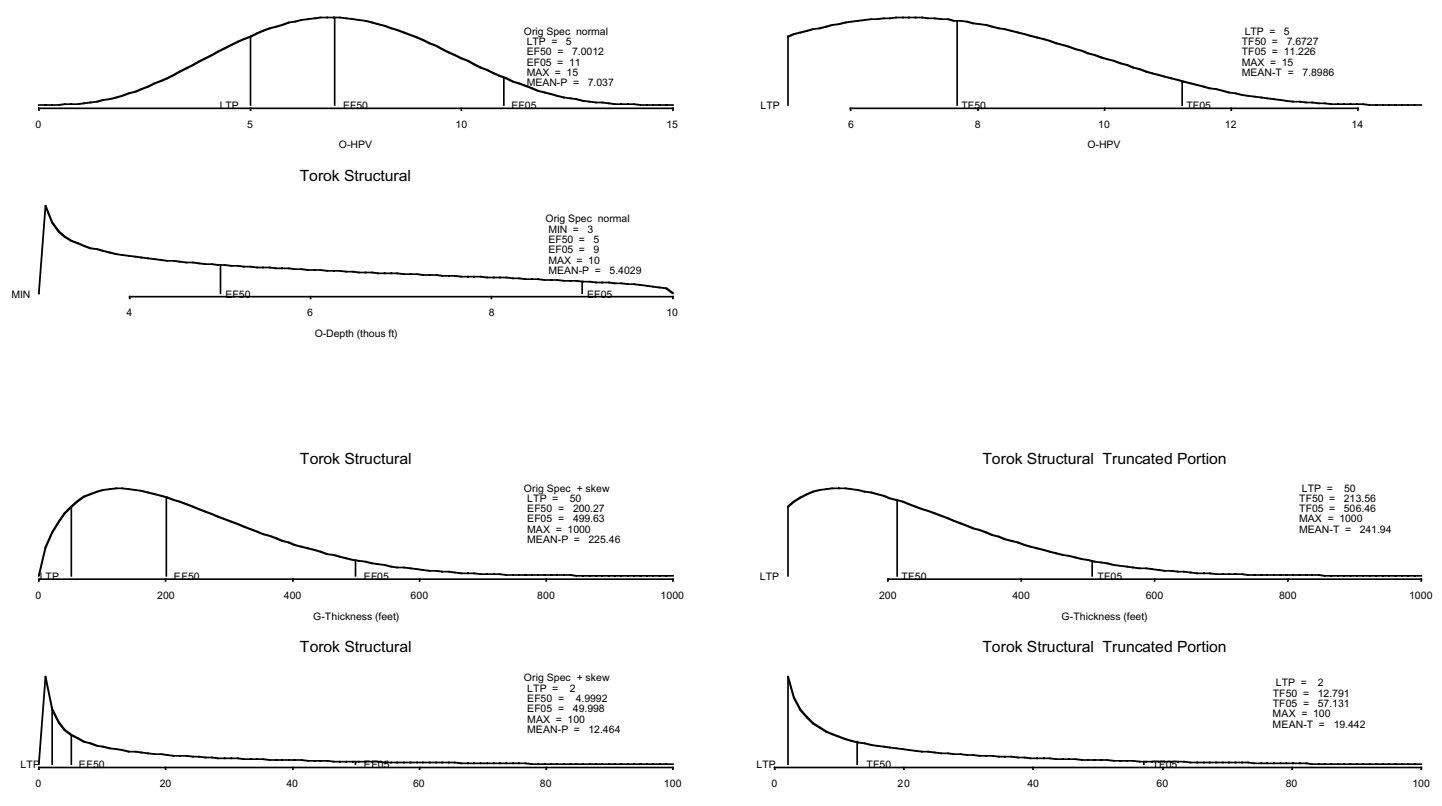

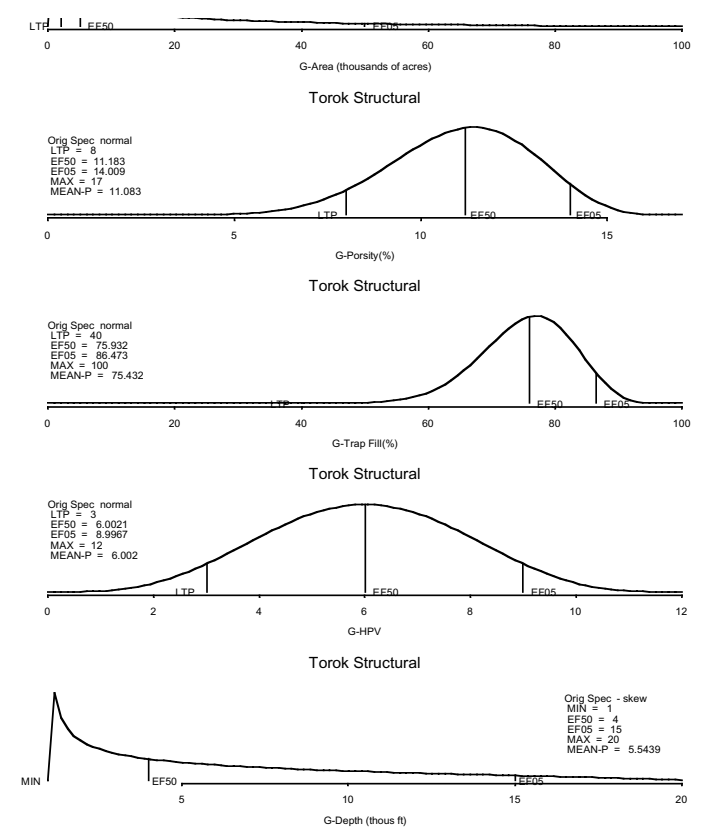
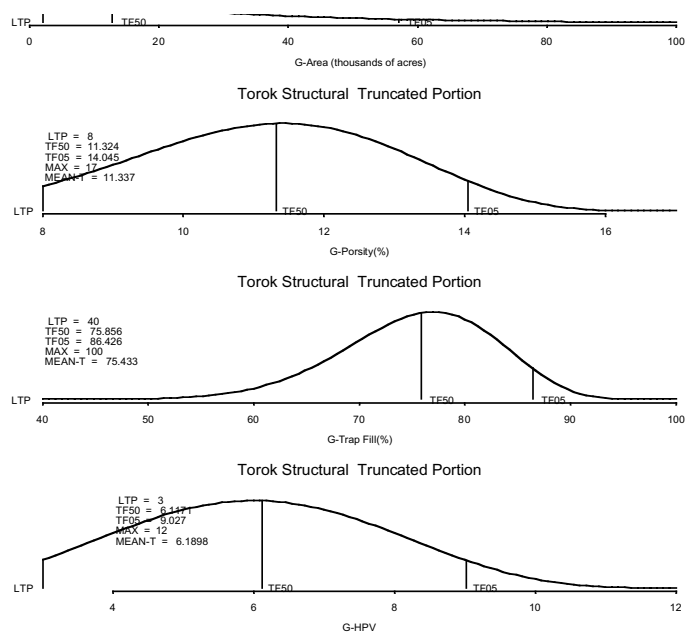

Torok Structural

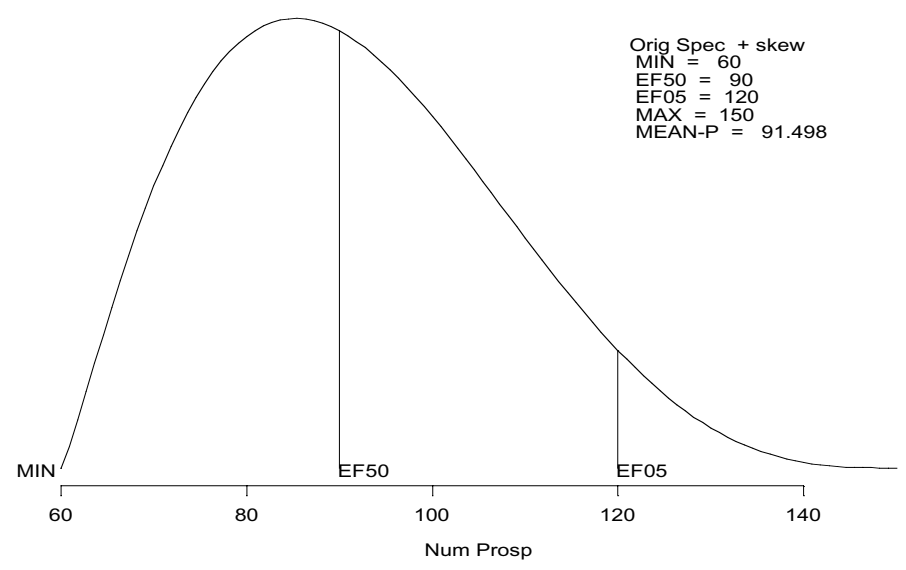


Table 21e: Estimated undiscovered oil and gas resources for the Torok Structural Play Deposit

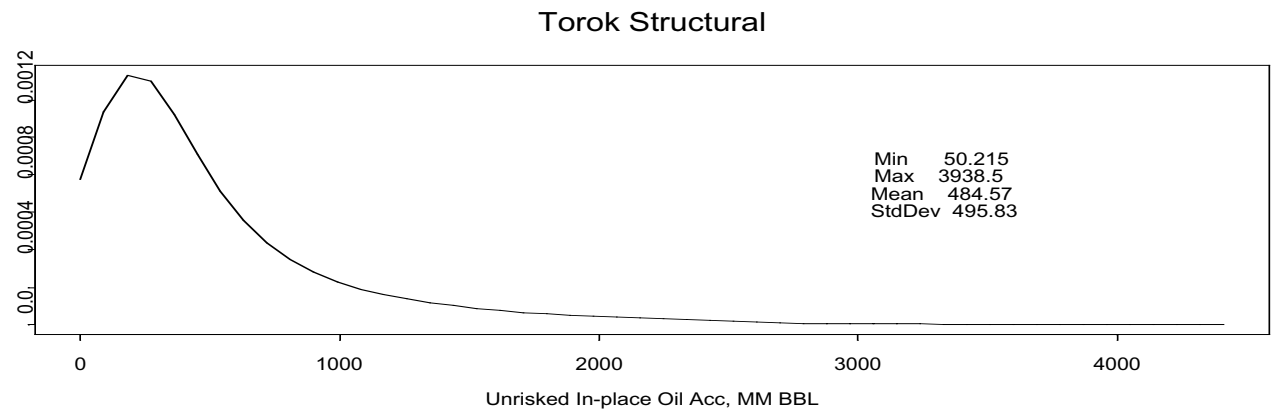

Torok Structural

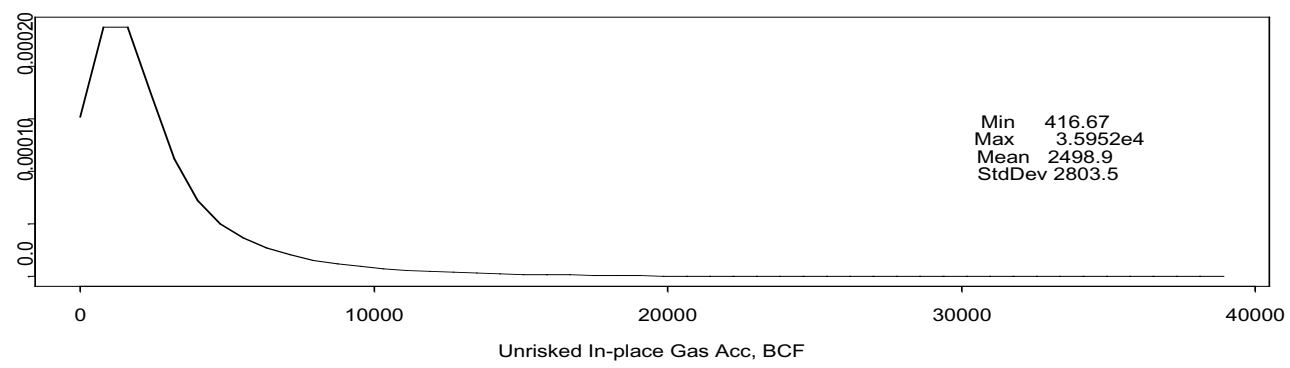

\begin{tabular}{|c|c|c|c|c|c|c|}
\hline \multicolumn{7}{|l|}{$\begin{array}{l}\text { Play } \\
\text { Play Stats }\end{array}$} \\
\hline Resource & Unit & Mean & StdDev & F95 & $\mathrm{F} 50$ & $\mathrm{~F} 05$ \\
\hline In-place oil & MMBO & 115.60 & 338.07 & 0.00 & 0.00 & 741.12 \\
\hline In-place NA gas & $\mathrm{BCFG}$ & 29842.46 & 16865.83 & 0.00 & 29614.90 & 58353.64 \\
\hline Recov oil & MMBO & 34.68 & 101.42 & 0.00 & 0.00 & 222.34 \\
\hline Recov assoc diss gas & $\mathrm{BCFG}$ & 18.70 & 55.45 & 0.00 & 0.00 & 121.83 \\
\hline Recov NA gas & BCFG & 17905.48 & 10119.50 & 0.00 & 17768.94 & 35012.18 \\
\hline Recov NGL (ADG) & MMBO & 0.48 & 1.45 & 0.00 & 0.00 & 3.14 \\
\hline Recov NGL (NAG) & MMBO & 263.70 & 174.80 & 0.00 & 244.18 & 577.07 \\
\hline Num oil deposits & & 0.24 & 0.49 & 0.00 & 0.00 & 1.00 \\
\hline Num NA gas deposits & & 11.94 & 5.53 & 0.00 & 12.00 & 20.00 \\
\hline
\end{tabular}

RNS $=70$

\section{Play Totals Torok Structural}
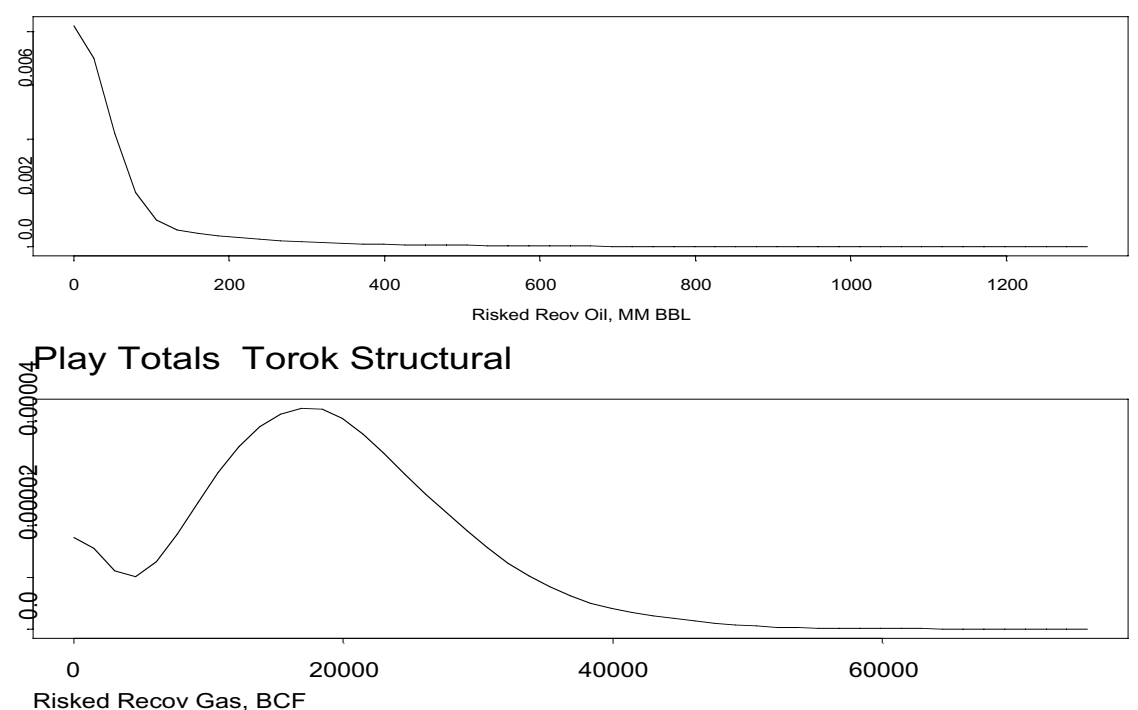
Table 21f: Estimated undiscovered oil and gas resources for the Torok Structural Play showing number of deposits and volumes by accumulation size class

\begin{tabular}{|c|c|c|c|c|c|}
\hline \multicolumn{3}{|c|}{ Oil (MMBO) } & \multicolumn{3}{|c|}{ NA Gas (BCFG) } \\
\hline Start size class & Oil deposits & Oil (MMBO) & Start size class & deposits & NA Gas (BCFG) \\
\hline 0 & 0.00 & 0.00 & 0 & 0.00 & 0.00 \\
\hline 8 & 0.00 & 0.04 & 48 & 0.00 & 0.00 \\
\hline 16 & 0.03 & 0.81 & 96 & 0.00 & 0.00 \\
\hline 32 & 0.05 & 2.34 & 192 & 1.93 & 606.78 \\
\hline 64 & 0.06 & 5.62 & 384 & 3.32 & 1841.27 \\
\hline 128 & 0.05 & 9.84 & 768 & 3.09 & 3399.06 \\
\hline 256 & 0.03 & 10.05 & 1536 & 2.17 & 4683.48 \\
\hline 512 & 0.01 & 5.69 & 3072 & 1.12 & 4672.24 \\
\hline 1024 & 0.00 & 0.30 & 6144 & 0.29 & 2343.48 \\
\hline 2048 & & & 12288 & 0.02 & 360.60 \\
\hline Totals & 0.24 & 34.68 & & 11.94 & 17906.91 \\
\hline
\end{tabular}
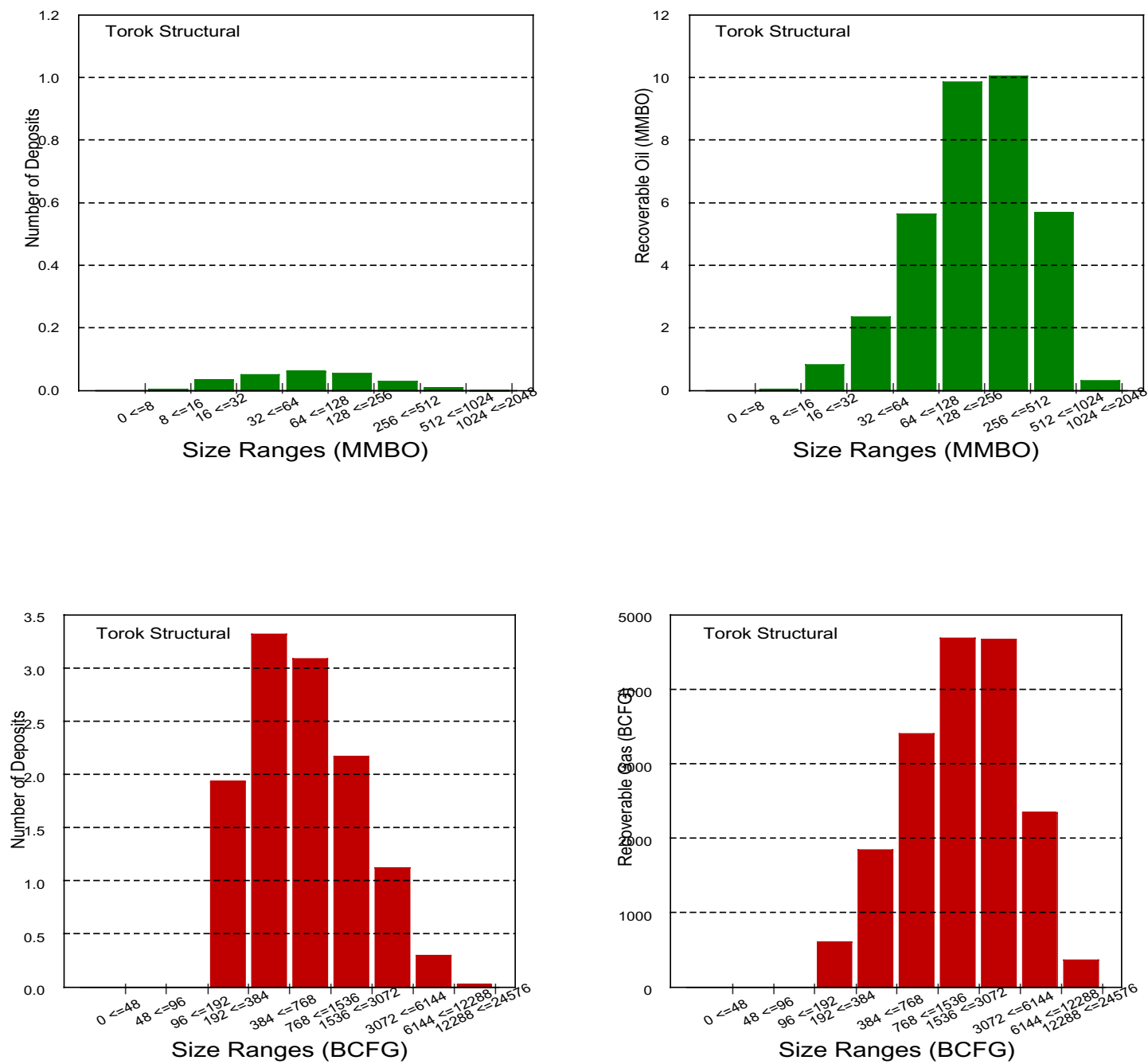
Table 22a: Input values for oil accumulations in the Ellesmarian Structural Play

NPRA Assessment Form-2001

PLAY: Ellesmerian Structural

Play area:

$10^{3}$ Acres

\section{OIL ACCUMULATION VOLUME PARAMETERS}

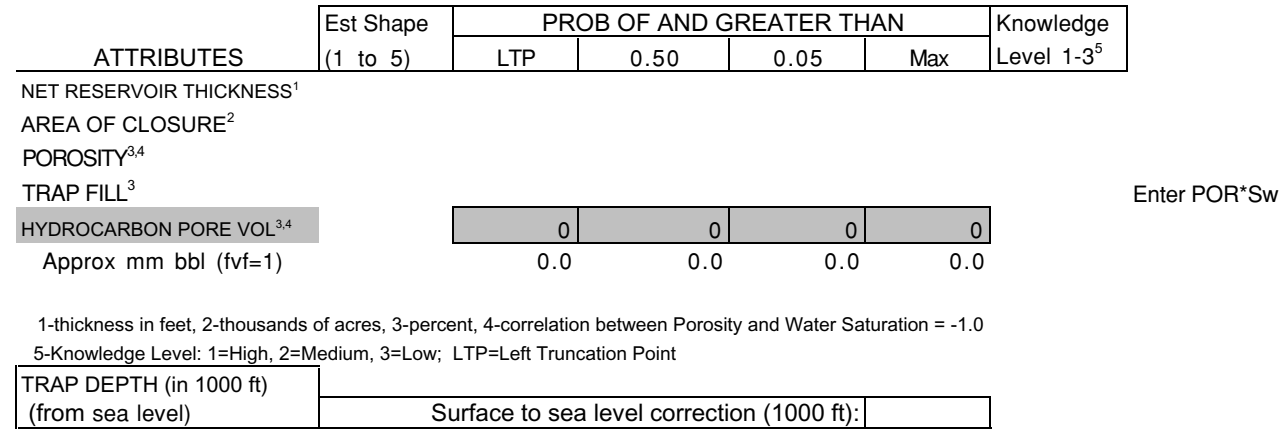

\section{OIL ACCUMULATION CHARACTERISTICS}

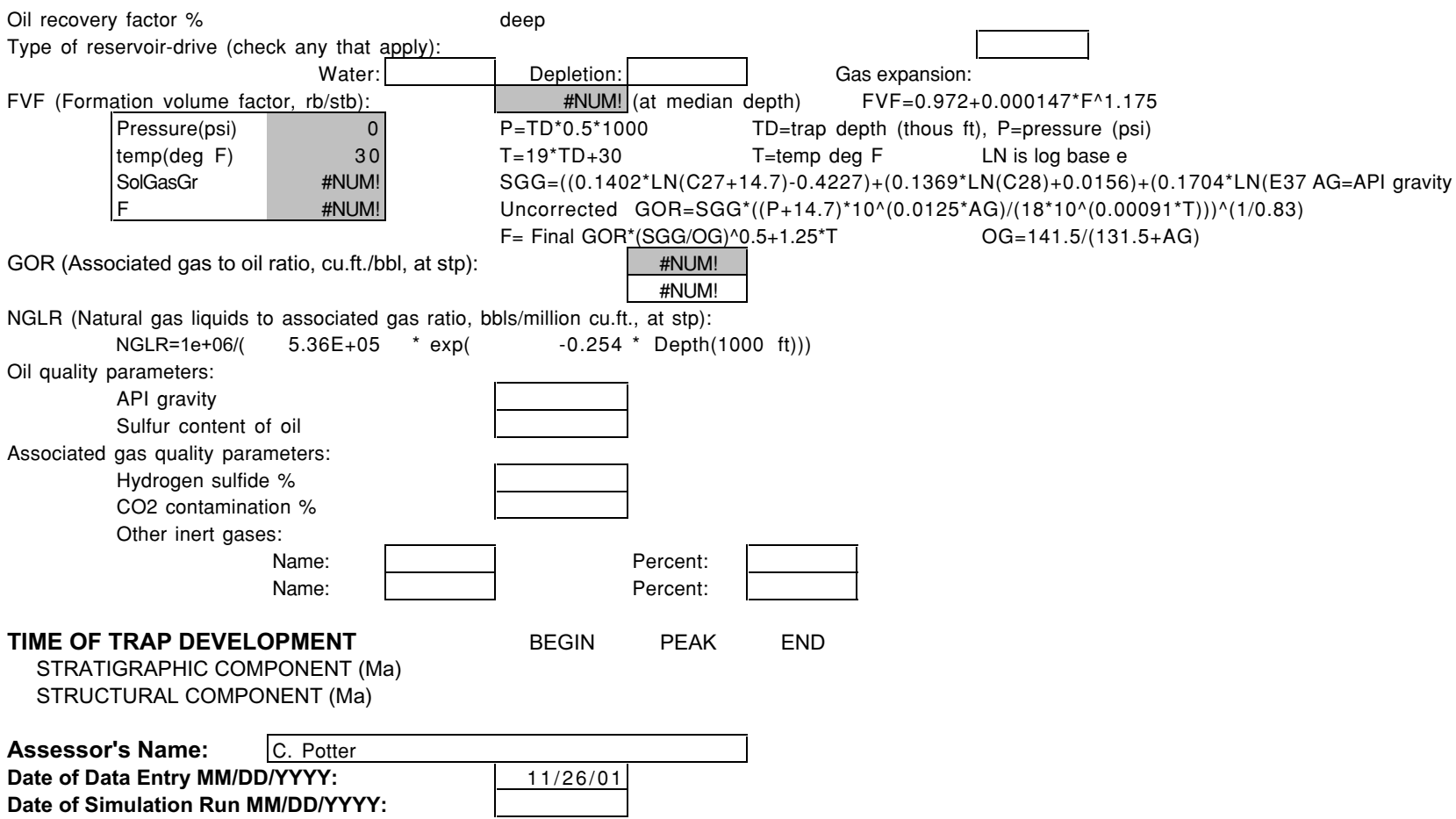

Note: only enter play name and assessor's name on Oil worksheet 
Table 22b: Input values for nonassociated gas accumulations in the Ellesmerian Structural Play NPRA Assessment Form-2001

PLAY: Ellesmerian Structural \begin{tabular}{ll|l|l|} 
Play area: $\quad 8125$ & $10^{3}$ Acres \\
\hline
\end{tabular}

\section{NONASSOCIATED GAS ACCUMULATION VOLUME PARAMETERS}

\begin{tabular}{|c|c|c|c|c|c|c|c|c|}
\hline \multirow[b]{2}{*}{ ATTRIBUTES } & \multirow{2}{*}{$\begin{array}{l}\text { Est Shape } \\
(1 \text { to } 5)\end{array}$} & \multicolumn{4}{|c|}{ PROB OF AND GREATER THAN } & \multirow{2}{*}{$\begin{array}{l}\text { Knowledge } \\
\text { Level } 1-3^{5}\end{array}$} & & \\
\hline & & LTP & 50 & 5 & Max & & & \\
\hline \multirow{4}{*}{$\begin{array}{l}\text { NET RESERVOIR THICKNESS } \\
\text { AREA OF CLOSURE }^{2} \\
\text { POROSITY }^{3,4} \\
\text { TRAP FILL }^{3}\end{array}$} & 2 & 40 & 100 & 200 & 600 & 3 & -0.8 & Corr(NRT,HPV) \\
\hline & 1 & 2.0 & 4.0 & 20.0 & 115.0 & 1 & 0.3 & Corr(NRT,AC) \\
\hline & 2 & 4 & 6 & 10 & 12 & 3 & \multirow{2}{*}{$\mathrm{POR}^{\star} \mathrm{Sw}$} & \\
\hline & 4 & 20 & 40 & 80 & 100 & 3 & & \\
\hline \multicolumn{2}{|l|}{ HYDROCARBON PORE VOL ${ }^{3,4}$} & 1 & 3 & 7 & 9 & & 3 & \\
\hline \multirow{2}{*}{\multicolumn{2}{|c|}{$\begin{array}{l}\text { Approx in place bcf } \\
\text { Recov bcf at surface }\end{array}$}} & 0.01 & 0.21 & 9.76 & 270.51 & & & \\
\hline & & \begin{tabular}{r|}
1.3 \\
\end{tabular} & 38.4 & 1789.9 & 49622.3 & & & \\
\hline \multicolumn{9}{|c|}{ 1-thickness in feet, 2-thousands of acres, 3-percent, 4-correlation between Porosity and Water Saturation $=-1.0$} \\
\hline \multicolumn{7}{|c|}{ 5-Knowledge Level: 1=High, 2=Medium, 3=Low; LTP=Left Truncation Point } & & \\
\hline \multirow{2}{*}{$\begin{array}{l}\text { TRAP DEPTH (in } 1000 \mathrm{ft} \text { ) } \\
\text { (from sea level) }\end{array}$} & 4 & \begin{tabular}{r|}
15 \\
\end{tabular} & 21 & 25 & 26 & 1 & & \\
\hline & & Surface to s & evel corre & $(1000 \mathrm{ft}):$ & 1.065 & & & \\
\hline
\end{tabular}

\section{NONASSOCIATED GAS ACCUMULATION CHARACTERISTICS}

NA Gas recovery factor \% 60

Type of reservoir-drive (check any that apply):

$$
\text { Water: Gas expansion: }
$$

Natural gas liquids plus condensate to non-associated gas (bbls/million cf) (in place): NGL-NAG $=1.785^{\star} \mathrm{TD}$ 37.5 (at median)

Non-associated gas quality parameters: Hydrogen sulfide \% CO2 contamination \% Other inert gases:

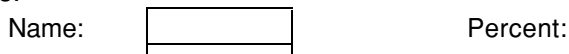

Name: $\quad$ Percent:

Gas fvf 305.7 Gas (at median depth)

$$
\begin{aligned}
& 113.3+21.1^{*} T D-0.812^{*} T^{\wedge} 2+0.0116^{*} T^{\wedge} 3 \quad 5.67<T D<=30 \\
& T D=\text { trap depth (thous } \mathrm{ft} \text { ) }
\end{aligned}
$$

TIME OF TRAP DEVELOPMENT STRATIGRAPHIC COMPONENT (Ma) STRUCTURAL COMPONENT (Ma)

\begin{tabular}{|r|r|r|}
\multicolumn{1}{|c}{ BEGIN } & \multicolumn{2}{c}{ PEAK } \\
\hline 360 & & 170 \\
\hline 70 & 60 & 45 \\
\hline
\end{tabular}

\begin{tabular}{l|r|} 
Assessor's Name: C. Potter & \\
Date of Data Entry MM/DD/YYYY: & $11 / 26 / 01$ \\
Date of Simulation Run MM/DD/YYYY: & $2 / 12 / 02$ \\
\hline
\end{tabular}


Table 22c:Input risking values for the Ellesmerian Structural Play

Play: Ellesmerian Structural

\section{RISKING}

MINIMUM ACCUMULATION SIZE, MAS (recov bcf)

PRERISKED FREQUENCY DISTRIBUTION (Oil plus Gas)

\begin{tabular}{l|r|r|r|r|r|r|}
\cline { 3 - 7 } & \multicolumn{4}{c|}{} & \multicolumn{3}{c|}{ PROB OF AND GREATER THAN } & Knowledge \\
NUM OF PROSPECTS & Est Shape & Min & 50 & 5 & Max & Level $^{1-3^{5}}$ \\
\hline MINIMUM SIZE & 2 & 25 & 35 & 45 & 60 & 2 \\
\hline
\end{tabular}

5-Knowledge Level: 1=High, 2=Medium, 3=Low; LTP=Left Truncation Point

\section{ATTRIBUTES}

PLAY

ATTRIBUTES

CHARGE (C)

TRAP (T)

TIMING (F)

Probability that play contains at least 1 reservoir $>=$ minimum size (CXTXF)

PROSPECT

ATTRIBUTES

CHARGE (c)

$\operatorname{TRAP}(\mathrm{t})$

TIMING (f)

Probability that a randomly chosen prospect is favorable (cxtxf)

Play Attributes x Prospect Attributes (CxTxFxcxtxf)

FRACTION OF ACCUMULATIONS BEING OIL

Fraction NA Gas=1-Fraction(Oil)

\section{PROBABILITY OF FAVORABLE}

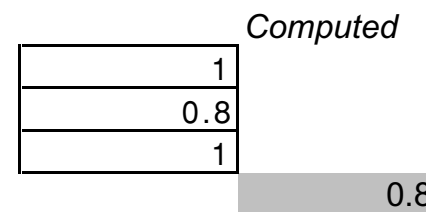

0.9

0.1

0.9

0.081

0.0648

0

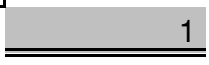

Allocation (percent):

\begin{tabular}{|l|r|r|r|}
\cline { 2 - 4 } \multicolumn{1}{c|}{} & \multicolumn{1}{c|}{ Land } & Oil & \multicolumn{1}{c|}{ Gas } \\
\hline Federal & 99 & & 99 \\
\hline State & 0 & & 0 \\
\hline Native & 1 & & 1 \\
\hline
\end{tabular}

Assessor's Name:

C. Potter

Date of Data Entry MM/DD/YYYY:

Date of Simulation Run MM/DD/YYYY:

\begin{tabular}{|r|}
\hline $11 / 26 / 01$ \\
\hline $2 / 12 / 02$ \\
\hline
\end{tabular} 
Table 22d: Distribution of fitted hydrocarbon volume attributes, trap depth, and number of prospects for the Ellesmerian Structural Play

Elles Structural

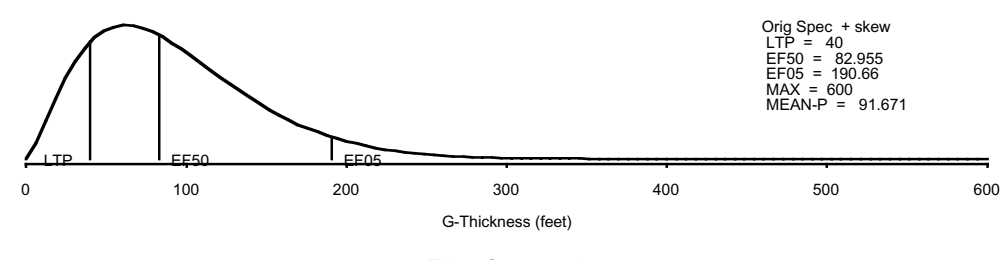

Elles Structural

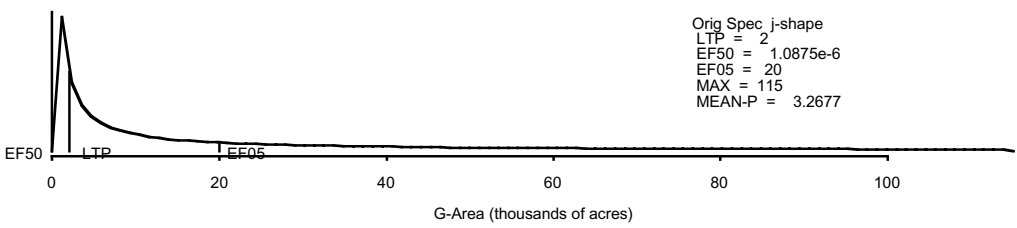

Elles Structural

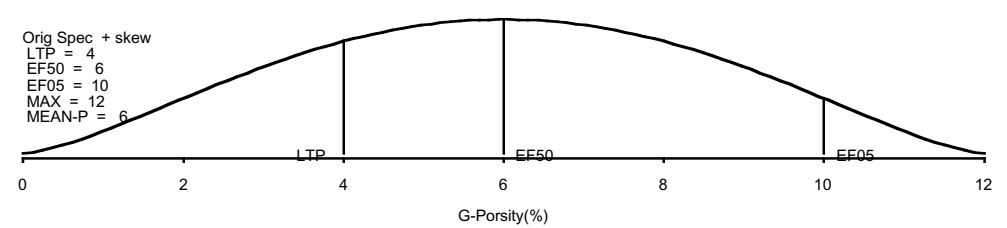

Elles Structural

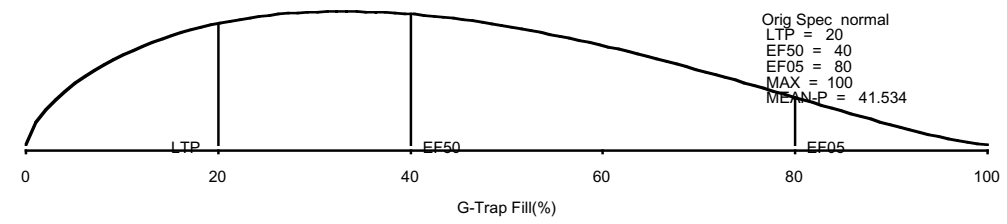

Elles Structural

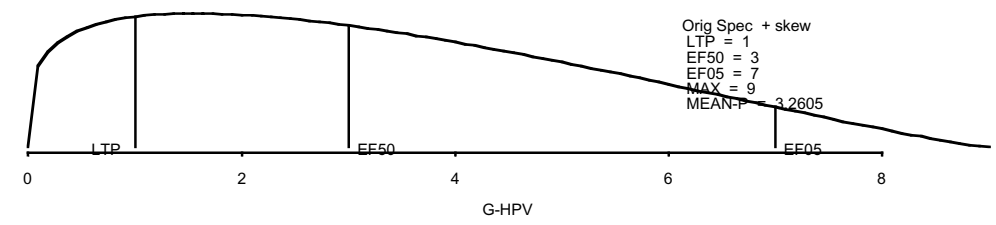

Elles Structural

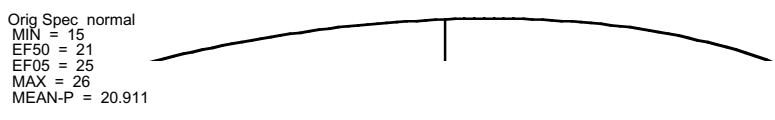

Elles Structural Truncated Portion

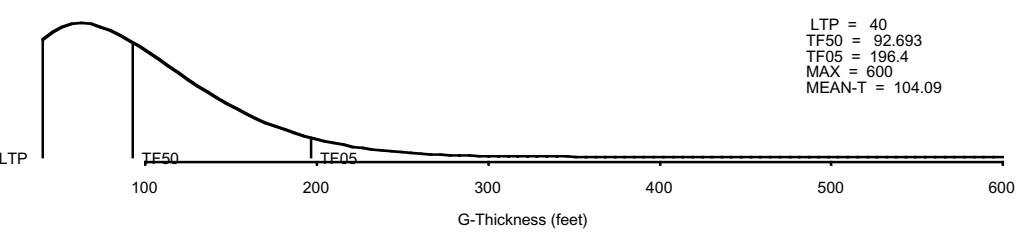

Elles Structural Truncated Portion

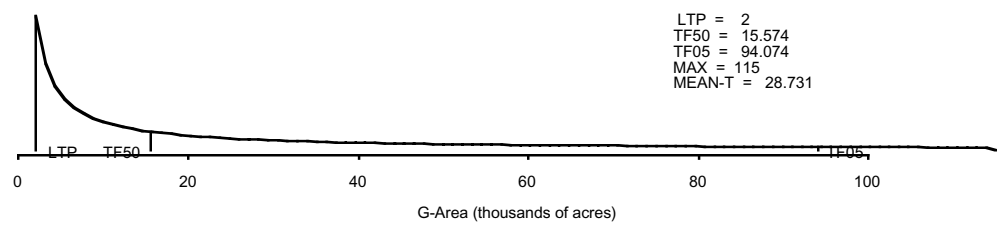

Elles Structural Truncated Portion

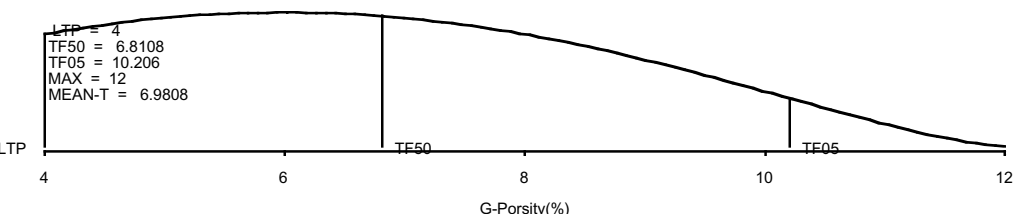

Elles Structural Truncated Portion

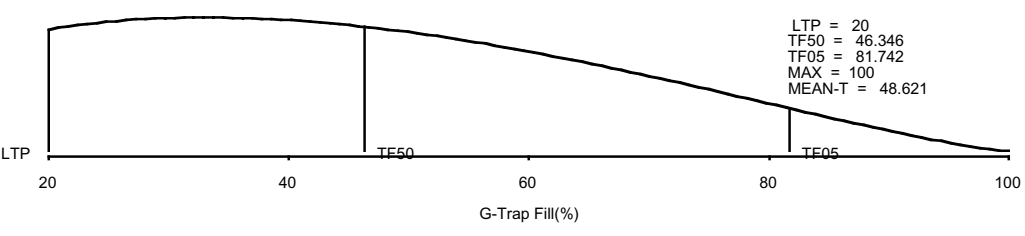

Elles Structural Truncated Portion

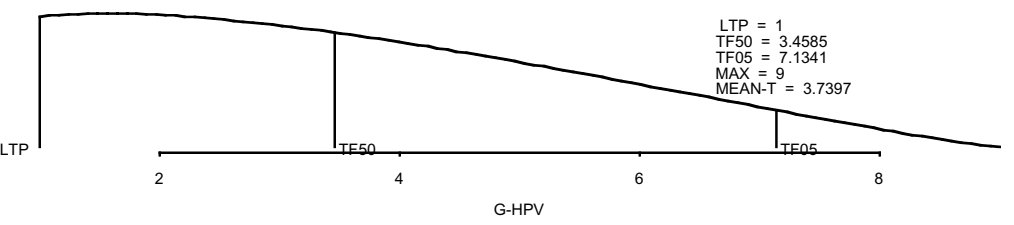


es Structural

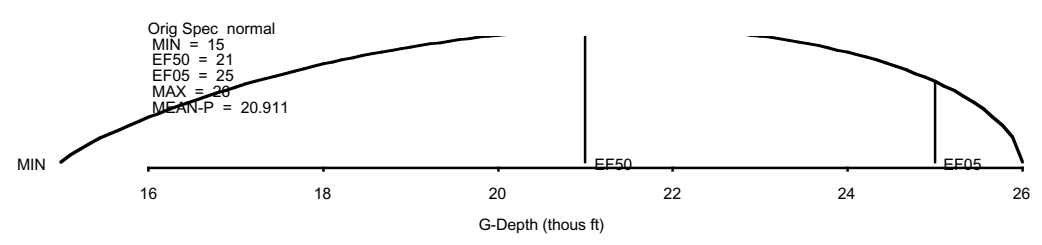

\section{Elles Structural}

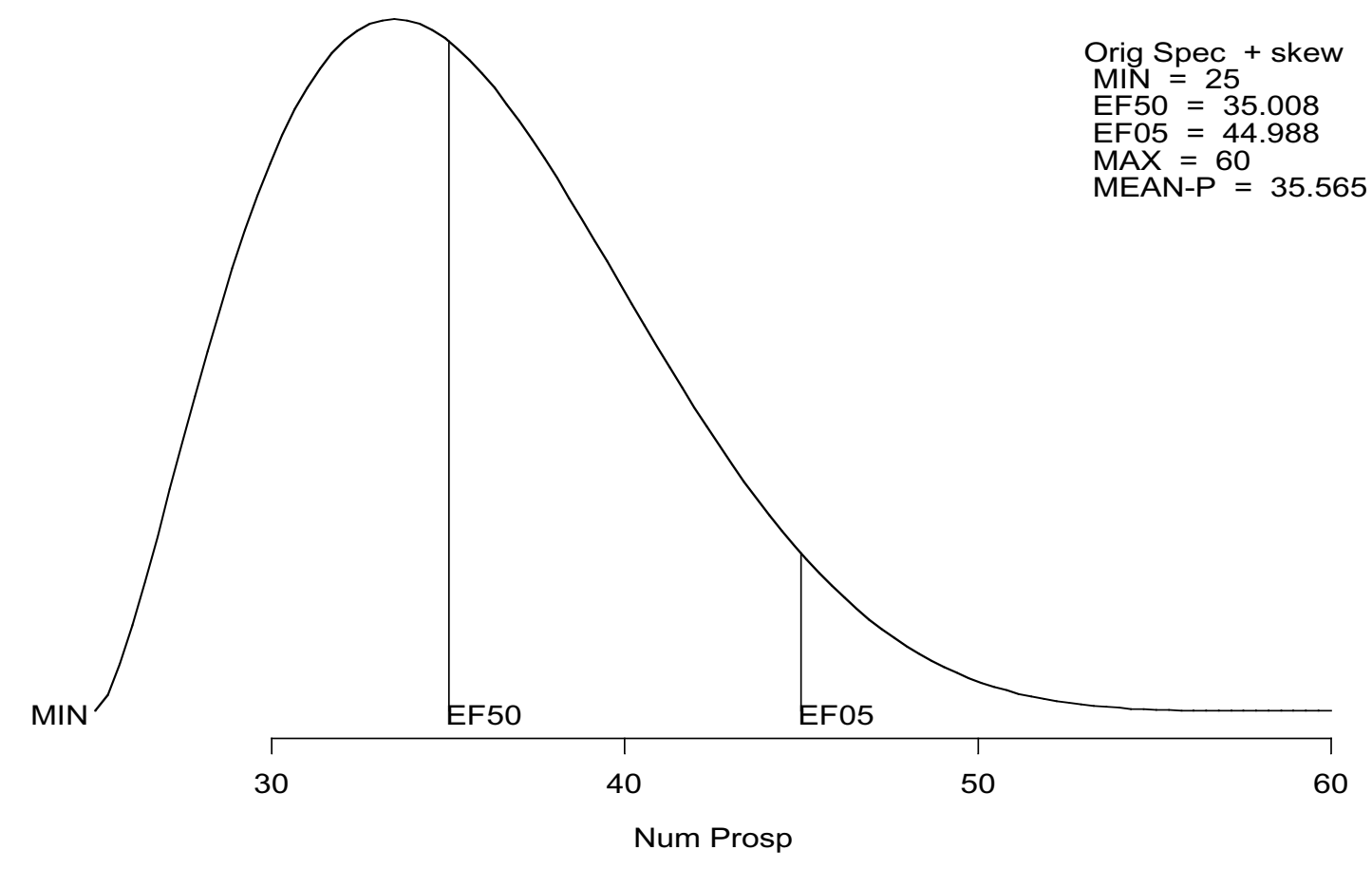


Table 22e: Estimated undiscovered oil and gas resources for the Ellesmerian Structural Play Deposits

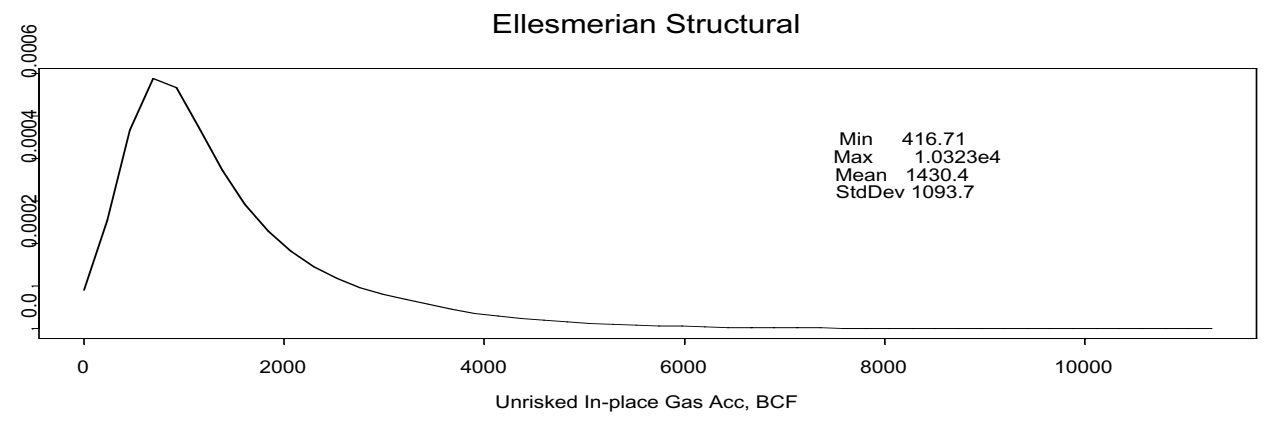

\begin{tabular}{|c|c|c|c|c|c|c|}
\hline $\begin{array}{l}\text { Play Stats } \\
\text { Resource }\end{array}$ & Unit & Mean & StdDev & F95 & F50 & F05 \\
\hline In-place oil & MMBO & & & & & \\
\hline In-place NA gas & BCFG & 3316.90 & 3200.49 & 0.00 & 2687.95 & 9457.68 \\
\hline Recov oil & MMBO & & & & & \\
\hline Recov assoc diss gas & BCFG & & & & & \\
\hline Recov NA gas & $\mathrm{BCFG}$ & 1990.14 & 1920.29 & 0.00 & 1612.77 & 5674.61 \\
\hline Recov NGL (ADG) & MMBO & & & & & \\
\hline Recov NGL (NAG) & MMBO & 78.18 & 75.93 & 0.00 & 63.22 & 225.50 \\
\hline Num oil deposits & & & & & & \\
\hline Num NA gas deposits & & 2.32 & 1.91 & 0.00 & 2.00 & 6.00 \\
\hline
\end{tabular}

Play Totals Ellesmerian Structural

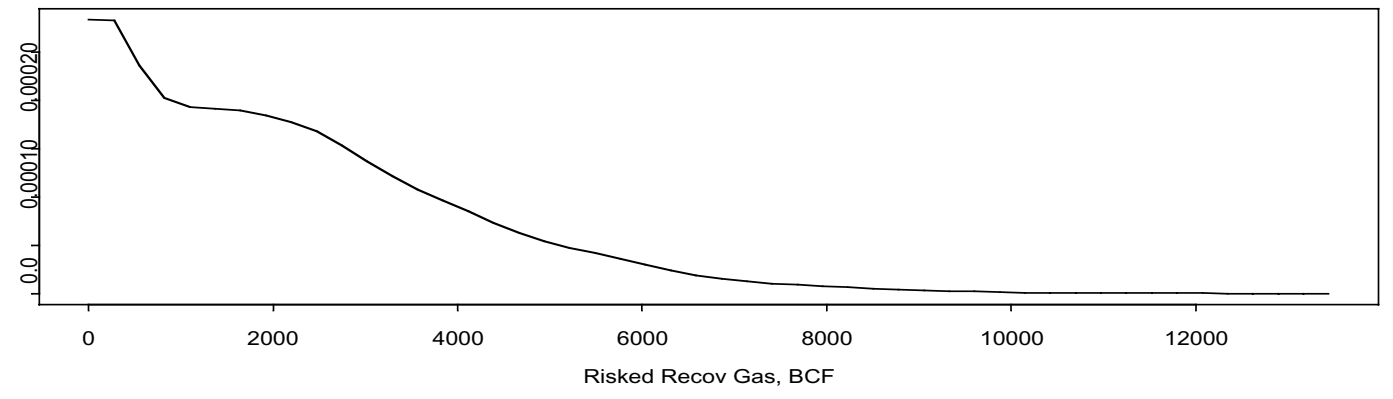


Table 22f: Estimated undiscovered oil and gas resources for the Ellesmerian Structural Play showing number of deposits and volumes by accumulation size class

Start size class

Oil deposits

Oil (MMBO_ Start size c Gas deposits

NA Gas (BCFG)

$\begin{array}{rrrr}0 & 0 & 0.00 & 0.00 \\ 8 & 48 & 0.00 & 0.00 \\ 16 & 96 & 0.00 & 0.00 \\ 32 & 192 & 0.54 & 167.02 \\ 64 & 384 & 0.83 & 460.90 \\ 128 & 768 & 0.65 & 693.92 \\ 256 & 1536 & 0.27 & 550.70 \\ 512 & 3072 & 0.03 & 117.27 \\ 1024 & 6144 & 0.00 & 0.50\end{array}$

\begin{tabular}{c|c|c|c|c|}
\hline Totals & 0.00 & 0.00 & 2.32 & 1990.30 \\
\hline
\end{tabular}
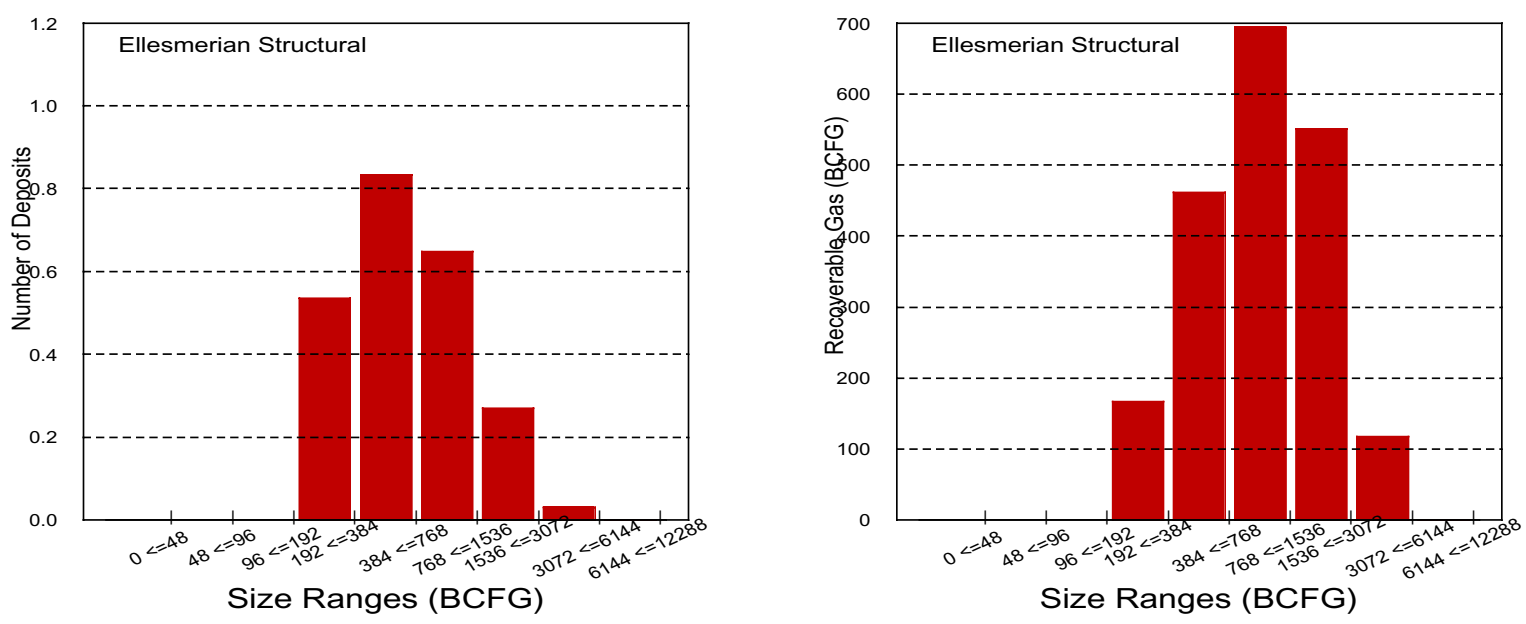
Table 23a: Input values for oil accumulations in the Ellesmerian Thrust Belt Play

NPRA Assessment Form-2001

PLAY: Ellesmerian Thrust Belt

Play area: $\quad 2579 \mid 10^{3}$ Acres

\section{OIL ACCUMULATION VOLUME PARAMETERS}

a few large vs many small

\begin{tabular}{|c|c|c|c|c|c|c|c|c|}
\hline \multirow[b]{3}{*}{ ATTRIBUTES } & \multirow{3}{*}{$\begin{array}{l}\text { Est Shape } \\
(1 \text { to } 6)\end{array}$} & & & & & \multirow{3}{*}{$\begin{array}{l}\text { Knowledge } \\
\text { Level } 1-3^{5}\end{array}$} & & \multirow[t]{3}{*}{ Dep NRT Por $=-0.5$} \\
\hline & & \multicolumn{4}{|c|}{ PROB OF AND GREATER THAN } & & & \\
\hline & & LTP & 50 & 5 & Max & & & \\
\hline \multirow{4}{*}{$\begin{array}{l}\text { NET RESERVOIR THICKNESS } \\
\text { AREA OF CLOSURE }^{1} \\
\text { POROSITY }^{3,4} \\
\text { TRAP FILL }^{3}\end{array}$} & 2 & 40 & 100 & 350 & 700 & 2 & -0.5 & Corr(NRT,HPV) \\
\hline & 2 & 1 & 4 & 10 & 12 & 2 & 0.5 & Corr(NRT,AC) \\
\hline & 1 & 4 & 5 & 8 & 13 & 2 & \multirow{2}{*}{\multicolumn{2}{|c|}{ Enter $\mathrm{POR}^{\star}{ }^{\mathrm{S} w}$}} \\
\hline & 4 & 30 & 40 & 80 & 100 & 3 & & \\
\hline HYDROCARBON PORE VOL ${ }^{3,4}$ & 4 & 3 & 4 & 7 & 12 & & \multirow[t]{3}{*}{1} & \\
\hline \multirow{2}{*}{$\begin{array}{l}\text { Approx } \mathrm{mm} \text { bbl }(\mathrm{fvf}=1) \\
\text { Recov } \mathrm{mm} \text { bbl at surface }\end{array}$} & & 2.8 & 43.4 & 1520.6 & 7820.1 & & & \\
\hline & & 0.7 & 10.6 & 371.0 & 1907.8 & & & \\
\hline \multicolumn{7}{|c|}{$\begin{array}{l}\text { 1-thickness in feet, 2-thousands of acres, 3-percent, 4-correlation between Porosity and Water Saturation }=-1.0 \\
\text { 5-Knowledge Level: 1=High, 2=Medium, 3=Low; LTP=Left Truncation Point }\end{array}$} & & \\
\hline \multirow{2}{*}{$\begin{array}{l}\text { TRAP DEPTH (in } 1000 \mathrm{ft} \text { ) } \\
\text { (from sea level) }\end{array}$} & 2 & 1 & 5 & 9 & 10 & 1 & & \\
\hline & Surf & ace to sea le & correctio & $1000 \mathrm{ft}$ ): & 2.228 & & & \\
\hline
\end{tabular}

\section{OIL ACCUMULATION CHARACTERISTICS}

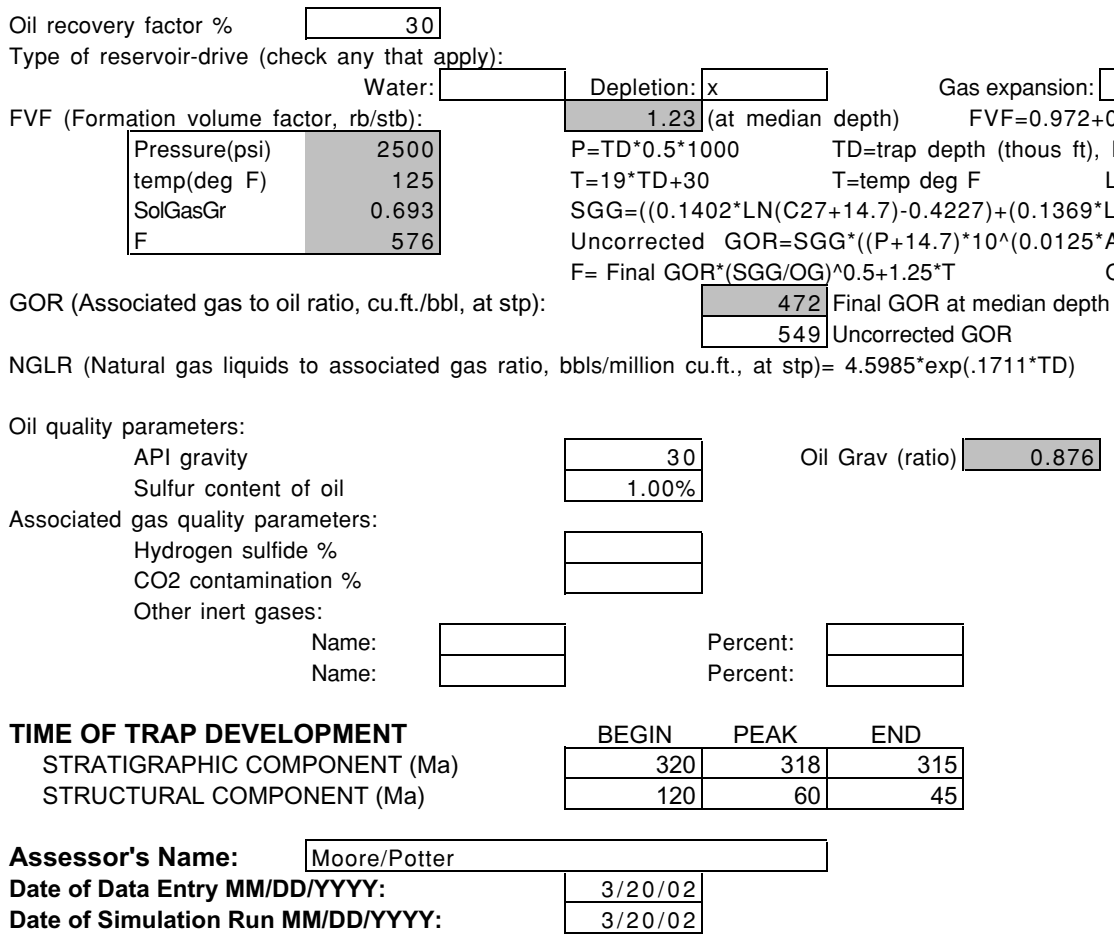


Table 23b: Input values for nonassociated gas accumulations in the Ellesmerian Thrust Belt NPRA Assessment Form-2001

PLAY: Ellesmerian Thrust Belt

\section{NONASSOCIATED GAS ACCUMULATION VOLUME PARAMETERS}

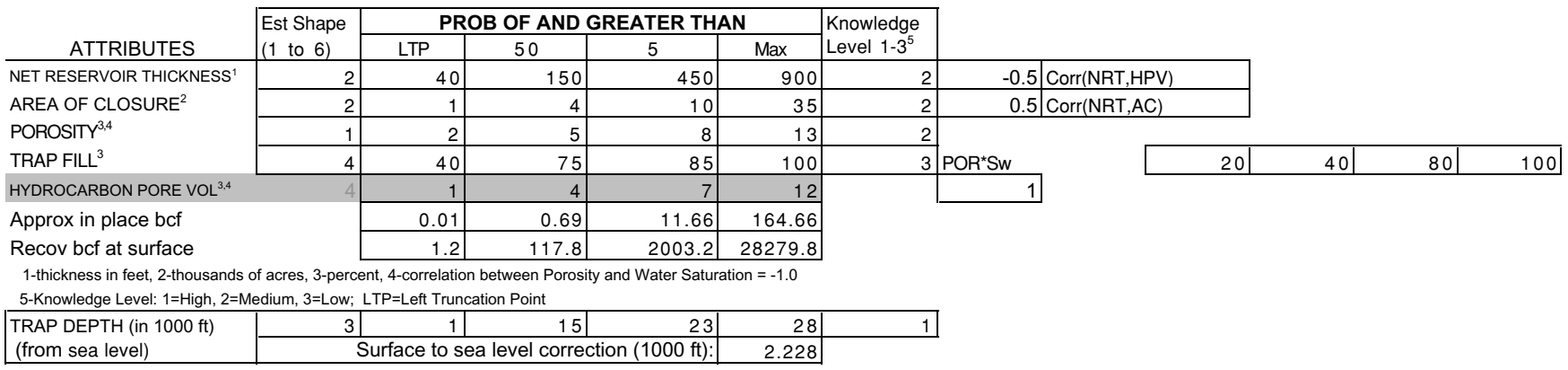

\section{NONASSOCIATED GAS ACCUMULATION CHARACTERISTICS}

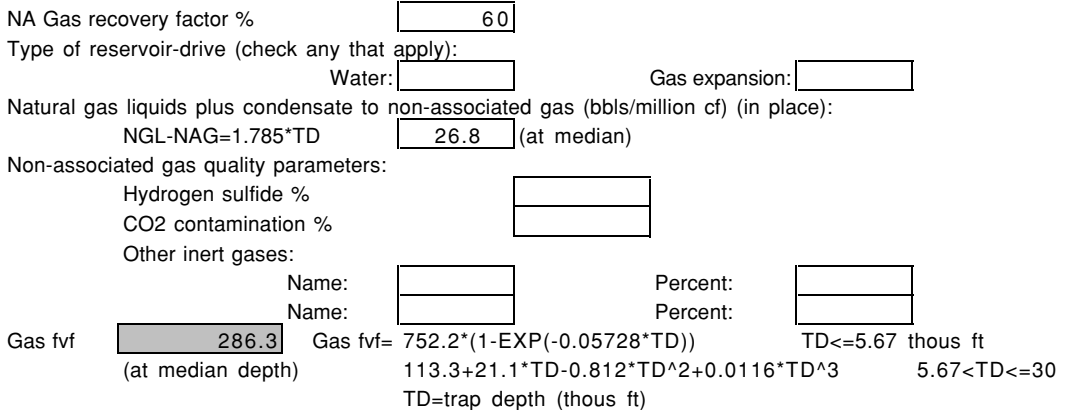

For Nonassociated Gas Accumulation:

TIME OF TRAP DEVELOPMEN 7 BEGIN STRATIGRAPHIC COMPONENT (Ma) STRUCTURAL COMPONENT (Ma)

Assessor's Name: Moore/Potter Date of Data Entry MM/DD/YYYY:

Date of Simulation Run MM/DD/YYYY: PEAK END

\begin{tabular}{|l|l|l|} 
& & \\
\hline & & \\
\hline
\end{tabular}

$3 / 20 / 02$

$3 / 20 / 02 \quad 12 / 6 / 01$


Table 23c: Input risking values for the Ellesmerian Thrust Belt Play NPRA Assessment Form-2001

Play: Ellesmerian Thrust Belt

\section{RISKING}

MINIMUM ACCUMULATION SIZE, MAS (bcf recov)

PRERISKED FREQUENCY DISTRIBUTION (Oil plus Gas)

\begin{tabular}{l|r|r|r|r|r|r|}
\cline { 3 - 7 } & \multicolumn{4}{c|}{ PROB OF AND GREATER THAN } & \multicolumn{1}{c|}{$\begin{array}{l}\text { Knowledge } \\
\text { NUM OF PROSPECTS }\end{array}$} \\
\cline { 2 - 7 } & Est Shape & Min & F50 & F05 & Max & Level $1-3^{5}$ \\
\hline 2 & 20 & 35 & 50 & 80 \\
\hline
\end{tabular}

5-Knowledge Level: 1=High, 2=Medium, 3=Low

\section{ATTRIBUTES}

$\begin{array}{ll}\text { PLAY } & \text { CHARGE }(\mathrm{C}) \\ \text { ATTRIBUTES } & \text { TRAP/ROCK (T) } \\ & \text { TIMING (F) }\end{array}$

Probability that play contains at least 1 reservoir >= minimum size (CxTxF)

PROSPECT

CHARGE (c)

TRAP/ROCK $(t)$

TIMING (f)

Probability that a randomly chosen prospect is favorable (cxtxf)

Play Attributes x Prospect Attributes (CxTxFxcxtxf)

FRACTION OF ACCUMULATIONS BEING OIL

Fraction NA Gas=1-Fraction(Oil)

\begin{tabular}{|l|r|r|r|}
\cline { 2 - 4 } \multicolumn{1}{c|}{} & \multicolumn{1}{c|}{ Land } & \multicolumn{1}{c|}{ Oil } & \multicolumn{1}{c|}{ Gas } \\
\hline Federal & 99 & 99 & 99 \\
\hline State & 0 & 0 & 0 \\
\hline Native & 1 & 1 & 1 \\
\hline
\end{tabular}

Assessor's Name:

Date of Data Entry MM/DD/YYYY:

Moore/Potter

Date of Simulation Run MM/DD/YYYY:

Allocation (percent):

\begin{tabular}{|l|l|}
\hline $3 / 20 / 02$ \\
\hline $3 / 20 / 02$ \\
\hline
\end{tabular}

\section{PROBABILITY}

OF FAVORABLE
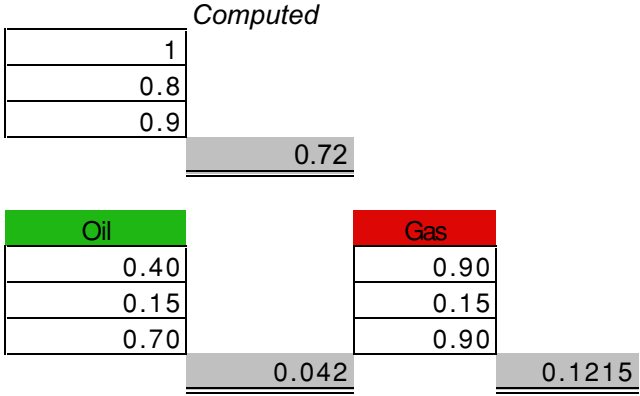

0.030

0.1

1 
Table 23d: Distribution of fitted hydrocarbon volume attributes, trap depth, and number of prospects for the Ellesmerian Thrust Belt Play

Elles Thurst Bel

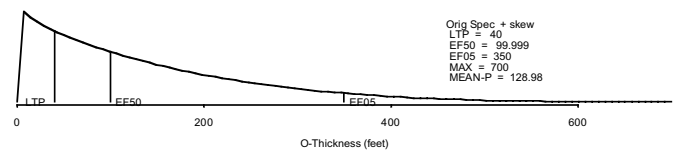

les Thurst Bel

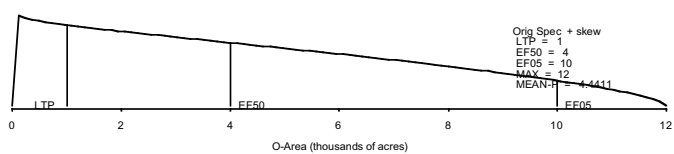

Elles Thurst Bel

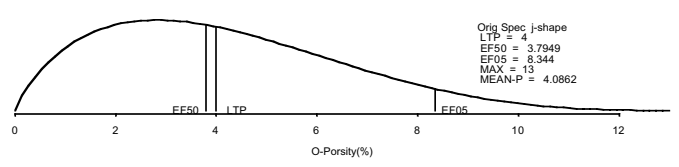

Elles Thurst Bell

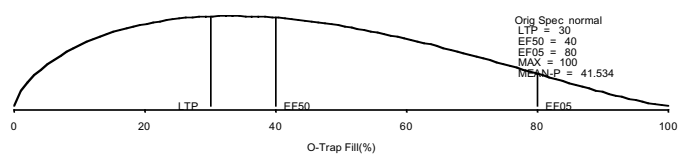

Elles Thurst Belt

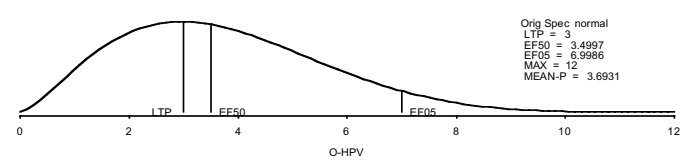

Elles Thurst Belt

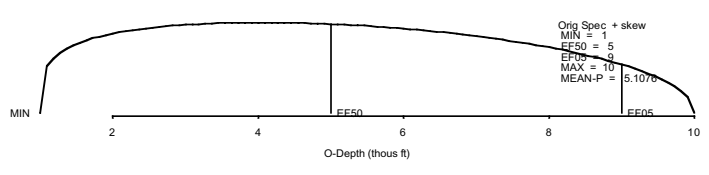

Elles Thurst Belt

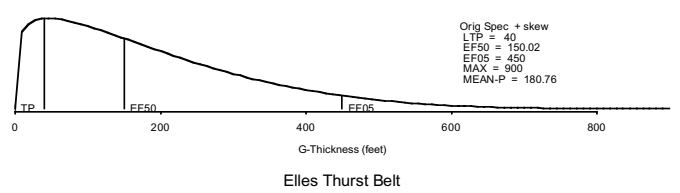

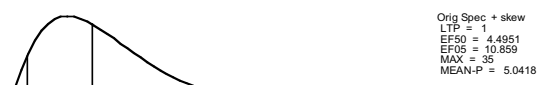

Elles Thurst Belt Truncated Portion
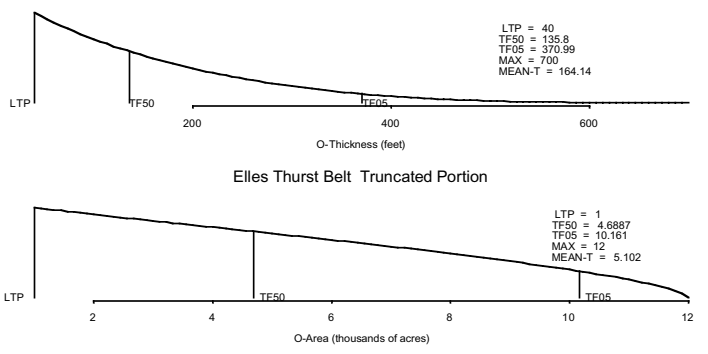

Elles Thurst Belt Truncated Portion

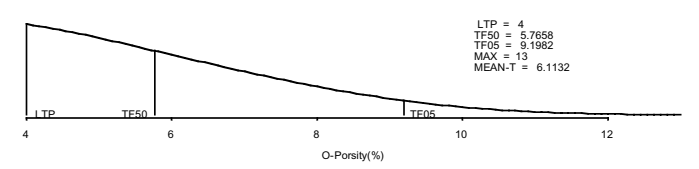

Elles Thurst Belt Truncated Portion

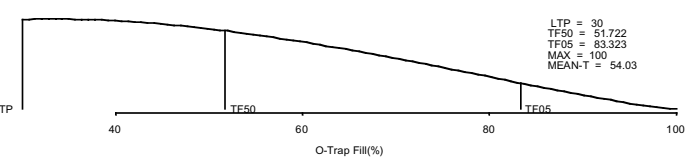

Elles Thurst Belt Truncated Portion

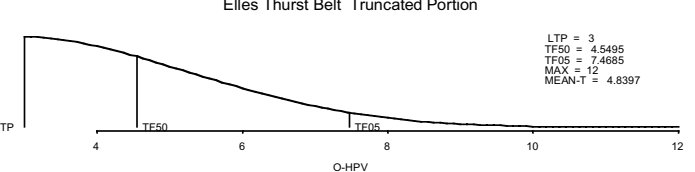

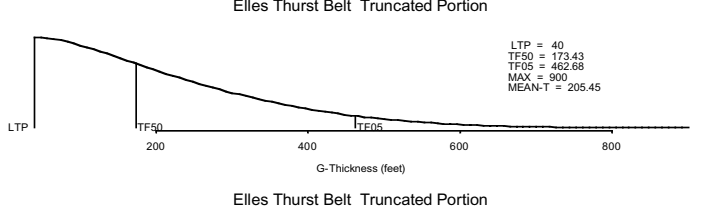

T1 

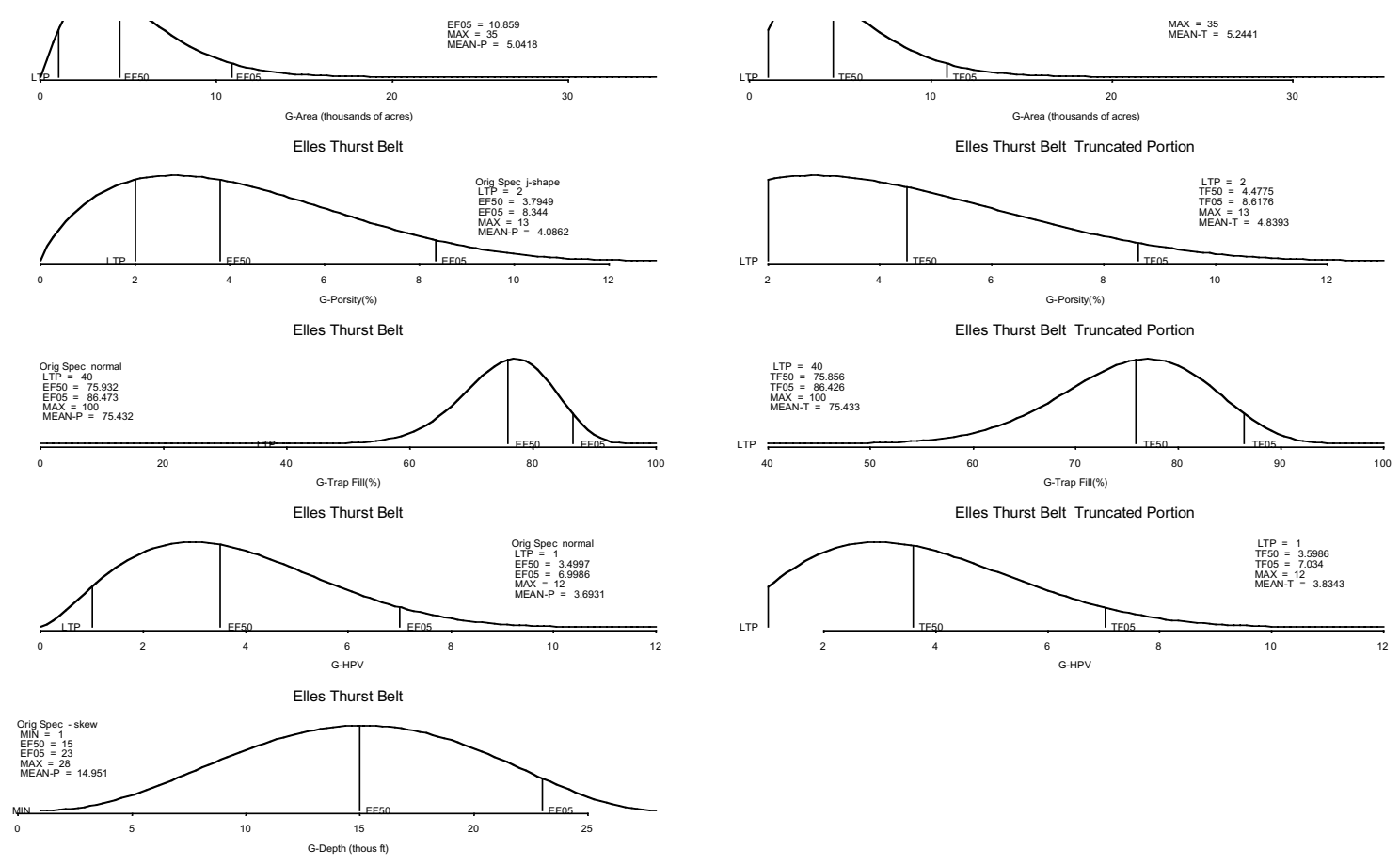

Elles Thurst Belt

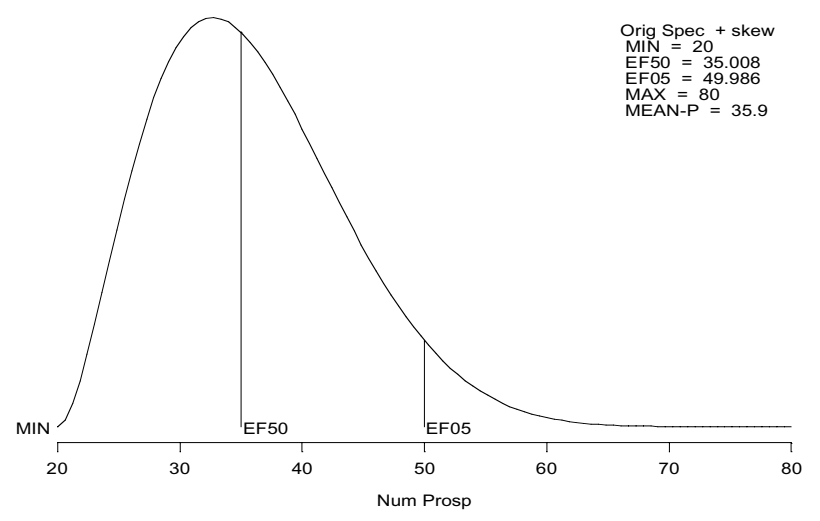


Table 23e: Estimated undiscovered oil and gas resources for the Ellesmerian Thrust Belt Play Deposit

Ellesmerian Thrust Belt

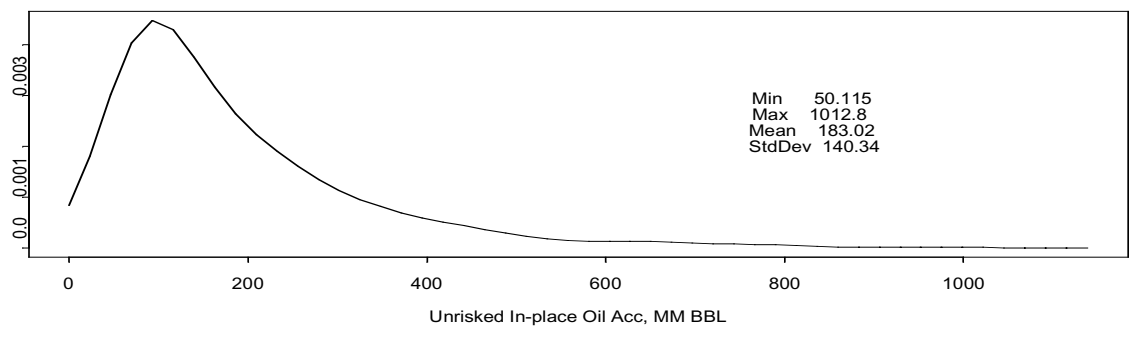

Ellesmerian Thrust Belt

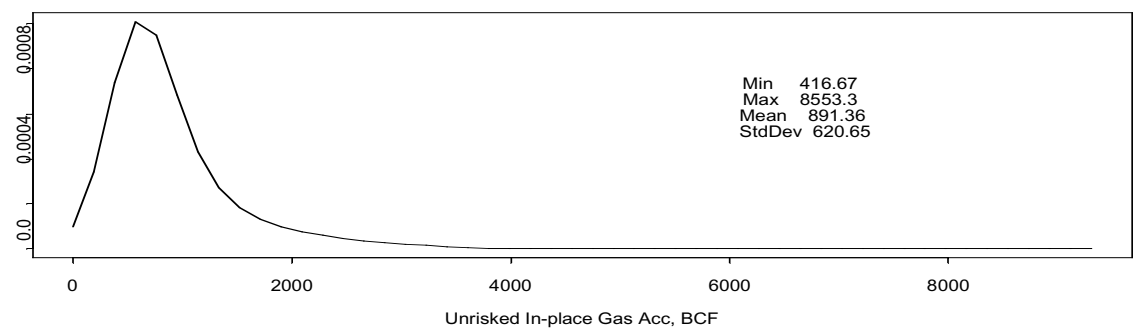

$\begin{aligned} & \text { Play } \\
& \text { Play Stats }\end{aligned}$
\begin{tabular}{|ll|rrrrr|} 
Resource & Unit & Mean & StdDev & F95 & F50 & F05 \\
\hline In-place oil & MMBO & 19.13 & 75.31 & 0.00 & 0.00 & 142.16 \\
In-place NA gas & BCFG & 2534.50 & 2455.67 & 0.00 & 2211.33 & 7065.88 \\
Recov oil & MMBO & 5.74 & 22.59 & 0.00 & 0.00 & 42.65 \\
Recov assoc diss gas & BCFG & 3.98 & 15.85 & 0.00 & 0.00 & 29.40 \\
Recov NA gas & BCFG & 1520.70 & 1473.40 & 0.00 & 1326.80 & 4239.53 \\
Recov NGL (ADG) & MMBO & 0.10 & 0.42 & 0.00 & 0.00 & 0.73 \\
Recov NGL (NAG) & MMBO & 49.28 & 48.73 & 0.00 & 41.93 & 140.14 \\
Num oil deposits & & 0.10 & 0.33 & 0.00 & 0.00 & 1.00 \\
Num NA gas deposits & & 2.84 & 2.49 & 0.00 & 3.00 & 7.00 \\
\hline
\end{tabular}

RNS $=528$

Play Totals Ellesmerian Thrust Belt

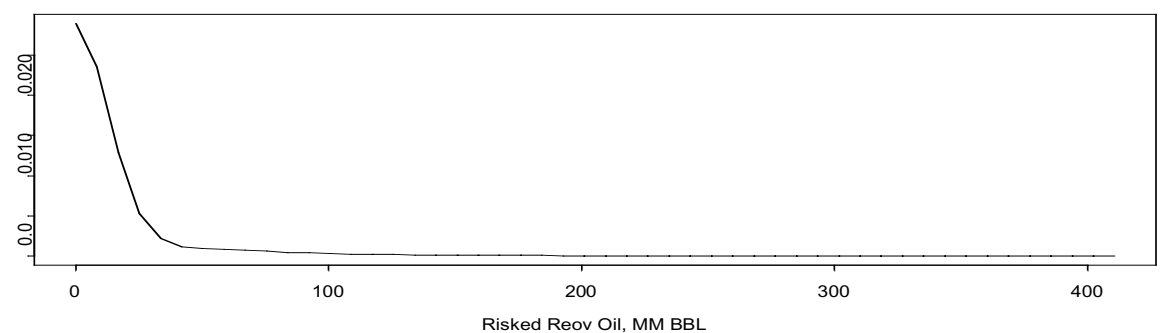

Play Totals Ellesmerian Thrust Belt

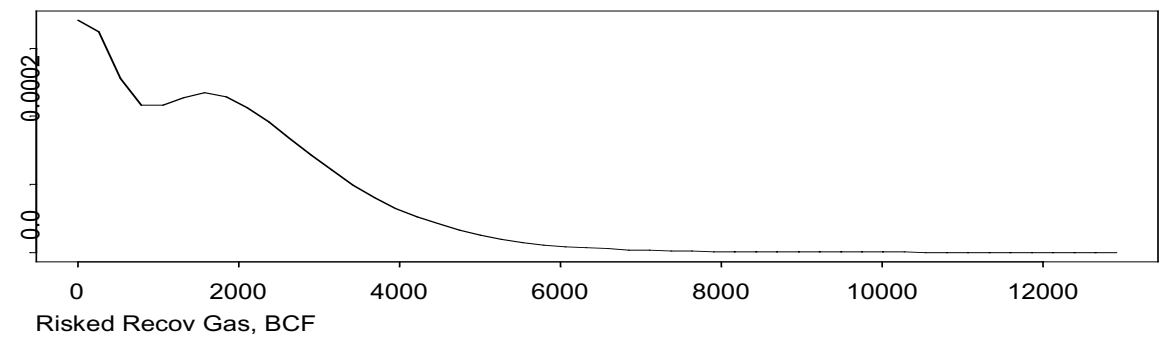


Table 23f: Estimated undiscovered oil and gas resources for the Ellesmerian Thrust Belt showing number of deposits and volumes by accumulation size class

\begin{tabular}{rrrrrr} 
Start size class & & Oil deposits & Oil (MMBO_Start size class & Gas deposits & NA Gas (BCFG) \\
0 & 0.00 & 0.00 & 0 & 0.00 & 0.00 \\
8 & 0.00 & 0.05 & 48 & 0.00 & 0.00 \\
16 & 0.04 & 0.84 & 96 & 0.00 & 0.00 \\
32 & 0.03 & 1.59 & 192 & 1.23 & 379.27 \\
64 & 0.02 & 2.06 & 384 & 1.16 & 599.03 \\
128 & 0.01 & 1.14 & 768 & 0.39 & 401.79 \\
256 & 0.00 & 0.06 & 1536 & 0.06 & 107.61 \\
512 & & & 3072 & 0.01 & 33.13 \\
\hline Totals & 0.10 & 5.74 & & 2.84 & 1520.82 \\
\hline
\end{tabular}
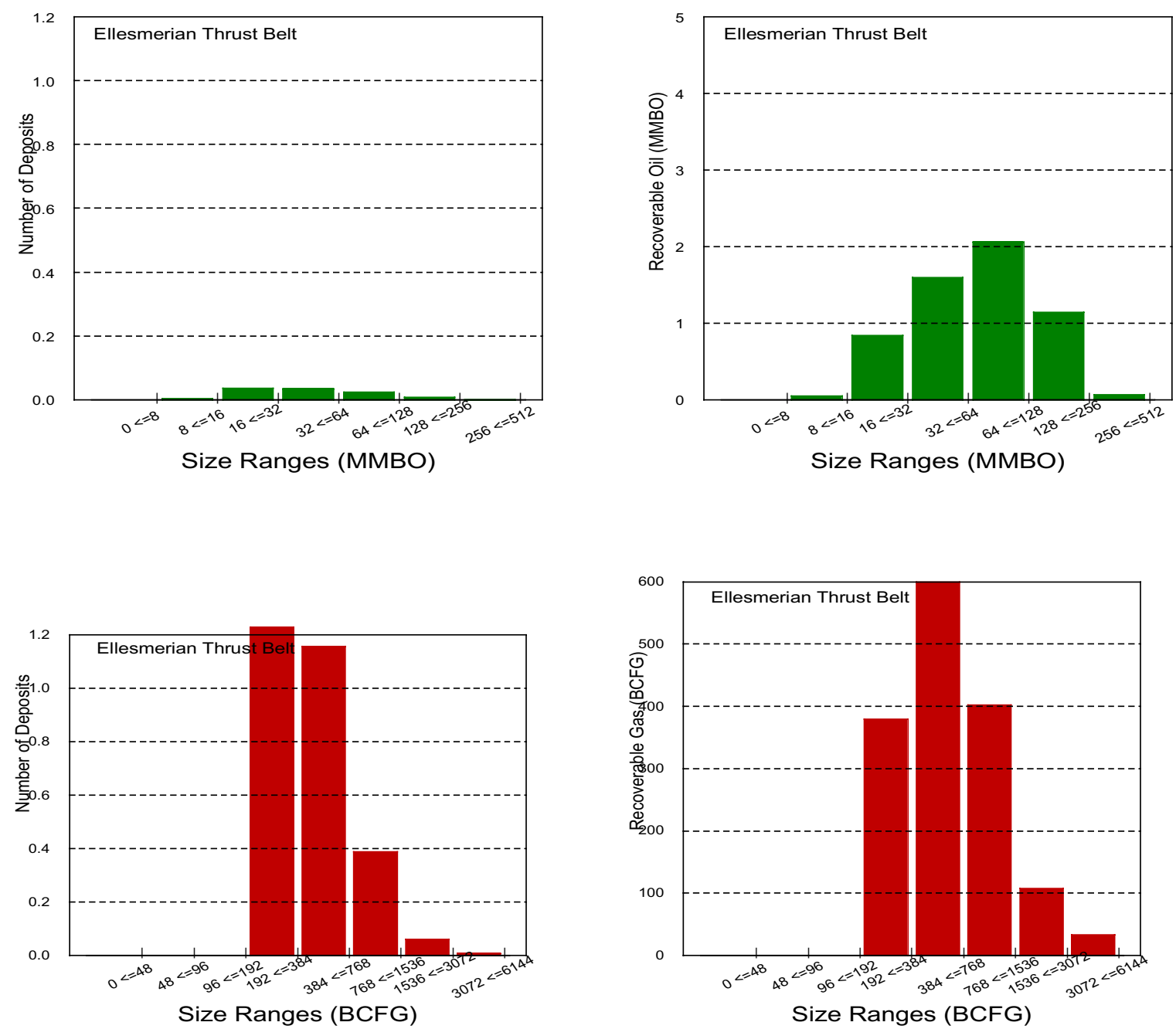
Table 24a: Input values for oil accumulations in the Ellesmerian Ivishak Play

NPRA Assessment Form-2001

PLAY: Ellesmerian-Ivishak

Play area: $12314 \mid 10^{3}$ Acres

\section{OIL ACCUMULATION VOLUME PARAMETERS}

\begin{tabular}{|c|c|c|c|c|c|c|c|}
\hline \multirow[b]{2}{*}{ ATTRIBUTES } & \multirow{2}{*}{$\begin{array}{l}\text { Est Shape } \\
(1 \text { to } 5)\end{array}$} & \multicolumn{4}{|c|}{ PROB OF AND GREATER THAN } & \multirow{2}{*}{$\begin{array}{l}\text { Knowledge } \\
\text { Level } 1-3^{5}\end{array}$} & \\
\hline & & LTP & 0.50 & 0.05 & Max & & \\
\hline \multirow{4}{*}{$\begin{array}{l}\text { NET RESERVOIR THICKNESS } \\
\text { AREA OF CLOSURE }^{2} \\
\text { POROSITY }^{3,4} \\
\text { TRAP FILL }^{3}\end{array}$} & 2 & 10 & 15 & 45 & 120 & 2 & 0.63 Corr (nrt,ac) \\
\hline & 2 & 2.0 & 4.0 & 15.0 & 22.0 & 2 & \multirow[b]{3}{*}{ Enter $\mathrm{POR}^{\star} \mathrm{Sw}$} \\
\hline & 2 & 9 & 12 & 19 & 22 & 1 & \\
\hline & 6 & 35 & 50 & 80 & 100 & 2 & \\
\hline \multicolumn{2}{|l|}{ HYDROCARBON PORE VOL ${ }^{3,4}$} & 5 & 8 & 15 & 18 & & 4 \\
\hline \multirow{2}{*}{\multicolumn{2}{|c|}{$\begin{array}{l}\text { Approx mm bbl (fvf }=1 \text { ) } \\
\text { Recov } \mathrm{mm} \text { bbl at surface }\end{array}$}} & 2.7 & 18.6 & 628.4 & 3686.6 & & \\
\hline & & 0.8 & 5.4 & 183.8 & 1078.4 & & \\
\hline \multicolumn{7}{|c|}{$\begin{array}{l}\text { 1-thickness in feet, 2-thousands of acres, 3-percent, 4-correlation between Porosity and Water Saturation }=-1.0 \\
\text { 5-Knowledge Level: 1=High, 2=Medium, 3=Low; LTP=Left Truncation Point }\end{array}$} & \\
\hline \multirow{2}{*}{$\begin{array}{l}\text { TRAP DEPTH (in } 1000 \mathrm{ft} \text { ) } \\
\text { (from sea level) } \\
\end{array}$} & \begin{tabular}{|r|}
4 \\
\end{tabular} & 6.5 & 9 & 13 & 15 & 1 & \\
\hline & \multicolumn{4}{|c|}{ Surface to sea level correction (1000 ft): } & 0.201 & & \\
\hline
\end{tabular}

\section{OIL ACCUMULATION CHARACTERISTICS}

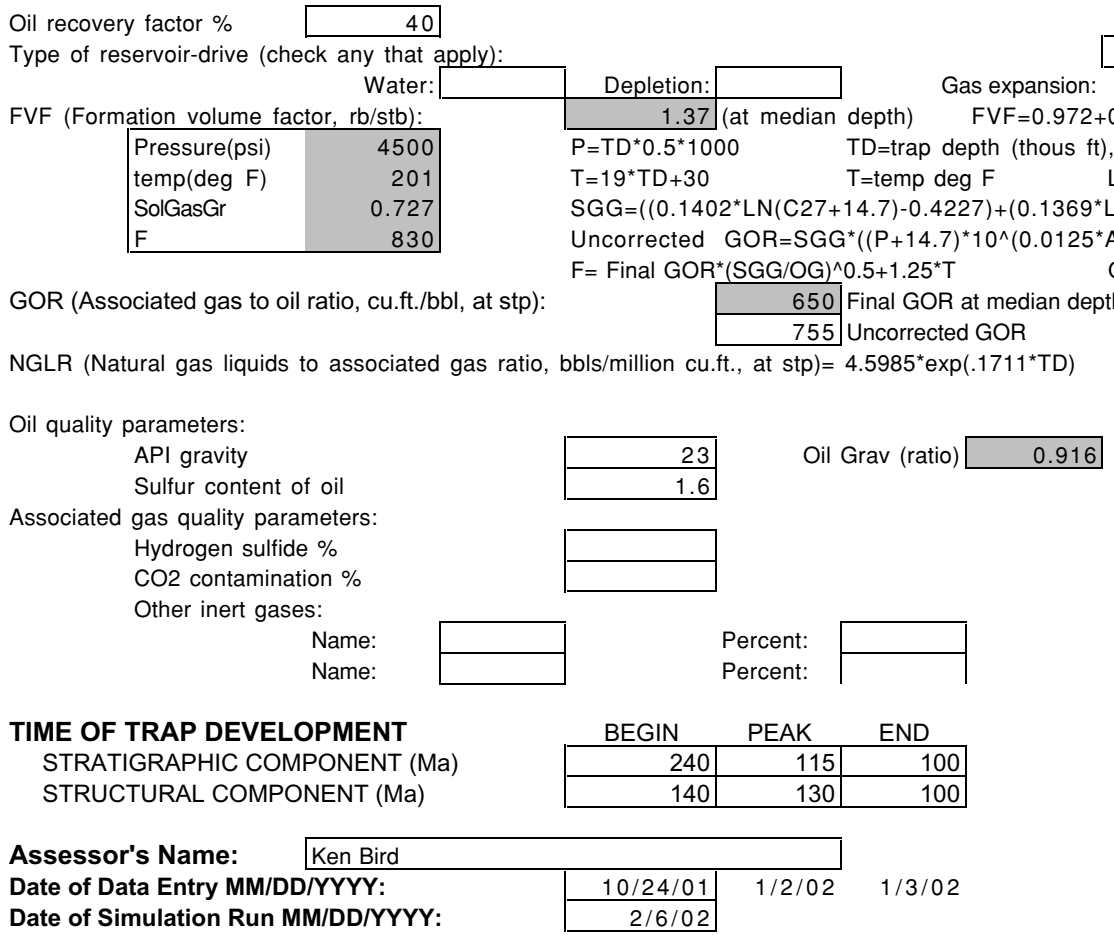

Note: only enter play name and assessor's name on Oil worksheet 
Table 24b: Input values for nonassociated gas accumulations in the Ellesmerian Ivishak Play NPRA Assessment Form-2001

PLAY: Ellesmerian-Ivishak

NONASSOCIATED GAS ACCUMULATION VOLUME PARAMETERS

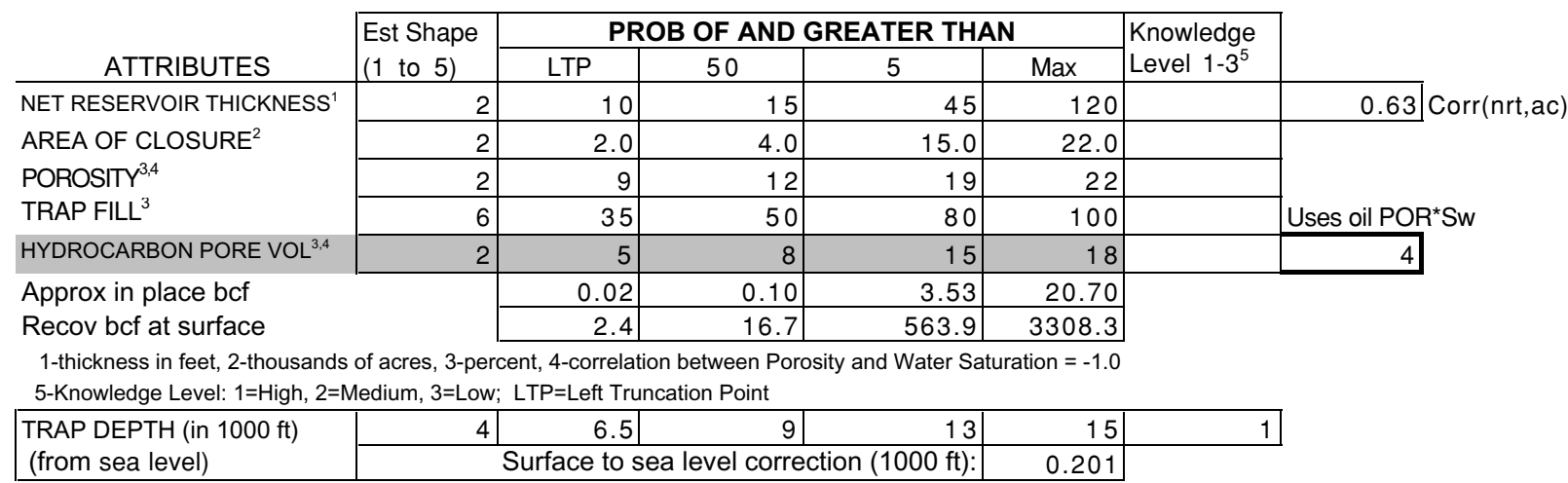

\section{NONASSOCIATED GAS ACCUMULATION CHARACTERISTICS}

NA Gas recovery factor \% 65

Type of reservoir-drive (check any that apply):

$$
\text { Water: Gas expansion: }
$$

Natural gas liquids plus condensate to non-associated gas (bbls/million cf) (in place): NGL-NAG $=1.785^{\star} \mathrm{TD}$ 16.1 (at median)

Non-associated gas quality parameters: Hydrogen sulfide \% CO2 contamination \% Other inert gases:

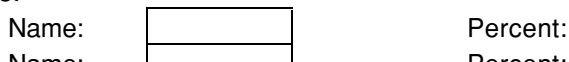
Name: $\quad$ Percent:

Gas fvf 245.9 (at median depth)

Gas $f v f=752.2^{*}\left(1-E X P\left(-0.05728^{*} T D\right)\right)$ $\mathrm{TD}=$ trap depth (thous $\mathrm{ft}$ )

\section{For Nonassociated Gas Accumulation:}

\begin{tabular}{|c|c|c|c|}
\hline TIME OF TRAP DEVELOPMEN 1 & BEGIN & PEAK & END \\
\hline STRATIGRAPHIC COMPONENT (M] & 240 & 115 & 100 \\
\hline STRUCTURAL COMPONENT (Ma) & 140 & 130 & 100 \\
\hline
\end{tabular}

Assessor's Name: Ken Bird Date of Data Entry MM/DD/YYYY: Date of Simulation Run MM/DD/YYYY: 
Table 24c: Input risking values for the Ellesmerian Ivishak Play

NPRA Assessment Form-2001

Play: Ellesmerian-Ivishak

\section{RISKING}

MINIMUM ACCUMULATION SIZE, MAS (Millions of BBL in place)

PRERISKED FREQUENCY DISTRIBUTION (Oil plus Gas)

NUM OF PROSPECTS

$>$ MINIMUM SIZE

\section{PROB OF AND GREATER THAN}

\begin{tabular}{|r|r|r|r|r|r|}
\hline Est Shape & Min & 50 & 5 & Max & Knowledge \\
Level $1-3^{5}$
\end{tabular}

5-Knowledge Level: 1=High, 2=Medium, 3=Low; LTP=Left Truncation Point

\section{ATTRIBUTES}

PLAY

ATTRIBUTES

CHARGE (C)

$\operatorname{TRAP}(\mathrm{T})$

TIMING (F)

Probability that play contains at least 1 reservoir $>=$ minimum size (CXTXF)

PROSPECT

ATTRIBUTES

CHARGE (c)

$\operatorname{TRAP}(\mathrm{t})$

TIMING (f)

Probability that a randomly chosen prospect is favorable (cxtxf)

Play Attributes x Prospect Attributes (CxTxFxcxtxf)

FRACTION OF ACCUMULATIONS BEING OIL

Fraction NA Gas=1-Fraction(Oil)

\section{PROBABILITY OF FAVORABLE}

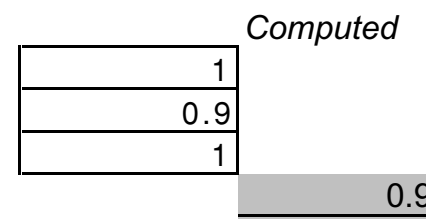

1

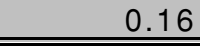

0.144

0.85

0.15

Allocation (percent):

\begin{tabular}{|l|r|r|r|}
\cline { 2 - 4 } \multicolumn{1}{c|}{} & \multicolumn{1}{c|}{ Land } & \multicolumn{1}{c|}{ Oil } & \multicolumn{1}{c|}{ Gas } \\
\hline Federal & 91 & 91 & 91 \\
\hline State & 6 & 6 & 6 \\
\hline Native & 3 & 3 & 3 \\
\hline
\end{tabular}

Assessor's Name:

Ken Bird

Date of Data Entry MM/DD/YYYY:

Date of Simulation Run MM/DD/YYYY:

$10 / 24 / 01$

$2 / 6 / 02$ 
Table 24d: Distribution of fitted hydrocarbon volume attributes, trap depth, and number of prospects for the Ellesmerian Ivishak Play

Elles Ivishak

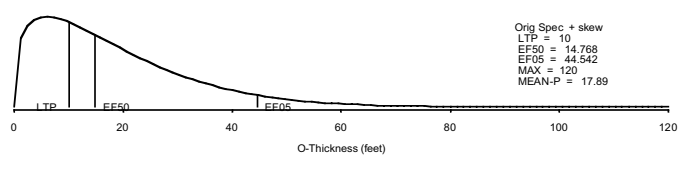

Elles Ivishak

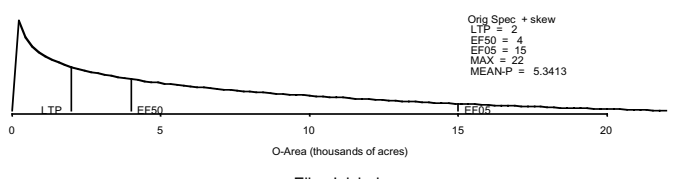

Elles lvishak
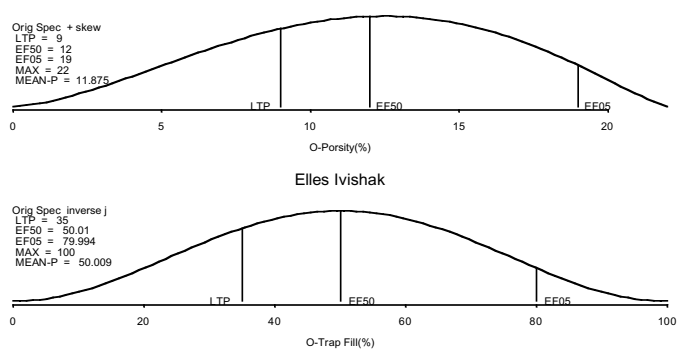

Elles Ivishak

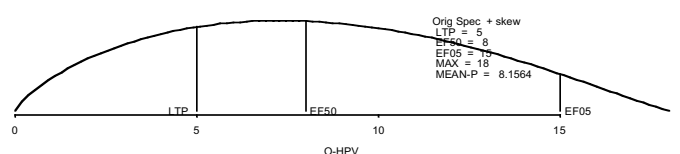

Olles Ivishak

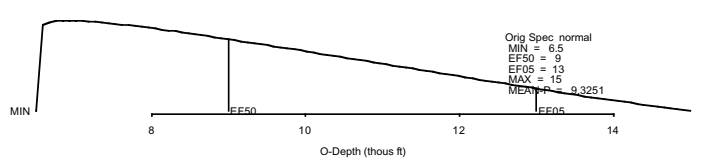

Elles Ivishak
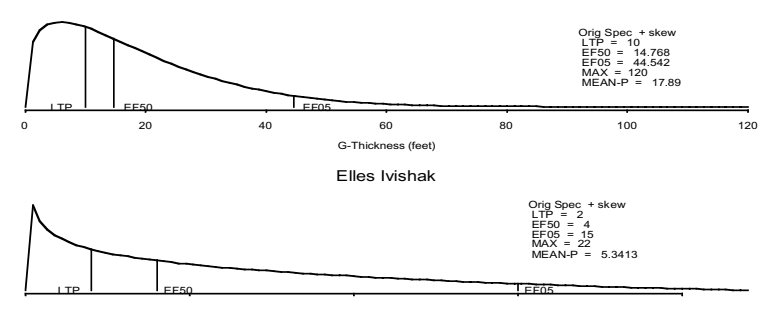

Elles Ivishak Truncated Portion

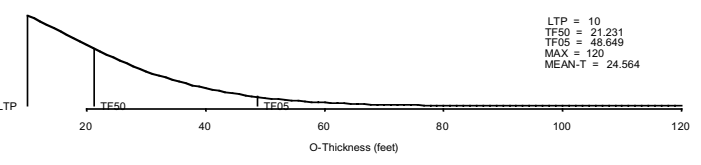

Elles Ivishak Truncated Portion

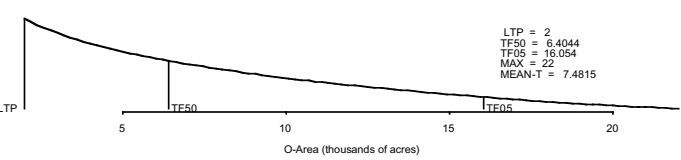

Elles Ivishak Truncated Portion

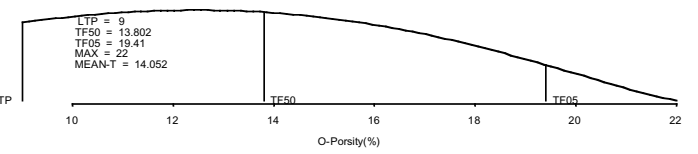

Elles lvishak Truncated Portion

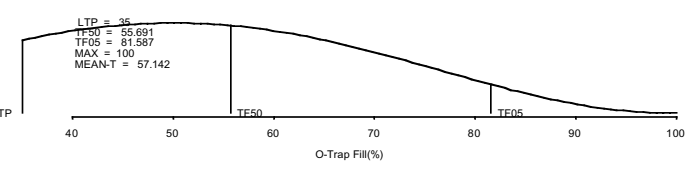

Elles Ivishak Truncated Portion

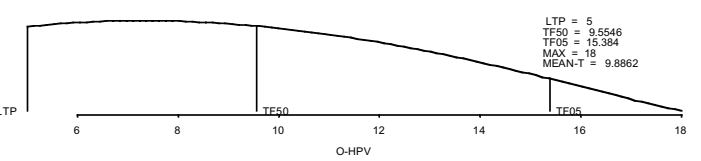

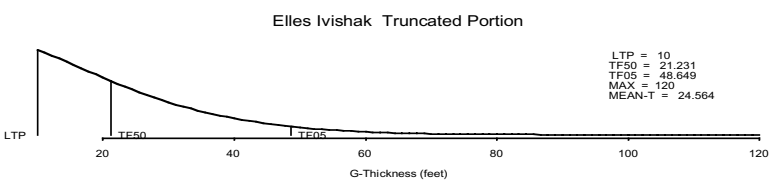

Elles Ivishak Truncated Portion 

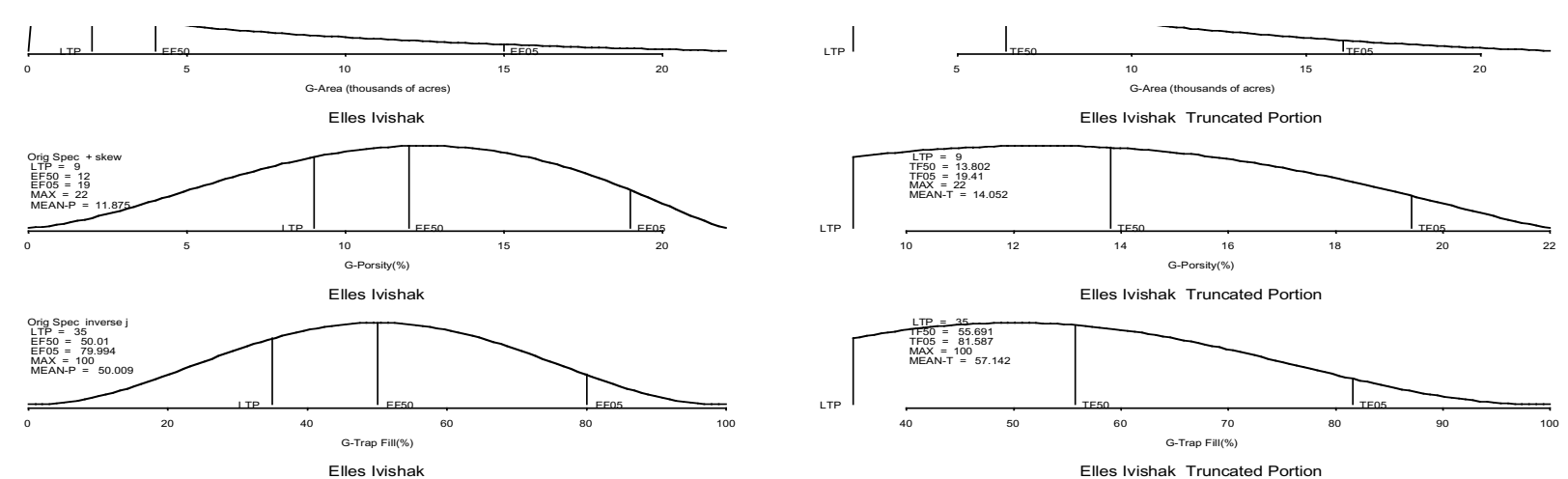

Elles Ivishak Truncated Portion
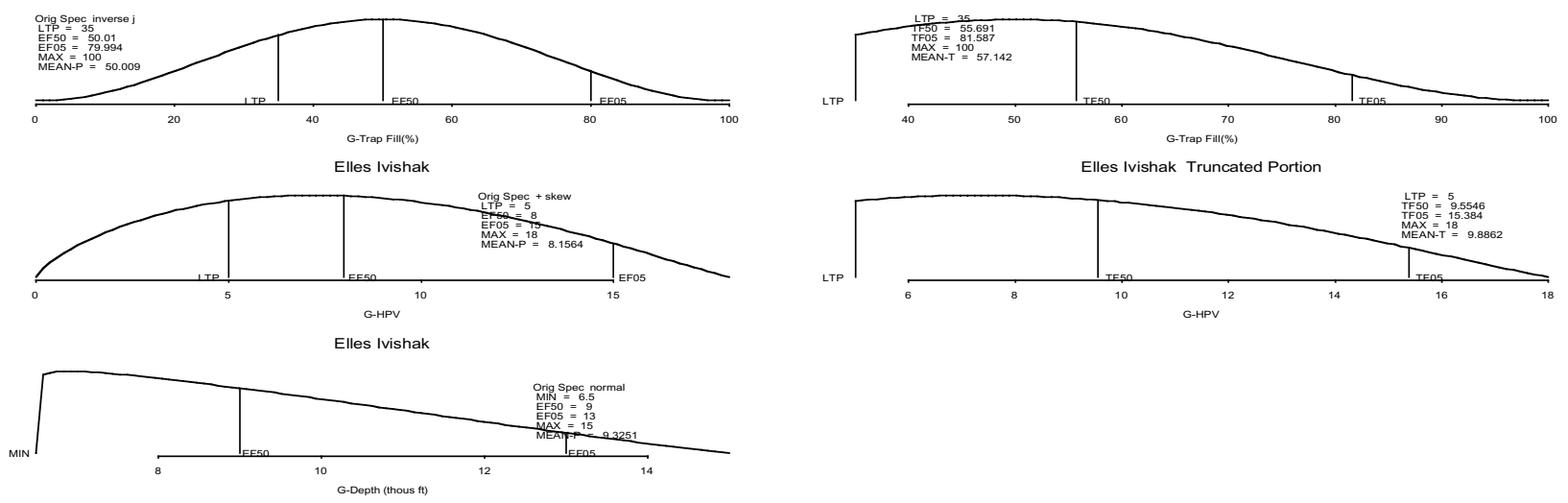

Elles Ivishak

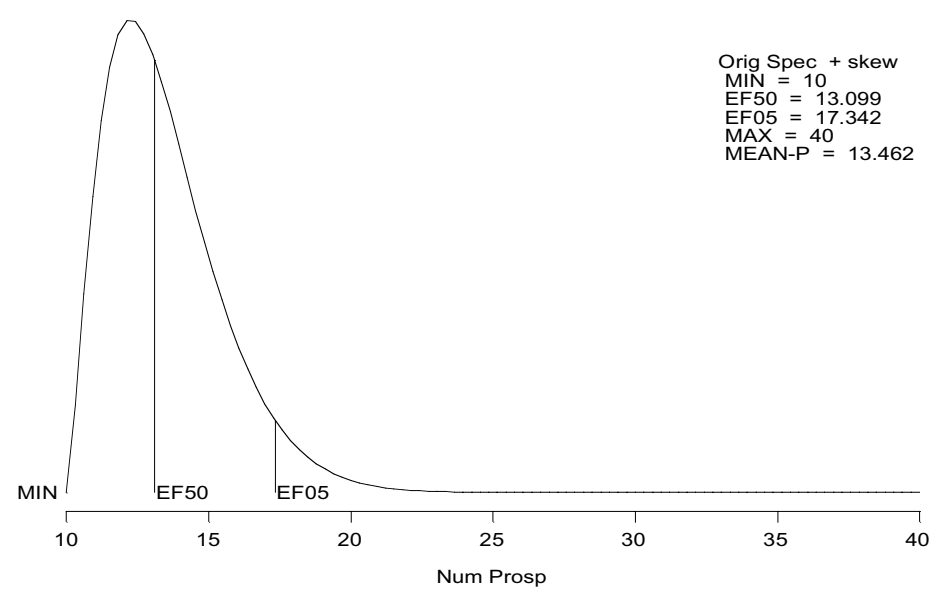


Table 24e: Estimated undiscovered oil and gas resources for Ellesmerian Ivishak Play

Deposit
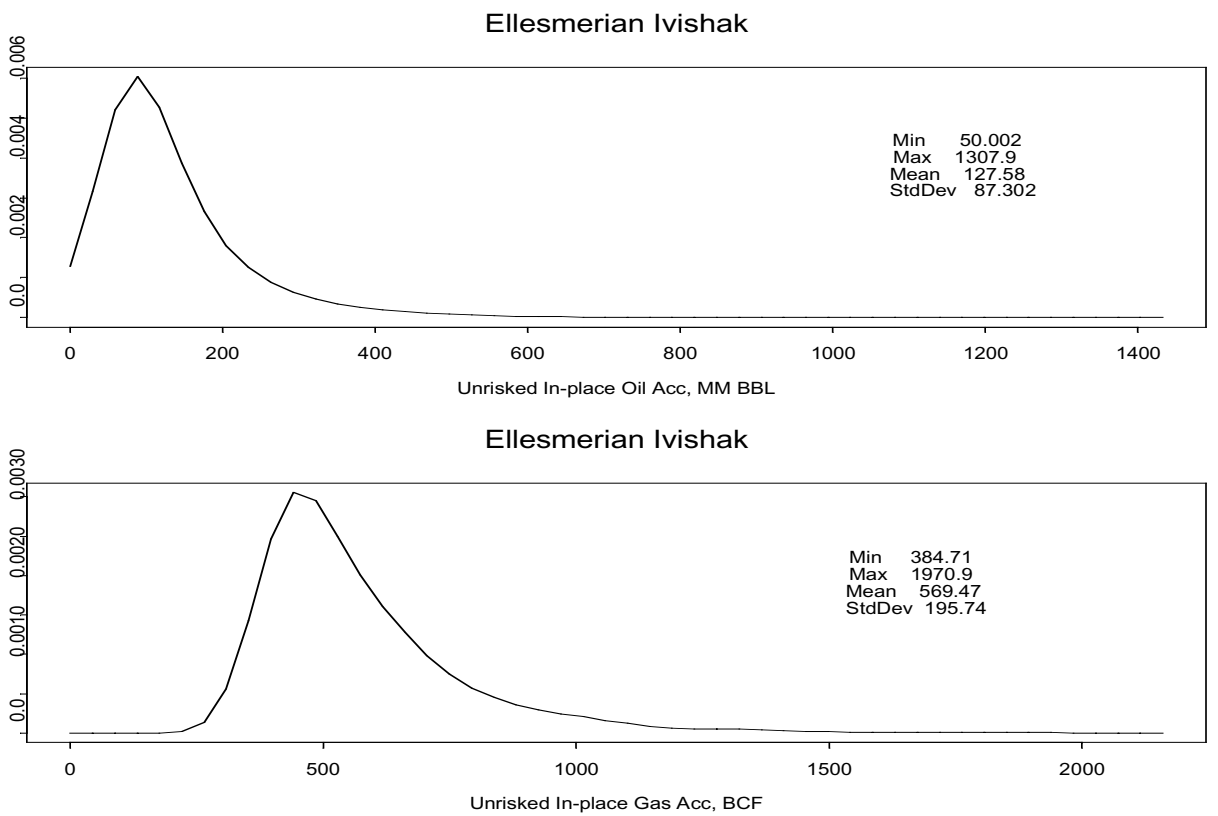

Play Stats
\begin{tabular}{|ll|rrrrr|} 
Resource & Unit & Mean & StdDev & F95 & F50 & F05 \\
\hline In-place oil & MMBO & 210.34 & 203.20 & 0.00 & 165.29 & 607.56 \\
In-place NA gas & BCFG & 162.46 & 324.93 & 0.00 & 0.00 & 868.62 \\
Recov oil & MMBO & 84.14 & 81.28 & 0.00 & 66.12 & 243.02 \\
Recov assoc diss gas & BCFG & 56.00 & 54.42 & 0.00 & 43.54 & 160.88 \\
Recov NA gas & BCFG & 105.60 & 211.20 & 0.00 & 0.00 & 564.60 \\
Recov NGL (ADG) & MMBO & 2.04 & 2.06 & 0.00 & 1.53 & 6.02 \\
Recov NGL (NAG) & MMBO & 1.86 & 3.82 & 0.00 & 0.00 & 9.88 \\
Num oil deposits & & 1.65 & 1.34 & 0.00 & 2.00 & 4.00 \\
Num NA gas deposits & & 0.29 & 0.54 & 0.00 & 0.00 & 1.00 \\
\hline
\end{tabular}

RNS $=715$

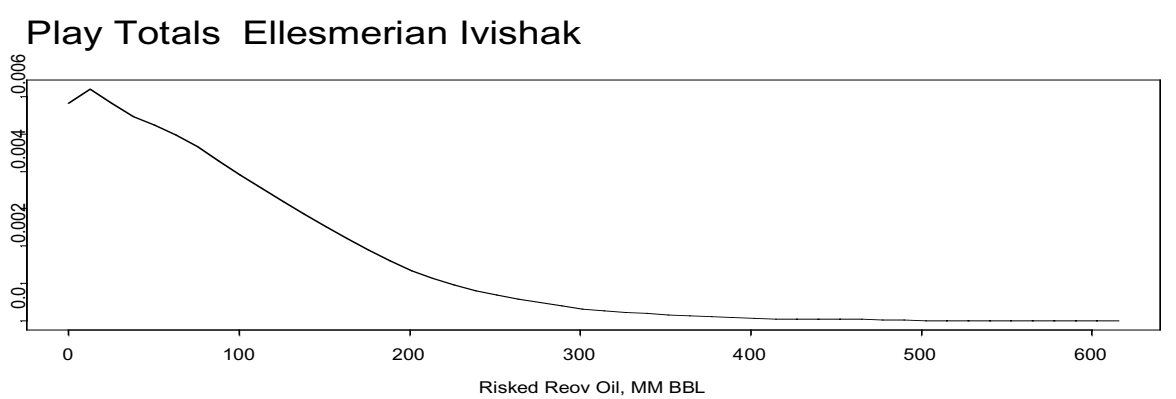

Play Totals Ellesmerian Ivishak

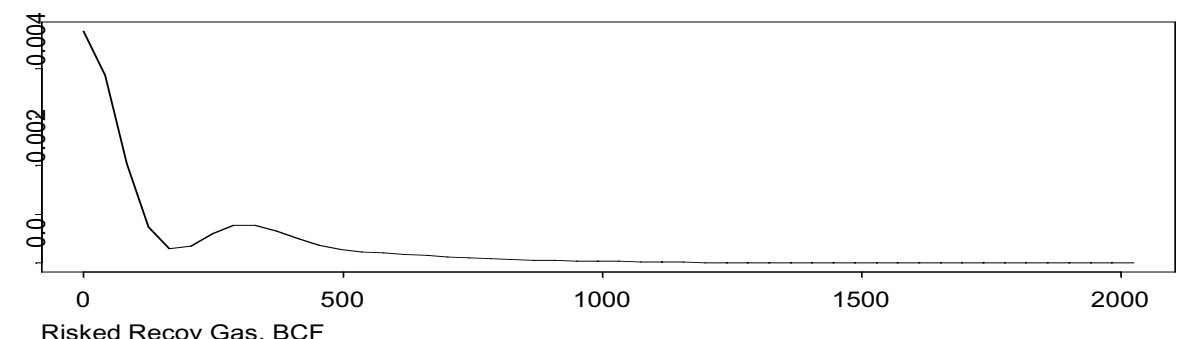


Table 24f: Estimated undiscovered oil and gas resources for the Ellesmerian Ivishak Play

showing number of deposits and volumes by accumulation size class

\begin{tabular}{rrrrrr|} 
Start size class & Oil deposits & Oil (MMBO_ Start size class & Gas deposits & NA Gas (BCFG) \\
0 & 0.00 & 0.00 & 0 & 0.00 & 0.00 \\
8 & 0.00 & 0.00 & 48 & 0.00 & 0.00 \\
16 & 0.58 & 14.90 & 96 & 0.00 & 0.00 \\
32 & 0.68 & 30.40 & 192 & 0.19 & 58.55 \\
64 & 0.32 & 27.38 & 384 & 0.09 & 42.73 \\
128 & 0.07 & 10.64 & 768 & 0.00 & 4.33 \\
256 & 0.00 & 0.78 & 1536 & & 105.61 \\
\hline 512 & 0.00 & 0.05 & 3072 & & 0.29 \\
\hline Totals & 1.65 & 84.14 & & & \\
\hline
\end{tabular}
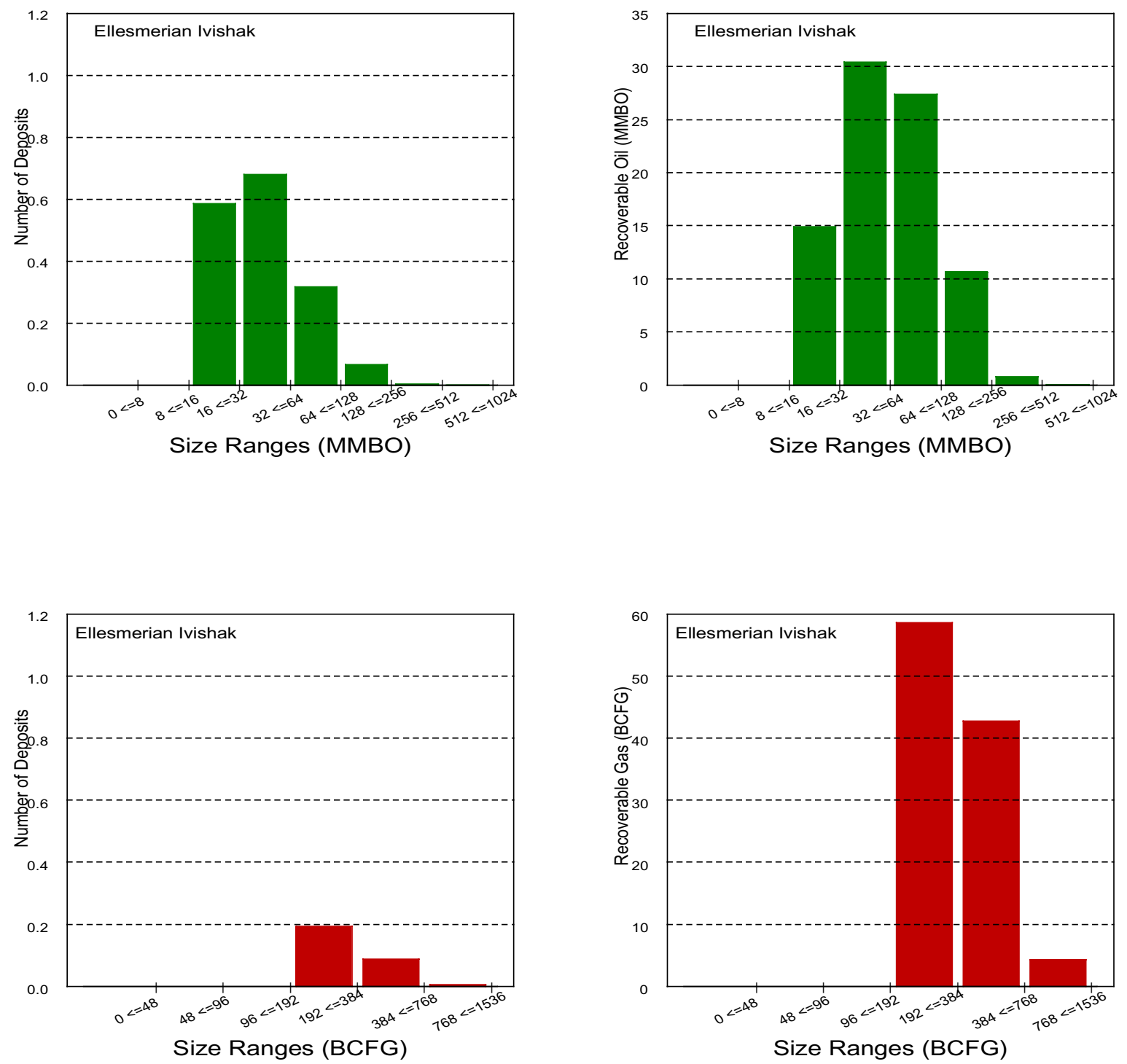
Table 25a. Input values for oil accumulations in the Ellesmerian Echooka North Play.

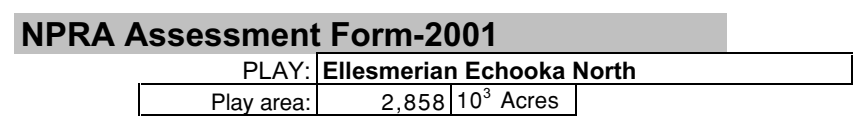

\section{OIL ACCUMULATION VOLUME PARAMETERS}

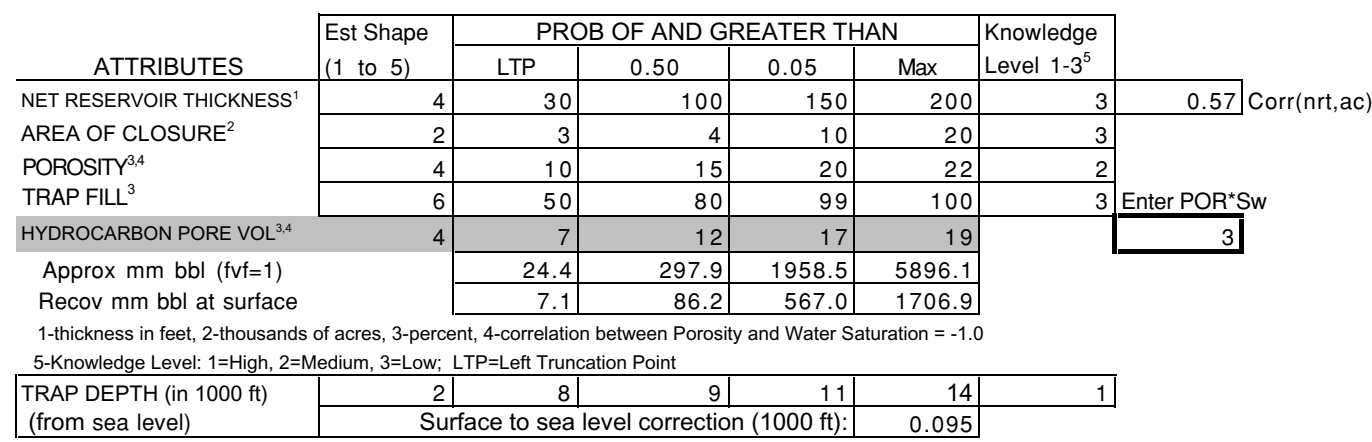

\section{OIL ACCUMULATION CHARACTERISTICS}

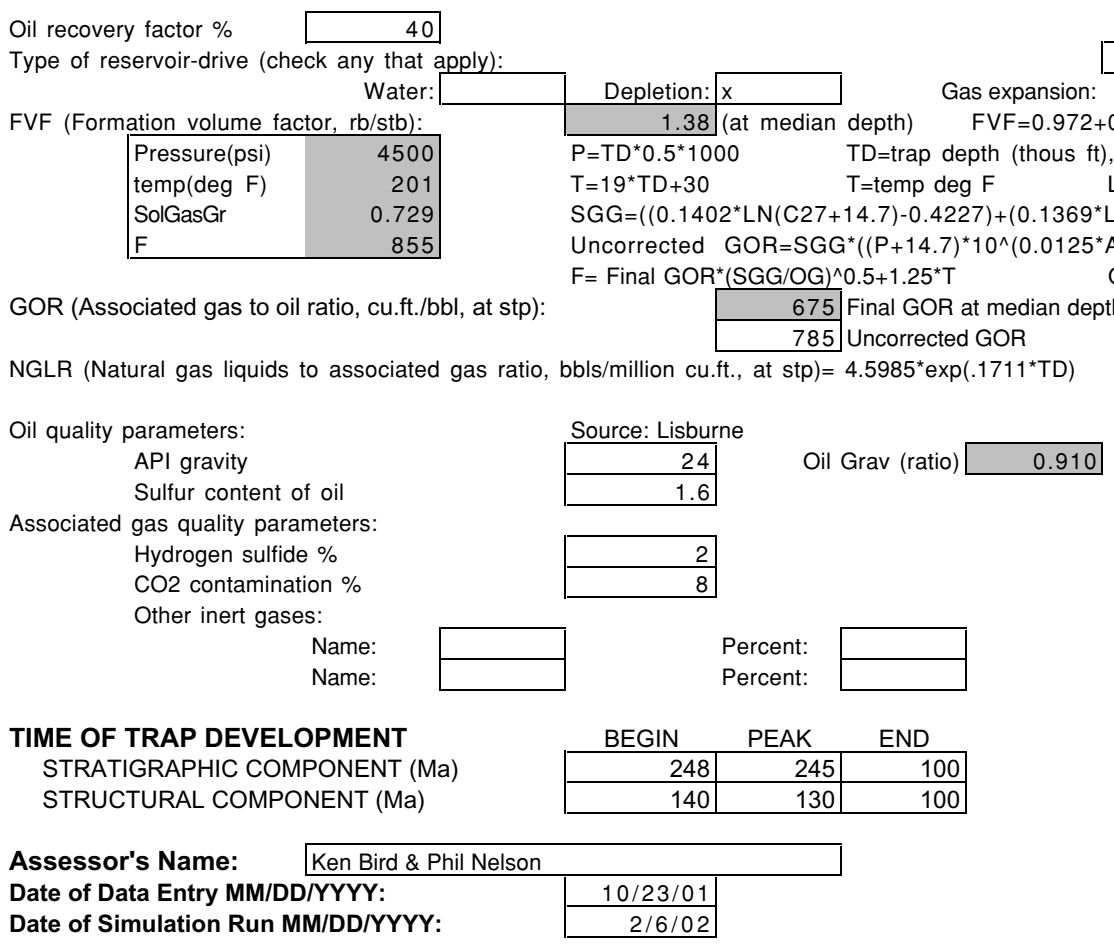

Note: only enter play name and assessor's name on Oil worksheet 


\section{Table 25b. Input values for nonassociated gas accumulations in the Ellesmerian Echooka North Play.}

NPRA Assessment Form-2001

PLAY: Ellesmerian Echooka North

\section{NONASSOCIATED GAS ACCUMULATION VOLUME PARAMETERS}

\begin{tabular}{|c|c|c|c|c|c|c|c|}
\hline \multirow[b]{2}{*}{ ATTRIBUTES } & \multirow{2}{*}{$\begin{array}{l}\text { Est Shape } \\
(1 \text { to } 5)\end{array}$} & \multicolumn{4}{|c|}{ PROB OF AND GREATER THAN } & \multirow{2}{*}{$\begin{array}{l}\text { Knowledge } \\
\text { Level } 1-3^{5}\end{array}$} & \\
\hline & & \begin{tabular}{|l|l|} 
LTP \\
\end{tabular} & 50 & \begin{tabular}{|l|l}
5 & \\
\end{tabular} & Max & & \\
\hline \multirow{4}{*}{$\begin{array}{l}\text { NET RESERVOIR THICKNESS } \\
\text { AREA OF CLOSURE }^{2} \\
\text { POROSITY,4 }^{3,4} \\
\text { TRAP FILL }^{3}\end{array}$} & 4 & 30 & 100 & 150 & 200 & 3 & $0.57 \operatorname{Corr}(\mathrm{nrt}, \mathrm{ac})$ \\
\hline & 2 & 3 & 4 & 10 & 20 & 3 & \multirow{3}{*}{ Uses oil POR ${ }^{\star} \mathrm{Sw}$} \\
\hline & 4 & 10 & 15 & 20 & 22 & 2 & \\
\hline & 6 & 50 & 80 & 99 & 100 & 3 & \\
\hline HYDROCARBON PORE VOL & 4 & 7 & 12 & 17 & 19 & & \multirow[t]{3}{*}{3} \\
\hline \multirow{2}{*}{\multicolumn{2}{|c|}{$\begin{array}{l}\text { Approx in place bcf } \\
\text { Recov bcf at surface }\end{array}$}} & 0.14 & 1.67 & 11.00 & 33.11 & & \\
\hline & & 21.9 & 267.3 & 1757.5 & 5291.1 & & \\
\hline \multicolumn{7}{|c|}{$\begin{array}{l}\text { 1-thickness in feet, 2-thousands of acres, 3-percent, 4-correlation between Porosity and Water Saturation }=-1.0 \\
\text { 5-Knowledge Level: } 1=\text { High, 2=Medium, 3=Low; LTP=Left Truncation Point }\end{array}$} & \\
\hline \multirow{2}{*}{$\begin{array}{l}\text { TRAP DEPTH (in } 1000 \mathrm{ft} \text { ) } \\
\text { (from sea level) }\end{array}$} & \begin{tabular}{r|}
2 \\
\end{tabular} & 8 & 9 & 11 & 14 & 3 & \\
\hline & & irface to $s$ & vel corre & $1000 \mathrm{ft}):$ & 0.095 & & \\
\hline
\end{tabular}

\section{NONASSOCIATED GAS ACCUMULATION CHARACTERISTICS}

NA Gas recovery factor \% 65

Type of reservoir-drive (check any that apply):

Water:

Gas expansion:

Natural gas liquids plus condensate to non-associated gas (bbls/million cf) (in place): NGL-NAG=1.785*TD $16.1 \quad$ (at median)

Non-associated gas quality parameters: Hydrogen sulfide \% CO2 contamination \% Other inert gases:

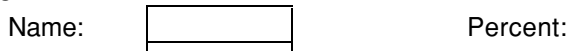
Name:

Gas fvf 245.9 (at median depth)

Gas $f v f=752.2^{*}\left(1-\operatorname{EXP}\left(-0.05728^{*} T D\right)\right)$ Percent:

$113.3+21.1^{*} T D-0.812^{\star} T^{\wedge} 2+0.0116^{*} T^{\wedge} 3$ $\mathrm{TD}=$ trap depth (thous $\mathrm{ft}$ )

\begin{tabular}{|c|c|c|c|}
\hline TIME OF TRAP DEVELOPMEN1 & BEGIN & PEAK & END \\
\hline STRATIGRAPHIC COMPONENT (M] & 248 & 245 & 100 \\
\hline STRUCTURAL COMPONENT (Ma) & 140 & 130 & 100 \\
\hline
\end{tabular}

Assessor's Name: $\quad$ Ken Bird \& Phil Nelson Date of Data Entry MM/DD/YYYY:

Date of Simulation Run MM/DD/YYYY:

$10 / 23 / 01$




\title{
Table 25c. Input risking values for the Ellesmerian Echooka North Play.
}

\author{
NPRA Assessment Form-2001 \\ Play: Ellesmerian Echooka North
}

\section{RISKING}

MINIMUM ACCUMULATION SIZE, MAS (Millions of BBL in place)

PRERISKED FREQUENCY DISTRIBUTION (Oil plus Gas)

NUM OF PROSPECTS

$>$ MINIMUM SIZE

\section{PROB OF AND GREATER THAN}

\begin{tabular}{|c|c|c|c|c|c|}
\hline & \multicolumn{4}{|c|}{ PROB OF AND GREATER THAN } & \multirow{2}{*}{$\begin{array}{l}\text { Knowledge } \\
\text { Level } 1-3^{5}\end{array}$} \\
\hline Est Shape & LTP & 50 & \begin{tabular}{|l|}
5 \\
\end{tabular} & Max & \\
\hline 2 & 2 & 4 & 6 & 20 & 3 \\
\hline
\end{tabular}

5-Knowledge Level: 1=High, 2=Medium, 3=Low; LTP=Left Truncation Point

\section{ATTRIBUTES}

PLAY

ATTRIBUTES

CHARGE (C)

TRAP (T)

TIMING (F)

Probability that play contains at least 1 reservoir $>=$ minimum size (CxTxF)

PROSPECT

ATTRIBUTES

CHARGE (c)

TRAP ( $\mathrm{t}$ )

TIMING (f)

Probability that a randomly chosen prospect is favorable (cxtxf)

Play Attributes x Prospect Attributes (CxTxFxcxtxf)

FRACTION OF ACCUMULATIONS BEING OIL

Fraction NA Gas=1-Fraction(Oil)
PROBABILITY

OF FAVORABLE

Computed

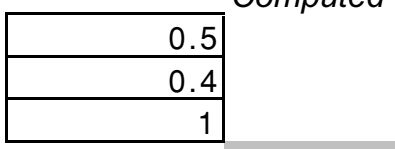

0.2

0.8

Allocation (percent):

\begin{tabular}{|c|c|c|c|}
\hline & Land & Oil & Gas \\
\hline Federal & 84 & 84 & 84 \\
\hline State & 12 & 12 & 12 \\
\hline Native & 4 & 4 & 4 \\
\hline
\end{tabular}

Assessor's Name:

Ken Bird \& Phil Nelson

Date of Data Entry MM/DD/YYYY:

Date of Simulation Run MM/DD/YYYY:

$10 / 23 / 01$

$2 / 6 / 02$ 
Table 25d. Distribution of fitted hydrocarbon volume attributes, trap depth, and number of prospects for the Ellesmerian Echooka North Play.
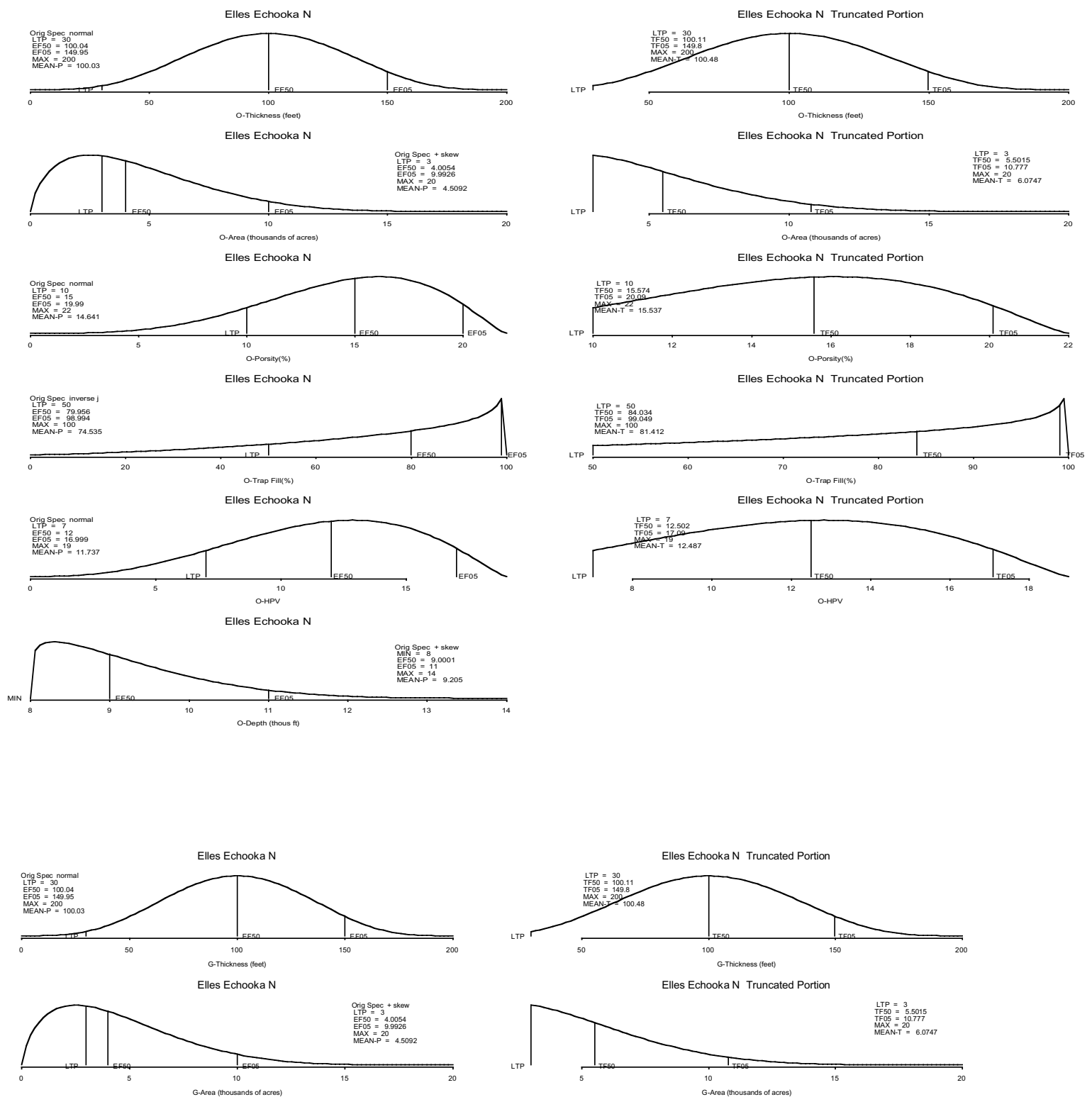

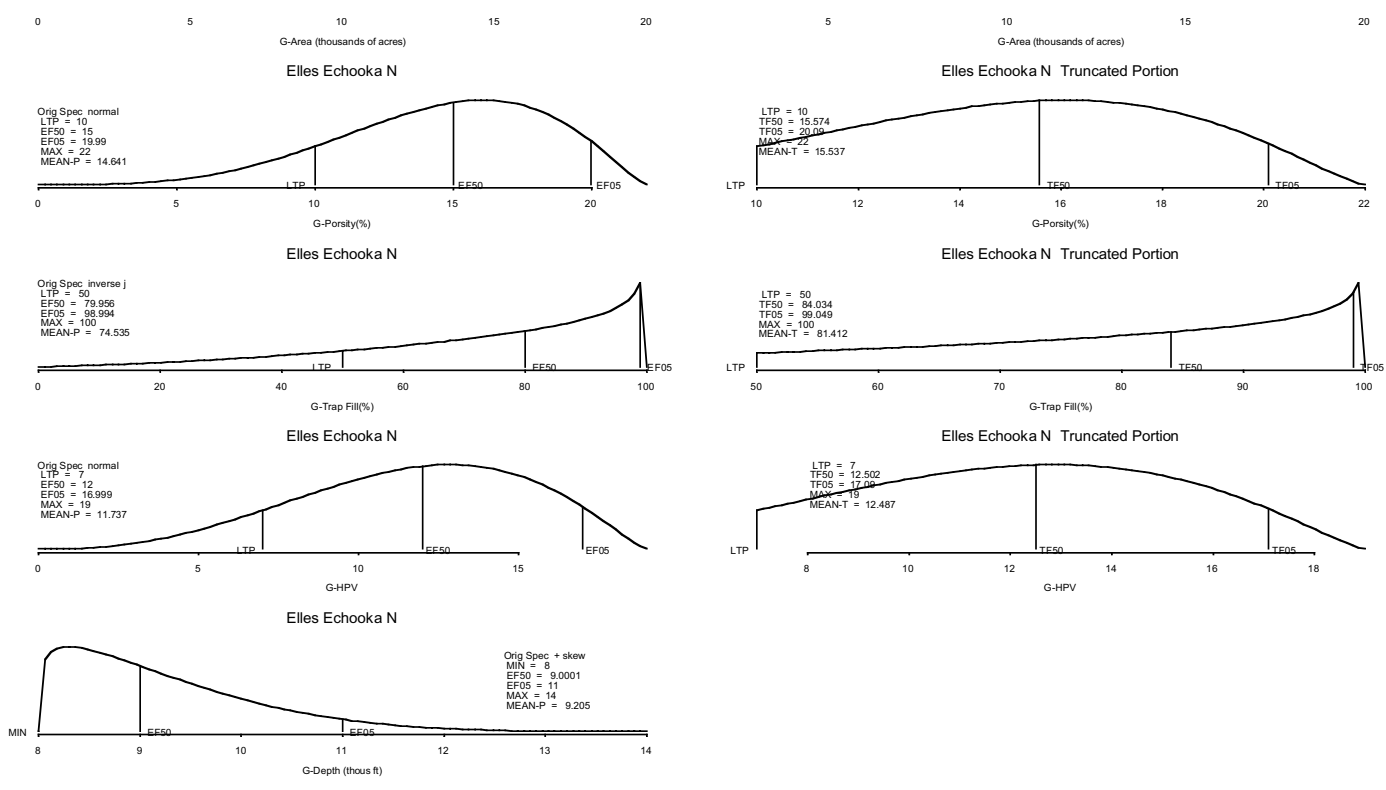

Elles Echooka N

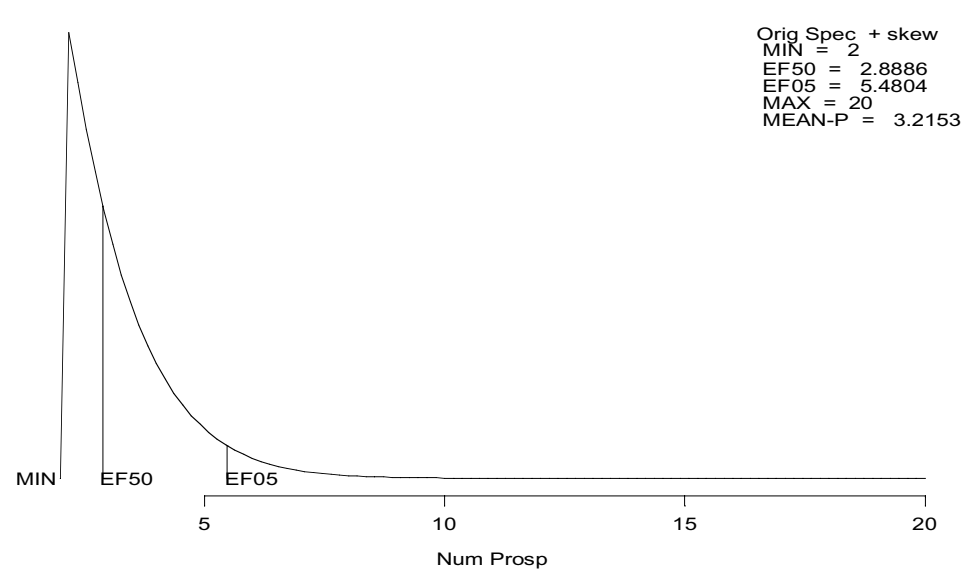


Table 25e. Estimated undiscovered oil and gas resources for the Ellesmerian Echooka North Play.

Ellesmerian Echooka $\mathrm{N}$

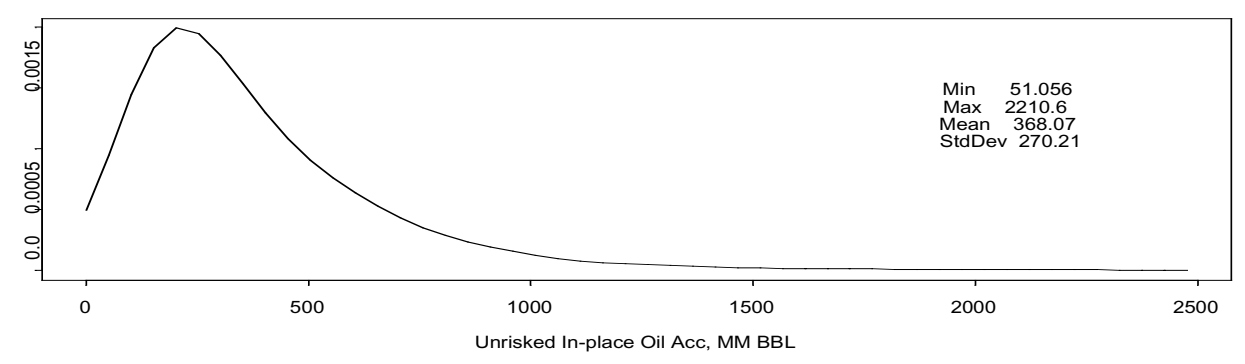

Ellesmerian Echooka N

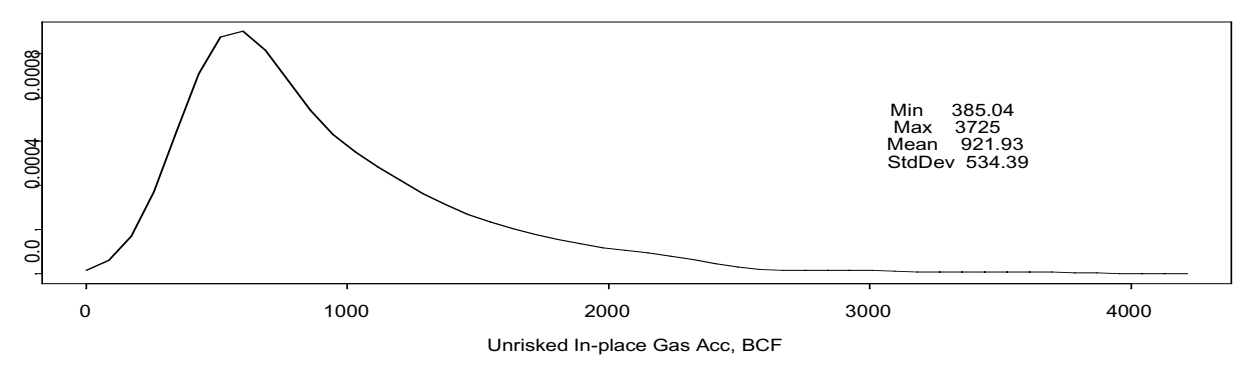

Play Stats
\begin{tabular}{|ll|rrrrr|} 
Resource & Unit & Mean & StdDev & F95 & F50 & F05 \\
\hline In-place oil & MMBO & 17.23 & 103.31 & 0.00 & 0.00 & 0.00 \\
In-place NA gas & BCFG & 11.16 & 119.70 & 0.00 & 0.00 & 0.00 \\
Recov oil & MMBO & 6.89 & 41.33 & 0.00 & 0.00 & 0.00 \\
Recov assoc diss gas & BCFG & 4.77 & 28.68 & 0.00 & 0.00 & 0.00 \\
Recov NA gas & BCFG & 7.25 & 77.81 & 0.00 & 0.00 & 0.00 \\
Recov NGL (ADG) & MMBO & 0.16 & 0.95 & 0.00 & 0.00 & 0.00 \\
Recov NGL (NAG) & MMBO & 0.12 & 1.31 & 0.00 & 0.00 & 0.00 \\
Num oil deposits & & 0.05 & 0.23 & 0.00 & 0.00 & 0.00 \\
Num NA gas deposits & & 0.01 & 0.11 & 0.00 & 0.00 & 0.00 \\
\hline
\end{tabular}

Play Totals Ellesmerian Echooka N

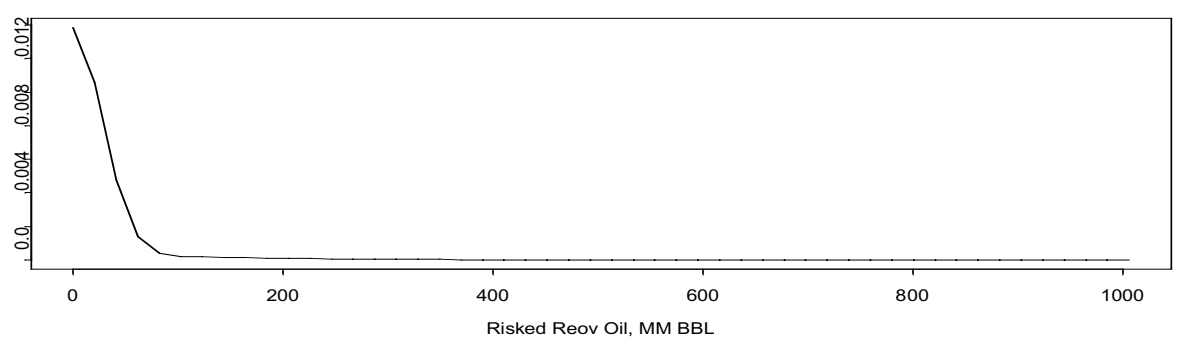

Play Totals Ellesmerian Echooka N

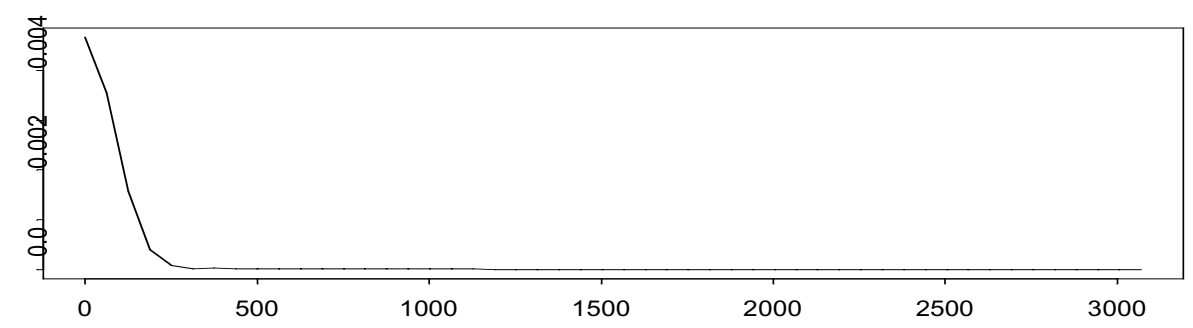

Risked Recov Gas, BCF 
Table 25f. Estimated undiscovered oil and gas resources for the Ellesmerian Echooka North Play showing number of deposits and volumes by accumulation size class.

\begin{tabular}{rrrrrr} 
Start size class & Oil deposits & Oil (MMBO_Start size class & Gas deposits Gas (BCFG) \\
0 & 0.00 & 0.00 & 0 & 0.00 & 0.00 \\
8 & 0.00 & 0.00 & 48 & 0.00 & 0.00 \\
16 & 0.00 & 0.03 & 96 & 0.00 & 0.00 \\
32 & 0.01 & 0.41 & 192 & 0.00 & 1.25 \\
64 & 0.02 & 1.51 & 384 & 0.01 & 2.86 \\
128 & 0.01 & 2.67 & 768 & 0.00 & 2.69 \\
256 & 0.01 & 1.92 & 1536 & 0.00 & 0.45 \\
512 & 0.00 & 0.36 & 3072 & & \\
\hline Totals & 0.05 & 6.89 & & 0.01 & 7.25 \\
\hline
\end{tabular}
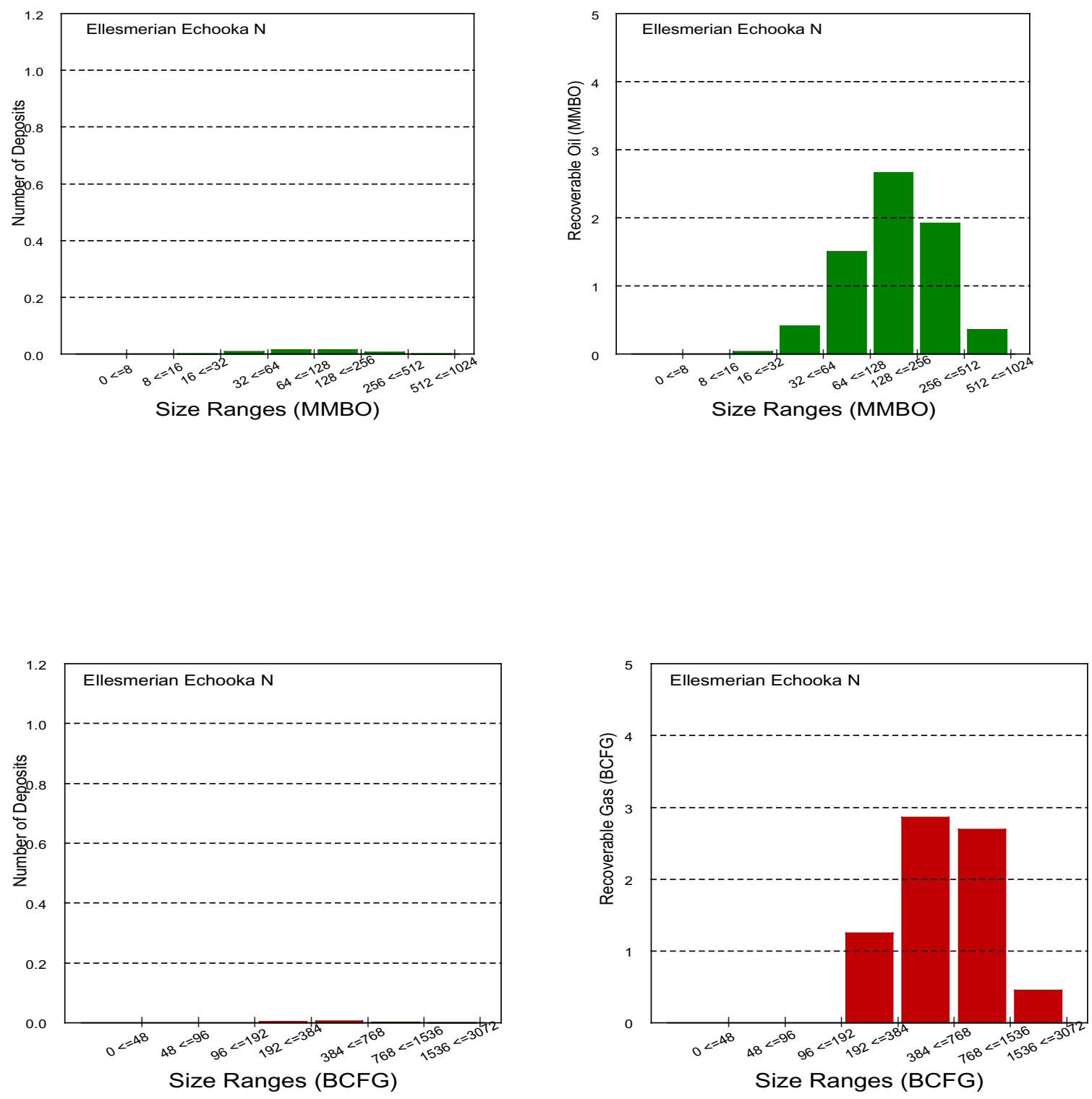
Table 26a: Input values for oil accumulations in the Ellesmerian Echooka South Play

NPRA Assessment Form-2001

PLAY: Ellesmerian Echooka South

Play area:

$716210^{3}$ Acres

\section{OIL ACCUMULATION VOLUME PARAMETERS}

\begin{tabular}{|c|c|c|c|c|c|c|c|}
\hline \multirow[b]{2}{*}{ ATTRIBUTES } & \multirow{2}{*}{$\begin{array}{l}\text { Est Shape } \\
(1 \text { to } 5)\end{array}$} & \multicolumn{4}{|c|}{ PROB OF AND GREATER THAN } & \multirow{2}{*}{$\begin{array}{l}\text { Knowledge } \\
\text { Level } 1-3^{5} \\
\end{array}$} & \multirow{7}{*}{ Enter POR*Sn } \\
\hline & & LTP & 0.50 & 0.05 & Max & & \\
\hline \multicolumn{7}{|l|}{ NET RESERVOIR THICKNESS ${ }^{1}$} & \\
\hline \multicolumn{7}{|l|}{ AREA OF CLOSURE ${ }^{2}$} & \\
\hline \multirow{2}{*}{$\begin{array}{l}\text { POROSITY, } \\
\text { TRAP FILL }\end{array}$} & & & & & & & \\
\hline & & & & & & & \\
\hline HYDROCARBON PORE VOL ${ }^{3,4}$ & & 0 & 0 & 0 & 0 & & \\
\hline Approx mm bbl $(f v f=1)$ & & 0.0 & 0.0 & 0.0 & 0.0 & & \\
\hline
\end{tabular}

1-thickness in feet, 2-thousands of acres, 3-percent, 4-correlation between Porosity and Water Saturation $=-1.0$

5-Knowledge Level: 1=High, 2=Medium, 3=Low; LTP=Left Truncation Point

TRAP DEPTH (in $1000 \mathrm{ft}$ )

(from sea level)

\begin{tabular}{r|r|r|r|}
\hline 4 & 10 & 11 & 15 \\
\hline Surface to sea level correction $(1000 \mathrm{ft}):$ & \\
\hline
\end{tabular}

\section{OIL ACCUMULATION CHARACTERISTICS}

Oil recovery factor \%

25

Type of reservoir-drive (check any that apply):

Water:

Depletion: $\mathrm{x}$

Gas expansion:

FVF (Formation volume factor, rb/stb):

GOR (Associated gas to oil ratio, cu.ft./bbl, at stp):

NGLR (Natural gas liquids to associated gas ratio, bbls/million cu.ft., at stp):
NGLR=1e+06/(
$5.36 \mathrm{E}+05$ * $\exp ($
$-0.254 * \operatorname{Depth}(1000 \mathrm{ft})))$

Oil quality parameters:

API gravity

Sulfur content of oil

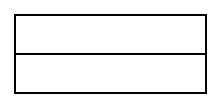

Associated gas quality parameters:

Hydrogen sulfide \%

CO2 contamination \%

Other inert gases:

Name:

Name:

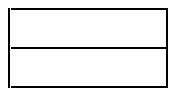

Percent:

TIME OF TRAP DEVELOPMENT

STRATIGRAPHIC COMPONENT (Ma)

STRUCTURAL COMPONENT (Ma)

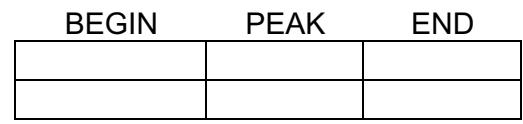

Assessor's Name: $\quad$ Ken Bird \& Phil Nelson

Date of Data Entry MM/DD/YYYY:

Date of Simulation Run MM/DD/YYYY:
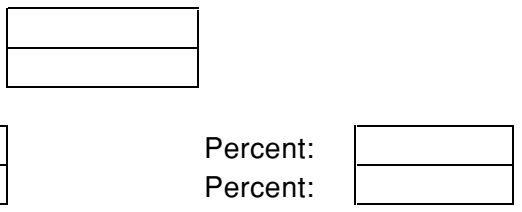

Note: only enter play name and assessor's name on Oil worksheet 
Table 26b: Input values for nonassociated gas accumulations in the Ellesmerian Echooka South Play NPRA Assessment Form-2001

PLAY: Ellesmerian Echooka South

\begin{tabular}{ll|l|} 
Play area: $\quad 7162$ & $10^{3}$ Acres \\
\hline
\end{tabular}

NONASSOCIATED GAS ACCUMULATION VOLUME PARAMETERS

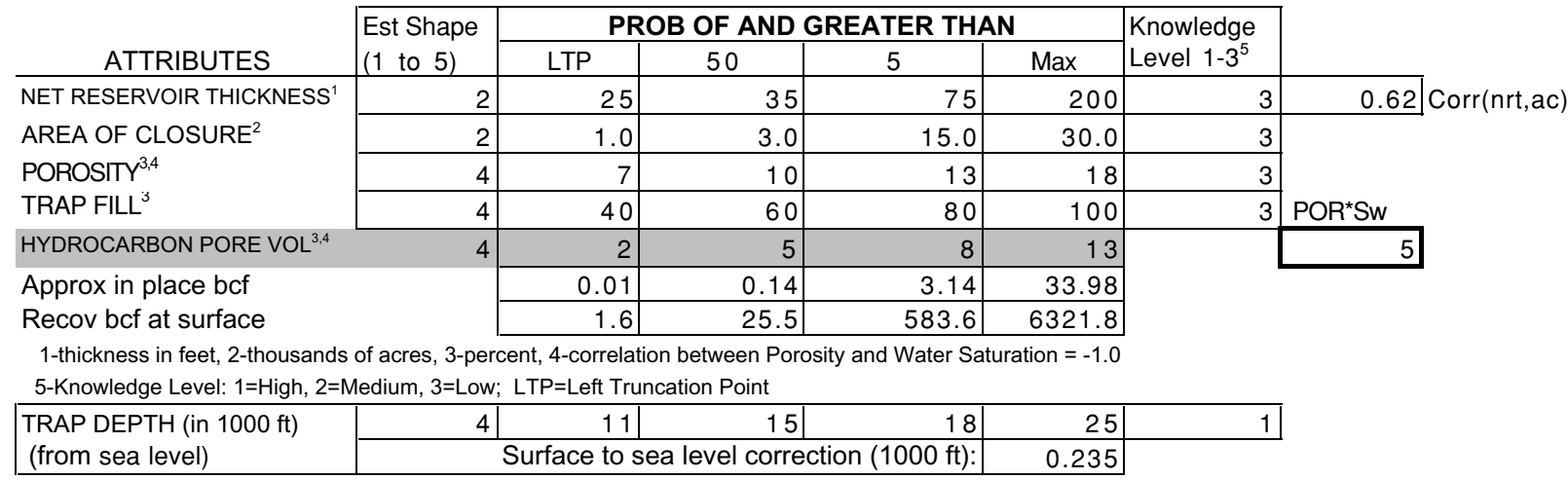

\section{NONASSOCIATED GAS ACCUMULATION CHARACTERISTICS}

NA Gas recovery factor \% 65

Type of reservoir-drive (check any that apply):

Water:

Gas expansion:

Natural gas liquids plus condensate to non-associated gas (bbls/million cf) (in place): NGL-NAG $=1.785^{\circ}$ TD 26.8 (at median)

Non-associated gas quality parameters: Hydrogen sulfide \% CO2 contamination \% Other inert gases:

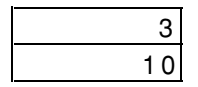

Name: Name:

Gas fvf 286.3 (at median depth)

Gas fvf $=7$

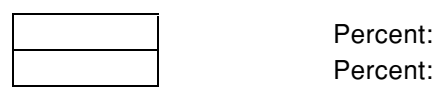

Percent:

752.2* $\left(1-\operatorname{EXP}\left(-0.05728^{*} \mathrm{TD}\right)\right)$

$113.3+21.1^{*} T D-0.812^{*} T^{\wedge} 2+0.0116^{*} T^{\wedge} 3$

$\mathrm{TD}=$ trap depth (thous $\mathrm{ft}$ )

TIME OF TRAP DEVELOPMENT

STRATIGRAPHIC COMPONENT (Ma)

STRUCTURAL COMPONENT (Ma)

\begin{tabular}{|r|r|r|}
\multicolumn{1}{c}{ BEGIN } & \multicolumn{1}{c}{ PEAK } & \multicolumn{1}{c|}{ END } \\
\hline 248 & 245 & 240 \\
\hline 200 & 130 & 100 \\
\hline
\end{tabular}

Assessor's Name: $\quad$ Ken Bird \& Phil Nelson

Date of Data Entry MM/DD/YYYY:

Date of Simulation Run MM/DD/YYYY:

\begin{tabular}{|r|}
\hline $10 / 23 / 01$ \\
\hline $2 / 6 / 02$ \\
\hline
\end{tabular}


Table 26c: Input risking values for the Ellesmerian Echooka South Play

NPRA Assessment Form-2001

Play: Ellesmerian Echooka South

\section{RISKING}

MINIMUM ACCUMULATION SIZE, MAS (bcf recov)

PRERISKED FREQUENCY DISTRIBUTION (Oil plus Gas)

NUM OF PROSPECTS

$>$ MINIMUM SIZE

\section{PROB OF AND GREATER THAN}

\begin{tabular}{|c|c|c|c|c|c|}
\hline & \multicolumn{4}{|c|}{ PROB OF AND GREATER THAN } & \multirow{2}{*}{$\begin{array}{l}\text { Knowledge } \\
\text { Level } 1-3^{5}\end{array}$} \\
\hline Est Shape & LTP & 50 & 5 & Max & \\
\hline 2 & 20 & 30 & 65 & 80 & 3 \\
\hline
\end{tabular}

5-Knowledge Level: 1=High, 2=Medium, 3=Low; LTP=Left Truncation Point

\section{ATTRIBUTES}

PLAY

ATTRIBUTES

CHARGE (C)

$\operatorname{TRAP}(\mathrm{T})$

TIMING (F)

Probability that play contains at least 1 reservoir $>=$ minimum size (CXTXF)

PROSPECT

ATTRIBUTES

CHARGE (c)

$\operatorname{TRAP}(\mathrm{t})$

TIMING (f)

Probability that a randomly chosen prospect is favorable (cxtxf)

Play Attributes x Prospect Attributes (CxTxFxcxtxf)

FRACTION OF ACCUMULATIONS BEING OIL

Fraction NA Gas=1-Fraction(Oil)

\section{PROBABILITY OF FAVORABLE}

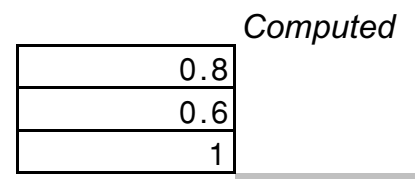

0.48

1

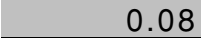

0.0384

0

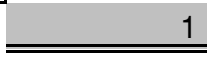

Allocation (percent):

\begin{tabular}{|l|r|r|r|}
\cline { 2 - 4 } \multicolumn{1}{c|}{} & \multicolumn{1}{c|}{ Land } & \multicolumn{1}{c|}{ Oil } & \multicolumn{2}{c|}{ Gas } \\
\hline Federal & 95 & & 95 \\
\hline State & 2 & & 2 \\
\hline Native & 3 & & 3 \\
\hline
\end{tabular}

Assessor's Name:

Ken Bird \& Phil Nelson

Date of Data Entry MM/DD/YYYY:

Date of Simulation Run MM/DD/YYYY:

$10 / 23 / 01$

$2 / 6 / 02$ 
Table 26d: Distribution of fited hydrocarbon volume attributes, trap depth, and number of prospects for the Ellesmerian Echooka South Play

Elles Echooka S
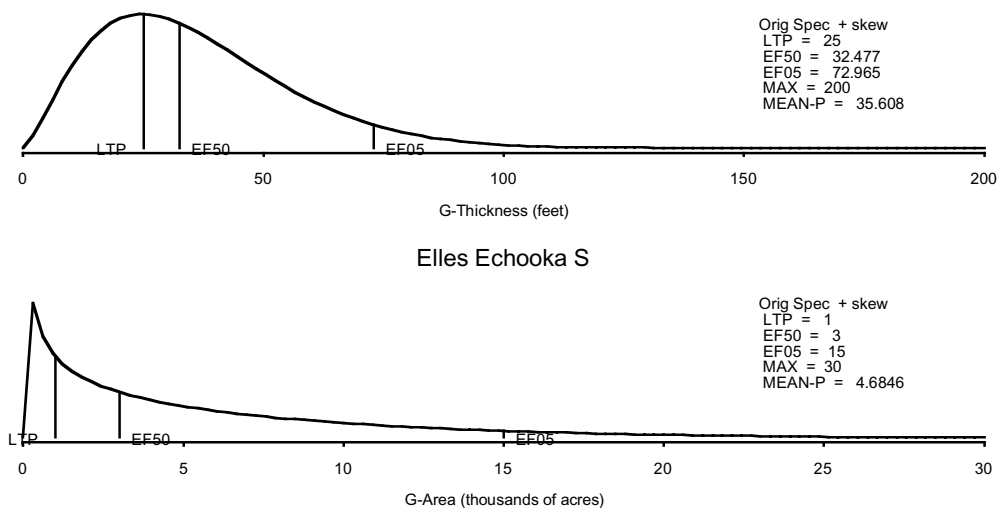

Elles Echooka S

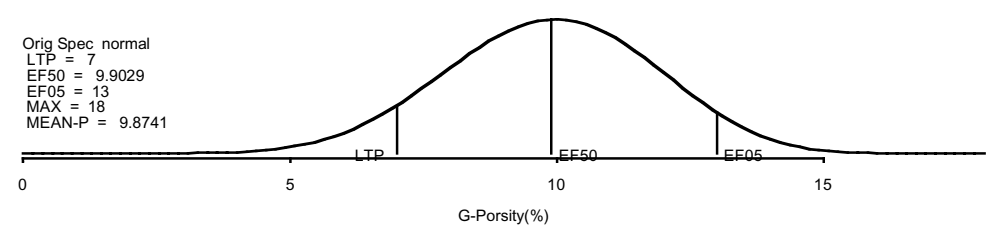

Elles Echooka S

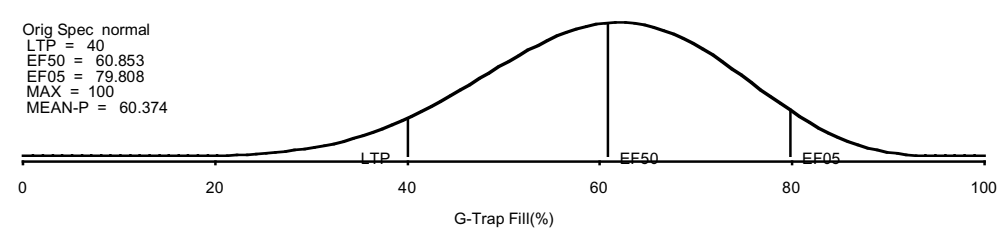

Elles Echooka S

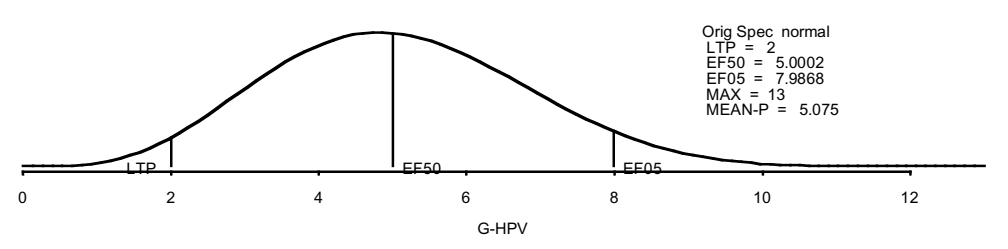

Elles Echooka S

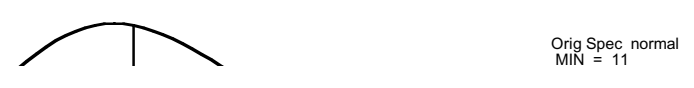

Elles Echooka S Truncated Portion

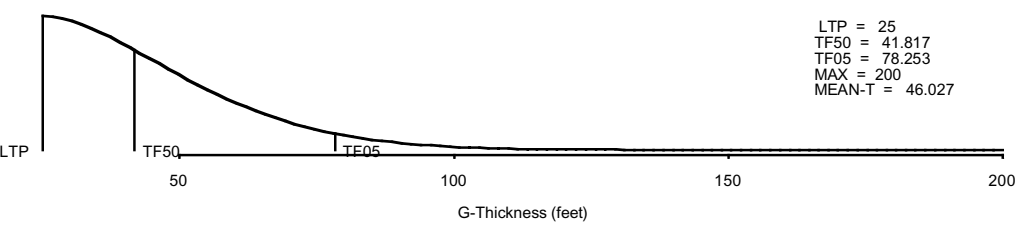

Elles Echooka S Truncated Portion

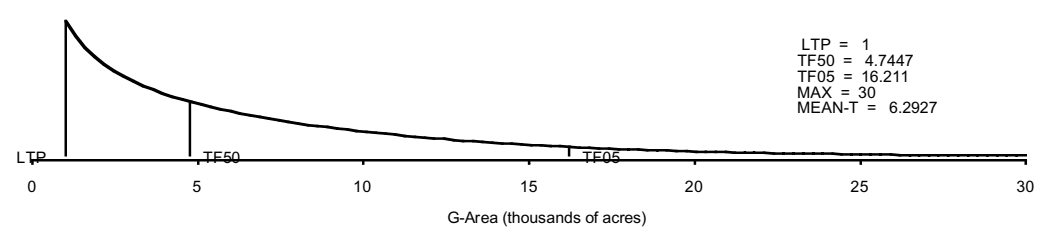

Elles Echooka S Truncated Portion

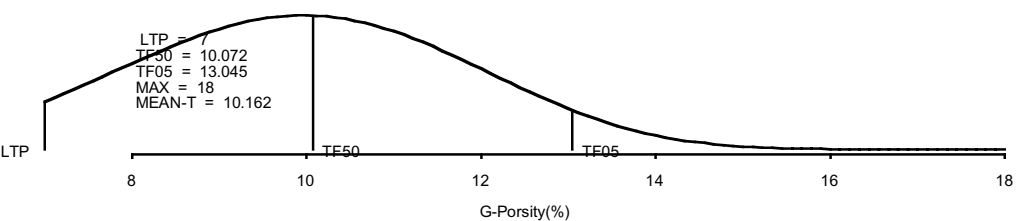

Elles Echooka S Truncated Portion

(TP

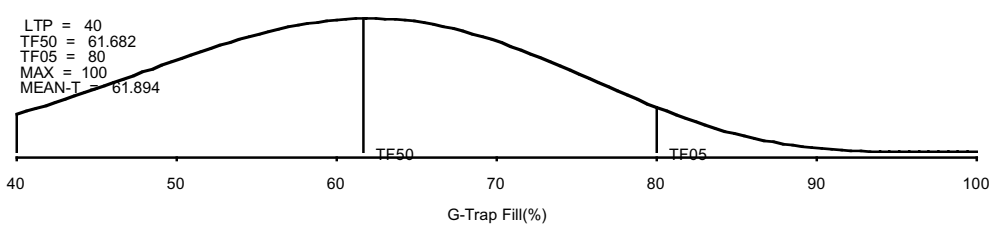

Elles Echooka S Truncated Portion

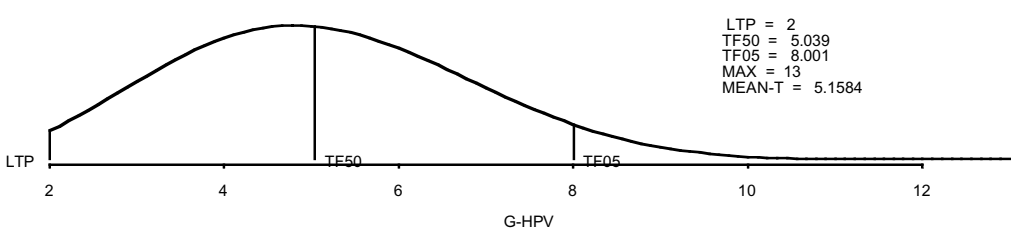




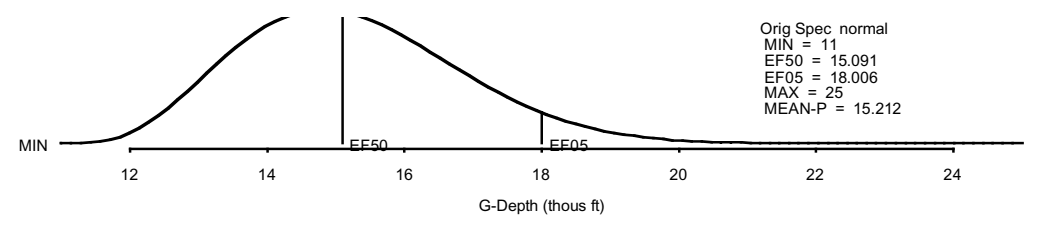

Elles Echooka S

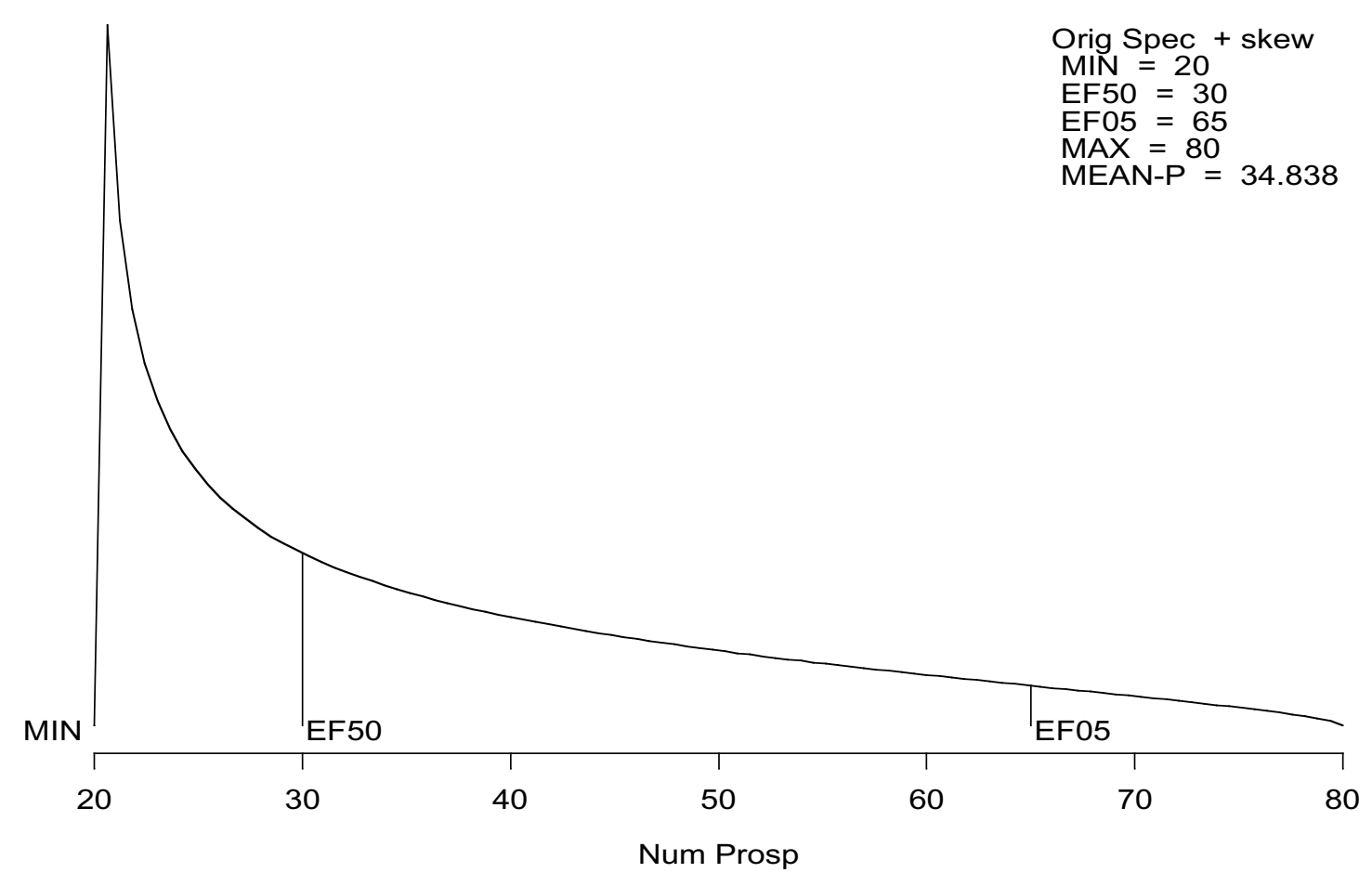


Table 26e: Estimated undiscovered oil and gas resources for the Ellesmerian Echooka South Play Deposit

\section{Ellesmerian Echooka S}

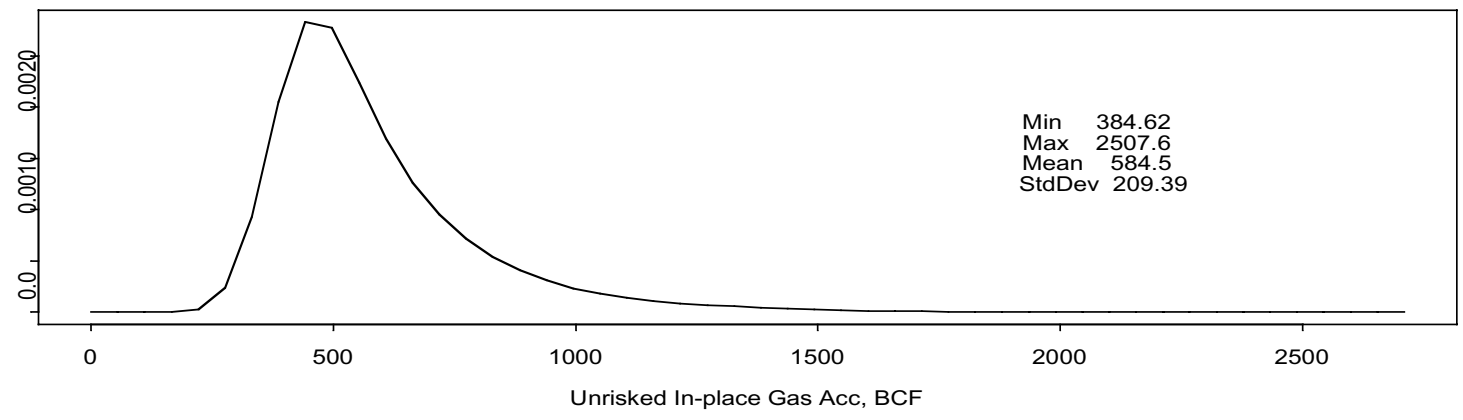

\begin{tabular}{|c|c|c|c|c|c|c|}
\hline \\
\hline \multicolumn{7}{|l|}{ Resource } \\
\hline In-place oil & MMBO & & & & & \\
\hline In-place NA gas & BCFG & 777.19 & 1162.52 & 0.00 & 0.00 & 3211.43 \\
\hline Recov oil & MMBO & & & & & \\
\hline Recov assoc diss gas & BCFG & & & & & \\
\hline Recov NA gas & BCFG & 505.17 & 755.64 & 0.00 & 0.00 & 2087.43 \\
\hline Recov NGL (ADG) & MMBO & & & & & \\
\hline Recov NGL (NAG) & MMBO & 14.00 & 21.00 & 0.00 & 0.00 & 58.31 \\
\hline \multicolumn{7}{|l|}{ Num oil deposits } \\
\hline Num NA gas deposits & & 1.33 & 1.94 & 0.00 & 0.00 & 5.00 \\
\hline
\end{tabular}

RNS $=525$

\section{Play Totals Ellesmerian Echooka S}

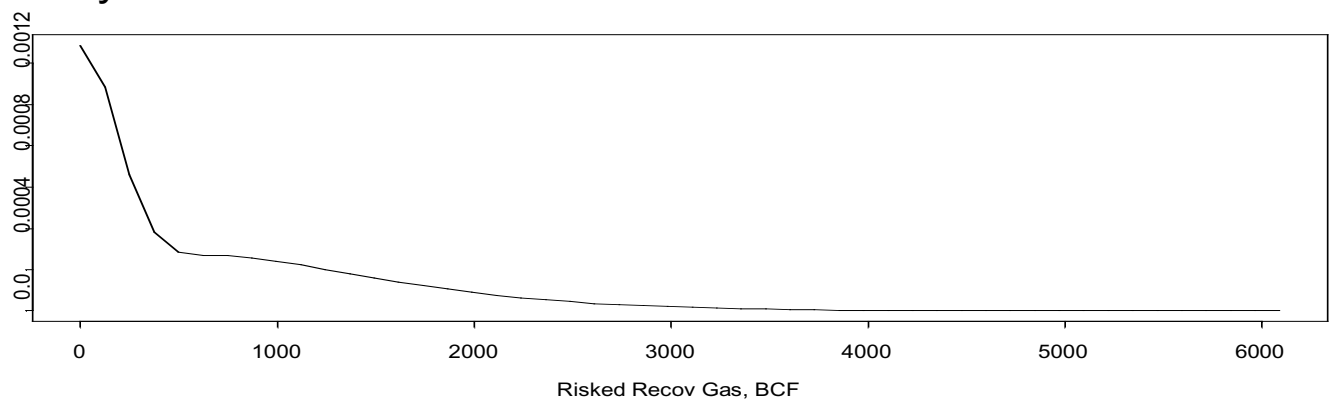


Table 26f: Estimated undiscovered oil and gas resources for the Ellesmerian Echooka South Play showing number of deposits and volumes by accumulation size class

\begin{tabular}{rrrrrr} 
Start size class & Oil deposits & Oil (MMBO_Start size class & Gas deposits & NA Gas (BCFG) \\
0 & & 0 & 0.00 & 0.00 \\
8 & & 48 & 0.00 & 0.00 \\
16 & & 96 & 0.00 & 0.00 \\
32 & & 192 & 0.87 & 264.72 \\
64 & & 384 & 0.43 & 212.48 \\
128 & & 768 & 0.03 & 27.84 \\
256 & & 1536 & 0.00 & 0.16 \\
512 & 0.00 & 3072 & \\
\hline Totals & 0.00 & & \\
\hline
\end{tabular}
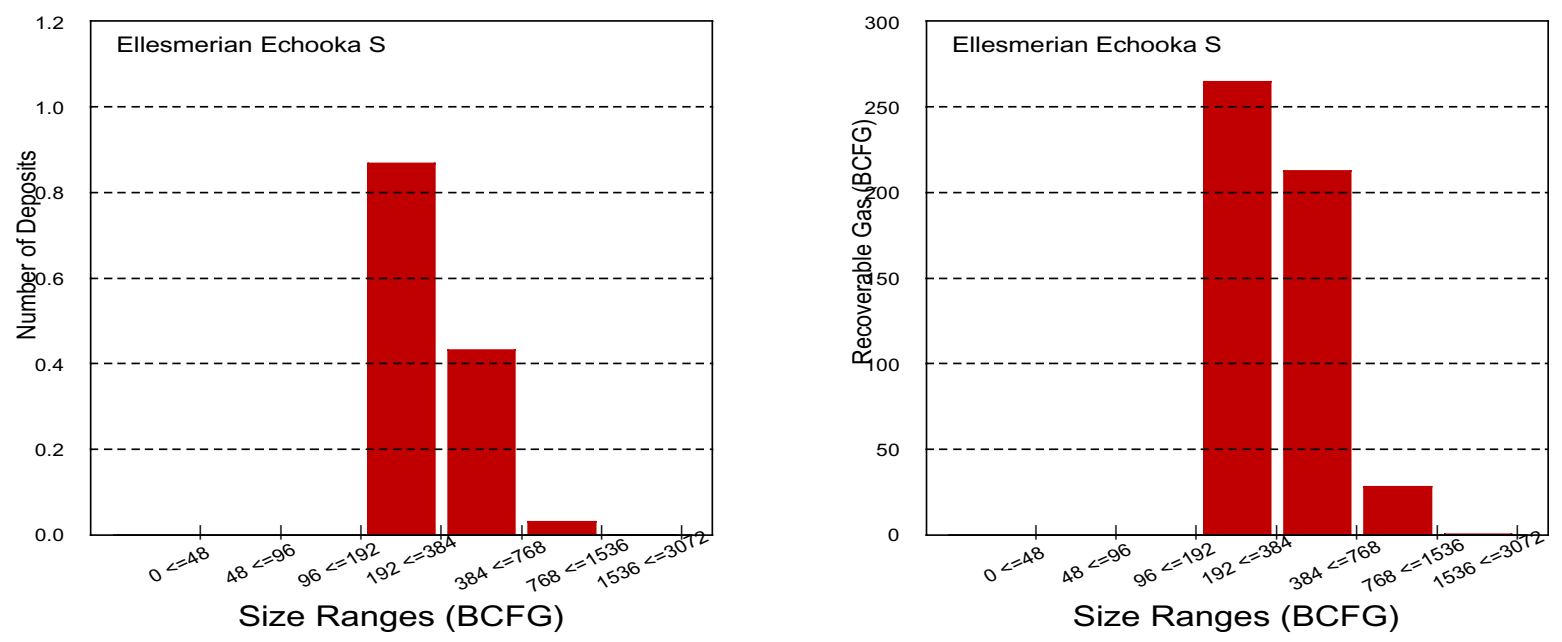
Table 27a: Input values for oil accumulations in the Ellesmerian Lisburne North Play

NPRA Assessment Form-2001

PLAY: Ellesmerian Lisburne North

\begin{tabular}{l|r|r|} 
Play area: & 3,394 & $10^{3}$ Acres \\
\hline
\end{tabular}

\section{OIL ACCUMULATION VOLUME PARAMETERS}

\begin{tabular}{|c|c|c|c|c|c|c|c|}
\hline \multirow[b]{2}{*}{ ATTRIBUTES } & \multirow{2}{*}{$\begin{array}{l}\text { Est Shape } \\
(1 \text { to } 5)\end{array}$} & \multicolumn{4}{|c|}{ PROB OF AND GREATER THAN } & \multirow{2}{*}{$\begin{array}{l}\text { Knowledge } \\
\text { Level } 1-3^{5}\end{array}$} & \\
\hline & & LTP & 0.50 & 0.05 & Max & & \\
\hline \multirow{4}{*}{$\begin{array}{l}\text { NET RESERVOIR THICKNESS } \\
\text { AREA OF CLOSURE }^{2} \\
\text { POROSITY }^{3,4} \\
\text { TRAP FILL }^{3}\end{array}$} & 4 & 40 & 100 & 200 & 300 & 3 & 0.56 Corr (nrt,ac) \\
\hline & 2 & 1.5 & 2.0 & 10.0 & 20.0 & 3 & \multirow[b]{3}{*}{ Enter POR*Sw } \\
\hline & 2 & 7 & 10 & 18 & 22 & 2 & \\
\hline & 6 & 50 & 80 & 99 & 100 & & \\
\hline \multicolumn{2}{|l|}{ HYDROCARBON PORE VOL ${ }^{3,4}$} & 5 & 8 & 16 & 20 & & 2 \\
\hline \multirow{2}{*}{\multicolumn{2}{|c|}{$\begin{array}{l}\text { Approx mm bbl (fvf }=1 \text { ) } \\
\text { Recov } \mathrm{mm} \text { bbl at surface }\end{array}$}} & 11.6 & 99.3 & 2457.7 & 9309.6 & & \\
\hline & & 2.4 & 20.8 & 515.6 & 1952.9 & & \\
\hline \multicolumn{7}{|c|}{$\begin{array}{l}\text { 1-thickness in feet, 2-thousands of acres, 3-percent, 4-correlation between Porosity and Water Saturation }=-1.0 \\
\text { 5-Knowledge Level: 1=High, 2=Medium, 3=Low; LTP=Left Truncation Point }\end{array}$} & \\
\hline \multirow{2}{*}{$\begin{array}{l}\text { TRAP DEPTH (in } 1000 \mathrm{ft} \text { ) } \\
\text { (from sea level) }\end{array}$} & \begin{tabular}{|r|}
4 \\
\end{tabular} & 8 & 10 & 11 & 15 & 1 & \\
\hline & \multicolumn{4}{|c|}{ Surface to sea level correction $(1000 \mathrm{ft})$ : } & 0.097 & & \\
\hline
\end{tabular}

\section{OIL ACCUMULATION CHARACTERISTICS}

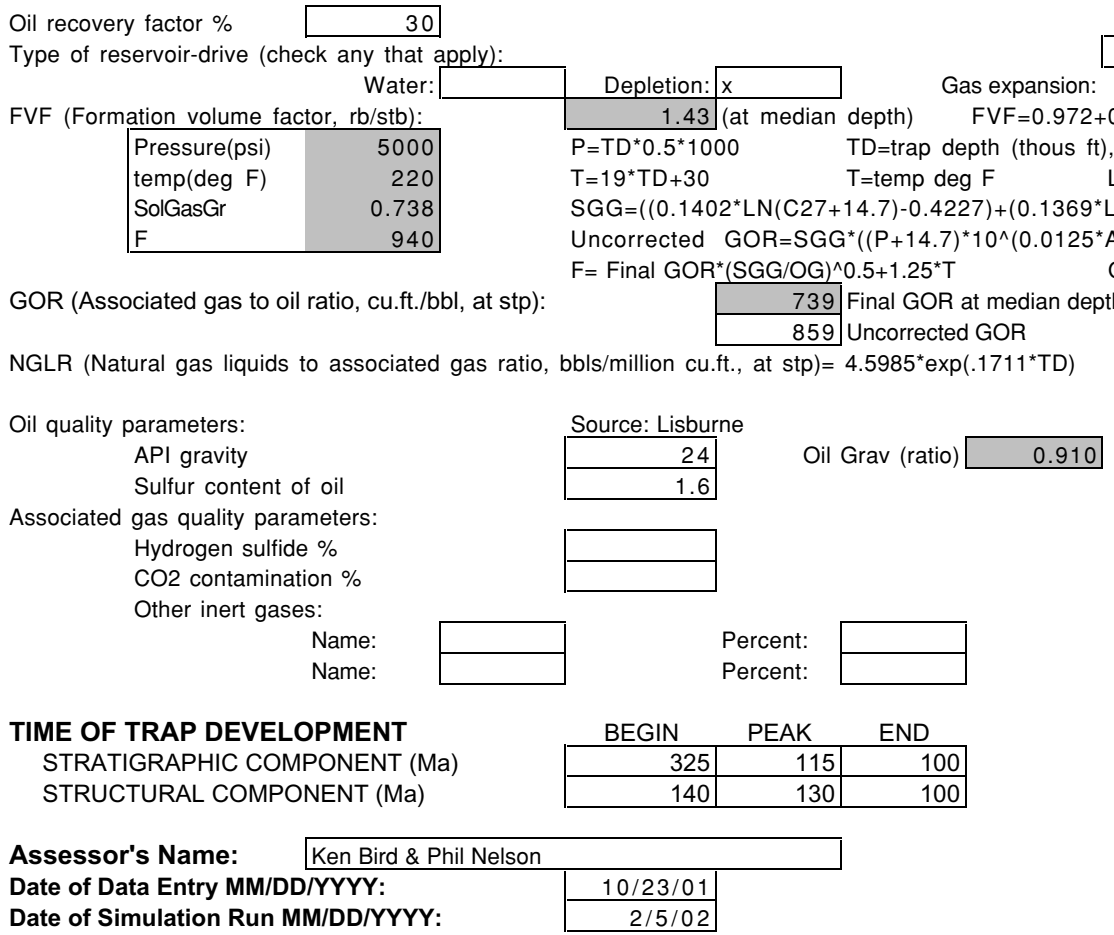

Note: only enter play name and assessor's name on Oil worksheet 
Table 27b: Input values for nonassociated gas accumulations in the Ellesmerian Lisburne North Play NPRA Assessment Form-2001

PLAY: Ellesmerian Lisburne North

NONASSOCIATED GAS ACCUMULATION VOLUME PARAMETERS

\begin{tabular}{|c|c|c|c|c|c|c|c|}
\hline \multirow[b]{2}{*}{ ATTRIBUTES } & \multirow{2}{*}{$\begin{array}{l}\text { Est Shape } \\
(1 \text { to } 5)\end{array}$} & \multicolumn{4}{|c|}{ PROB OF AND GREATER THAN } & \multirow{2}{*}{$\begin{array}{l}\text { Knowledge } \\
\text { Level } 1-3^{5}\end{array}$} & \\
\hline & & LTP & 50 & 5 & Max & & \\
\hline NET RESERVOIR THICKNESS ${ }^{1}$ & 4 & 40 & 100 & 200 & 300 & 3 & 0.56 Corr $(\mathrm{nrt}, \mathrm{ac})$ \\
\hline \multirow{3}{*}{$\begin{array}{l}\text { AREA OF CLOSURE } \\
\text { POROSITY,4 } \\
\text { TRAP FILL }^{3}\end{array}$} & 2 & 1.5 & 2.0 & 10.0 & 20.0 & 3 & \multirow[b]{3}{*}{ Uses oil POR ${ }^{\star}$ Sw } \\
\hline & 2 & 7 & 10 & 18 & 22 & 2 & \\
\hline & 6 & 50 & 80 & 99 & 100 & & \\
\hline \multicolumn{2}{|l|}{ HYDROCARBON PORE VOL 3.4} & 5 & 8 & 16 & 20 & & 2 \\
\hline \multirow{2}{*}{\multicolumn{2}{|c|}{$\begin{array}{l}\text { Approx in place bcf } \\
\text { Recov bcf at surface }\end{array}$}} & 0.07 & 0.56 & 13.80 & 52.27 & & \\
\hline & & 10.8 & 92.3 & 2284.6 & 8653.9 & & \\
\hline \multicolumn{7}{|c|}{$\begin{array}{l}\text { 1-thickness in feet, 2-thousands of acres, 3-percent, 4-correlation between Porosity and Water Saturation }=-1.0 \\
\text { 5-Knowledge Level: 1=High, 2=Medium, 3=Low; LTP=Left Truncation Point }\end{array}$} & \\
\hline \multirow{2}{*}{$\begin{array}{l}\text { TRAP DEPTH (in } 1000 \mathrm{ft} \text { ) } \\
\text { (from sea level) }\end{array}$} & 4 & 8 & 10 & 11 & 15 & 1 & \\
\hline & & Surface to $s$ & evel corre & $(1000 \mathrm{ft}):$ & 0.097 & & \\
\hline
\end{tabular}

\section{NONASSOCIATED GAS ACCUMULATION CHARACTERISTICS}

NA Gas recovery factor \% 65

Type of reservoir-drive (check any that apply):

$$
\text { Water: Gas expansion: }
$$

Natural gas liquids plus condensate to non-associated gas (bbls/million cf) (in place):

$$
\text { NGL-NAG }=1.785^{\star} \mathrm{TD} \quad 17.9 \text { (at median) }
$$

Non-associated gas quality parameters:

Hydrogen sulfide \%

CO2 contamination \%

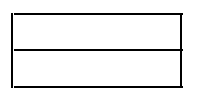

Other inert gases:

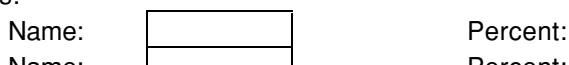

$$
\text { Name: } \quad \text { Percent: }
$$

Gas fvf

$$
254.7 \text { Gas } f v f=752.2^{*}\left(1-\operatorname{EXP}\left(-0.05728^{*} T D\right)\right)
$$
(at median depth)

$$
\begin{aligned}
& 113.3+21.1^{\star} T D-0.812^{*} T D^{\wedge} 2+0.0116^{*} T D^{\wedge} 3 \quad 5.67<T D<=30 \\
& T D=\text { trap depth (thous } \mathrm{ft} \text { ) }
\end{aligned}
$$

TIME OF TRAP DEVELOPMENT

STRATIGRAPHIC COMPONENT (Ma) STRUCTURAL COMPONENT (Ma)

\begin{tabular}{|r|r|r|}
\multicolumn{1}{c}{ BEGIN } & \multicolumn{1}{c}{ PEAK } & \multicolumn{1}{l|}{ END } \\
\hline 325 & 115 & 100 \\
\hline 140 & 130 & 100 \\
\hline
\end{tabular}

Assessor's Name: Ken Bird \& Phil Nelson

Date of Data Entry MM/DD/YYYY:

Date of Simulation Run MM/DD/YYYY: 
Table 27c: Input risking values for the Ellesmerian Lisburne North Play

NPRA Assessment Form-2001

Play: Ellesmerian Lisburne North

\section{RISKING}

MINIMUM ACCUMULATION SIZE, MAS (Millions of BBL in place)

50

PRERISKED FREQUENCY DISTRIBUTION (Oil plus Gas)

NUM OF PROSPECTS

$>$ MINIMUM SIZE

\section{PROB OF AND GREATER THAN}

\begin{tabular}{|c|c|c|c|c|c|}
\hline & \multicolumn{4}{|c|}{ PROB OF AND GREATER THAN } & \multirow{2}{*}{$\begin{array}{l}\text { Knowledge } \\
\text { Level } 1-3^{5}\end{array}$} \\
\hline Est Shape & Min & 50 & 5 & Max & \\
\hline 2 & 15 & 20 & 30 & 40 & 3 \\
\hline
\end{tabular}

5-Knowledge Level: 1=High, 2=Medium, 3=Low; LTP=Left Truncation Point

\section{ATTRIBUTES}

PLAY

ATTRIBUTES

Probability that play conta
PROBABILITY OF FAVORABLE

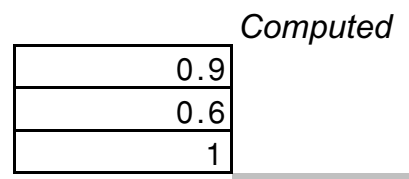

0.54

$\begin{array}{ll}\text { PROSPECT } & \text { CHARGE (c) } \\ \text { ATTRIBUTES } & \text { TRAP (t) } \\ & \text { TIMING (f) }\end{array}$

Probability that a randomly chosen prospect is favorable (cxtxf)

0.03

Play Attributes x Prospect Attributes (CxTxFxcxtxf)

0.0162

FRACTION OF ACCUMULATIONS BEING OIL

Fraction NA Gas=1-Fraction(Oil)

0.9

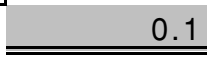

Allocation (percent):

\begin{tabular}{|l|r|r|r|}
\cline { 2 - 4 } \multicolumn{1}{c|}{} & \multicolumn{1}{c|}{ Land } & \multicolumn{1}{c|}{ Oil } & \multicolumn{1}{c|}{ Gas } \\
\hline Federal & 90 & 90 & 90 \\
\hline State & 8 & 8 & 8 \\
\hline Native & 2 & 2 & 2 \\
\hline
\end{tabular}

Assessor's Name:

Ken Bird \& Phil Nelson

Date of Data Entry MM/DD/YYYY:

Date of Simulation Run MM/DD/YYYY:

$10 / 23 / 01$

$2 / 5 / 02$ 
Table 27d: Distribution of fitted hydrocarbon volume attributes, trap depth, and number of prospects for the Ellesmerian Lisburne North Play

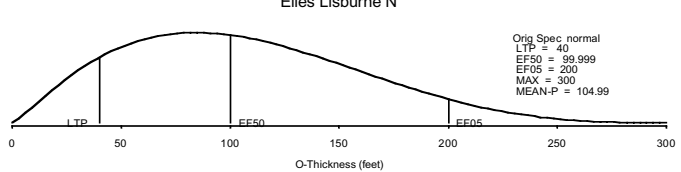

Elles Lisburne N

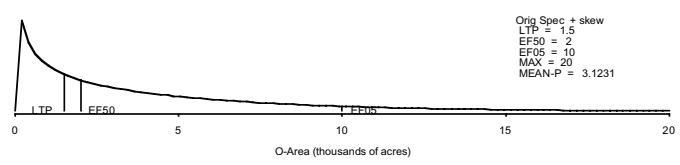

Elles Lisburne $N$

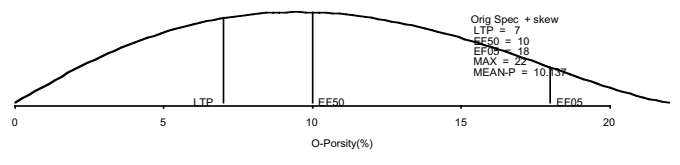

Elles Lisburne $\mathrm{N}$

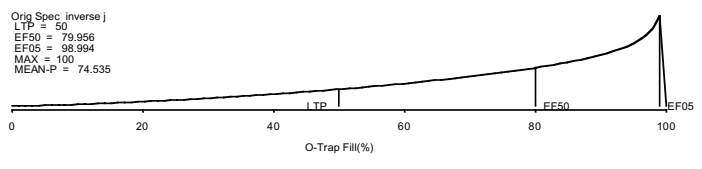

Elles Lisburne $\mathrm{N}$

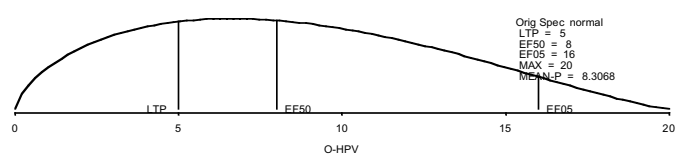

Elles Lisburne N

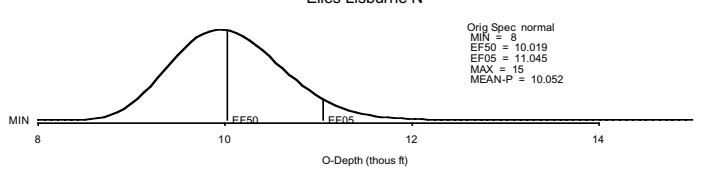

Elles Lisburne $\mathrm{N}$
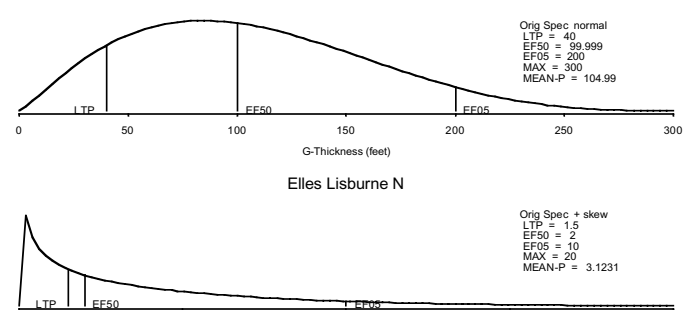

Elles Lisburne N Truncated Portion

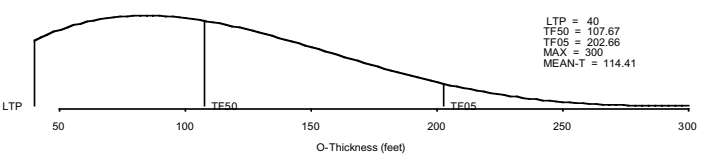

Elles Lisburne N Truncated Portion

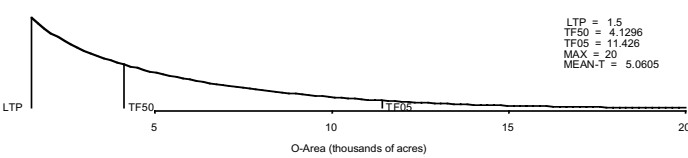

Elles Lisburne N Truncated Portion

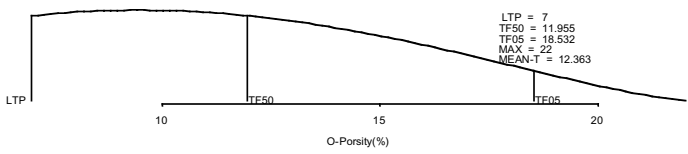

Elles Lisburne N Truncated Portio
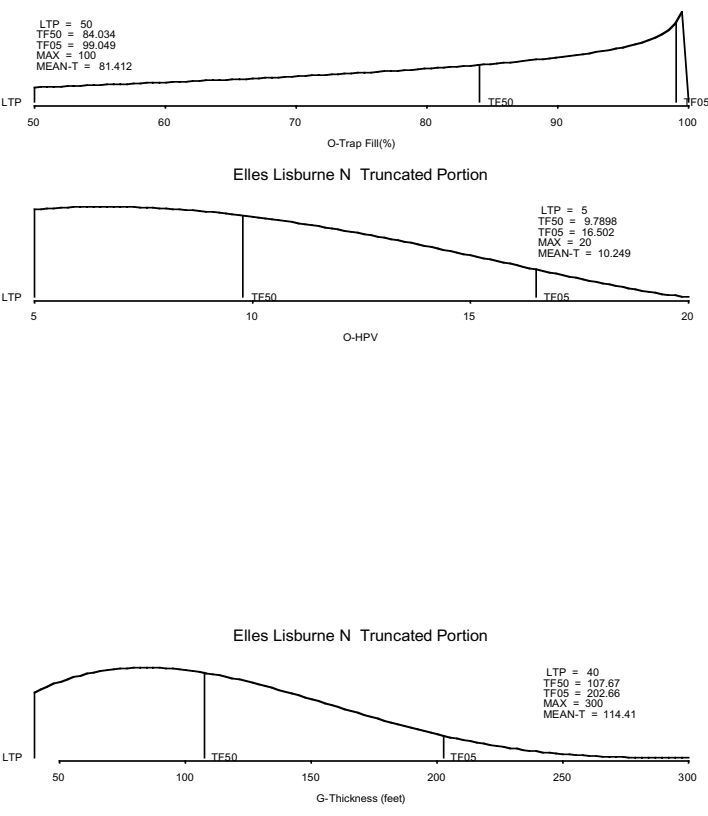

Elles Lisburne N Truncated Portion

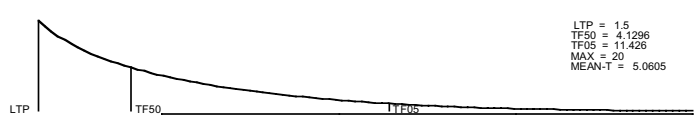



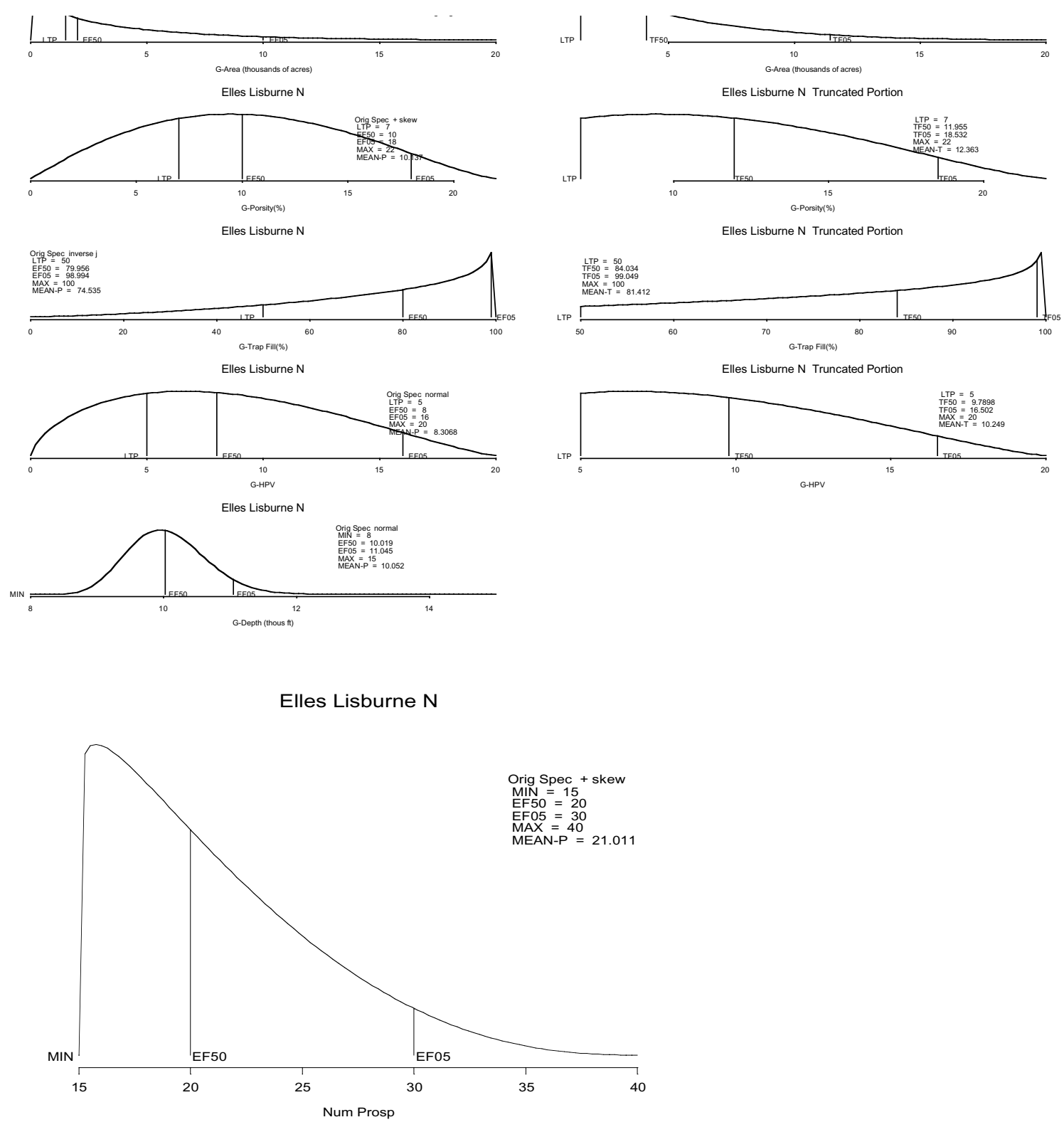
Table 27e: Estimated undiscovered oil and gas resources for the Ellesmerian Lisburne North Play Deposit

Ellesmerian Lisburne $\mathrm{N}$

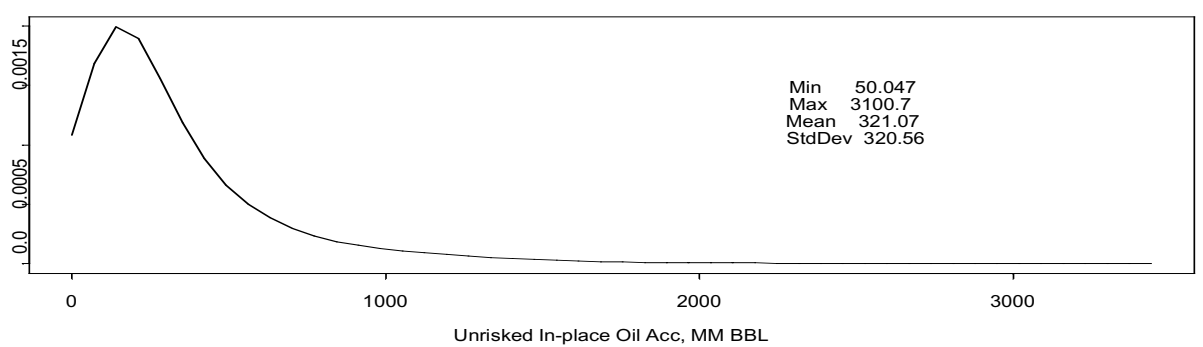

Ellesmerian Lisburne $\mathrm{N}$

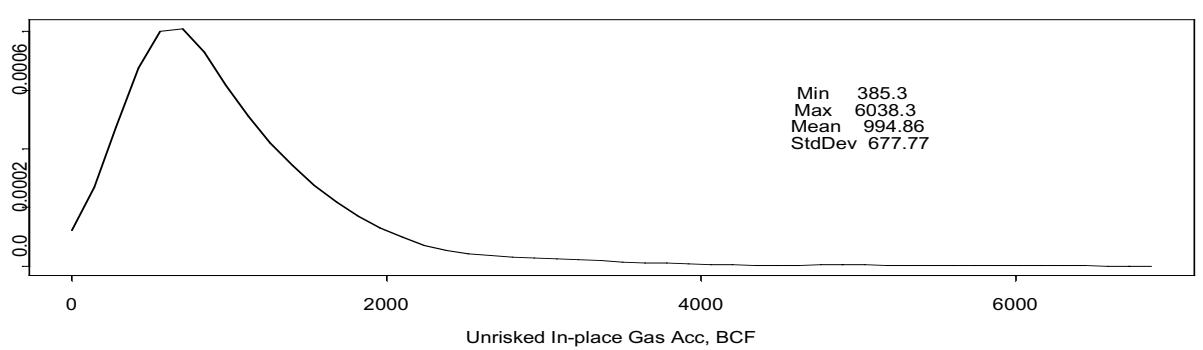

\begin{tabular}{|c|c|c|c|c|c|c|}
\hline $\begin{array}{l}\text { Play Stats } \\
\text { Resource }\end{array}$ & Unit & Mean & StdDev & $\mathrm{F} 95$ & $\mathrm{~F} 50$ & F05 \\
\hline In-place oil & $\mathrm{MMBO}$ & 95.68 & 262.52 & 0.00 & 0.00 & 601.71 \\
\hline In-place NA gas & BCFG & 33.52 & 224.72 & 0.00 & 0.00 & 0.00 \\
\hline Recov oil & MMBO & 28.70 & 78.76 & 0.00 & 0.00 & 180.51 \\
\hline Recov assoc diss gas & BCFG & 21.45 & 58.86 & 0.00 & 0.00 & 135.35 \\
\hline Recov NA gas & $\mathrm{BCFG}$ & 21.79 & 146.07 & 0.00 & 0.00 & 0.00 \\
\hline Recov NGL (ADG) & MMBO & 0.75 & 2.07 & 0.00 & 0.00 & 4.70 \\
\hline Recov NGL (NAG) & MMBO & 0.40 & 2.66 & 0.00 & 0.00 & 0.00 \\
\hline Num oil deposits & & 0.30 & 0.61 & 0.00 & 0.00 & 2.00 \\
\hline Num NA gas deposits & & 0.03 & 0.18 & 0.00 & 0.00 & 0.00 \\
\hline
\end{tabular}

RNS $=891$

Play Totals Ellesmerian Lisburne N

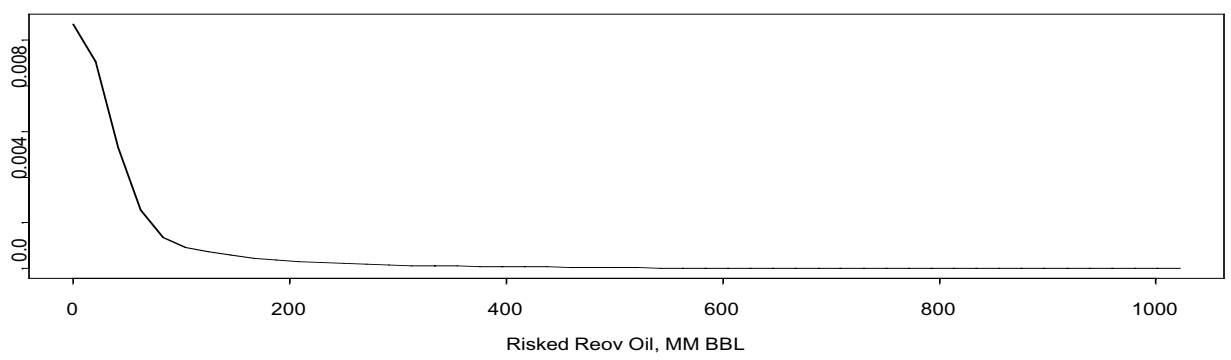

Play Totals Ellesmerian Lisburne N

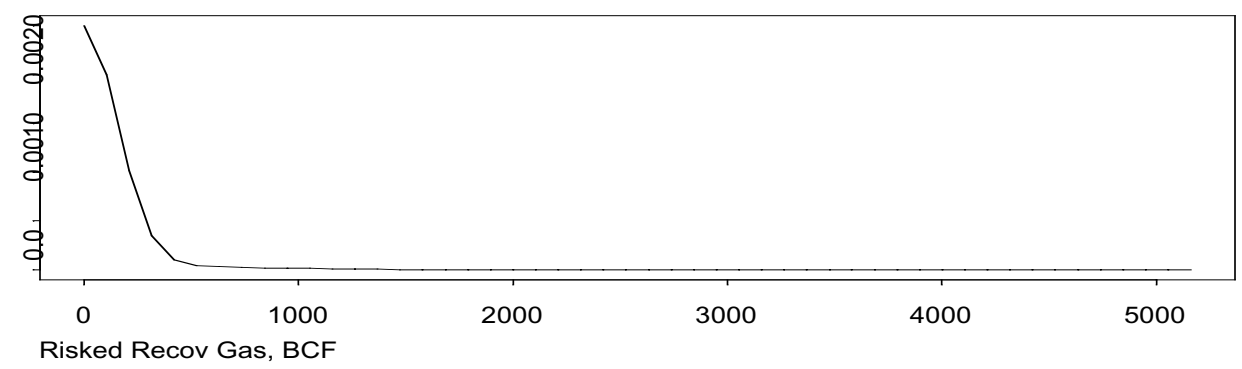


Table 27f: Estimated undiscovered oil and gas resources for the Ellesmerian Lisburne North Play showing number of deposits and volumes by accumulation size class

\begin{tabular}{rrrrrr} 
Start size class & Oil deposits & Oil (MMBO_ Start size class & Gas deposits & NA Gas (BCFG) \\
0 & 0.00 & 0.00 & 0 & 0.00 & 0.00 \\
8 & 0.00 & 0.07 & 48 & 0.00 & 0.00 \\
16 & 0.06 & 1.50 & 96 & 0.00 & 0.00 \\
32 & 0.08 & 3.87 & 192 & 0.01 & 3.31 \\
64 & 0.08 & 7.15 & 384 & 0.01 & 7.39 \\
128 & 0.05 & 8.43 & 768 & 0.01 & 7.99 \\
256 & 0.02 & 6.46 & 1536 & 0.00 & 2.55 \\
512 & 0.00 & 1.24 & 3072 & 0.00 & 0.55 \\
\hline Totals & 0.30 & 28.71 & & 0.03 & 21.79 \\
\hline
\end{tabular}
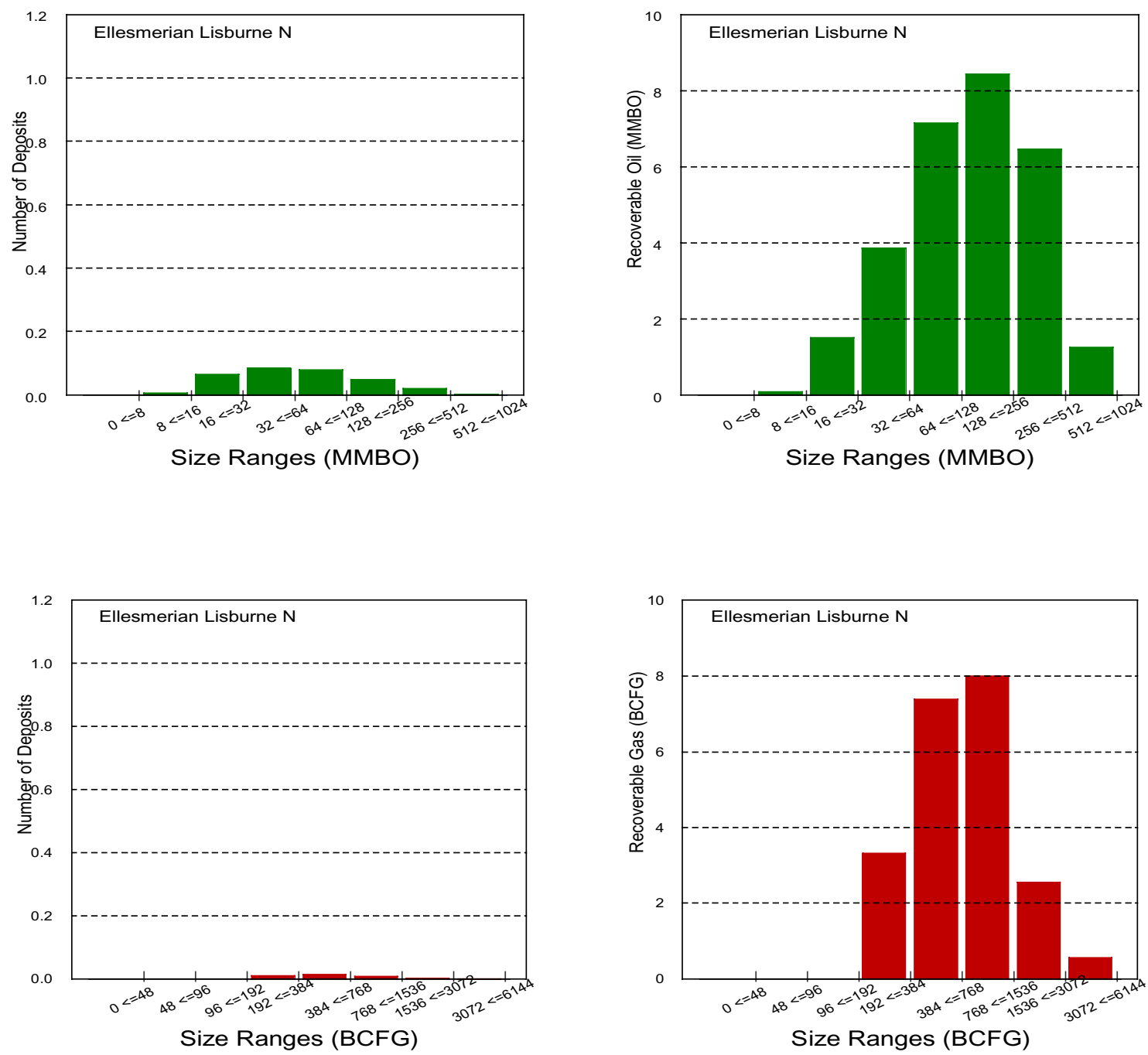
Table 28a: Input values for oil accumulations in the Ellesmerian Lisburne South Play

NPRA Assessment Form-2001

PLAY: Ellesmerian Lisburne South

Play area:
$10^{3}$ Acres

\section{OIL ACCUMULATION VOLUME PARAMETERS}

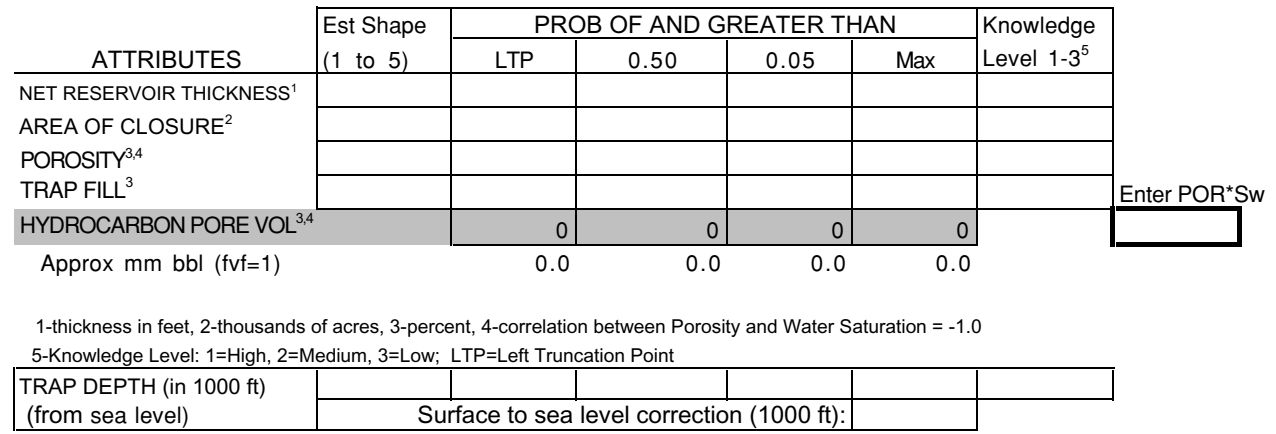

\section{OIL ACCUMULATION CHARACTERISTICS}

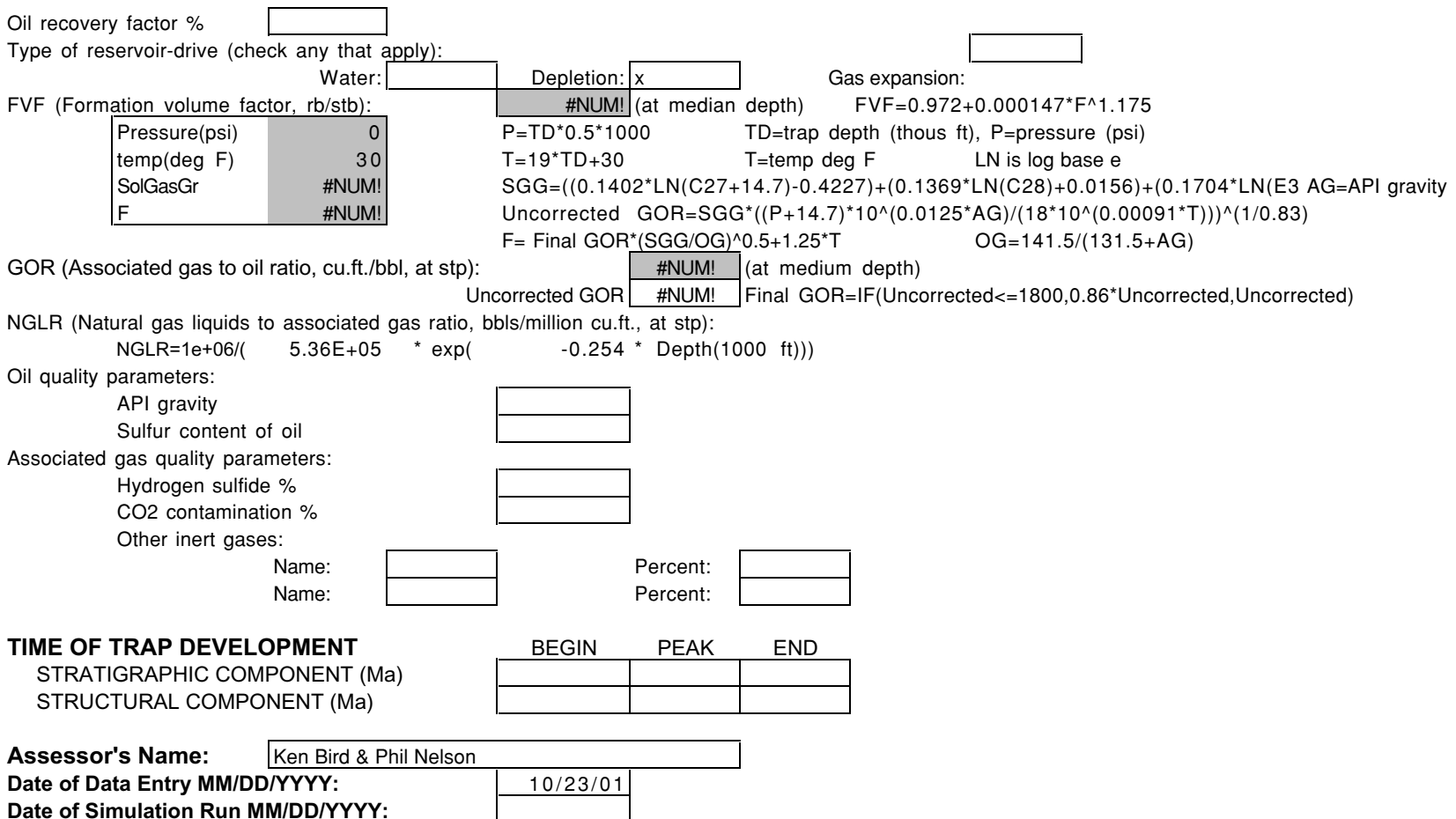

Note: only enter play name and assessor's name on Oil worksheet 
Table 28b: Input values for nonassociated gas accumulations in the Ellesmerian Lisburne South Play NPRA Assessment Form-2001

PLAY: Ellesmerian Lisburne South
Play area:
\begin{tabular}{l|l|l|l}
6,952 & $10^{3}$ Acres \\
\hline
\end{tabular}

\section{NONASSOCIATED GAS ACCUMULATION VOLUME PARAMETERS}

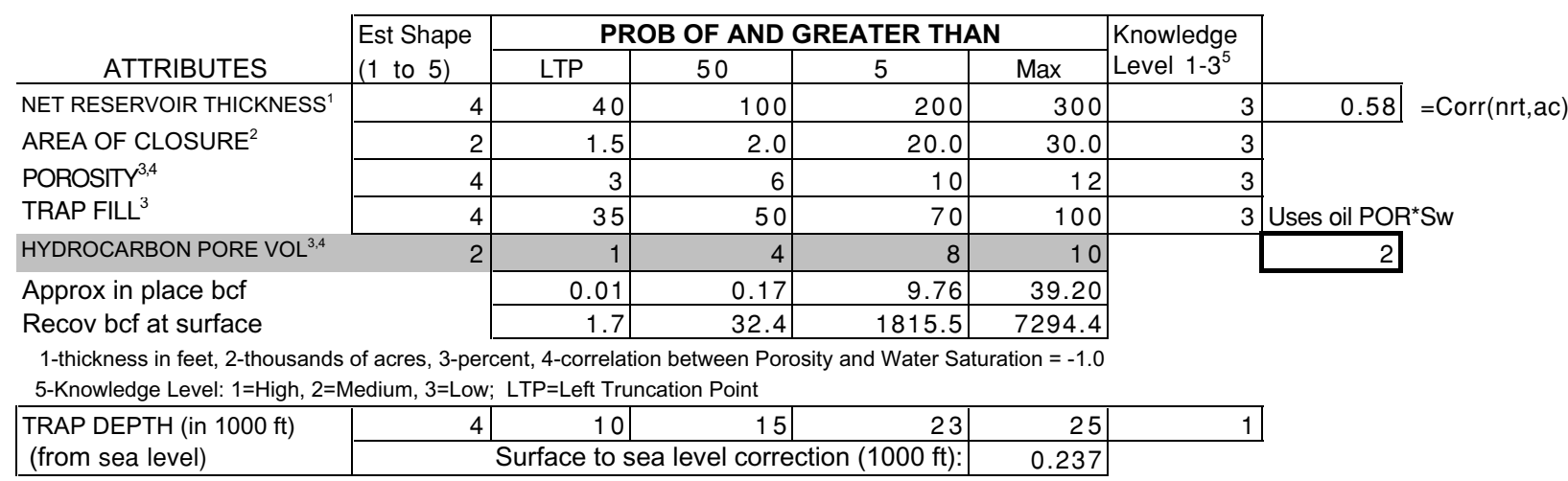

\section{NONASSOCIATED GAS ACCUMULATION CHARACTERISTICS}

NA Gas recovery factor \%

$65 \quad 14-\mathrm{Dec}$

Type of reservoir-drive (check any that apply):

$$
\text { Water: }
$$

Gas expansion:

Natural gas liquids plus condensate to non-associated gas (bbls/million cf) (in place): NGL-NAG $=1.785^{\star} \mathrm{TD}$ 26.8 (at median)

Non-associated gas quality parameters: Hydrogen sulfide \% CO2 contamination \% Other inert gases:

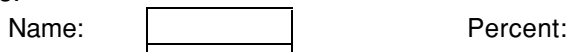

$$
\text { Name: } \quad \text { Percent: }
$$

Gas fvf 286.3 Gas $f v f=752.2^{*}\left(1-\operatorname{EXP}\left(-0.05728^{*} T D\right)\right.$ (at median depth)

$$
113.3+21.1^{*} T D-0.812^{*} T D
$$

TD=trap depth (thous $\mathrm{ft}$ )

\begin{tabular}{l|r|r|r|} 
TIME OF TRAP DEVELOPMEN1 & \multicolumn{1}{c}{ BEGIN } & \multicolumn{1}{r}{ PEAK } & \multicolumn{1}{c}{ END } \\
STRATIGRAPHIC COMPONENT (M & 350 & 275 & 245 \\
\hline STRUCTURAL COMPONENT (Ma) & 350 & 300 & 100 \\
\hline
\end{tabular}

\begin{tabular}{l|r|}
\hline Assessor's Name: $\quad$ Ken Bird \& Phil Nelson \\
\hline Date of Data Entry MM/DD/YYYY: & $10 / 23 / 01$ \\
\hline Date of Simulation Run MM/DD/YYYY: & $2 / 7 / 02$ \\
\hline
\end{tabular}


Table 28c: Input risking values for the Ellesmerian Lisburne South Play

NPRA Assessment Form-2001

Play: Ellesmerian Lisburne South

\section{RISKING}

MINIMUM ACCUMULATION SIZE, MAS (bcf recov)

PRERISKED FREQUENCY DISTRIBUTION (Oil plus Gas)

NUM OF PROSPECTS

$>$ MINIMUM SIZE

\section{PROB OF AND GREATER THAN}

5-Knowledge Level: 1=High, 2=Medium, 3=Low; LTP=Left Truncation Point

\section{ATTRIBUTES}

\section{OF FAVORABLE}

PLAY

CHARGE (C)

ATTRIBUTES

TRAP (T)

TIMING (F)

Probability that play contains at least 1 reservoir $>=$ minimum size (CXTXF)

$\begin{array}{ll}\text { PROSPECT } & \text { CHARGE (c) } \\ \text { ATTRIBUTES } & \text { TRAP ( } \mathrm{t} \text { ) } \\ & \text { TIMING (f) }\end{array}$

Probability that a randomly chosen prospect is favorable (cxtxf)

Play Attributes $x$ Prospect Attributes (CxTxFxcxtxf)

FRACTION OF ACCUMULATIONS BEING OIL

Fraction NA Gas=1-Fraction(Oil)

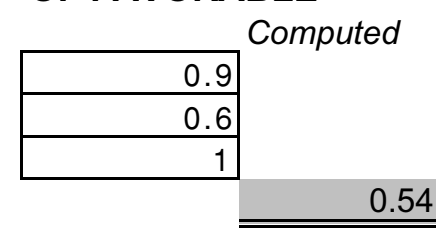

0

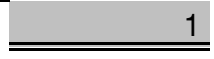

Allocation (percent):

\begin{tabular}{|l|r|r|r|}
\cline { 2 - 4 } \multicolumn{1}{c|}{} & \multicolumn{1}{c|}{ Land } & \multicolumn{1}{c|}{ Oil } & \multicolumn{2}{c|}{ Gas } \\
\hline Federal & 94 & & 97 \\
\hline State & 2 & & 1 \\
\hline Native & 4 & & 2 \\
\hline
\end{tabular}

Assessor's Name:

Ken Bird \& Phil Nelson

Date of Data Entry MM/DD/YYYY:

Date of Simulation Run MM/DD/YYYY:

\begin{tabular}{r|}
$10 / 23 / 01$ \\
\hline $2 / 7 / 02$ \\
\hline
\end{tabular} 
Table 28d: Distribution of fitted hydrocarbon volume attributes, trap depth, and number of prospects for the Ellesmerian Lisburne South Play
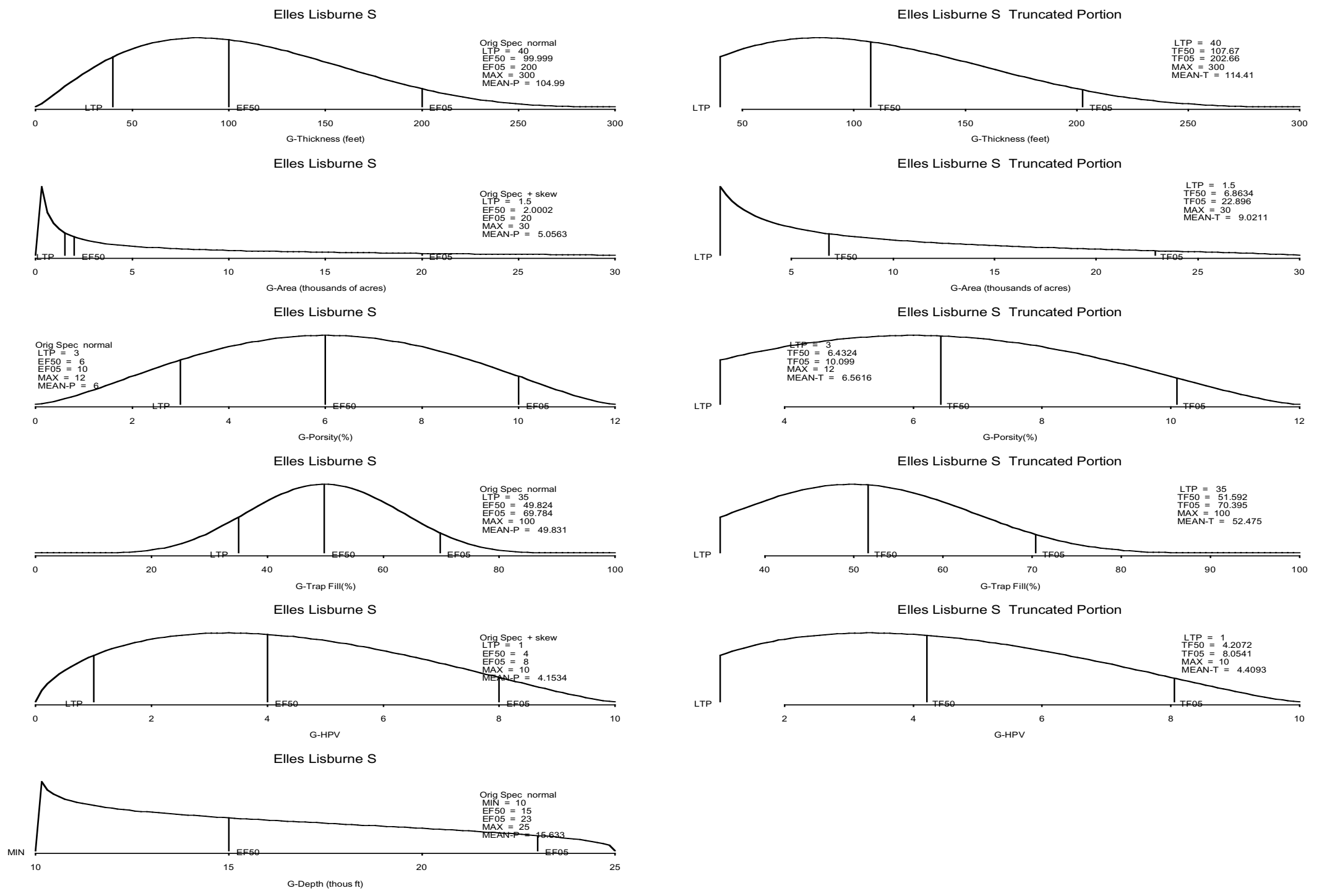


\section{Elles Lisburne S}

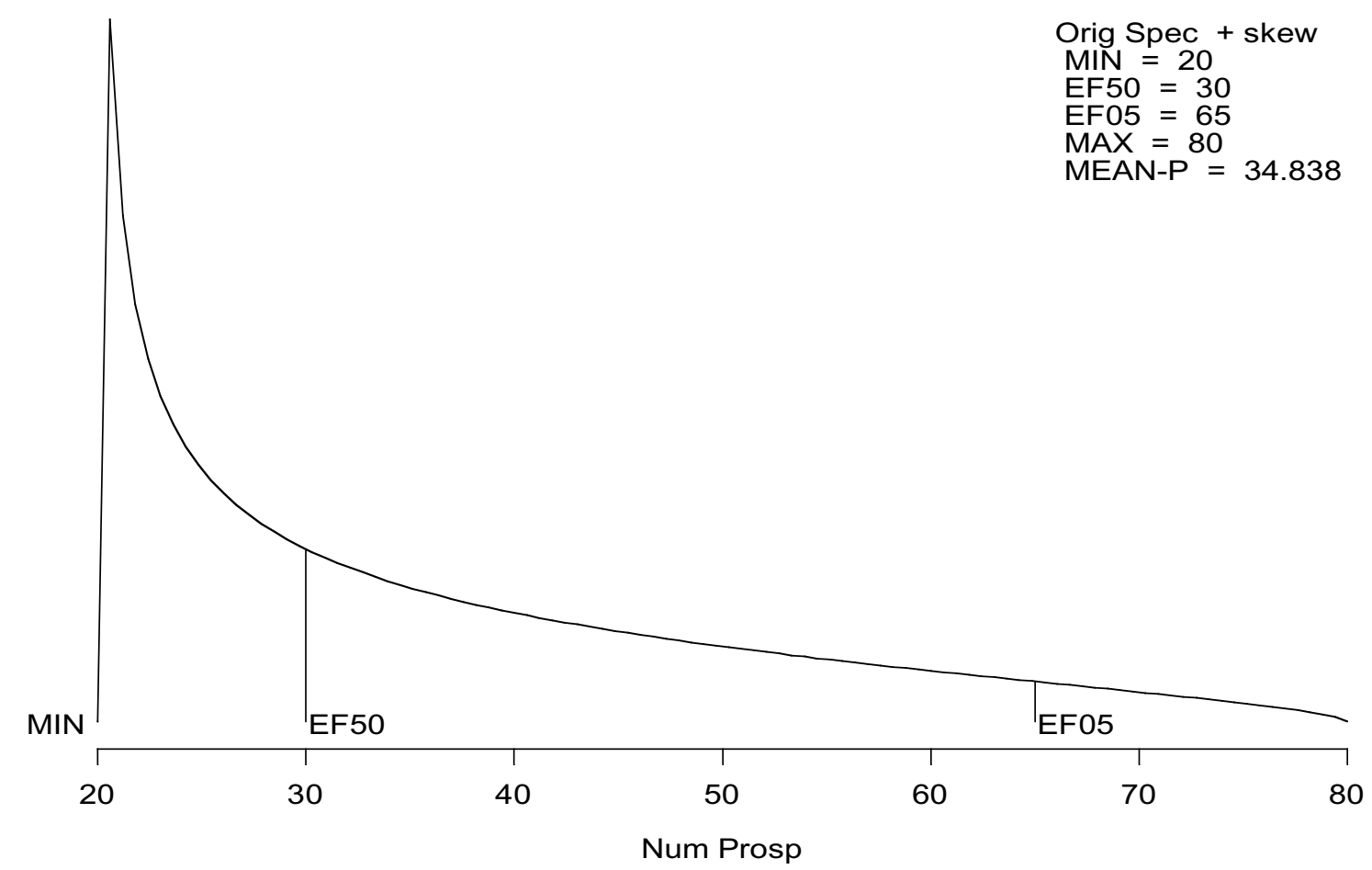


Table 28e: Estimated undiscovered oil and gas resources for the Ellesmerian Lisburne South Play Deposit

Ellesmerian Lisburne $\mathrm{S}$

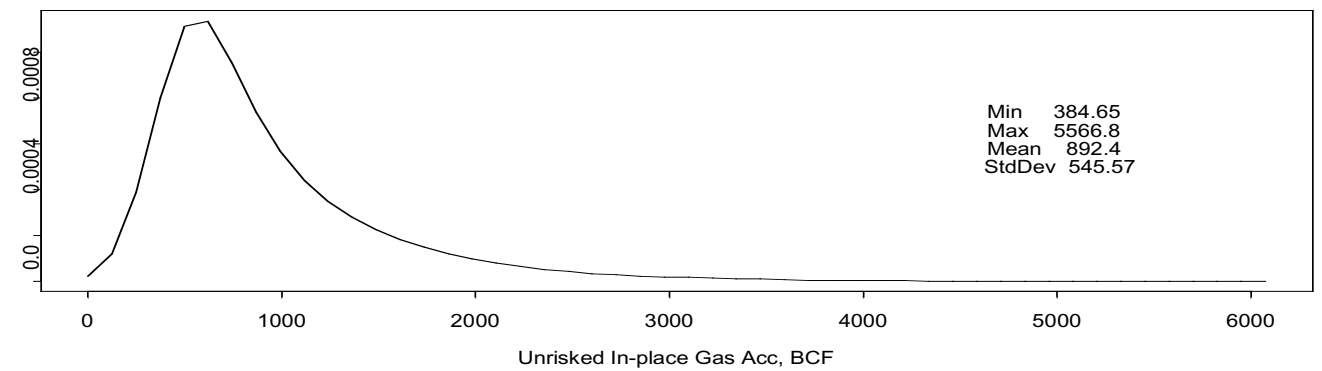

\begin{tabular}{|c|c|c|c|c|c|c|}
\hline $\begin{array}{l}\text { Play Stats } \\
\text { Resource }\end{array}$ & Unit & Mean & StdDev & F95 & F50 & F05 \\
\hline In-place oil & MMBO & & & & & \\
\hline In-place NA gas & BCFG & 993.83 & 1510.96 & 0.00 & 0.00 & 4203.00 \\
\hline Recov oil & MMBO & & & & & \\
\hline Recov assoc diss gas & BCFG & & & & & \\
\hline Recov NA gas & BCFG & 645.99 & 982.12 & 0.00 & 0.00 & 2731.95 \\
\hline Recov NGL (ADG) & MMBO & & & & & \\
\hline Recov NGL (NAG) & MMBO & 18.64 & 28.98 & 0.00 & 0.00 & 80.54 \\
\hline Num oil deposits & & & & & & \\
\hline Num NA gas deposits & & 1.11 & 1.57 & 0.00 & 0.00 & 4.00 \\
\hline
\end{tabular}

Play Totals Ellesmerian Lisburne $\mathrm{S}$

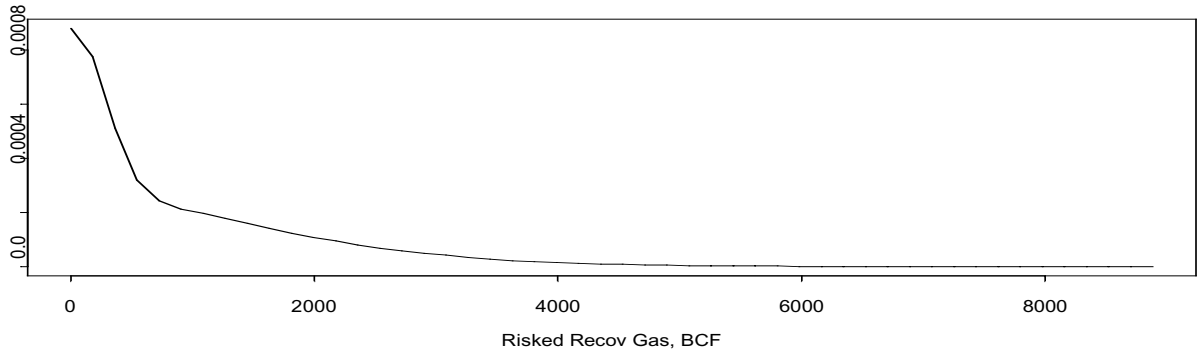


Table 28f: Estimated undiscovered oil and gas resources for the Ellesmerian Lisburne South Play showing number of deposits and volumes by accumulation size class

\begin{tabular}{rrrrrr|} 
Start size class & Oil deposits & Oil (MMBO_Start size class & Gas deposits & NA Gas (BCFG) \\
0 & & 0 & 0.00 & 0.00 \\
8 & & 48 & 0.00 & 0.00 \\
16 & & 96 & 0.00 & 0.00 \\
32 & & 192 & 0.41 & 128.02 \\
64 & & 384 & 0.47 & 249.96 \\
128 & & 768 & 0.20 & 210.95 \\
256 & & 1536 & 0.03 & 56.57 \\
512 & & 3072 & 0.00 & 0.55 \\
\hline Totals & 0.00 & 0.00 & & 1.11 & 646.04 \\
\hline
\end{tabular}
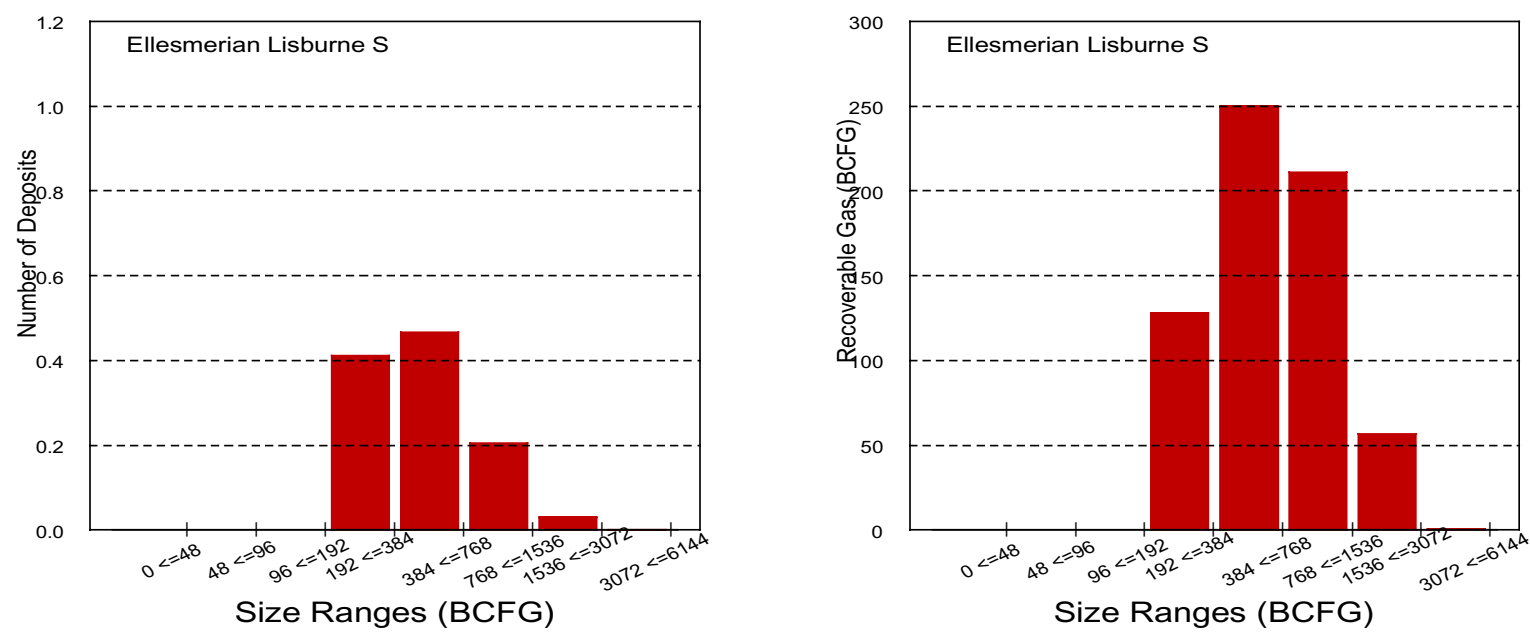
Table 29a: Input values for oil accumulations in the Ellesmerian Endicott Basins North Play

NPRA Assessment Form-2001

PLAY:|Ellesmerian Endicott Basins North

Play area: $357 \mid 10^{3}$ Acres

\section{OIL ACCUMULATION VOLUME PARAMETERS}

\begin{tabular}{l}
\cline { 2 - 7 } \\
\cline { 2 - 7 } \multicolumn{1}{c|}{$\begin{array}{l}\text { Est Shape } \\
\text { ATtRIBUTES }\end{array}$}
\end{tabular}

\section{OIL ACCUMULATION CHARACTERISTICS}

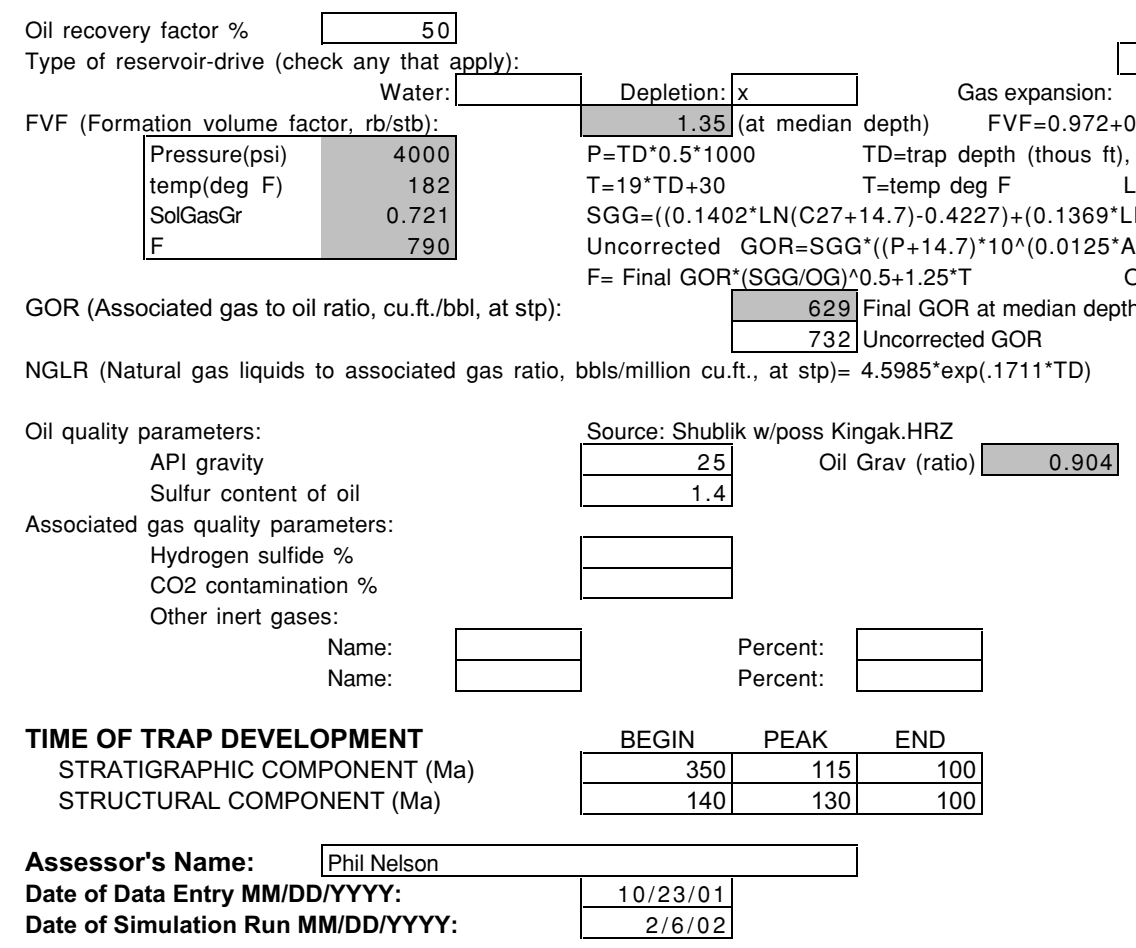

Date of Simulation Run MM/DD/YYYY:

$\mathrm{N}(\mathrm{C} 28)+0.0156)+\left(0.1704^{*} \mathrm{LN}(\mathrm{E3} \mathrm{AG}=\mathrm{API}\right.$ gravity

(0.00091*T)) $)^{\wedge}(1 / 0.83)$

$\mathrm{G}=141.5 /(131.5+A G)$

$$
\begin{array}{r|l}
629 & \text { Final GOR at median depth } \\
732 & \text { Uncorrected GOR }
\end{array}
$$

NGLR (Natural gas liquids to associated gas ratio, bbls/million cu.ft., at $\operatorname{stp})=4.5985^{\star} \exp \left(.1711^{\star} T D\right.$ )

18.1 (median depth)

Note: only enter play name and assessor's name on Oil worksheet 
Table 29b: Input values for nonassociated gas accumulations in the Ellesmerian Endicott North Play NPRA Assessment Form-2001

PLAY:

NONASSOCIATED GAS ACCUMULATION VOLUME PARAMETERS

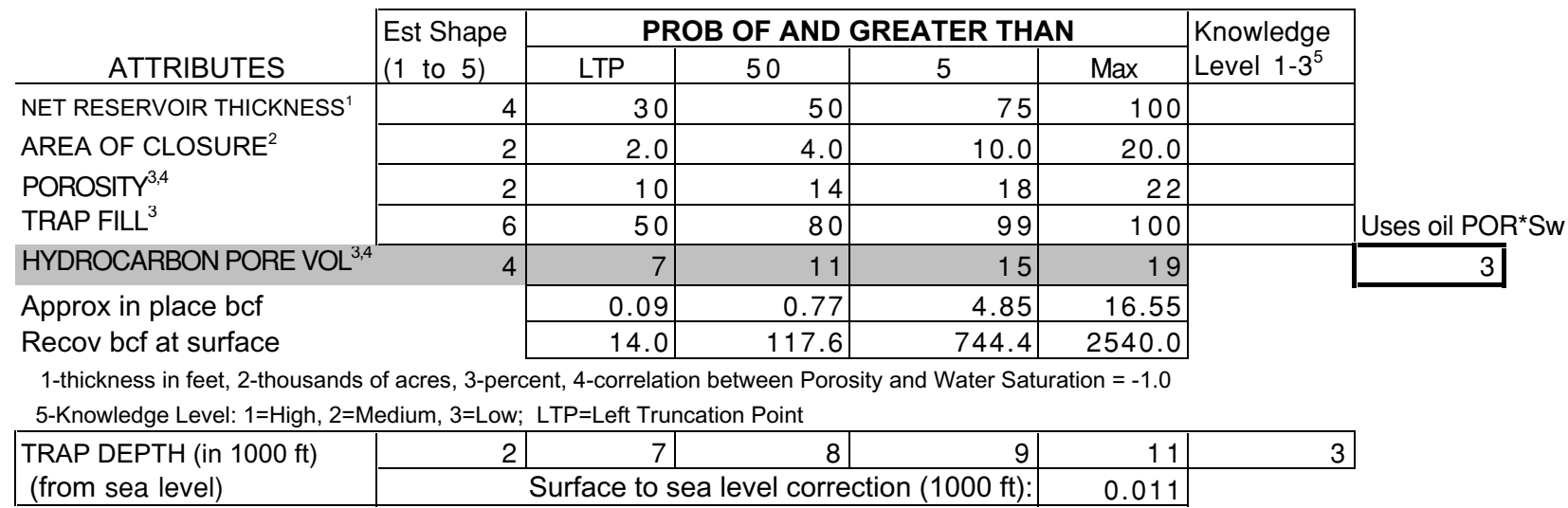

\section{NONASSOCIATED GAS ACCUMULATION CHARACTERISTICS}

NA Gas recovery factor \% 65

Type of reservoir-drive (check any that apply):

$$
\text { Water: }
$$

Gas expansion:

Natural gas liquids plus condensate to non-associated gas (bbls/million cf) (in place): NGL-NAG $=1.785^{*} \mathrm{TD}$

$$
14.3 \text { (at median) }
$$

Non-associated gas quality parameters: Hydrogen sulfide \% CO2 contamination \% Other inert gases:

Name: Name:

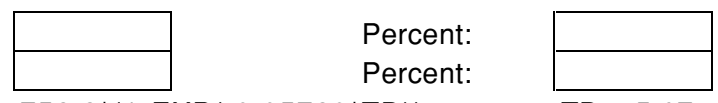

Gas fvf 236.1 (at median depth)

Gas $f v f=752.2^{*}\left(1-\operatorname{EXP}\left(-0.05728^{*} T D\right)\right)$ $\mathrm{TD}<=5.67$ thous $\mathrm{ft}$ $113.3+21.1^{*} T D-0.812^{*} T^{\wedge} 2+0.0116^{*} T^{\wedge} 3 \quad 5.67<T D<=30$ $\mathrm{TD}=$ trap depth (thous $\mathrm{ft}$ )

TIME OF TRAP DEVELOPMENT STRATIGRAPHIC COMPONENT (Ma)

\begin{tabular}{|c|c|c|}
\hline BEGIN & PEAK & END \\
\hline 350 & 115 & 100 \\
\hline 140 & 130 & 100 \\
\hline
\end{tabular}
STRUCTURAL COMPONENT (Ma)

Assessor's Name: Phil Nelson Date of Data Entry MM/DD/YYYY: Date of Simulation Run MM/DD/YYYY: $10 / 23 / 01$ 
Table 29c: Input risking values for the Ellesmerian Endicott Basins North Play

NPRA Assessment Form-2001

Play: Ellesmerian Endicott Basins North

\section{RISKING}

MINIMUM ACCUMULATION SIZE, MAS (Millions of BBL in place)

50

PRERISKED FREQUENCY DISTRIBUTION (Oil plus Gas)

NUM OF PROSPECTS

$>$ MINIMUM SIZE

PROB OF AND GREATER THAN

5-Knowledge Level: 1=High, 2=Medium, 3=Low; LTP=Left Truncation Point

\section{ATTRIBUTES}

PLAY

ATTRIBUTES

CHARGE (C)

$\operatorname{TRAP}(\mathrm{T})$

TIMING (F)

Probability that play contains at least 1 reservoir $>=$ minimum size (CXTXF)

PROSPECT

ATTRIBUTES

CHARGE (c)

$\operatorname{TRAP}(\mathrm{t})$

TIMING (f)

Probability that a randomly chosen prospect is favorable (cxtxf)

Play Attributes x Prospect Attributes (CxTxFxcxtxf)

FRACTION OF ACCUMULATIONS BEING OIL

Fraction NA Gas=1-Fraction(Oil)

\section{PROBABILITY OF FAVORABLE}

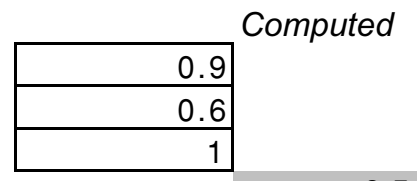

0.54

0.1

0.0108

0.9

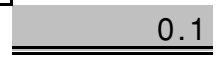

Allocation (percent):

\begin{tabular}{|l|r|r|r|}
\cline { 2 - 4 } \multicolumn{1}{c|}{} & \multicolumn{1}{c|}{ Land } & \multicolumn{1}{c|}{ Oil } & \multicolumn{2}{c|}{ Gas } \\
\hline Federal & 75 & 70 & 70 \\
\hline State & 24 & 30 & 30 \\
\hline Native & 1 & 0 & 0 \\
\hline
\end{tabular}

Assessor's Name:

Phil Nelson

Date of Data Entry MM/DD/YYYY:

Date of Simulation Run MM/DD/YYYY:

$10 / 23 / 01$

$2 / 6 / 02$ 
Table 29d: Distribution of fitted hydrocarbon volume attributes, trap depth, and number of prosects for the Ellesmerian Endicott North Play
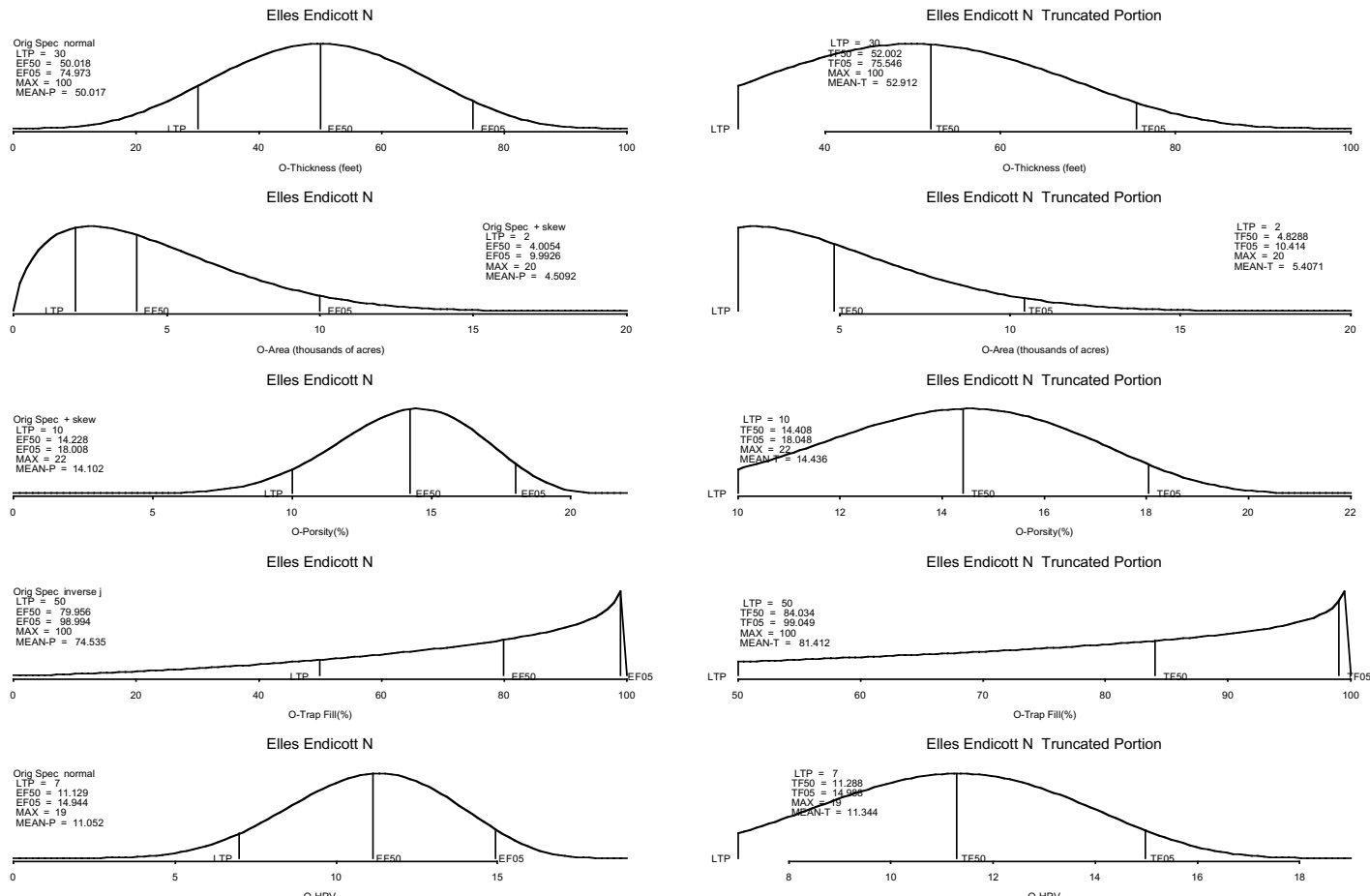

Elles Endicott N
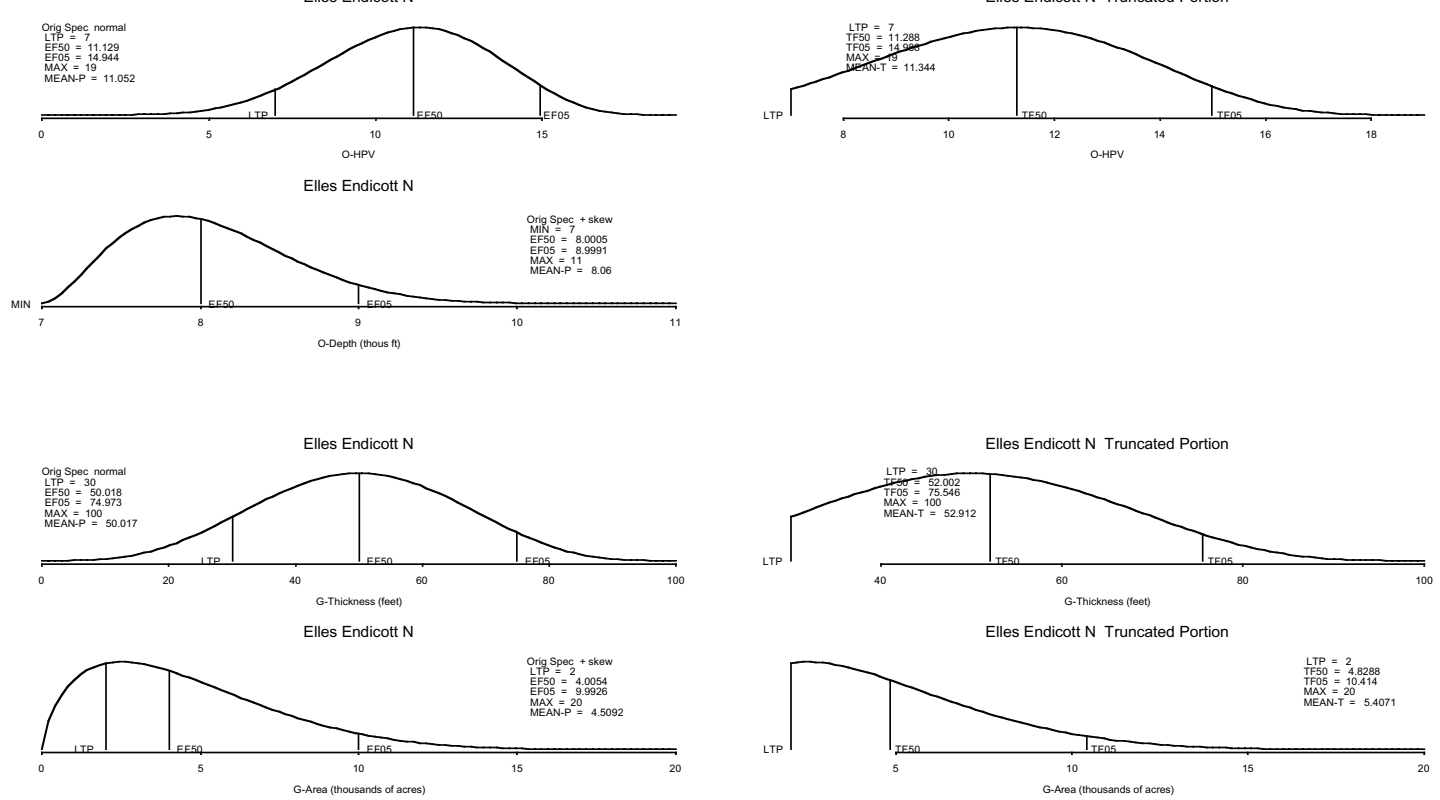

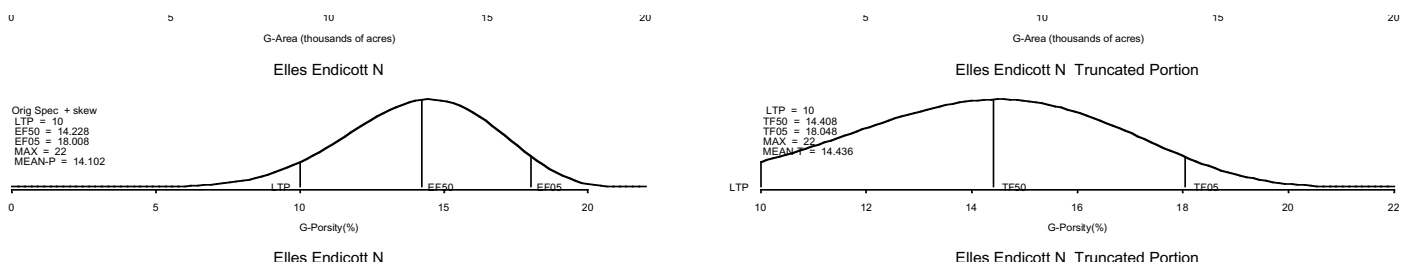

In place pre-risked accumulation
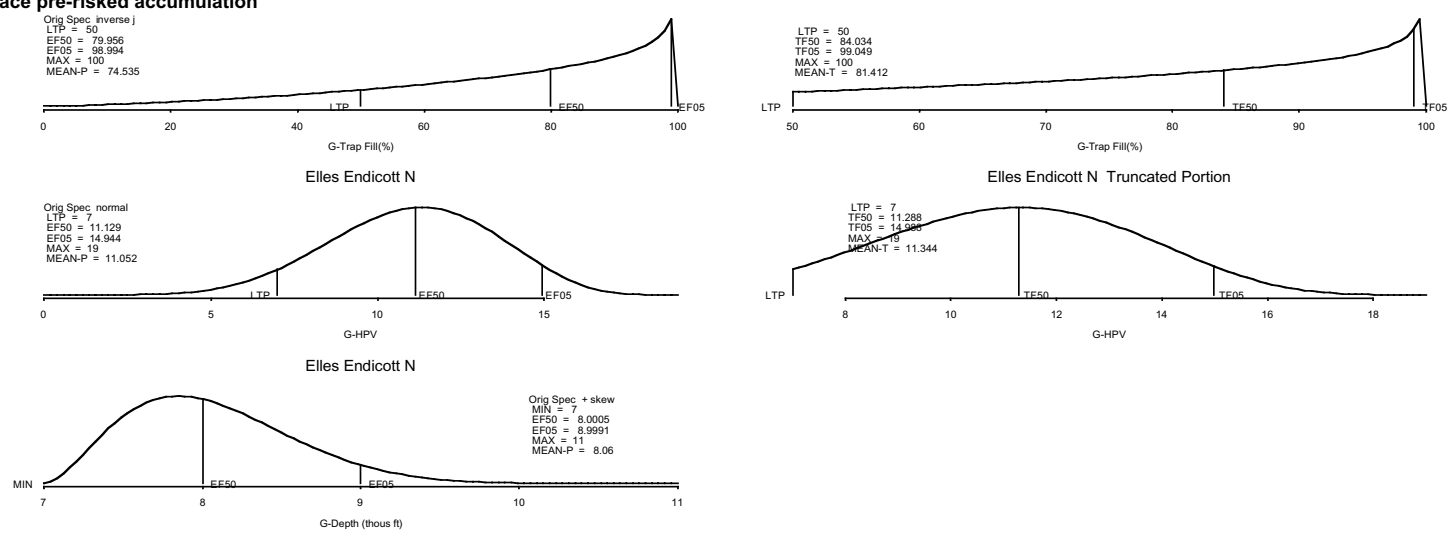

Elles Endicott N

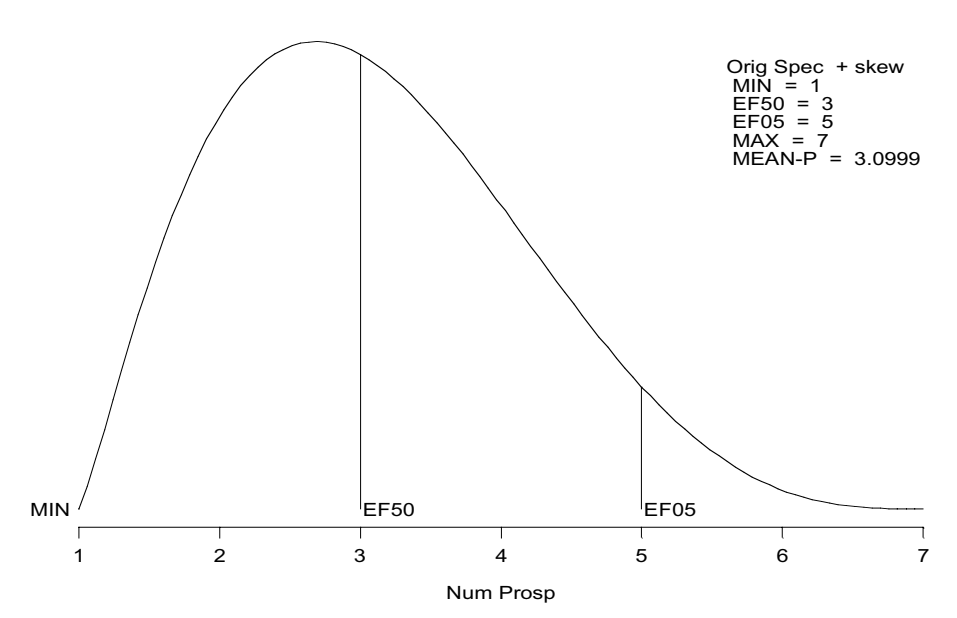


Table 29e: Estimated undiscovered oil and gas resources for the Ellesmerian Endicott North Play Deposit

Ellesmerian Endicott $\mathrm{N}$

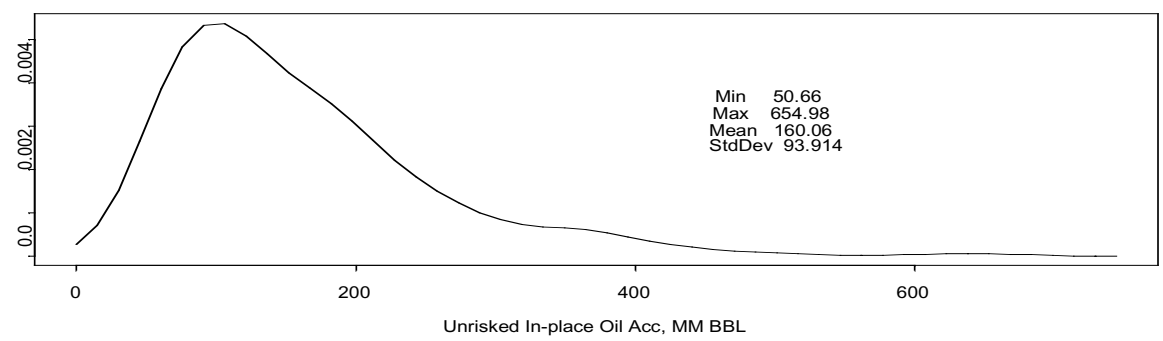

Ellesmerian Endicott $\mathrm{N}$

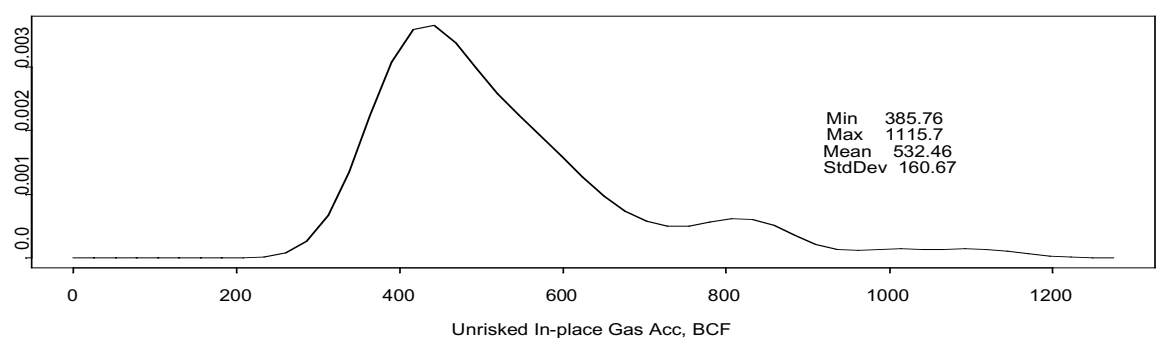

Play Stats
\begin{tabular}{|ll|rrrrr|} 
Resource & Unit & Mean & StdDev & F95 & F50 & F05 \\
\hline In-place oil & MMBO & 5.00 & 32.99 & 0.00 & 0.00 & 0.00 \\
In-place NA gas & BCFG & 1.73 & 31.59 & 0.00 & 0.00 & 0.00 \\
Recov oil & MMBO & 2.50 & 16.50 & 0.00 & 0.00 & 0.00 \\
Recov assoc diss gas & BCFG & 1.59 & 10.49 & 0.00 & 0.00 & 0.00 \\
Recov NA gas & BCFG & 1.12 & 20.53 & 0.00 & 0.00 & 0.00 \\
Recov NGL (ADG) & MMBO & 0.05 & 0.30 & 0.00 & 0.00 & 0.00 \\
Recov NGL (NAG) & MMBO & 0.02 & 0.30 & 0.00 & 0.00 & 0.00 \\
Num oil deposits & & 0.03 & 0.18 & 0.00 & 0.00 & 0.00 \\
Num NA gas deposits & & 0.00 & 0.06 & 0.00 & 0.00 & 0.00 \\
\hline RNS=121 & & & & & & \\
\hline
\end{tabular}

Play Totals Ellesmerian Endicott $\mathrm{N}$

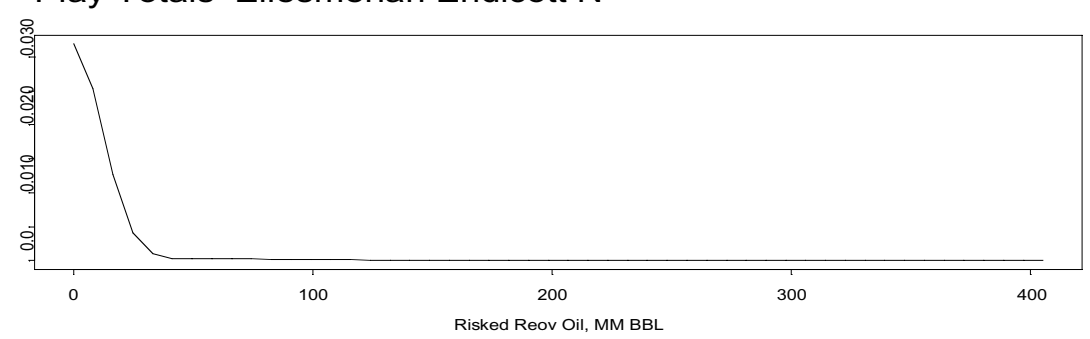

Play Totals Ellesmerian Endicott $\mathrm{N}$

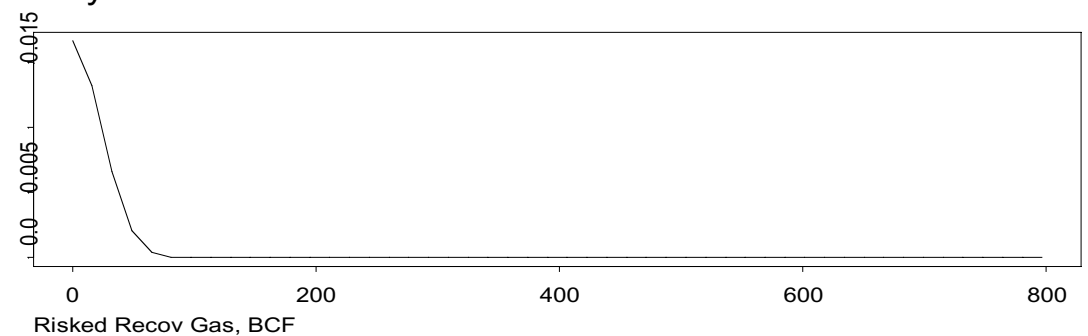


Table 29f: Estimated undiscovered oil and gas resources for the Ellesmerian Endicott North Play

showing number of deposits and volumes by accumulation size class

\begin{tabular}{|rrrrrr|} 
Start size c & Oil deposits & Oil (MMBO_ Start size class & Gas deposits & NA Gas (BCFG) \\
0 & 0.00 & 0.00 & 0 & 0.00 & 0.00 \\
8 & 0.00 & 0.00 & 48 & 0.00 & 0.00 \\
16 & 0.00 & 0.07 & 96 & 0.00 & 0.00 \\
32 & 0.01 & 0.58 & 192 & 0.00 & 0.74 \\
64 & 0.01 & 1.11 & 384 & 0.00 & 0.38 \\
128 & 0.00 & 0.69 & 768 & & \\
256 & 0.00 & 0.05 & 1536 & & \\
512 & 0.03 & 2.50 & 3072 & & \\
\hline Totals & & & & 0.00 & 1.12 \\
\hline
\end{tabular}
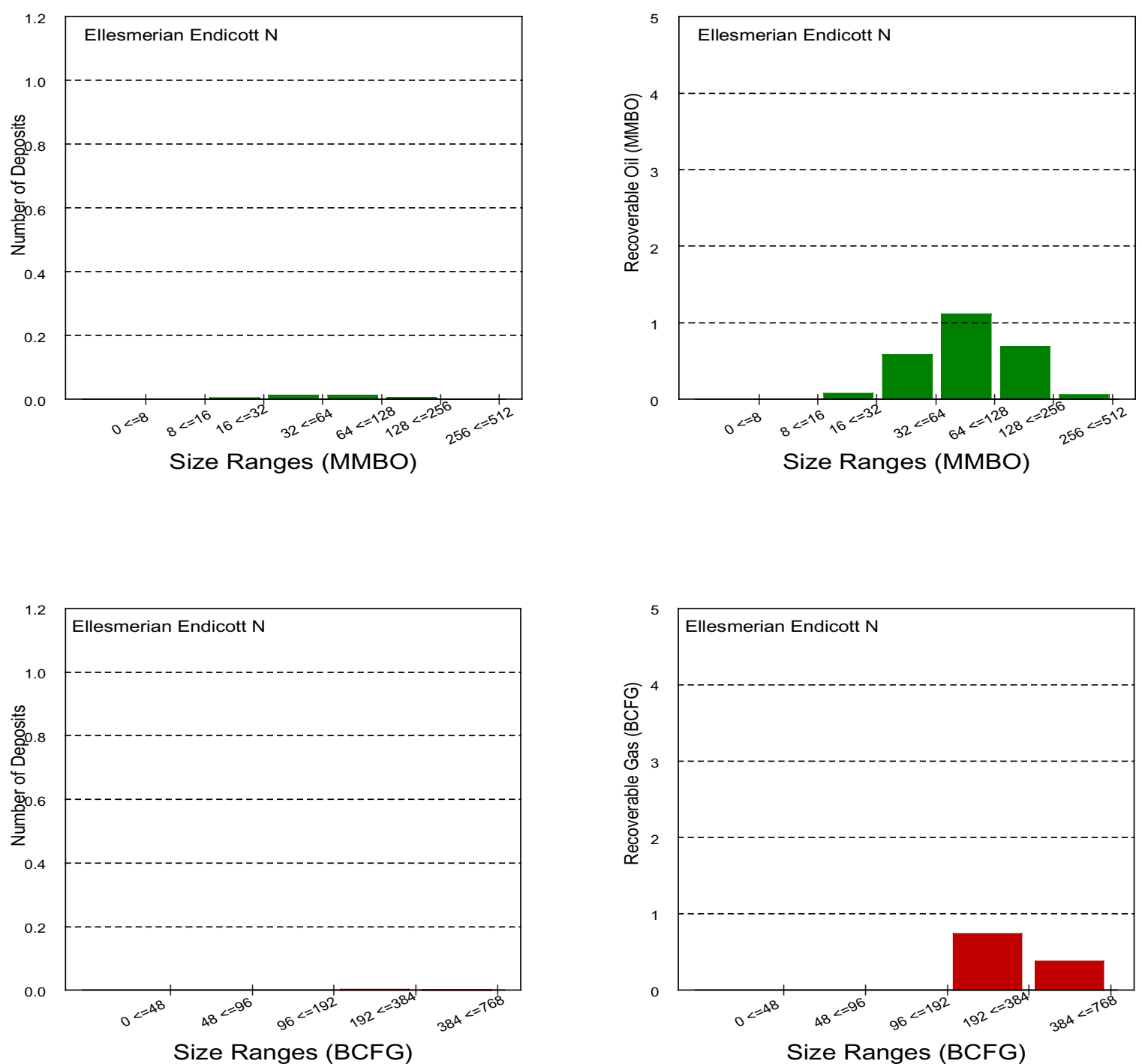
Table 30a: Input values for oil accumulations in the Ellesmerian Endicott South Play

NPRA Assessment Form-2001

PLAY: Ellesmerian Endicott South

Play area:

$10^{3}$ Acres

\section{OIL ACCUMULATION VOLUME PARAMETERS}

\begin{tabular}{|c|c|c|c|c|c|c|c|}
\hline \multirow[b]{2}{*}{ ATTRIBUTES } & \multirow{2}{*}{$\begin{array}{l}\text { Est Shape } \\
(1 \text { to } 5)\end{array}$} & \multicolumn{4}{|c|}{ PROB OF AND GREATER THAN } & \multirow{2}{*}{$\begin{array}{l}\text { Knowledge } \\
\text { Level } 1-3^{5}\end{array}$} & \multirow[b]{6}{*}{ Enter POR ${ }^{\star} \mathrm{Sy}$} \\
\hline & & LTP & 0.50 & 0.05 & Max & & \\
\hline \multicolumn{8}{|l|}{ NET RESERVOIR THICKNESS ${ }^{1}$} \\
\hline \multicolumn{7}{|l|}{ AREA OF CLOSURE ${ }^{2}$} & \\
\hline POROSITY, & & & & & & & \\
\hline TRAP FILL $^{3}$ & & & & & & & \\
\hline HYDROCARBON PORE VOL ${ }^{3,4}$ & & 0 & 0 & 0 & 0 & & \\
\hline Approx mm bbl $(f v f=1)$ & & 0.0 & 0.0 & 0.0 & 0.0 & & \\
\hline
\end{tabular}

1-thickness in feet, 2-thousands of acres, 3-percent, 4-correlation between Porosity and Water Saturation $=-1.0$

5-Knowledge Level: 1=High, 2=Medium, 3=Low; LTP=Left Truncation Point

TRAP DEPTH (in $1000 \mathrm{ft}$ )

(from sea level)

\section{OIL ACCUMULATION CHARACTERISTICS}

Oil recovery factor \%

Type of reservoir-drive (check any that apply):

$$
\text { Water: }
$$

Depletion: $\mathrm{x}$

Gas expansion:

FVF (Formation volume factor, rb/stb):

GOR (Associated gas to oil ratio, cu.ft./bbl, at stp):

NGLR (Natural gas liquids to associated gas ratio, bbls/million cu.ft., at stp):

Oil quality parameters:

API gravity

Sulfur content of oil

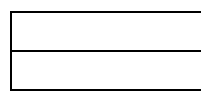

Associated gas quality parameters:

Hydrogen sulfide \%

CO2 contamination \%

Other inert gases:

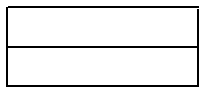

Name:

Name:

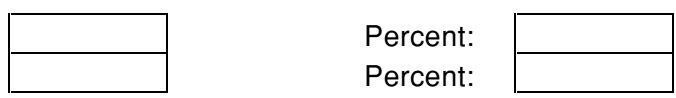

TIME OF TRAP DEVELOPMENT

STRATIGRAPHIC COMPONENT (Ma)

STRUCTURAL COMPONENT (Ma)

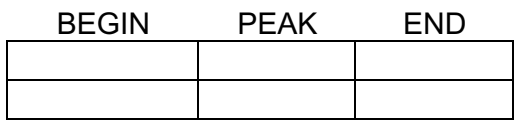

Assessor's Name: Ken Bird \& Phil Nelson

Date of Data Entry MM/DD/YYYY:

Date of Simulation Run MM/DD/YYYY:

$10 / 23 / 01$

$2 / 6 / 02$

Note: only enter play name and assessor's name on Oil worksheet 
Table 30b: Input values for nonassociated gas accumulations in the Ellesmerian Endicott South Play NPRA Assessment Form-2001

PLAY: Ellesmerian Endicott South Play area: $\quad 6,993 \mid 10^{3}$ Acres

\section{NONASSOCIATED GAS ACCUMULATION VOLUME PARAMETERS}

\begin{tabular}{|c|c|c|c|c|c|c|c|c|}
\hline \multirow[b]{2}{*}{ ATTRIBUTES } & \multirow{2}{*}{$\begin{array}{l}\text { Est Shape } \\
(1 \text { to } 5)\end{array}$} & \multicolumn{4}{|c|}{ PROB OF AND GREATER THAN } & \multirow{2}{*}{$\begin{array}{l}\text { Knowledge } \\
\text { Level } 1-3^{5}\end{array}$} & & \multirow[b]{3}{*}{ Corr(nrt,ac) } \\
\hline & & LTP & 50 & 5 & Max & & & \\
\hline \multirow{4}{*}{$\begin{array}{l}\text { NET RESERVOIR THICKNESS } \\
\text { AREA OF CLOSURE }^{2} \\
\text { POROSITY }^{3,4} \\
\text { TRAP FILL }^{3}\end{array}$} & 2 & 50 & 100 & 175 & 350 & 3 & 0.86 & \\
\hline & 2 & 2.0 & 3.5 & 40.0 & 50.0 & 2 & \multirow{3}{*}{$\mathrm{POR}^{\star} \mathrm{Sw}$} & \\
\hline & 4 & 10 & 14 & 18 & 20 & 3 & & \\
\hline & 3 & 35 & 50 & 70 & 100 & 3 & & \\
\hline \multicolumn{2}{|l|}{ HYDROCARBON PORE VOL ${ }^{3,4}$} & 4 & 8 & 12 & 14 & & 6 & \\
\hline \multirow{2}{*}{\multicolumn{2}{|c|}{$\begin{array}{l}\text { Approx in place bcf } \\
\text { Recov bcf at surface }\end{array}$}} & 0.06 & 0.61 & 25.61 & 106.72 & & & \\
\hline & & $\begin{array}{r}12.0 \\
\end{array}$ & 120.2 & 5049.5 & 21039.7 & & & \\
\hline \multicolumn{9}{|c|}{ 1-thickness in feet, 2-thousands of acres, 3-percent, 4-correlation between Porosity and Water Saturation $=-1.0$} \\
\hline \multicolumn{7}{|c|}{ 5-Knowledge Level: 1=High, 2=Medium, 3=Low; LTP=Left Truncation Point } & & \\
\hline \multirow{2}{*}{$\begin{array}{l}\text { TRAP DEPTH (in } 1000 \mathrm{ft} \text { ) } \\
\text { (from sea level) }\end{array}$} & 4 & 10 & 20 & 22 & 25 & 1 & & \\
\hline & & Surface to s & evel corre & (1000 ft): & 0.23 & & & \\
\hline
\end{tabular}

\section{NONASSOCIATED GAS ACCUMULATION CHARACTERISTICS}

NA Gas recovery factor \% 65

Type of reservoir-drive (check any that apply):

$$
\text { Water: Gas expansion: }
$$

Natural gas liquids plus condensate to non-associated gas (bbls/million cf) (in place): NGL-NAG $=1.785^{\star} \mathrm{TD}$ 35.7 (at median)

Non-associated gas quality parameters: Hydrogen sulfide \% CO2 contamination \% Other inert gases:

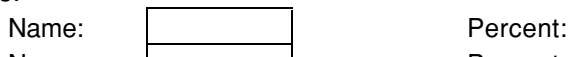

$$
\text { Name: } \quad \text { Percent: }
$$

Gas fvf 303.3 Gas $f v f=752.2^{*}\left(1-\operatorname{EXP}\left(-0.05728^{*} \mathrm{TD}\right)\right.$ $113.3+21.1^{*} T D-0.812^{*} T^{\wedge} 2+0.0116^{*} T^{\wedge} 3 \quad 5.67<T D<=30$

$$
\begin{array}{ll}
\text { (at median depth) } & 113.3+21.1^{*} \mathrm{TD}-0.812^{*} \mathrm{~T} \\
& \text { TD }=\text { trap depth (thous } \mathrm{ft} \text { ) }
\end{array}
$$

TIME OF TRAP DEVELOPMENT STRATIGRAPHIC COMPONENT (Ma) STRUCTURAL COMPONENT (Ma)

\begin{tabular}{|r|r|r|}
\multicolumn{1}{c}{ BEGIN } & \multicolumn{1}{c}{ PEAK } & \multicolumn{1}{c|}{ END } \\
\hline 360 & 355 & 350 \\
\hline 360 & 300 & 100 \\
\hline
\end{tabular}

Assessor's Name: Ken Bird \& Phil Nelson Date of Data Entry MM/DD/YYYY: Date of Simulation Run MM/DD/YYYY: 
Table 30c: Input risking values for the Ellesmerian Endicott South Play

NPRA Assessment Form-2001

Play: Ellesmerian Endicott South

\section{RISKING}

MINIMUM ACCUMULATION SIZE, MAS (bcf recov)

PRERISKED FREQUENCY DISTRIBUTION (Oil plus Gas)

NUM OF PROSPECTS

$>$ MINIMUM SIZE

\section{PROB OF AND GREATER THAN}

\begin{tabular}{|r|r|r|r|r|r|}
\hline Est Shape & Min & 50 & 5 & Max & Knowledge \\
\hline 2 & 30 & 50 & 80 & 90 & 2 \\
\hline
\end{tabular}

5-Knowledge Level: 1=High, 2=Medium, 3=Low; LTP=Left Truncation Point

\section{ATTRIBUTES}

PLAY

ATTRIBUTES

CHARGE (C)

$\operatorname{TRAP}(\mathrm{T})$

TIMING (F)

Probability that play contains at least 1 reservoir $>=$ minimum size (CXTXF)

PROSPECT

ATTRIBUTES

CHARGE (c)

TRAP ( $\mathrm{t}$ )

TIMING (f)

Probability that a randomly chosen prospect is favorable (cxtxf)

Play Attributes x Prospect Attributes (CxTxFxcxtxf)

FRACTION OF ACCUMULATIONS BEING OIL

Fraction NA Gas=1-Fraction(Oil)

\section{PROBABILITY OF FAVORABLE}

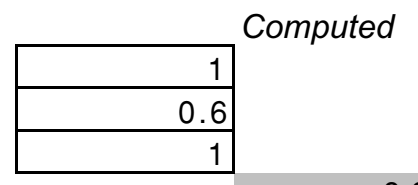

0.6

Allocation (percent):

\begin{tabular}{|l|r|r|r|}
\cline { 2 - 4 } \multicolumn{1}{c|}{} & \multicolumn{1}{c|}{ Land } & Oil & \multicolumn{1}{c|}{ Gas } \\
\hline Federal & 95 & & 95 \\
\hline State & 4 & & 4 \\
\hline Native & 1 & & 1 \\
\hline
\end{tabular}

Assessor's Name:

Ken Bird \& Phil Nelson

Date of Data Entry MM/DD/YYYY:

Date of Simulation Run MM/DD/YYYY:

\begin{tabular}{|r|}
\hline 0.3 \\
\hline 0.1 \\
\hline 0.9 \\
\hline
\end{tabular}

0.9

0

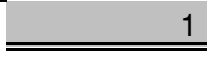


Table 30d: Distribution of fitted hydrocarbon volume attributes, trap depth, and number of prospects for the Ellesmerian Endicott South Play
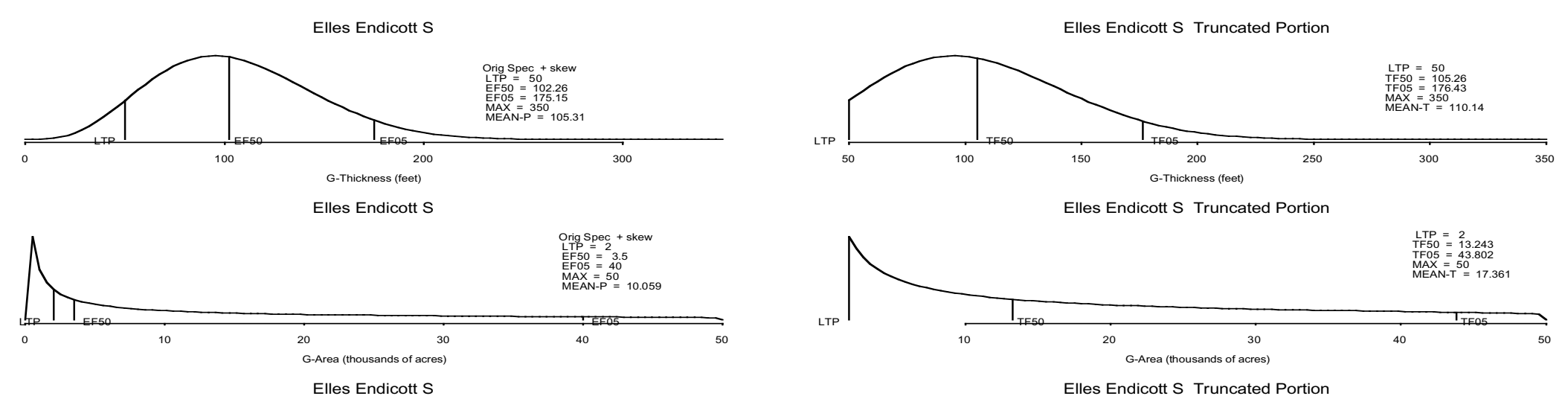

Elles Endicott S Truncated Portion
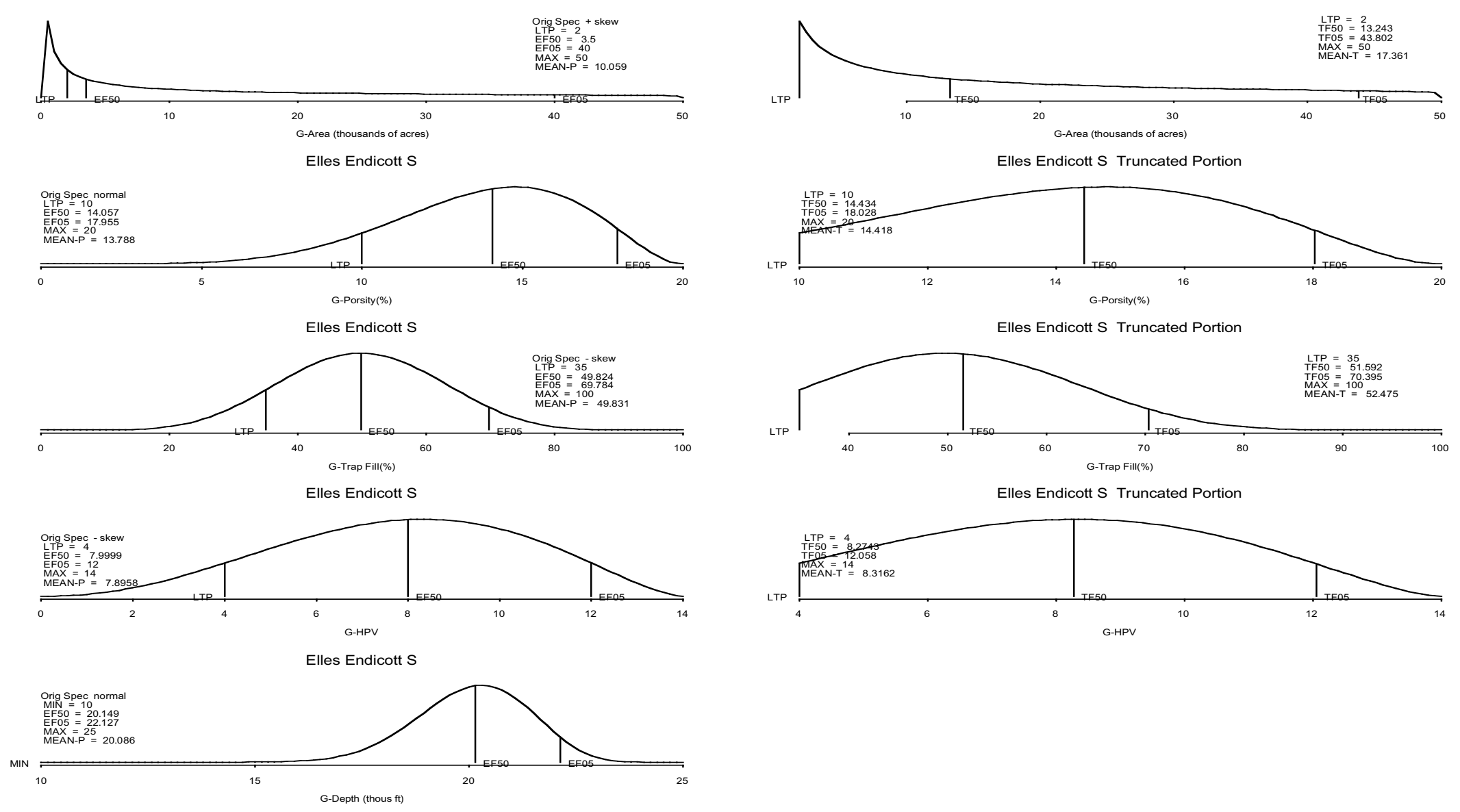
Elles Endicott S

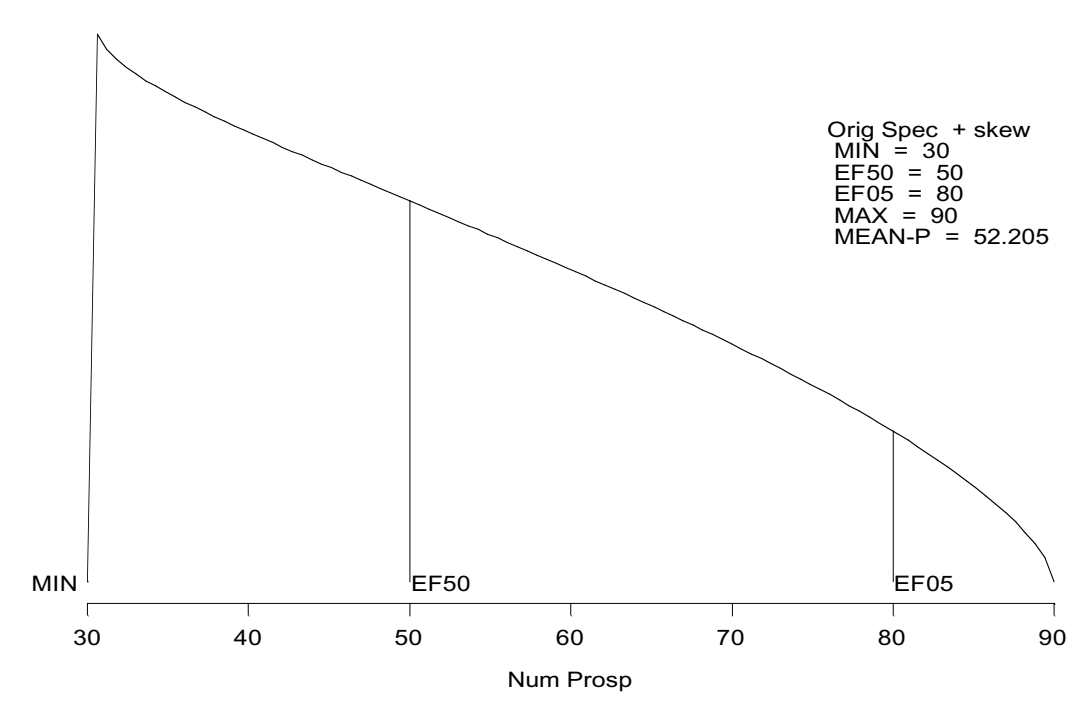


Table 30e: Estimated undiscovered oil and gas resources for the Ellesmerian Endicott South Play Deposit

\section{Ellesmerian Endicott S}

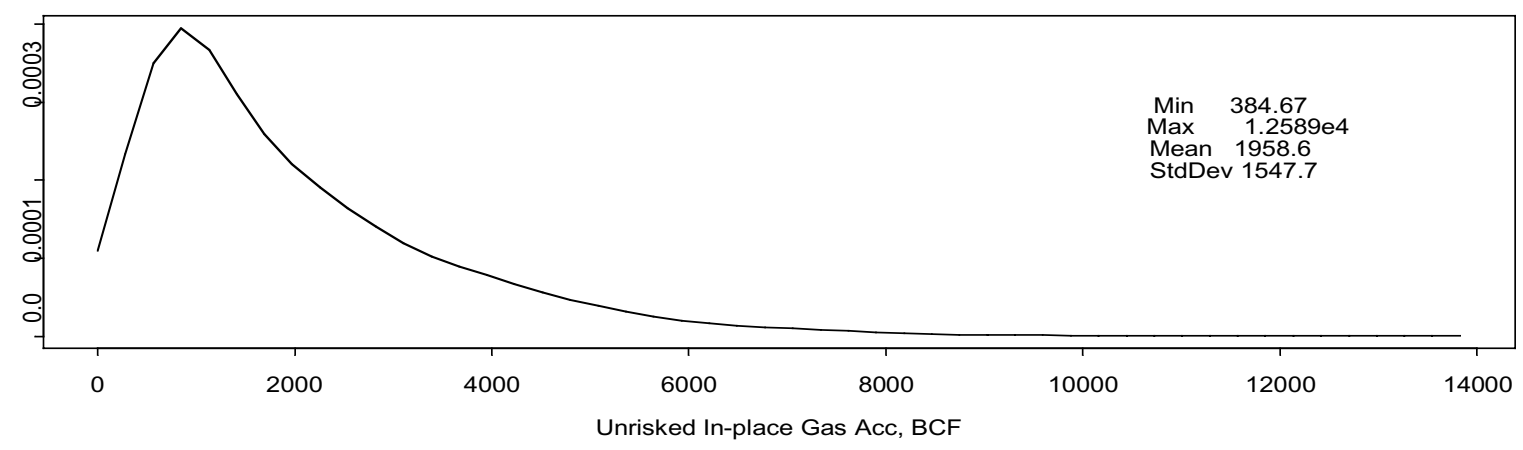

\begin{tabular}{|c|c|c|c|c|c|c|}
\hline \multicolumn{7}{|l|}{ Play Stats } \\
\hline Resource & Unit & Mean & StdDev & F95 & F50 & F05 \\
\hline In-place oil & MMBO & & & & & \\
\hline In-place NA gas & BCFG & 1649.41 & 2722.31 & 0.00 & 0.00 & 7409.01 \\
\hline Recov oil & MMBO & & & & & \\
\hline Recov assoc diss gas & BCFG & & & & & \\
\hline Recov NA gas & BCFG & 1072.12 & 1769.50 & 0.00 & 0.00 & 4815.86 \\
\hline Recov NGL (ADG) & MMBO & & & & & \\
\hline Recov NGL (NAG) & MMBO & 38.93 & 64.31 & 0.00 & 0.00 & 174.05 \\
\hline Num oil deposits & & & & & & \\
\hline Num NA gas deposits & & 0.84 & 1.18 & 0.00 & 0.00 & 3.00 \\
\hline
\end{tabular}

RNS $=588$

\section{Play Totals Ellesmerian Endicott S}

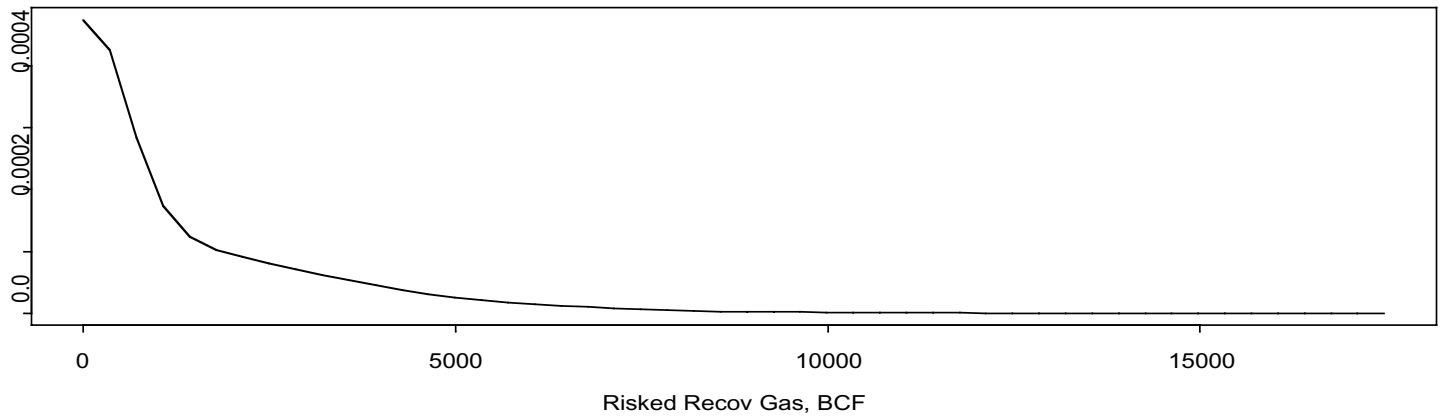


Table 30f: Estimated undiscovered oil and gas resources for the Ellesmerian Endicott South Play showing number of deposits and volumes by accumulation size class

\begin{tabular}{rrrrrr} 
Start size class & Oil deposits & Oil (MMBO_Start size class & Gas deposits & NA Gas (BCFG) \\
0 & & 0 & 0.00 & 0.00 \\
8 & & 48 & 0.00 & 0.00 \\
16 & & 96 & 0.00 & 0.00 \\
32 & & 192 & 0.12 & 38.56 \\
64 & & 384 & 0.22 & 125.66 \\
128 & & 768 & 0.24 & 269.27 \\
256 & & 1536 & 0.20 & 422.89 \\
512 & & 3072 & 0.05 & 206.94 \\
& & 6144 & 0.00 & 8.88 \\
\hline Totals & 0.00 & 0.00 & & 0.84 & 1072.21 \\
\hline
\end{tabular}
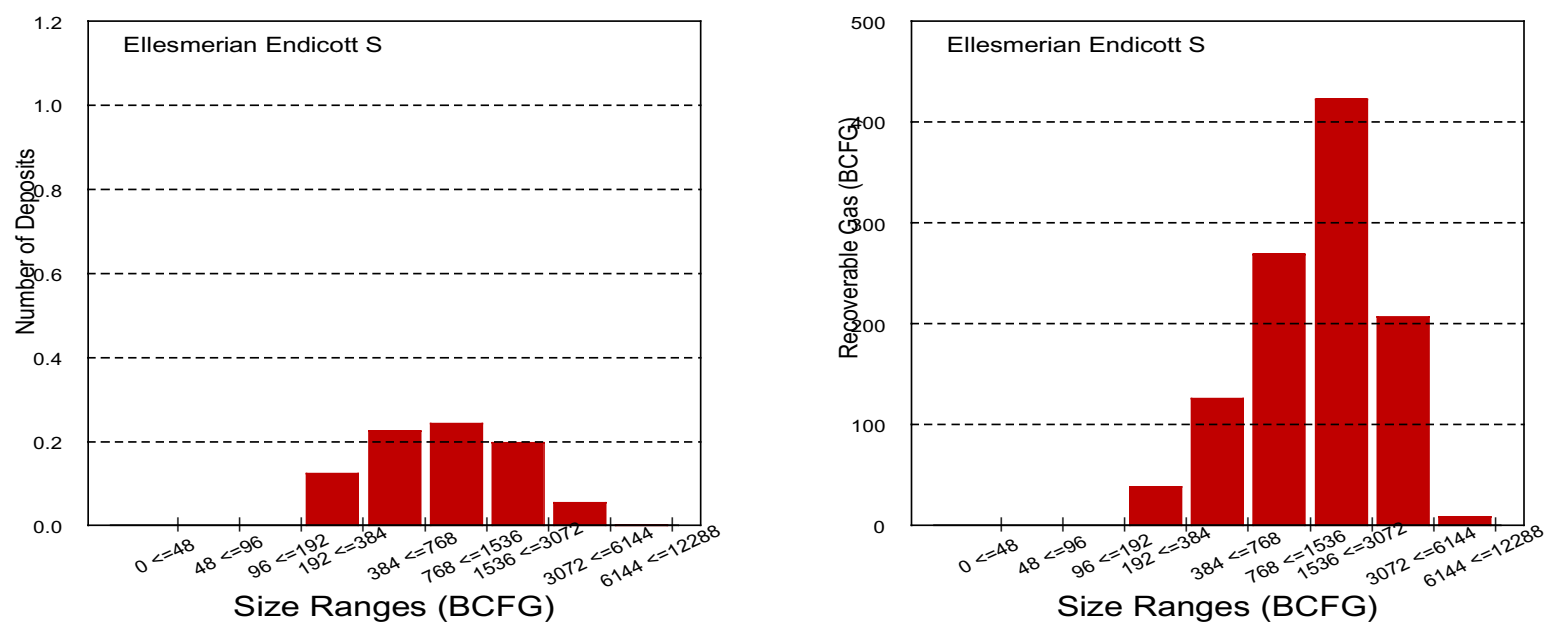


\begin{tabular}{|c|c|c|c|c|c|c|c|c|c|}
\hline \multirow[b]{2}{*}{ Play } & \multicolumn{2}{|c|}{ Oil Mean } & \multicolumn{2}{|c|}{ NA Gas Mean } & \multicolumn{2}{|c|}{ ADG Mean } & \multicolumn{2}{|c|}{ NGL(adg) Mean } & \multirow{2}{*}{$\frac{\text { NGL(na }}{\text { Total }}$} \\
\hline & Total & Federal & Total & Federal & Total & Federal & Total & Federal & \\
\hline Brookian Topset & 683.1 & 587.4 & 295.7 & 272.1 & 431.3 & 370.9 & 8.3 & 7.1 & 4.0 \\
\hline Brookian Clinoform North & $3,732.2$ & 2985.8 & $1,037.0$ & 850.3 & $3,178.1$ & 2542.5 & 57.5 & 46.0 & 13.3 \\
\hline Brookian Clinoform Central & $2,779.1$ & 2723.5 & $8,315.3$ & 7816.4 & $3,515.5$ & 3445.2 & 74.5 & 73.0 & 181.9 \\
\hline Brookian Clinoform South-Shallow & $1,692.0$ & 1692.0 & $3,699.4$ & 3699.4 & $1,207.0$ & 1207.0 & 26.9 & 26.9 & 73.6 \\
\hline Brookian Clinoform South-Deep & & & $5,827.8$ & 5827.8 & & & & & 171.4 \\
\hline Beaufortian Cretaceous Topset North & 294.5 & 220.8 & 577.9 & 450.7 & 226.3 & 169.7 & 5.5 & 4.1 & 8.5 \\
\hline Beaufortian Cretaceous Topset South & & & $3,042.4$ & 2859.9 & & & & & 67.4 \\
\hline Beaufortian Upper Jurassic Topset Northeast & $10,352.1$ & 9523.9 & & & $12,626.0$ & 11615.9 & 227.8 & 209.6 & \\
\hline Beaufortian Upper Jurassic Topset Southeast & & & $7,903.3$ & 7903.3 & & & & & 190.0 \\
\hline Beaufortian Upper Jurassic Topset Northwest & $3,718.8$ & 2789.1 & & & $4,506.1$ & 3379.5 & 81.1 & 60.8 & \\
\hline Beaufortian Upper Jurassic Topset Southwest & & & $8,030.0$ & 7467.9 & & & & & 193.1 \\
\hline Beaufortian Lower Jurassic Topset & 275.6 & 223.3 & $1,219.9$ & 975.9 & 8.4 & 6.8 & 3.2 & 2.6 & 21.3 \\
\hline Beaufortian Clinoform & 33.5 & 21.8 & $1,264.6$ & 1188.7 & 42.7 & 27.7 & 0.8 & 0.5 & 29.0 \\
\hline Brookian Topset Structural & 391.6 & 387.7 & $17,676.1$ & 17499.4 & 171.1 & 169.4 & 3.9 & 3.9 & 196.6 \\
\hline Torok Structural & 115.6 & 114.4 & $29,842.5$ & 29544.0 & 62.3 & 61.7 & 1.6 & 1.6 & 439.5 \\
\hline Ellesmerian Structural & & & $3,316.9$ & 3283.7 & & & & & 130.3 \\
\hline Thrust Belt & 19.1 & 18.9 & $2,534.5$ & 2509.2 & 13.3 & 13.1 & 0.3 & 0.3 & 82.1 \\
\hline Ellesmerian Ivishak & 210.3 & 191.4 & 162.5 & 147.8 & 140.0 & 127.4 & 5.1 & 4.6 & 2.9 \\
\hline Ellesmerian Echooka North & 17.2 & 14.5 & 11.2 & 9.4 & 11.9 & 10.0 & 0.4 & 0.3 & 0.2 \\
\hline Ellesmerian Echooka South & & & 777.2 & 738.3 & & & & & 21.5 \\
\hline Ellesmerian Lisburne North & 95.7 & 86.1 & 33.5 & 30.2 & 71.5 & 64.4 & 2.5 & 2.3 & 0.6 \\
\hline Ellesmerian Lisburne South & & & 993.8 & 964.0 & & & & & 28.7 \\
\hline Ellesmerian Endicott North & 5.0 & 3.5 & 1.7 & 1.2 & 3.2 & 2.2 & 0.1 & 0.1 & 0.0 \\
\hline Ellesmerian Endicott South & & & $1,649.4$ & 1566.9 & & & & & 59.9 \\
\hline Sum & $24,415.5$ & $21,584.2$ & $98,212.5$ & $95,606.5$ & $26,214.6$ & $23,213.5$ & 499.4 & 443.7 & $1,915.8$ \\
\hline
\end{tabular}




\begin{tabular}{|c|c|c|c|c|c|c|c|c|c|}
\hline \multirow[b]{2}{*}{ Play } & \multicolumn{2}{|c|}{ Oil Mean } & \multicolumn{2}{|c|}{ NA Gas Mean } & \multicolumn{2}{|c|}{ ADG Mean } & \multicolumn{2}{|c|}{ NGL(adg) Mean } & \multirow{2}{*}{$\begin{array}{l}\text { NGL(nag } \\
\text { Total }\end{array}$} \\
\hline & Total & Federal & Total & Federal & Total & Federal & Total & Federal & \\
\hline Brookian Topset & 239.1 & 205.6 & 192.2 & 176.8 & 150.9 & 129.8 & 2.9 & 2.5 & 2.6 \\
\hline Brookian Clinoform North & $1,306.3$ & 1045.0 & 674.0 & 552.7 & $1,112.3$ & 889.9 & 20.1 & 16.1 & 8.6 \\
\hline Brookian Clinoform Central & 972.7 & 953.2 & $5,404.9$ & 5080.6 & $1,230.4$ & $1,205.8$ & 26.1 & 25.5 & 118.3 \\
\hline Brookian Clinoform South-Shallow & 507.6 & 507.6 & $2,404.6$ & 2404.6 & 362.1 & 362.1 & 8.1 & 8.1 & 47.9 \\
\hline Brookian Clinoform South-Deep & 0.0 & 0.0 & $3,788.1$ & 3788.1 & 0.0 & 0.0 & 0.0 & 0.0 & 111.4 \\
\hline Beaufortian Cretaceous Topset North & 103.1 & 77.3 & 404.5 & 315.5 & 79.2 & 59.4 & 1.9 & 1.4 & 5.9 \\
\hline Beaufortian Cretaceous Topset South & 0.0 & 0.0 & $2,129.7$ & 2001.9 & 0.0 & 0.0 & 0.0 & 0.0 & 47.2 \\
\hline Beaufortian Upper Jurassic Topset Northeast & $5,176.1$ & 4762.0 & 0.0 & 0.0 & $6,313.0$ & $5,808.0$ & 113.9 & 104.8 & 0.0 \\
\hline Beaufortian Upper Jurassic Topset Southeast & 0.0 & 0.0 & $5,137.1$ & 5137.1 & 0.0 & 0.0 & 0.0 & 0.0 & 123.5 \\
\hline Beaufortian Upper Jurassic Topset Northwest & $1,859.4$ & 1394.6 & 0.0 & 0.0 & $2,253.0$ & $1,689.8$ & 40.5 & 30.4 & 0.0 \\
\hline Beaufortian Upper Jurassic Topset Southwest & 0.0 & 0.0 & $5,219.5$ & 4854.1 & 0.0 & 0.0 & 0.0 & 0.0 & 125.5 \\
\hline Beaufortian Lower Jurassic Topset & 82.7 & 67.0 & 792.9 & 634.3 & 2.5 & 2.0 & 1.0 & 0.8 & 13.9 \\
\hline Beaufortian Clinoform & 11.7 & 7.6 & 822.0 & 772.7 & 14.9 & 9.7 & 0.3 & 0.2 & 18.8 \\
\hline Brookian Topset Structural & 137.1 & 135.7 & $10,605.7$ & 10499.6 & 59.9 & 59.3 & 1.4 & 1.4 & 117.9 \\
\hline Torok Structural & 34.7 & 34.3 & $17,905.5$ & 17726.4 & 18.7 & 18.5 & 0.5 & 0.5 & 263.7 \\
\hline Ellesmerian Structural & 0.0 & 0.0 & $1,990.1$ & 1970.2 & 0.0 & 0.0 & 0.0 & 0.0 & 78.2 \\
\hline Thrust Belt & 5.7 & 5.7 & $1,520.7$ & 1505.5 & 4.0 & 3.9 & 0.1 & 0.1 & 49.3 \\
\hline Ellesmerian Ivishak & 84.1 & 76.6 & 105.6 & 96.1 & 56.0 & 51.0 & 2.0 & 1.9 & 1.9 \\
\hline Ellesmerian Echooka North & 6.9 & 5.8 & 7.3 & 6.1 & 4.8 & 4.0 & 0.2 & 0.1 & 0.1 \\
\hline Ellesmerian Echooka South & 0.0 & 0.0 & 505.2 & 479.9 & 0.0 & 0.0 & 0.0 & 0.0 & 14.0 \\
\hline Ellesmerian Lisburne North & 28.7 & 25.8 & 21.8 & 19.6 & 21.5 & 19.3 & 0.8 & 0.7 & 0.4 \\
\hline Ellesmerian Lisburne South & 0.0 & 0.0 & 646.0 & 626.6 & 0.0 & 0.0 & 0.0 & 0.0 & 18.6 \\
\hline Ellesmerian Endicott North & 2.5 & 1.8 & 1.1 & 0.8 & 1.6 & 1.1 & 0.1 & 0.0 & 0.0 \\
\hline Ellesmerian Endicott South & 0.0 & 0.0 & $1,072.1$ & 1018.5 & 0.0 & 0.0 & 0.0 & 0.0 & 38.9 \\
\hline Sum & $10,558.3$ & $9,305.5$ & $61,350.7$ & $59,668.0$ & $11,684.9$ & $10,313.6$ & 219.7 & 194.4 & $1,206.7$ \\
\hline
\end{tabular}


Table 33. Aggregate summary of in-place and technically recoverable resource estimates of the NPRA.

Oil in total assessment area

Oil in total Federal land

Non-associated gas in total assessment area

Non-associated gas in Federal land

NGL from ADG in total assessment area

NGL from ADG in Federal land

ADG in total assessment area

$A D G$ in Federal land
Volume of

In-place resources

\begin{tabular}{|c|c|c|c|c|c|c|c|}
\hline \multicolumn{4}{|c|}{ In-place resources } & \multicolumn{4}{|c|}{ recoverable resources } \\
\hline & \multicolumn{3}{|c|}{ Fractiles } & & \multicolumn{3}{|c|}{ Fractiles } \\
\hline Means & 95 & 50 & 5 & Means & 95 & 50 & 5 \\
\hline 24,416 & 15,594 & 24,020 & 34,509 & 10,558 & 6,673 & 10,388 & 15,007 \\
\hline 21,584 & 13,756 & 21,226 & 30,611 & 9,306 & 5,873 & 9,154 & 13,235 \\
\hline 98,213 & 64,103 & 96,880 & 137,304 & 61,351 & 40,372 & 60,559 & 85,317 \\
\hline 95,607 & 62,115 & 94,271 & 134,023 & 59,668 & 39,071 & 58,880 & 83,208 \\
\hline & & & & 1,426 & & & \\
\hline & & & & 1,367 & & & \\
\hline & & & & 11,685 & & & \\
\hline & & & & 10,314 & & & \\
\hline
\end{tabular}

